\title{
Multiple Absolute Stereocontrol in Cascade Lactone Formation via Dynamic Kinetic Resolution Driven by the Asymmetric Transfer Hydrogenation of Keto Acids with Oxo-Tethered Ruthenium Catalysts
}

Taichiro Touge*, ${ }^{[a]}$ Kazuhiko Sakaguchi, ${ }^{[a]}$ Nao Tamaki, ${ }^{[a]}$ Hideki Nara, ${ }^{[a]}$ Tohru Yokozawa, ${ }^{[a]}$ Kazuhiko Matsumura, ${ }^{[a]}$ and Yoshihito Kayaki* ${ }^{[b]}$

${ }^{\text {[a] }}$ Corporate Research \& Development Division, Takasago International Corporation, 1-4-11 Nishi-yawata, Hiratsuka City, Kanagawa 254-0073, Japan

[b]

Department of Chemical Science and Engineering, School of Materials and Chemical Technology, Tokyo Institute of Technology, 2-12-1-E4-1 O-okayama, Meguro-ku, Tokyo 152-8552, Japan

ykayaki@o.cc.titech.ac.jp

taichiro_touge@takasago.com 


\section{Contents}

$\begin{array}{ll}\text { General Information } & \text { S2 }\end{array}$

A. Ancillary Data for Optimization of Reaction Conditions S3

B. Ancillary Data for ATH-DKR/Lactonization $\quad \mathbf{S 1 2}$

C. Synthesis of Ketone Substrates $\quad$ S13

D. Procedures for Catalytic Reactions $\quad$ S29

E. Characterization Data for Products $\quad \mathbf{S 3 0}$

F. Synthesis of $18 \mathrm{c}$ and $19 \mathrm{c}$

G. Synthesis of Wine Lactone (6) S50

H. Synthesis of $(R, R)$-TIPPs-DENEB $((R, R)-5) \quad S 55$

I. Deterimination of Absolute Configurateion of Products S57

$\begin{array}{ll}\text { J. References } & \mathbf{S 7 4}\end{array}$

K. NMR Spectra for Ketones and Reduction Products $\quad \mathbf{S 7 5}$

L. Chiral HPLC Charts for Reduction Products $\quad$ S173

M. HR-MS Spectra for Substrates and Products $\quad$ S211

\section{$\underline{\text { General Information }}$}

All reactions and manipulations were conducted under a nitrogen atmosphere unless otherwise noted. All reactions were performed in commercial anhydrous solvents. NMR Spectra were obtained on Agilent 400-MR DD2 and Bruker BioSpin Avance III 500 Systems. NMR chemical shifts are reported in ppm relative to $\mathrm{CHCl}_{3}\left(7.26 \mathrm{ppm}\right.$ for ${ }^{1} \mathrm{H}$ and $77.0 \mathrm{ppm}$ for $\left.{ }^{13} \mathrm{C}\right), \mathrm{CH}_{3} \mathrm{OH}$ (3.30 ppm for ${ }^{1} \mathrm{H}$ and $49.0 \mathrm{ppm}$ for ${ }^{13} \mathrm{C}$ ), or DMSO (2.49 ppm for ${ }^{1} \mathrm{H}$ and $39.51 \mathrm{ppm}$ for ${ }^{13} \mathrm{C}$ ). The following abbreviations were used to designate peak splitting patterns: $\mathrm{s}=$ singlet, $\mathrm{d}=$ doublet, $\mathrm{t}=$ triplet, $\mathrm{q}=$ quartet, $\mathrm{sep}=$ septet, $\mathrm{dd}=\mathrm{a}$ doublet of doublets, $\mathrm{ddd}=\mathrm{a}$ doublet of doublets of doublets, $\mathrm{m}=$ multiplet, $\mathrm{br}=$ broad. Optical rotations were obtained on a JASCO P-1020 polarimeter. Mass spectra were recorded on Bruker Impact II and JEOL JMS-T100GCV spectrometers. High performance liquid chromatography (HPLC) analysis was performed using a system comprised of a GL-Science GL-7400 series; a column oven (GL-7430), a gradient unit, a pump, degasser (GL-7430), a UV detector (GL-7450), and an auto sampler (GL-7420).

Ketones $(\mathbf{8 a}, \mathbf{8 b})$ were purchased from Sigma-Aldrich. 
A. Ancillary Data for Optimization of Reaction Conditions.

(1) Asymmetric Transfer Hydrogenation of 2-(2-Oxo-1,2,3,4-tetrahydronaphthalen-1yl)-2-phenylacetic Acid (14d).

Table S1. Amine and Solvent Effects on the Tandem ATH/Lactonization. ${ }^{[a]}$
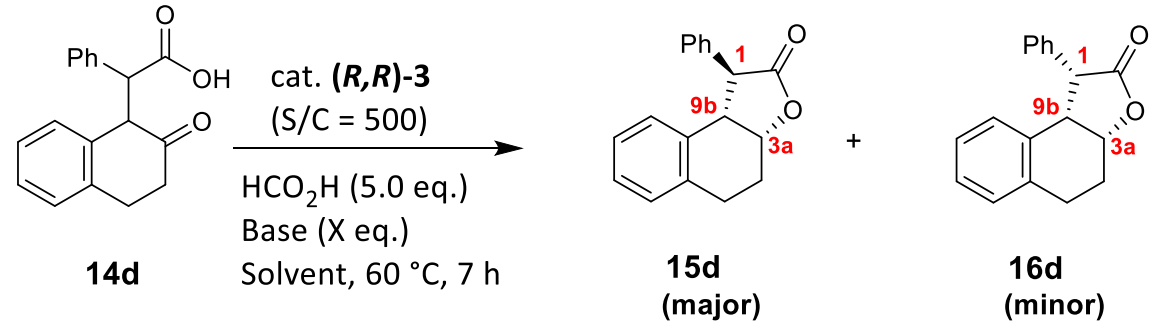

\begin{tabular}{|c|c|c|c|c|c|}
\hline Entry & $\begin{array}{c}\text { Base } \\
(X \text { equiv) }\end{array}$ & Solvent & Yield (\%) $^{[\mathrm{b}]}$ & D.r. (\%) $)^{[\mathrm{c}] \mathrm{d}]}$ & Ee (\%) ${ }^{[\mathrm{e}]}$ \\
\hline 1 & $\mathrm{Et}_{3} \mathrm{~N}(2)$ & none & $>90$ & $98.2 / 1.8$ & 92.2 \\
2 & $\mathrm{Et}_{3} \mathrm{~N}(2)$ & none & $>90$ & $93.1 / 6.9$ & 93.9 \\
3 & $\mathrm{DABCO}(3)$ & $\mathrm{CH}_{3} \mathrm{CN}$ & 80 & $95.0 / 5.0$ & 93.9 \\
4 & $\mathrm{DBU}(3)$ & $\mathrm{CH}_{3} \mathrm{CN}$ & 70 & $97.9 / 2.1$ & 94.9 \\
5 & $\mathrm{DBU}(3)$ & $\mathrm{DMF}$ & 78 & $96.3 / 3.7$ & 98.0 \\
6 & $\mathrm{DBU}(3)$ & EtOAC & $\mathbf{8 0}$ & $96.5 / 3.5$ & 98.1 \\
\hline
\end{tabular}

[a] Typical reaction conditions: substrate $(1.0 \mathrm{mmol})$, catalyst $(0.002 \mathrm{mmol})$, and solvent $(1.0 \mathrm{~mL})$ [b] Isolated yield. [c] Relative stereochemistry of the 3a- and 9b-positions in all products was determined to be cis (>99\% de). [d] D.r. ratios of 15 and 16 were determined by ${ }^{1} \mathrm{H}-\mathrm{NMR}$ analysis of the crude products. [e] E.e. values based on the ratio of $9 \mathrm{~b}-(S)$ and $-(R)$ were determined by HPLC analysis using a Chiralpak AD-H column. 
(2) Diastereoselective Hydrogenation of $(3 \mathrm{a} S, 9 \mathrm{~b} R)-3-M e t h y l e n e-3 \mathrm{a}, 4,5,9 \mathrm{~b}-$ tetrahydronaphtho[1,2-b]furan-2(3H)-one (20).

Table S2. Catalyst Screening Experiments for the Stereoselective Hydrogenation of 20. ${ }^{\text {[a] }}$

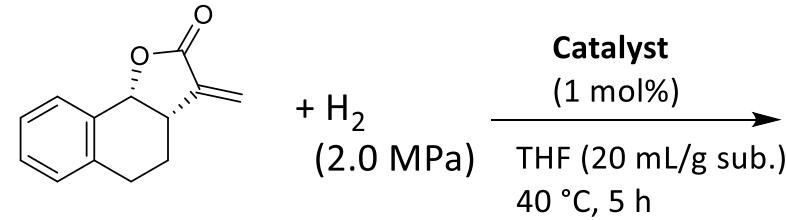

20 $40{ }^{\circ} \mathrm{C}, 5 \mathrm{~h}$

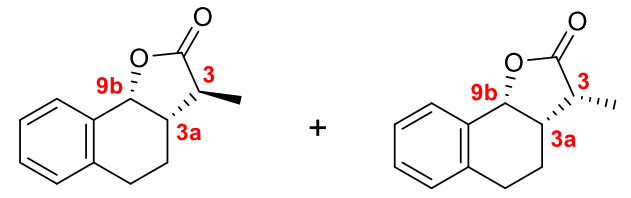

$18 \mathrm{c}(3 S)$

$19 \mathrm{c}(3 R)$

\begin{tabular}{|c|c|c|c|}
\hline Entry & Catalyst & Yield $(\%)^{[b]}$ & $\begin{array}{l}\text { D.r. }(\%)^{[c]} \\
(18 c / 19 c)\end{array}$ \\
\hline 1 & {$\left[\mathrm{NH}_{2} \mathrm{Me}_{2}\right]\left[\{\mathrm{RuCl}((S) \text {-segphos })\}_{2}(\mu-\mathrm{Cl})_{3}\right]$} & 100 & $0 / 100$ \\
\hline 2 & {$\left[\mathrm{NH}_{2} \mathrm{Me}_{2}\right]\left[\{\mathrm{RuCl}((R) \text {-segphos })\}_{2}(\mu-\mathrm{Cl})_{3}\right]$} & 88 & $34 / 66$ \\
\hline 3 & $\mathrm{Ru}(\mathrm{OAc})_{2}[(R)$-segphos $\left.)\right]$ & 75 & $15 / 85$ \\
\hline 4 & $\mathrm{Ru}(\mathrm{OAc})_{2}[(R)$-binap $\left.)\right]$ & 29 & $4 / 96$ \\
\hline 5 & $\mathrm{Ru}(\mathrm{OAc})_{2}[(R)$-xyl-binap $\left.)\right]$ & 86 & $3 / 97$ \\
\hline 6 & $\mathrm{Ru}(\mathrm{OAc})_{2}[(R)-\mathrm{dtbm}$-segphos $\left.)\right]$ & 97 & $2 / 98$ \\
\hline 7 & $\mathrm{RuCl}(p$-cymene $)[(R)$-dtbm-segphos $)] \mathrm{Cl}$ & 54 & $1 / 99$ \\
\hline 8 & $\mathrm{RuCl}(p$-cymene $)\left[(R)-\mathrm{H}_{8}\right.$-binap $\left.)\right] \mathrm{Cl}$ & 98 & $64 / 36$ \\
\hline 9 & $\mathrm{Ru}(\mathrm{OAc})_{2}\left[(R)-\mathrm{H}_{8}\right.$-binap $\left.)\right]$ & 98 & $53 / 47$ \\
\hline 10 & {$\left[\mathrm{NH}_{2} \mathrm{Me}_{2}\right]\left[\left\{\mathrm{RuCl}\left((R)-\mathrm{H}_{8}-\text { binap }\right)\right\}_{2}(\mu-\mathrm{Cl})_{3}\right]$} & 98 & $71 / 29$ \\
\hline
\end{tabular}

[a] Typical reaction conditions: substrate $(0.2 \mathrm{mmol})$, catalyst $(0.002 \mathrm{mmol})$, and solvent $(1.0 \mathrm{~mL})$. [b] Determined by GC internal standard method. [c] Determined by GC area normalization method. 
Figure S1. Structure of Ru Catalysts Employed in Table S2.

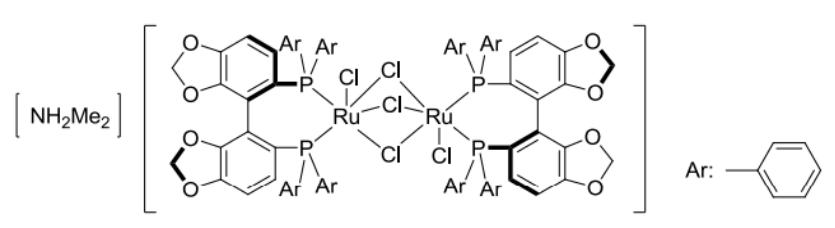

$\left[\mathrm{NH}_{2} \mathrm{Me}_{2}\right]\left[\{\mathrm{RuCl}((R)-\operatorname{segphos})\}_{2}(\mu-\mathrm{Cl})_{3}\right]$

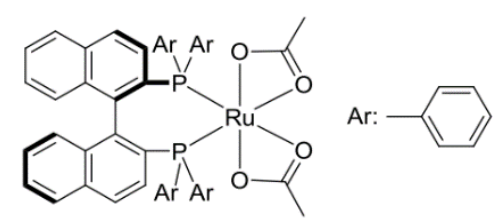

$\mathrm{Ru}(\mathrm{OAc})_{2}[(R)$-binap) $]$

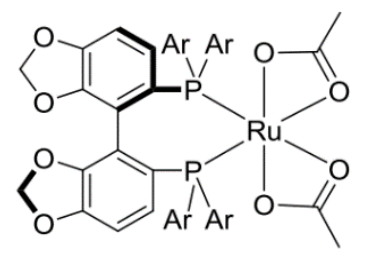

$\mathrm{Ru}(\mathrm{OAc})_{2}[(R)-\mathrm{dtbm}$-segphos $\left.)\right]$

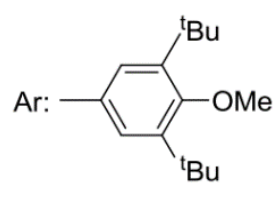

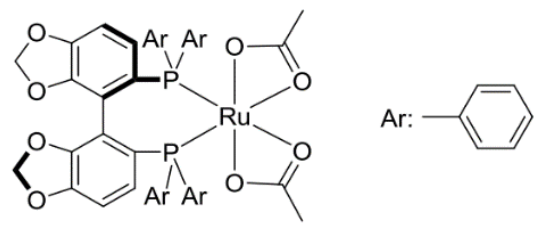

$\mathrm{Ru}(\mathrm{OAc})_{2}[(R)$-segphos $\left.)\right]$

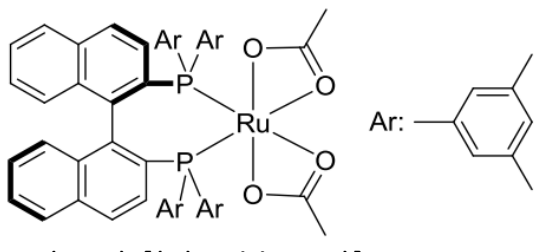

$\mathrm{Ru}(\mathrm{OAc})_{2}[(R)-x y l-$ binap $\left.)\right]$

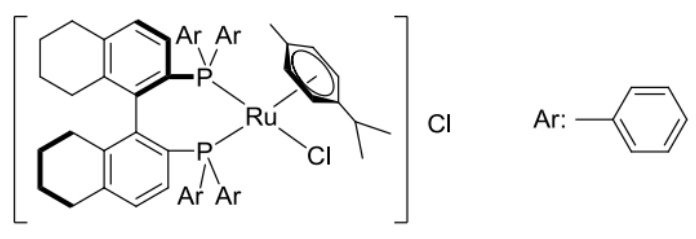

$\mathrm{RuCl}(p$-cymene $)\left[(R)-\mathrm{H}_{8}\right.$-binap $\left.)\right] \mathrm{Cl}$

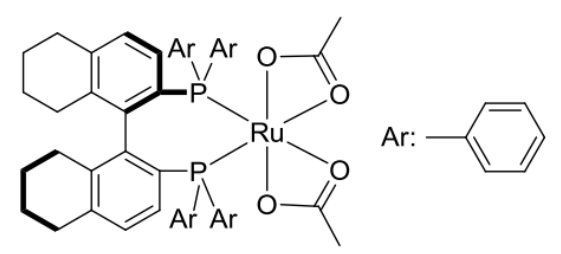

$\mathrm{Ru}(\mathrm{OAc})_{2}\left[(R)-\mathrm{H}_{8}\right.$-binap $\left.)\right]$ 
Table S3. Screening of Solvents for the Stereoselective Hydrogenation of 20. ${ }^{[\mathrm{a}]}$

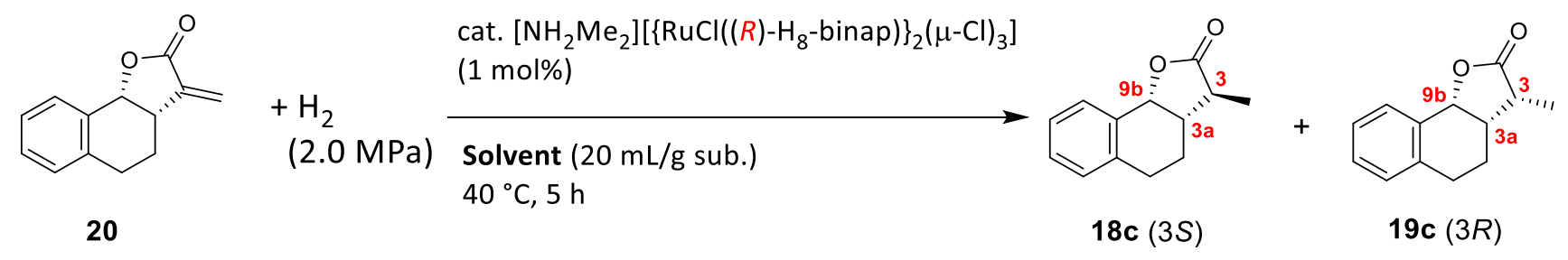

\begin{tabular}{|c|c|c|c|}
\hline Entry & Solvent & Yield $(\%)^{[b]}$ & $\begin{array}{l}\text { D.r. }(\%)^{[c]} \\
(20 c / 21 c)\end{array}$ \\
\hline 1 & THF & 98 & $71 / 29$ \\
\hline 2 & Acetone & 100 & $59 / 41$ \\
\hline 3 & EtOAc & 91 & $69 / 31$ \\
\hline 4 & 2-Me-THF & 100 & $74 / 26$ \\
\hline 5 & Toluene & 100 & $75 / 25$ \\
\hline 6 & 4-Me-THP & 99 & $77 / 23$ \\
\hline 7 & CPME & 99 & $74 / 26$ \\
\hline 8 & IPA & 100 & $62 / 38$ \\
\hline 9 & Dioxane & 100 & $83 / 17$ \\
\hline 10 & IPE & 93 & $79 / 21$ \\
\hline 11 & TBME & 87 & $79 / 21$ \\
\hline
\end{tabular}

[a] Typical reaction conditions: substrate $(0.2 \mathrm{mmol})$, catalyst $(0.002 \mathrm{mmol})$, and solvent $(1.0 \mathrm{~mL})$. [b] Determined by GC internal standard method. [c] Determined by GC area normalization method. 
Table S4. Effects of Hydrogen Pressure and Reaction Temperature on the Stereoselective Hydrogenation of 20. ${ }^{\text {[a] }}$

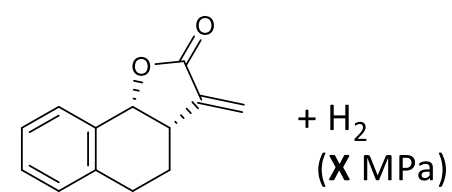

20 cat. $\left[\mathrm{NH}_{2} \mathrm{Me}_{2}\right]\left[\left\{\mathrm{RuCl}\left((R)-\mathrm{H}_{8}-\text { binap }\right)\right\}_{2}(\mu-\mathrm{Cl})_{3}\right]$

(1 mol\%)

Dioxane (20 mL/g sub.)

$\mathrm{Y}^{\circ} \mathrm{C}, 5 \mathrm{~h}$

\begin{tabular}{|c|c|c|c|c|}
\hline Entry & $\mathrm{H}_{2}(\mathrm{XMPa})$ & Temp. $\left(\mathbf{Y}^{\circ} \mathrm{C}\right)$ & Yield (\%) ${ }^{[b]}$ & $\begin{array}{l}\text { D.r. }(\%)^{[c]} \\
(18 c / 19 c)\end{array}$ \\
\hline 1 & 4.0 & 40 & 100 & $80 / 20$ \\
\hline 2 & 2.0 & 40 & 100 & $83 / 17$ \\
\hline 3 & 1.0 & 40 & 100 & $82 / 18$ \\
\hline 4 & 0.5 & 40 & 91.7 & $85 / 15$ \\
\hline 5 & 2.0 & 60 & 100 & $77 / 23$ \\
\hline 6 & 2.0 & 40 & 100 & $83 / 17$ \\
\hline 7 & 2.0 & 20 & 95.3 & $87 / 13$ \\
\hline 8 & 2.0 & 10 & 37.8 & $>90 /<10$ \\
\hline
\end{tabular}

[a] Typical reaction conditions: substrate $(0.2 \mathrm{mmol})$, catalyst $(0.002 \mathrm{mmol})$, and solvent $(1.0 \mathrm{~mL})$ [b] Determined by GC internal standard method. [c] Determined by GC area normalization method. 
(3) Asymmetric Transfer Hydrogenation of 2-(4-Methyl-2-oxocyclohex-3-en-1-yl)acetic acid (23).

Table S5. Screening of Catalysts for the ATH of 23. ${ }^{[a]}$<smiles>CC1=CC(=O)C(CC(=O)O)CC1</smiles>

23

Catalyst
$(1$ mol\% $)$
$\mathrm{HCO}_{2} \mathrm{H}$ (5 equiv)
$\mathrm{Et}_{3} \mathrm{~N}(2$ equiv)
$\mathrm{EtOAC}$

$60{ }^{\circ} \mathrm{C} 6 \mathrm{~h}$, then $80{ }^{\circ} \mathrm{C} 2 \mathrm{~h}$

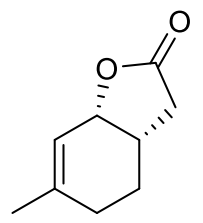

24

\begin{tabular}{|c|c|c|}
\hline Entry & Catalyst & $\begin{array}{c}\text { Ee }(\%)^{[\mathrm{b}]} \\
\text { of } \mathbf{2 4}\end{array}$ \\
\hline 1 & $(R, R)$-Ts-DENEB, $(R, R)-3$ & $55^{[\mathrm{c}]}$ \\
2 & $(R, R)$-Ms-DENEB, $(R, R)-\mathbf{4}$ & 57 \\
3 & $(R, R)-$-o-CF 3 Ps-DENEB & 55 \\
4 & $(R, R)$-TIPPs-DENEB, $(R, R)-5$ & 90 \\
5 & $\eta^{6}$-Benzene-(R,R)-Ts-DENEB & 52 \\
6 & $\eta^{6}$-Benzene-(R,R)-Ms-DENEB & 32 \\
\hline
\end{tabular}

[a] Typical reaction conditions: substrate $(1 \mathrm{mmol})$, catalyst $(0.01 \mathrm{mmol})$, and solvent $(1.0 \mathrm{~mL})$. In all cases, yields were within a range of ca. 60-70\%. [b] Determined by GC analysis. [c] Isolated yield was $70 \%$. 
Figure S2. Structure of Catalysts Employed in Table S5.

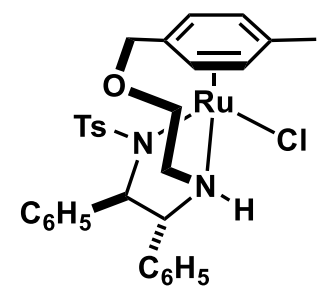

$(R, R)$-Ts-DENEB $((R, R)-3)$

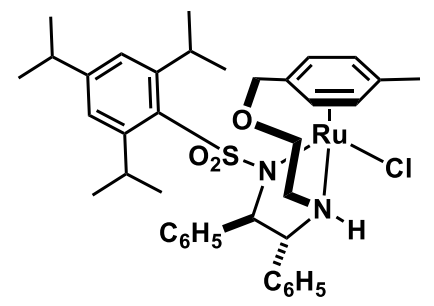

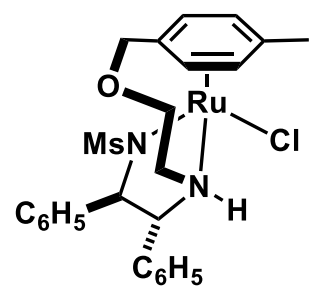

$(R, R)$-Ms-DENEB $((R, R)-4)$

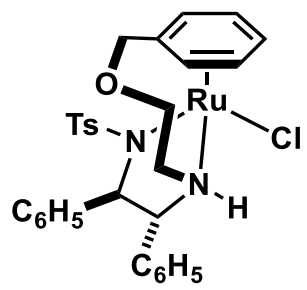

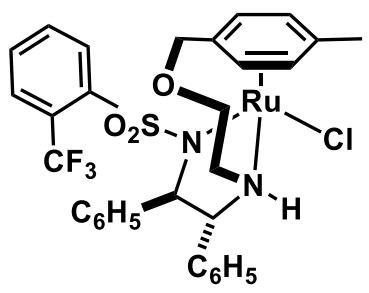

$(R, R)-0-\mathrm{CF}_{3} \mathrm{Ps}-\mathrm{DENEB}$

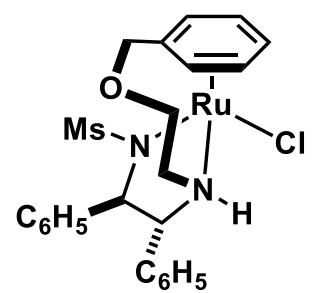

$(R, R)$-TIPPs-DENEB $((R, R)-5) \quad \eta^{6}$-Benzene-( $\left.R, R\right)$-Ts-DENEB $\quad \eta^{6}$-Benzene- $(R, R)$-Ms-DENEB 
Table S6. Screening of Solvents for the ATH of 23. ${ }^{[a]}$<smiles>CC1=CC(=O)C(CC(=O)O)CC1</smiles>

23

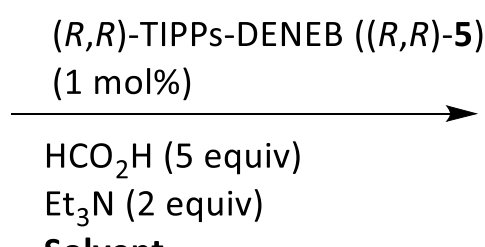

Solvent

$60{ }^{\circ} \mathrm{C}, 6 \mathrm{~h}$ then $80^{\circ} \mathrm{C}, 2 \mathrm{~h}$

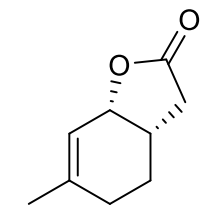

24

\begin{tabular}{|c|c|c|}
\hline Entry & Solvent & $\begin{array}{c}\text { Ee }(\%)^{[\mathrm{b}]} \\
\text { of } \mathbf{2 4}\end{array}$ \\
\hline 1 & EtOAc & $\mathbf{9 0}$ \\
2 & $\mathrm{MeOH}$ & 84 \\
3 & $\mathrm{CH}_{3} \mathrm{CN}$ & $\mathbf{9 0}^{[\mathrm{c}]}$ \\
4 & $\mathrm{DMF}$ & 85 \\
5 & $\mathrm{THF}$ & 86 \\
6 & $\mathrm{CHCl}{ }_{3}$ & 88 \\
8 & Toluene & 84 \\
9 & Dioxane & 84 \\
\hline
\end{tabular}

[a] Typical reaction conditions: substrate $(1 \mathrm{mmol})$, catalyst $(0.01 \mathrm{mmol})$, and solvent $(1.0 \mathrm{~mL})$. In all cases, yields were within a range of ca. $60-70 \%$. [b] Determined by GC analysis. [c] Isolated yield was $68 \%$. 
Table S7. Optimization of the Amounts of Base and $\mathrm{HCO}_{2} \mathrm{H}$ in the ATH of 23. ${ }^{\text {[a] }}$<smiles>CC1=CC(=O)C(CC(=O)O)CC1</smiles>

23

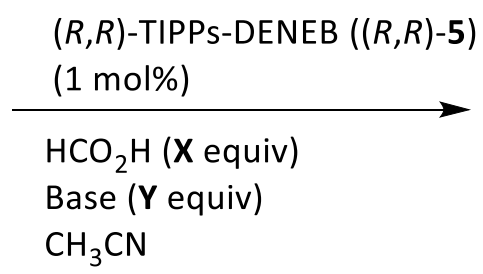

$60{ }^{\circ} \mathrm{C} 6 \mathrm{~h}$, then $80^{\circ} \mathrm{C} 2 \mathrm{~h}$

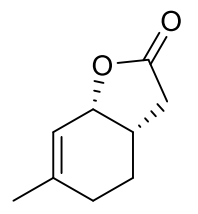

24

\begin{tabular}{|c|c|c|c|c|}
\hline Entry & Base & $\begin{array}{c}\mathrm{HCO}_{2} \mathrm{H} \\
\text { (X equiv) }\end{array}$ & $\begin{array}{c}\text { Base } \\
\text { (Y equiv) }\end{array}$ & $\begin{array}{c}\text { Ee }(\%)^{[b]} \\
\text { of } \mathbf{2 4}\end{array}$ \\
\hline 1 & $\mathrm{Et}_{3} \mathrm{~N}$ & 5 & 2 & 90 \\
\hline 2 & DABCO & 5 & 3 & $92^{[\mathrm{c}]}$ \\
\hline 3 & DABCO & 3 & 3 & 90 \\
\hline 4 & DABCO & 10 & 6 & 92 \\
\hline 5 & DBU & 5 & 3 & 91 \\
\hline 6 & DBU & 3 & 3 & 74 \\
\hline
\end{tabular}

[a] Typical reaction conditions: substrate $(1 \mathrm{mmol})$, catalyst $(0.01 \mathrm{mmol})$, and solvent $(1.0 \mathrm{~mL})$. In all cases, yields were within a range of 60-70\%. [b] Determined by GC analysis.

[c] Isolated yield was $70 \%$. 


\section{B. Ancillary Data for ATH-DKR/Lactonization.}

Table S8. Time dependence of yield, dr, and ee in the ATH-DKR/Lactonization of $\mathbf{1 4 f}$.

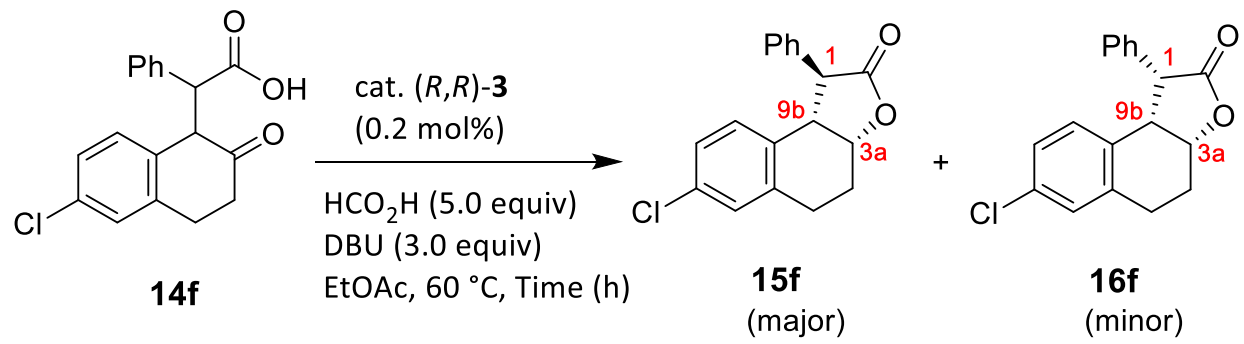

\begin{tabular}{|c|c|c|c|c|}
\hline Entry & Time (h) & Yield (\%) $^{[a]}$ & $\begin{array}{c}\text { D.r. }(\%)^{[b]} \\
(\mathbf{1 5 f} / \mathbf{1 6 f})\end{array}$ & $\begin{array}{c}\text { Ee (\%) } \\
\text { of 15f }\end{array}$ \\
\hline 1 & 2 & ca. 25 & $97 / 3$ & 94 \\
2 & 4 & ca. 60 & $97 / 3$ & 94 \\
3 & 7 & ca. 90 & $96 / 4$ & 94 \\
\hline
\end{tabular}

[a] Monitored by TLC analysis. [b] Determined by GC-MS analysis. [c] Determined by HPLC analysis (Chiralpak AD-H).

During the ATH-DKR/lactonization, the stereoselectivities were not mostly changed. These results indicate that these stereocenters were established during the lactonization and subsequent epimerization could not proceed under the reaction conditions. 


\section{Synthesis of Ketone Substrates.}

(a) Synthesis of 6-Methyl-6,7,8,9-tetrahydro-5H-benzo[7]annulen-5-one $(8 \mathrm{c})^{[1]}$<smiles>O=C1CCCCc2ccccc21</smiles>

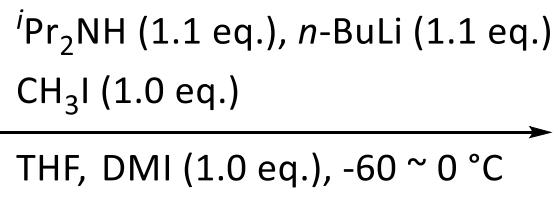

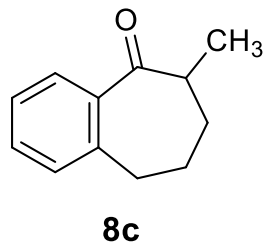

A THF solution $(1.64 \mathrm{M})$ of $n$-BuLi $(25.4 \mathrm{~mL}, 41.6 \mathrm{mmol})$ was added slowly to a THF solution $(100 \mathrm{~mL})$ containing $N, N$-diisopropylamine $(5.37 \mathrm{~g}, 41.6 \mathrm{mmol})$ at $-60{ }^{\circ} \mathrm{C}$ under $\mathrm{N}_{2}$ atmosphere and stirred for $1 \mathrm{~h}$. The resulting mixture was warmed to $-20{ }^{\circ} \mathrm{C}$, then $6,7,8,9$ tetrahydro-5H-benzo[7]annulen-5-one $(6.06 \mathrm{~g}, 37.8 \mathrm{mmol})$ was added and stirred for $1 \mathrm{~h}$ at $0{ }^{\circ} \mathrm{C}$. Subsequently, iodomethane $(5.37 \mathrm{~g}, 37.8 \mathrm{mmol})$ and 1, 3-dimethyl-2-imidazolidinone were added to the reaction mixture. After stirring further for $5 \mathrm{~h}$ at $0{ }^{\circ} \mathrm{C}$, water $(50 \mathrm{~mL})$ was added and followed by extraction with $\mathrm{CHCl}_{3}(3 \times 50 \mathrm{~mL})$. The combined organic layer was washed with brine $(100 \mathrm{~mL})$, dried over anhydrous $\mathrm{MgSO}_{4}$, and concentrated under reduced pressure to afford the crude product. Purification by silica gel column chromatography (eluent: hexane/AcOEt $=20 / 1)$ gave the product $(\mathbf{8 c})$ as a colorless oil $(3.9 \mathrm{~g}, 59 \%$ yield $)$.

${ }^{1} \mathrm{H}$ NMR $\left(400 \mathrm{MHz}, \mathrm{CDCl}_{3}\right) \delta$ 7.68-7.66 (m, 1H), 7.39-7.35 (m, 1H), 7.27 (t, J= 7.6 Hz, $1 \mathrm{H}), 7.20(\mathrm{~d}, J=7.6 \mathrm{~Hz}, 1 \mathrm{H}), 3.04-2.89(\mathrm{~m}, 3 \mathrm{H}), 2.10-2.04(\mathrm{~m}, 1 \mathrm{H}), 1.95-1.87(\mathrm{~m}, 1 \mathrm{H})$, 1.74-1.57 (m, 2H), $1.22(\mathrm{~d}, J=6.8 \mathrm{~Hz}, 3 \mathrm{H}) ;{ }^{13} \mathrm{C} \mathrm{NMR}\left(125 \mathrm{MHz}, \mathrm{CDCl}_{3}\right) \delta 207.8,141.8$, 139.7, 131.3, 129.7, 128.4, 126.3, 44.1, 33.6, 32.0, 25.5, 16.5 .

HRMS (ESI) calcd for $\mathrm{C}_{12} \mathrm{H}_{15} \mathrm{O}[\mathrm{M}+\mathrm{H}]^{+}:$175.1117. Found: 175.1099.

\section{(b) Synthesis of Methyl 1-oxo-1,2,3,4-tetrahydronaphthalene-2-carboxylate $(8 d)^{[2]}$}

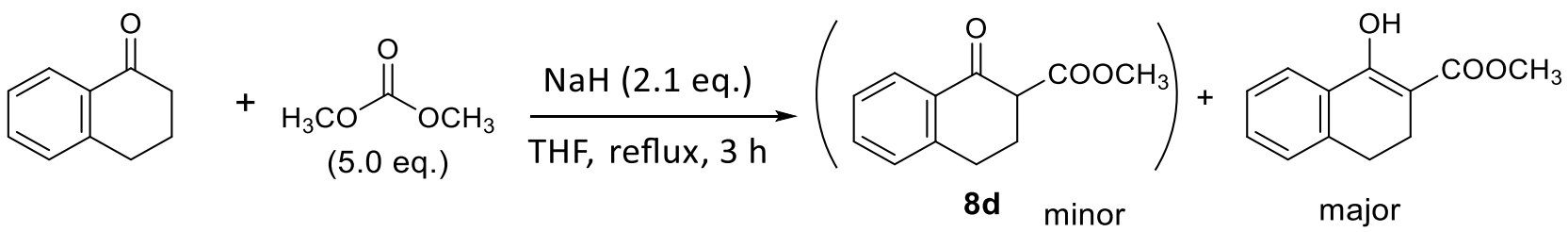

A THF solution $(120 \mathrm{~mL})$ containing $\alpha$-tetralone $(5.00 \mathrm{~g}, 34.2 \mathrm{mmol})$ and $\mathrm{NaH}(60 \%$ dispersion in mineral oil; $2.88 \mathrm{~g}, 71.8 \mathrm{mmol}$ ) was added dimethyl carbonate (14.05 g, 156.0 mmol) slowly and heated under $\mathrm{N}_{2}$ atmosphere at $65{ }^{\circ} \mathrm{C}$ for $3 \mathrm{~h}$. After cooling the reaction mixture, $1 \mathrm{~N} \mathrm{HCl}$ solution was added to neutralize the reaction mixture, followed by extraction with $\mathrm{CHCl}_{3}(100 \mathrm{~mL}$ and $2 \times 50 \mathrm{~mL})$. The combined organic layer was washed with brine $(100 \mathrm{~mL})$, dried over anhydrous $\mathrm{MgSO}_{4}$, and concentrated under reduced pressure to afford the crude product. Purification by silica gel column chromatography (eluent: 
hexane/AcOEt $=20 / 1)$ gave the product $(\mathbf{8 d})$ primarily in the enol form as a pale yellow oil (6.6 $\mathrm{g}, 95 \%$ yield).

${ }^{1} \mathrm{H}$ NMR $\left(400 \mathrm{MHz}, \mathrm{CDCl}_{3}\right) \delta 12.41(\mathrm{~s}, 1 \mathrm{H}), 7.80-7.78(\mathrm{~m}, 1 \mathrm{H}), 7.33-7.20(\mathrm{~m}, 2 \mathrm{H}), 7.17-$ $7.15(\mathrm{~m}, 1 \mathrm{H}), 3.81(\mathrm{~s}, 3 \mathrm{H}), 2.80(\mathrm{t}, J=7.4 \mathrm{~Hz}, 2 \mathrm{H}), 2.56(\mathrm{t}, J=7.4 \mathrm{~Hz}, 2 \mathrm{H}) ;{ }^{13} \mathrm{C}$ NMR $(125$ $\left.\mathrm{MHz} \mathrm{CDCl}_{3}\right) \delta 193.0,165.0,139.3,133.8,130.5,127.7,126.5,124.3,54.4,51.5,27.7,20.4$. HRMS (ESI) calcd for $\mathrm{C}_{12} \mathrm{H}_{13} \mathrm{O}_{3}[\mathrm{M}+\mathrm{H}]^{+}:$205.0859. Found: 205.0861 .

(c) Synthesis of Methyl 2-(1-oxo-1,2,3,4-tetrahydronaphthalen-2-yl)acetate (8e)<smiles>COC(=O)CC1CCc2ccccc2C1=O</smiles>

A THF solution $(1.57 \mathrm{M})$ of $n$-BuLi $(33.5 \mathrm{~mL}, 52.7 \mathrm{mmol})$ was added slowly to a THF solution $(200 \mathrm{~mL})$ containing $N, N$-diisopropylamine $(6.82 \mathrm{~g}, 52.7 \mathrm{mmol})$ at $-60{ }^{\circ} \mathrm{C}$ under $\mathrm{N}_{2}$ atmosphere and stirred for $1 \mathrm{~h}$. The resulting mixture was warmed to $-20{ }^{\circ} \mathrm{C}$, then $\alpha$-tetralone $(7.00 \mathrm{~g}, 47.9 \mathrm{mmol})$ was added and stirred for $1 \mathrm{~h}$ at $0{ }^{\circ} \mathrm{C}$. Subsequently, methyl 2bromoacetate (7.32 g, $47.9 \mathrm{mmol})$ and 1,3-dimethyl-2-imidazolidinone (5.47 g, $47.9 \mathrm{mmol})$ were added to the reaction mixture. After stirring further for $4 \mathrm{~h}$ at $0{ }^{\circ} \mathrm{C}$, saturated aqueous $\mathrm{NH}_{4} \mathrm{Cl}$ solution $(100 \mathrm{~mL})$ and $\mathrm{CHCl}_{3}(100 \mathrm{~mL})$ were added and stirred for $5 \mathrm{~min}$. The organic layer was separated and the aqueous layer was extracted with $\mathrm{CHCl}_{3}(2 \times 50 \mathrm{~mL})$. The combined organic layer was washed with brine $(100 \mathrm{~mL})$, dried over anhydrous $\mathrm{MgSO}_{4}$, and concentrated under reduced pressure to afford the crude product. Purification by silica gel column chromatography (eluent: hexane/AcOEt $=15 / 1$ to $10 / 1)$ gave the product $(\mathbf{8 e})$ as a pale orange solid ( $8.9 \mathrm{~g}, 85 \%$ yield).

${ }^{1} \mathrm{H}$ NMR $\left(500 \mathrm{MHz}, \mathrm{CDCl}_{3}\right) \delta 8.03(\mathrm{dd}, J=7.9,1.1 \mathrm{~Hz}, 1 \mathrm{H}), 7.47(\mathrm{dt}, J=7.5,1.4 \mathrm{~Hz}, 1 \mathrm{H})$, 7.32-7.28 (m, 1H), 7.25-7.23 (m, 1H), $3.73(\mathrm{~s}, 3 \mathrm{H}), 3.16-2.95(\mathrm{~m}, 4 \mathrm{H}), 2.48-2.40(\mathrm{~m}, 1 \mathrm{H})$, $2.58-2.21(\mathrm{~m}, 1 \mathrm{H}), 1.97(\mathrm{ddd}, J=21.6,17.3,4.3 \mathrm{~Hz}, 1 \mathrm{H}) ;{ }^{13} \mathrm{C} \mathrm{NMR}\left(125 \mathrm{MHz}, \mathrm{CDCl}_{3}\right) \delta$ 198.3, 172.9, 143.9, 133.4, 132.0, 128.7, 127.4, 126.6, 51.7, 44.7, 34.8, 29.19, 29.18.

HRMS (APPI) calcd for $\mathrm{C}_{13} \mathrm{H}_{15} \mathrm{O}_{3}[\mathrm{M}+\mathrm{H}]^{+}:$219.1016. Found: 219.1015. 
(d) Synthesis of 8-Bromo-7-methoxy-3,4-dihydronaphthalen-1(2H)-one<smiles>COc1ccc2c(c1)C(=O)CC[CH+]2C</smiles>

To a solution of 7-methoxy-3,4-dihydronaphthalen-1(2H)-one $(7.00 \mathrm{~g}, 39.7 \mathrm{mmol})$ in acetonitrile $(55 \mathrm{~mL})$ was added portionwise $N$-bromosuccinimide $(10.60 \mathrm{~g}, 59.55 \mathrm{mmol}, 1.5$ equiv) under stirring at rt. The reaction suspension was stirred over $10 \mathrm{~h}$. After the solvent were removed under reduced pressure, the red crude product was purified by column chromatography (Hexane:EtOAc $=10: 1$ to $3: 1)$ to yield the desired ketone as a brown solid (9.42 g, 94\% yield).

${ }^{1} \mathrm{H}$ NMR $\left(500 \mathrm{MHz}, \mathrm{CDCl}_{3}\right) \delta 7.17(\mathrm{~d}, J=8.4 \mathrm{~Hz}, 2 \mathrm{H}), 7.01(\mathrm{~d}, J=8.4 \mathrm{~Hz}, 2 \mathrm{H}), 3.91(\mathrm{~s}$, $3 \mathrm{H}), 2.91(\mathrm{t}, J=6.1 \mathrm{~Hz}, 2 \mathrm{H}), 2.69(\mathrm{t}, J=6.1 \mathrm{~Hz}, 2 \mathrm{H}), 2.11-2.05(\mathrm{~m}, 2 \mathrm{H}) ;{ }^{13} \mathrm{C} \mathrm{NMR}(125$ $\left.\mathrm{MHz}, \mathrm{CDCl}_{3}\right) \delta 197.3,155.5,138.7,132.5,128.4,115.9,111.7,56.8,40.1,30.0,22.7$. HRMS (ESI) calcd for $\mathrm{C}_{11} \mathrm{H}_{11} \mathrm{BrO}_{2} \mathrm{Na}[\mathrm{M}+\mathrm{Na}]^{+}: 276.9835$. Found: 276.9845 .

(e) Synthesis of 2-Substituted $\alpha$-Tetralones and Indanones Functionalized with Carboxylic Acids (11a-m) ${ }^{[3]}$

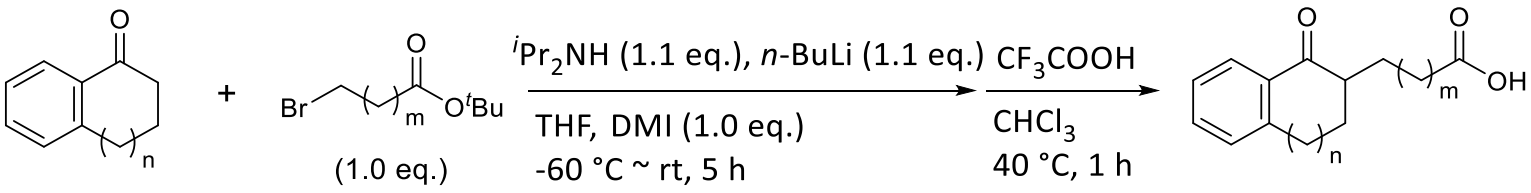

A THF solution $(1.55 \mathrm{M})$ of $n$-BuLi $(72.7 \mathrm{~mL}, 112.9 \mathrm{mmol})$ was added slowly to a THF solution $(200 \mathrm{~mL})$ containing $N, N$-diisopropylamine $(14.6 \mathrm{~g}, 112.9 \mathrm{mmol})$ at $-60{ }^{\circ} \mathrm{C}$ under $\mathrm{N}_{2}$ atmosphere and stirred for $1 \mathrm{~h}$. The resulting mixture was warmed to $-20{ }^{\circ} \mathrm{C}$, then cyclic ketone $(102.6 \mathrm{mmol})$ was added and stirred for $1 \mathrm{~h}$ at $0{ }^{\circ} \mathrm{C}$. Subsequently, alkyl bromide (102.6 mmol) and 1,3-dimethyl-2-imidazolidinone $(102.6 \mathrm{mmol})$ were added to the reaction mixture. After stirring further for $5 \mathrm{~h}$ at $0{ }^{\circ} \mathrm{C}$, the resulting mixture was concentrated by rotary evaporator. The residue was added trifluoroacetic acid $(25.7 \mathrm{~g}, 225.7 \mathrm{mmol})$ and $\mathrm{CHCl}_{3}(150$ $\mathrm{mL})$. After stirring the reaction mixture for $2 \mathrm{~h}$ at $40{ }^{\circ} \mathrm{C}$, water $(50 \mathrm{~mL})$ was added and followed by extraction with $\mathrm{CHCl}_{3}(3 \times 50 \mathrm{~mL})$. The combined organic layer was washed with brine $(100 \mathrm{~mL})$, dried over anhydrous $\mathrm{MgSO}_{4}$, and concentrated under reduced pressure to afford the crude product. Purification by silica gel column chromatography (eluent: hexane/AcOEt = 2/1) gave the product $(11 \mathrm{a}, 11 \mathrm{~b}, 11 \mathrm{c}, 11 \mathrm{~d}, 11 \mathrm{e}, 11 \mathrm{f}, 11 \mathrm{~g}, 11 \mathrm{~h}, 11 \mathbf{i}, 11 \mathbf{j}, 11 \mathrm{k}$, 111, 11m and 11n). 
<smiles>O=C(O)CC1CCc2ccccc2C1=O</smiles>

(14.7 g, 70\% yield from $102.6 \mathrm{mmol}$ of $\alpha$-tetralone, pale yellow powder) ${ }^{1} \mathrm{H}$ NMR (500 MHz, DMSO- $\left.d_{6}\right) \delta 12.2(\mathrm{br}, 1 \mathrm{H}), 7.88-7.60(\mathrm{~m}, 1 \mathrm{H}), 7.54(\mathrm{dt}, J=7.5,1.4 \mathrm{~Hz}$, 1H), 7.36-7.33 (m, 2H), 3.12-3.05 (m, 1H), 2.97-2.89 (m, 2H), 2.72 (dd, J = 16.6, 6.0 Hz, $1 \mathrm{H}), 2.43$ (dd, $J=16.6,6.0 \mathrm{~Hz}, 1 \mathrm{H}), 2.18-2.12(\mathrm{~m}, 1 \mathrm{H}), 1.95$ (ddd, $J=21.7,17.3,4.3 \mathrm{~Hz}$, $1 \mathrm{H}) ;{ }^{13} \mathrm{C} \mathrm{NMR}\left(125 \mathrm{MHz}, \mathrm{CDCl}_{3}\right) \delta 198.2,173.3,144.4,133.4,131.8,129.0,126.52,126.52$, 40.2, 34.6, 28.57, 28.57.

HRMS (ESI) calcd for $\mathrm{C}_{12} \mathrm{H}_{13} \mathrm{O}_{3}[\mathrm{M}+\mathrm{H}]^{+}:$205.0859. Found: 205.0855.

\section{2-(7-Methoxy-1-oxo-1,2,3,4-tetrahydronaphthalen-2-yl)acetic acid (11b)}<smiles>COc1ccc2c(c1)C(=O)C(CC(=O)O)CC2</smiles>

(10.0 g, 75\% yield from 56.7 mmol of 7-methoxy-1-oxo-1,2,3,4-tetrahydronaphthalene, pale orange powder)

${ }^{1} \mathrm{H}$ NMR $\left(400 \mathrm{MHz}, \mathrm{CDCl}_{3}\right) \delta 7.51(\mathrm{~d}, J=3.0 \mathrm{~Hz}, 1 \mathrm{H}), 7.16(\mathrm{~d}, J=8.5 \mathrm{~Hz}, 1 \mathrm{H}), 7.07(\mathrm{dd}, J$ $=8.5,3.0 \mathrm{~Hz}, 1 \mathrm{H}), 3.83(\mathrm{~s}, 3 \mathrm{H}), 3.07-2.90(\mathrm{~m}, 4 \mathrm{H}), 2.51-2.48(\mathrm{~m}, 1 \mathrm{H}), 2.25-2.23(\mathrm{~m}, 1 \mathrm{H})$, 2.05-1.95 (m, 1H); ${ }^{13} \mathrm{C}$ NMR $\left(125 \mathrm{MHz}, \mathrm{CDCl}_{3}\right) \delta 198.4,178.2,158.3,136.7,132.7,130.0$, $122.0,109.4,55.5,44.5,35.0,29.5,28.4$.

HRMS (ESI) calcd for $\mathrm{C}_{13} \mathrm{H}_{15} \mathrm{O}_{4}[\mathrm{M}+\mathrm{H}]^{+}:$235.0964. Found: 235.0967.

\section{2-(7-Chloro-1-oxo-1,2,3,4-tetrahydronaphthalen-2-yl)acetic acid (11c)}<smiles>O=C(O)CC1CCc2ccc(Cl)cc2C1=O</smiles>

(9.5 g, 72\% yield from $55.3 \mathrm{mmol}$ of 7-chloro-1-oxo-1,2,3,4-tetrahydronaphthalene, light brown powder)

${ }^{1} \mathrm{H}$ NMR (400 MHz, $\left.\mathrm{CDCl}_{3}\right) \delta 12.2(\mathrm{br}, 1 \mathrm{H}), 7.77(\mathrm{~d}, J=2.4 \mathrm{~Hz}, 1 \mathrm{H}), 7.60(\mathrm{dd}, J=8.4,2.4$ $\mathrm{Hz}, 1 \mathrm{H}), 7.40(\mathrm{~d}, J=8.4 \mathrm{~Hz}, 1 \mathrm{H}), 3.10-2.92(\mathrm{~m}, 3 \mathrm{H}), 2.71(\mathrm{dd}, J=16.8,5.6 \mathrm{~Hz}, 1 \mathrm{H}), 2.52-$ $2.43(\mathrm{~m}, 1 \mathrm{H}), 2.18-2.12(\mathrm{~m}, 1 \mathrm{H}), 2.00-1.90(\mathrm{~m}, 1 \mathrm{H}) ;{ }^{13} \mathrm{C}$ NMR $\left(125 \mathrm{MHz}, \mathrm{CDCl}_{3}\right) \delta 197.2$, 173.1, 143.2, 133.3, 133.0, 131.34, 131.27, 125.7, 43.9, 34.5, 28.2, 27.9. HRMS (ESI) calcd for $\mathrm{C}_{12} \mathrm{H}_{10} \mathrm{ClO}_{3}$ [M-H] : 237.0324. Found: 237.0314. 
<smiles>O=C(O)CC1CCc2ccc(Br)cc2C1=O</smiles>

(6.9 g, 55\% yield from $44.4 \mathrm{mmol}$ of 7-bromo-1-oxo-1,2,3,4-tetrahydronaphthalene, white powder)

${ }^{1} \mathrm{H}$ NMR (400 MHz, DMSO-d $) \delta 12.2(\mathrm{br}, 1 \mathrm{H}), 7.91(\mathrm{~d}, J=2.2 \mathrm{~Hz}, 1 \mathrm{H}), 7.73$ (dd, $J=8.2$, $2.2 \mathrm{~Hz}, 1 \mathrm{H}), 7.33(\mathrm{~d}, J=8.2 \mathrm{~Hz}, 1 \mathrm{H}), 3.05-2.85(\mathrm{~m}, 3 \mathrm{H}), 2.70-2.65(\mathrm{~m}, 1 \mathrm{H}), 2.50-2.42(\mathrm{~m}$, 1H), 2.20-2.10 (m, 1H), 2.00-1.90 (m, 1H); ${ }^{13} \mathrm{C}$ NMR (125 MHz, DMSO-d6) $\delta 197.1,173.1$, 143.6, 135.8, 133.6, 131.5, 128.7, 119.5, 43.8, 34.5, 28.1, 27.9.

HRMS (APPI) calcd for $\mathrm{C}_{12} \mathrm{H}_{10} \mathrm{BrO}_{3}[\mathrm{M}-\mathrm{H}]^{-:}: 280.9819$. Found: 280.9821.

\section{2-(8-Bromo-7-methoxy-1-oxo-1,2,3,4-tetrahydronaphthalen-2-yl)acetic acid (11e)}<smiles>COc1ccc2c(c1Br)C(=O)C(CC(=O)O)CC2</smiles>

(6.4 g, 65\% yield from 31.4 mmol of 8-bromo-7-methoxy-3,4-dihydronaphthalen-1(2H)-one, gray powder)

${ }^{1} \mathrm{H}$ NMR (500 MHz, DMSO-d $) \delta 12.2(\mathrm{br}, 1 \mathrm{H}), 7.33-7.27(\mathrm{~m}, 2 \mathrm{H}), 3.85(\mathrm{~s}, 3 \mathrm{H}), 3.08-2.86$ (m, 3H), 2.67-2.60 (m, 1H), 2.44-2.40 (m, 1H), 2.13-2.09 (m, 1H), 1.88-1.77 (m, 1H); ${ }^{13} \mathrm{C}$ NMR (125 MHz, DMSO-d6) 197.9, 173.1, 154.8, 138.0, 131.8, 129.1, 116.7, 109.9, 56.7, 45.0, 35.0, 28.5, 28.3

HRMS (ESI) calcd for $\mathrm{C}_{13} \mathrm{H}_{13} \mathrm{BrO}_{4} \mathrm{Na}[\mathrm{M}+\mathrm{Na}]^{+}: 334.9889$. Found: 334.9899.

\section{2-(6-Hydroxy-1-oxo-1,2,3,4-tetrahydronaphthalen-2-yl)acetic acid (11f)}<smiles>O=C(O)CC1CCc2cc(O)ccc2C1=O</smiles>

An excess amount (2.2 equiv each) of $N, N$-diisopropylamine and $n$-BuLi was used. (7.2 g, $53 \%$ yield from $61.7 \mathrm{mmol}$ of 6-hydroxy-1-oxo-1,2,3,4-tetrahydronaphthalene, light brown powder)

${ }^{1} \mathrm{H}$ NMR (400 MHz, DMSO-d $)_{6} \delta 12.10$ (br, 1H), 10.32 (br, 1H), 7.73 (d, J=8.6 Hz, 1H), $6.71(\mathrm{dd}, J=8.6,2.2 \mathrm{~Hz}, 1 \mathrm{H}), 6.63(\mathrm{~d}, J=2.2 \mathrm{~Hz}, 1 \mathrm{H}), 3.02-2.95(\mathrm{~m}, 1 \mathrm{H}), 2.85-2.78(\mathrm{~m}$, 2H), $2.70(\mathrm{dd}, J=16.5,5.6 \mathrm{~Hz}, 1 \mathrm{H}), 2.34(\mathrm{dd}, J=16.5,6.8 \mathrm{~Hz}, 1 \mathrm{H}), 2.12-2.06(\mathrm{~m}, 1 \mathrm{H})$, $1.92-1.82(\mathrm{~m}, 1 \mathrm{H}) ;{ }^{13} \mathrm{C}$ NMR $\left(125 \mathrm{MHz}, \mathrm{DMSO}-d_{6}\right) \delta 196.4,173.4,162.0,146.9,129.3$, 124.1, 114.3, 114.1, 43.8, 34.7, 28.8, 28.7.

HRMS (APCI) calcd for $\mathrm{C}_{12} \mathrm{H}_{11} \mathrm{O}_{4}[\mathrm{M}-\mathrm{H}]^{-}: 219.0663$. Found: 219.0665. 


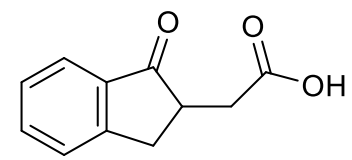

(3.9 g, 60\% yield from $34.2 \mathrm{mmol}$ of 1 -indanone, off-white powder)

${ }^{1} \mathrm{H}$ NMR (400 MHz, $\left.\mathrm{CDCl}_{3}\right) \delta 7.77(\mathrm{~d}, J=7.6 \mathrm{~Hz}, 1 \mathrm{H}), 7.64-7.60(\mathrm{~m}, 1 \mathrm{H}), 7.48-7.46(\mathrm{~m}$, 1H), 7.41-7.37 (m, 1H), 3.49 (dd, $J=17.2,7.6 \mathrm{~Hz}, 1 \mathrm{H}), 3.08-3.01(\mathrm{~m}, 2 \mathrm{H}), 2.90(\mathrm{dd}, J=$ 17.2, 4.4 Hz, 1H), 2.69-2.62 (m, 1H); $\left.{ }^{13} \mathrm{C} \mathrm{NMR} \mathrm{(125} \mathrm{MHz,} \mathrm{CDCl}_{3}\right) \delta 206.7,177.3,153.2$, 136.1, 135.1, 127.6, 126.5, 124.1, 43.4, 34.9, 33.0.

HRMS (ESI) calcd for $\mathrm{C}_{11} \mathrm{H}_{11} \mathrm{O}_{3}[\mathrm{M}+\mathrm{H}]^{+}:$191.0703. Found: 191.0703.

\section{2-(5,6-Dimethoxy-1-oxo-2,3-dihydro-1H-inden-2-yl)acetic acid (11h)}<smiles>COc1cc2c(cc1OC)C(=O)C(CC(=O)O)C2</smiles>

(5.9 g, 45\% yield from $57.2 \mathrm{mmol}$ of 5,6-dimethoxy-1-oxo-2,3-dihydro- $1 H$-indene, yellow powder)

${ }^{1} \mathrm{H}$ NMR $\left(400 \mathrm{MHz}, \mathrm{CDCl}_{3}\right) \delta 7.20(\mathrm{~s}, 1 \mathrm{H}), 6.88(\mathrm{~s}, 1 \mathrm{H}), 3.98(\mathrm{~s}, 3 \mathrm{H}), 3.91(\mathrm{~s}, 3 \mathrm{H}), 3.39$ (dd, $J=17.0,7.2 \mathrm{~Hz}, 1 \mathrm{H}), 3.06-3.00(\mathrm{~m}, 2 \mathrm{H}), 2.81(\mathrm{dd}, J=17.0,3.6 \mathrm{~Hz}, 1 \mathrm{H}), 2.60(\mathrm{dd}, J=17.8$, $10.0 \mathrm{~Hz}, 1 \mathrm{H}) ;{ }^{13} \mathrm{C} \mathrm{NMR}\left(125 \mathrm{MHz}, \mathrm{CDCl}_{3}\right) \delta 205.5,177.1,155.9,149.6,148.8,128.7,107.3$, 104.5, 56.2, 56.1, 43.5, 35.3, 32.8.

HRMS (ESI) calcd for $\mathrm{C}_{13} \mathrm{H}_{15} \mathrm{O}_{5}[\mathrm{M}+\mathrm{H}]^{+}:$251.0914. Found: 251.0916.

\section{2-(6-Fluoro-1-oxo-2,3-dihydro-1H-inden-2-yl)acetic acid (11i)}<smiles>O=C(O)CC1Cc2ccc(F)cc2C1=O</smiles>

(10.0 g, 72\% yield from $66.6 \mathrm{mmol}$ of 6-fluoro-1-oxo-2,3-dihydro- $1 H$-indene, light orange powder)

${ }^{1} \mathrm{H}$ NMR (400 MHz, DMSO- $\left.d_{6}\right) \delta 12.3(\mathrm{br}, 1 \mathrm{H}), 7.64-7.60(\mathrm{~m}, 1 \mathrm{H}), 7.56-7.51(\mathrm{~m}, 1 \mathrm{H}), 7.41-$ $7.38(\mathrm{~m}, 1 \mathrm{H}), 3.33(\mathrm{~d}, J=16.8,8.0 \mathrm{~Hz}, 1 \mathrm{H}), 3.01-2.95(\mathrm{~m}, 1 \mathrm{H}), 2.87-2.64(\mathrm{~m}, 3 \mathrm{H}) ;{ }^{13} \mathrm{C} \mathrm{NMR}$ $\left(125 \mathrm{MHz}, \mathrm{DMSO}-d_{6}\right) \delta 205.6(\mathrm{~d}, J=2.5 \mathrm{~Hz}), 172.6,161.5(\mathrm{~d}, J=244 \mathrm{~Hz}), 149.2(\mathrm{~d}, J=2.5$ $\mathrm{Hz}), 137.8$ (d, $J=7.5 \mathrm{~Hz}), 128.5$ (d, $J=8.8 \mathrm{~Hz}), 121.9$ (d, $J=22.5 \mathrm{~Hz}), 108.5$ (d, $J=21.3$ $\mathrm{Hz}), 44.0,34.1,31.8$.

HRMS (ESI) calcd for $\mathrm{C}_{11} \mathrm{H}_{8} \mathrm{FO}_{3}[\mathrm{M}-\mathrm{H}]^{-}: 207.0463$. Found: 207.0460 . 
<smiles>O=C(O)CC1Cc2ccc(Cl)cc2C1=O</smiles>

(9.2 g, 68\% yield from $60.0 \mathrm{mmol}$ of 6-chloro-1-oxo-2,3-dihydro- $1 H$-indene, orange solid) ${ }^{1} \mathrm{H}$ NMR (400 MHz, DMSO-d6) $\delta 12.3(\mathrm{br}, 1 \mathrm{H}), 7.72-7.69(\mathrm{~m}, 1 \mathrm{H}), 7.65-7.60(\mathrm{~m}, 2 \mathrm{H}), 3.35$ $(\mathrm{dd}, J=17.2,8.0 \mathrm{~Hz}, 1 \mathrm{H}), 3.00-2.90(\mathrm{~m}, 1 \mathrm{H}), 2.88-2.60(\mathrm{~m}, 3 \mathrm{H}) ;{ }^{13} \mathrm{C} \mathrm{NMR}(125 \mathrm{MHz}$, DMSO- $\left.d_{6}\right) \delta 205.5,172.8,152.2,138.0,134.3,132.7,128.6,122.5,48.0,34.1,32.0$.

HRMS (APPI) calcd for $\mathrm{C}_{11} \mathrm{H}_{8} \mathrm{ClO}_{3}[\mathrm{M}-\mathrm{H}]^{-}:$223.0167. Found: 223.0173.

\section{2-(5-Oxo-6,7,8,9-tetrahydro-5H-benzo[7]annulen-6-yl)acetic acid (11k)}<smiles>O=C(O)CC1CCCc2ccccc2C1=O</smiles>

(6.6 g, 80\% yield from $37.8 \mathrm{mmol}$ of 1-benzosuberone, light yellow solid) ${ }^{1} \mathrm{H}$ NMR (400 MHz, $\left.\mathrm{CDCl}_{3}\right) \delta 9.45(\mathrm{br}, 1 \mathrm{H}), 7.70(\mathrm{~d}, J=6.4 \mathrm{~Hz}, 1 \mathrm{H}), 7.41-7.38(\mathrm{~m}, 1 \mathrm{H})$, 7.30-7.21 (m, 2H), 3.37-3.30 (m, 1H), 3.10-2.92 (m, 3H), $2.52(\mathrm{dd}, J=17.0,5.2 \mathrm{~Hz}, 1 \mathrm{H})$, 2.20-2.05 (m, 1H), 2.00-1.90 (m, 1H), 1.78-1.60 (m, 2H); ${ }^{13} \mathrm{C}$ NMR (125 MHz, $\left.\mathrm{CDCl}_{3}\right) \delta$ 205.1, 178.3, 142.1, 138.9, 131.7, 129.9, 128.8, 126.5, 45.5, 36.0, 33.5, 29.5, 25.5. HRMS (ESI) calcd for $\mathrm{C}_{13} \mathrm{H}_{15} \mathrm{O}_{3}[\mathrm{M}+\mathrm{H}]^{+}: 219.1016$. Found: 219.1015.

\section{3-(1-Oxo-1,2,3,4-tetrahydronaphthalen-2-yl)propanoic acid (11I)}<smiles>O=C(O)CCC1CCc2ccccc2C1=O</smiles>

(4.3 g, 52\% yield from $37.8 \mathrm{mmol}$ of $\alpha$-tetralone, light brown powder) ${ }^{1} \mathrm{H}$ NMR $\left(400 \mathrm{MHz}, \mathrm{CDCl}_{3}\right) \delta 8.80(\mathrm{br}, 1 \mathrm{H}), 8.01(\mathrm{~d}, J=7.8,1.6 \mathrm{~Hz}, 1 \mathrm{H}), 7.48-7.44(\mathrm{~m}, 1 \mathrm{H})$, 7.32-7.22 (m, 2H), 3.04-3.01 (m, 2H), 2.59-2.54 (m, 2H), 2.31-1.83 (m, 5H); ${ }^{13} \mathrm{C}$ NMR $\left(125 \mathrm{MHz}, \mathrm{CDCl}_{3}\right) \delta 199.7,179.4,143.8,133.3,132.3,128.7,127.4,126.6,46.6,31.6,28.7$, 28.6, 24.8.

HRMS (ESI) calcd for $\mathrm{C}_{13} \mathrm{H}_{13} \mathrm{O}_{3}[\mathrm{M}-\mathrm{H}]^{-}: 217.0870$. Found: 217.0868. 
<smiles>COc1ccc2c(c1)C(=O)C(CCC(=O)O)CC2</smiles>

(5.3 g, 45\% yield from $47.7 \mathrm{mmol}$ of 7-methoxy-1-oxo-1,2,3,4-tetrahydronaphthalene, light brown powder)

${ }^{1} \mathrm{H}$ NMR $\left(400 \mathrm{MHz}, \mathrm{CDCl}_{3}\right) \delta 7.49(\mathrm{~d}, J=3.0 \mathrm{~Hz}, 1 \mathrm{H}), 7.14(\mathrm{~d}, J=9.2 \mathrm{~Hz}, 1 \mathrm{H}), 7.05(\mathrm{~d}, J$ $=9.2,3.0 \mathrm{~Hz}, 1 \mathrm{H}), 3.83(\mathrm{~s}, 3 \mathrm{H}), 2.95(\mathrm{~d}, J=7.6,4.8 \mathrm{~Hz}, 2 \mathrm{H}), 2.58-2.53(\mathrm{~m}, 3 \mathrm{H}), 2.29-2.19$ (m, 2H), 1.90-1.83 (m, 2H); $\left.{ }^{13} \mathrm{C} \mathrm{NMR} \mathrm{(125} \mathrm{MHz,} \mathrm{CDCl}_{3}\right) \delta$ 199.7, 179.4, 158.3, 136.4, 133.1, 129.9, 121.7, 109.3, 55.5, 46.4, 31.6, 29.0, 27.7, 24.9.

HRMS (ESI) calcd for $\mathrm{C}_{14} \mathrm{H}_{15} \mathrm{O}_{4}[\mathrm{M}-\mathrm{H}]=: 247.0976$. Found: 247.0973.

\section{3-(7-Chloro-1-oxo-1,2,3,4-tetrahydronaphthalen-2-yl)propanoic acid (11n)}<smiles>O=C(O)CCC1CCc2ccc(Cl)cc2C1=O</smiles>

(7.1 g, 51\% yield from $55.4 \mathrm{mmol}$ of 7-chloro-1-oxo-1,2,3,4-tetrahydronaphthalene, offwhite powder)

${ }^{1} \mathrm{H}$ NMR $\left(400 \mathrm{MHz}, \mathrm{DMSO}-d_{6}\right) \delta 12.10(\mathrm{br}, 1 \mathrm{H}), 7.78(\mathrm{~d}, J=2.2 \mathrm{~Hz}, 1 \mathrm{H}), 7.60(\mathrm{dd}, J=8.0$, $2.2 \mathrm{~Hz}, 1 \mathrm{H}), 7.39(\mathrm{~d}, J=8.0 \mathrm{~Hz}, 1 \mathrm{H}), 2.99-2.96(\mathrm{~m}, 2 \mathrm{H}), 2.60-2.55(\mathrm{~m}, 1 \mathrm{H}), 2.40-2.35(\mathrm{~m}$, $2 \mathrm{H}), 2.20-2.02(\mathrm{~m}, 2 \mathrm{H}), 1.82-1.70(\mathrm{~m}, 1 \mathrm{H}), 1.70-1.60(\mathrm{~m}, 1 \mathrm{H}) ;{ }^{13} \mathrm{C}$ NMR $(125 \mathrm{MHz}$, DMSO- $\left.d_{6}\right) \delta 198.2,174.3,143.0,133.4,132.9,131.32,131.25,125.7,45.8,31.0,27.6,27.3$, 24.5.

HRMS (ESI) calcd for $\mathrm{C}_{13} \mathrm{H}_{12} \mathrm{ClO}_{3}[\mathrm{M}-\mathrm{H}]^{-}:$251.0480. Found: 251.0478.

(f) Synthesis of 1-Substituted $\beta$-Tetralones Functionalized with Carboxylic Acids (11or) ${ }^{[4]}$<smiles>[R][X]1ccc2c(c1)CCC(=O)C2</smiles>

A THF solution $(200 \mathrm{~mL})$ containing $\beta$-tetralone derivatives $(56.7 \mathrm{mmol})$ and $\mathrm{NaH}(60 \%$ dispersion in mineral oil; $2.27 \mathrm{~g}, 56.7 \mathrm{mmol}$ ) under $\mathrm{N}_{2}$ atmosphere was stirred for $15 \mathrm{~min}$ at $30{ }^{\circ} \mathrm{C}$. Subsequently, alkyl bromide $(56.7 \mathrm{mmol})$ and 1,3-dimethyl-2-imidazolidinone (102.6 
mmol, 1.0 equiv) were slowly added. After stirring for $3 \mathrm{~h}$ at $\mathrm{rt}$, the resulting mixture was concentrated by rotary evaporator. The residue was added trifluoroacetic acid (12.9 g, 113.4 mmol) and $\mathrm{CHCl}_{3}(50 \mathrm{~mL})$. After stirring the reaction mixture for $2 \mathrm{~h}$ at $40{ }^{\circ} \mathrm{C}$, water $(50$ $\mathrm{mL})$ was added and followed by extraction with $\mathrm{CHCl}_{3}(3 \times 50 \mathrm{~mL})$. The combined organic layer was washed with brine $(50 \mathrm{~mL})$, dried over anhydrous $\mathrm{MgSO}_{4}$, and concentrated under reduced pressure to afford the crude product. Purification by silica gel column chromatography (eluent: hexane/AcOEt $=2 / 1)$ gave the product $(\mathbf{1 1 0}-11 \mathbf{r})$.

\section{2-(2-Oxo-1,2,3,4-tetrahydronaphthalen-1-yl)acetic acid (110)}<smiles>O=C(O)CC1C(=O)CCc2ccccc21</smiles>

(12.3 g, 88\% yield from $68.4 \mathrm{mmol}$ of $\beta$-tetralone, light brown powder)

${ }^{1} \mathrm{H}$ NMR (400 MHz, DMSO- $\left.d_{6}\right) \delta 12.22(\mathrm{br}, 1 \mathrm{H}), 7.40-7.10(\mathrm{~m}, 4 \mathrm{H}), 3.90-3.85(\mathrm{~m}, 1 \mathrm{H})$,

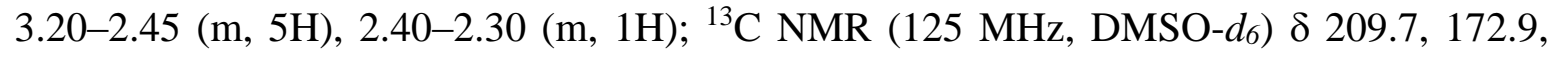
137.5, 136.0, 126.6, 126.4, 126.3, 125.5, 48.0, 36.9, 32.5, 27.3.

HRMS (APPI) calcd for $\mathrm{C}_{12} \mathrm{H}_{11} \mathrm{O}_{3}[\mathrm{M}-\mathrm{H}]^{-}: 203.0714$. Found: 203.0719.

\section{2-(5-Methoxy-2-oxo-1,2,3,4-tetrahydronaphthalen-1-yl)acetic acid (11p)}<smiles>COc1cccc2c1CCC(=O)C2CC(=O)O</smiles>

(9.0 g, 68\% yield from $56.7 \mathrm{mmol}$ of 5-methoxy-2-oxo-1,2,3,4-tetrahydronaphthalene, white powder)

${ }^{1} \mathrm{H}$ NMR $\left(400 \mathrm{MHz}, \mathrm{CDCl}_{3}\right) \delta 12.19(\mathrm{br}, 1 \mathrm{H}), 7.20(\mathrm{t}, J=8.0 \mathrm{~Hz}, 2 \mathrm{H}), 6.88(\mathrm{~d}, J=8.0,1 \mathrm{H})$, $3.82(\mathrm{t}, J=5.6 \mathrm{~Hz}, 1 \mathrm{H}), 3.80(\mathrm{~s}, 3 \mathrm{H}), 3.15-2.80(\mathrm{~m}, 4 \mathrm{H}), 2.65-2.60(\mathrm{~m}, 1 \mathrm{H}), 2.36-2.30(\mathrm{~m}$, $1 \mathrm{H}) ;{ }^{13} \mathrm{C}$ NMR (125 MHz, DMSO- $\left.d_{6}\right) \delta 209.5,172.6,155.6,137.3,127.2,125.1,118.1,108.7$, 55.4, 47.6, 36.5, 33.5, 20.0.

HRMS (ESI) calcd for $\mathrm{C}_{13} \mathrm{H}_{13} \mathrm{O}_{4}[\mathrm{M}-\mathrm{H}]=233.0819$. Found: 233.0818 . 
<smiles>O=C(O)CC1C(=O)CCc2cc(Br)ccc21</smiles>

(7.4 g, 59\% yield from $44.4 \mathrm{mmol}$ of 6-bromo-2-oxo-1,2,3,4-tetrahydronaphthalene, pale orange powder)

${ }^{1} \mathrm{H}$ NMR (400 MHz, $\left.\mathrm{CDCl}_{3}\right) \delta 12.27(\mathrm{br}, 1 \mathrm{H}), 7.55-7.35(\mathrm{~m}, 2 \mathrm{H}), 7.19-7.10(\mathrm{~m}, 1 \mathrm{H}), 3.84$ (t, $J=5.6 \mathrm{~Hz}, 1 \mathrm{H}), 3.20-2.80(\mathrm{~m}, 4 \mathrm{H}), 2.70-2.60(\mathrm{~m}, 1 \mathrm{H}), 2.40-2.30(\mathrm{~m}, 1 \mathrm{H}) ;{ }^{13} \mathrm{C}$ NMR $\left(125 \mathrm{MHz}, \mathrm{CDCl}_{3}\right) \delta 209.0,172.7,140.3,135.8,130.0,129.2,127.9,119.4,47.5,36.6,32.6$, 26.9

HRMS (APPI) calcd for $\mathrm{C}_{12} \mathrm{H}_{10} \mathrm{BrO}_{3}[\mathrm{M}-\mathrm{H}]^{-}: 280.9819$. Found: 280.9824.

\section{2-(6-Chloro-2-oxo-1,2,3,4-tetrahydronaphthalen-1-yl)acetic acid (11r)}<smiles>O=C(O)CC1C(=O)CCc2cc(Cl)ccc21</smiles>

(9.8 g, 74\% yield from $55.4 \mathrm{mmol}$ of 6-chloro-2-oxo-1,2,3,4-tetrahydronaphthalene, light yellow solid)

${ }^{1} \mathrm{H}$ NMR (400 MHz, $\left.\mathrm{CDCl}_{3}\right) \delta 12.27(\mathrm{br}, 1 \mathrm{H}), 7.36-7.10(\mathrm{~m}, 3 \mathrm{H}), 3.86(\mathrm{t}, J=5.2 \mathrm{~Hz}, 1 \mathrm{H})$, 3.20-2.60 (m, 4H), 2.78-2.60 (m, 1H), 2.45-2.37 (m, 1H); $\left.{ }^{13} \mathrm{C} \mathrm{NMR} \mathrm{(125} \mathrm{MHz,} \mathrm{CDCl}_{3}\right) \delta$ 209.1, 172.7, 139.9, 135.2, 130.9, 127.6, 127.2, 126.3, 47.5, 36.6, 32.7, 27.0.

HRMS (APPI) calcd for $\mathrm{C}_{12} \mathrm{H}_{10} \mathrm{ClO}_{3}[\mathrm{M}-\mathrm{H}]^{-}: 237.0324$. Found: 237.0324. 
(g) Synthesis of 1-Substituted $\beta$-Tetralones Functionalized with Carboxylic Acids (14) ${ }^{[4],[5]}$

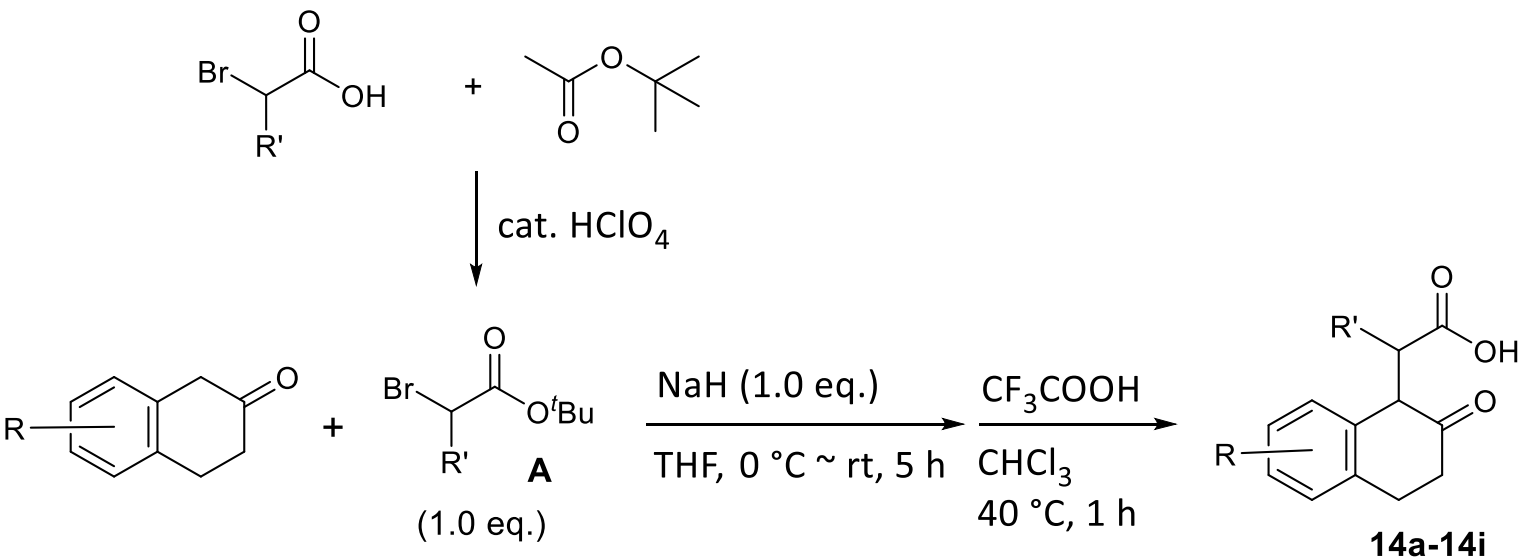

A tert-butyl acetate solution $(200 \mathrm{~mL})$ containing $\alpha$-bromocarboxylic acids $(45.1 \mathrm{mmol})$ and $\mathrm{HClO}_{4}(1.0 \mathrm{~mL})$ under $\mathrm{N}_{2}$ atmosphere was stirred for $12 \mathrm{~h}$ at $25^{\circ} \mathrm{C}$. Subsequently, saturated aqueous $\mathrm{NaHCO}_{3}$ solution $(700 \mathrm{~mL})$ was added, and organic layer was separated. The aqueous layer was extracted with AcOEt $(3 \times 2.0 \mathrm{~L})$ and the combined organic layer was washed with brine $(50 \mathrm{~mL})$, dried over anhydrous $\mathrm{MgSO}_{4}$, and concentrated under reduced pressure to afford the desired tert-butyl ester $(\mathbf{A})$.

A THF solution $(200 \mathrm{~mL})$ containing $\beta$-tetralone derivatives $(45.1 \mathrm{mmol})$ and $\mathrm{NaH}(60 \%$ dispersion in mineral oil; $45.1 \mathrm{mmol}$ ) under $\mathrm{N}_{2}$ atmosphere was stirred for $15 \mathrm{~min}$ at $30{ }^{\circ} \mathrm{C}$. Subsequently, $\alpha$-bromoester (A) $(45.1 \mathrm{mmol})$ and 1, 3-dimethyl-2-imidazolidinone (45.1 mmol) were added slowly to the solution and stirred for $3 \mathrm{~h}$ at $\mathrm{rt}$. The resulting mixture was concentrated by rotary evaporator. The residue was added trifluoroacetic acid $(90.2 \mathrm{mmol})$ and $\mathrm{CHCl}_{3}(50 \mathrm{~mL})$ and stirred for $2 \mathrm{~h}$ at $40{ }^{\circ} \mathrm{C}$. Water $(50 \mathrm{~mL})$ was added and followed by extraction with $\mathrm{CHCl}_{3}(3 \times 50 \mathrm{~mL})$. The combined organic layer was washed with brine (50 $\mathrm{mL}$ ), dried over anhydrous $\mathrm{MgSO}_{4}$, and concentrated under reduced pressure to afford the crude product. Purification by silica gel column chromatography (eluent: hexane/AcOEt = 2/1) gave the product (14a-14i).

\section{2-(2-Oxo-1,2,3,4-tetrahydronaphthalen-1-yl)propanoic acid (14a)}<smiles>CC(C(=O)O)C1C(=O)CCc2ccccc21</smiles>

(6.6 g, 55\% yield from $55.4 \mathrm{mmol}$ of $\beta$-tetralone, orange solid)

${ }^{1} \mathrm{H}$ NMR (500 MHz, DMSO-d6) $\delta 7.45(\mathrm{br}, 1 \mathrm{H}), 7.23-7.15(\mathrm{~m}, 4 \mathrm{H}), 3.10(\mathrm{~d}, J=10.3 \mathrm{~Hz}$, $1 \mathrm{H}), 2.82-2.78(\mathrm{~m}, 2 \mathrm{H}), 2.67-2.62(\mathrm{~m}, 1 \mathrm{H}), 2.06-2.02(\mathrm{~m}, 2 \mathrm{H}), 1.36(\mathrm{~d}, J=7.1 \mathrm{~Hz}, 3 \mathrm{H}) ;{ }^{13} \mathrm{C}$ NMR $\left(125 \mathrm{MHz}, \mathrm{DMSO}-d_{6}\right) \delta 176.1,135.3,134.8,128.64,128.58,126.7,126.3,105.8,51.8$, 
HRMS (ESI) calcd for $\mathrm{C}_{13} \mathrm{H}_{15} \mathrm{O}_{3}[\mathrm{M}+\mathrm{H}]^{+}:$219.1016. Found: 219.1011.

\section{2-(2-Oxo-1,2,3,4-tetrahydronaphthalen-1-yl)butanoic acid (14b)}<smiles>CCC(C(=O)O)C1C(=O)CCc2ccccc21</smiles>

(6.8 g, 53\% yield from $55.4 \mathrm{mmol}$ of $\beta$-tetralone, orange solid)

${ }^{1} \mathrm{H}$ NMR (500 MHz, DMSO-d $\left.d_{6}\right) \delta 7.47(\mathrm{br}, 1 \mathrm{H}), 7.22-7.16(\mathrm{~m}, 3 \mathrm{H}), 7.12-7.09(\mathrm{~m}, 1 \mathrm{H}), 3.26$ $(\mathrm{d}, J=8.7 \mathrm{~Hz}, 1 \mathrm{H}), 2.80-2.77(\mathrm{~m}, 2 \mathrm{H}), 2.61-2.58(\mathrm{~m}, 1 \mathrm{H}), 2.15-2.10(\mathrm{~m}, 1 \mathrm{H}), 2.00-1.90(\mathrm{~m}$, $2 \mathrm{H}), 1.82-1.77(\mathrm{~m}, 1 \mathrm{H}), 1.07(\mathrm{t}, J=7.4 \mathrm{~Hz}, 3 \mathrm{H}) ;{ }^{13} \mathrm{C}$ NMR $\left(125 \mathrm{MHz}, \mathrm{DMSO}-d_{6}\right) \delta 175.8$, $135.9,135.1,128.7,128.5,126.6,126.5,105.9,51.0,47.6,32.0,26.5,21.6,10.4$.

HRMS (ESI) calcd for $\mathrm{C}_{14} \mathrm{H}_{17} \mathrm{O}_{3}[\mathrm{M}+\mathrm{H}]^{+}:$233.1172. Found: 233.1168.

\section{2-(2-Oxo-1,2,3,4-tetrahydronaphthalen-1-yl)-3-phenylpropanoic acid (14c)}<smiles>O=C(O)C(Cc1ccccc1)C1C(=O)CCc2ccccc21</smiles>

(5.8 g, 56\% yield from $35.1 \mathrm{mmol}$ of $\beta$-tetralone, brown powder) ${ }^{1} \mathrm{H}$ NMR (500 MHz, DMSO-d $) \delta 7.57$ (br, 1H), 7.43-7.04 (m, 8H), 6.60-6.57 (m, 1H), 3.21$3.18(\mathrm{~m}, 3 \mathrm{H}), 2.93-2.88(\mathrm{~m}, 1 \mathrm{H}), 2.77-2.72(\mathrm{~m}, 2 \mathrm{H}), 2.25-2.20(\mathrm{~m}, 1 \mathrm{H}), 1.95-1.88(\mathrm{~m}, 1 \mathrm{H})$; ${ }^{13} \mathrm{C}$ NMR $\left(125 \mathrm{MHz}, \mathrm{DMSO}-d_{6}\right) \delta 176.2,167.6,138.4,135.2,129.5,128.6,128.3,128.2$, 126.5, 126.4, 119.2, 106.6, 52.1, 47.8, 35.8, 32.5, 26.3.

HRMS (APPI) calcd for $\mathrm{C}_{19} \mathrm{H}_{19} \mathrm{O}_{3}[\mathrm{M}+\mathrm{H}]^{+}:$295.1329. Found: 295.1324.

\section{2-(2-Oxo-1,2,3,4-tetrahydronaphthalen-1-yl)-2-phenylacetic acid (14d)}<smiles>O=C(O)C(c1ccccc1)C1C(=O)CCc2ccccc21</smiles>

(7.8 g, 62\% yield from $45.1 \mathrm{mmol}$ of $\beta$-tetralone, orange powder)

${ }^{1} \mathrm{H}$ NMR (500 MHz, DMSO- $\left.d_{6}\right) \delta 7.67$ (br, 1H), 7.46-7.42 (m, 2H), 7.39-7.35 (m, 1H), 7.32- 
$7.29(\mathrm{~m}, 2 \mathrm{H}), 7.20-7.18(\mathrm{~m}, 1 \mathrm{H}), 7.13(\mathrm{dt}, J=7.2,0.8 \mathrm{~Hz}, 1 \mathrm{H}), 6.95(\mathrm{t}, J=7.2 \mathrm{~Hz}, 1 \mathrm{H}), 6.33$ $(\mathrm{d}, J=7.7 \mathrm{~Hz}, 1 \mathrm{H}), 4.07(\mathrm{~d}, J=11.6 \mathrm{~Hz}, 1 \mathrm{H}), 3.70(\mathrm{~d}, J=11.6 \mathrm{~Hz}, 1 \mathrm{H}), 2.89-2.85(\mathrm{~m}, 2 \mathrm{H})$, 2.20-2.14 (m, 2H); ${ }^{13} \mathrm{C}$ NMR (125 MHz, DMSO- $\left.d_{6}\right) \delta 173.7,137.0,134.8,134.5,129.3$, $128.8,128.7,128.0,127.6,126.7,125.9,105.6,56.1,52.5,31.1,26.6$.

HRMS (ESI) calcd for $\mathrm{C}_{18} \mathrm{H}_{17} \mathrm{O}_{3}[\mathrm{M}+\mathrm{H}]^{+}:$281.1172. Found: 281.1168.

\section{2-(6-Bromo-2-oxo-1,2,3,4-tetrahydronaphthalen-1-yl)-2-phenylacetic acid (14e)}<smiles>O=C(O)C(c1ccccc1)C1C(=O)CCc2cc(Br)ccc21</smiles>

(6.4 g, 80\% yield from $22.2 \mathrm{mmol}$ of 6-bromo-2-oxo-1,2,3,4-tetrahydronaphthalene, orange powder)

${ }^{1} \mathrm{H}$ NMR (500 MHz, DMSO- $\left.d_{6}\right) \delta 7.70(\mathrm{br}, 1 \mathrm{H}), 7.46-7.42(\mathrm{~m}, 3 \mathrm{H}), 7.39-7.36(\mathrm{~m}, 1 \mathrm{H}), 7.31-$ $7.28(\mathrm{~m}, 2 \mathrm{H}), 7.17(\mathrm{dd}, J=8.3,2.1 \mathrm{~Hz}, 1 \mathrm{H}), 6.24(\mathrm{~d}, J=8.3 \mathrm{~Hz}, 1 \mathrm{H}), 4.10(\mathrm{~d}, J=11.5 \mathrm{~Hz}$, $1 \mathrm{H}), 3.65(\mathrm{~d}, J=11.5 \mathrm{~Hz}, 1 \mathrm{H}), 2.88-2.85(\mathrm{~m}, 2 \mathrm{H}), 2.19-2.10(\mathrm{~m}, 2 \mathrm{H}) ;{ }^{13} \mathrm{C}$ NMR $(125 \mathrm{MHz}$, DMSO- $\left.d_{6}\right) \delta 173.5,137.8,136.6,133.8,131.2,130.0,129.3,128.9,128.8,127.7,119.9$, 105.3, 55.8, 52.1, 30.7, 26.4.

HRMS (ESI) calcd for $\mathrm{C}_{18} \mathrm{H}_{16} \mathrm{BrO}_{3}[\mathrm{M}+\mathrm{H}]^{+}:$359.0277. Found: 359.0278 .

\section{2-(6-Chloro-2-oxo-1,2,3,4-tetrahydronaphthalen-1-yl)-2-phenylacetic acid (14f)}<smiles>O=C(O)C(c1ccccc1)C1C(=O)CCc2cc(Cl)ccc21</smiles>

(6.6 g, 78\% yield from 26.9 mmol of 6-chloro-2-oxo-1,2,3,4-tetrahydronaphthalene, orange powder)

${ }^{1} \mathrm{H}$ NMR (500 MHz, DMSO- $\left.d_{6}\right) \delta 7.71(\mathrm{br}, 1 \mathrm{H}), 7.45-7.25(\mathrm{~m}, 6 \mathrm{H}), 7.05(\mathrm{dd}, J=8.4,2.2 \mathrm{~Hz}$, $1 \mathrm{H}), 6.32(\mathrm{~d}, J=8.4 \mathrm{~Hz}, 1 \mathrm{H}), 4.08(\mathrm{~d}, J=11.6 \mathrm{~Hz}, 1 \mathrm{H}), 3.69$ (d, $J=11.6 \mathrm{~Hz}, 1 \mathrm{H}), 2.91-2.85$ (m, 2H), 2.19-2.12 (m, 2H); ${ }^{13} \mathrm{C}$ NMR (125 MHz, DMSO-d6) $\delta 173.5,137.4,136.7,133.4$, 131.3, 130.0, 129.3, 128.9, 128.3, 127.7, 126.0, 105.3, 55.8, 52.1, 30.7, 26.4. HRMS (ESI) calcd for $\mathrm{C}_{18} \mathrm{H}_{16} \mathrm{ClO}_{3}[\mathrm{M}+\mathrm{H}]^{+}: 315.0782$. Found: 315.0778. 
<smiles>COc1cccc2c1CCC(=O)C2C(C(=O)O)c1ccccc1</smiles>

(7.1 g, 81\% yield from $28.4 \mathrm{mmol}$ of 5-methoxy-2-oxo-1,2,3,4-tetrahydronaphthalene, brown powder)

${ }^{1} \mathrm{H}$ NMR (500 MHz, DMSO-d 6$) \delta 7.65(\mathrm{br}, 1 \mathrm{H}), 7.45-7.10(\mathrm{~m}, 5 \mathrm{H}), 6.93(\mathrm{~d}, J=7.8 \mathrm{~Hz}, 1 \mathrm{H})$, 6.80-6.77 (m, 1H), $5.91(\mathrm{~d}, J=7.8 \mathrm{~Hz}, 1 \mathrm{H}), 4.08(\mathrm{~d}, J=11.6 \mathrm{~Hz}, 1 \mathrm{H}), 3.68(\mathrm{~s}, 3 \mathrm{H}), 3.67$ (d, $J=11.6 \mathrm{~Hz}, 1 \mathrm{H}), 2.98-2.93(\mathrm{~m}, 1 \mathrm{H}), 2.58-2.52(\mathrm{~m}, 1 \mathrm{H}), 2.22-2.17(\mathrm{~m}, 1 \mathrm{H}), 2.10-2.05(\mathrm{~m}$, $1 \mathrm{H}) ;{ }^{13} \mathrm{C}$ NMR $\left(125 \mathrm{MHz}, \mathrm{DMSO}-d_{6}\right) \delta 173.8,156.5,137.1,135.4,129.3,128.2,127.5$, $126.7,123.2,120.1,108.4,105.4,56.0,55.3,52.3,30.3,20.5$.

HRMS (ESI) calcd for $\mathrm{C}_{19} \mathrm{H}_{19} \mathrm{O}_{4}[\mathrm{M}+\mathrm{H}]^{+}: 311.1278$. Found: 311.1272 .

\section{2-(2-Chlorophenyl)-2-(2-oxo-1,2,3,4-tetrahydronaphthalen-1-yl)acetic acid (14h)}<smiles>O=C(O)C(c1ccccc1Cl)C1C(=O)CCc2ccccc21</smiles>

( $8.0 \mathrm{~g}, 74 \%$ yield from $34.2 \mathrm{mmol}$ of $\beta$-tetralone, orange solid) ${ }^{1} \mathrm{H}$ NMR (500 MHz, DMSO-d6) $\delta 7.73(\mathrm{br}, 1 \mathrm{H}), 7.58-7.13(\mathrm{~m}, 6 \mathrm{H}), 7.00-6.96(\mathrm{~m}, 1 \mathrm{H}), 6.27$ $(\mathrm{d}, J=7.5 \mathrm{~Hz}, 1 \mathrm{H}), 4.38(\mathrm{~d}, J=11.6 \mathrm{~Hz}, 1 \mathrm{H}), 3.87(\mathrm{~d}, J=11.6 \mathrm{~Hz}, 1 \mathrm{H}), 2.90-2.85(\mathrm{~m}, 2 \mathrm{H})$, 2.22-1.98 (m, 2H); ${ }^{13} \mathrm{C}$ NMR (125 MHz, DMSO- $\left.d_{6}\right) \delta 171.5,134.8,134.0,133.7,133.3$, 132.2, 129.7, 129.0, 128.7, 127.7, 127.1, 126.8, 126.0, 106.0, 79.2, 38.6, 31.1, 26.6.

HRMS (ESI) calcd for $\mathrm{C}_{18} \mathrm{H}_{16} \mathrm{ClO}_{3}[\mathrm{M}+\mathrm{H}]^{+}:$315.0782. Found: 315.0780 .

\section{2-(7-Methoxy-2-oxo-1,2,3,4-tetrahydronaphthalen-1-yl)-2-phenylacetic acid (14i)}<smiles>COc1ccc2c(c1)C(C(C(=O)O)c1ccccc1)C(=O)CC2</smiles>

(13.2 g, 75\% yield from $56.8 \mathrm{mmol}$ of 7-methoxy-2-oxo-1,2,3,4-tetrahydronaphthalene, light brown solid)

${ }^{1} \mathrm{H}$ NMR (500 MHz, DMSO-d6) $\delta 7.66$ (s, 1H), 7.46-7.43 (m, 2H), 7.40-7.36 (m, 1H), 7.32- 
$7.30(\mathrm{~m}, 2 \mathrm{H}), 7.11-7.08(\mathrm{~m}, 1 \mathrm{H}), 6.76-6.72(\mathrm{~m}, 1 \mathrm{H}), 5.81(\mathrm{~d}, J=2.5 \mathrm{~Hz}, 1 \mathrm{H}), 4.07(\mathrm{~d}, J=$ $11.6 \mathrm{~Hz}, 1 \mathrm{H}), 3.62(\mathrm{~d}, J=11.6 \mathrm{~Hz}, 1 \mathrm{H}), 3.40(\mathrm{~s}, 3 \mathrm{H}), 2.80-2.77(\mathrm{~m}, 2 \mathrm{H}), 2.20-2.07(\mathrm{~m}, 2 \mathrm{H})$; ${ }^{13} \mathrm{C}$ NMR $\left(125 \mathrm{MHz}\right.$, DMSO-d $d_{6} \delta$ 173.7, 157.1, 137.1, 135.5, 129.6, 129.4, 128.8, 127.6, 126.6, 113.0, 112.7, 105.7, 56.1, 54.5, 52.8, 31.3, 25.8 .

HRMS (FD) calcd for $\mathrm{C}_{19} \mathrm{H}_{18} \mathrm{O}_{4}[\mathrm{M}]^{+}: 310.1205$. Found: 310.1233 .

(h) Synthesis of 2-Substituted $\alpha$-Tetralones Functionalized with Carboxylic Acids $(17)^{[3]}$

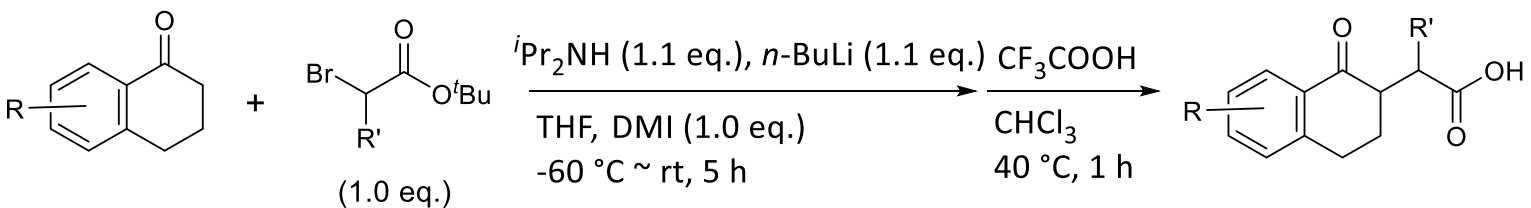

A THF solution $(1.57 \mathrm{M})$ of $n$-BuLi $(38.8 \mathrm{~mL}, 60.9 \mathrm{mmol})$ was added slowly to a THF solution $(150 \mathrm{~mL})$ containing $N, N$-diisopropylamine $(7.88 \mathrm{~g}, 60.9 \mathrm{mmol})$ at $-60{ }^{\circ} \mathrm{C}$ under $\mathrm{N}_{2}$ atmosphere and stirred for $1 \mathrm{~h}$. The resulting mixture was warmed to $-20^{\circ} \mathrm{C}$, then cyclic ketone $(55.4 \mathrm{mmol})$ was added and stirred for $1 \mathrm{~h}$ at $0{ }^{\circ} \mathrm{C}$. Subsequently, alkyl bromide $(55.4$ mmol.) and 1,3-dimethyl-2-imidazolidinone $(55.4 \mathrm{mmol})$ were added to the reaction mixture. After stirring further for $5 \mathrm{~h}$ at $0{ }^{\circ} \mathrm{C}$, the resulting mixture was concentrated by rotary evaporator. The residue was added trifluoroacetic acid $(25.7 \mathrm{~g}, 225.7 \mathrm{mmol})$ and $\mathrm{CHCl}_{3}(150$ $\mathrm{mL})$. After stirring the reaction mixture for $2 \mathrm{~h}$ at $40{ }^{\circ} \mathrm{C}$, water $(50 \mathrm{~mL})$ was added and followed by extraction with $\mathrm{CHCl}_{3}(3 \times 50 \mathrm{~mL})$. The combined organic layer was washed with brine $(100 \mathrm{~mL})$, dried over anhydrous $\mathrm{MgSO}_{4}$, and concentrated under reduced pressure to afford the crude product. Purification by silica gel column chromatography (eluent: hexane/AcOEt $=2 / 1)$ gave the product $(\mathbf{1 7 a - 1 7 c})$.

\section{2-(1-Oxo-1,2,3,4-tetrahydronaphthalen-2-yl)-2-phenylacetic acid (17a)}<smiles>O=C1c2ccccc2CCC1C(C(=O)O)c1ccccc1</smiles>

(7.1 g, 45\% yield from $56.4 \mathrm{mmol}$ of $\alpha$-tetralone, brown solid)

${ }^{1} \mathrm{H}$ NMR $\left(500 \mathrm{MHz}, \mathrm{CDCl}_{3}\right) \delta 8.40(\mathrm{br}, 1 \mathrm{H}), 7.48-7.20(\mathrm{~m}, 9 \mathrm{H}), 3.84(\mathrm{~d}, J=10.3 \mathrm{~Hz}, 1 \mathrm{H})$, 3.44-3.39 (m, 1H), 3.37-2.83 (m, 2H), 1.82-1.60 (m, 1H), 1.31-1.25 (m, 1H); ${ }^{13} \mathrm{C}$ NMR $\left(125 \mathrm{MHz} \mathrm{CDCl}_{3}\right) \delta 198.8,177.6,143.9,133.3,129.1,128.7,128.5,128.3,127.9,127.5$, 127.3, 126.7, 50.9, 41.0, 29.4, 26.8.

HRMS (APCI) calcd for $\mathrm{C}_{18} \mathrm{H}_{17} \mathrm{O}_{3}[\mathrm{M}+\mathrm{H}]^{+}:$281.1172. Found: 281.1182. 
2-(7-Chloro-1-oxo-1,2,3,4-tetrahydronaphthalen-2-yl)-2-phenylacetic acid (17b)<smiles>O=C1c2cc(Cl)ccc2CCC1C(C(=O)O)c1ccccc1</smiles>

(12.5 g, 72\% yield from $55.4 \mathrm{mmol}$ of 7-chloro-1-oxo-1,2,3,4-tetrahydronaphthalene, offwhite powder)

${ }^{1} \mathrm{H}$ NMR $\left(500 \mathrm{MHz}, \mathrm{DMSO}-d_{6}\right) \delta 7.80-7.78(\mathrm{~m}, 1 \mathrm{H}), 7.61-7.59(\mathrm{~m}, 1 \mathrm{H}), 7.38-7.24(\mathrm{~m}, 6 \mathrm{H})$, $3.55(\mathrm{~d}, J=10.2 \mathrm{~Hz}, 1 \mathrm{H}), 3.47-3.40(\mathrm{~m}, 1 \mathrm{H}), 3.10-2.80(\mathrm{~m}, 2 \mathrm{H}), 1.62-1.58(\mathrm{~m}, 2 \mathrm{H}) ;{ }^{13} \mathrm{C}$ NMR $\left(125 \mathrm{MHz}, \mathrm{DMSO}-d_{6}\right) \delta 197.5,174.1,143.1,137.3,133.4,133.1,131.3,131.2,128.6$, 128.5, 127.3, 125.6, 51.3, 49.7, 27.9, 26.0.

HRMS (APCI) calcd for $\mathrm{C}_{18} \mathrm{H}_{16} \mathrm{ClO}_{3}[\mathrm{M}+\mathrm{H}]^{+}: 315.0782$. Found: 315.0790.

\section{2-(1-Oxo-1,2,3,4-tetrahydronaphthalen-2-yl)propanoic acid (17c)}<smiles>CC(C(=O)O)C1CCc2ccccc2C1=O</smiles>

(15.7 g, 70\% yield from $102.6 \mathrm{mmol}$ of $\alpha$-tetralone, light orange solid)

${ }^{1} \mathrm{H}$ NMR $\left(500 \mathrm{MHz}, \mathrm{DMSO}-d_{6}\right) \delta 7.88-7.85(\mathrm{~m}, 1 \mathrm{H}), 7.56-7.51(\mathrm{~m}, 1 \mathrm{H}), 7.38-7.30(\mathrm{~m}, 2 \mathrm{H})$, 3.15-2.96 (m, 2H), 2.88-2.82 (m, 1H), 2.15-2.00 (m, 2H), 1.92-1.85 (m, 1H), $1.05(\mathrm{~d}, J=$ $6.9 \mathrm{~Hz}, 3 \mathrm{H}) ;{ }^{13} \mathrm{C}$ NMR $\left(125 \mathrm{MHz}, \mathrm{DMSO}-d_{6}\right) \delta 197.8,176.7,144.4,133.5,132.0,128.9$, 126.6, 126.5, 49.7, 38.2, 28.5, 24.9, 13.2.

HRMS (FD) calcd for $\mathrm{C}_{13} \mathrm{H}_{14} \mathrm{O}_{3}[\mathrm{M}]^{+}:$218.0943. Found: 218.0967 . 


\section{Procedures for Catalytic Reactions.}

(1) Asymmetric Transfer Hydrogenation of Substututed Ketones Using $(R, R)-3$ or $(R, R)-4$ (Tables 1 and 2)

General procedures under the conditions of $\mathrm{S} / \mathrm{C}=500,60{ }^{\circ} \mathrm{C}$, and $9 \mathrm{~h}$.

Under $\mathrm{N}_{2}$ atmosphere, a mixture of ketone $(1.0 \mathrm{mmol})$ and the $\mathrm{Ru}$ catalyst $(0.002 \mathrm{mmol})$ in an azeotropic mixture of formic acid and triethylamine $(5: 2,0.5 \mathrm{~mL})$ was stirred at $60{ }^{\circ} \mathrm{C}$ for $9 \mathrm{~h}$. After the reaction completion, water $(3 \mathrm{~mL})$ and EtOAc $(5 \mathrm{~mL})$ was added. The biphasic layers were separated, the aqueous layer was extracted with EtOAc $(3 \times 5 \mathrm{~mL})$, and the combined organic portions were washed with brine $(3 \mathrm{~mL})$. After drying over $\mathrm{MgSO}_{4}$, filtration, and solvent removal under reduced pressure, the crude residue was purified by silica gel column chromatography to afford the desired product. The optical purity of the products was determined by chiral HPLC analysis using a Daicel Chiralcel OD-H, OJ-H, OJ3 or Chiralpak AD-H column $(4.6 \mathrm{~mm} \times 25 \mathrm{~cm})$ and YMC Alcyon SFC CPS Cellulose-SC column $(4.6 \mathrm{~mm} \times 25 \mathrm{~cm})$ with hexane/2-propanol as the eluent where a clear base-line separation was obtained.

(2) Asymmetric Transfer Hydrogenation and Sequential Lactonization of Substituted Ketones Using $(R, R)-3$ (Tables 3 and 4)

General procedures under the conditions of $\mathrm{S} / \mathrm{C}=500,60{ }^{\circ} \mathrm{C}$, and $7 \mathrm{~h}$.

Under $\mathrm{N}_{2}$ atmosphere, a mixture of ketone $(1.0 \mathrm{mmol})$ and the $\mathrm{Ru}$ catalyst $(0.002 \mathrm{mmol})$ in formic acid $(0.23 \mathrm{~g}, 0.19 \mathrm{~mL}, 5.0 \mathrm{mmol})$, DBU $(0.46 \mathrm{~g}, 0.45 \mathrm{~mL}, 3.0 \mathrm{mmol})$ and ethyl acetate $(1.0 \mathrm{~mL})$ was stirred at $60{ }^{\circ} \mathrm{C}$ for $7 \mathrm{~h}$. After the reaction completion, water $(3 \mathrm{~mL})$ and EtOAc $(5 \mathrm{~mL})$ was added. The biphasic layers were separated, the aqueous layer was extracted with EtOAc $(3 \times 5 \mathrm{~mL})$, and the combined organic portions were washed with brine $(3 \mathrm{~mL})$. After drying over $\mathrm{MgSO}_{4}$, filtration, and solvent removal under reduced pressure, the crude residue was purified by silica gel column chromatography to afford the desired product. The optical purity of product was determined by chiral HPLC analysis using a Daicel Chiralcel OD-H, OZ-3 or Chiralpak AD-H, IB column $(4.6 \mathrm{~mm} \times 25 \mathrm{~cm})$ and YMC Alcyon SFC CPS Cellulose-SC column $(4.6 \mathrm{~mm} \times 25 \mathrm{~cm})$ with hexane/2-propanol as the eluent where a clear base-line separation was obtained. 


\section{E. Characterization Data for Products.}

\section{$(1 R, 2 R)-2-M e t h y l-1,2,3,4-t e t r a h y d r o n a p h t h a l e n-1-o l ~(9 a)$}<smiles>C[C@@H]1CCc2ccccc2[C@H]1O</smiles>

According to the general procedure (ketone 8a: $0.160 \mathrm{~g}(1.0 \mathrm{mmol})$ and cat. $(\boldsymbol{R}, \boldsymbol{R})-\mathbf{3}), 0.146$ g of 9a was obtained as a pale yellow oil (90\% yield, 97\% ee, $84 \%$ de).

${ }^{1} \mathrm{H}$ NMR $\left(400 \mathrm{MHz}, \mathrm{CDCl}_{3}\right) \delta$ 7.36-7.34 (m, 1H), 7.23-7.17 (m, 2H), 7.17-7.11 (m, 1H), $4.55(\mathrm{~m}, 1 \mathrm{H}), 2.89-2.72(\mathrm{~m}, 2 \mathrm{H}), 1.93-1.62(\mathrm{~m}, 3 \mathrm{H}), 1.53(\mathrm{~m}, 1 \mathrm{H}), 1.13(\mathrm{~d}, J=4.0 \mathrm{~Hz}, 3 \mathrm{H})$; ${ }^{13} \mathrm{C}$ NMR $\left(125 \mathrm{MHz}, \mathrm{CDCl}_{3}\right) \delta 138.7,136.7,129.8,129.0,127.8,126.1,71.5,34.2,28.9$, 24.7, 16.9 .

HRMS (FI) calcd for $\mathrm{C}_{11} \mathrm{H}_{14} \mathrm{O}[\mathrm{M}]^{+}:$162.1045. Found: 162.1051.

The enantiomeric excess was determined by HPLC analysis (YMC Alcyon SFC CPS Cellulose-SC, 250×4.6 mm column, hexane/2-propanol 99:1, $1.0 \mathrm{~mL} / \mathrm{min}, 220 \mathrm{~nm}, 40{ }^{\circ} \mathrm{C}$, $(1 S, 2 S)$ isomer $11.2 \mathrm{~min}$ (minor), $(1 R, 2 R)$ isomer $12.4 \mathrm{~min}$ (major), $(1 S, 2 R)$ and $(1 R, 2 S)$ isomers $13.4 \mathrm{~min}$ or $18.1 \mathrm{~min}$ ); IR (neat) 3221, 3095, 3072, 3025, 2966, 2894, 1459, 1420, 1332, 1323, 1299, 1283, 1273, 1207, 1199, 1107, 1071, 1002, 962, 900, 757, 746, $731 \mathrm{~cm}^{-1}$; $[\alpha]_{\mathrm{D}}{ }^{20}+61.0\left(\mathrm{c} 0.15\right.$ in $\left.\mathrm{C}_{6} \mathrm{H}_{6}\right) 97 \%$ ee, $84 \%$ de $(1 R, 2 R)\left(\right.$ lit. $^{[6]}[\alpha]_{\mathrm{D}}{ }^{20}+62.5\left(\mathrm{c} 2.10\right.$ in $\left.\mathrm{C}_{6} \mathrm{H}_{6}\right)$ $87 \%$ ee $(1 R, 2 R)$, cis:trans $=98.6: 1.4)$.

\section{(1R,2R)-2-Methyl-2,3-dihydro-1H-inden-1-ol (9b)}<smiles>C[C@H]1Cc2ccccc2[C@H]1O</smiles>

According to the general procedure (ketone 8b: $0.292 \mathrm{~g} \mathrm{(1.0} \mathrm{mmol),} \mathrm{cat.}(\boldsymbol{R}, \boldsymbol{R})-3), 0.290 \mathrm{~g}$ of 9b was obtained as a white solid (98\% yield, $98 \%$ ee, $96 \%$ de).

${ }^{1} \mathrm{H}$ NMR $\left(400 \mathrm{MHz}, \mathrm{CDCl}_{3}\right) \delta 7.41-7.40(\mathrm{~m}, 1 \mathrm{H}), 7.25-7.20(\mathrm{~m}, 3 \mathrm{H}), 5.00(\mathrm{~m}, 1 \mathrm{H}), 2.95(\mathrm{dd}$, $J=15.6,7.2 \mathrm{~Hz}, 1 \mathrm{H}), 2.67(\mathrm{dd}, J=15.6,7.2 \mathrm{~Hz}, 1 \mathrm{H}), 2.58-2.53(\mathrm{~m}, 1 \mathrm{H}), 1.60$ (br, $1 \mathrm{H}), 1.14$ $(\mathrm{d}, J=7.2 \mathrm{~Hz}, 3 \mathrm{H}) ;{ }^{13} \mathrm{C} \mathrm{NMR}\left(125 \mathrm{MHz}, \mathrm{CDCl}_{3}\right) \delta 144.7,143.3,128.4,126.6,125.0,124.7$, 77.7, 39.2, 37.9, 13.6.

HRMS (FI) calcd for $\mathrm{C}_{10} \mathrm{H}_{12} \mathrm{O}[\mathrm{M}]^{+}:$148.0888. Found: 148.0900 .

The enantiomeric excess was determined by HPLC analysis (Chiralcel OJ-H, $250 \times 4.6 \mathrm{~mm}$ column, hexane/2-propanol 98:2, $1.0 \mathrm{~mL} / \mathrm{min}, 220 \mathrm{~nm}, 30^{\circ} \mathrm{C},(1 S, 2 S)$ isomer $7.8 \mathrm{~min}$ (minor), $(1 R, 2 R)$ isomer $10.7 \mathrm{~min}$ (major), $(1 S, 2 R)$ and $(1 R, 2 S)$ isomers $7.2 \mathrm{~min}$ or $11.6 \mathrm{~min})$; IR (neat) 3074, 3048, 3022, 2971, 2955, 2923, 2866, 2843, 1478, 1457, 1435, 1342, 1317, 1272, 1217 , 1163, 1104, 1074, 1049, 1021, $738 \mathrm{~cm}^{-1} ;[\alpha]_{\mathrm{D}}{ }^{20}-35.2$ (c 0.89 in $\left.\mathrm{CHCl}_{3}\right) 98 \%$ ee, $96 \%$ de $(1 R, 2 R)\left(\right.$ lit. $^{[7]}[\alpha]_{\mathrm{D}}{ }^{20}+40.5\left(\mathrm{c} 0.5\right.$ in $\left.\mathrm{CHCl}_{3}\right) 97 \%$ ee, $94 \%$ de $\left.(1 S, 2 S)\right)$ 
<smiles>C[C@H]1CCCc2ccccc2[C@@H]1O</smiles>

According to the general procedure (ketone 8c: $0.174 \mathrm{~g}(1.0 \mathrm{mmol})$, cat. $(\boldsymbol{R}, \boldsymbol{R})-4), 0.150 \mathrm{~g}$ of 9c was obtained as a colorless oil ( $85 \%$ yield, $98 \%$ ee, $88 \%$ de).

${ }^{1} \mathrm{H}$ NMR $\left(400 \mathrm{MHz}, \mathrm{CDCl}_{3}\right) \delta 7.32-7.06(\mathrm{~m}, 4 \mathrm{H}), 4.87(\mathrm{~m}, 1 \mathrm{H}), 3.00-2.94(\mathrm{~m}, 1 \mathrm{H}), 2.70-$ $2.63(\mathrm{~m}, 1 \mathrm{H}), 2.10-2.00(\mathrm{~m}, 1 \mathrm{H}), 1.95-1.78(\mathrm{~m}, 3 \mathrm{H}), 1.75-1.50(\mathrm{~m}, 2 \mathrm{H}), 0.84(\mathrm{~d}, J=6.8 \mathrm{~Hz}$, $3 \mathrm{H}) ;{ }^{13} \mathrm{C} \mathrm{NMR}\left(125 \mathrm{MHz}, \mathrm{CDCl}_{3}\right) \delta 142.0,141.2,129.5,127.1,126.8,125.8,78.2,37.7$, 35.6, 35.1, 34.4, 24.8.

HRMS (FI) calcd for $\mathrm{C}_{12} \mathrm{H}_{16} \mathrm{O}[\mathrm{M}]^{+}:$176.1201. Found: 176.1220 .

The enantiomeric excess was determined by HPLC analysis (Chiralcel OJ-H, 250×4.6 mm column, hexane/2-propanol 98:2, $1.0 \mathrm{~mL} / \mathrm{min}, 220 \mathrm{~nm}, 30{ }^{\circ} \mathrm{C},(5 R, 6 R)$ isomer $10.5 \mathrm{~min}$ (major), $(5 S, 6 S)$ isomer $13.3 \mathrm{~min}$ (minor), $(5 S, 6 R)$ and $(5 R, 6 S)$ isomers $11.6 \mathrm{~min}$ or $22.0 \mathrm{~min}$ ); IR (neat) 3019, 2959, 2921, 2852, 1489, 1452, 1381, 1362, 1321, 1302 , 1285, 1207, 1188, $1051,1032,995,976,946,769,747,735 \mathrm{~cm}^{-1}[\alpha]_{\mathrm{D}}^{20}+24.2\left(\mathrm{c} 1.01\right.$ in $\left.\mathrm{CHCl}_{3}\right) 98 \%$ ee , 86\% de $(5 R, 6 R)\left(\right.$ lit. $^{[7]}[\alpha]_{\mathrm{D}}{ }^{23}-115.0\left(\mathrm{c} 0.80\right.$ in $\left.\mathrm{CHCl}_{3}\right) 58 \%$ ee, $66 \%$ de $\left.(5 S, 6 S)\right)$.

Methyl (1R,2R)-1-hydroxy-1,2,3,4-tetrahydronaphthalene-2-carboxylate (9d)<smiles>CC(=O)[C@H]1CCc2ccccc2[C@@H]1O</smiles>

According to the general procedure (ketone 8d: $0.204 \mathrm{~g}(1.0 \mathrm{mmol})$, cat. $(\boldsymbol{R}, \boldsymbol{R})-\mathbf{3}), 0.204 \mathrm{~g}$ of 9d was obtained as a colorless oil (99\% yield, >99.9\% ee, $>99 \%$ de).

${ }^{1} \mathrm{H}$ NMR (400 MHz, $\left.\mathrm{CDCl}_{3}\right) \delta$ 7.40-7.38 (m, 1H), 7.24-7.21 (m, 2H), 7.13-7.11 (m, 1H), $5.03(\mathrm{~m}, 1 \mathrm{H}), 3.77(\mathrm{~s}, 3 \mathrm{H}), 2.99(\mathrm{~d}, J=5.2 \mathrm{~Hz}, 1 \mathrm{H}), 2.95-2.90(\mathrm{~m}, 1 \mathrm{H}), 2.84-2.77(\mathrm{~m}, 2 \mathrm{H})$, 2.24-2.18 (m, 1H), 2.13-2.10 (m, 1H); $\left.{ }^{13} \mathrm{C} \mathrm{NMR} \mathrm{(125} \mathrm{MHz,} \mathrm{CDCl}_{3}\right) \delta$ 174.7, 136.6, 136.0, 129.8, 128.9, 128.1, 126.3, 67.7, 51.9, 45.4, 28.3, 19.8.

HRMS (FI) calcd for $\mathrm{C}_{12} \mathrm{H}_{14} \mathrm{O}_{3}[\mathrm{M}]^{+}:$206.0943. Found: 206.0951.

The enantiomeric excess was determined by HPLC analysis (Chiralcel OJ-H, 250×4.6 mm column, hexane/2-propanol 96:4, $1.0 \mathrm{~mL} / \mathrm{min}, 220 \mathrm{~nm}, 30{ }^{\circ} \mathrm{C},(1 S, 2 S)$ isomer $16.9 \mathrm{~min}$ (minor), (1R,2R) isomer 19.9 min (major)); IR (neat) 3021, 2950, 2839, 1724, 1491, 1454, 1435, 1378, 1308, 1369, 1221, 1175, 158, 1113, 1104, 1060, 1038, 1019, 1008, 961, 948, 901, $773,734 \mathrm{~cm}^{-1} ;[\alpha]_{\mathrm{D}}{ }^{20}+118.2\left(\mathrm{c} 1.55\right.$ in $\left.\mathrm{CHCl}_{3}\right)>99.9 \%$ ee, $>99 \%$ de $(1 R, 2 R)\left(\right.$ lit. $^{[8]}[\alpha]_{\mathrm{D}}+84.2$ (c 2.21 in $\mathrm{EtOH}) 72 \%$ ee $(1 R, 2 R),>98 \%$ de). 
<smiles>O=C1C[C@H]2CCc3ccccc3[C@H]2O1</smiles>

According to the general procedure (ketone 11a: $0.300 \mathrm{~g}(1.47 \mathrm{mmol})$, cat. $(\boldsymbol{R}, \boldsymbol{R})-3), 0.249 \mathrm{~g}$ of 13a was obtained as a colorless oil (90\% yield, $99.7 \%$ ee, $>99 \%$ de).

${ }^{1} \mathrm{H}$ NMR (400 MHz, $\left.\mathrm{CDCl}_{3}\right) \delta$ 7.46-7.44 (m, 1H), 7.29-7.26 (m, 2H), 7.18-7.15 (m, 1H), $5.43(\mathrm{~d}, J=6.0 \mathrm{~Hz}, 1 \mathrm{H}), 2.96-2.73(\mathrm{~m}, 4 \mathrm{H}), 2.42(\mathrm{dd}, J=17.4,2.4 \mathrm{~Hz}, 1 \mathrm{H}), 1.95-1.91(\mathrm{~m}$, 1H), 1.65-1.60 (m, 1H); ${ }^{13} \mathrm{C}$ NMR $\left(125 \mathrm{MHz}, \mathrm{CDCl}_{3}\right) \delta 176.2,137.5,131.1,130.9,128.8$, 128.7, 126.7, 78.5, 36.3, 34.7, 27.7, 25.1.

HRMS (ESI) calcd for $\mathrm{C}_{12} \mathrm{H}_{13} \mathrm{O}_{2}[\mathrm{M}+\mathrm{H}]^{+}:$189.0910. Found: 189.0909.

The enantiomeric excess was determined by HPLC analysis (Chiralcel OD-H, 250×4.6 mm column, hexane/2-propanol 95:5, $1.0 \mathrm{~mL} / \mathrm{min}, 220 \mathrm{~nm}, 30{ }^{\circ} \mathrm{C},(3 \mathrm{aS}, 9 \mathrm{~b} R)$ isomer $17.9 \mathrm{~min}$ (major), (3aR,9bS) isomer $25.7 \mathrm{~min}$ (minor)); IR (neat) 3852, 3500, 3059, 3023, 2930, 2906, 2854, 2844, 1781, 1752, 1737, 1606, 1495, 1456, 1437, 1414, 1376, 1350, 1327, 1291, 1264, 1223, 1194, 1171, 1161, 1084, 1052, 1033, 993, 951, 927, 871, 852, 804, 775, 744, 732, 679 $\mathrm{cm}^{-1} ;[\alpha]_{\mathrm{D}}{ }^{20}-102.6$ (c 2.55 in $\left.\mathrm{CHCl}_{3}\right) 95 \%$ ee, $>99 \%$ de $(3 \mathrm{aS}, 9 \mathrm{bR})\left(\right.$ lit. $^{[9]}[\alpha]_{\mathrm{D}}{ }^{20}-93.0$ (c 1.02 in $\left.\mathrm{CHCl}_{3}\right) 96 \%$ ee $\left.(3 \mathrm{a} S, 9 \mathrm{~b} R)\right)$.

(3aS,9bR)-8-Methoxy-3a,4,5,9b-tetrahydronaphtho[1,2-b]furan-2(3H)-one (13b)<smiles>COc1ccc2c(c1)[C@H]1OC(=O)C[C@H]1CC2</smiles>

According to the general procedure (ketone 11b: $0.234 \mathrm{~g}(1.0 \mathrm{mmol})$, cat. $(\boldsymbol{R}, \boldsymbol{R})-\mathbf{3}), 0.181 \mathrm{~g}$ of $\mathbf{1 3 b}$ was obtained as a white powder ( $83 \%$ yield, $99.4 \%$ ee, $>99 \%$ de).

${ }^{1} \mathrm{H}$ NMR $\left(400 \mathrm{MHz}, \mathrm{CDCl}_{3}\right) \delta 7.07(\mathrm{~d}, J=8.4 \mathrm{~Hz}, 1 \mathrm{H}), 6.96(\mathrm{~m}, 1 \mathrm{H}), 6.87-6.85(\mathrm{~m}, 1 \mathrm{H})$, $5.39(\mathrm{~d}, J=6.0 \mathrm{~Hz}, 1 \mathrm{H}), 3.80(\mathrm{~s}, 3 \mathrm{H}), 2.90(\mathrm{dd}, J=17.6,8.0 \mathrm{~Hz}, 1 \mathrm{H}), 2.79-2.63(\mathrm{~m}, 3 \mathrm{H})$, $2.41(\mathrm{dd}, J=17.6,2.8 \mathrm{~Hz}, 1 \mathrm{H}), 1.94-1.89(\mathrm{~m}, 1 \mathrm{H}), 1.63-1.58(\mathrm{~m}, 1 \mathrm{H}) ;{ }^{13} \mathrm{C}$ NMR $(125 \mathrm{MHz}$, $\left.\mathrm{CDCl}_{3}\right) \delta 176.2,158.2,132.0,129.7,129.5,116.1,114.4,78.6,55.4,36.1,34.6,26.8,25.4$. HRMS (APCI) calcd for $\mathrm{C}_{13} \mathrm{H}_{15} \mathrm{O}_{3}[\mathrm{M}+\mathrm{H}]^{+}:$219.1016. Found: 219.1005.

The enantiomeric excess was determined by HPLC analysis (Chiralcel OD-H, 250×4.6 mm column, hexane/2-propanol 95:5, $0.8 \mathrm{~mL} / \mathrm{min}, 220 \mathrm{~nm}, 30{ }^{\circ} \mathrm{C},(3 \mathrm{a} S, 9 \mathrm{bR})$ isomer $26.8 \mathrm{~min}$ (major), (3aR,9bS) isomer 28.7 min (minor)); IR (neat) 2925, 1759, 1505, 1445, 1429, 1266, $1218,1161,1127,1038,1028,954,935,920,883,828 \mathrm{~cm}^{-1} ;[\alpha]_{\mathrm{D}}{ }^{20}-145.3\left(\mathrm{c} 1.01\right.$ in $\left.\mathrm{CHCl}_{3}\right)$ $99.4 \%$ ee, $>99 \%$ de $(3 \mathrm{a} S, 9 \mathrm{~b} R)$. 
<smiles>O=C1C[C@H]2CCc3ccc(Cl)cc3[C@H]2O1</smiles>

According to the general procedure (ketone 11c: $0.238 \mathrm{~g}(1.0 \mathrm{mmol})$, cat. $(\boldsymbol{R}, \boldsymbol{R})-\mathbf{3}), 0.155 \mathrm{~g}$ of 13c was obtained as a white solid (70\% yield, $99.8 \%$ ee, $>99 \%$ de).

${ }^{1} \mathrm{H} \mathrm{NMR}\left(400 \mathrm{MHz}, \mathrm{CDCl}_{3}\right) \delta 7.45(\mathrm{~d}, J=2.4 \mathrm{~Hz}, 1 \mathrm{H}), 7.27-7.24(\mathrm{~m}, 1 \mathrm{H}), 7.10(\mathrm{~d}, J=8.4$ $\mathrm{Hz}, 1 \mathrm{H}), 5.37(\mathrm{~d}, J=6.0 \mathrm{~Hz}, 1 \mathrm{H}), 2.92(\mathrm{dd}, J=17.5,8.0 \mathrm{~Hz}, 1 \mathrm{H}), 2.83-2.63(\mathrm{~m}, 3 \mathrm{H}), 2.42$ $(\mathrm{dd}, J=17.5,2.4 \mathrm{~Hz}, 1 \mathrm{H}), 1.97-1.92(\mathrm{~m}, 1 \mathrm{H}), 1.62-1.58(\mathrm{~m}, 1 \mathrm{H}) ;{ }^{13} \mathrm{C}$ NMR $(125 \mathrm{MHz}$, $\left.\mathrm{CDCl}_{3}\right) \delta 175.8,135.8,132.9,132.2,130.6,130.0,129.0,77.6,36.0,34.4,27.2,25.0$.

HRMS (APCI) calcd for $\mathrm{C}_{12} \mathrm{H}_{12} \mathrm{ClO}_{2}[\mathrm{M}+\mathrm{H}]^{+}:$223.0520. Found: 223.0509.

The enantiomeric excess was determined by HPLC analysis (Chiralcel OD-H, 250×4.6 mm column, hexane/2-propanol 93:7, $1.0 \mathrm{~mL} / \mathrm{min}, 220 \mathrm{~nm}, 30{ }^{\circ} \mathrm{C},(3 \mathrm{a} S, 9 \mathrm{bR})$ isomer $18.8 \mathrm{~min}$ (major), (3aR,9bS) isomer $20.9 \mathrm{~min}$ (minor)); IR (neat) 2857, 1777, 1756, 1488, 1323, 1224, 1216, 1195, 1168, 1157, 950, 937, 892, 877, 821, $665 \mathrm{~cm}^{-1} ;[\alpha]_{\mathrm{D}}{ }^{20}-153.5\left(\mathrm{c} 1.6\right.$ in $\left.\mathrm{CHCl}_{3}\right)$ $99.6 \%$ ee, $>99 \%$ de $(3 \mathrm{a} S, 9 \mathrm{~b} R)$.

(3aS,9bR)-8-Bromo-3a,4,5,9b-tetrahydronaphtho[1,2-b]furan-2(3H)-one (13d)<smiles>O=C1C[C@H]2CCc3ccc(Br)cc3[C@H]2O1</smiles>

According to the general procedure (ketone 11d: $0.283 \mathrm{~g}(1.0 \mathrm{mmol})$, cat. $(\boldsymbol{R}, \boldsymbol{R})-\mathbf{3}), 0.182 \mathrm{~g}$ of 13d was obtained as a white powder ( $68 \%$ yield, $99.7 \%$ ee, $>99 \% \mathrm{de}$ ).

${ }^{1} \mathrm{H}$ NMR $\left(400 \mathrm{MHz}, \mathrm{CDCl}_{3}\right) \delta 7.60(\mathrm{~d}, J=2.0 \mathrm{~Hz}, 1 \mathrm{H}), 7.40(\mathrm{dd}, J=8.3,2.0 \mathrm{~Hz}, 1 \mathrm{H}), 7.04$ $(\mathrm{d}, J=8.4 \mathrm{~Hz}, 1 \mathrm{H}), 5.37(\mathrm{~d}, J=6.0 \mathrm{~Hz}, 1 \mathrm{H}), 2.92(\mathrm{dd}, J=17.5,8.4 \mathrm{~Hz}, 1 \mathrm{H}), 2.82-2.62(\mathrm{~m}$, $3 \mathrm{H}), 2.41(\mathrm{dd}, J=17.5,2.4 \mathrm{~Hz}, 1 \mathrm{H}), 1.97-1.92(\mathrm{~m}, 1 \mathrm{H}), 1.65-1.56(\mathrm{~m}, 1 \mathrm{H}) ;{ }^{13} \mathrm{C}$ NMR $(125$ $\left.\mathrm{MHz}, \mathrm{CDCl}_{3}\right) \delta 175.7,136.3,133.5,133.3,131.9,130.3,120.1,77.5,36.0,34.4,27.2,25.0$. HRMS (ESI) calcd for $\mathrm{C}_{12} \mathrm{H}_{12} \mathrm{BrO}_{2}[\mathrm{M}+\mathrm{H}]^{+}:$267.0015. Found: 267.0012.

The enantiomeric excess was determined by HPLC analysis (Chiralcel OD-H, 250×4.6 mm column, hexane/2-propanol 94:6, $1.0 \mathrm{~mL} / \mathrm{min}, 220 \mathrm{~nm}, 35^{\circ} \mathrm{C},(3 \mathrm{aS}, 9 \mathrm{bR})$ isomer $18.9 \mathrm{~min}$ (major), (3aR,9bS) isomer $21.6 \mathrm{~min}$ (minor)); IR (neat) 3072, 3048, 2937, 2915, 2856, 1767, 1593, 1482, 1450, 1436, 1418, 1372, 1344, 1319, 1296, 1261, 1220, 1192, 1171, 1075, 1051, $1035,990,951,930,896,877,828,770,714,674 \mathrm{~cm}^{-1} ;[\alpha]_{\mathrm{D}}^{20}-145.7$ (c 2.5 in $\left.\mathrm{CHCl}_{3}\right) 99.7 \%$ ee, $>99 \%$ de $(3 \mathrm{a} S, 9 \mathrm{~b} R)$. 
<smiles>COc1ccc2c(c1Br)[C@H]1OC(=O)C[C@H]1CC2</smiles>

According to the general procedure (ketone 11e: $0.313 \mathrm{~g}(1.0 \mathrm{mmol})$, cat. $(\boldsymbol{R}, \boldsymbol{R})-3), 0.182 \mathrm{~g}$ of 13e was obtained as a light gray powder (65\% yield, $99.6 \%$ ee, $>99 \%$ de).

${ }^{1} \mathrm{H} \mathrm{NMR}\left(500 \mathrm{MHz}, \mathrm{CDCl}_{3}\right) \delta 7.10(\mathrm{~d}, J=8.5 \mathrm{~Hz}, 1 \mathrm{H}), 6.90(\mathrm{~d}, J=8.5,1 \mathrm{H}), 5.63(\mathrm{~d}, J=5.2$ $\mathrm{Hz}, 1 \mathrm{H}), 3.90(\mathrm{~s}, 3 \mathrm{H}), 2.97(\mathrm{dd}, J=17.2,7.5 \mathrm{~Hz}, 1 \mathrm{H}), 2.87-2.82(\mathrm{~m}, 1 \mathrm{H}), 2.75-2.66(\mathrm{~m}, 2 \mathrm{H})$, $2.43(\mathrm{~d}, J=17.2,1 \mathrm{H}), 1.90-1.84(\mathrm{~m}, 1 \mathrm{H}), 1.65-1.58(\mathrm{~m}, 1 \mathrm{H}) ;{ }^{13} \mathrm{C}$ NMR $\left(125 \mathrm{MHz}, \mathrm{CDCl}_{3}\right)$ $\delta 175.9,154.8,132.0,131.7,128.5,116.6,113.0,77.9,56.6,36.8,35.1,27.8,24.9$.

HRMS (ESI) calcd for $\mathrm{C}_{13} \mathrm{H}_{13} \mathrm{BrO}_{3} \mathrm{Na}[\mathrm{M}+\mathrm{Na}]^{+}: 318.9940$. Found: 318.9945.

The enantiomeric excess was determined by HPLC analysis (Chiralcel OD-H, 250×4.6 mm column, hexane/2-propanol 88:12, $1.0 \mathrm{~mL} / \mathrm{min}, 220 \mathrm{~nm}, 35^{\circ} \mathrm{C},(3 \mathrm{a} R, 9 \mathrm{bS})$ isomer $18.7 \mathrm{~min}$ (minor), (3aS,9bR) isomer 26.4 min (major)); IR (neat) 3308, 3160, 3012, 2936, 2910, 2850, 2838, 1778, 1598, 1558, 1479, 1451, 1435, 1417, 1347, 1322, 1308, 1296, 1280, 1253, 1236, $1217,1205,1158,1132,1096,1065,1032,999,962,927,891,869,813,762,735,710,667$ $\mathrm{cm}^{-1} ;[\alpha]_{\mathrm{D}}{ }^{20}-146.8$ (c 1.3 in $\left.\mathrm{CHCl}_{3}\right) 99.6 \%$ ee, $>99 \%$ de $(3 \mathrm{aS}, 9 \mathrm{~b} R)$.

(3aS,9bR)-7-Hydroxy-3a,4,5,9b-tetrahydronaphtho[1,2-b]furan-2(3H)-one (13f)<smiles>O=C1C[C@H]2CCc3cc(O)ccc3[C@H]2O1</smiles>

According to the general procedure (ketone 11f: $0.220 \mathrm{~g}(1.0 \mathrm{mmol})$, cat. $(\boldsymbol{R}, \boldsymbol{R})-3), 0.143 \mathrm{~g}$ of $\mathbf{1 3 f}$ was obtained as a white solid (70\% yield, $93 \%$ ee, $>99 \% \mathrm{de})$.

${ }^{1} \mathrm{H}$ NMR $\left(400 \mathrm{MHz}, \mathrm{CD}_{3} \mathrm{OD}\right) \delta 7.19(\mathrm{~d}, J=8.4 \mathrm{~Hz}, 1 \mathrm{H}), 6.56(\mathrm{dd}, J=8.4,2.6 \mathrm{~Hz}, 1 \mathrm{H}), 6.56$ $(\mathrm{d}, J=2.6 \mathrm{~Hz}, 1 \mathrm{H}), 5.38(\mathrm{~d}, J=5.6 \mathrm{~Hz}, 1 \mathrm{H}), 2.96(\mathrm{dd}, J=17.6,7.6 \mathrm{~Hz}, 1 \mathrm{H}), 2.72-2.63(\mathrm{~m}$, $3 \mathrm{H}), 2.31(\mathrm{dd}, J=17.6,1.6 \mathrm{~Hz}, 1 \mathrm{H}), 1.86-1.79(\mathrm{~m}, 1 \mathrm{H}), 1.48-1.39(\mathrm{~m}, 1 \mathrm{H}) ;{ }^{13} \mathrm{C}$ NMR $(125$ $\left.\mathrm{MHz}, \mathrm{CD}_{3} \mathrm{OD}\right) \delta 180.0,159.5,141.3,133.8,124.0,116.1,115.5,81.4,38.2,36.6,29.5,26.7$. HRMS (APCI) calcd for $\mathrm{C}_{12} \mathrm{H}_{13} \mathrm{O}_{3}[\mathrm{M}+\mathrm{H}]^{+}:$205.0859. Found: 205.0843.

The enantiomeric excess was determined by HPLC analysis (Chiralpak AD-H, 250×4.6 mm column, hexane/2-propanol 85:15, $1.0 \mathrm{~mL} / \mathrm{min}, 220 \mathrm{~nm}, 40^{\circ} \mathrm{C},(3 \mathrm{a} S, 9 \mathrm{bR})$ isomer $18.0 \mathrm{~min}$ (major), (3aR,9bS) isomer 19.4 min (minor)); IR (neat) 3262, 2996, 2940, 2910, 2854, 2428, 1736, 1618, 1581, 1505, 1473, 1451, 1435, 1422, 1372, 1350, 1322, 1295, 1281, 1257, 1233, $1189,1164,1121,1023,997,948,929,911,882,864,820,793,738,682 \mathrm{~cm}^{-1} ;[\alpha]_{\mathrm{D}}{ }^{20}-30.0$ (c 0.51 in $\mathrm{MeOH}) 93 \%$ ee, $>99 \%$ de $(3 \mathrm{aS}, 9 \mathrm{~b} R)$. 
<smiles>O=C1C[C@H]2Cc3ccccc3[C@H]2O1</smiles>

According to the general procedure (ketone 11g: $0.190 \mathrm{~g}(1.0 \mathrm{mmol})$, cat. $(\boldsymbol{R}, \boldsymbol{R})-3), 0.148 \mathrm{~g}$ of $\mathbf{1 3 g}$ was obtained as a white solid (85\% yield, $99 \%$ ee, $>99 \% \mathrm{de}$ ).

${ }^{1} \mathrm{H}$ NMR $\left(400 \mathrm{MHz}, \mathrm{CDCl}_{3}\right) \delta$ 7.49-7.47 (m, 1H), 7.35-7.26 (m, 3H), $5.88(\mathrm{~d}, J=6.8 \mathrm{~Hz}$, $1 \mathrm{H}), 3.36-3.28(\mathrm{~m}, 2 \mathrm{H}), 2.94-2.86(\mathrm{~m}, 2 \mathrm{H}), 2.38(\mathrm{dd}, J=18.0,5.6 \mathrm{~Hz}, 1 \mathrm{H}) ;{ }^{13} \mathrm{C} \mathrm{NMR}(125$ $\left.\mathrm{MHz}, \mathrm{CDCl}_{3}\right) \delta 176.9,142.5,138.7,129.9,127.5,126.3,125.3,87.6,37.9,37.3,35.6$.

HRMS (ESI) calcd for $\mathrm{C}_{11} \mathrm{H}_{11} \mathrm{O}_{2}[\mathrm{M}+\mathrm{H}]^{+}:$175.0754. Found: 175.0755 .

The enantiomeric excess was determined by HPLC analysis (Chiralcel OJ-H, 250×4.6 mm column, hexane/2-propanol 95:5, $1.0 \mathrm{~mL} / \mathrm{min}, 220 \mathrm{~nm}, 30{ }^{\circ} \mathrm{C},(3 \mathrm{a} R, 8 \mathrm{bS})$ isomer $29.6 \mathrm{~min}$ (minor), (3aS,8bR) isomer 35.3 min (major)); IR (neat) 2933, 1763, 1482, 1462, 1443, 1417, 1347, 1313, 1194, 1161, 1094, 1016, 950, 934, 856, 758, $741 \mathrm{~cm}^{-1} ;[\alpha]_{\mathrm{D}}{ }^{20}-97.5(\mathrm{c} 0.63$ in $\left.\mathrm{CHCl}_{3}\right) 99 \%$ ee, $>99 \%$ de $(3 \mathrm{aS}, 8 \mathrm{bR})$. (lit. ${ }^{[10]}[\alpha]_{\mathrm{D}}{ }^{20}-109\left(\mathrm{c} 0.985\right.$ in $\left.\mathrm{CHCl}_{3}\right) 99 \%$ ee, $99 \%$ de $(3 \mathrm{a} S, 8 \mathrm{~b} R))$.

(3aS,8bR)-6,7-Dimethoxy-3,3a,4,8b-tetrahydro-2H-indeno[1,2-b]furan-2-one (13h)<smiles>COc1cc2c(cc1OC)[C@H]1OC(=O)C[C@H]1C2</smiles>

According to the general procedure (ketone 11h: $0.250 \mathrm{~g}(1.0 \mathrm{mmol})$, cat. $(\boldsymbol{R}, \boldsymbol{R})-\mathbf{3}), 0.183 \mathrm{~g}$ of $\mathbf{1 3 h}$ was obtained as a white powder (78\% yield, $94 \%$ ee, $>99 \%$ de).

${ }^{1} \mathrm{H} \mathrm{NMR}\left(400 \mathrm{MHz}, \mathrm{CDCl}_{3}\right) \delta 6.96(\mathrm{~s}, 1 \mathrm{H}), 6.74(\mathrm{~s}, 1 \mathrm{H}), 5.85(\mathrm{~d}, J=7.2 \mathrm{~Hz}, 1 \mathrm{H}), 3.89(\mathrm{~s}$, $3 \mathrm{H}), 3.88(\mathrm{~s}, 3 \mathrm{H}), 3.39-3.36(\mathrm{~m}, 1 \mathrm{H}), 3.26(\mathrm{dd}, J=16.0,8.4 \mathrm{~Hz}, 1 \mathrm{H}), 2.90(\mathrm{dd}, J=18.4,10.0$ $\mathrm{Hz}, 1 \mathrm{H}), 2.82(\mathrm{dd}, J=16.0,3.2 \mathrm{~Hz}, 1 \mathrm{H}), 2.40(\mathrm{dd}, J=18.4,6.0 \mathrm{~Hz}, 1 \mathrm{H}) ;{ }^{13} \mathrm{C} \mathrm{NMR}(125 \mathrm{MHz}$, $\left.\mathrm{CDCl}_{3}\right) \delta 177.0,151.0,149.1,134.5,130.5,108.1,107.3,88.2,56.00,55.95,38.0,37.7,35.9$. HRMS (APCI) calcd for $\mathrm{C}_{13} \mathrm{H}_{15} \mathrm{O}_{4}[\mathrm{M}+\mathrm{H}]^{+}:$235.0965. Found: 235.0956.

The enantiomeric excess was determined by HPLC analysis (Chiralcel OD-H, 250×4.6 mm column, hexane/2-propanol 90:10, $1.0 \mathrm{~mL} / \mathrm{min}, 220 \mathrm{~nm}, 30^{\circ} \mathrm{C},(3 \mathrm{aS}, 8 \mathrm{~b} R)$ isomer $24.6 \mathrm{~min}$ (major), (3aR,8bS) isomer 28.7 min (minor)); IR (neat) 1760, 1504, 1452, 1317, 1297, 1229, $1202,1191,1156,1103,997,988,936 \mathrm{~cm}^{-1} ;[\alpha]_{\mathrm{D}}{ }^{20}-100.25$ (c 1.03 in $\left.\mathrm{CHCl}_{3}\right) 94 \%$ ee, $>99 \%$ $\mathrm{de}(3 \mathrm{a} S, 8 \mathrm{~b} R)$. 
<smiles>O=C1C[C@H]2Cc3ccc(F)cc3[C@H]2O1</smiles>

According to the general procedure (ketone 11i: $0.208 \mathrm{~g}(1.0 \mathrm{mmol})$, cat. $(\boldsymbol{R}, \boldsymbol{R})-\mathbf{3}), 0.146 \mathrm{~g}$ of $13 \mathrm{i}$ was obtained as a off-white solid (76\% yield, $96 \%$ ee, $>99 \%$ de).

${ }^{1} \mathrm{H}$ NMR $\left(400 \mathrm{MHz}, \mathrm{CDCl}_{3}\right) \delta$ 7.24-7.20 (m, 1H), 7.17-7.14 (m, 1H), 7.08-7.03 (m, 1H), $5.85(\mathrm{~d}, J=7.2 \mathrm{~Hz}, 1 \mathrm{H}), 3.44-3.40(\mathrm{~m}, 1 \mathrm{H}), 3.28(\mathrm{dd}, J=16.2,8.4 \mathrm{~Hz}, 1 \mathrm{H}), 2.94-2.82(\mathrm{~m}$, $2 \mathrm{H}), 2.38(\mathrm{dd}, J=18.4,6.0 \mathrm{~Hz}, 1 \mathrm{H}) ;{ }^{13} \mathrm{C} \mathrm{NMR}\left(125 \mathrm{MHz}, \mathrm{CDCl}_{3}\right) \delta 176.5,162.4(\mathrm{~d}, J=244$ $\mathrm{Hz}), 140.6(\mathrm{~d}, J=8.8 \mathrm{~Hz}), 137.7(\mathrm{~d}, J=2.5 \mathrm{~Hz}), 126.5(\mathrm{~d}, J=7.5 \mathrm{~Hz}), 117.3(\mathrm{~d}, J=22.5 \mathrm{~Hz})$, $112.9(\mathrm{~d}, J=22.5 \mathrm{~Hz}), 87.0(\mathrm{~d}, J=2.5 \mathrm{~Hz}), 37.9,37.1,35.4$.

HRMS (APCI) calcd for $\mathrm{C}_{11} \mathrm{H}_{10} \mathrm{FO}_{2}[\mathrm{M}+\mathrm{H}]^{+}:$193.0659. Found: 193.0645.

The enantiomeric excess was determined by HPLC analysis (Chiralcel OJ-H, $250 \times 4.6 \mathrm{~mm}$ column, hexane/2-propanol 93:7, $1.0 \mathrm{~mL} / \mathrm{min}, 220 \mathrm{~nm}, 30{ }^{\circ} \mathrm{C},(3 \mathrm{a} R, 8 \mathrm{bS})$ isomer $25.9 \mathrm{~min}$ (minor), (3aS,8bR) isomer 27.5 min (major)); IR (neat) 1752, 1487, 1448, 1296, 1245, 1180, 1133, 1096, 1002, 927, 887, 821, $751 \mathrm{~cm}^{-1}$; $[\alpha]_{\mathrm{D}}{ }^{20}-83.3$ (c 1.14 in $\left.\mathrm{CHCl}_{3}\right) 96 \%$ ee, $>99 \%$ de $(3 \mathrm{a} S, 8 \mathrm{~b} R)$.

(3aS,8bR)-7-Chloro-3,3a,4,8b-tetrahydro-2H-indeno[1,2-b]furan-2-one (13j)<smiles>O=C1C[C@H]2Cc3ccc(Cl)cc3[C@H]2O1</smiles>

According to the general procedure (ketone 11j: $0.224 \mathrm{~g}(1.0 \mathrm{mmol})$, cat. $(\boldsymbol{R}, \boldsymbol{R})-\mathbf{3}), 0.159 \mathrm{~g}$ of 13j was obtained as a off-white solid (76\% yield, $96 \%$ ee, $>99 \%$ de).

${ }^{1} \mathrm{H}$ NMR $\left(400 \mathrm{MHz}, \mathrm{CDCl}_{3}\right) \delta 7.46(\mathrm{~s}, 1 \mathrm{H}), 7.32(\mathrm{~d}, J=8.2 \mathrm{~Hz}, 1 \mathrm{H}), 7.20(\mathrm{~d}, J=8.2 \mathrm{~Hz}$, $1 \mathrm{H}), 5.84(\mathrm{~d}, J=7.2 \mathrm{~Hz}, 1 \mathrm{H}), 3.43-3.38(\mathrm{~m}, 1 \mathrm{H}), 3.28(\mathrm{dd}, J=16.6,8.4 \mathrm{~Hz}, 1 \mathrm{H}), 2.95-2.84$ $(\mathrm{m}, 2 \mathrm{H}), 2.39(\mathrm{dd}, J=18.2,5.6 \mathrm{~Hz}, 1 \mathrm{H}) ;{ }^{13} \mathrm{C} \mathrm{NMR}\left(125 \mathrm{MHz}, \mathrm{CDCl}_{3}\right) \delta 176.4,140.8,140.6$, $133.3,130.2,126.5,126.5,86.8,37.7,37.4,35.4$.

HRMS (ESI) calcd for $\mathrm{C}_{11} \mathrm{H}_{10} \mathrm{ClO}_{2}[\mathrm{M}+\mathrm{H}]^{+}:$209.0364. Found: 209.0361.

The enantiomeric excess was determined by HPLC analysis (Chiralpak AD-H, $250 \times 4.6 \mathrm{~mm}$ column, hexane/2-propanol 94:6, $1.0 \mathrm{~mL} / \mathrm{min}, 220 \mathrm{~nm}, 35^{\circ} \mathrm{C},(3 \mathrm{a} R, 8 \mathrm{bS})$ isomer $16.3 \mathrm{~min}$ (minor), (3aS,8bR) isomer $21.0 \mathrm{~min}$ (major)); IR (neat) 2925, 1759, 1473, 1440, 1418, 1349, $1296,1249,1165,1107,1071,1014,963,881,858,816,685 \mathrm{~cm}^{-1}$; $[\alpha]_{\mathrm{D}}{ }^{20}-154.8(\mathrm{c} 1.07$ in $\left.\mathrm{CHCl}_{3}\right) 96 \%$ ee $(3 \mathrm{aS}, 8 \mathrm{~b} R),>99 \%$ de. 
<smiles>O=C1C[C@H]2CCCc3ccccc3[C@H]2O1</smiles>

According to the general procedure (ketone 11k: $0.218 \mathrm{~g}(1.0 \mathrm{mmol})$, cat. $(\boldsymbol{R}, \boldsymbol{R})-\mathbf{4}), 0.172 \mathrm{~g}$ of 13k was obtained as a colorless oil ( $85 \%$ yield, $96 \%$ ee, $80 \% \mathrm{de})$.

${ }^{1} \mathrm{H}$ NMR $\left(400 \mathrm{MHz}, \mathrm{CDCl}_{3}\right) \delta$ 7.34-7.25 (m, 3H), 7.14-7.12 (m, 1H), $5.85(\mathrm{~d}, J=9.2 \mathrm{~Hz}$, 1H), 2.85-2.60 (m, 4H), 2.10-2.05 (m, 1H), 1.80-1.60 (m, 3H), 1.00-0.85 (m, 1H); ${ }^{13} \mathrm{C} \mathrm{NMR}$ $\left(125 \mathrm{MHz} \mathrm{CDCl}_{3}\right) \delta 176.5,135.6,135.4,128.6,128.1,126.8,123.2,81.1,36.3,34.0,30.2$, $27.9,22.8$.

HRMS (FI) calcd for $\mathrm{C}_{13} \mathrm{H}_{14} \mathrm{O}_{2}[\mathrm{M}]^{+}:$202.0994. Found: 202.1009.

The enantiomeric excess was determined by HPLC analysis (Chiralcel OD-H, $250 \times 4.6 \mathrm{~mm}$ column, hexane/2-propanol 90:10, $1.0 \mathrm{~mL} / \mathrm{min}, 220 \mathrm{~nm}, 40^{\circ} \mathrm{C},(3 \mathrm{a} S, 10 \mathrm{~b} R)$ isomer $9.0 \mathrm{~min}$ (major), $(3 \mathrm{a} R, 10 \mathrm{bS})$ isomer $12.0 \mathrm{~min}$ (minor), $(3 \mathrm{a} S, 10 \mathrm{bS})$ and $(3 \mathrm{a} R, 10 \mathrm{~b} R)$ isomers $10.3 \mathrm{~min}$ and 11.1 min); IR (neat) 2929, 2860, 1775, 1453, 1336, 1286, 1180, 1167, 1045, 1029, 1005, $754,734 \mathrm{~cm}^{-1} ;[\alpha]_{\mathrm{D}}{ }^{20}+45.3\left(\mathrm{c} 1.02\right.$ in $\left.\mathrm{CHCl}_{3}\right) 96 \%$ ee, $80 \%$ de $(3 \mathrm{aS}, 10 \mathrm{~b} R)$.

(4aS,10bR)-3,4,4a,5,6,10b-Hexahydro-2H-benzo[h]chromen-2-one (131)<smiles>O=C1CC[C@H]2CCc3ccccc3[C@H]2O1</smiles>

According to the general procedure (ketone 11l: $0.327 \mathrm{~g}(1.5 \mathrm{mmol})$, cat. $(\boldsymbol{R}, \boldsymbol{R})-3), 0.212 \mathrm{~g}$ of 131 was obtained as a off-white solid (70\% yield, $>99.9 \%$ ee, $>99 \%$ de).

${ }^{1} \mathrm{H}$ NMR $\left(400 \mathrm{MHz}, \mathrm{CDCl}_{3}\right) \delta$ 7.44-7.42 (m, 1H), 7.28-7.20 (m, 2H), 7.15-7.13 (m, 1H), $5.31(\mathrm{~d}, J=4.4 \mathrm{~Hz}, 1 \mathrm{H}), 2.95-2.75(\mathrm{~m}, 2 \mathrm{H}), 2.63-2.50(\mathrm{~m}, 2 \mathrm{H}), 2.35-2.19(\mathrm{~m}, 2 \mathrm{H}), 1.87-$ $1.70(\mathrm{~m}, 3 \mathrm{H}) ;{ }^{13} \mathrm{C} \mathrm{NMR}\left(125 \mathrm{MHz}, \mathrm{CDCl}_{3}\right) \delta 171.7,136.7,132.8,130.4,128.7,128.6,126.3$, 77.8, 31.4, 28.1, 27.6, 24.2, 24.1.

HRMS (ESI) calcd for $\mathrm{C}_{13} \mathrm{H}_{15} \mathrm{O}_{2}[\mathrm{M}+\mathrm{H}]^{+}:$203.1067. Found: 203.1070.

The enantiomeric excess was determined by HPLC analysis (Chiralcel OD-H, 250×4.6 mm column, hexane/2-propanol 95:5, $1.0 \mathrm{~mL} / \mathrm{min}, 220 \mathrm{~nm}, 35^{\circ} \mathrm{C},(4 \mathrm{a} S, 10 \mathrm{bR})$ isomer $23.7 \mathrm{~min}$ (major), (4aR,10bS) isomer $42.7 \mathrm{~min}$ (minor)); IR (neat) 2928, 1726, 1456, 1338, 1245, 1188, $1172,1096,1052,1037,1013,961,945,742 \mathrm{~cm}^{-1}$; $[\alpha]_{\mathrm{D}}{ }^{20}-59.8\left(\mathrm{c} 1.39\right.$ in $\left.\mathrm{CHCl}_{3}\right)>99.9 \%$ ee, $>99 \%$ de $(4 \mathrm{a} S, 10 \mathrm{~b} R)$. 
<smiles>COc1ccc2c(c1)[C@H]1OC(=O)CC[C@H]1CC2</smiles>

According to the general procedure (ketone 11m: $0.248 \mathrm{~g}(1.0 \mathrm{mmol})$, cat. $(\boldsymbol{R}, \boldsymbol{R})-3), 0.158 \mathrm{~g}$ of 13m was obtained as an off-white solid (68\% yield, $>99.9 \%$ ee, $>99 \%$ de).

${ }^{1} \mathrm{H}$ NMR $\left(400 \mathrm{MHz}, \mathrm{CDCl}_{3}\right) \delta 7.05(\mathrm{~d}, J=8.4 \mathrm{~Hz}, 1 \mathrm{H}), 6.96(\mathrm{~d}, J=2.8 \mathrm{~Hz}, 1 \mathrm{H}), 6.86-6.83$ $(\mathrm{m}, 1 \mathrm{H}), 5.29(\mathrm{~d}, J=4.0 \mathrm{~Hz}, 1 \mathrm{H}), 3.79(\mathrm{~s}, 3 \mathrm{H}), 2.86-2.81(\mathrm{~m}, 1 \mathrm{H}), 2.76-2.72(\mathrm{~m}, 1 \mathrm{H}), 2.61-$ $2.51(\mathrm{~m}, 2 \mathrm{H}), 2.31-2.28(\mathrm{~m}, 1 \mathrm{H}), 2.22(\mathrm{ddd}, J=14.0,14.0,8.2 \mathrm{~Hz}, 1 \mathrm{H}), 1.85-1.67(\mathrm{~m}, 3 \mathrm{H})$; ${ }^{13} \mathrm{C} \mathrm{NMR}\left(125 \mathrm{MHz}, \mathrm{CDCl}_{3}\right) \delta 171.7,158.0,133.8,129.8,128.7,116.0,113.8,78.1,55.4$, 31.4, 27.8, 27.2, 24.5, 24.0.

HRMS (APCI) calcd for $\mathrm{C}_{14} \mathrm{H}_{17} \mathrm{O}_{3}[\mathrm{M}+\mathrm{H}]^{+}:$233.1172. Found: 233.1161.

The enantiomeric excess was determined by HPLC analysis (Chiralcel OJ-3, 250×4.6 mm column, hexane/2-propanol 94:6, $1.0 \mathrm{~mL} / \mathrm{min}, 220 \mathrm{~nm}, 40{ }^{\circ} \mathrm{C},(4 \mathrm{a} S, 10 \mathrm{~b} R)$ isomer $24.5 \mathrm{~min}$ (major), $(4 \mathrm{a} R, 10 \mathrm{bS}$ ) isomer $26.3 \mathrm{~min}$ (minor)); IR (neat) 3005, 2951, 2930, 2871, 2840, 1728, 1614, 1582, 1504, 1454, 1440, 1430, 1352, 1330, 1292, 12634, 1239, 1216, 1180, 1152, 1127 , 1105, 1049, 1034, 1014, 968, 947, 925, 883, 868, 842, 816, 795, 768, 738, 714, $662 \mathrm{~cm}^{-1}$; $[\alpha]_{\mathrm{D}}^{20}-106.4\left(\mathrm{c} 0.75\right.$ in $\left.\mathrm{CHCl}_{3}\right)>99.9 \%$ ee, $>99 \%$ de $(4 \mathrm{aS}, 10 \mathrm{~b} R)$.

(4aS,10bR)-9-Chloro-3,4,4a,5,6,10b-hexahydro-2H-benzo[h]chromen-2-one (13n)<smiles>O=C1CC[C@H]2CCc3ccc(Cl)cc3[C@H]2O1</smiles>

According to the general procedure (ketone 11n: $0.253 \mathrm{~g}(1.0 \mathrm{mmol})$, cat. $(\boldsymbol{R}, \boldsymbol{R})-\mathbf{3}), 0.161 \mathrm{~g}$ of 13n was obtained as a colorless oil (68\% yield, $99.7 \%$ ee, $>99 \%$ de).

${ }^{1} \mathrm{H} \mathrm{NMR}\left(400 \mathrm{MHz}, \mathrm{CDCl}_{3}\right) \delta 7.43(\mathrm{~d}, J=2.4 \mathrm{~Hz}, 1 \mathrm{H}), 7.23(\mathrm{dd}, J=8.0,2.4 \mathrm{~Hz}, 1 \mathrm{H}), 7.08$ $(\mathrm{d}, J=8.0 \mathrm{~Hz}, 1 \mathrm{H}), 5.27(\mathrm{~d}, J=4.0 \mathrm{~Hz}, 1 \mathrm{H}), 2.89-2.84(\mathrm{~m}, 1 \mathrm{H}), 2.79-2.63(\mathrm{~m}, 1 \mathrm{H}), 2.61-$ $2.51(\mathrm{~m}, 2 \mathrm{H}), 2.31-2.20(\mathrm{~m}, 2 \mathrm{H}), 1.85-1.70(\mathrm{~m}, 3 \mathrm{H}) ;{ }^{13} \mathrm{C} \mathrm{NMR}\left(125 \mathrm{MHz}, \mathrm{CDCl}_{3}\right) \delta 171.2$, 135.1, 134.6, 131.9, 130.14, 130.07, 128.8, 77.3, 31.2, 27.7, 27.6, 24.1, 23.9.

HRMS (APCI) calcd for $\mathrm{C}_{13} \mathrm{H}_{14} \mathrm{ClO}_{2}[\mathrm{M}+\mathrm{H}]^{+}:$: 237.0677. Found: 237.0655 .

The enantiomeric excess was determined by HPLC analysis (Chiralcel OD-H, 250×4.6 mm column, hexane/2-propanol 94:6, $1.0 \mathrm{~mL} / \mathrm{min}, 220 \mathrm{~nm}, 40^{\circ} \mathrm{C},(4 \mathrm{a} S, 10 \mathrm{~b} R)$ isomer $16.1 \mathrm{~min}$ (major), $(4 \mathrm{a} R, 10 \mathrm{bS})$ isomer $21.1 \mathrm{~min}$ (minor)); IR (neat) 2939, 2872, 1739, 1599, 1576, 1483, 1456, 1430, 1371, 1356, 1326, 1290, 1247, 1184, 1168, 1148, 1127, 1100, 1086, 1054, 1011, 966, 930, 874, 844, 816, 766, 720, 705, $667 \mathrm{~cm}^{-1}$; $[\alpha]_{\mathrm{D}}{ }^{20}-99.9$ (c 0.28 in $\left.\mathrm{CHCl}_{3}\right) 99.7 \%$ ee, $>99 \%$ de $(4 \mathrm{a} S, 10 \mathrm{~b} R)$. 
<smiles>O=C1C[C@H]2c3ccccc3CC[C@H]2O1</smiles>

According to the general procedure (ketone 11o: $0.204 \mathrm{~g}(1.0 \mathrm{mmol})$, cat. $(\boldsymbol{R}, \boldsymbol{R})-3), 0.169 \mathrm{~g}$ of 130 was obtained as a colorless oil (90\% yield, $97 \%$ ee, $>99 \%$ de).

${ }^{1} \mathrm{H}$ NMR (400 MHz, $\mathrm{CDCl}_{3}$ ) $\delta 7.26-7.10(\mathrm{~m}, 4 \mathrm{H}), 4.98$ (ddd, $\left.J=6.8,6.8,3.2 \mathrm{~Hz}, 1 \mathrm{H}\right), 3.81$ (m, 1H), 3.09 (dd, $J=17.6,9.6 \mathrm{~Hz}, 1 \mathrm{H}), 2.92-2.87(\mathrm{~m}, 1 \mathrm{H}), 2.73-2.67(\mathrm{~m}, 1 \mathrm{H}), 2.57$ (dd, $J$ $=17.6,5.6 \mathrm{~Hz}, 1 \mathrm{H}), 2.23-2.15(\mathrm{~m}, 1 \mathrm{H}), 2.03-1.95(\mathrm{~m}, 1 \mathrm{H}) ;{ }^{13} \mathrm{C} \mathrm{NMR}\left(125 \mathrm{MHz}, \mathrm{CDCl}_{3}\right) \delta$ $176.3,135.9,135.5,129.0,128.8,127.0,126.8,78.7,37.7,37.6,26.4,24.5$.

HRMS (ESI) calcd for $\mathrm{C}_{12} \mathrm{H}_{13} \mathrm{O}_{2}[\mathrm{M}+\mathrm{H}]^{+}:$189.0910. Found: 189.0910 .

The enantiomeric excess was determined by HPLC analysis (Chiralcel OJ-H, 250×4.6 mm column, hexane/2-propanol 95:5, $1.0 \mathrm{~mL} / \mathrm{min}, 220 \mathrm{~nm}, 30{ }^{\circ} \mathrm{C},(3 \mathrm{a} R, 9 \mathrm{bS})$ isomer $28.1 \mathrm{~min}$ (major), (3aS,9bR) isomer $36.5 \mathrm{~min}$ (minor)); IR (neat) 2962, 2941, 1771, 1488, 1450, 1422, $1353,1332,1248,1184,1151,1132,1110,1024,892,772,747 \mathrm{~cm}^{-1} ;[\alpha]_{\mathrm{D}}{ }^{20}-163.6(\mathrm{c} 0.99$ in $\left.\mathrm{CHCl}_{3}\right) 97 \%$ ee, $>99 \%$ de $(3 \mathrm{aR}, 9 \mathrm{bS})$. (lit. ${ }^{[11]}[\alpha]_{\mathrm{D}}{ }^{23}+118.4$ (c 0.11 in $\left.\mathrm{CHCl}_{3}\right) 76 \%$ ee $(3 \mathrm{a} R, 9 \mathrm{~b} S))$. In the reported works on the synthesis of $\mathbf{1 3 0}$, the dextrorotatory product has been assigned as the $(3 \mathrm{a} R, 9 \mathrm{~b} S)$-configuration; however, the obtained levorotatory isomer was determined to be $(3 \mathrm{a} R, 9 \mathrm{~b} S)$ by its delivatization to $(1 R, 3 \mathrm{a} R, 9 \mathrm{~b} S)-\mathbf{1 5} \mathbf{a}$.

\section{$(3 \mathrm{a} R, 9 \mathrm{~b} S)-6-$ Methoxy-3a,4,5,9b-tetrahydronaphtho[2,1-b]furan-2(1H)-one (13p)}<smiles>COc1cccc2c1CC[C@@H]1OC(=O)C[C@H]21</smiles>

According to the general procedure (ketone 11p: $0.234 \mathrm{~g}(1.0 \mathrm{mmol})$, cat. $(\boldsymbol{R}, \boldsymbol{R})-\mathbf{3}), 0.192 \mathrm{~g}$ of 13p was obtained as a white powder ( $88 \%$ yield, $98 \%$ ee, $>99 \%$ de).

${ }^{1} \mathrm{H}$ NMR $\left(400 \mathrm{MHz}, \mathrm{CDCl}_{3}\right) \delta 7.18(\mathrm{t}, J=8.0 \mathrm{~Hz}, 1 \mathrm{H}), 6.72(\mathrm{~d}, J=8.0 \mathrm{~Hz}, 2 \mathrm{H}), 4.93(\mathrm{ddd}$, $J=6.9,6.9,3.4 \mathrm{~Hz}, 1 \mathrm{H}), 3.82(\mathrm{~s}, 3 \mathrm{H}), 3.80-3.77(\mathrm{~m}, 1 \mathrm{H}), 3.06(\mathrm{dd}, J=17.5,9.2 \mathrm{~Hz}, 1 \mathrm{H})$, 2.76-2.73 (m, 2H), 2.57 (dd, $J=17.5,6.0 \mathrm{~Hz}, 1 \mathrm{H}), 2.15-2.10(\mathrm{~m}, 1 \mathrm{H}), 1.20-1.95(\mathrm{~m}, 1 \mathrm{H})$; ${ }^{13} \mathrm{C} \mathrm{NMR}\left(125 \mathrm{MHz}, \mathrm{CDCl}_{3}\right) \delta 176.4,156.7,136.5,127.3,125.0,120.9,107.9,78.5,55.4$, 37.7, 37.6, 25.6, 17.7 .

HRMS (APCI) calcd for $\mathrm{C}_{13} \mathrm{H}_{15} \mathrm{O}_{3}[\mathrm{M}+\mathrm{H}]^{+}:$219.1016. Found: 219.1000.

The enantiomeric excess was determined by HPLC analysis (Chiralcel OJ-H, 250×4.6 mm column, hexane/2-propanol 94:6, $1.0 \mathrm{~mL} / \mathrm{min}, 220 \mathrm{~nm}, 40{ }^{\circ} \mathrm{C},(3 \mathrm{a} R, 9 \mathrm{bS})$ isomer $25.9 \mathrm{~min}$ (major), (3aS,9bR) isomer $28.8 \mathrm{~min}$ (minor)); IR (neat) 1798, 1583, 1468, 1440, 1346, 1332, 1258, 1246, 1189, 1176, 1161, 1096, 1078, 1029, 992, $778 \mathrm{~cm}^{-1} ;[\alpha]_{\mathrm{D}}^{20}-110.8$ (c 1.29 in $\left.\mathrm{CHCl}_{3}\right) 98 \%$ ee, $>99 \%$ de $(3 \mathrm{a} R, 9 \mathrm{bS})$. 
<smiles>O=C1C[C@H]2c3ccc(Br)cc3CC[C@H]2O1</smiles>

According to the general procedure (ketone 11q: $0.283 \mathrm{~g}(1.0 \mathrm{mmol})$, cat. $(\boldsymbol{R}, \boldsymbol{R})-3), 0.240 \mathrm{~g}$ of 13q was obtained as a pale yellow oil (90\% yield, $94 \%$ ee, $>99 \%$ de).

${ }^{1} \mathrm{H}$ NMR $\left(400 \mathrm{MHz}, \mathrm{CDCl}_{3}\right) \delta$ 7.35-7.26 (m, 2H), $6.99(\mathrm{~d}, J=8.0 \mathrm{~Hz}, 1 \mathrm{H}), 4.97$ (ddd, $J=$ 6.6, 6.6, 3.2 Hz, 1H), $3.74(\mathrm{ddd}, J=8.0,8.0,5.6 \mathrm{~Hz}, 1 \mathrm{H}), 3.09(\mathrm{dd}, J=17.6,9.6 \mathrm{~Hz}, 1 \mathrm{H})$, 2.89 (ddd, $J=11.0,11.0,5.2 \mathrm{~Hz}, 1 \mathrm{H}), 2.67$ (ddd, $J=11.0,11.0,5.2 \mathrm{~Hz}, 1 \mathrm{H}), 2.52$ (dd, $J=$ 17.6, 5.6 Hz, 1H), 2.24-2.16 (m, 1H), 1.99-1.91 (m, 1H); $\left.{ }^{13} \mathrm{C} \mathrm{NMR} \mathrm{(125} \mathrm{MHz,} \mathrm{CDCl}_{3}\right) \delta$ $175.9,138.1,134.5,131.6,130.6,130.2,120.6,78.2,37.5,37.2,26.0,24.2$.

HRMS (ESI) calcd for $\mathrm{C}_{12} \mathrm{H}_{12} \mathrm{BrO}_{2}[\mathrm{M}+\mathrm{H}]^{+}: 267.0015$. Found: 267.0012 .

The enantiomeric excess was determined by HPLC analysis (Chiralcel OD-H, 250×4.6 mm column, hexane/2-propanol 94:6, $1.0 \mathrm{~mL} / \mathrm{min}, 220 \mathrm{~nm}, 35^{\circ} \mathrm{C},(3 \mathrm{a} R, 9 \mathrm{bS})$ isomer $19.6 \mathrm{~min}$ (major), (3aS,9bR) isomer $23.0 \mathrm{~min}$ (minor)); IR (neat) 2939, 1765, 1592, 1481, 1434, 1407, $1348,1259,1169,1081,1047,1022,956,886,818,770,669 \mathrm{~cm}^{-1} ;[\alpha]_{\mathrm{D}}^{20}-104.7$ (c 0.74 in $\left.\mathrm{CHCl}_{3}\right) 94 \%$ ee, $>99 \%$ de $(3 \mathrm{a} R, 9 \mathrm{bS})$.

\section{(3aR,9bS)-7-Chloro-3a,4,5,9b-tetrahydronaphtho[2,1-b]furan-2(1H)-one (13r)}<smiles>O=C1C[C@H]2c3ccc(Cl)cc3CC[C@H]2O1</smiles>

According to the general procedure (ketone 11r: $0.239 \mathrm{~g}(1.0 \mathrm{mmol})$, cat. $(\boldsymbol{R}, \boldsymbol{R})-\mathbf{3}), 0.186 \mathrm{~g}$ of 13r was obtained as a white solid ( $84 \%$ yield, $95 \%$ ee, $>99 \%$ de).

${ }^{1} \mathrm{H}$ NMR (400 MHz, $\left.\mathrm{CDCl}_{3}\right) \delta 7.26-7.13(\mathrm{~m}, 2 \mathrm{H}), 7.04(\mathrm{~d}, J=8.4 \mathrm{~Hz}, 1 \mathrm{H}), 4.98$ (ddd, $J=$ 6.7, 6.7, 3.4 Hz, 1H), $3.76(\mathrm{ddd}, J=7.8,7.8,6.0 \mathrm{~Hz}, 1 \mathrm{H}), 3.09$ (dd, $J=17.6,9.2 \mathrm{~Hz}, 1 \mathrm{H})$, 2.89 (ddd, $J=11.0,11.0,5.2 \mathrm{~Hz}, 1 \mathrm{H}), 2.67$ (ddd, $J=11.0,11.0,5.2 \mathrm{~Hz}, 1 \mathrm{H}), 2.53(\mathrm{dd}, J=$ 17.6, 5.2 Hz, 1H), 2.24-2.17 (m, 1H), 2.00-1.92 (m, 1H); $\left.{ }^{13} \mathrm{C} \mathrm{NMR} \mathrm{(125} \mathrm{MHz,} \mathrm{CDCl}_{3}\right) \delta$ 175.9, 137.8, 134.0, 132.5, 130.3, 128.6, 127.3, 78.3, 37.6, 37.2, 26.0, 24.3.

HRMS (APCI) calcd for $\mathrm{C}_{12} \mathrm{H}_{12} \mathrm{ClO}_{2}[\mathrm{M}+\mathrm{H}]^{+}:$223.0520. Found: 223.0507.

The enantiomeric excess was determined by HPLC analysis (Chiralcel OD-H, 250×4.6 mm column, hexane/2-propanol 94:6, $1.0 \mathrm{~mL} / \mathrm{min}, 220 \mathrm{~nm}, 40{ }^{\circ} \mathrm{C},(3 \mathrm{a} R, 9 \mathrm{bS})$ isomer $17.9 \mathrm{~min}$ (major), (3aS,9bR) isomer 22.5 min (minor)); IR (neat) 3049, 2933, 2841, 1766, 1597, 1486, 1456, 1435, 1413, 1339, 1309, 1248, 1190, 1164, 1133, 1089, 1018, 979, 891, 847, 831, 817, $723,676 \mathrm{~cm}^{-1} ;[\alpha]_{\mathrm{D}}{ }^{20}-98.4\left(\mathrm{c} 1.67\right.$ in $\left.\mathrm{CHCl}_{3}\right) 95 \%$ ee, $>99 \%$ de $(3 \mathrm{a} R, 9 \mathrm{bS})$. 
<smiles>C[C@H]1C(=O)O[C@H]2CCc3ccccc3[C@H]21</smiles>

According to the general procedure (ketone 14a: $0.218 \mathrm{~g}(1.0 \mathrm{mmol})$, cat. $(\boldsymbol{R}, \boldsymbol{R})-\mathbf{3}), 0.162 \mathrm{~g}$ of 15a was obtained as a white powder ( $80 \%$ yield, $98 \%$ ee, $58 \%$ de).

${ }^{1} \mathrm{H}$ NMR $\left(500 \mathrm{MHz}, \mathrm{CDCl}_{3}\right) \delta 7.23-7.14(\mathrm{~m}, 4 \mathrm{H}), 4.91(\mathrm{ddd}, J=7.5,7.5,5.0 \mathrm{~Hz}, 1 \mathrm{H}), 3.36$ $(\mathrm{t}, J=8.4 \mathrm{~Hz}, 1 \mathrm{H}), 2.85(\mathrm{ddd}, J=14.8,9.5,5.1 \mathrm{~Hz}, 1 \mathrm{H}), 2.75(\mathrm{ddd}, J=14.8,9.5,5.1 \mathrm{~Hz}$, $1 \mathrm{H}), 2.55(\mathrm{ddd}, J=14.5,8.8,7.2 \mathrm{~Hz}, 1 \mathrm{H}), 2.10-2.00(\mathrm{~m}, 2 \mathrm{H}), 1.48(\mathrm{~d}, J=2.4 \mathrm{~Hz}, 3 \mathrm{H}) ;{ }^{13} \mathrm{C}$ NMR $\left(125 \mathrm{MHz}, \mathrm{CDCl}_{3}\right) \delta 178.9,135.8,135.4,128.8,128.6,127.0,126.7,76.6,45.7,42.7$, 27.1, 25.9, 15.5 .

HRMS (ESI) calcd for $\mathrm{C}_{13} \mathrm{H}_{15} \mathrm{O}_{2}[\mathrm{M}+\mathrm{H}]^{+}:$203.1067. Found: 203.1062 .

The enantiomeric excess was determined by HPLC analysis (Chiralpak IB, $250 \times 4.6 \mathrm{~mm}$ column, hexane/2-propanol 94:6, $1.0 \mathrm{~mL} / \mathrm{min}, 220 \mathrm{~nm}, 35^{\circ} \mathrm{C},(1 R, 3 \mathrm{a} R, 9 \mathrm{bS})$ isomer $9.5 \mathrm{~min}$ (major), $(1 S, 3 \mathrm{a} S, 9 \mathrm{~b} R)$ isomer $10.2 \mathrm{~min}$ (minor), $(1 R, 3 \mathrm{a} S, 9 \mathrm{~b} R)$ and $(1 S, 3 \mathrm{a} R, 9 \mathrm{~b} S)$ isomers 11.8 min or 12.4 min (minor)); IR (neat) 3025, 3963, 2932, 2880, 2842, 1756, 1490, 1452, 1436, 1377, 1350, 1336, 1292, 1258, 1230, 1178, 1162, 1150, 1135, 1112, 1085, 1054, 1040, 1008, 936, 901, 876, 816, 776, 766, 745, $681 \mathrm{~cm}^{-1}$; $[\alpha]_{\mathrm{D}}^{20}-61.3\left(\mathrm{c} 1.97\right.$ in $\left.\mathrm{CHCl}_{3}\right) 98 \%$ ee, $99 \%$ de $(1 R, 3 \mathrm{a} R, 9 \mathrm{~b} S)$.

\section{$(1 R, 3 a R, 9 b S)-1-E t h y l-3 a, 4,5,9 b-t e t r a h y d r o n a p h t h o[2,1-b]$ furan-2(1H)-one (15b)}<smiles>CC[C@H]1C(=O)O[C@H]2CCc3ccccc3[C@H]21</smiles>

According to the general procedure (ketone 14b: $0.465 \mathrm{~g}(2.0 \mathrm{mmol})$, cat. $(\boldsymbol{R}, \boldsymbol{R})-3), 0.398 \mathrm{~g}$ of $\mathbf{1 5 b}$ was obtained as a white solid (95\% yield, $97 \%$ ee, $70 \% \mathrm{de}$ ).

${ }^{1} \mathrm{H}$ NMR $\left(500 \mathrm{MHz}, \mathrm{CDCl}_{3}\right) \delta$ 7.23-7.09 (m, 4H), 4.97 (ddd, $\left.J=7.2,5.7,3.6 \mathrm{~Hz}, 1 \mathrm{H}\right), 3.52$ $(\mathrm{t}, J=6.6 \mathrm{~Hz}, 1 \mathrm{H}), 2.88(\mathrm{ddd}, J=10.7,10.7,5.2 \mathrm{~Hz}, 1 \mathrm{H}), 2.70(\mathrm{ddd}, J=10.7,10.7,5.2 \mathrm{~Hz}$, $1 \mathrm{H}), 2.53(\mathrm{q}, J=6.2 \mathrm{~Hz}, 1 \mathrm{H}), 2.26-2.20(\mathrm{~m}, 1 \mathrm{H}), 1.95-1.89(\mathrm{~m}, 3 \mathrm{H}), 1.18(\mathrm{t}, J=7.4 \mathrm{~Hz}$, $3 \mathrm{H}) ;{ }^{13} \mathrm{C} \mathrm{NMR}\left(125 \mathrm{MHz}, \mathrm{CDCl}_{3}\right) \delta 178.7,136.2,135.9,128.8,128.7,126.9,126.8,76.7$, 50.6, 42.1, 26.9, 24.5, 23.2, 11.2.

HRMS (ESI) calcd for $\mathrm{C}_{14} \mathrm{H}_{17} \mathrm{O}_{2}[\mathrm{M}+\mathrm{H}]^{+}:$217.1223. Found: 217.1218.

The enantiomeric excess was determined by HPLC analysis (Chiralcel OZ-3, 250×4.6 mm column, hexane/2-propanol 97:3, $1.0 \mathrm{~mL} / \mathrm{min}, 220 \mathrm{~nm}, 35^{\circ} \mathrm{C},(1 S, 3 \mathrm{a} S, 9 \mathrm{~b} R)$ isomer $14.9 \mathrm{~min}$ (minor), $(1 R, 3 \mathrm{a} R, 9 \mathrm{bS})$ isomer $15.5 \mathrm{~min}$ (major), $(1 R, 3 \mathrm{a} S, 9 \mathrm{~b} R)$ and $(1 S, 3 \mathrm{a} R, 9 \mathrm{~b} S)$ isomers 22.0 min or 22.8 min (minor)); IR (neat) 3060, 3027, 2961, 2930, 2902, 2889, 2874, 1771, 1577, 1494, 1471, 1450, 1435, 1381, 1355, 1333, 1320, 1298, 1257, 1175, 1155, 1135, 1113, 1079, $1040,1003,915,851,825,761,750,731,679 \mathrm{~cm}^{-1}$; $[\alpha]_{\mathrm{D}}^{20}-110.9$ (c 1.23 in $\left.\mathrm{CHCl}_{3}\right) 97 \%$ ee, $99 \%$ de $(1 R, 3 \mathrm{a} R, 9 \mathrm{~b} S)$. 
<smiles>O=C1O[C@@H]2CCc3ccccc3[C@H]2C1Cc1ccccc1</smiles>

According to the general procedure (ketone 14c: $0.465 \mathrm{~g}(2.0 \mathrm{mmol})$, cat. $(\boldsymbol{R}, \boldsymbol{R})-3), 0.398 \mathrm{~g}$ of $\mathbf{1 5 c}$ was obtained as a colorless oil ( $92 \%$ yield, $97 \%$ ee, $90 \%$ de).

${ }^{1} \mathrm{H}$ NMR $\left(500 \mathrm{MHz}, \mathrm{CDCl}_{3}\right) \delta 7.40-7.36(\mathrm{~m}, 2 \mathrm{H}), 7.34-7.29(\mathrm{~m}, 3 \mathrm{H}), 7.12-7.05(\mathrm{~m}, 3 \mathrm{H})$, 6.63-6.60 (m, 1H), 4.80 (ddd, $J=5.5,5.5,3.9 \mathrm{~Hz}, 1 \mathrm{H}), 3.49$ (dd, $J=7.1,4.5 \mathrm{~Hz}, 1 \mathrm{H}), 3.23$ $(\mathrm{dd}, J=13.8,5.0 \mathrm{~Hz}, 1 \mathrm{H}), 3.10(\mathrm{dd}, J=13.8,8.4 \mathrm{~Hz}, 1 \mathrm{H}), 2.89-2.80(\mathrm{~m}, 2 \mathrm{H}), 2.61(\mathrm{dt}, J=$ 16.1, 4.4 Hz, 1H), 2.29-2.23 (m, 1H), 1.80-1.73 (m, 1H); $\left.{ }^{13} \mathrm{C} \mathrm{NMR} \mathrm{(125} \mathrm{MHz,} \mathrm{CDCl}_{3}\right) \delta$ $178.5,137.7,136.2$, 135.8, 129.4, 128.9, 128.6, 128.4, 127.1, 126.9, 126.6, 77.0, 51.9, 41.1, $36.3,26.9,23.8$.

HRMS (ESI) calcd for $\mathrm{C}_{19} \mathrm{H}_{19} \mathrm{O}_{2}[\mathrm{M}+\mathrm{H}]^{+}:$279.1380. Found: 279.1376.

The enantiomeric excess was determined by HPLC analysis (YMC Alcyon SFC CPS Cellulose-SC, $250 \times 4.6 \mathrm{~mm}$ column, hexane/2-propanol 94:6, $1.0 \mathrm{~mL} / \mathrm{min}, 220 \mathrm{~nm}, 35^{\circ} \mathrm{C}$, $(1 R, 3 \mathrm{a} R, 9 \mathrm{~b} S)$ isomer $19.1 \mathrm{~min}$ (major), $(1 S, 3 \mathrm{a} S, 9 \mathrm{~b} R)$ isomer $21.9 \mathrm{~min}$ (minor), $(1 S, 3 \mathrm{a} R, 9 \mathrm{~b} S)$ and $(1 R, 3 \mathrm{a} S, 9 \mathrm{~b} R)$ isomers $32.7 \mathrm{~min}$ or $46.8 \mathrm{~min}$ (minor)); IR (neat) 3026, 2938, $2850,1763,1602,1494,1454,1356,1258,1164,1043,1020,958,745,701 \mathrm{~cm}^{-1} ;[\alpha]_{\mathrm{D}}{ }^{20}-$ 26.1 (c 1.29 in $\mathrm{CHCl}_{3}$ ) 97\% ee, $99 \%$ de $(1 R, 3 \mathrm{a} R, 9 \mathrm{bS})$.

\section{$(1 S, 3 a R, 9 b R)-1-P h e n y l-3 a, 4,5,9 b-t e t r a h y d r o n a p h t h o[2,1-b]$ furan-2(1H)-one (15d)}<smiles>O=C1O[C@@H]2CCc3ccccc3[C@H]2[C@H]1c1ccccc1</smiles>

According to the general procedure (ketone 14d: $0.280 \mathrm{~g}(1.0 \mathrm{mmol})$, cat. $(\boldsymbol{R}, \boldsymbol{R})-\mathbf{3}), 0.211 \mathrm{~g}$ of $15 \mathbf{d}$ was obtained as a white powder ( $80 \%$ yield, $98 \%$ ee, $94 \% \mathrm{de}$ ).

${ }^{1} \mathrm{H}$ NMR $\left(500 \mathrm{MHz}, \mathrm{CDCl}_{3}\right) \delta$ 7.44-7.34 (m, 3H), 7.28-7.25 (m, 2H), 7.20-7.16 (m, 2H), $7.09-7.05(\mathrm{~m}, 1 \mathrm{H}), 6.73(\mathrm{~d}, J=7.7 \mathrm{~Hz}, 1 \mathrm{H}), 5.05$ (ddd, $J=8.0,8.0,4.4 \mathrm{~Hz}, 1 \mathrm{H}), 3.88(\mathrm{~m}$, $1 \mathrm{H}), 3.77$ (m, 1H), 2.95 (ddd, $J=14.0,9.3,4.8 \mathrm{~Hz}, 1 \mathrm{H}), 2.81$ (ddd, $J=15.1,10.3,4.6 \mathrm{~Hz}$, 1H), 2.22-2.08 (m, 2H); $\left.{ }^{13} \mathrm{C} \mathrm{NMR} \mathrm{(125} \mathrm{MHz,} \mathrm{CDCl}_{3}\right) \delta 176.7,136.8,135.6,134.7,129.2$, $128.8,128.8,128.7,127.9,127.1,126.7,77.1,54.9,46.8,27.0,25.9$.

HRMS (ESI) calcd for $\mathrm{C}_{18} \mathrm{H}_{17} \mathrm{O}_{3}[\mathrm{M}+\mathrm{H}]^{+}:$265.1223. Found: 265.1218.

The enantiomeric excess was determined by HPLC analysis (Chiralpak AD-H, 250×4.6 mm column, hexane/2-propanol 94:6, $1.0 \mathrm{~mL} / \mathrm{min}, 220 \mathrm{~nm}, 35^{\circ} \mathrm{C},(1 R, 3 \mathrm{aS}, 9 \mathrm{~b} R)$ isomer $13.3 \mathrm{~min}$ (minor), (1S,3aR,9bS) isomer $24.2 \mathrm{~min}$ (major)); IR (neat) 3060, 2957, 2927, 2900, 2869, 1764, 1603, 1498, 1485, 1452, 1434, 1352, 1330, 1293, 1269, 1258, 1191, 1151, 1126, 1111 , $1079,1065,1017,952,937,918,857,762,743,724,707 \mathrm{~cm}^{-1} ;[\alpha]_{\mathrm{D}}^{20}+62.5\left(\mathrm{c} 0.29\right.$ in $\left.\mathrm{CHCl}_{3}\right)$ $98 \%$ ee, $99 \%$ de $(1 S, 3 \mathrm{a} R, 9 \mathrm{~b} R)$. 
<smiles>O=C1O[C@@H]2CCc3cc(Br)ccc3[C@H]2[C@H]1c1ccccc1</smiles>

According to the general procedure (ketone 14e: $0.359 \mathrm{~g}(1.0 \mathrm{mmol})$, cat. $(\boldsymbol{R}, \boldsymbol{R})-3), 0.316 \mathrm{~g}$ of 15e was obtained as a white powder ( $92 \%$ yield, $95 \%$ ee, $92 \%$ de).

${ }^{1} \mathrm{H}$ NMR $\left(500 \mathrm{MHz}, \mathrm{CDCl}_{3}\right) \delta 7.43-7.33(\mathrm{~m}, 4 \mathrm{H}), 7.25-7.17(\mathrm{~m}, 3 \mathrm{H}), 6.57(\mathrm{~d}, J=8.3 \mathrm{~Hz}$, $1 \mathrm{H}), 5.03(\mathrm{ddd}, J=8.1,8.1,4.4 \mathrm{~Hz}, 1 \mathrm{H}), 3.81(\mathrm{t}, J=8.9 \mathrm{~Hz}, 1 \mathrm{H}), 3.70(\mathrm{~d}, J=8.9 \mathrm{~Hz}, 1 \mathrm{H})$, 2.91 (ddd, $J=14.0,9.2,4.8 \mathrm{~Hz}, 1 \mathrm{H}$ ), 2.77 (ddd, $J=15.3,10.5,4.5 \mathrm{~Hz}, 1 \mathrm{H}$ ), 2.21-2.14 (m, 1H), 2.12-2.05 (m, 1H); ${ }^{13} \mathrm{C}$ NMR (125 MHz, $\left.\mathrm{CDCl}_{3}\right) \delta 176.2,137.8,136.3,133.6,131.6$, $130.4,129.8,129.2,128.6,128.0,121.0,76.6,54.6,46.5,26.6,25.7$.

HRMS (ESI) calcd for $\mathrm{C}_{18} \mathrm{H}_{16} \mathrm{BrO}_{2}[\mathrm{M}+\mathrm{H}]^{+}:$343.0328. Found: 343.0326.

The enantiomeric excess was determined by HPLC analysis (Chiralpak AD-H, 250×4.6 mm column, hexane/2-propanol 94:6, $1.0 \mathrm{~mL} / \mathrm{min}, 220 \mathrm{~nm}, 35^{\circ} \mathrm{C},(1 R, 3 \mathrm{a} S, 9 \mathrm{~b} R)$ isomer $16.3 \mathrm{~min}$ (minor), $(1 S, 3 \mathrm{a} R, 9 \mathrm{~b} S)$ isomer $30.5 \mathrm{~min}$ (major), $(1 S, 3 \mathrm{a} S, 9 \mathrm{~b} R)$ and $(1 R, 3 \mathrm{a} R, 9 \mathrm{~b} S)$ isomers 15.5 $\min$ or $19.8 \mathrm{~min}$ (minor)); IR (neat) 3065, 2953, 2925, 2897, 2867, 1771, 1760, 1589, 1497 , 1483, 1452, 1435, 1405, 1348, 1268, 1187, 1163, 1151, 1127, 1079, 1024, 979, 937, 920, 885, 860, 834, 815, 786, 755, $705 \mathrm{~cm}^{-1} ;[\alpha]_{\mathrm{D}}{ }^{20}+98.7$ (c 1.15 in $\left.\mathrm{CHCl}_{3}\right)$ 95\% ee, 92\% de $(1 S, 3 \mathrm{a} R, 9 \mathrm{~b} S)$.

$(1 S, 3 \mathrm{a} R, 9 \mathrm{~b} S)$-7-Chloro-1-phenyl-3a,4,5,9b-tetrahydronaphtho[2,1-b]furan-2(1H)-one (15f)<smiles>O=C1O[C@@H]2CCc3cc(Cl)ccc3[C@H]2[C@H]1c1ccccc1</smiles>

According to the general procedure (ketone 14f: $0.472 \mathrm{~g}(1.5 \mathrm{mmol})$, cat. $(\boldsymbol{R}, \boldsymbol{R})-3), 0.412 \mathrm{~g}$ of $\mathbf{1 5 f}$ was obtained as a white powder ( $92 \%$ yield, $94 \%$ ee, $94 \%$ de).

${ }^{1} \mathrm{H}$ NMR $\left(500 \mathrm{MHz}, \mathrm{CDCl}_{3}\right) \delta$ 7.44-7.35 (m, 3H), 7.26-7.23 (m, 2H), 7.17 (d, J = 2.2 Hz, $1 \mathrm{H}), 7.04$ (dd, $J=8.3,2.2 \mathrm{~Hz}, 1 \mathrm{H}), 6.63(\mathrm{~d}, J=8.3 \mathrm{~Hz}, 1 \mathrm{H}), 5.05$ (ddd, $J=8.4,7.7,4.4 \mathrm{~Hz}$, $1 \mathrm{H}), 3.84(\mathrm{t}, J=8.9 \mathrm{~Hz}, 1 \mathrm{H}), 3.70(\mathrm{~d}, J=8.9 \mathrm{~Hz}, 1 \mathrm{H}), 2.93(\mathrm{ddd}, J=14.0,9.3,4.8 \mathrm{~Hz}, 1 \mathrm{H})$, $2.78(\mathrm{ddd}, J=15.3,10.6,4.6 \mathrm{~Hz}, 1 \mathrm{H}), 2.22-2.16(\mathrm{~m}, 1 \mathrm{H}), 2.13-2.02(\mathrm{~m}, 1 \mathrm{H}) ;{ }^{13} \mathrm{C} \mathrm{NMR}(125$ $\left.\mathrm{MHz} \mathrm{CDCl}_{3}\right) \delta 176.3,137.4,136.4,133.1,132.9,130.1,129.3,129.3,128.7,128.0,126.9$, 76.7, 54.7, 46.5, 26.6, 25.8.

HRMS (ESI) calcd for $\mathrm{C}_{18} \mathrm{H}_{16} \mathrm{ClO}_{2}[\mathrm{M}+\mathrm{H}]^{+}:$299.0833. Found: 299.0832.

The enantiomeric excess was determined by HPLC analysis (Chiralpak AD-H, 250×4.6 mm column, hexane/2-propanol 94:6, $1.0 \mathrm{~mL} / \mathrm{min}, 220 \mathrm{~nm}, 35^{\circ} \mathrm{C},(1 R, 3 \mathrm{a} S, 9 \mathrm{~b} R)$ isomer $15.2 \mathrm{~min}$ (minor), (1S,3aR,9bS) isomer 28.9 min (major)); IR (neat) 3068, 2959, 2928, 2856, 1770, 1596, 1569, 1498, 1481, 1453, 1441, 1406, 1335, 1311, 1297, 1282, 1256, 1216, 1190, 1143, 
$1126,1083,1027,983,939,924,907,875,866,838,827,796,752,740,703 \mathrm{~cm}^{-1} ;[\alpha]_{\mathrm{D}^{20}}$ +94.4 (c 1.61 in $\left.\mathrm{CHCl}_{3}\right) 94 \%$ ee, $99 \%$ de $(1 S, 3 \mathrm{a}, 9 \mathrm{bS})$.

$(1 S, 3 a R, 9 b S)-9-M e t h o x y-1-p h e n y l-3 a, 4,5,9 b-t e t r a h y d r o n a p h t h o[2,1-b] f u r a n-2(1 H)-$ one $(15 \mathrm{~g})$<smiles>COc1cccc2c1CC[C@@H]1OC(=O)[C@@H](c3ccccc3)[C@H]21</smiles>

According to the general procedure (ketone $14 \mathrm{~g}: 0.310 \mathrm{~g}(1.0 \mathrm{mmol})$, cat. $(\boldsymbol{R}, \boldsymbol{R})-\mathbf{3}), 0.265 \mathrm{~g}$ of $\mathbf{1 5} \mathbf{g}$ was obtained as a white powder (90\% yield, $98 \%$ ee, $92 \% \mathrm{de}$ ).

${ }^{1} \mathrm{H}$ NMR $\left(500 \mathrm{MHz}, \mathrm{CDCl}_{3}\right) \delta$ 7.44-7.39 (m, 2H), 7.37-7.33 (m, 1H), 7.28-7.25 (m, 2H), $7.04(\mathrm{t}, J=8.0 \mathrm{~Hz}, 1 \mathrm{H}), 6.72(\mathrm{~d}, J=8.0 \mathrm{~Hz}, 1 \mathrm{H}), 6.32(\mathrm{~d}, J=7.8 \mathrm{~Hz}, 1 \mathrm{H}), 5.00$ (ddd, $J=9.2$, 7.4, $4.2 \mathrm{~Hz}, 1 \mathrm{H}), 3.85$ (t, $J=8.8 \mathrm{~Hz}, 1 \mathrm{H}), 3.83$ (s, $3 \mathrm{H}), 3.77$ (d, $J=8.8 \mathrm{~Hz}, 1 \mathrm{H}), 2.97$ (ddd, $J$ $=11.5,11.5,5.6 \mathrm{~Hz}, 1 \mathrm{H}), 2.65(\mathrm{ddd}, J=16.0,11.0,4.9 \mathrm{~Hz}, 1 \mathrm{H}), 2.22-2.16(\mathrm{~m}, 1 \mathrm{H}), 2.07-$ $1.99(\mathrm{~m}, 1 \mathrm{H}) ;{ }^{13} \mathrm{C} \mathrm{NMR}\left(125 \mathrm{MHz}, \mathrm{CDCl}_{3}\right) \delta 176.8,156.8,136.9,135.8,129.1,128.7,127.8$, 127.1, 124.6, 120.8, 108.2, 76.9, 55.4, 54.8, 46.8, 26.1, 19.1.

HRMS (ESI) calcd for $\mathrm{C}_{19} \mathrm{H}_{19} \mathrm{O}_{3}[\mathrm{M}+\mathrm{H}]^{+}:$295.1329. Found: 295.1326.

The enantiomeric excess was determined by HPLC analysis (Chiralpak AD-H, $250 \times 4.6 \mathrm{~mm}$ column, hexane/2-propanol 94:6, $1.0 \mathrm{~mL} / \mathrm{min}, 220 \mathrm{~nm}, 35^{\circ} \mathrm{C},(1 R, 3 \mathrm{a} S, 9 \mathrm{~b} R)$ isomer $15.3 \mathrm{~min}$ (minor), (1S,3aR,9bS) isomer $23.2 \mathrm{~min}$ (major)); IR (neat) 3106, 3065, 3007, 2960, 2889, 2839, 2161, 1815, 1783, 1604, 1583, 1500, 1468, 1453, 1340, 1325, 1260, 1224, 1203, 1186, 1162, 1127, 1091, 1064, 1028, 1012, 990, 951, 934, 882, 865, 805, 784, 753, 725, $698 \mathrm{~cm}^{-1}$; $[\alpha]_{\mathrm{D}}^{20}+78.2\left(\mathrm{c} 1.20\right.$ in $\left.\mathrm{CHCl}_{3}\right) 96 \%$ ee, $99 \%$ de $(1 S, 3 \mathrm{a} R, 9 \mathrm{~b} S)$.

\section{$(1 S, 3 \mathrm{a} R, 9 \mathrm{~b} S)$-1-(2-Chlorophenyl)-3a,4,5,9b-tetrahydronaphtho[2,1-b]furan-2(1H)-one} (15h)<smiles>O=C1O[C@H]2CCc3ccccc3[C@H]2[C@H]1c1ccccc1Cl</smiles>

According to the general procedure (ketone $14 \mathrm{~h}: 0.315 \mathrm{~g}(1.0 \mathrm{mmol})$, cat. $(\boldsymbol{R}, \boldsymbol{R})-\mathbf{3}), 0.242 \mathrm{~g}$ of $\mathbf{1 5 h}$ was obtained as a off-white solid ( $81 \%$ yield, $93 \%$ ee, $76 \%$ de).

${ }^{1} \mathrm{H}$ NMR $\left(500 \mathrm{MHz}, \mathrm{CDCl}_{3}\right) \delta$ 7.48-7.46 (m, 1H), 7.34-7.29 (m, 2H), 7.22-7.16 (m, 3H), 7.10-7.07 (m, 1H), $6.80(\mathrm{~d}, J=7.7 \mathrm{~Hz}, 1 \mathrm{H}), 5.10(\mathrm{ddd}, J=7.5,7.5,4.9 \mathrm{~Hz}, 1 \mathrm{H}), 4.10(\mathrm{~d}, J$ $=6.6 \mathrm{~Hz}, 1 \mathrm{H}), 4.00(\mathrm{t}, J=8.0 \mathrm{~Hz}, 1 \mathrm{H}), 2.95(\mathrm{ddd}, J=14.4,9.1,5.2 \mathrm{~Hz}, 1 \mathrm{H}), 2.80$ (ddd, $J=$ 14.4, 9.1, 5.2 Hz, 1H), 2.21-2.11 (m, 2H); $\left.{ }^{13} \mathrm{C} \mathrm{NMR} \mathrm{(125} \mathrm{MHz,} \mathrm{CDCl}_{3}\right) \delta 175.7,135.8$, 134.6, 134.5, 134.1, 131.2, 130.5, 129.4, 128.8, 128.7, 127.4, 127.1, 126.7, 77.3, 53.3, 45.2, 
27.1, 25.7.

HRMS (ESI) calcd for $\mathrm{C}_{18} \mathrm{H}_{16} \mathrm{ClO}_{2}[\mathrm{M}+\mathrm{H}]^{+}:$299.0833. Found: 299.0832 .

The enantiomeric excess was determined by HPLC analysis (Chiralcel OD-H, $250 \times 4.6 \mathrm{~mm}$ column, hexane/2-propanol 94:6, $1.0 \mathrm{~mL} / \mathrm{min}, 220 \mathrm{~nm}, 35^{\circ} \mathrm{C},(1 R, 3 \mathrm{a} S, 9 \mathrm{~b} R)$ isomer $12.0 \mathrm{~min}$ (minor), $(1 S, 3 \mathrm{a} R, 9 \mathrm{~b} S)$ isomer $14.2 \mathrm{~min}$ (major), $(1 R, 3 \mathrm{a} R, 9 \mathrm{~b} S)$ and $(1 S, 3 \mathrm{a} S, 9 \mathrm{~b} R)$ isomers 9.7 min or 10.6 min (minor)); IR (neat) 3419, 3065, 3025, 2936, 2866, 2848, 2156, 1771, 1573, 1484, 1454, 1436, 1351, 1331, 1299, 1260, 1205, 1189, 1157, 1138, 1121, 1053, 1035, 1018, 984, 949, 933, 868, 829, 761, 750, 723, $708 \mathrm{~cm}^{-1} ;[\alpha]_{\mathrm{D}}^{20}-6.1$ (c 1.04 in $\left.\mathrm{CHCl}_{3}\right) 93 \%$ ee, $99 \%$ de $(1 S, 3 \mathrm{a} R, 9 \mathrm{~b} S)$.

$(1 R, 3 \mathrm{a} S, 9 \mathrm{~b} R)$-8-Methoxy-1-phenyl-3a,4,5,9b-tetrahydronaphtho[2,1-b]furan-2(1H)one (7)<smiles>COc1ccc2c(c1)C1C(CC2)OC(=O)[C@H]1c1ccccc1</smiles>

According to the general procedure (ketone $14 \mathrm{i}: 0.31 \mathrm{~g}(1.0 \mathrm{mmol})$, cat. $(\boldsymbol{S}, \boldsymbol{S})-3), 0.251 \mathrm{~g}$ of 7 was obtained as a off-white solid ( $85 \%$ yield, $95 \%$ ee, $90 \%$ de).

${ }^{1} \mathrm{H}$ NMR (500 MHz, $\left.\mathrm{CDCl}_{3}\right) \delta 7.42(\mathrm{t}, J=7.5 \mathrm{~Hz}, 2 \mathrm{H}), 7.36(\mathrm{t}, J=7.4 \mathrm{~Hz}, 1 \mathrm{H}), 7.28-7.25$ $(\mathrm{m}, 2 \mathrm{H}), 7.07(\mathrm{~d}, J=8.5 \mathrm{~Hz}, 1 \mathrm{H}), 6.74(\mathrm{dd}, J=8.4,2.6 \mathrm{~Hz}, 1 \mathrm{H}), 6.19(\mathrm{~d}, J=2.6 \mathrm{~Hz}, 1 \mathrm{H})$, 5.01 (ddd, $J=8.3,8.2,4.3 \mathrm{~Hz}, 1 \mathrm{H}), 3.85-3.79(\mathrm{~m}, 1 \mathrm{H}), 3.77-3.75(\mathrm{~m}, 1 \mathrm{H}), 3.57(\mathrm{~s}, 3 \mathrm{H})$, 2.91-2.84 (m, 1H), 2.78-2.70 (m, 1H), 2.21-2.17 (m, 1H), 2.12-2.02 (m, 1H); ${ }^{13} \mathrm{C}$ NMR $\left(125 \mathrm{MHz}, \mathrm{CDCl}_{3}\right) \delta 176.6,158.0,136.8,135.6,129.7,129.1,128.8,127.9,127.5,113.7$, 113.1, 77.1, 55.0, 54.7, 47.2, 27.2, 25.2.

HRMS (APCI) calcd for $\mathrm{C}_{19} \mathrm{H}_{19} \mathrm{O}_{3}[\mathrm{M}+\mathrm{H}]^{+}:$295.1329. Found: 295.1321.

The enantiomeric excess was determined by HPLC analysis (Chiralcel OZ-3, 250×4.6 mm column, hexane/2-propanol 95:5, $1.0 \mathrm{~mL} / \mathrm{min}, 220 \mathrm{~nm}, 35^{\circ} \mathrm{C},(1 R, 3 \mathrm{a} S, 9 \mathrm{~b} R)$ isomer $36.1 \mathrm{~min}$ (major), $(1 S, 3 \mathrm{a} R, 9 \mathrm{~b} S)$ isomer $41.6 \mathrm{~min}$ (minor), $(1 R, 3 \mathrm{a} R, 9 \mathrm{~b} S)$ and $(1 S, 3 \mathrm{a} S, 9 \mathrm{~b} R)$ isomers 57.7 min or 69.1 min (minor)); IR (neat) 3064, 3022, 2953, 2935, 2842, 1774, 1609, 1589, 1504, 1454, 1445, 1354, 1445, 1354, 1343, 1327, 1296, 1263, 1247, 1211, 1184, 1137, 1109, 1080, $1031,1009,944,859,842,818,756,702 \mathrm{~cm}^{-1} ;[\alpha]_{\mathrm{D}}^{20}-14.3$ (c 0.85 in $\left.\mathrm{CHCl}_{3}\right)$ 95\% ee, 99\% de $(1 R, 3 \mathrm{a} S, 9 \mathrm{~b} R)$.

\section{(3R,3aS,9bR)-3-Phenyl-3a,4,5,9b-tetrahydronaphtho[1,2-b]furan-2(3H)-one (18a)}<smiles>O=C1O[C@@H]2c3ccccc3CC[C@H]2[C@H]1c1ccccc1</smiles>

According to the general procedure (ketone 17a: $0.280 \mathrm{~g}(1.0 \mathrm{mmol})$, cat. $(\boldsymbol{R}, \boldsymbol{R})-\mathbf{3}), 0.193 \mathrm{~g}$ of 18a was obtained as a off-white solid (73\% yield, $98.3 \%$ ee, $67 \%$ de). 
${ }^{1} \mathrm{H}$ NMR $\left(500 \mathrm{MHz}, \mathrm{CDCl}_{3}\right) \delta$ 7.57-7.49 (m, 1H), 7.42-7.38 (m, 2H), 7.35-7.28 (m, 5H), 7.20-7.17 (m, 1H), $5.63(\mathrm{~d}, J=7.0 \mathrm{~Hz}, 1 \mathrm{H}), 3.68(\mathrm{~d}, J=7.5 \mathrm{~Hz}, 1 \mathrm{H}), 3.04-2.92(\mathrm{~m}, 2 \mathrm{H})$, 2.82-2.76 (m, 1H), 2.11-2.05 (m, 1H), 2.89-1.82 (m, 1H); ${ }^{13} \mathrm{C}$ NMR (125 MHz, $\left.\mathrm{CDCl}_{3}\right) \delta$ 176.7, 136.6, 136.0, 131.9, 130.4, 129.1, 128.7, 128.7, 128.0, 127.7, 126.9, 76.9, 51.1, 43.0, 26.0, 23.7.

HRMS (APCI) calcd for $\mathrm{C}_{18} \mathrm{H}_{17} \mathrm{O}_{2}[\mathrm{M}+\mathrm{H}]^{+}:$265.1223. Found: 265.1231.

The enantiomeric excess was determined by HPLC analysis (Chiralcel OZ-3, $250 \times 4.6 \mathrm{~mm}$ column, hexane/2-propanol 95:5, $1.0 \mathrm{~mL} / \mathrm{min}, 215 \mathrm{~nm}, 35^{\circ} \mathrm{C},(3 R, 3 \mathrm{a} S, 9 \mathrm{~b} R)$ isomer $17.1 \mathrm{~min}$ (major), $(3 S, 3 \mathrm{a} R, 9 \mathrm{~b} S)$ isomer $18.9 \mathrm{~min}$ (minor), $(3 S, 3 \mathrm{a} S, 9 \mathrm{~b} R)$ and $(3 R, 3 \mathrm{a} R, 9 \mathrm{~b} S)$ isomers 28.4 min or $33.9 \min$ (minor)); IR (neat) 3019, 2934, 2920, 2858, 1770, 1605, 1491, 1454, 1441, 1376, 1354, 1343, 1321, 1184, 1173, 1137, 1115, 1093, 1005, 985, 944, 919, 854, 759, 744, 728, 709, 703, $683 \mathrm{~cm}^{-1}$; $[\alpha]_{\mathrm{D}}{ }^{20}-81.5\left(\mathrm{c} 0.13\right.$ in $\left.\mathrm{CHCl}_{3}\right) 98.3 \%$ ee, $99 \%$ de $(3 R, 3 \mathrm{a} S, 9 \mathrm{~b} R)$.

\section{(3R,3aS,9bR)-8-Chloro-3-phenyl-3a,4,5,9b-tetrahydronaphtho[1,2-b]furan-2(3H)-one} (18b)<smiles>O=C1O[C@@H]2c3cc(Cl)ccc3CC[C@H]2[C@H]1c1ccccc1</smiles>

According to the general procedure (ketone 17b: $0.315 \mathrm{~g}(1.0 \mathrm{mmol})$, cat. $(\boldsymbol{R}, \boldsymbol{R})-\mathbf{3}), 0.224 \mathrm{~g}$ of $\mathbf{1 8 b}$ was obtained as a off-white solid (75\% yield, $99.7 \%$ ee, $60 \% \mathrm{de})$.

${ }^{1} \mathrm{H}$ NMR $\left(500 \mathrm{MHz}, \mathrm{CDCl}_{3}\right) \delta 7.50(\mathrm{t}, J=2.2 \mathrm{~Hz}, 1 \mathrm{H}), 7.42-7.38(\mathrm{~m}, 2 \mathrm{H}), 7.35-7.25(\mathrm{~m}$, 4H), 7.13-7.11 (m, 1H), $5.57(\mathrm{~d}, J=7.1 \mathrm{~Hz}, 1 \mathrm{H}), 3.65(\mathrm{~d}, J=7.8 \mathrm{~Hz}, 1 \mathrm{H}), 3.02-2.99(\mathrm{~m}$, $1 \mathrm{H}), 2.95-2.88(\mathrm{~m}, 1 \mathrm{H}), 2.78-2.72(\mathrm{~m}, 1 \mathrm{H}), 2.11-2.05(\mathrm{~m}, 1 \mathrm{H}), 1.87-1.79(\mathrm{~m}, 1 \mathrm{H}) ;{ }^{13} \mathrm{C} \mathrm{NMR}$ $\left(125 \mathrm{MHz}, \mathrm{CDCl}_{3}\right) \delta 176.3,135.7,134.9,133.6,132.6,130.08,130.05,129.1,128.9,128.0$, $127.9,76.1,50.8,42.7,25.4,23.5$.

HRMS (APCI) calcd for $\mathrm{C}_{18} \mathrm{H}_{16} \mathrm{ClO}_{2}[\mathrm{M}+\mathrm{H}]^{+}:$299.0833. Found: 299.0842.

The enantiomeric excess was determined by HPLC analysis (Chiralcel AD-H, $250 \times 4.6 \mathrm{~mm}$ column, hexane/2-propanol 95:5, $1.0 \mathrm{~mL} / \mathrm{min}, 220 \mathrm{~nm}, 35^{\circ} \mathrm{C},(3 R, 3 \mathrm{a} S, 9 \mathrm{~b} R)$ isomer $22.7 \mathrm{~min}$ (major), $(3 S, 3 \mathrm{a} R, 9 \mathrm{~b} S)$ isomer $29.4 \mathrm{~min}$ (minor), $(3 S, 3 \mathrm{a} S, 9 \mathrm{~b} R)$ and $(3 R, 3 \mathrm{a} R, 9 \mathrm{~b} S)$ isomers 40.1 min and 48.5 min (minor)); IR (neat) 3031, 2932, 2851, 2361, 1769, 1601, 1488, 1453, 1321, 1185, 1169, 1140, 1091, 1009, 986, 923, 875, 858, 816, 789, 760, 732, 717, 701, $663 \mathrm{~cm}^{-1}$; $[\alpha]_{\mathrm{D}}{ }^{20}-103.4\left(\mathrm{c} 0.15\right.$ in $\left.\mathrm{CHCl}_{3}\right) 99.7 \%$ ee, $99 \%$ de $(3 R, 3 \mathrm{a} S, 9 \mathrm{~b} R)$. 


\section{F. Synthesis of 18c and 19c.}

(1) Synthesis of (3aS,9bR)-3-Methylene-3a,4,5,9b-tetrahydronaphtho[1,2-b]furan$2(3 H)$-one (20)

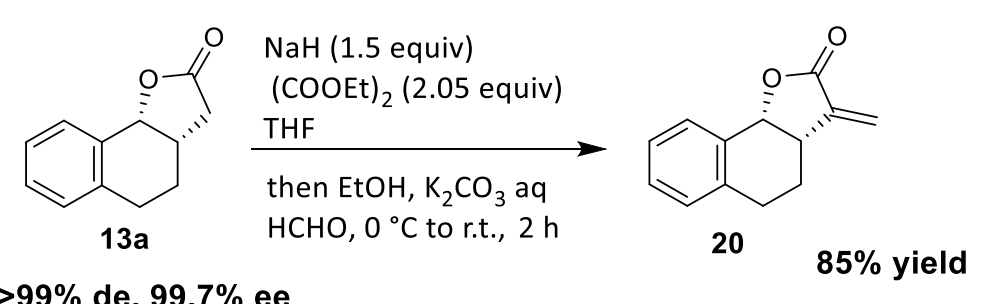

To a suspension of $\mathrm{NaH}$ ( $60 \mathrm{wt} \%$ dispersion in mineral oil, $0.80 \mathrm{~g}, 19.9 \mathrm{mmol}$ ) in THF (78 $\mathrm{mL})$ cooled at $0{ }^{\circ} \mathrm{C}$, a solution of $13 \mathrm{a}(2.50 \mathrm{~g}, 13.3 \mathrm{mmol})$ and diethyl oxalate $(3.70 \mathrm{~mL}, 27.2$ $\mathrm{mmol})$ in THF $(78 \mathrm{~mL})$ was added dropwise. After complete addition, ethanol $(3.5 \mathrm{~mL})$ was added and stirred at ambient temperature for $4 \mathrm{~h}$. After cooling the reaction mixture to $0{ }^{\circ} \mathrm{C}$, a solution of $\mathrm{K}_{2} \mathrm{CO}_{3}(7.50 \mathrm{~g})$ in water $(11.0 \mathrm{~mL})$ and formaldehyde ( $37 \mathrm{wt} \%$ aqueous solution, $14.7 \mathrm{~g}$ ) were added followed by stirring for $0.25 \mathrm{~h}$ at this temperature. The mixture was diluted with brine $(40 \mathrm{~mL})$, and the resulting aqueous layer was extracted twice with ethyl acetate $(50 \mathrm{~mL}$ each). The combined organic extracts were washed with brine, dired with $\mathrm{MgSO}_{4}$, filtered, and evaporated. The residue was purified by column chromatography on silica, using hexane/MTBE mixtures of increasing polarity as eluent, to furnish $\mathbf{2 0}$ ( $2.26 \mathrm{~g}$, $85 \%$ yield) as a light brown solid.

${ }^{1} \mathrm{H}$ NMR $\left(500 \mathrm{MHz}, \mathrm{CDCl}_{3}\right) \delta$ 7.50-7.47 (m, 1H), 7.29-7.24 (m, 2H), 7.16-7.12 (m, 1H), $6.33(\mathrm{~d}, J=2.1 \mathrm{~Hz}, 1 \mathrm{H}), 5.71(\mathrm{~d}, J=2.1 \mathrm{~Hz}, 1 \mathrm{H}), 5.51(\mathrm{~d}, J=7.5 \mathrm{~Hz}, 1 \mathrm{H}), 3.45-3.38(\mathrm{~m}$, $1 \mathrm{H}), 2.82-2.76(\mathrm{~m}, 1 \mathrm{H}), 2.70-2.64(\mathrm{~m}, 1 \mathrm{H}), 2.10-2.03(\mathrm{~m}, 1 \mathrm{H}), 1.85-1.78(\mathrm{~m}, 1 \mathrm{H}) ;{ }^{13} \mathrm{C}$ NMR $\left(125 \mathrm{MHz}, \mathrm{CDCl}_{3}\right) \delta 170.0,139.4,137.7,131.7,130.5,128.7,128.5,126.8,122.2,76.2,38.8$, 27.1, 26.7.

HRMS (APCI) calcd for $\mathrm{C}_{13} \mathrm{H}_{13} \mathrm{O}_{2}[\mathrm{M}+\mathrm{H}]^{+}:$201.0910. Found: 201.0912.

IR (neat) 3480, 3106, 3062, 3045, 3021, 2938, 1931, 1742, 1668, 1605, 1578, 1495, 1455, $1433,1366,1325,1313,1302,1272,1260,1249,1223,1168,1144,1087,1044,1005,980$, $968,959,942,908,880,828,796,771,742,681 \mathrm{~cm}^{-1} ;[\alpha]_{\mathrm{D}}^{20}-246.4$ (c 1.40 in $\left.\mathrm{CHCl}_{3}\right)$ $>99.9 \%$ ee, $>99 \%$ de $(3 \mathrm{aS}, 9 \mathrm{~b} R)$.

\section{(2) Diastereoselective Hydrogenation of 20 (Table S2, S3, S4)}

General procedures under the conditions of $\mathrm{S} / \mathrm{C}=100,40{ }^{\circ} \mathrm{C}$ for $5 \mathrm{~h}$ with $2.0 \mathrm{MPa}$ of $\mathrm{H}_{2}$. (3aS,9bR)-3-Methylene-3a,4,5,9b-tetrahydronaphtho[1,2-b]furan-2(3H)-one (20) (40 mg, $0.2 \mathrm{mmol})$ and $\mathrm{Ru}$ catalyst $(0.002 \mathrm{mmol})$ were placed in a $50 \mathrm{~mL}$ stainless steel autoclave equipped with a glass inner tube and Teflon-coated magnetic stir bar. The atmosphere was replaced with argon gas, and solvent $(1.0 \mathrm{~mL})$ was added to the mixture. Hydrogen was initially introduced into the autoclave at a pressure of $0.5 \mathrm{MPa}$, before being reduced to 0.1 MPa. This procedure was repeated three times. Then the autoclave was pressurized with $\mathrm{H}_{2}$ 
gas (2.0 MPa), and the solution was stirred vigorously at $40{ }^{\circ} \mathrm{C}$ for $5 \mathrm{~h}$. After completion of the reaction, hydrogen gas was carefully vented. Diastereoselctivity of the products was determined by GC analysis and enantioselectivity of products was determined HPLC analysis. The product was purified by silica gel chromatography (hexane/TBME $=5 / 1$ ).

\section{(3S,3aS,9bR)-3-Methyl-3a,4,5,9b-tetrahydronaphtho[1,2-b]furan-2(3H)-one (18c)}<smiles>C[C@H]1C(=O)O[C@H]2c3ccccc3CC[C@@H]12</smiles>

According to the general procedure (ketone 20: $0.300 \mathrm{~g} \quad(1.5 \mathrm{mmol})$, cat. $\left[\mathrm{NH}_{2} \mathrm{Me}_{2}\right]\left[\left\{\mathrm{RuCl}\left((R)-\mathrm{H}_{8} \text {-binap }\right\}_{2}(\mu-\mathrm{Cl})_{3}\right]\right.$, dioxane solvent $), 0.260 \mathrm{~g}$ of 18c was obtained as a white solid ( $86 \%$ yield, $>99 \%$ ee, $>99 \%$ de).

${ }^{1} \mathrm{H}$ NMR $\left(500 \mathrm{MHz}, \mathrm{CDCl}_{3}\right) \delta$ 7.48-7.45 (m, 1H), 7.27-7.24 (m, 2H), 7.15-7.13 (m, 1H), $5.51(\mathrm{t}, J=6.5 \mathrm{~Hz}, 1 \mathrm{H}), 2.84(\mathrm{ddd}, J=14.6,9.7,4.9 \mathrm{~Hz}, 1 \mathrm{H}), 2.73(\mathrm{ddd}, J=14.6,9.7,4.9$ $\mathrm{Hz}, 1 \mathrm{H}), 2.55-2.45(\mathrm{~m}, 2 \mathrm{H}), 2.05-1.99(\mathrm{~m}, 1 \mathrm{H}), 1.82-1.75(\mathrm{~m}, 1 \mathrm{H}), 1.36$ (ddd, $J=7.1 \mathrm{~Hz}$, $3 \mathrm{H}) ;{ }^{13} \mathrm{C}$ NMR $\left(125 \mathrm{MHz}, \mathrm{CDCl}_{3}\right) \delta 179.4,136.8,132.1,130.3,128.6,128.5,126.8,76.6$, 41.8, 39.4, 26.0, 23.8, 14.4.

HRMS (APCI) calcd for $\mathrm{C}_{13} \mathrm{H}_{15} \mathrm{O}_{2}[\mathrm{M}+\mathrm{H}]^{+}:$203.1067. Found: 203.1072.

The enantiomeric excess was determined by HPLC analysis (Chiralcel OJ-3, 250×4.6 mm column, hexane/2-propanol 95:5, $1.0 \mathrm{~mL} / \mathrm{min}, 220 \mathrm{~nm}, 35^{\circ} \mathrm{C},(3 R, 3 \mathrm{a} R, 9 \mathrm{bS})$ isomer $13.9 \mathrm{~min}$ (minor), $(3 S, 3 \mathrm{a} S, 9 \mathrm{~b} R)$ isomer $15.8 \mathrm{~min}$ (major), $(3 S, 3 \mathrm{a} R, 9 \mathrm{bS})$ isomer $16.5 \mathrm{~min}$ (minor), $(3 R, 3 \mathrm{a} S, 9 \mathrm{~b} R)$ isomer $24.8 \mathrm{~min}$ (minor)); IR (neat) 3490, 3027, 2984, 2944, 2922, 2876, 2859, 1757, 1745, 1605, 1496, 1487, 1457, 1441, 1380, 1323, 1279, 1223, 1211, 1179, 1164, 1118, $1085,1063,1047,1015,997,944,912,846,778,749,715,698,670 \mathrm{~cm}^{-1} ;[\alpha]_{\mathrm{D}}{ }^{20}-121.2(\mathrm{c}$ 0.85 in $\left.\mathrm{CHCl}_{3}\right)>99 \%$ ee, $>99 \%$ de $(3 S, 3 \mathrm{a} S, 9 \mathrm{bR})$.

\section{(3R,3aS,9bR)-3-Methyl-3a,4,5,9b-tetrahydronaphtho[1,2-b]furan-2(3H)-one (19c)}<smiles>C[C@H]1C(=O)O[C@H]2c3ccccc3CC[C@@H]12</smiles>

According to the general procedure (ketone 20: $0.150 \mathrm{~g}(0.75 \mathrm{mmol})$, cat. $\left[\mathrm{NH}_{2} \mathrm{Me}_{2}\right]\left[\{\mathrm{RuCl}((S) \text {-segphos })\}_{2}(\mu-\mathrm{Cl})_{3}\right]$, THF solvent $),, 0.151 \mathrm{~g}$ of 19c was obtained as a white solid (>99\% yield, $>99 \%$ ee, $>99 \%$ de).

${ }^{1} \mathrm{H}$ NMR $\left(500 \mathrm{MHz}, \mathrm{CDCl}_{3}\right) \delta$ 7.48-7.43 (m, 1H), 7.30-7.24 (m, 2H), 7.17-7.15 (m, 1H), $5.29(\mathrm{t}, J=5.0 \mathrm{~Hz}, 1 \mathrm{H}), 3.08-3.01(\mathrm{~m}, 1 \mathrm{H}), 2.90-2.84(\mathrm{~m}, 1 \mathrm{H}), 2.75-2.63(\mathrm{~m}, 2 \mathrm{H}), 1.93-$ $1.87(\mathrm{~m}, 1 \mathrm{H}), 1.42-1.33(\mathrm{~m}, 1 \mathrm{H}), 1.26(\mathrm{t}, J=7.3 \mathrm{~Hz}, 3 \mathrm{H}) ;{ }^{13} \mathrm{C} \mathrm{NMR}\left(125 \mathrm{MHz}, \mathrm{CDCl}_{3}\right) \delta$ 178.5, 137.8, 131.1, 130.8, 128.9, 128.7, 126.6, 76.7, 40.5, 39.6, 28.2, 20.2, 9.4.

HRMS (APCI) calcd for $\mathrm{C}_{13} \mathrm{H}_{15} \mathrm{O}_{2}[\mathrm{M}+\mathrm{H}]^{+}:$203.1067. Found: 203.1071. 
The enantiomeric excess was determined by HPLC analysis (Chiralcel OJ-3, 250×4.6 mm column, hexane/2-propanol 95:5, $1.0 \mathrm{~mL} / \mathrm{min}, 220 \mathrm{~nm}, 35^{\circ} \mathrm{C},(3 R, 3 \mathrm{a} R, 9 \mathrm{~b} S)$ isomer $13.9 \mathrm{~min}$ (minor), (3S,3aS,9bR) isomer $15.8 \mathrm{~min}$ (minor), $(3 S, 3 \mathrm{a} R, 9 \mathrm{~b} S)$ isomer $16.5 \mathrm{~min}$ (minor), $(3 R, 3 \mathrm{a} S, 9 \mathrm{~b} R)$ isomer $24.8 \mathrm{~min}$ (major)); IR (neat) 3494, 3036, 2985, 2969, 2935, 2905, 2853, 1752, 1606, 1497, 1458, 1448, 1377, 1350, 1320, 1311, 1284, 1267, 1225, 1192, 1178, 1119, $1057,1007,995,941,911,880,862,836,769,746,693 \mathrm{~cm}^{-1} ;[\alpha]_{\mathrm{D}}^{20}-73.2\left(\mathrm{c} 0.99\right.$ in $\left.\mathrm{CHCl}_{3}\right)$ $>99 \%$ ee, $>99 \%$ de $(3 R, 3 \mathrm{a} S, 9 \mathrm{~b} R)$. 


\section{G. Synthesis of Wine Lactone (6).}

(1) Synthesis of 2-(4-Methyl-2-oxocyclohex-3-en-1-yl)acetic acid (23)

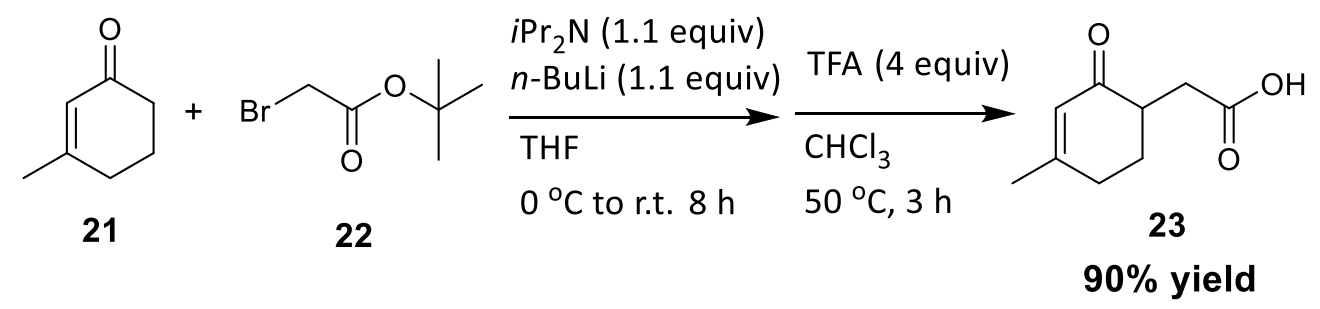

A THF solution $(1.57 \mathrm{M})$ of $n$-BuLi $(159.0 \mathrm{~mL}, 249.6 \mathrm{mmol})$ was added slowly to a THF solution $(400 \mathrm{~mL})$ containing $N, N$-diisopropylamine $(32.3 \mathrm{~g}, 249.6 \mathrm{mmol})$ at $-60{ }^{\circ} \mathrm{C}$ under $\mathrm{N}_{2}$ atmosphere and stirred for $1 \mathrm{~h}$. The resulting mixture was warmed to $-20{ }^{\circ} \mathrm{C}$, then 3methylcyclohexenone (21) $(25.0 \mathrm{~g}, 226.9 \mathrm{mmol})$ was added and stirred for $1 \mathrm{~h}$ at $0{ }^{\circ} \mathrm{C}$. Subsequently, tert-butyl 2-bromoacetate (22) (44.3 g, $226.9 \mathrm{mmol})$ and 1,3-dimethyl-2imidazolidinone $(26.1 \mathrm{~g}, 226.94 \mathrm{mmol}, 1.0$ equiv) were added to the reaction mixture. After stirring further for $5 \mathrm{~h}$ at $0{ }^{\circ} \mathrm{C}$, the resulting mixture was concentrated by rotary evaporator. The residue was added trifluoroacetic acid (103.5 g, $907.8 \mathrm{mmol})$ and $\mathrm{CHCl}_{3}(250 \mathrm{~mL})$. After stirring the reaction mixture for $2 \mathrm{~h}$ at $50{ }^{\circ} \mathrm{C}$, water $(50 \mathrm{~mL})$ was added and followed by extraction with $\mathrm{CHCl}_{3}(3 \times 50 \mathrm{~mL})$. The combined organic layer was washed with brine (100 $\mathrm{mL}$ ), dried over anhydrous $\mathrm{MgSO}_{4}$, and concentrated under reduced pressure to afford the crude product. Purification by silica gel column chromatography (eluent: hexane/AcOEt $=$ $1 / 1$ to $2 / 3$ ) gave 23 (34.4 g, 90\% yield) as a light brown solid.

${ }^{1} \mathrm{H}$ NMR $\left(500 \mathrm{MHz}, \mathrm{CDCl}_{3}\right) \delta 10.0(\mathrm{br}, 1 \mathrm{H}), 5.91(\mathrm{~s}, 1 \mathrm{H}), 2.95(\mathrm{dd}, J=16.6,5.8 \mathrm{~Hz}, 1 \mathrm{H})$, 2.78-2.72 (m, 1H), 2.51-2.43 (m, 1H), 2.35-2.28 (m, 2H), 2.18-2.12 (m, 1H), 1.97 (s, 3H), $1.84-1.76(\mathrm{~m}, 1 \mathrm{H}) ;{ }^{13} \mathrm{C}$ NMR $\left(125 \mathrm{MHz}, \mathrm{CDCl}_{3}\right) \delta 199.5,178.0,162.9,125.8,42.4,34.6$, 31.0, 28.5, 24.2.

HRMS (APCI) calcd for $\mathrm{C}_{9} \mathrm{H}_{13} \mathrm{O}_{3}[\mathrm{M}+\mathrm{H}]^{+}:$169.0859. Found: 169.0864 .

IR (neat) 2984, 2932, 2850, 1712, 1621, 1451, 1412, 1381, 1320, 1270, 1214, 1173, 1144, $1112,1069,1037,1017,993,918,872,764,688,666 \mathrm{~cm}^{-1}$

(2) Synthesis of $(3 \mathrm{a} S, 7 \mathrm{a} R)$-6-Methyl-3a,4,5,7a-tetrahydrobenzofuran-2(3H)-one (24)

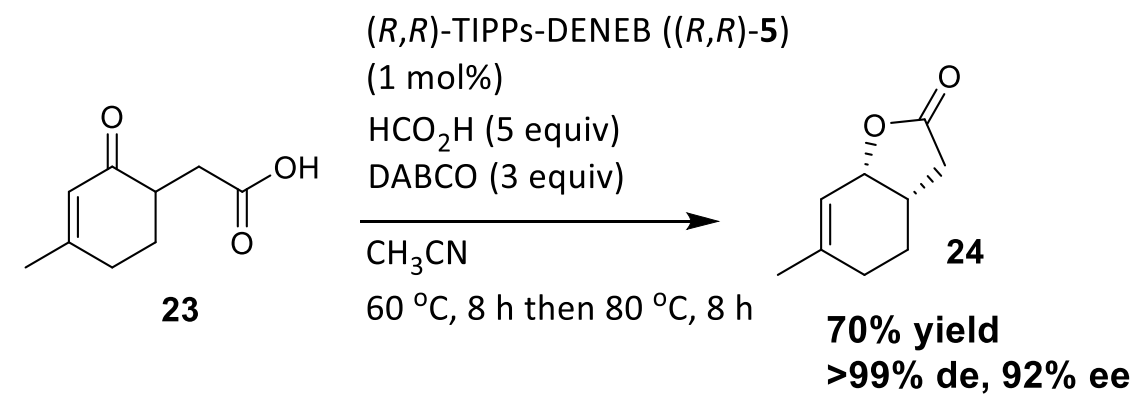


Under $\mathrm{N}_{2}$ atmosphere, a mixture of ketone $(\mathbf{2 3})(5.0 \mathrm{~g}, 30.0 \mathrm{mmol})$ and the $(R, R)$-TIPPs$\operatorname{DENEB}((R, R)-7)(0.24 \mathrm{~g}, 0.30 \mathrm{mmol})$ in formic acid $(6.84 \mathrm{~g}, 5.60 \mathrm{~mL}, 149 \mathrm{mmol}), \mathrm{DABCO}$ $(10.0 \mathrm{~g}, 89.2 \mathrm{mmol})$ and acetonitrile $(30 \mathrm{~mL})$ was stirred at $60{ }^{\circ} \mathrm{C}$ for $8 \mathrm{~h}$, and then $80{ }^{\circ} \mathrm{C}$ for another $8 \mathrm{~h}$. After the reaction completion, water $(20 \mathrm{~mL})$ and chloroform $(20 \mathrm{~mL})$ was added. The biphasic layers were separated, the aqueous layer was extracted with chlroform $(3 \times 15 \mathrm{~mL})$, and the combined organic portions were washed with brine $(15 \mathrm{~mL})$. After drying over $\mathrm{MgSO}_{4}$, filtration, and solvent removal under reduced pressure, the crude residue was purified by silica gel column chromatography (hexane/EtOAc $=5 / 1$ to $2 / 1$ ) to afford the desired product (24) (3.17 g, 70\% yield, 92\% ee). The optical purity of product was determined by chiral GC analysis using a CHIRALSIL-DEX-CB column.

${ }^{1} \mathrm{H}$ NMR $\left(500 \mathrm{MHz}, \mathrm{CDCl}_{3}\right) \delta 5.63-5.62(\mathrm{~m}, 1 \mathrm{H}), 4.81-4.79(\mathrm{~m}, 1 \mathrm{H}), 2.71(\mathrm{dd}, J=17.3,8.0$ $\mathrm{Hz}, 1 \mathrm{H}), 2.52-2.46(\mathrm{~m}, 1 \mathrm{H}), 2.32(\mathrm{dd}, J=17.3,3.5 \mathrm{~Hz}, 1 \mathrm{H}), 2.04-1.95(\mathrm{~m}, 2 \mathrm{H}), 1.78(\mathrm{~s}, 3 \mathrm{H})$, $1.77-1.73(\mathrm{~m}, 1 \mathrm{H}), 1.55-1.47(\mathrm{~m}, 1 \mathrm{H}) ;{ }^{13} \mathrm{C} \mathrm{NMR}\left(125 \mathrm{MHz}, \mathrm{CDCl}_{3}\right) \delta 176.7,142.9,117.6$, 77.0, 35.3, 33.0, 27.9, 24.0, 23.8.

HRMS (APCI) calcd for $\mathrm{C}_{9} \mathrm{H}_{13} \mathrm{O}_{2}[\mathrm{M}+\mathrm{H}]^{+}:$153.0910. Found: 153.0912 .

The enantiomeric excess was determined by Chiral-GC analysis (CHIRALSIL-DEX-CB, $0.25 \mathrm{~mm} \times 25 \mathrm{~m}$ column, $\mathrm{DF}=0.25, \mathrm{Temp} .=130^{\circ} \mathrm{C}$ const.,$(3 \mathrm{a} R, 7 \mathrm{a} S)$ isomer $13.3 \mathrm{~min}$ (minor), $(3 \mathrm{a} S, 7 \mathrm{a} R$ ) isomer $13.7 \mathrm{~min}$ (major)); IR (neat) 3496, 3021, 2942, 2911, 2857, 2842, 1748, 1668, 1450, 1434, 1419, 1385, 1353, 1334, 1310, 1293, 1252, 1232, 1215, 1154, 1120, 1070, $1051,1033,942,924,877,824,799,758,705,673 \mathrm{~cm}^{-1} ;[\alpha]_{\mathrm{D}}{ }^{20}+52.4\left(\mathrm{c} 0.90\right.$ in $\left.\mathrm{CHCl}_{3}\right) 92 \%$ ee, $>99 \%$ de $(3 \mathrm{a} S, 7 \mathrm{a} R)$.

\section{(3) Synthesis of (3aS,7aS)-6-Methyl-3-methylene-3a,4,5,7a-tetrahydrobenzofuran-} $2(3 H)$-one (25)

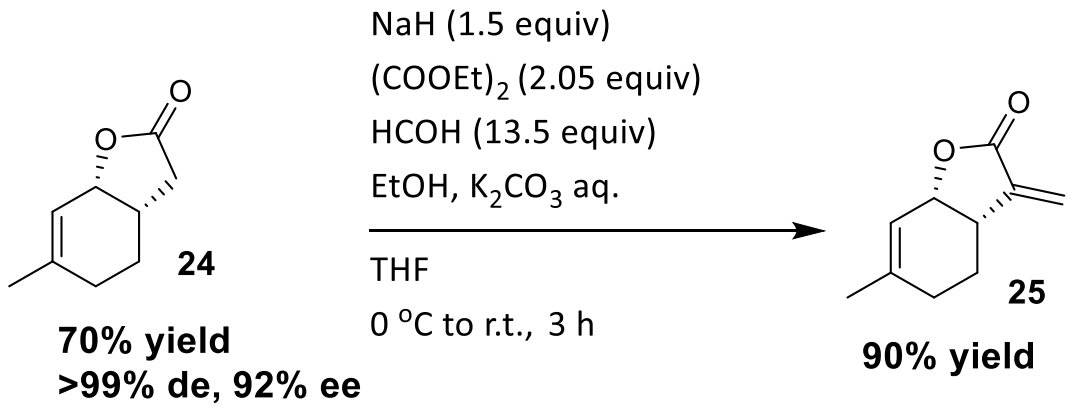

To a suspension of $\mathrm{NaH}$ ( $60 \mathrm{wt} \%$ dispersion in mineral oil, $0.24 \mathrm{~g}, 5.91 \mathrm{mmol}$ ) in THF (24 $\mathrm{mL})$ cooled at $0{ }^{\circ} \mathrm{C}$, a solution of $24(0.6 \mathrm{~g}, 3.94 \mathrm{mmol})$ and diethyl oxalate $(1.18 \mathrm{~mL}, 8.08$ $\mathrm{mmol})$ in THF (24 mL) was added dropwise. After complete addition, ethanol $(3.5 \mathrm{~mL})$ was added and stirred at ambient temperature for $4 \mathrm{~h}$. After cooling the reaction mixture to $0{ }^{\circ} \mathrm{C}$, a solution of $\mathrm{K}_{2} \mathrm{CO}_{3}(2.23 \mathrm{~g})$ in water $(3.3 \mathrm{~mL})$ and formaldehyde $(37 \mathrm{wt} \%$ aqueous solution, $4.37 \mathrm{~g}$ ) were added followed by stirring for $0.25 \mathrm{~h}$ at this temperature. The mixture was diluted with brine $(10 \mathrm{~mL})$, and the resulting aqueous layer was extracted twice with ethyl acetate $(50 \mathrm{~mL}$ each). The combined organic extracts were washed with brine, dired with $\mathrm{MgSO}_{4}$, filtered, and evaporated. The residue was purified by column chromatography on 
silica, using hexane/MTBE (3/1) as eluent, to furnish 25 (0.58 g, 90\% yield) as a clear oil.

${ }^{1} \mathrm{H}$ NMR $\left(500 \mathrm{MHz}, \mathrm{CDCl}_{3}\right) \delta 6.24(\mathrm{~d}, J=2.5 \mathrm{~Hz}, 1 \mathrm{H}), 5.62-5.59(\mathrm{~m}, 2 \mathrm{H}), 4.92-4.88(\mathrm{~m}$, 1H), 3.13-3.08 (m, 1H), 2.05-2.00 (m, 1H), 1.95-1.86 (m, 2H), 1.78-1.71 (m, 1H), $1.76(\mathrm{~s}$, $3 \mathrm{H}) ;{ }^{13} \mathrm{C} \mathrm{NMR}\left(125 \mathrm{MHz}, \mathrm{CDCl}_{3}\right) \delta 170.5,142.3,139.1,121.1,118.5,74.9,37.4,26.7,24.5$, 23.9.

HRMS (APCI) calcd for $\mathrm{C}_{10} \mathrm{H}_{13} \mathrm{O}_{2}[\mathrm{M}+\mathrm{H}]^{+}:$165.0910. Found: 165.0916.

IR (neat) 2915, 1753, 1669, 1447, 1404, 1381, 1362, 1314, 1294, 1266, 1247, 1214, 1188, $1145,1109,1047,1002,987,944,899,865,817,786,753 \mathrm{~cm}^{-1} ;[\alpha]_{\mathrm{D}}^{20}-63.3(\mathrm{c} 1.03$ in $\left.\mathrm{CHCl}_{3}\right) 92 \%$ ee, $>99 \%$ de $(3 \mathrm{aS}, 7 \mathrm{aS})$.

\section{(4) Selective Synthesis of Wine Lactone (3S,3aS,9bS)-6 via Asymmetric Hydrogenation}
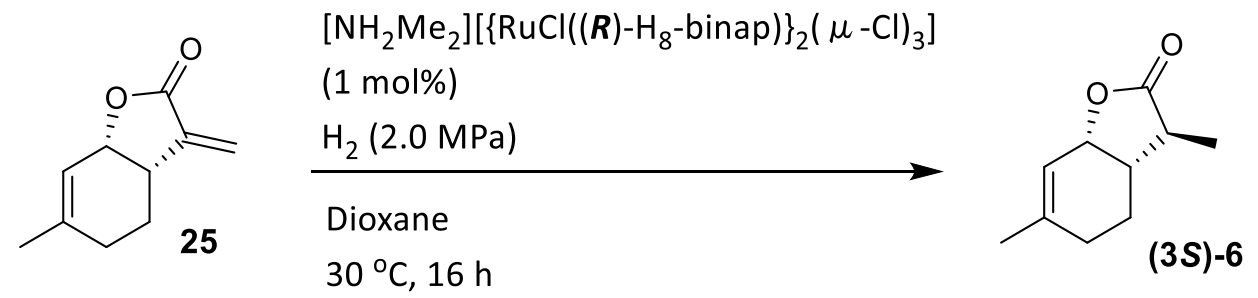

(3aS,7aS)-6-Methyl-3-methylene-3a,4,5,7a-tetrahydrobenzofuran-2(3H)-one (25) $(54.7 \mathrm{mg}$, $0.33 \mathrm{mmol})$ and $\left[\mathrm{NH}_{2} \mathrm{Me}_{2}\right]\left[\left\{\mathrm{RuCl}\left((R)-\mathrm{H}_{8} \text {-binap }\right)\right\}_{2}(\mu-\mathrm{Cl})_{3}\right](2.8 \mathrm{mg}, 0.0033 \mathrm{mmol})$ were placed in a $50 \mathrm{~mL}$ stainless steel autoclave equipped with a glass inner tube and Teflon-coated magnetic stir bar. The atmosphere was replaced with argon gas, and dioxane $(1.0 \mathrm{~mL})$ was added to the mixture. Hydrogen was initially introduced into the autoclave at a pressure of $0.5 \mathrm{MPa}$, before being reduced to $0.1 \mathrm{MPa}$. This procedure was repeated three times. Then the autoclave was pressurized with $\mathrm{H}_{2}$ gas $(2.0 \mathrm{MPa})$, and the solution was stirred vigorously at $30{ }^{\circ} \mathrm{C}$ for $16 \mathrm{~h}$. After completion of the reaction, hydrogen gas was carefully vented. Diastereoselctivity of the products was determined by GC analysis $(\mathrm{dr}=(\mathbf{3 S})-\mathbf{6}:(\mathbf{3 R})-\mathbf{6}=7: 3)$ and enantioselectivity of products was determined HPLC analysis $(>99.9 \%$ ee). The crude product was purified by silica gel chromatography (hexane/TBME $=15 / 1$ to $5 / 1$ ) to give product ((3S)-6) (38.2 $\mathrm{mg}, 69 \%$ yield) as a colorless solid.

${ }^{1} \mathrm{H}$ NMR $\left(500 \mathrm{MHz}, \mathrm{CDCl}_{3}\right) \delta 5.51(\mathrm{~d}, J=1.5 \mathrm{~Hz}, 1 \mathrm{H}), 4.89(\mathrm{ddd}, J=3.4,3.4,1.5 \mathrm{~Hz}, 1 \mathrm{H})$, 2.45-2.39 (m, 1H), 2.30-2.24 (m, 1H), 2.08-1.92 (m, 2H), 1.87-1.70 (m, 2H), $1.74(\mathrm{~s}, 3 \mathrm{H})$, $1.26(\mathrm{~d}, J=7.3 \mathrm{~Hz}, 3 \mathrm{H}) ;{ }^{13} \mathrm{C} \mathrm{NMR}\left(125 \mathrm{MHz}, \mathrm{CDCl}_{3}\right) \delta 179.6,140.7,118.7,75.3,40.3,37.5$, 25.9, 23.6, 22.2, 13.9.

HRMS (APCI) calcd for $\mathrm{C}_{10} \mathrm{H}_{15} \mathrm{O}_{2}[\mathrm{M}+\mathrm{H}]^{+}$: 167.1067. Found: 167.1069.

The enantiomeric excess was determined by HPLC analysis (Chiralcel OZ-3, 250×4.6 mm column, hexane/2-propanol 97:3, $1.0 \mathrm{~mL} / \mathrm{min}, 210 \mathrm{~nm}, 35^{\circ} \mathrm{C},(3 S, 3 \mathrm{a} S, 7 \mathrm{a} R)$ isomer $16.4 \mathrm{~min}$ (major), $(3 R, 3 \mathrm{a} R, 7 \mathrm{a} S)$ isomer $17.3 \mathrm{~min}$ (minor), $(3 R, 3 \mathrm{a} S, 7 \mathrm{a} R)$ isomer $24.4 \mathrm{~min}$ (minor), and $(3 S, 3 \mathrm{a} R, 7 \mathrm{a} S$ ) isomer $25.9 \mathrm{~min}$ (minor)); IR (neat) 2965, 2929, 1763, 1275, 1673, 1448, 1382, 1352, 1322, 1287, 1210, 1171, 1148, 1085, 1056, 1010, 971, 949, 898, 840, 821, 745, 711, 
$663 \mathrm{~cm}^{-1} ;[\alpha]_{\mathrm{D}}{ }^{20}-11.6\left(\mathrm{c} 0.42\right.$ in $\left.\mathrm{CHCl}_{3}\right) 99 \%$ ee, $99 \%$ de $(3 S, 3 \mathrm{aS}, 7 \mathrm{aR})\left(\right.$ lit. $^{[12]}[\alpha]_{\mathrm{D}}{ }^{20}-13.1(\mathrm{c}$ 1 in $\left.\mathrm{CHCl}_{3}\right) 99 \%$ de $\left.(3 S, 3 \mathrm{aS}, 7 \mathrm{a} R)\right)$.

(5) Selective Synthesis of an Epimer of Wine Lactone (3R,3aS,9bS)-6<smiles>C=C1C(=O)O[C@@H]2C=C(C)CC[C@H]12</smiles>
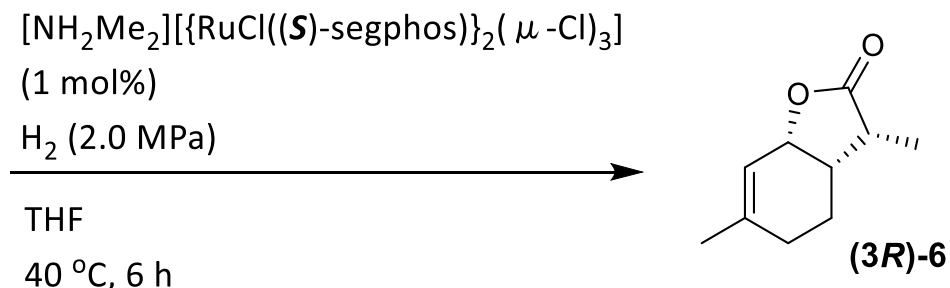

(3aS,7aS)-6-Methyl-3-methylene-3a,4,5,7a-tetrahydrobenzofuran-2(3H)-one (25) $(54.7 \mathrm{mg}$, $0.33 \mathrm{mmol})$ and $\left[\mathrm{NH}_{2} \mathrm{Me}_{2}\right]\left[\{\mathrm{RuCl}((S) \text {-segphos })\}_{2}(\mu-\mathrm{Cl})_{3}(2.7 \mathrm{mg}, 0.0033 \mathrm{mmol})\right.$ were placed in a $50 \mathrm{~mL}$ stainless steel autoclave equipped with a glass inner tube and Teflon-coated magnetic stir bar. The atmosphere was replaced with argon gas, and dioxane $(1.0 \mathrm{~mL})$ was added to the mixture. Hydrogen was initially introduced into the autoclave at a pressure of $0.5 \mathrm{MPa}$, before being reduced to $0.1 \mathrm{MPa}$. This procedure was repeated three times. Then the autoclave was pressurized with $\mathrm{H}_{2}$ gas $(2.0 \mathrm{MPa})$, and the solution was stirred vigorously at $40{ }^{\circ} \mathrm{C}$ for $6 \mathrm{~h}$. After completion of the reaction, hydrogen gas was carefully vented. Diastereoselctivity of the products was determined by GC analysis $(\mathrm{dr}=(\mathbf{3 S})-\mathbf{6}:(\mathbf{3 R})-\mathbf{6}=$ $<1:>99)$ and enantioselectivity of products was determined HPLC analysis ( $94.3 \%$ ee). The crude product was purified by silica gel chromatography (hexane/TBME $=15 / 1$ to $5 / 1$ ) to give product ((3R)-6) (101.0 $\mathrm{mg},>99 \%$ yield) as a white solid.

${ }^{1} \mathrm{H}$ NMR $\left(500 \mathrm{MHz}, \mathrm{CDCl}_{3}\right) \delta$ 5.69-5.62 (m, 1H), 4.66-4.62 (m, 1H), 2.93-2.88 (m, 1H), 2.39-2.32 (m, 1H), 2.08-1.95 (m, 2H), 1.79 (s, 3H), 1.72-1.66 (m, 1H), 1.19 (d, J = 7.3 Hz, $3 \mathrm{H}) ;{ }^{13} \mathrm{C} \mathrm{NMR}\left(125 \mathrm{MHz}, \mathrm{CDCl}_{3}\right) \delta 178.8,144.0,117.0,74.7,40.2,37.8,28.7,23.7,19.7$, 9.3 .

HRMS (APPI) calcd for $\mathrm{C}_{10} \mathrm{H}_{15} \mathrm{O}_{2}[\mathrm{M}+\mathrm{H}]^{+}$: 167.1067. Found: 167.1066.

The enantiomeric excess was determined by HPLC analysis (Chiralcel OZ-3, 250×4.6 mm column, hexane/2-propanol 97:3, $1.0 \mathrm{~mL} / \mathrm{min}, 210 \mathrm{~nm}, 30^{\circ} \mathrm{C},(3 S, 3 \mathrm{a} S, 7 \mathrm{a} R)$ isomer $16.4 \mathrm{~min}$ (minor), (3R,3aR,7aS) isomer $17.3 \mathrm{~min}$ (minor), $(3 R, 3 \mathrm{a} S, 7 \mathrm{a} R)$ isomer $24.4 \mathrm{~min}$ (major), and $(3 S, 3 \mathrm{a} R, 7 \mathrm{a} S$ ) isomer $25.9 \mathrm{~min}$ (minor)); IR (neat) 3018, 2984, 2969, 2935, 2856, 1747, 1670, 1448, 1434, 1383, 1350, 1327, 1312, 1287, 1254, 1215, 1201, 1167, 1095, 1064, 1043, 1028, $1004,973,940,887,866,822,783,754,736,709,683 \mathrm{~cm}^{-1} ;[\alpha]_{\mathrm{D}}^{20}+103.5\left(\mathrm{c} 1.10\right.$ in $\left.\mathrm{CHCl}_{3}\right)$ $94 \%$ ee, $99 \%$ de $(3 R, 3 \mathrm{aS}, 7 \mathrm{a} R)\left(\right.$ lit. $^{[12]}[\alpha]_{\mathrm{D}}{ }^{20}+121.2\left(\mathrm{c} 1\right.$ in $\left.\mathrm{CHCl}_{3}\right) 99 \%$ de $\left.(3 R, 3 \mathrm{aS}, 7 \mathrm{a} R)\right)$. 


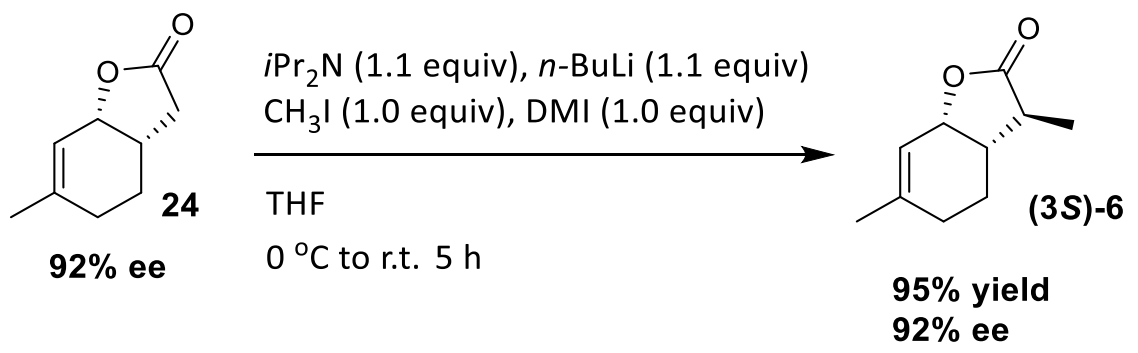

A THF solution $(1.57 \mathrm{M})$ of $n$-BuLi $(4.6 \mathrm{~mL}, 7.2 \mathrm{mmol})$ was added slowly to a THF solution $(25 \mathrm{~mL})$ containing $N, N$-diisopropylamine $(0.94 \mathrm{~g}, 7.2 \mathrm{mmol})$ at $-60{ }^{\circ} \mathrm{C}$ under $\mathrm{N}_{2}$ atmosphere and stirred for $1 \mathrm{~h}$. The resulting mixture was warmed to $-20{ }^{\circ} \mathrm{C}$, then $(3 \mathrm{aS}, 7 \mathrm{a} R)-6$-methyl3a,4,5,7a-tetrahydrobenzofuran-2(3H)-one $(\mathbf{2 4})(1.0 \mathrm{~g}, 6.57 \mathrm{mmol})$ was added and stirred for $1 \mathrm{~h}$ at $0{ }^{\circ} \mathrm{C}$. Subsequently, $\mathrm{CH}_{3} \mathrm{I}(0.93 \mathrm{~g}, 6.6 \mathrm{mmol})$ and 1,3 -dimethyl-2-imidazolidinone $(0.75$ $\mathrm{g}, 6.6 \mathrm{mmol}$ ) were added to reaction mixture and stirred at $0{ }^{\circ} \mathrm{C}$. After stirring further for $5 \mathrm{~h}$ at ambient temperature, the resulting mixture was diluted with saturated aqueous $\mathrm{NH}_{4} \mathrm{Cl}$ solution $(10 \mathrm{~mL})$. The layers were separated, and the aqueous layer was extracted twice with $\mathrm{CHCl}_{3}$ (30 mL each). The combined organic extracts were washed with brine, dired with $\mathrm{MgSO}_{4}$, filtered, and evaporated. The residue was purified by column chromatography on silica, usine hexane/MTBE (5/1 to 4/1) as eluent, to furnish (3S,3aS,9bS)-6 (0.98 g, $90 \%$ yield) as a colorless solid. 


\section{H. Synthesis of $(R, R)$-TIPPs-DENEB $((R, R)-5)$.}

\section{(1) Synthesis of $(R, R)$-TIPPs-DPEN}
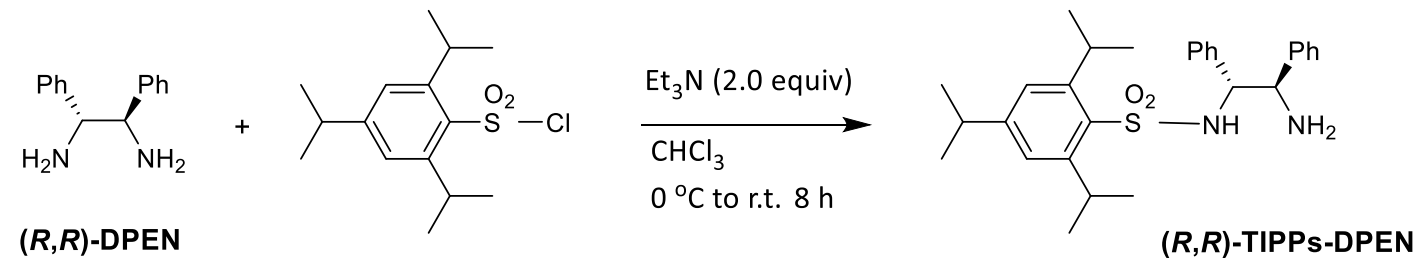

To a suspension of $(\boldsymbol{R}, \boldsymbol{R})$-DPEN $(17.5 \mathrm{~g}, 82.6 \mathrm{mmol})$ and triethylamine $(12.5 \mathrm{~g}, 124 \mathrm{mmol})$ in chloroform $(350 \mathrm{~mL})$ was added 2,4,6-triisopropylbenzenesulfonyl chloride $(25.0 \mathrm{~g}, 82.6$ $\mathrm{mmol})$ chloroform $(100 \mathrm{~mL})$ solution slowly at $0{ }^{\circ} \mathrm{C}$. The reaction mixture was stirred at room temperature for $8 \mathrm{~h}$. After the reaction completion, water $(10 \mathrm{~mL})$ was added and the biphasic layers were separated. The aqueous layer was extracted with chlroform $(3 \times 50 \mathrm{~mL})$, and the combined organic portions were washed with brine $(15 \mathrm{~mL})$. After drying over $\mathrm{MgSO}_{4}$, filtration, and solvent removal under reduced pressure, the crude residue was purified by silica gel column chromatography (hexane/EtOAc $=2 / 1$ to $1 / 1$ ) to afford the desired product $((\boldsymbol{R}, \boldsymbol{R})$-TIPPs-DPEN $)(38.3 \mathrm{~g}, 95 \%$ yield $)$.

${ }^{1} \mathrm{H}$ NMR $\left(500 \mathrm{MHz}, \mathrm{CDCl}_{3}\right) \delta$ 7.17-7.13 (m, 3H), 7.04-6.92 (m, 7H), 6.82-6.80 (m, 2H), 6.20 (br, $1 \mathrm{H}), 4.49$ (d, $J=7.7 \mathrm{~Hz}, 1 \mathrm{H}), 4.00-3.85$ (m, 3H), $2.83(\mathrm{sep}, J=7.0 \mathrm{~Hz}, 1 \mathrm{H}), 1.20$ $(\mathrm{d}, J=7.0 \mathrm{~Hz}, 6 \mathrm{H}), 1.17(\mathrm{~d}, J=6.8 \mathrm{~Hz}, 6 \mathrm{H}), 1.09(\mathrm{~d}, J=6.8 \mathrm{~Hz}, 6 \mathrm{H}) ;{ }^{13} \mathrm{C} \mathrm{NMR}(125 \mathrm{MHz}$, $\left.\mathrm{CDCl}_{3}\right) \delta 152.2,149.6,141.9,138.6,133.9,128.2,127.7,127.4,127.2,127.2,126.7,123.2$, 63.5, 61.1, 34.0, 29.7, 24.8, 24.7, 23.6, 23.6.

HRMS (APCI) calcd for $\mathrm{C}_{29} \mathrm{H}_{39} \mathrm{~N}_{2} \mathrm{O}_{2} \mathrm{~S}[\mathrm{M}+\mathrm{H}]^{+}:$479.2727. Found: 479.2737.

IR (neat) 3297, 3063, 3030, 2958, 2928, 2868, 1600, 1562, 1494, 1455, 1424, 1383, 1363, $1313,1255,1195,1148,1105,1059,1041,1028,926,881,756,733,697,662 \mathrm{~cm}^{-1}$

(2) Synthesis of 2,4,6-Triisopropyl- $N$-((1R,2R)-2-((2-((4-methylcyclohexa-1,4-dien-1yl)methoxy)ethyl)amino)-1,2-diphenylethyl)benzenesulfonamide

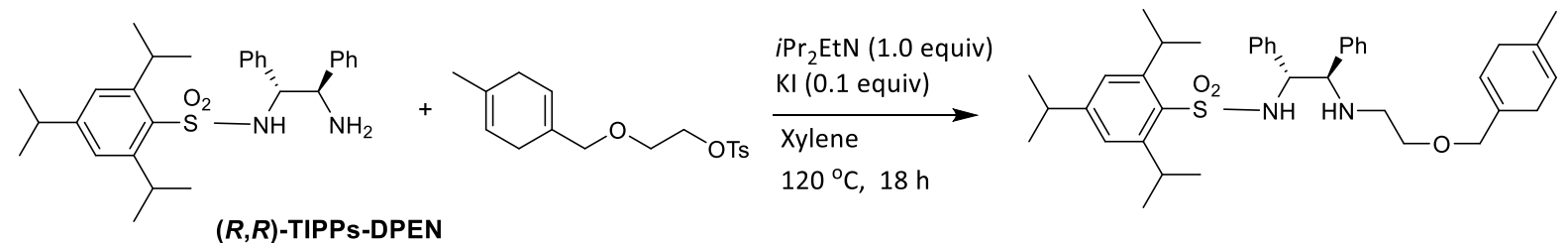

To a suspension of 4-(4-methylcyclohexa-1,4-dienyl)butyl 4-methylbenzenesulfonate (13.94 $\mathrm{g}, 43.5 \mathrm{mmol})$ and diisopropylethylamine $(5.40 \mathrm{~g}, 43.5 \mathrm{mmol})$ and $\mathrm{KI}(0.72 \mathrm{~g}, 4.35 \mathrm{mml})$ in xylene $(60 \mathrm{~mL})$ was added $(R, R)$-TIPPsDPEN $(20.8 \mathrm{~g}, 43.5 \mathrm{mmol})$. The reaction mixture was heated at $125^{\circ} \mathrm{C}$ for $14 \mathrm{~h}$, after which the reaction mixture was concentrated under reduced pressure. The residue was purified by flash chromatography (hexane/EtOAc $=20 / 1$ to $15 / 1$ ) to give the desired diamine $(18.0 \mathrm{~g}, 66 \%$ yield $)$ as a pale yellow amorphous. 
${ }^{1} \mathrm{H}$ NMR $\left(500 \mathrm{MHz}, \mathrm{CDCl}_{3}\right) \delta 7.12-7.10(\mathrm{~m}, 3 \mathrm{H}), 6.99(\mathrm{~s}, 2 \mathrm{H}), 6.94-6.92(\mathrm{~m}, 1 \mathrm{H}), 6.89-$ $6.86(\mathrm{~m}, 4 \mathrm{H}), 6.77-6.74(\mathrm{~m}, 2 \mathrm{H}), 5.63(\mathrm{~s}, 1 \mathrm{H}), 5.44(\mathrm{~s}, 1 \mathrm{H}), 4.41(\mathrm{~d}, J=9.0 \mathrm{~Hz}, 1 \mathrm{H}), 4.00$ $3.92(\mathrm{~m}, 2 \mathrm{H}), 3.80-3.75(\mathrm{~m}, 2 \mathrm{H}), 3.56(\mathrm{~d}, J=9.0 \mathrm{~Hz}, 1 \mathrm{H}), 3.49-3.44(\mathrm{~m}, 1 \mathrm{H}), 3.40-3.36(\mathrm{~m}$, $1 \mathrm{H}), 2.83$ (sep, $J=6.9 \mathrm{~Hz}, 1 \mathrm{H}), 2.70-2.50(\mathrm{~m}, 6 \mathrm{H}), 1.70(\mathrm{~s}, 3 \mathrm{H}), 1.20(\mathrm{~d}, J=7.0 \mathrm{~Hz}, 12 \mathrm{H})$, $1.07(\mathrm{~d}, J=6.7 \mathrm{~Hz}, 6 \mathrm{H}) ;{ }^{13} \mathrm{C}$ NMR $\left(125 \mathrm{MHz}, \mathrm{CDCl}_{3}\right) \delta 152.2,149.8,139.3,138.1,133.8$, 132.1, 130.8, 129.0, 128.1, 128.1, 127.6, 127.5, 127.4, 127.4, 127.0, 123.1, 121.9, 118.4, 74.9, $68.9,68.4,63.0,46.8,34.1,31.2,29.6,27.7,24.9,24.6,23.6,23.5,23.0$.

HRMS (APCI) calcd for $\mathrm{C}_{39} \mathrm{H}_{53} \mathrm{~N}_{2} \mathrm{O}_{3} \mathrm{~S}[\mathrm{M}+\mathrm{H}]^{+}:$629.3771. Found: 629.3766 .

IR (neat) 3220, 3063, 3029, 2958, 2927, 2867, 2819, 2361, 1600, 1562, 1494, 1455, 1425, $1382,1362,1314,1255,1195,1148,1104,1059,1041,1027,941,919,880,843,756,698$, $662 \mathrm{~cm}^{-1}$

\section{(3) Synthesis of $(R, R)$-TIPPs-DENEB $((R, R)-5)$}
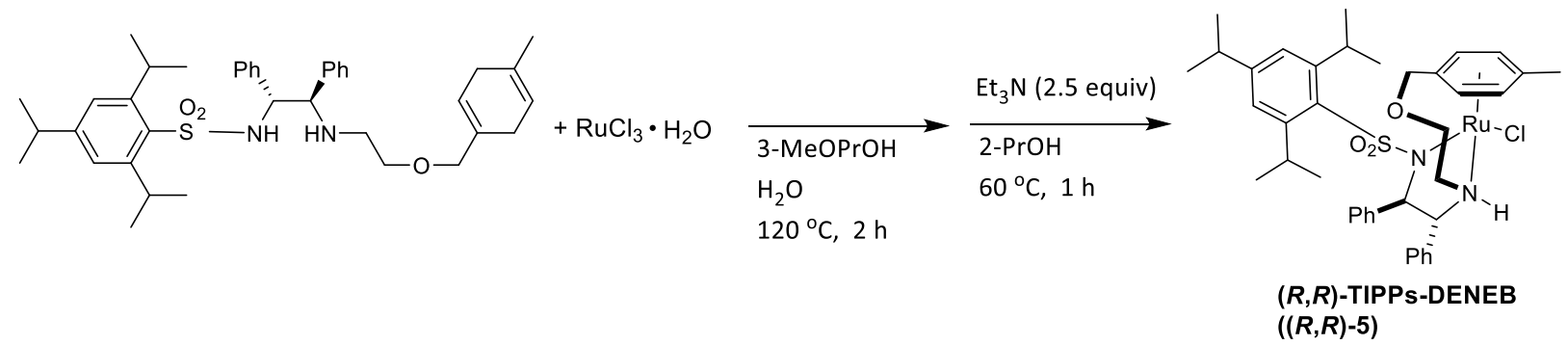

To a suspension of 2,4,6-triisopropyl- $N-((1 R, 2 R)-2-((2-((4-m e t h y l c y c l o h e x a-1,4-d i e n-1-$ yl)methoxy)ethyl)amino)-1,2-diphenylethyl)benzenesulfonamide (3.60 g, $5.72 \mathrm{mmol})$ in 3methoxypropanol $(54 \mathrm{~mL})$ and $\mathrm{H}_{2} \mathrm{O}(18 \mathrm{~mL})$ was added ruthenium(III) trichroride hydrate $(1.29 \mathrm{~g}, 4.92 \mathrm{mmol})$. The reaction mixuture was heated at $120^{\circ} \mathrm{C}$ for $2 \mathrm{~h}$. The resulting mixture was concentrated under reduced pressure. Triethylamine $(1.37 \mathrm{~g}, 13.5 \mathrm{mmol})$ and 2-propanol $(61 \mathrm{~mL})$ were added to the residue. After stirring at $60^{\circ} \mathrm{C}$ for $1 \mathrm{~h}$, the reaction mixture was concentrated under reduced pressure. The residue was purified by flash chromatography (hexane/EtOAc $=2 / 1$ to $\mathrm{CHCl}_{3} / \mathrm{MeOH}=20 / 1$ ) to give the desired ruthenium complex, $(\boldsymbol{R}, \boldsymbol{R})$-TIPPs-DENEB, $((\boldsymbol{R}, \boldsymbol{R})-5 ; 3.0 \mathrm{~g}, 80 \%$ yield $)$ as a brown solid.

${ }^{1} \mathrm{H}$ NMR (500 MHz, $\left.\mathrm{CD}_{2} \mathrm{Cl}_{2}\right) \delta 7.20-6.30(\mathrm{~m}, 12 \mathrm{H}), 5.63(\mathrm{~d}, J=6.2 \mathrm{~Hz}, 1 \mathrm{H}), 5.49(\mathrm{~d}, J=$ $6.2 \mathrm{~Hz}, 1 \mathrm{H}), 5.31-5.29(\mathrm{~m}, 1 \mathrm{H}), 5.00(\mathrm{~d}, J=15.1 \mathrm{~Hz}, 1 \mathrm{H}), 4.85-4.80(\mathrm{~m}, 1 \mathrm{H}), 4.33(\mathrm{~d}, J=$ $15.1 \mathrm{~Hz}, 1 \mathrm{H}), 4.05(\mathrm{~d}, J=10.9 \mathrm{~Hz}, 1 \mathrm{H}), 3.90-3.00(\mathrm{~m}, 7 \mathrm{H}), 2.66-2.55(\mathrm{~m}, 1 \mathrm{H}), 2.45(\mathrm{~s}, 3 \mathrm{H})$, $1.30-0.95(\mathrm{~m}, 18 \mathrm{H}) ;{ }^{13} \mathrm{C} \mathrm{NMR}\left(125 \mathrm{MHz}, \mathrm{CD}_{2} \mathrm{Cl}_{2}\right) \delta 149.8,140.1,138.5,136.1,129.3,129.1$, $128.9,128.6,128.5,128.3,128.0,126.9,126.4,122.5,94.7,92.4,91.1,90.4,77.8,75.6,74.5$, 74.4, 69.5, 68.1, 56.8, 34.3, 32.5, 28.8, 23.8, 23.6, 23.6, 18.9.

HRMS (FD) calcd for $\mathrm{C}_{39} \mathrm{H}_{49} \mathrm{ClN}_{2} \mathrm{O}_{3} \mathrm{RuS}[\mathrm{M}]^{+}:$762.2196. Found: 762.2215 .

IR (neat) 3446, 3172, 3061, 3029, 2955, 2868, 2361, 1934, 1733, 1601, 1559, 1494, 1455, 1423, 1380, 1360, 1319, 1272, 1249, 1193, 1117, 1074, 1059, 1037, 962, 940, 913, 872, 843, $792,756,700,681 \mathrm{~cm}^{-1}$ 


\section{Determination of Absolute Configuration of Products}

\section{(1) X-ray analysis}

$\underline{X-\text { ray Structure Determination for } \mathbf{1 3 d}, \mathbf{1 5 e} \text {, and } \mathbf{1 5 f}}$

Measurements were made on a Rigaku Saturn CCD area detector equipped with graphitemonochromated Mo- $K \alpha$ radiation $(\lambda=0.71070 \AA)$ under nitrogen stream at $93 \mathrm{~K}$. Single crystals suitable for X-ray analyses were mounted on glass fibers. Indexing was performed from eighteen images. The crystal-to-detector distance was $45.05 \mathrm{~mm}$. The data were collected to a maximum $2 \theta$ value of $55.0^{\circ}$. A total of 720 oscillation images were collected. A sweep of data was carried out using $\omega$ scans from 720 oscillation images were $\operatorname{collec} \chi=$ 45.0from $7 \phi=0.00 \mathrm{fr}$ A second sweep was performed using $\omega$ scans from ep was performed using were collec $\chi=45.0$ from $\phi=90.0$ from ep was performed using were for Lorentzpolarization effects as well as absorption. Structure solution and refinements were performed with the CrystalStructure program package. ${ }^{[13]}$ The heavy atom positions were determined by direct methods (SIR2002), and the remaining non-hydrogen atoms were found by subsequent Fourier techniques. An empirical absorption correction based on equivalent reflections was applied to all data. All non-hydrogen atoms other than solvent molecules were refined anisotropically by full-matrix least-square techniques based on $F^{2}$ using the SHELXL-2014/7 program. ${ }^{[14]}$ All hydrogen atoms were constrained to ride on their parent atom. Relevant crystallographic data are compiled in Tables S1-S3.

Table S9. Crystallographic Data for 13d, 15e, and $15 f$

\begin{tabular}{|c|c|c|c|}
\hline & 13d & $15 \mathrm{e}$ & $15 f$ \\
\hline empirical formula & $\mathrm{C}_{12} \mathrm{H}_{11} \mathrm{BrO}_{2}$ & $\mathrm{C}_{18} \mathrm{H}_{15} \mathrm{BrO}_{2}$ & $\mathrm{C}_{18} \mathrm{H}_{15} \mathrm{ClO}_{2}$ \\
\hline formula weight & 267.12 & 343.22 & 298.77 \\
\hline crystal color & Colorless & Colorless & Colorless \\
\hline crystal system & Orthorhombic & Monoclinic & Monoclinic \\
\hline space group & $P 2{ }_{1}{ }_{1} 2_{1}(\# 19)$ & $P 2_{1}(\# 4)$ & $P 2_{1}(\# 4)$ \\
\hline$a, \AA$ & $5.018(2)$ & $8.342(3)$ & $5.662(8)$ \\
\hline$b, \AA$ & $12.447(4)$ & $8.299(3)$ & $11.423(15)$ \\
\hline$c, \AA$ & $16.893(5)$ & $10.721(4)$ & $11.586(16)$ \\
\hline$\beta, \operatorname{deg}$ & & $101.619(4)$ & 101.932(19) \\
\hline$V, \AA^{3}$ & $1055.1(6)$ & $727.0(4)$ & $733.2(18)$ \\
\hline$Z$ & 4 & 2 & 2 \\
\hline$D_{\text {calcd }}, \mathrm{g} \mathrm{cm}^{-3}$ & 1.682 & 1.568 & 1.353 \\
\hline $\mathrm{F}_{000}$ & 536.00 & 348.00 & 312.00 \\
\hline$\mu, \mathrm{cm}^{-1}(\mathrm{MoK} \alpha)$ & 38.820 & 28.365 & 2.613 \\
\hline Exposure rate & $32.0 \mathrm{sec} /{ }^{\circ}$ & $10.0 \mathrm{sec} /{ }^{\circ}$ & $16.0 \mathrm{sec} /{ }^{\circ}$ \\
\hline $\begin{array}{l}\text { no. of reflections } \\
\text { measured }\end{array}$ & 8633 & 4698 & 5771 \\
\hline $\begin{array}{l}\text { no. of unique } \\
\text { reflections }\end{array}$ & 2412 & 2994 & 2537 \\
\hline no. of variables & 981 & 190 & 206 \\
\hline$R 1(I>2.00 \sigma(I))$ & 0.0462 & 0.0386 & 0.0505 \\
\hline $\begin{array}{l}\mathrm{w} R 2 \\
\text { reflections) }\end{array}$ & 0.1044 & 0.0963 & 0.1203 \\
\hline GOF on $F^{2}$ & 1.000 & 1.000 & 1.010 \\
\hline Flack parameter & $-0.017(16)$ & $0.047(14)$ & $-0.08(11)$ \\
\hline
\end{tabular}


Figure S3. X-ray crystallographic structure of 13d.

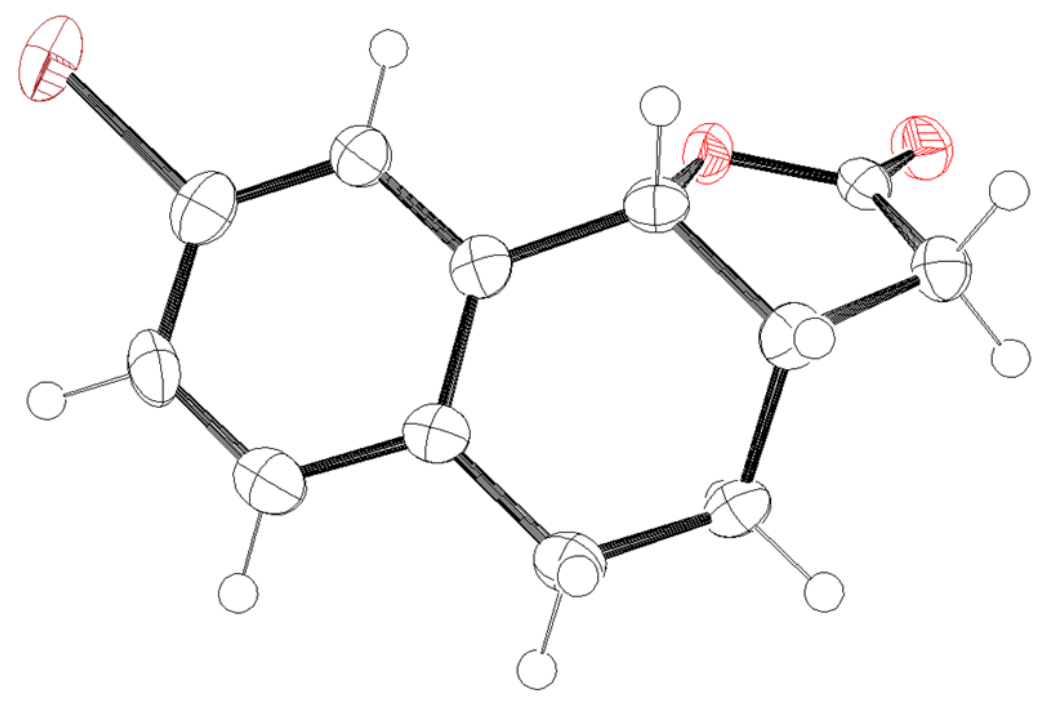<smiles>O=C1C[C@H]2CCc3ccc(Br)cc3[C@H]2O1</smiles>

Figure S4. X-ray crystallographic structure of $\mathbf{1 5 e .}$

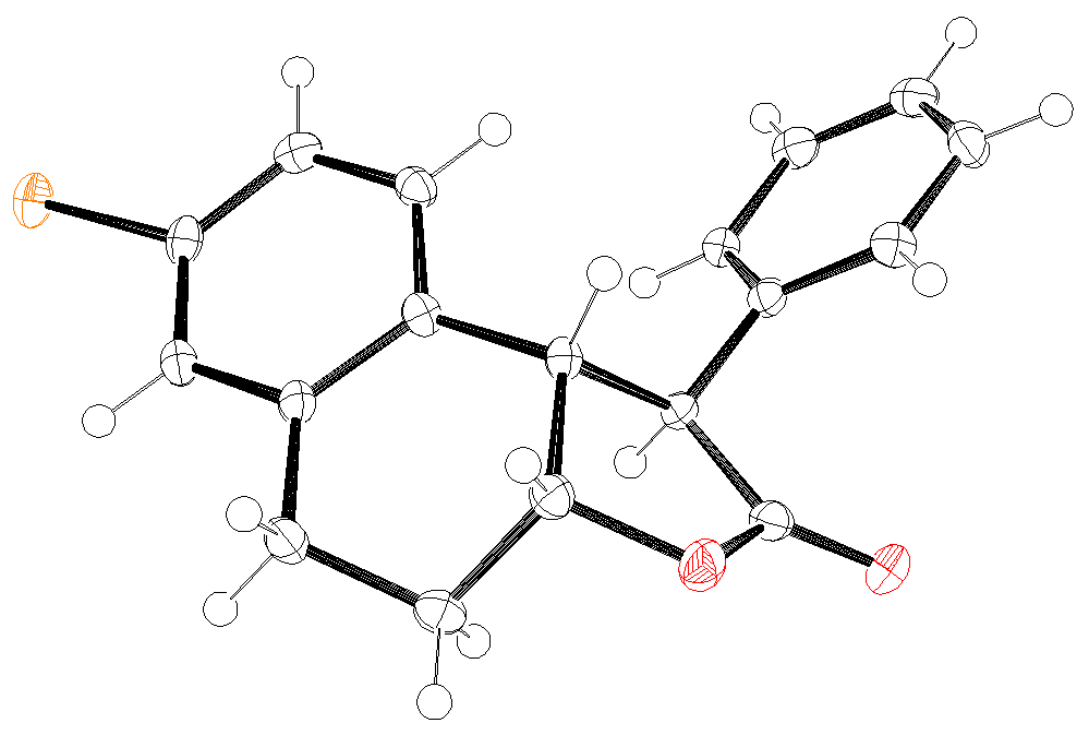<smiles>O=C1O[C@@H]2CCc3cc(Br)ccc3[C@H]2[C@H]1c1ccccc1</smiles> 
Figure S5. X-ray crystallographic structure of $\mathbf{1 5 f}$.

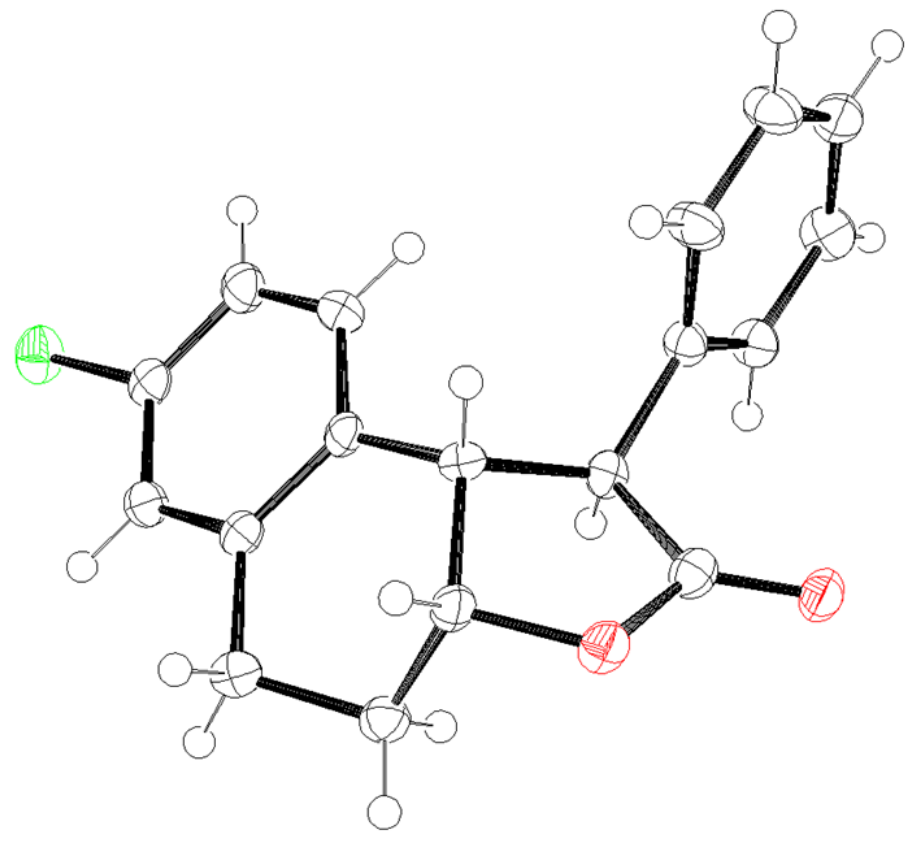<smiles>O=C1O[C@@H]2CCc3cc(Cl)ccc3[C@H]2[C@H]1c1ccccc1</smiles> 


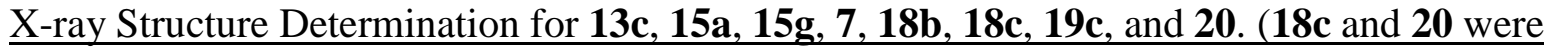
determined by the crystalline sponge method.)

The crystalline sponge $(\mathrm{CS})\left[\left(\mathrm{ZnI}_{2}\right)_{3}(\mathrm{tpt})_{2} \cdot \mathrm{tpt}\right)$ rystalline $\mathrm{n}(\mathbf{H}, \mathrm{tpt}=2,4,6$-tris $(4$-pyridyl $)$ 1,3,5-triazine) was prepared according to the reported procedure. ${ }^{[15]}$ A screw-top microvial (GL sciensce, cat. no. 1030-51023), a screw cap with a septum seal (Thermo scientific, cat. no. C5000-51B), and a syringe needle (Top corporation, cat. no. 00823) were used for guest inclusion into the porous crystals.

Preparation of guest-absorbed crystals.

The guest-absorbed crystals $\mathbf{H} \cdot \mathbf{x}$ were prepared by immersing a single crystal of $\mathbf{H}$ in $50 \mu \mathrm{L}$ cyclohexane and $1 \mu \mathrm{L}$ MTBE containing $1 \mu \mathrm{g}$ of $\mathbf{x}$. The solvent evaporation was conducted at $50^{\circ} \mathrm{C}$ for $2 \mathrm{~d}$ with a needle.

Single crystal diffraction data were collected on a Synergy-S (Rigaku Oxford Diffraction) diffractometer equipped with a micro-focus $\mathrm{Cu} K \alpha$ radiation source $(\lambda=1.5418 \AA$ ), a hybrid pixel array detector (HPAD), and a low temperature system using cold nitrogen stream (100 $\mathrm{K})$. CrysAlisPro performed data reduction, integration, and scaling. Empirical and numerical absorption corrections were applied in this process. All crystal structures were solved using SHELXT ver. $2014 / 5^{[16]}$ and refined using SHELXL ver.2014/7 ${ }^{[14]}$ programs. All the nonhydrogen atoms were refined anisotropically. All the hydrogen atoms were grown using the proper HFIX command and refined isotropically using the riding model.

In ths structure analyses of the crystalline sponge method (18c and 20), Minimum number of restraints was applied. Iodine atoms in the framework were refined with SIMU command. Large void and residual unassignable electron densities remained in the crystal structure, and we assumed that the void is filled with solvent cyclohexane molecules. These molecules were expected to be severely disordered for their high thermal motion and have an averaged structure of various geometry and orientation and refined using the restraints (DFIX, DANG, SIMU, RIGU, EADP and ISOR). This is a reason why some cyclohexane molecules are distorted to energetically-unfavorable (boat-shaped or twisted) structure. Some “Alert A" notifications were found in the validation program CheckCIF. Those alerts are derived from short intermolecular contacts of hydrogen atoms between solvent molecules (cyclohexane) and the crystalline sponge framework and other solvent molecules, and are unavoidable due to severe disorder of solvent molecules. The comments for the alerts are described in the CIFs using the validation response form (vrf). 
Table S10. Crystallographic Data for 13c, 15a, 15g, and $18 b$.

\begin{tabular}{|c|c|c|c|c|c|}
\hline & $13 c$ & $15 \mathbf{a}$ & $15 g$ & $18 b$ & \\
\hline $\begin{array}{ll}\text { formula } & \text { of } \\
\text { asymmetric unit }\end{array}$ & $\mathrm{C}_{12} \mathrm{H}_{11} \mathrm{ClO}_{2}$ & $\mathrm{C}_{13} \mathrm{H}_{14} \mathrm{O}_{2}$ & $\mathrm{C}_{19} \mathrm{H}_{18} \mathrm{O}_{3}$ & $2\left(\mathrm{C}_{18} \mathrm{H}_{15} \mathrm{ClO}_{2}\right)$ & \\
\hline formula weight & 222.66 & 202.24 & 294.33 & 597.5 & \\
\hline crystal color & Colorless & Colorless & Colorless & Colorless & \\
\hline crystal system & Orthorhombic & Monoclinic & Monoclinic & Monoclinic & \\
\hline space group & $P 2_{1} 2_{1} 2_{1}(\# 19)$ & $P 2_{1}(\# 4)$ & $P 2_{1}(\# 4)$ & $P 2_{1}(\# 4)$ & \\
\hline$a, \AA$ & $4.98860(10)$ & $7.79570(10)$ & $7.70360(10)$ & $6.74007(4)$ & \\
\hline$b, \AA$ & $7.73610(10)$ & $13.9632(2)$ & $6.16330(10)$ & $24.03214(17)$ & \\
\hline$c, \AA$ & $26.4469(4)$ & $9.8653(2)$ & $15.5653(2)$ & $8.74986(6)$ & \\
\hline$\beta, \operatorname{deg}$ & - & $106.276(2)$ & $90.6700(10)$ & $91.2367(6)$ & \\
\hline$V, \AA^{3}$ & $1020.65(3)$ & $1030.83(3)$ & 738.984(18) & $1416.956(16)$ & \\
\hline$Z$ & 4 & 4 & 2 & 2 & \\
\hline$D_{\text {calcd }}, \mathrm{g} \mathrm{cm}^{-3}$ & 1.449 & 1.303 & 1.323 & 1.400 & \\
\hline $\begin{array}{l}\text { theta }(\theta) \text { range for data } \\
\text { collection }\end{array}$ & $\begin{array}{l}3.342^{\circ}<\theta< \\
76.400^{\circ}\end{array}$ & $\begin{array}{l}4.669^{\circ}<\theta< \\
76.477^{\circ}\end{array}$ & $\begin{array}{l}2.839^{\circ}<\theta< \\
77.268^{\circ}\end{array}$ & $\begin{array}{l}3.678^{\circ}<\theta \\
77.361^{\circ}\end{array}$ & $<$ \\
\hline $\begin{array}{l}\text { linear absorption } \\
\text { coefficient }\end{array}$ & 3.109 & 0.693 & 0.712 & 2.393 & \\
\hline $\begin{array}{l}\text { no. of reflections } \\
\text { measured }\end{array}$ & 9699 & 19007 & 15067 & 29371 & \\
\hline $\begin{array}{l}\text { no. of unique } \\
\text { reflections }\end{array}$ & 2088 & 4148 & 3072 & 5950 & \\
\hline number of parameters & 136 & 273 & 200 & 379 & \\
\hline number of restraints & 0 & 1 & 1 & 1 & \\
\hline $\begin{array}{l}\text { highest electron } \\
\text { density maximum }\end{array}$ & 0.193 & 0.329 & 0.371 & 0.300 & \\
\hline $\begin{array}{l}\text { deepest electron } \\
\text { density hole }\end{array}$ & -0.409 & -0.271 & -0.355 & -0.199 & \\
\hline $\mathrm{R}_{\text {int }}$ & 0.0418 & 0.0469 & 0.0549 & 0.0347 & \\
\hline$R 1 F^{2}>2 \sigma\left(F^{2}\right)$ & 0.0322 & 0.0431 & 0.0527 & 0.0264 & \\
\hline $\mathrm{w} R 2$ & 0.1057 & 0.1159 & 0.1375 & 0.0702 & \\
\hline GOF & 0.94 & 1.025 & 1.109 & 1.036 & \\
\hline $\begin{array}{l}\text { Flack parameter } \\
\text { (Parsons) }\end{array}$ & $-0.003(8)$ & $0.04(9)$ & $-0.08(8)$ & $-0.001(3)$ & \\
\hline CCDC number & 1914657 & 1914658 & 1914659 & 1914660 & \\
\hline
\end{tabular}


Table S11. Crystallographic Data for 7 and 19c.

\begin{tabular}{|c|c|c|}
\hline & 7 & $19 \mathrm{c}$ \\
\hline formula of asymmetric unit & $0.67\left(\mathrm{C}_{19} \mathrm{H}_{18} \mathrm{O}_{3}\right)$ & $\mathrm{C}_{13} \mathrm{H}_{14} \mathrm{O}_{2}$ \\
\hline formula weight & 192.22 & 202.24 \\
\hline crystal color & Colorless & Colorless \\
\hline crystal system & Monoclinic & Orthorhombic \\
\hline space group & $P 2_{1}(\# 4)$ & $P 2{ }_{1} 2_{1} 2_{1}(\# 19)$ \\
\hline$a, \AA$ & $9.8340(2)$ & $5.03220(10)$ \\
\hline$b, \AA$ & $5.85050(10)$ & $13.0715(2)$ \\
\hline$c, \AA$ & $12.9552(2)$ & $16.2633(2)$ \\
\hline$\beta, \operatorname{deg}$ & $102.401(2)$ & - \\
\hline$V, \AA^{3}$ & $727.97(2)$ & $1069.77(3)$ \\
\hline$Z$ & 3 & 4 \\
\hline$D_{\text {calcd }}, \mathrm{g} \mathrm{cm}^{-3}$ & 1.449 & 1.256 \\
\hline $\begin{array}{l}\text { theta }(\theta) \text { range for data } \\
\text { collection }\end{array}$ & $3.493^{\circ}<\theta<76.560^{\circ}$ & $4.339^{\circ}<\theta<77.500^{\circ}$ \\
\hline linear absorption coefficient & 0.723 & 0.668 \\
\hline no. of reflections measured & 13484 & 10688 \\
\hline no. of unique reflections & 2959 & 2238 \\
\hline number of parameters & 200 & 137 \\
\hline number of restraints & 1 & 0 \\
\hline $\begin{array}{l}\text { highest electron density } \\
\text { maximum }\end{array}$ & 0.274 & 0.198 \\
\hline deepest electron density hole & -0.296 & -0.145 \\
\hline $\mathrm{R}_{\text {int }}$ & 0.0451 & 0.0283 \\
\hline$R 1 F^{2}>2 \sigma\left(F^{2}\right)$ & 0.0396 & 0.0309 \\
\hline $\mathrm{w} R 2$ & 0.1082 & 0.0861 \\
\hline GOF & 1.063 & 1.096 \\
\hline Flack parameter (Parsons) & $-0.05(8)$ & $0.07(9)$ \\
\hline CCDC number & 1914664 & 1914658 \\
\hline
\end{tabular}


Table S12. Crystallographic Data for 18c and 20 (crystalline sponge method).

\begin{tabular}{|c|c|c|}
\hline & $18 \mathrm{c}$ & 20 \\
\hline formula of asymmetric unit & $\mathrm{C}_{143} \mathrm{H}_{130} \mathrm{I}_{12} \mathrm{~N}_{24} \mathrm{O}_{10} \mathrm{Zn}_{6}$ & $\mathrm{C}_{104} \mathrm{H}_{84} \mathrm{I}_{12} \mathrm{~N}_{24} \mathrm{O}_{4} \mathrm{Zn}_{6}$ \\
\hline formula weight & 4259.72 & 3648.97 \\
\hline crystal color & light yellow & light yellow \\
\hline crystal system & Monoclinic & Monoclinic \\
\hline space group & $C 2(\# 5)$ & $C 2(\# 5)$ \\
\hline$a, \AA$ & $36.2576(4)$ & $36.2700(3)$ \\
\hline$b, \AA$ & $14.34286(13)$ & $14,50280(10)$ \\
\hline$c, \AA$ & $34.8216(4)$ & $34.6393(3)$ \\
\hline$\beta, \operatorname{deg}$ & $108.3979(11)$ & $109.1430(10)$ \\
\hline$V, \AA^{3}$ & $17183.0(3)$ & $17213.3(3)$ \\
\hline$Z$ & 4 & 4 \\
\hline$D_{\text {calcd }}, \mathrm{g} \mathrm{cm}^{-3}$ & 1.647 & 1.408 \\
\hline $\begin{array}{l}\text { theta }(\theta) \text { range for data } \\
\text { collection }\end{array}$ & $2.493^{\circ}<\theta<77.475^{\circ}$ & $2.488^{\circ}<\theta<77.501^{\circ}$ \\
\hline linear absorption coefficient & 18.303 & 18.147 \\
\hline no. of reflections measured & 125166 & 171259 \\
\hline no. of unique reflections & 34121 & 35636 \\
\hline number of parameters & 1868 & 1425 \\
\hline number of restraints & 1141 & 151 \\
\hline $\begin{array}{l}\text { highest electron density } \\
\text { maximum }\end{array}$ & 1.192 & 1.339 \\
\hline deepest electron density hole & -1.830 & -1.546 \\
\hline $\mathrm{R}_{\text {int }}$ & 0.0391 & 0.059 \\
\hline$R 1 F^{2}>2 \sigma\left(F^{2}\right)$ & 0.0733 & 0.0798 \\
\hline $\mathrm{w} R 2$ & 0.2096 & 0.2317 \\
\hline GOF & 1.083 & 1.042 \\
\hline Flack parameter (Parsons) & $0.108(5)$ & $0.033(4)$ \\
\hline CCDC number & 1914661 & 1914663 \\
\hline
\end{tabular}


Figure S6. X-ray crystallographic structure of 13c.

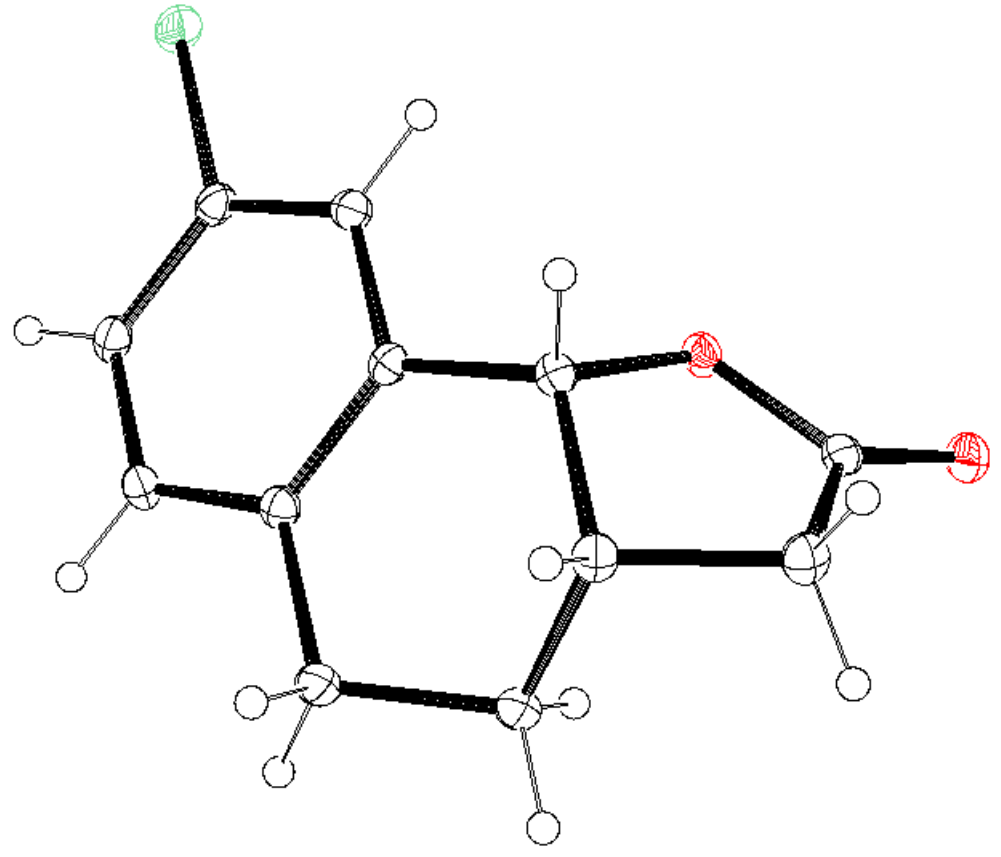<smiles>O=C1C[C@H]2CCc3ccc(Cl)cc3[C@H]2O1</smiles>

Figure S7. X-ray crystallographic structure of $\mathbf{1 5 a}$.

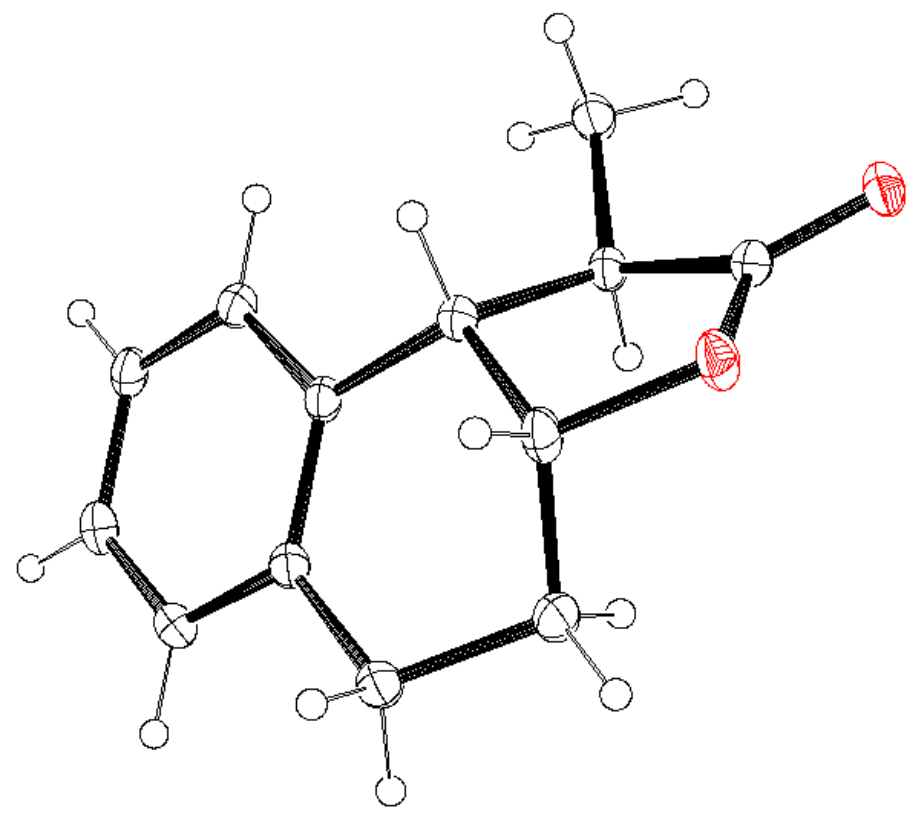<smiles>C[C@H]1C(=O)O[C@H]2CCc3ccccc3[C@H]21</smiles> 
Figure S8. X-ray crystallographic structure of $\mathbf{1 5 g}$.
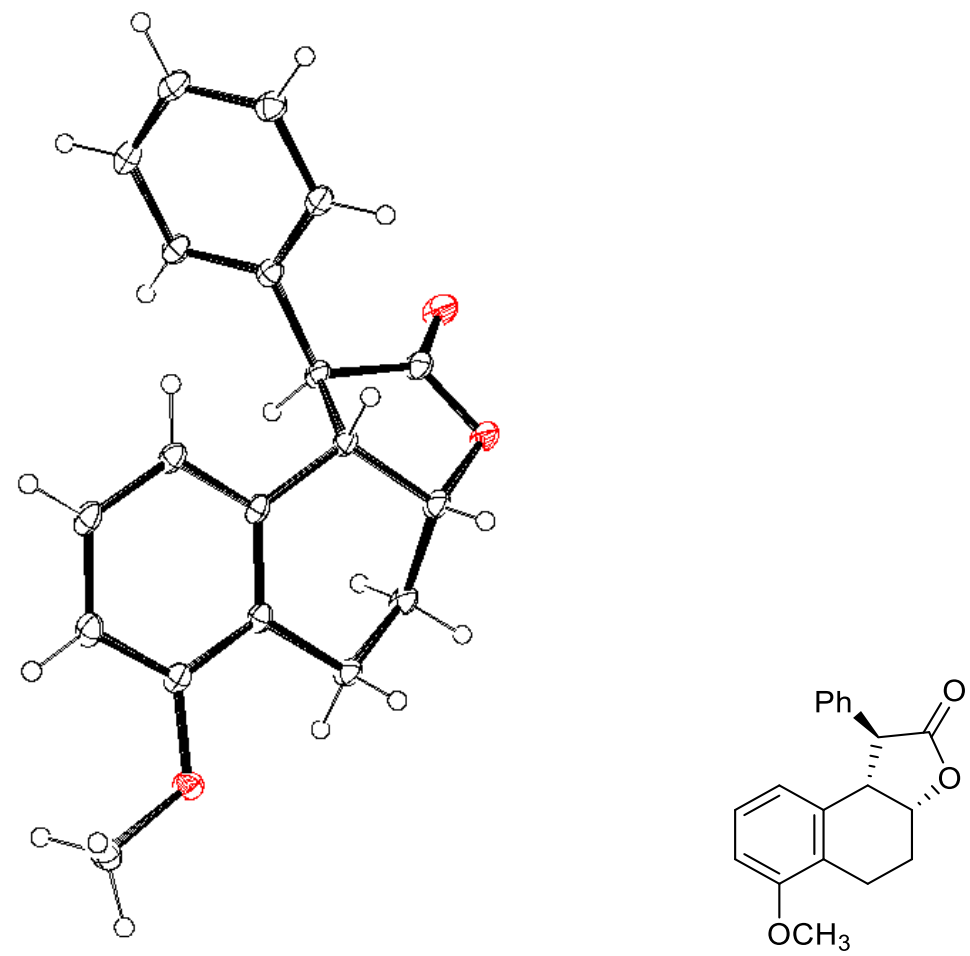

Figure S9. X-ray crystallographic structure of 7.

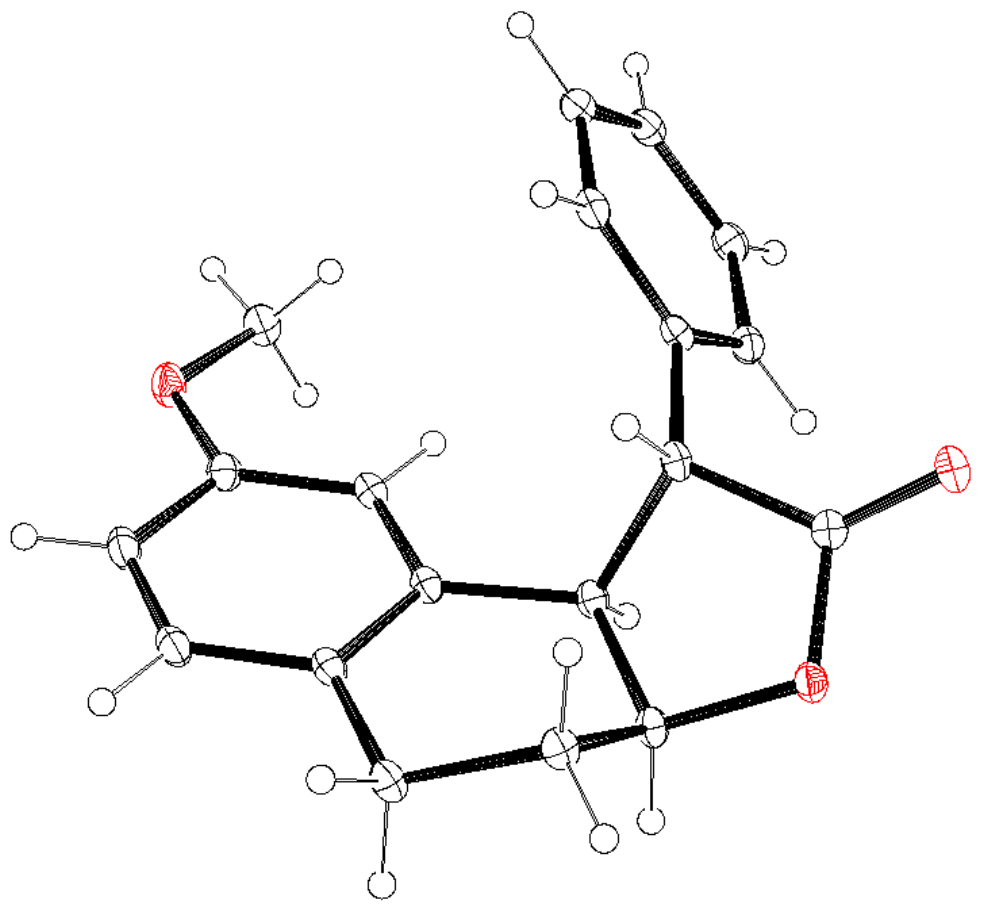<smiles>COc1ccc2c(c1)C1C(CC2)OC(=O)C1c1ccccc1</smiles> 
Figure S10. X-ray crystallographic structure of $\mathbf{1 8 b}$.
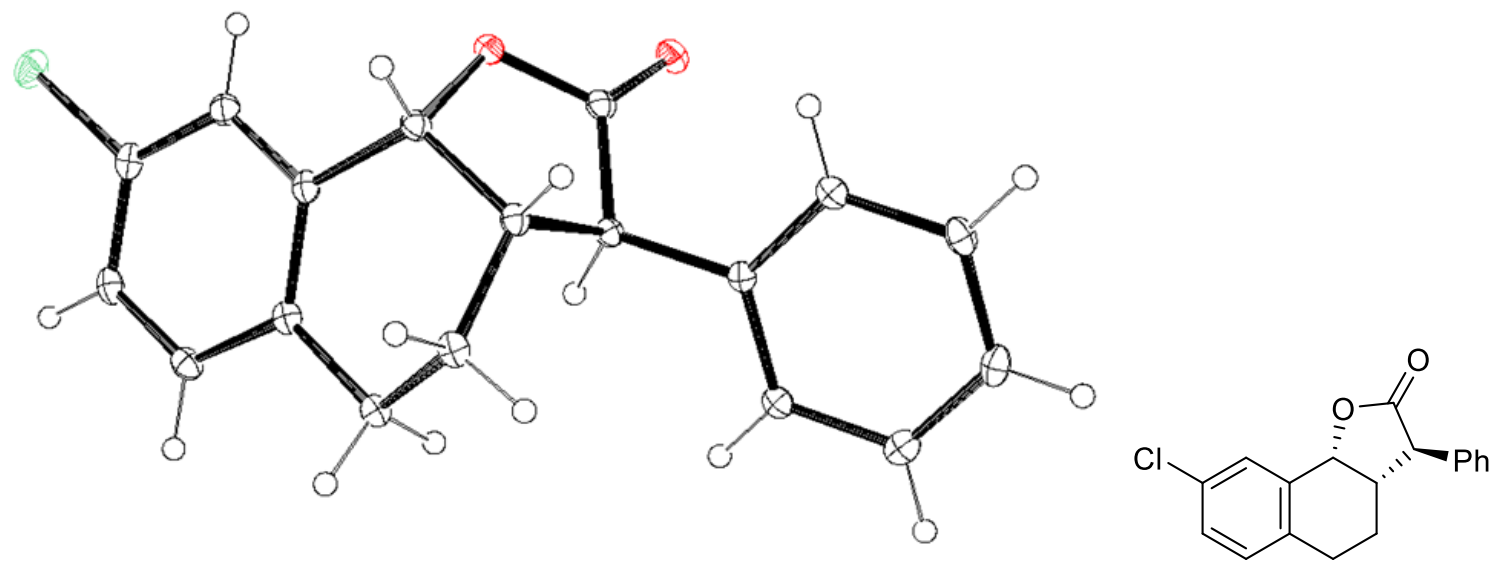

Figure S11. X-ray crystallographic structure of 18c.

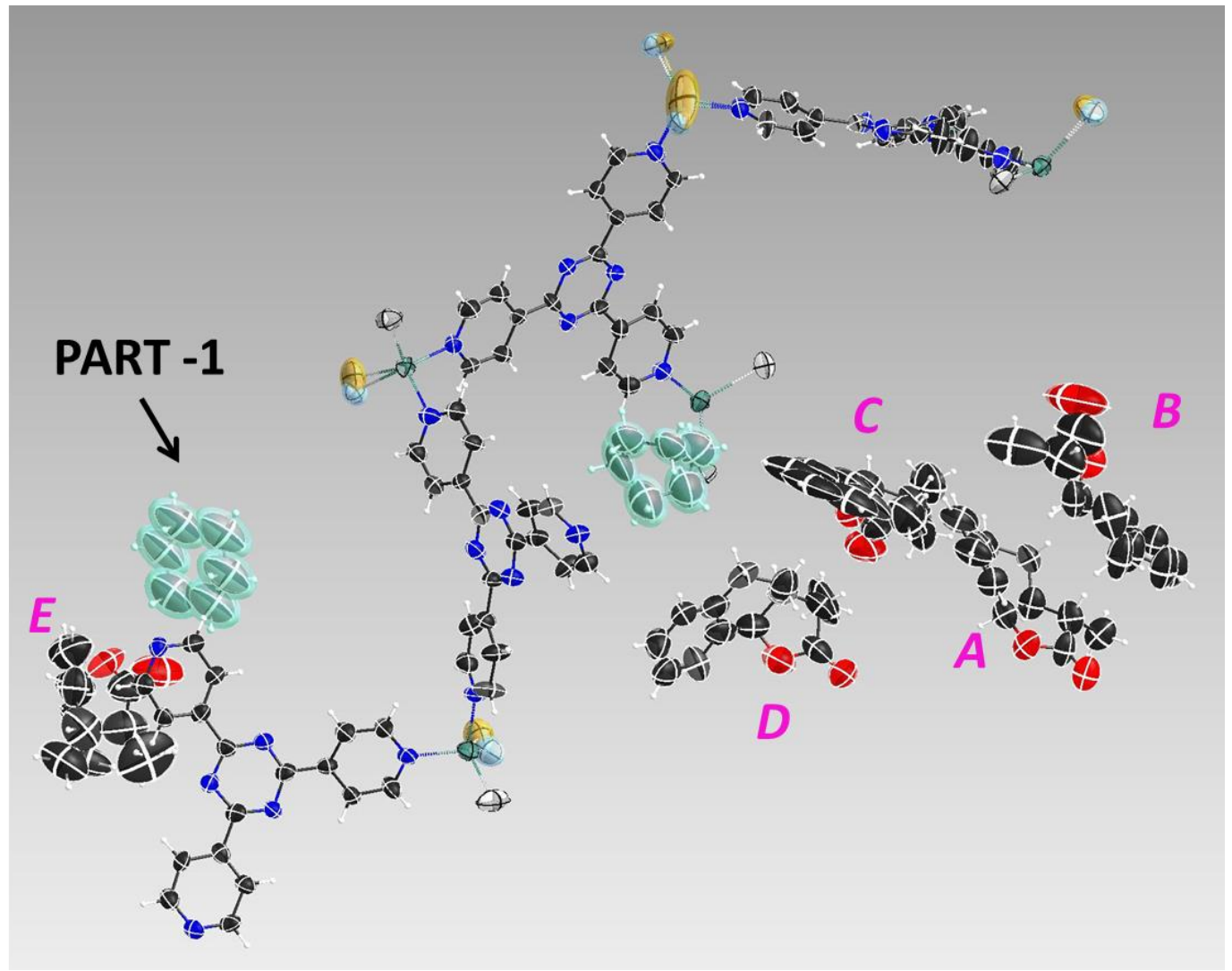



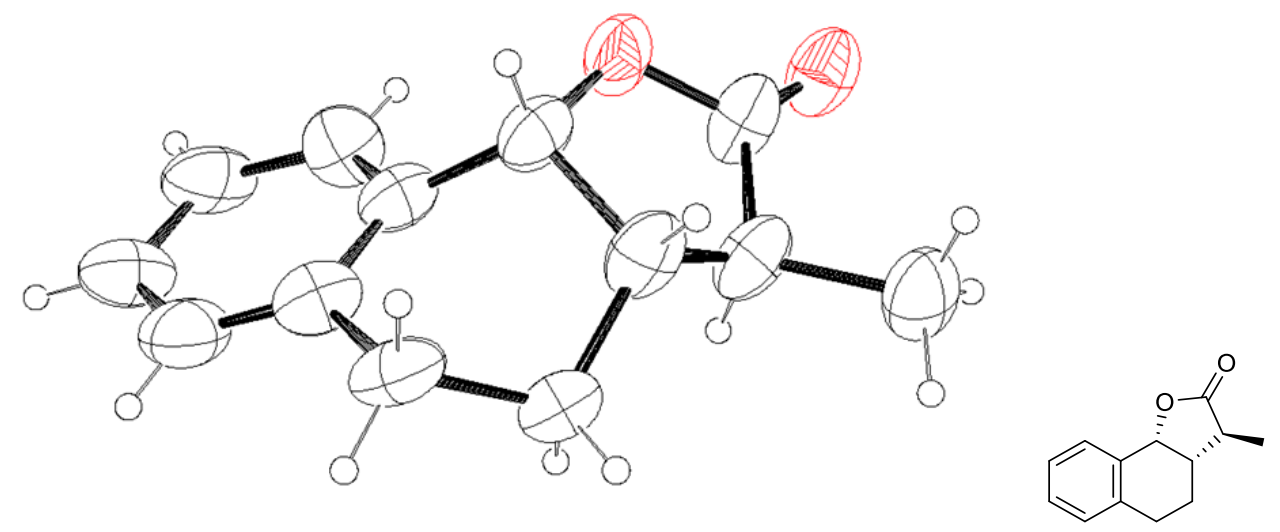

ORTEP diagram with $50 \%$ probability in the asymmetric unit of $\mathbf{H} \cdot \mathbf{1 8 c}$. Enclosed atoms by the PART command in SHELXL were represented using the difference color code.

\section{Crystal Structure Analysis of 18c.}

ORTEP diagram of the asymmetric unit is shown in Figure S11. In this crystal structure, five guest molecules were found. All the guests are ordered at general position and have occupancies of $100 \%$. All guest molecules were refined with applying SIMU (for the whole molecules). The geometry of A was related with applying SAME for B-E (for the whole molecule).

Figure S12. X-ray crystallographic structure of 19c.

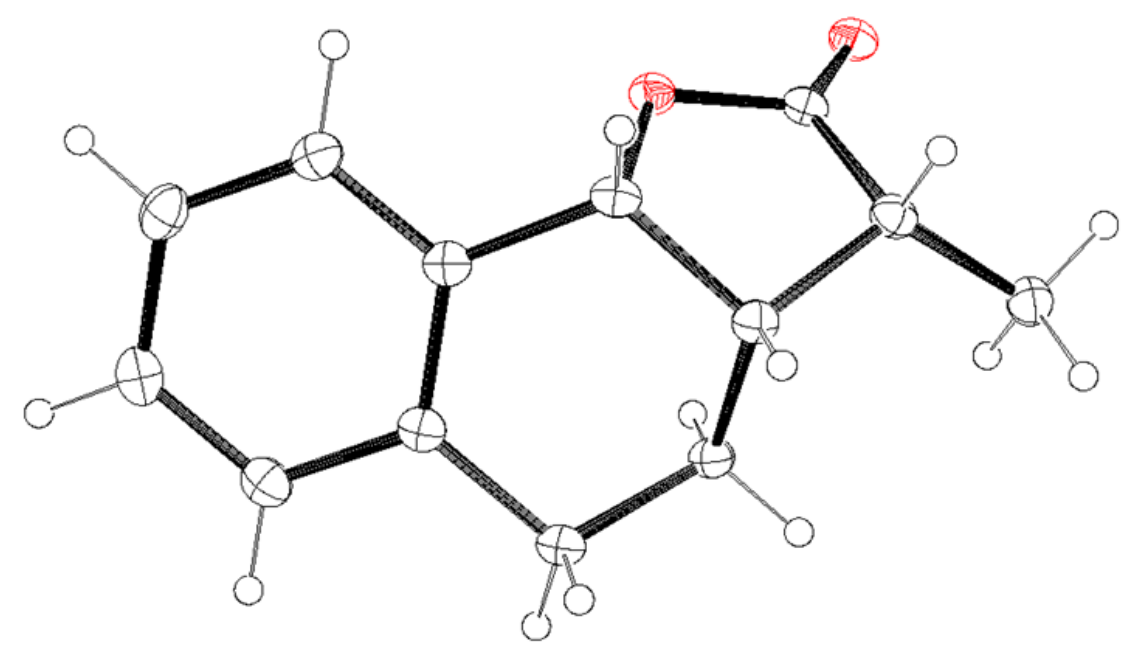<smiles>C[C@H]1C(=O)O[C@H]2c3ccccc3CC[C@@H]12</smiles> 
Figure S13. X-ray crystallographic structure of 20.
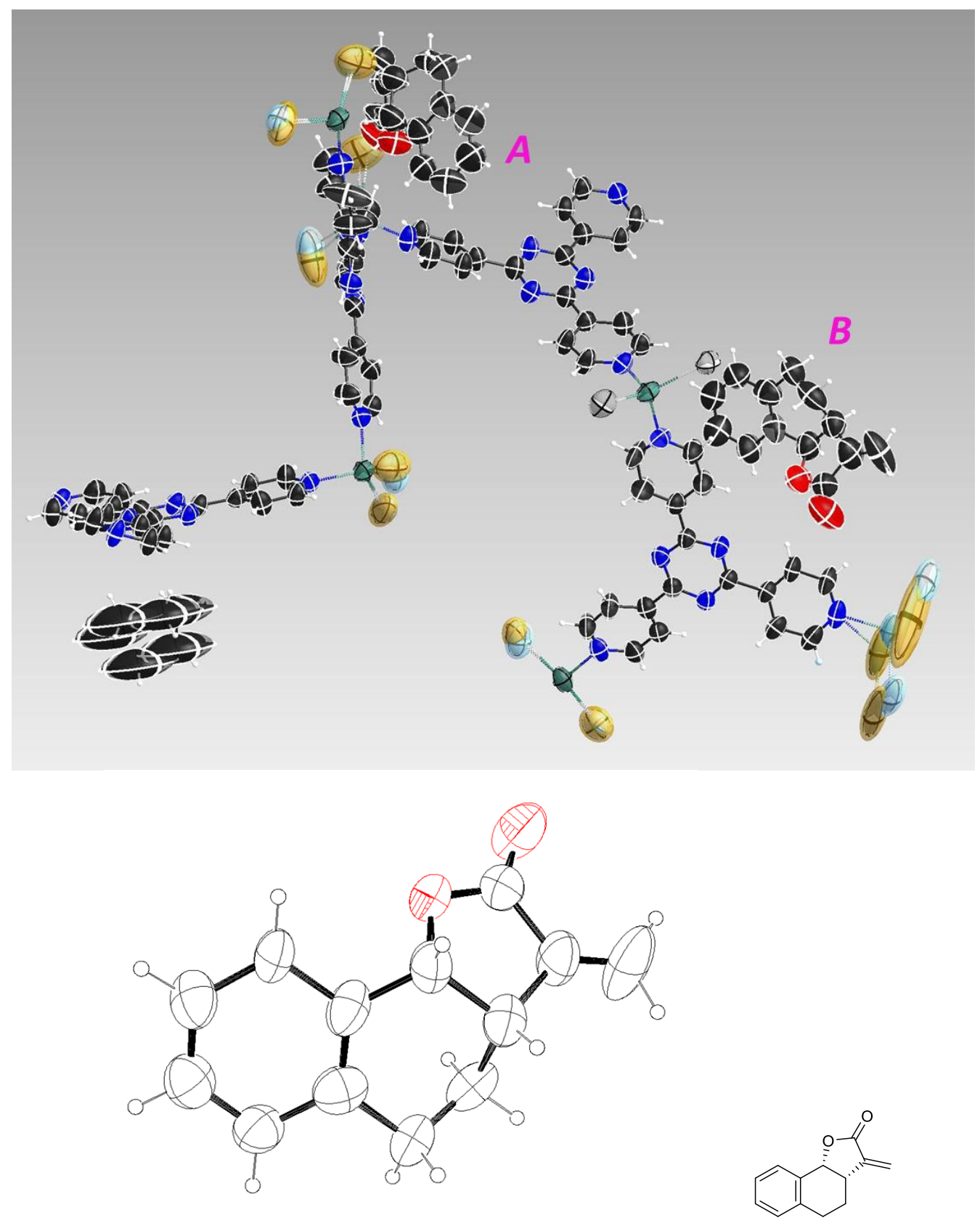

ORTEP diagram with $50 \%$ probability in the asymmetric unit of $\mathbf{H} \cdot \mathbf{2 0}$. Enclosed atoms by the PART command in SHELXL were represented using the difference color code.

\section{Crystal Structure Analysis of 20.}

ORTEP diagram of the asymmetric unit is shown in Figure S13. In this crystal structure, two guest molecules were found. All the guests are ordered at general position and have occupancies of $100 \%$. SIMU was used for five carbon atoms. The restraints used for the 
refinement are summarized in Figure S14.

Figure S14. Restraints applied in the refinement of $\mathbf{H \bullet 2 0 .}$

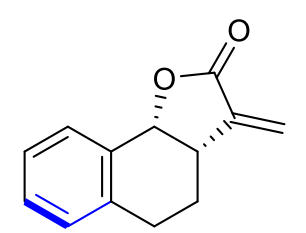

A<smiles>C=C1C(=O)O[C@H]2c3ccccc3CC[C@H]12</smiles>

B

\section{SIMU}




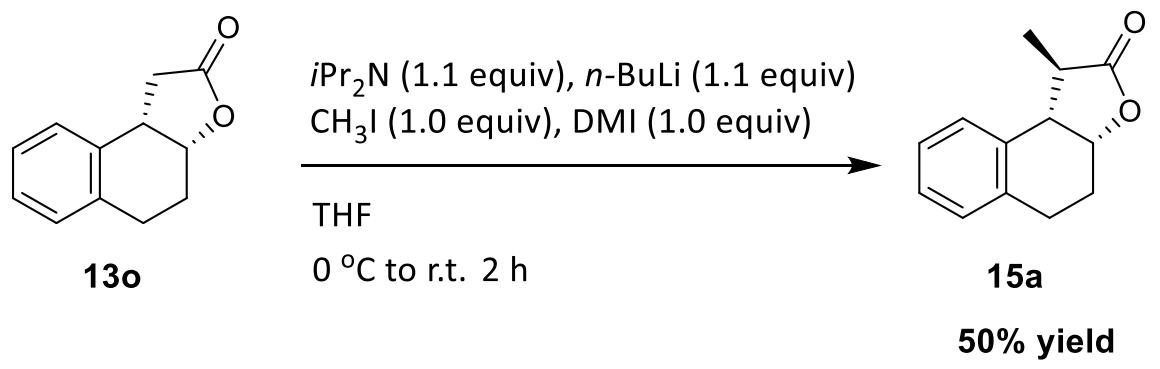

The absolute configuration of $\mathbf{1 3 0}$ was confirmed by diastereoselective methylation of $\mathbf{1 3 0}$ into 15a.

$<$ Synthetic procedure>

A THF solution $(1.57 \mathrm{M})$ of $n$-BuLi $(0.45 \mathrm{~mL}, 0.71 \mathrm{mmol})$ was added slowly to a THF solution $(2.5 \mathrm{~mL})$ containing $N, N$-diisopropylamine $(91.9 \mathrm{mg}, 0.71 \mathrm{mmol})$ at $-60{ }^{\circ} \mathrm{C}$ under $\mathrm{N}_{2}$ atmosphere and stirred for $1 \mathrm{~h}$. The resulting mixture was warmed to $-20{ }^{\circ} \mathrm{C}$, then (3aR,9bS)-3a,4,5,9b-tetrahydronaphtho[2,1-b]furan-2(1H)-one (13o) $(120 \mathrm{mg}, 0.65 \mathrm{mmol})$ was added and stirred for $1 \mathrm{~h}$ at $0{ }^{\circ} \mathrm{C}$. Subsequently, $\mathrm{CH}_{3} \mathrm{I}(92.0 \mathrm{mg}, 0.65 \mathrm{mmol})$ and 1,3dimethyl-2-imidazolidinone $(74.0 \mathrm{mg}, 0.65 \mathrm{mmol})$ were added to the reaction mixture and stirred at $0{ }^{\circ} \mathrm{C}$. After stirring further for $2 \mathrm{~h}$ at ambient temperature, the resulting mixture was diluted with a saturated aqueous solution of $\mathrm{NH}_{4} \mathrm{Cl}(2 \mathrm{~mL})$. The layers were separated, and the aqueous layer was extracted twice with $\mathrm{CHCl}_{3}(3 \mathrm{~mL}$ each). The combined organic phase was washed with brine, dried with $\mathrm{MgSO}_{4}$, filtered, and evaporated. The residue was purified by column chromatography on silica, using hexane/EtOAc (5/1 to 3/1) as eluent, to furnish $(1 R, 3 \mathrm{a} R, 9 \mathrm{~b} S)$-1-methyl-3a,4,5,9b-tetrahydronaphtho[2,1-b]furan-2(1H)-one (15a) $(65.3 \mathrm{mg}$, $50 \%$ yield) as a white solid.

The HPLC data of the methylated product matched well with that of structurally welldefined $(1 R, 3 \mathrm{a} R, 9 \mathrm{~b} S)$-15a which was confirmed by X-ray crystallographic analysis (Figure S7). 
Chart S1. HPLC chart of racemic compound of $\mathbf{1 5 a}$

(HPLC conditons) Column: Chiralpak IB (250 x 4.6 mm), Eluent: Hexane/2-propanol = 94/6, Flow = 1.0 $\mathrm{mL} / \mathrm{min}$, Detector $=220 \mathrm{~nm}$, Temp. $=35^{\circ} \mathrm{C}$

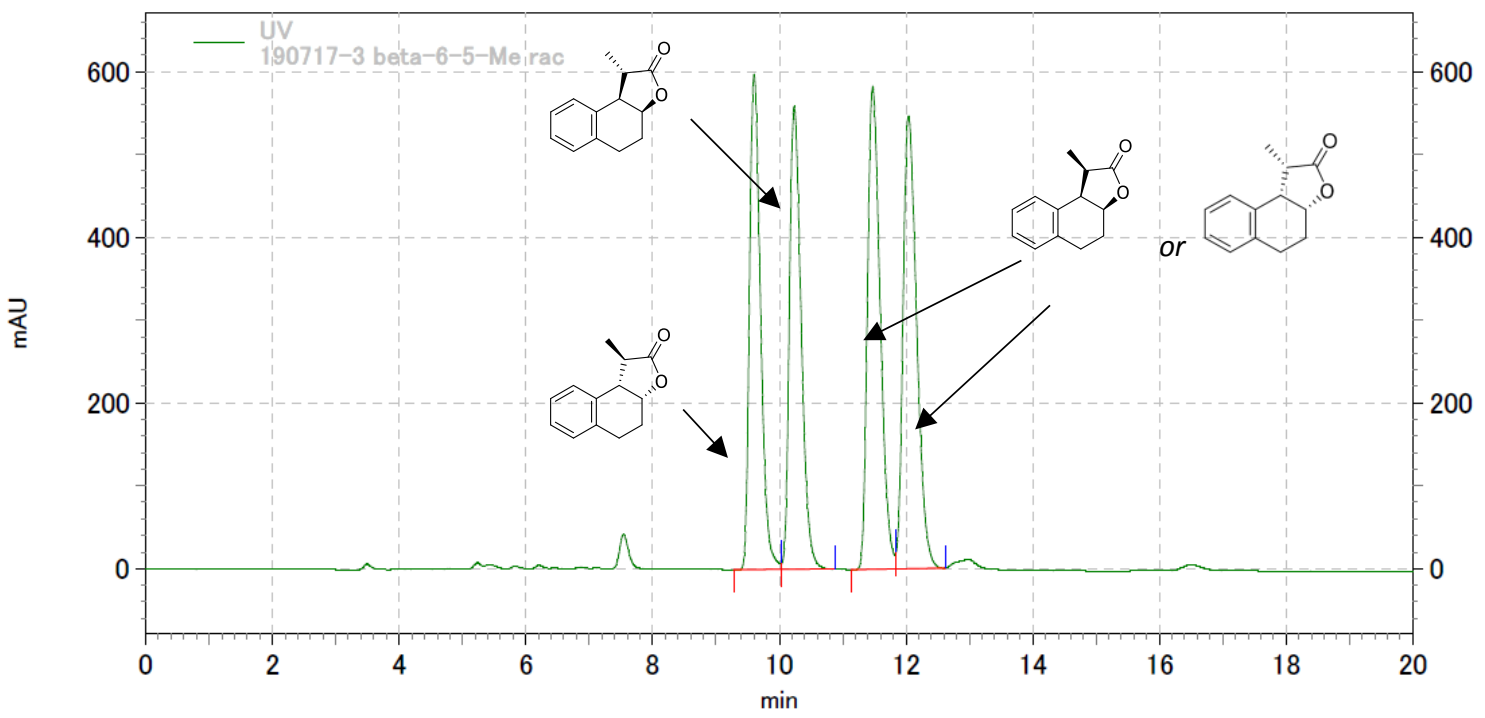

190717-3 beta-6-5-Me rac

\begin{tabular}{|c|c|c|c|c|c|}
\hline $\mathrm{Pk}$ & R. T. & Area & Area\% & B. C. & Symmetry \\
\hline $\begin{array}{l}1 \\
2 \\
3 \\
4\end{array}$ & $\begin{array}{r}9.603 \\
10.237 \\
11.477 \\
12.040\end{array}$ & $\begin{array}{l}28898226 \\
28898612 \\
32649443 \\
32771455\end{array}$ & $\begin{array}{l}23.453 \\
23.453 \\
26.497 \\
26.596\end{array}$ & $\begin{array}{l}\mathrm{Mx} \\
\mathrm{xM} \\
\mathrm{Mx} \\
\mathrm{xM}\end{array}$ & $\begin{array}{l}0.00000 \\
0.00000 \\
0.00000 \\
0.00000\end{array}$ \\
\hline & & 123217736 & 100.000 & & \\
\hline
\end{tabular}


Chart S2. HPLC chart of authentic sample of $(1 R, 3 \mathrm{a} R, 9 \mathrm{~b} S)-\mathbf{1 5 a}$.

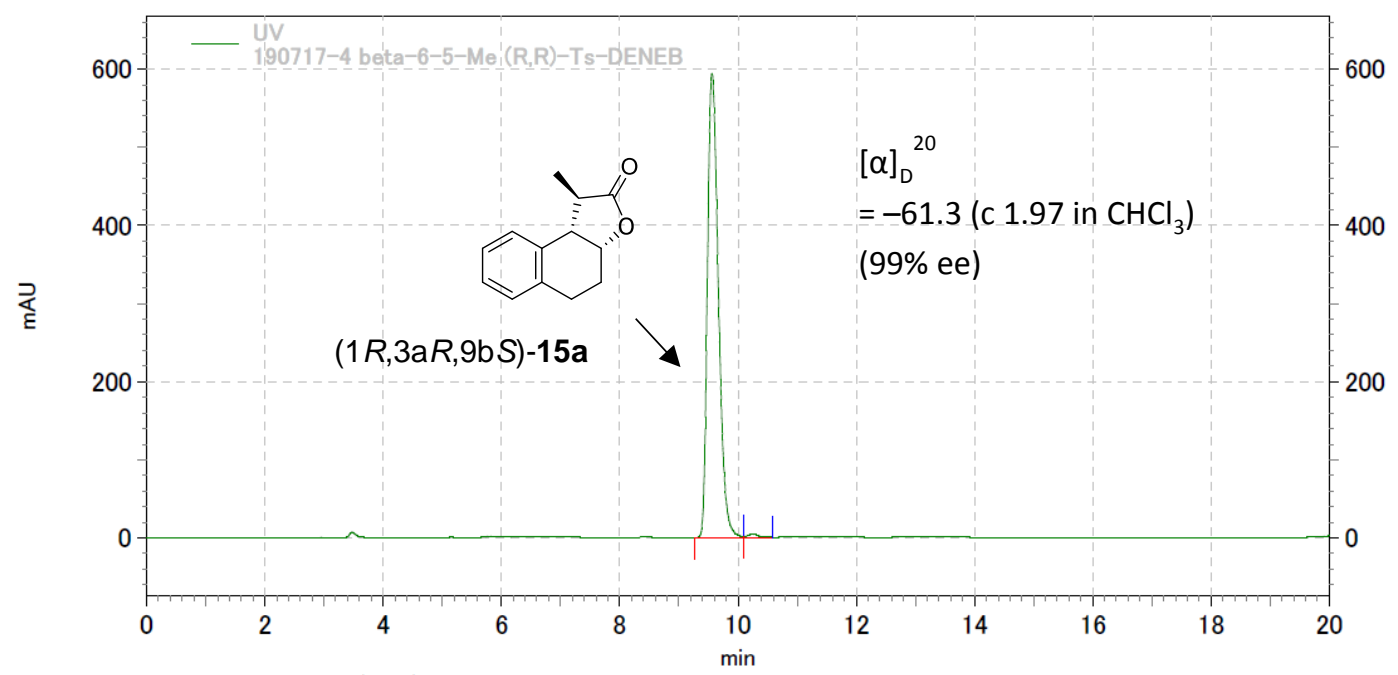

190717-4 beta-6-5-Me (R, R)-Ts-DENEB

\begin{tabular}{rrrrrr}
$\begin{array}{r}\text { Pk } \\
\text { No. }\end{array}$ & \multicolumn{1}{c}{ R. T. } & Area & Area\% & B. C. & Symmetry \\
\hline 1 & 9.560 & 30488640 & 99.331 & Mx & 0.00000 \\
2 & 10.247 & 205400 & 0.669 & xM & 0.00000 \\
\hline & & & & & \\
& & 30694040 & 100.000 & & \\
\hline
\end{tabular}

Chart S3. HPLC chart of 130.

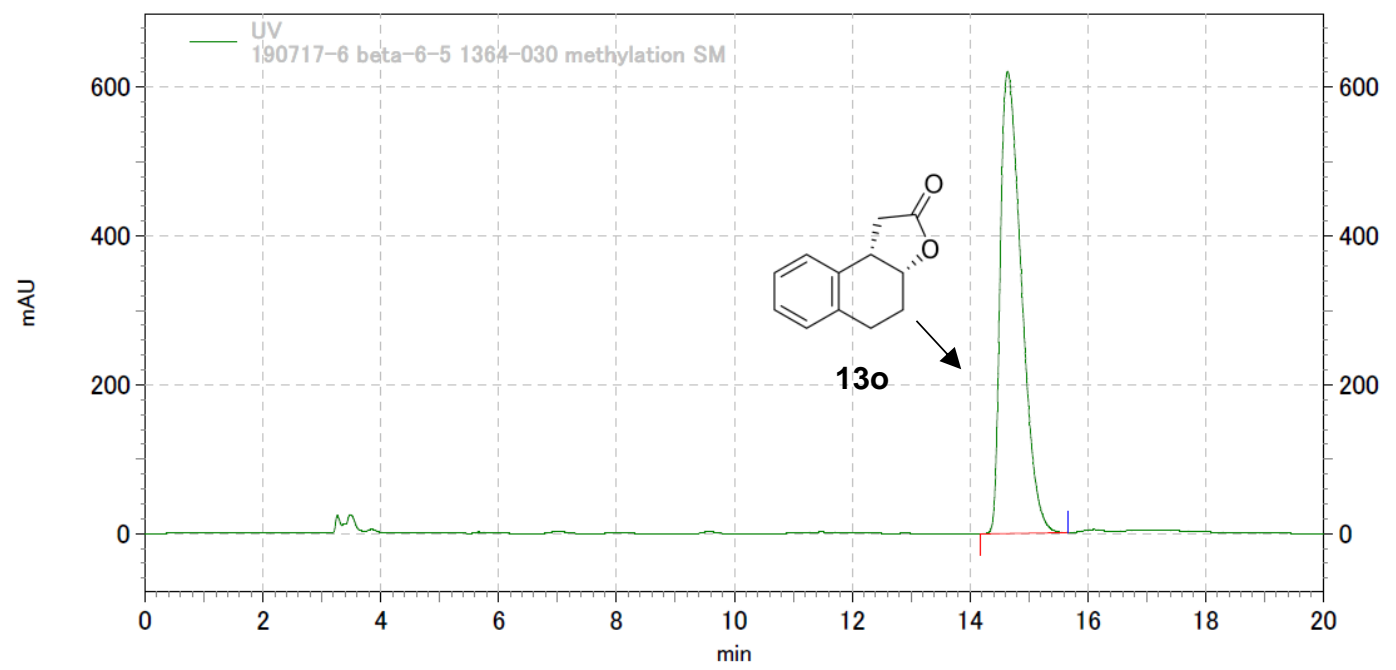

190717-6 beta-6-5 1364-030 methylation SM

\begin{tabular}{rlll|l|l|}
$\begin{array}{r}\text { Pk } \\
\text { No. }\end{array}$ & R. T. & Area & Area\% & B. C. & Symmetry \\
\hline 1 & 14.643 & 61738758 & 100.000 & MM & 0.00000 \\
\hline & & & & & \\
\hline & 61738758 & 100.000 & & \\
\hline
\end{tabular}


Chart S3. HPLC chart of crude mixture of after diastereoselective methylation of $\mathbf{1 3 0 .}$

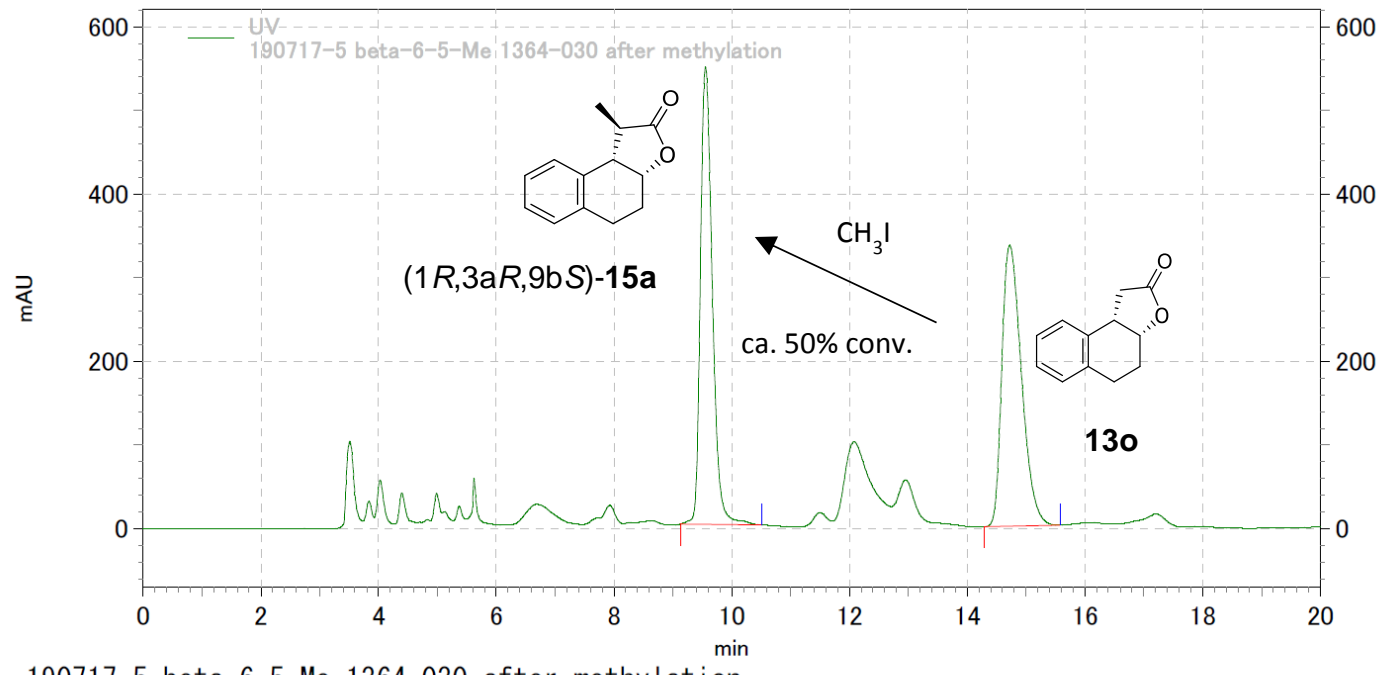

190717-5 beta-6-5-Me 1364-030 after methylation

\begin{tabular}{rrrrrrr}
$\begin{array}{rllll}\text { Pk } \\
\text { No. }\end{array}$ & R. T. & Area & Area\% & B. C. & Symmetry \\
\hline 1 & 9.553 & 30758426 & 49.830 & MM & 0.00000 \\
2 & 14.723 & 30968851 & 50.170 & MM & 0.00000 \\
\hline & & & & & \\
& & 61727277 & 100.000 & & \\
\hline
\end{tabular}

Chart S4. HPLC chart of purified 15a derivatized from 130 by diastereoselective methylation.

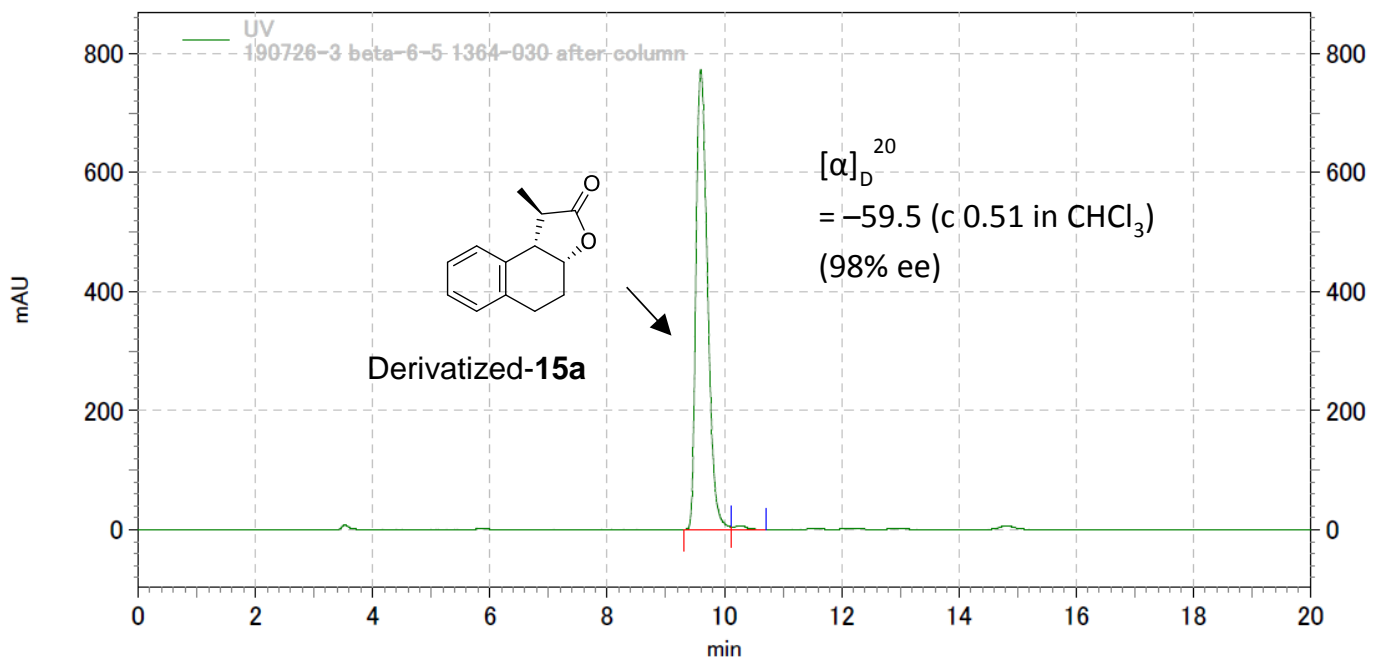

190726-3 beta-6-5 1364-030 after column

\begin{tabular}{rrrrrr}
$\begin{array}{r}\text { Pk } \\
\text { No. }\end{array}$ & \multicolumn{1}{c}{ R. T. } & Area & \multicolumn{1}{l}{ Area\% } & B. C. & Symmetry \\
\hline 1 & 9.597 & 42216556 & 99.131 & Mx & 0.00000 \\
2 & 10.273 & 370224 & 0.869 & xM & 0.00000 \\
\hline & & & & & \\
& & 42586780 & 100.000 & & \\
\hline
\end{tabular}




\section{J. References}

(1) Eames, J.; Weerasooriya, N.; Coumbarides, G. S. Investigations on the Efficiency of Regioselective C-Deuteration of Endocyclic Enolates. Eur. J. Org. Chem. 2002, 181-187.

(2) Pan, C.; Zeng, X.; Guan, Y.; Jiang, X.; Li, L.; Zhang, H. Desing and Synthesis of Brazilinlike Compounds. Synlett 2011, 3, 425-429.

(3) Terada, M.; Moriya, K.; Kanomata, K.; Sorimachi, K. Chiral Brønsted Acid Catalyzed Stereoselective Addition of Azlactones to 3-Vinylindoles for Facile Access to Enantioenriched Tryptophan Derivatives. Angew. Chem. Int. Ed. 2011, 52, 12586-12590.

(4) Malaquin, S.; Jida, M.; Courtin, J.; Laconde, G.; Willand, N.; Deprez, B.; Deprez-Poulain, R. Tetrahedron Lett. Water-based Conditions for the Microscale Parallel Synthesis of Bicyclic Lactams. 2013, 54, 562-567.

(5) Kolb, M.; Barth, J.; Heydt, J.-G.; Jung, M. J. Synthesis and Evaluation of Mono-, Di-, and Trifluoroethenyl-GABA Derivatives as GABA-T Inhibitors. J. Med. Chem, 1987, 30, 267-272.

(6) Ohkuma, T.; Hattori, T.; Ooka, H.; Inoue, T.; Noyori, R. BINAP/1,4-Diamine-ruthenium (II) Complexes for Efficient Asymmetric Hydrogenation of 1-Tetralones and Analogues. Org. Lett. 2004, 6, 2681-2683.

(7) Fernández, R.; Ros, A.; Magriz, A.; Dietrich, H.; Lassaletta, J. M. Enantioselective Synthesis of cis- $\alpha$-Substituted Cycloalkanols and trans-Cycloalkyl Amines Thereof. Tetrahedron 2007, 63, 6755-6763.

(8) Seebach, D.; Roggo, S.; Maetzke, T. Diasterio-und Enantioselektive Reduktion von $\beta$ Ketoestern mit Cyclopentanon-, Cyclohexanon-, Piperidon- und Tetralon-struktur Durch nicht Fermentierende Bäcker-Hefe. Helv. Chem. Acta 1987, 70, 1605-1615.

(9) Fujii, A.; Hashiguchi, S.; Uematsu, N.; Ikariya, T.; Noyori, R. Ruthenium (II)-catalyzed Asymmetric Transfer Hydrogenation of Ketones Using a Formic Acid-Triethylamine Mixture. J. Am. Chem. Soc. 1996, 118, 2521-2522.

(10) Bromhead, L. J.; Visser, J.; McErlean, C. S. P. J. Enantioselective Synthesis of the Strigolactone Mimic (+)-GR24. J. Org. Chem, 2014, 79, 1516-1520.

(11) Watanabe, A.; Uchida, T.; Irie, R.; Katsuki, T. Zr[bis(salicylidene)ethylenediaminate]mediated Baeyer-Villiger Oxidation: Stereospecific Synthesis of Abnormal and Normal Lactones. Proc. Natl. Acad. Sci. U. S. A. 2004, 101, 5737-5742.

(12) Serra, S.; Fuganti, C. Natural p-Menthene Monoterpenes: Synthesis of the Enantiomeric Forms of Wine Lactone, Epi-winelactone, Dill Ether, and Epi-dill Ether Starting from a Common Intermediate. Helv. Chim. Acta 2004, 87, 2100-2109.

(13) CrystalStructure 4.1: Crystal Structure Analysis Package; Rigaku Coorporation, Tokyo 196-8666, Japan, 2000-2015.

(14) Sheldrick, G. M. Crystal Structure Refinement with SHELXL. Acta Crystallogr., Sect. C 2015, 71, 3-8.

(15) Inokuma, Y.; Yoshioka, S.; Ariyoshi, J.; Arai T.; M. Fujita. Preparation and Guest-uptake Protocol for a Porous Complex Useful for 'Crystal-free' Crystallography. Nat. Protoc. 2014, 9, 246-252.

(16) Sheldrick, G. M. SHELXT-Integrated space-group and crystal-structure determination. Acta Crystallogr. Sect. A 2015, 71, 3-8. 


\section{J. NMR Spectra for Ketones and Reduction Products.}

6-Methyl-6,7,8,9-tetrahydro-5H-benzo[7]annulen-5-one (8c)

${ }^{1} \mathrm{H}-\mathrm{NMR}\left(400 \mathrm{MHz}, \mathrm{CDCl}_{3}\right)$

$$
\text { 170906-10 }
$$

\begin{tabular}{|c|c|c|c|}
\hline $\begin{array}{ll}\text { Sample Name } & 170906-10 \\
\text { Date collected } & 2017-09-06\end{array}$ & $\begin{array}{l}\text { Pulse sequence PROTON } \\
\text { Solvent cdcl3 }\end{array}$ & $\begin{array}{l}\text { Temperature } 25 \\
\text { Spectrometer Agllent-NMR-vnmrs400 }\end{array}$ & $\begin{array}{l}\text { Study owner vnmr1 } \\
\text { Operator vnmr1 }\end{array}$ \\
\hline
\end{tabular}

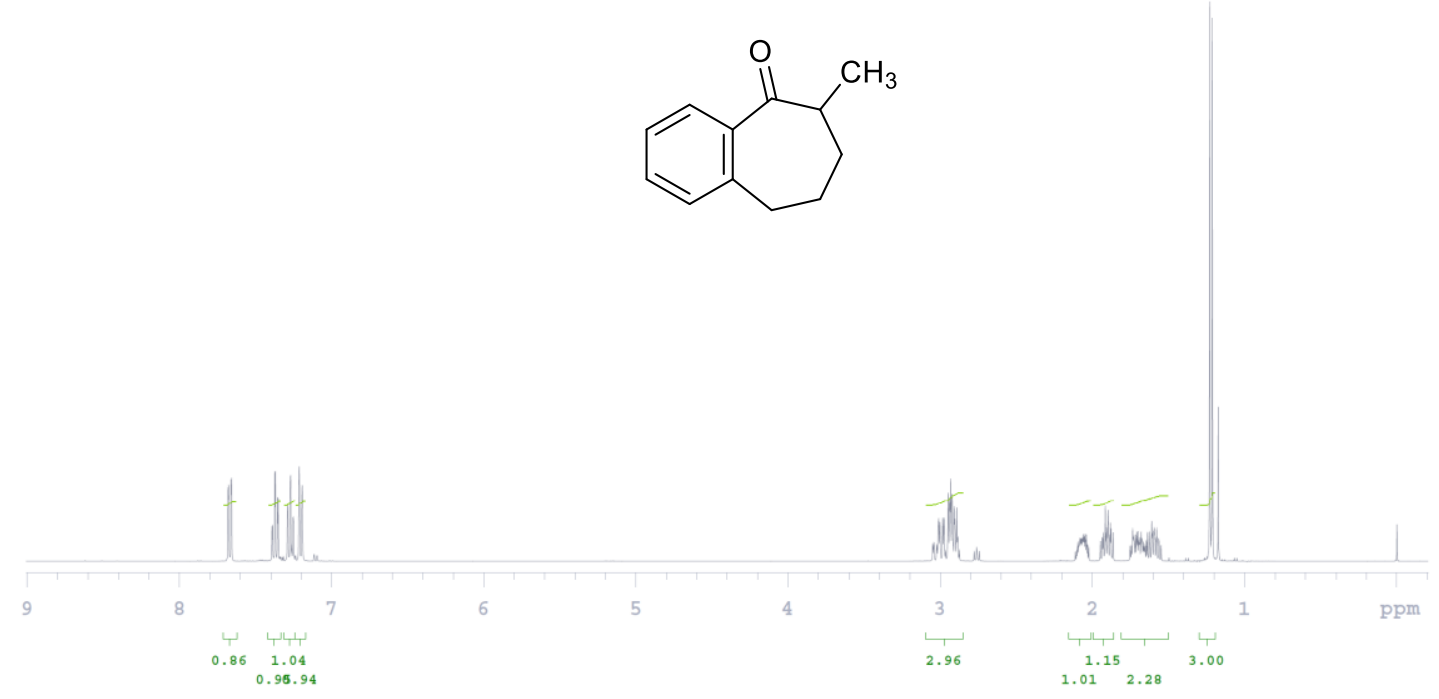

${ }^{13} \mathrm{C}$ NMR $\left(125 \mathrm{MHz}, \mathrm{CDCl}_{3}\right)$
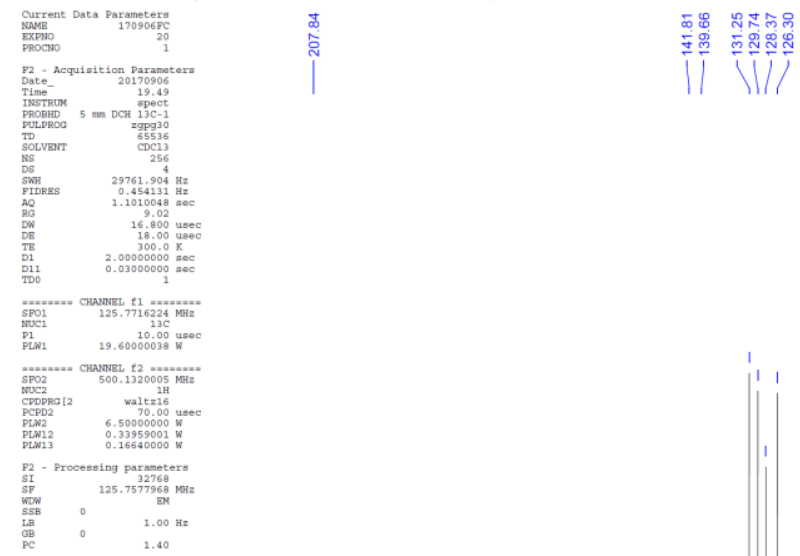

$$
\text { Hz }
$$


Methyl 1-oxo-1,2,3,4-tetrahydronaphthalene-2-carboxylate (8d)

${ }^{1} \mathrm{H}-\mathrm{NMR}\left(400 \mathrm{MHz}, \mathrm{CDCl}_{3}\right)$

$170830-7$

\begin{tabular}{|c|c|c|c|c|c|}
\hline $\begin{array}{l}\text { Sample Name } \\
\text { Date collectec }\end{array}$ & $\begin{array}{l}\begin{array}{l}170830-7 \\
2017-08-30\end{array}\end{array}$ & $\begin{array}{l}\text { Pulse sequence PROTON } \\
\text { Solvent cdcl3 }\end{array}$ & $\begin{array}{l}\text { Temperature } \\
\text { Spectrometer }\end{array}$ & $\begin{array}{l}25 \\
\text { Agilent-NMR-vnmrs400 }\end{array}$ & $\begin{array}{l}\text { Study owner vnmr1 } \\
\text { Operator vnmr1 }\end{array}$ \\
\hline
\end{tabular}

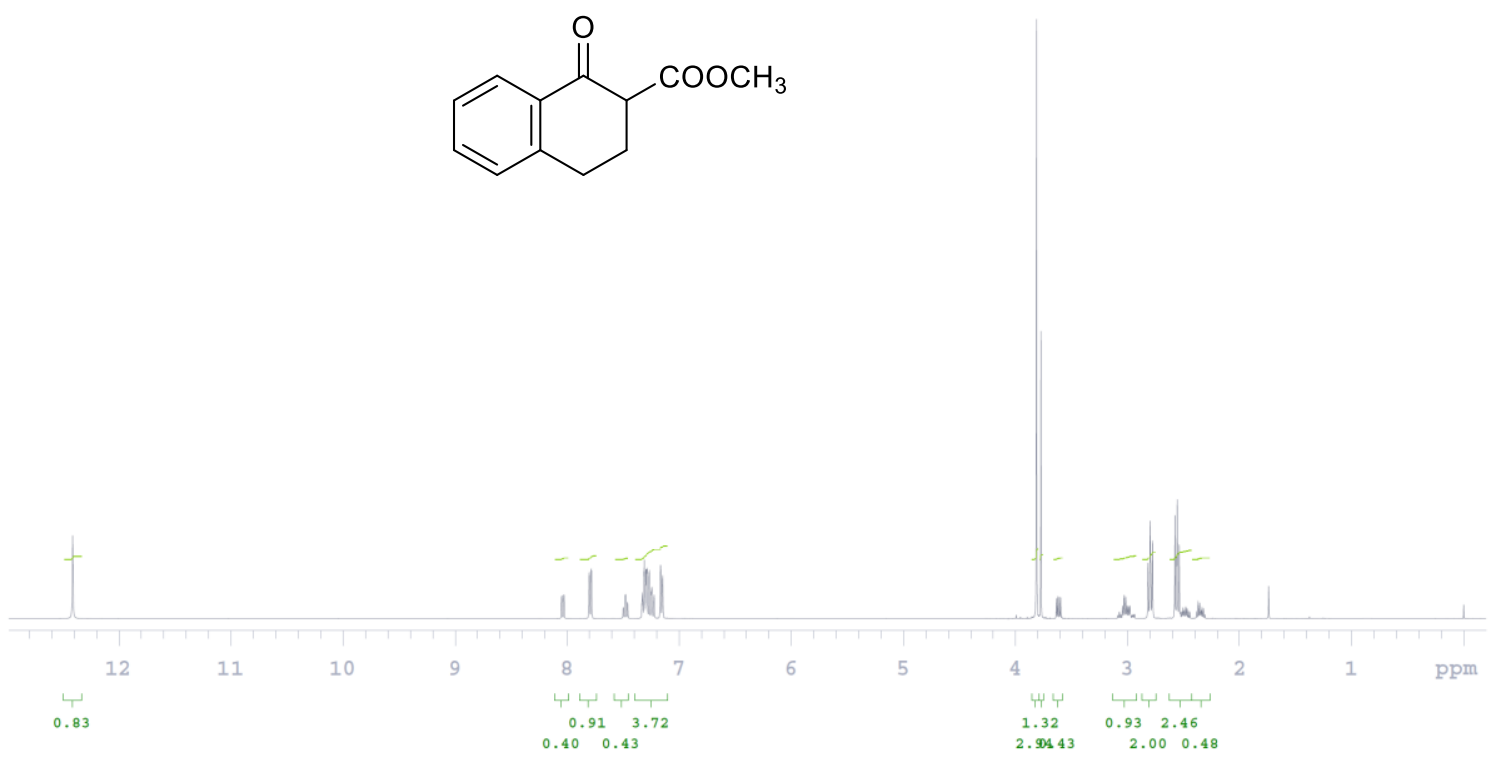

${ }^{13} \mathrm{C} \mathrm{NMR}\left(125 \mathrm{MHz}, \mathrm{CDCl}_{3}\right)$

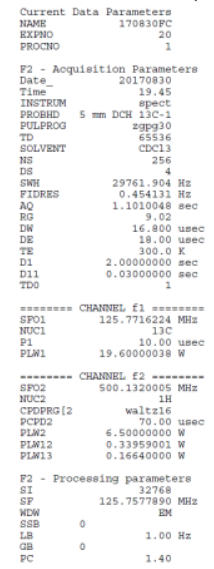


Methyl 2-(1-oxo-1,2,3,4-tetrahydronaphthalen-2-yl)acetate (8e)

${ }^{1} \mathrm{H}-\mathrm{NMR}\left(500 \mathrm{MHz}, \mathrm{CDCl}_{3}\right)$
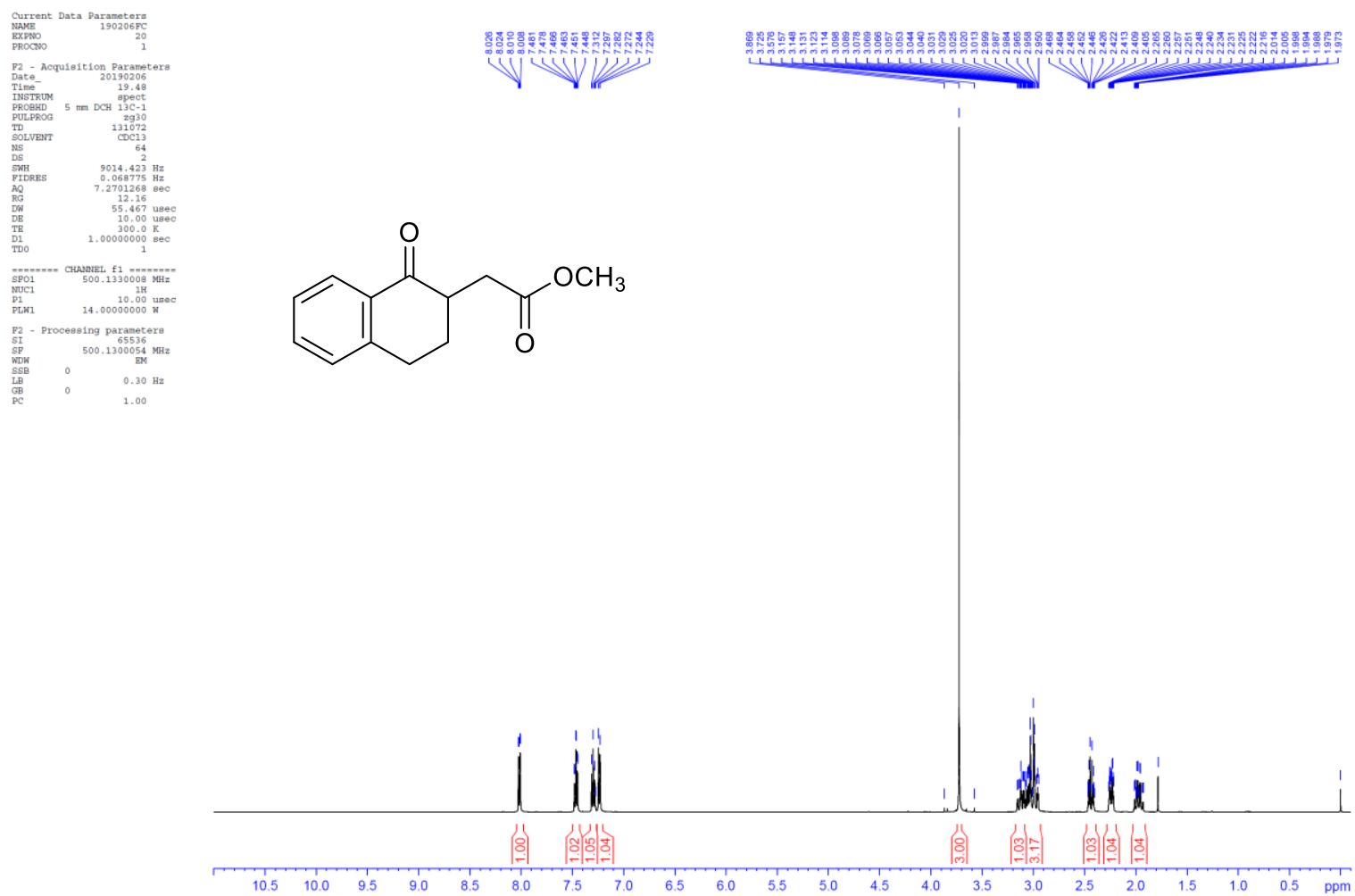

${ }^{13} \mathrm{C} \mathrm{NMR}\left(125 \mathrm{MHz}, \mathrm{CDCl}_{3}\right)$
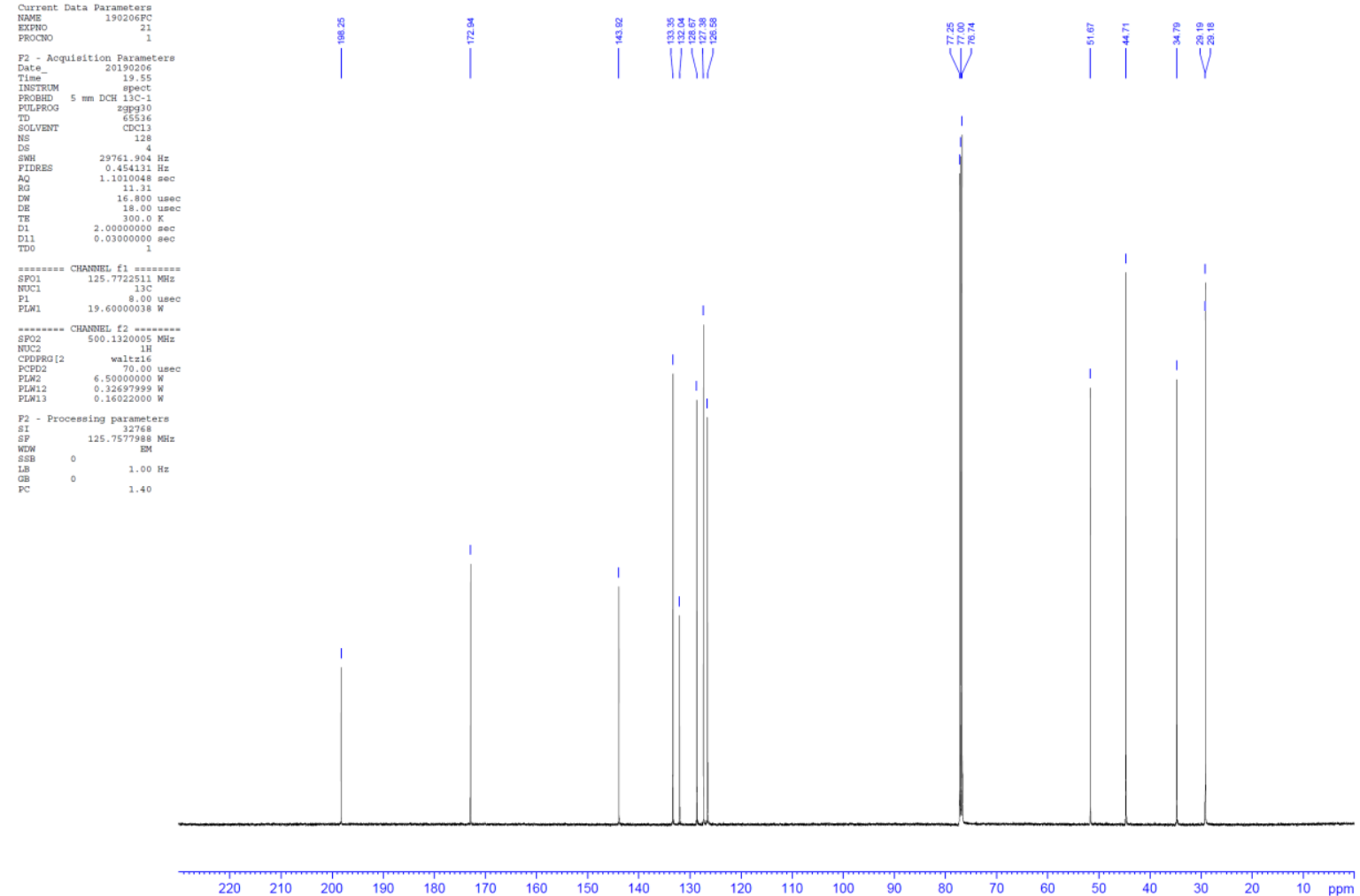
8-Bromo-7-methoxy-3,4-dihydronaphthalen-1(2H)-one

${ }^{1} \mathrm{H}-\mathrm{NMR}\left(500 \mathrm{MHz}, \mathrm{CDCl}_{3}\right)$
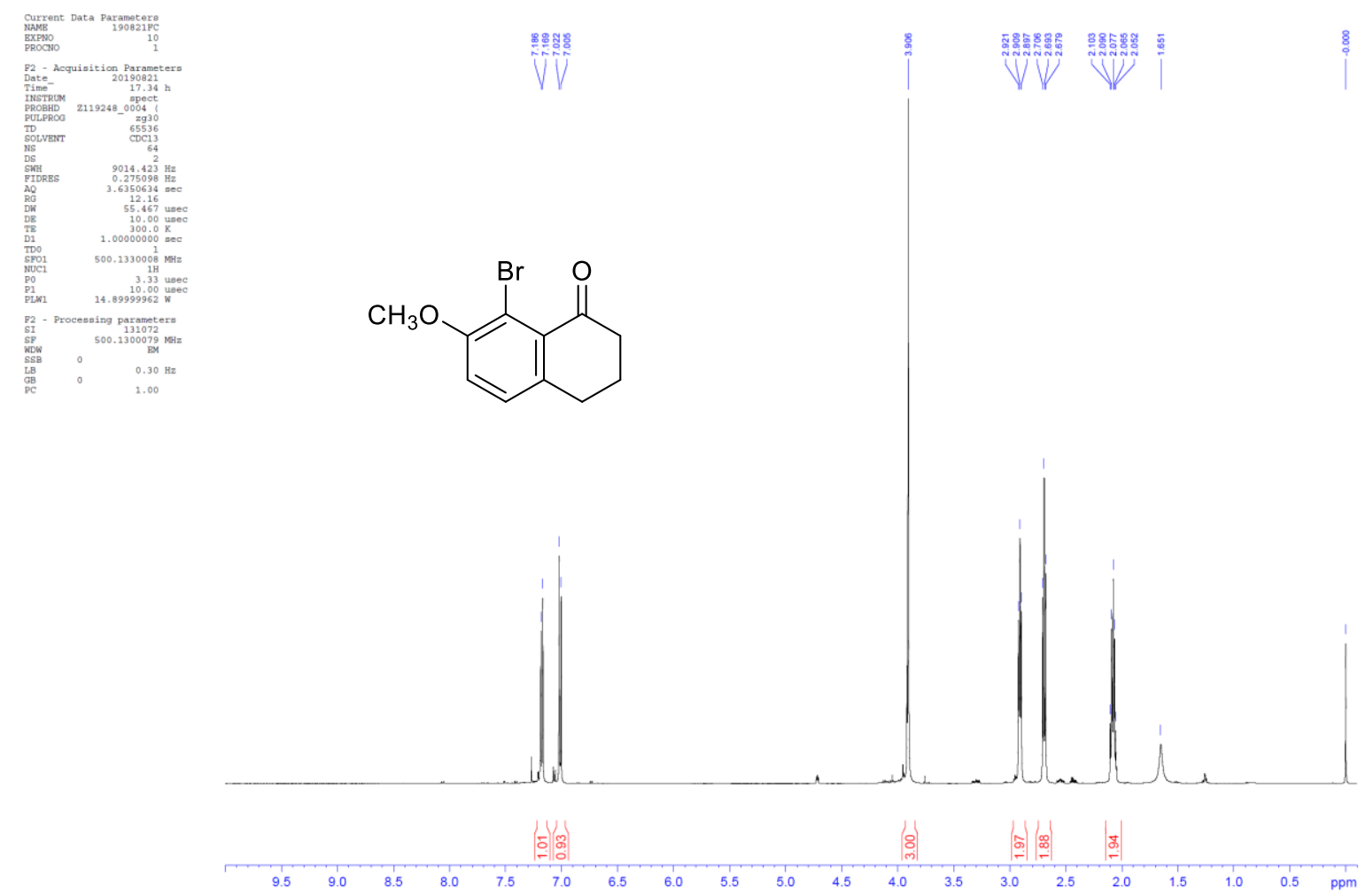

${ }^{13} \mathrm{C}$ NMR $\left(125 \mathrm{MHz}, \mathrm{CDCl}_{3}\right)$
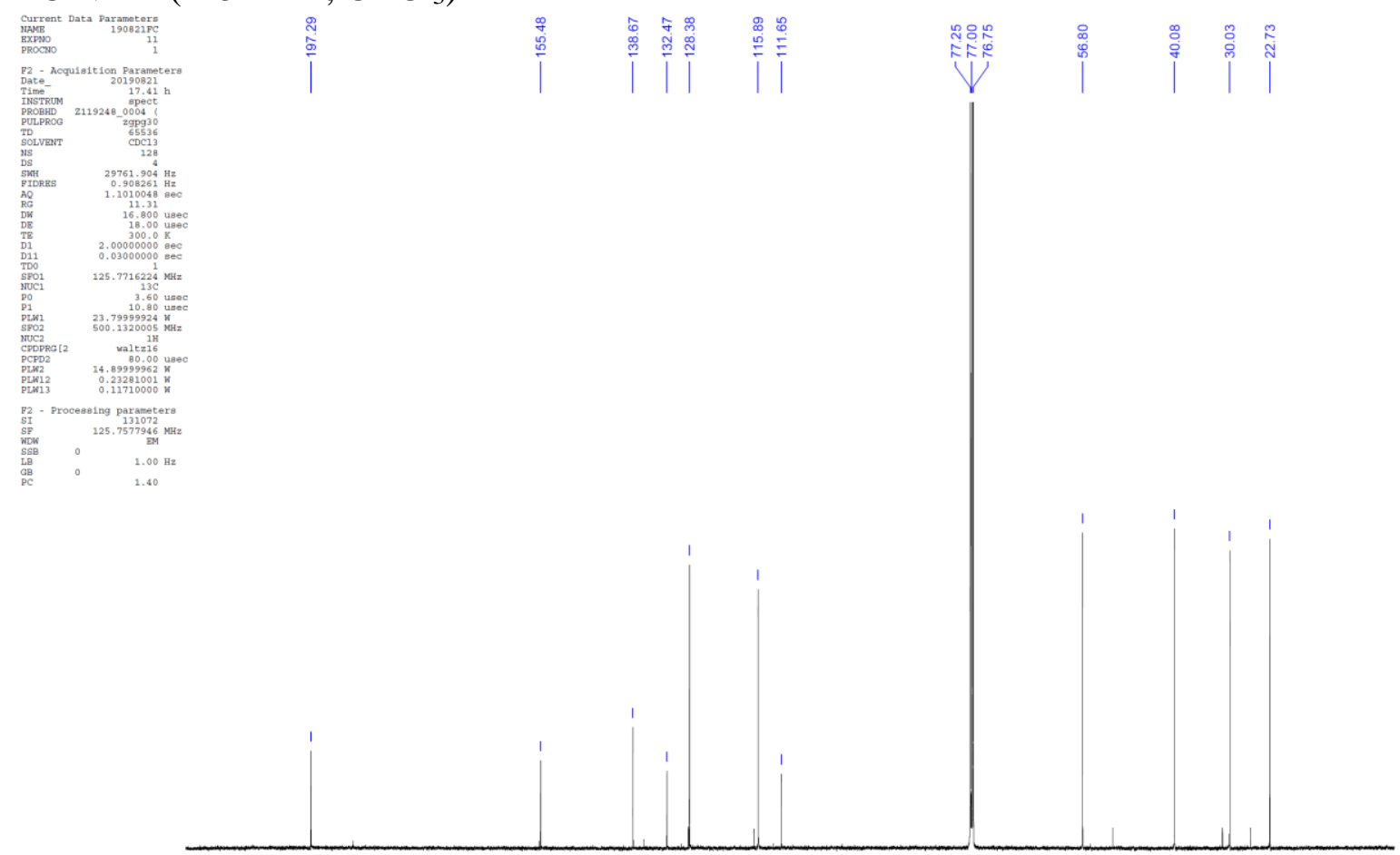
2-(1-Oxo-1,2,3,4-tetrahydronaphthalen-2-yl)acetic acid (11a)

${ }^{1} \mathrm{H}-\mathrm{NMR}\left(500 \mathrm{MHz}, \mathrm{DMSO}-d_{6}\right)$
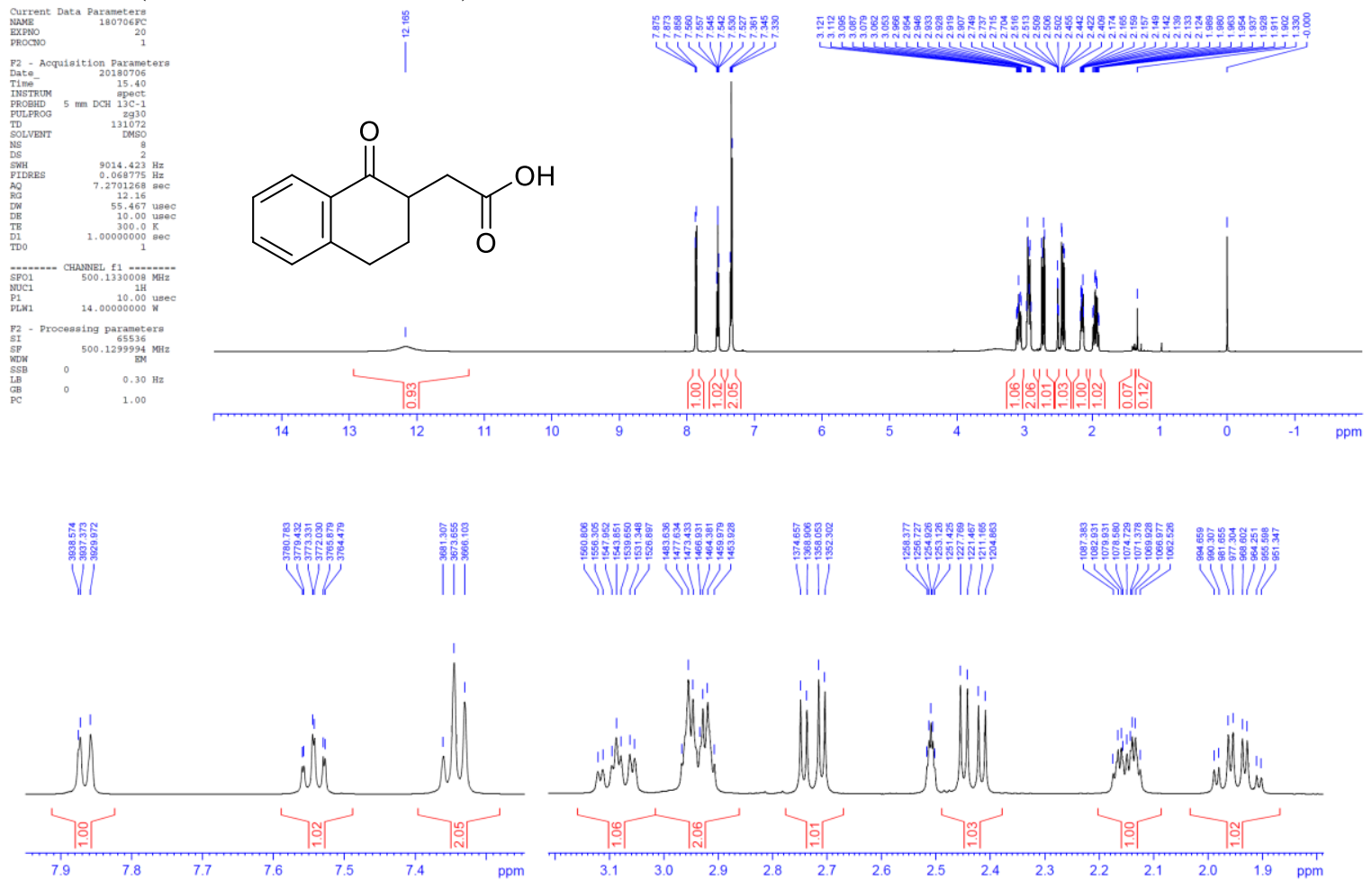

${ }^{13} \mathrm{C}$ NMR (125 MHz, DMSO- $\left.d_{6}\right)$

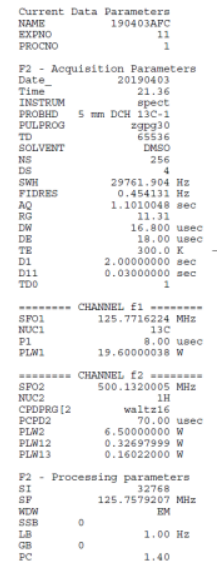

||$^{1} \mid$

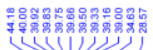

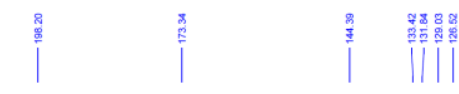

+

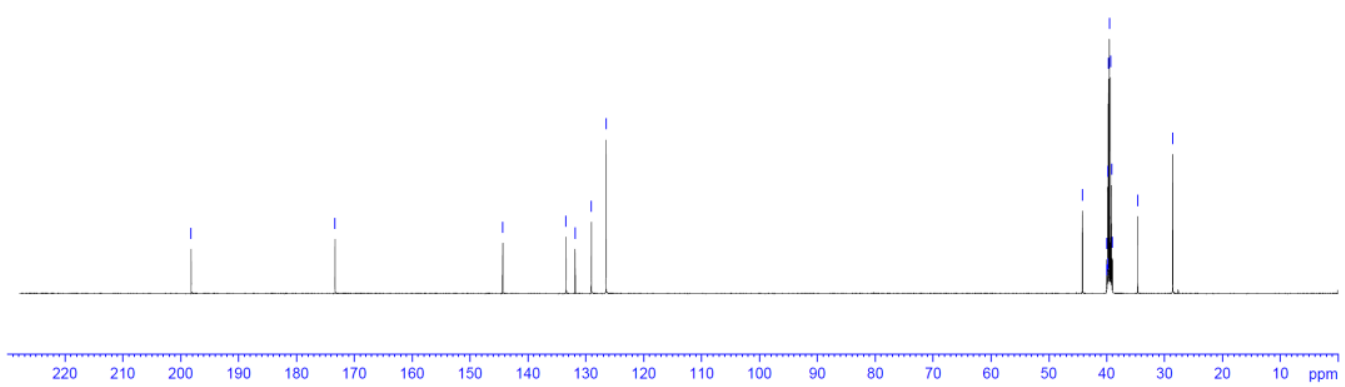


HMBC (DMSO- $d_{6}$ )

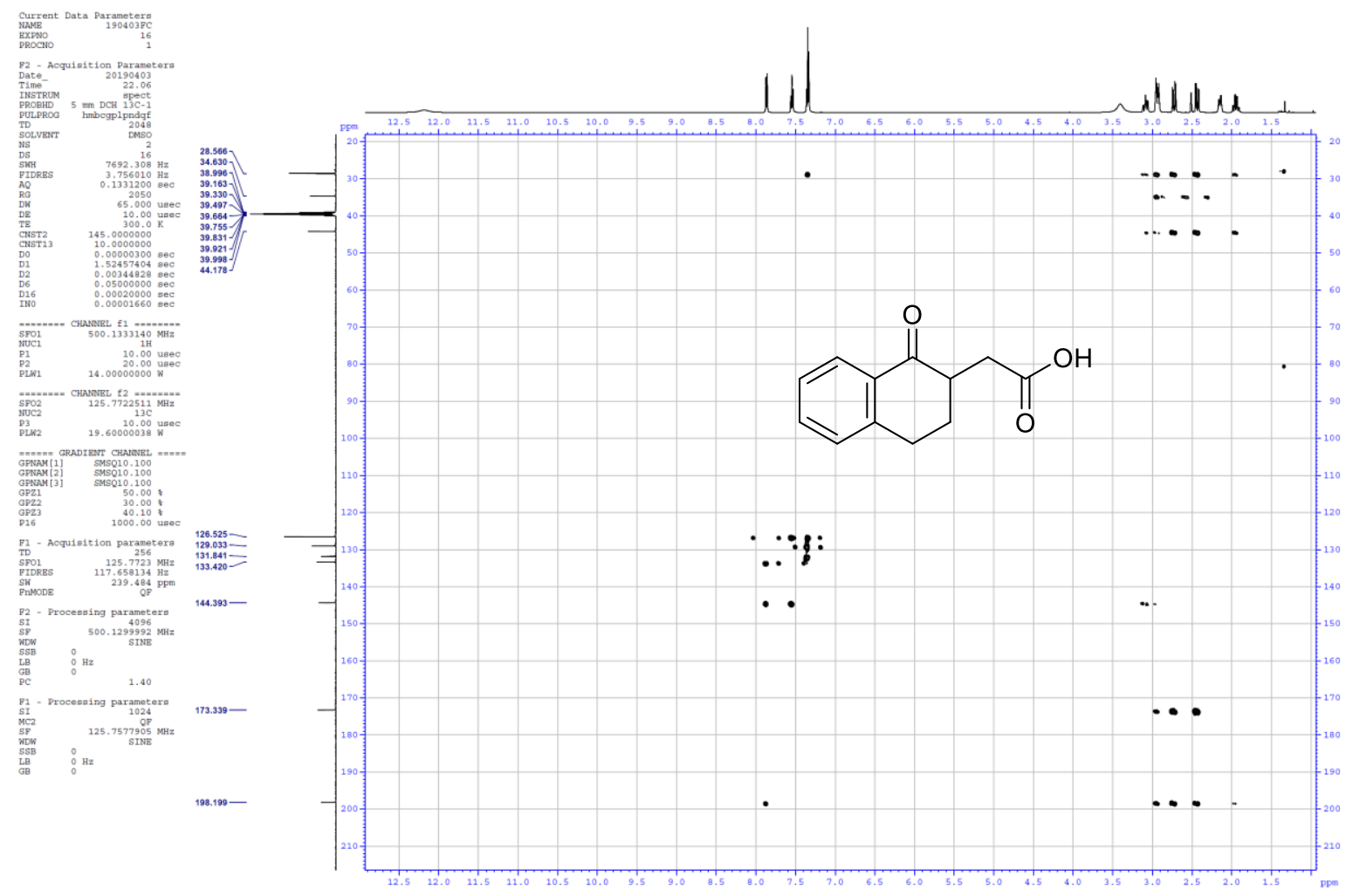

HMQC DMSO- $d_{6}$ )

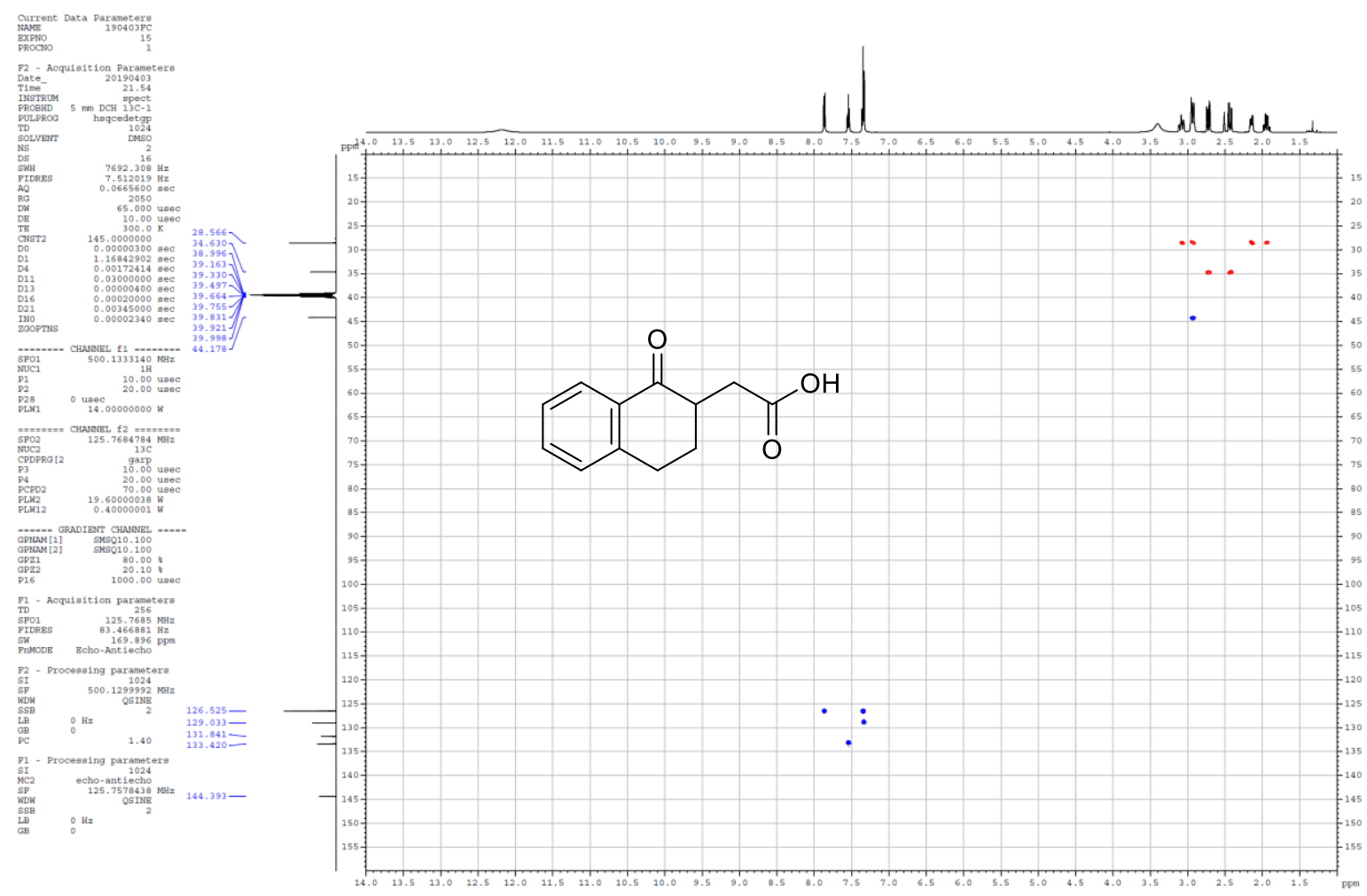


2-(7-Methoxy-1-oxo-1,2,3,4-tetrahydronaphthalen-2-yl)acetic acid (11b)

${ }^{1} \mathrm{H}-\mathrm{NMR}\left(400 \mathrm{MHz}, \mathrm{CDCl}_{3}\right)$

170830-4

Sample Name $170830-4$

Pulse sequence PROTON
Solvent cdcl3

Temperature 25
Spectrometer Agilent-NMR-vnmrs400

Study owner vnmr1<smiles>COc1ccc2c(c1)C(=O)C(CC(=O)O)CC2</smiles>

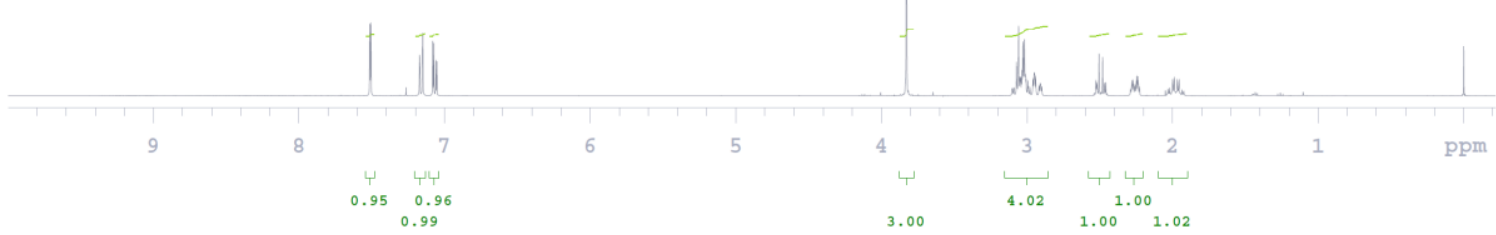

${ }^{13} \mathrm{C} \mathrm{NMR}\left(125 \mathrm{MHz}, \mathrm{CDCl}_{3}\right)$
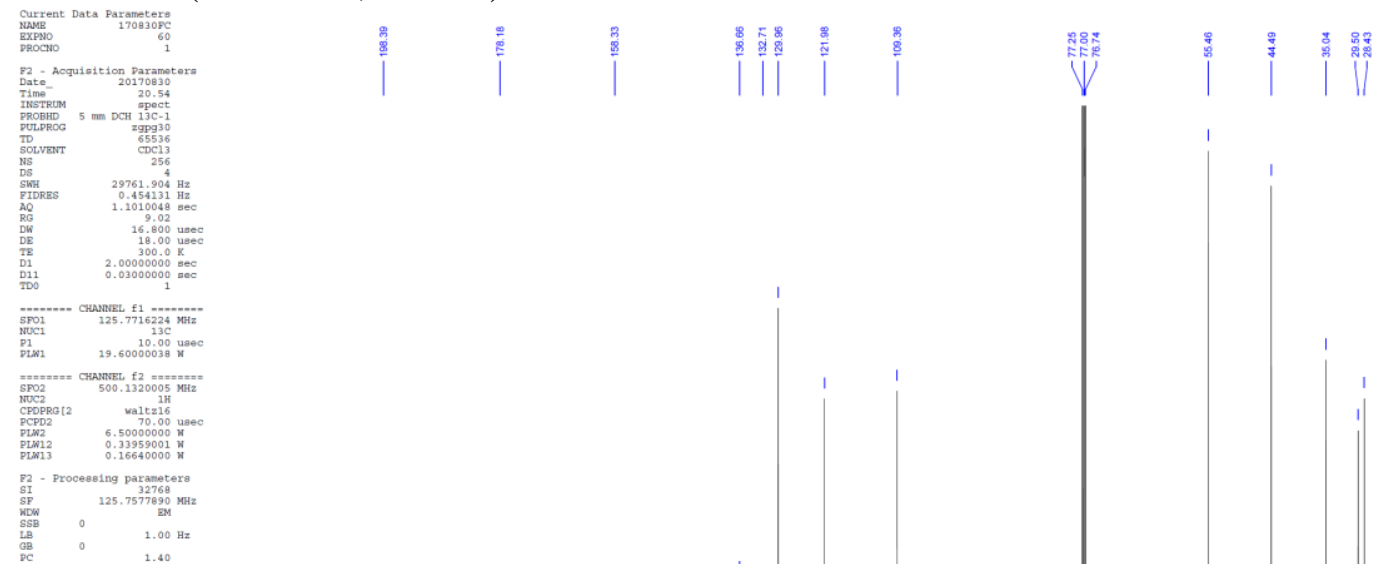

.
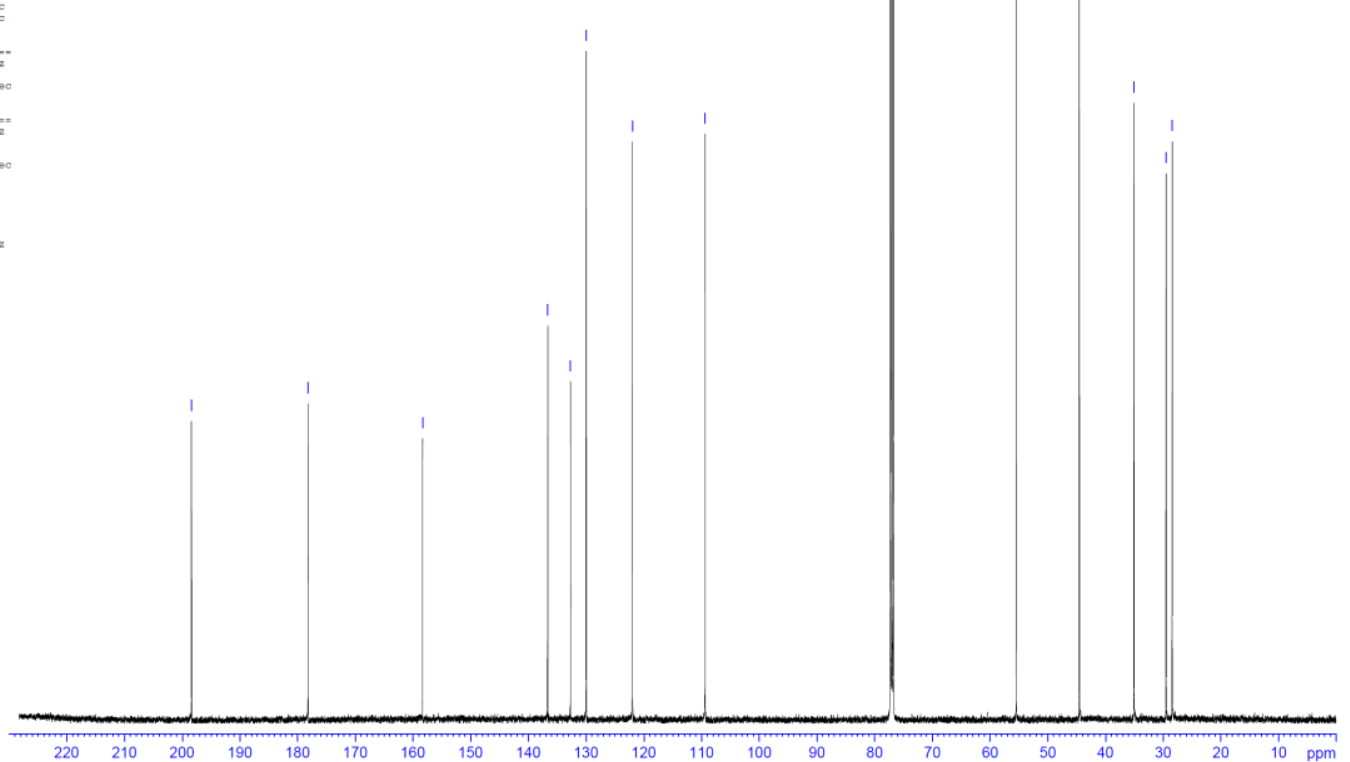
2-(7-Chloro-1-oxo-1,2,3,4-tetrahydronaphthalen-2-yl)acetic acid (11c)

${ }^{1} \mathrm{H}-\mathrm{NMR}\left(400 \mathrm{MHz}, \mathrm{DMSO}-d_{6}\right)$

$$
\begin{array}{ll}
\text { 170830-6 } & \\
\hline \text { Sample Name } & 170830-6 \\
\text { Date collected } & 2017-08-30
\end{array}
$$

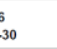
Pulse sequence PROTON
Solvent dmso Temperature 25
Spectrometer $\mathbf{A g}$ il Agilent-NMR-vnmrs400 Study owner vnmr1
Operator vnmr1

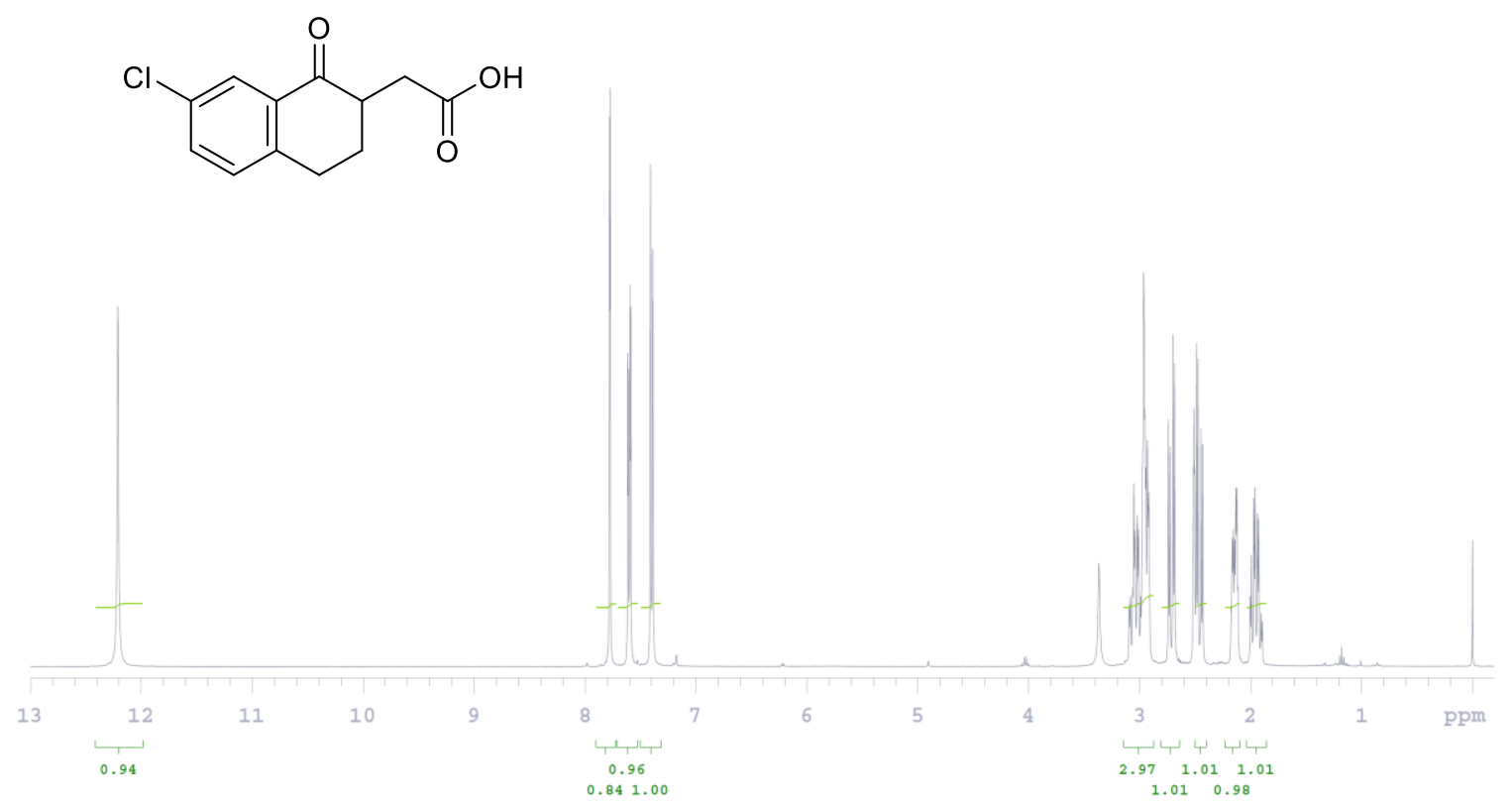

${ }^{13} \mathrm{C}$ NMR (125 MHz, DMSO- $d_{6}$ )
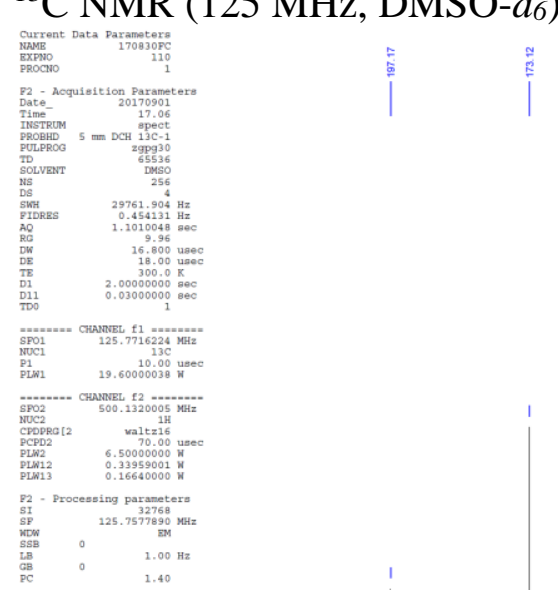

$$
\text { (1) }
$$
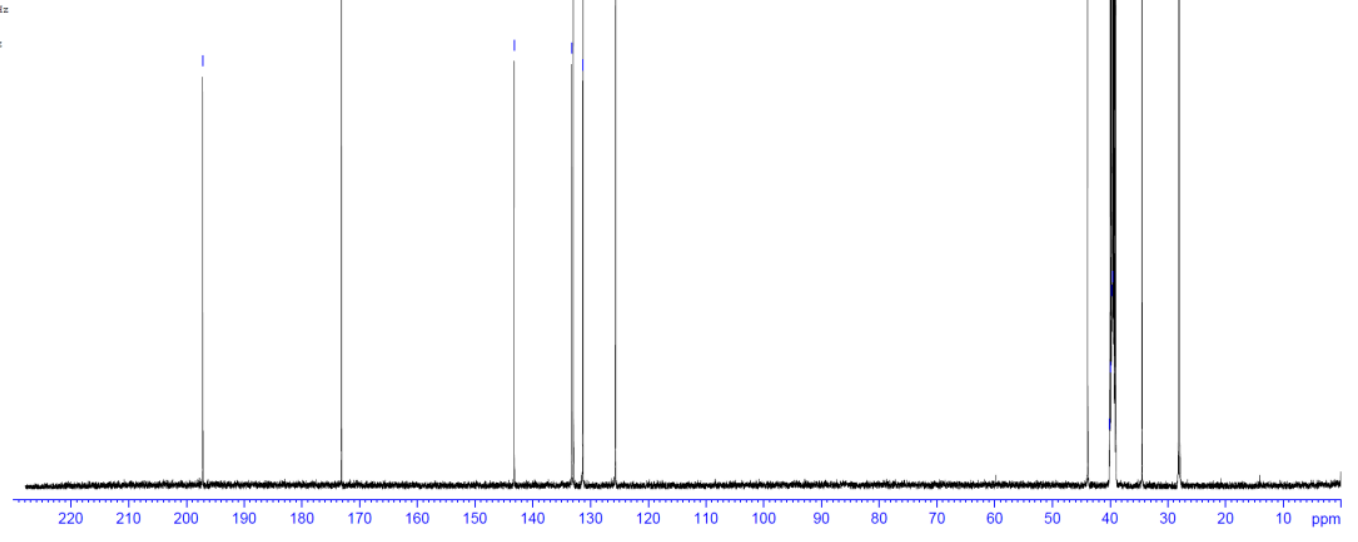
2-(7-Bromo-1-oxo-1,2,3,4-tetrahydronaphthalen-2-yl)acetic acid (11d)

${ }^{1} \mathrm{H}-\mathrm{NMR}\left(400 \mathrm{MHz}, \mathrm{DMSO}-d_{6}\right)$

$171109-2$

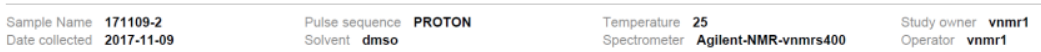

$\underbrace{O H}_{O}$

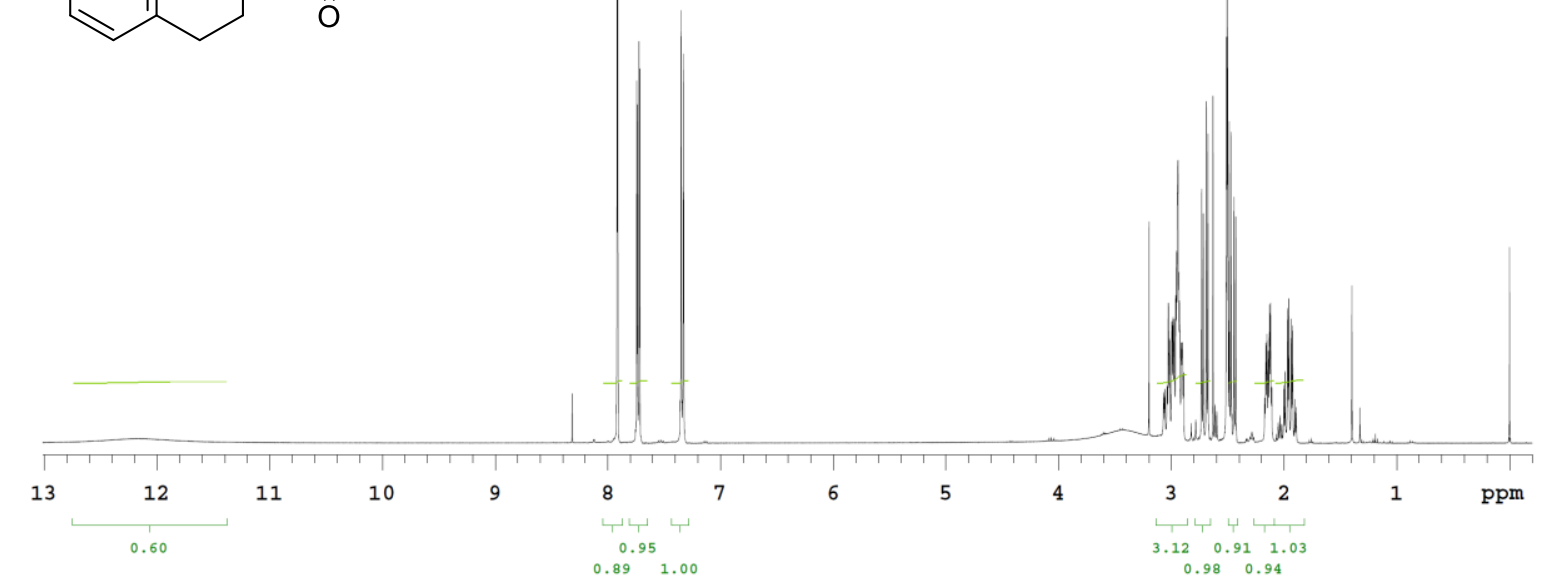

${ }^{13} \mathrm{C}$ NMR (125 MHz, DMSO-d6)

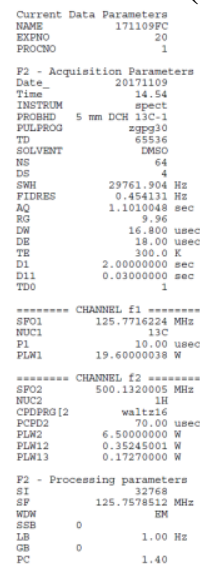


2-(8-Bromo-7-methoxy-1-oxo-1,2,3,4-tetrahydronaphthalen-2-yl)acetic acid (11e)

${ }^{1} \mathrm{H}-\mathrm{NMR}\left(500 \mathrm{MHz}, \mathrm{DMSO}-d_{6}\right)$
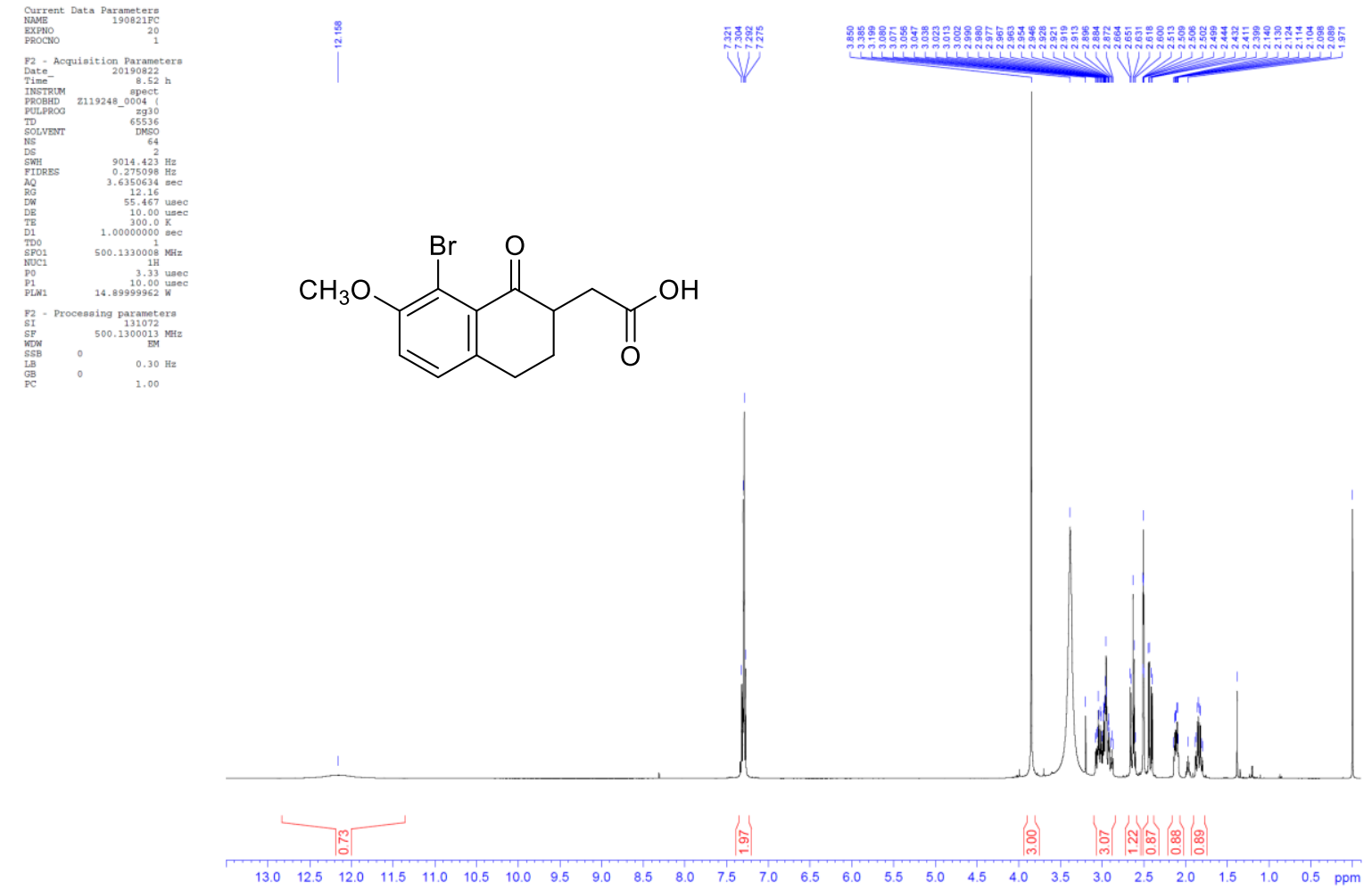

${ }^{13} \mathrm{C}$ NMR (125 MHz, DMSO- $\left.d_{6}\right)$
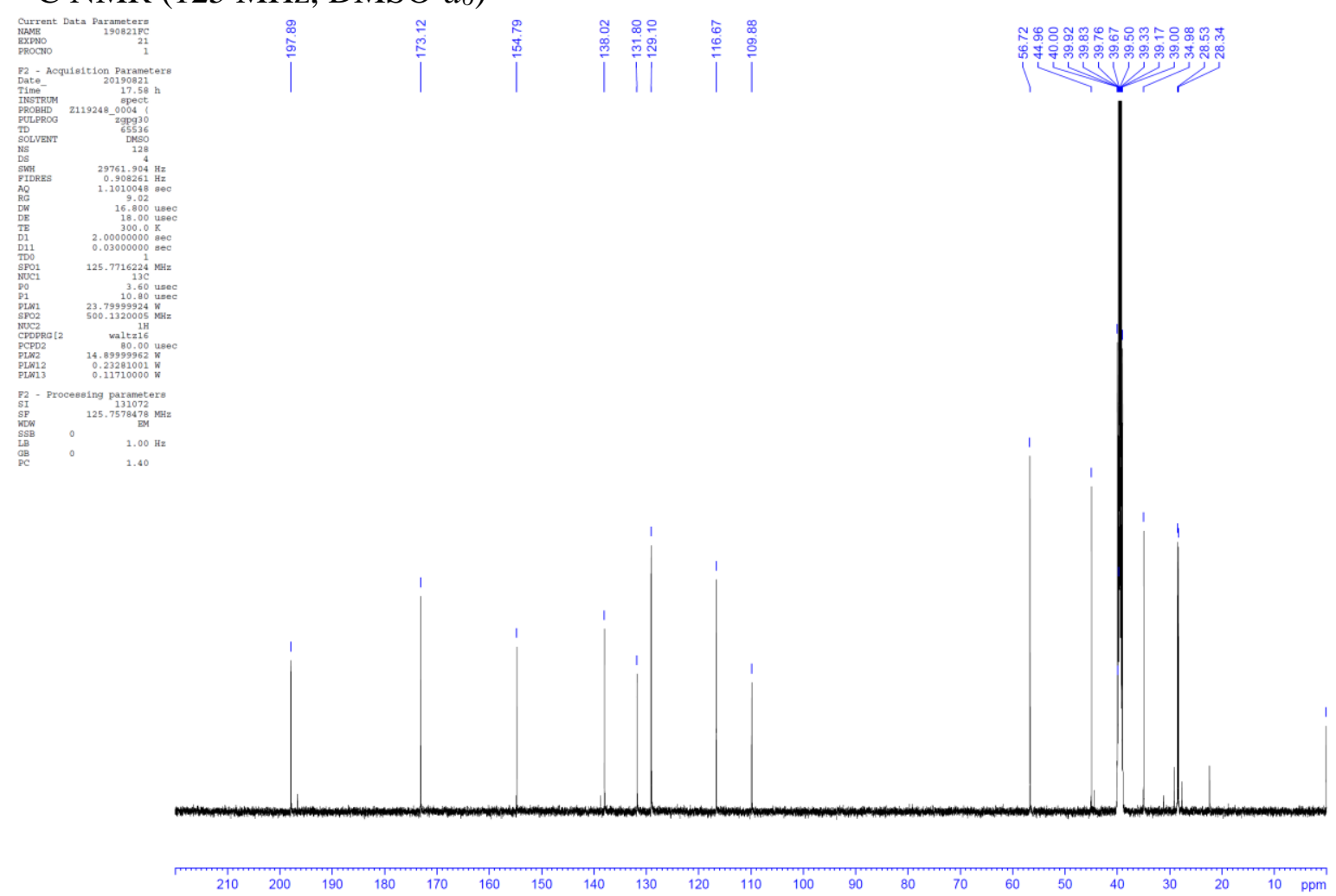
2-(6-Hydroxy-1-oxo-1,2,3,4-tetrahydronaphthalen-2-yl)acetic acid (11f)

${ }^{1} \mathrm{H}-\mathrm{NMR}\left(400 \mathrm{MHz}, \mathrm{DMSO}-d_{6}\right)$

170913-1

Sample Name $170913-1$

Pulse sequence PROTON
Solvent dmso

Temperature 25
Spectrometer Agilent-NMR-vnmrs40

Study owner vnmr

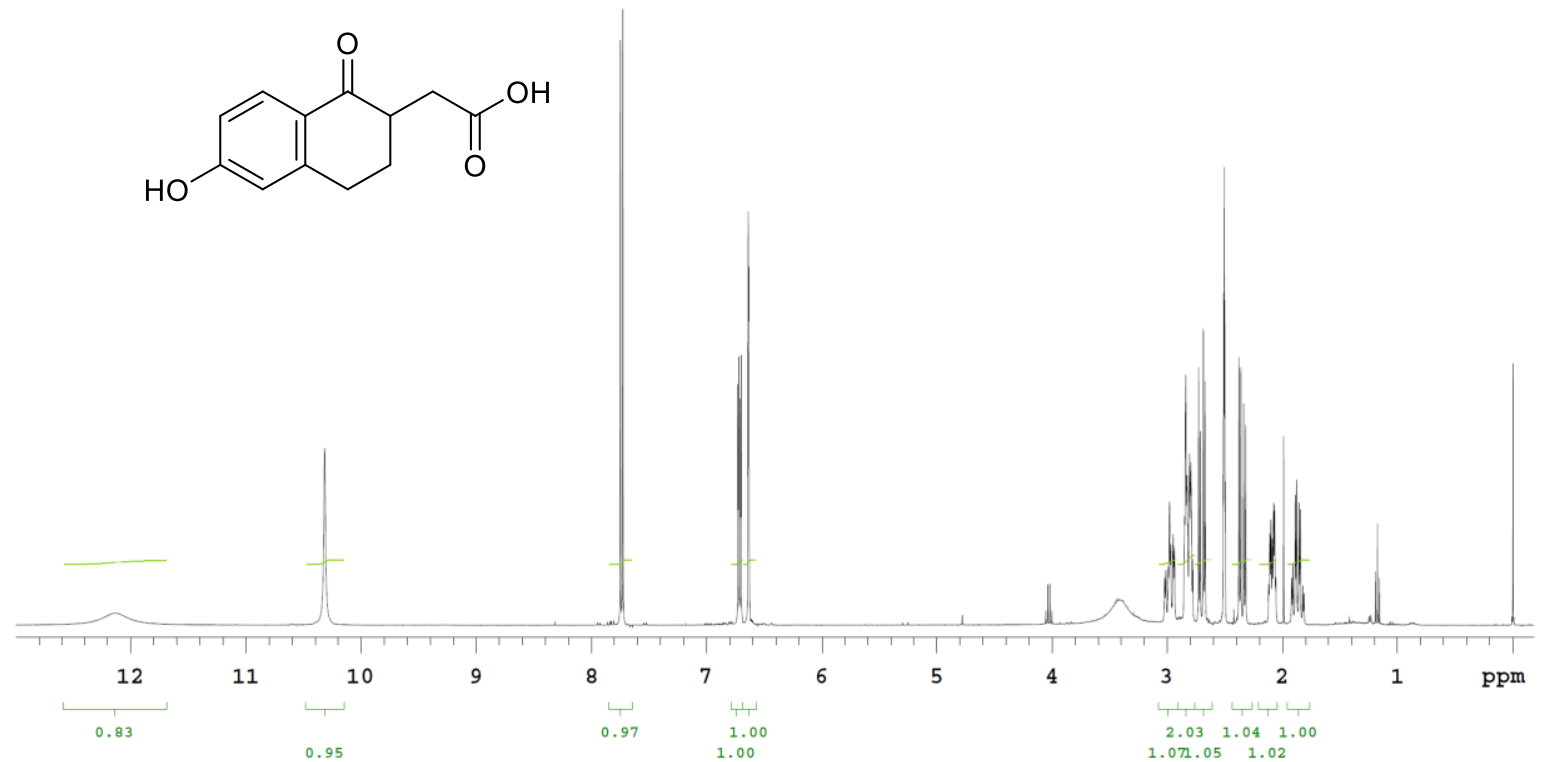

${ }^{13} \mathrm{C}$ NMR (125 MHz, DMSO- $\left.d_{6}\right)$
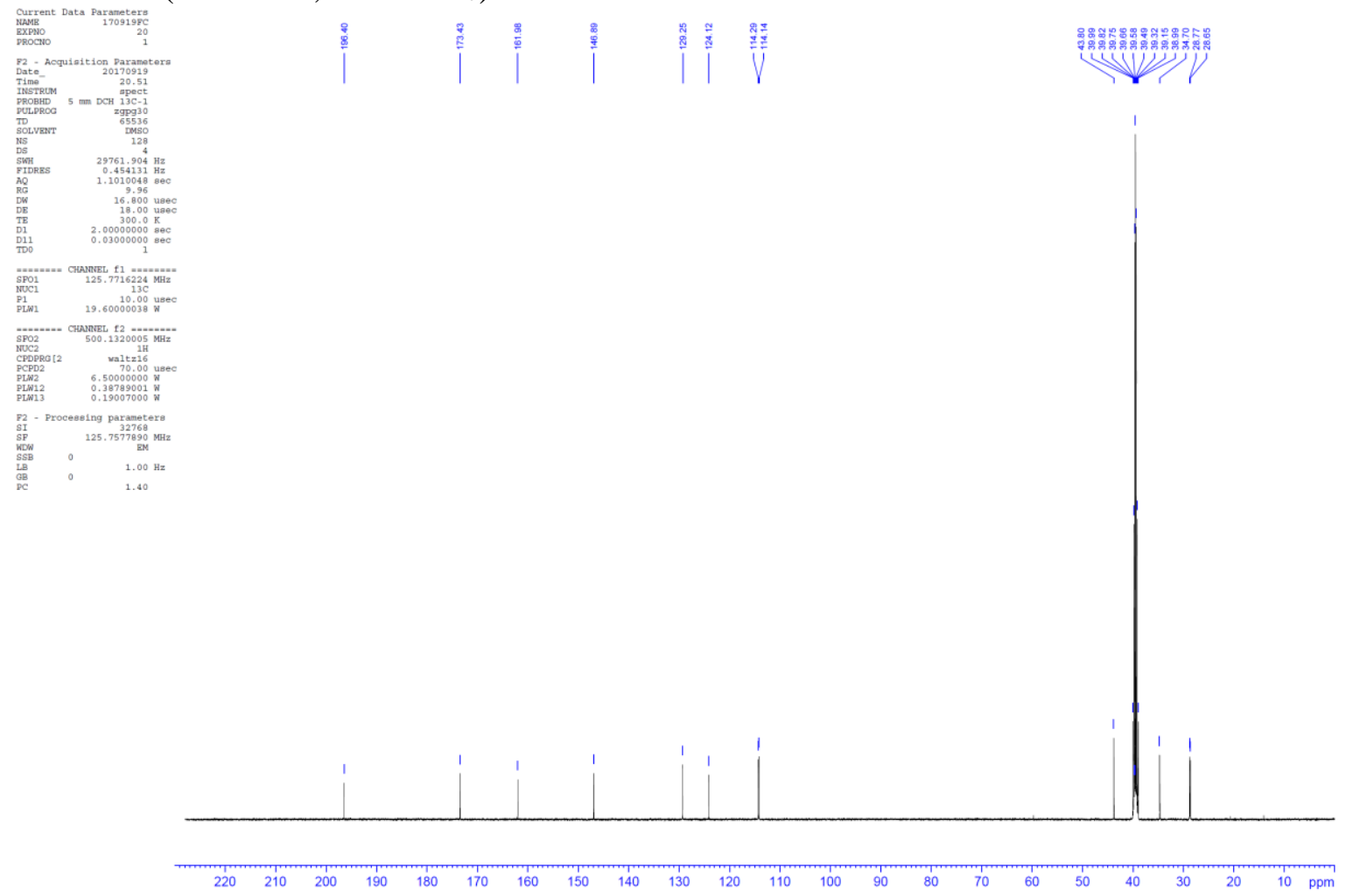
2-(1-Oxo-2,3-dihydro-1H-inden-2-yl)acetic acid (11g)

${ }^{1} \mathrm{H}-\mathrm{NMR}\left(400 \mathrm{MHz}, \mathrm{CDCl}_{3}\right)$

170824-7

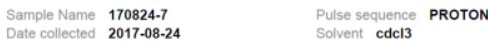

Temperature 25
Spectrometer Agilent-NMR-vnmrs40

Study owner vnmr
Operator vnmr1
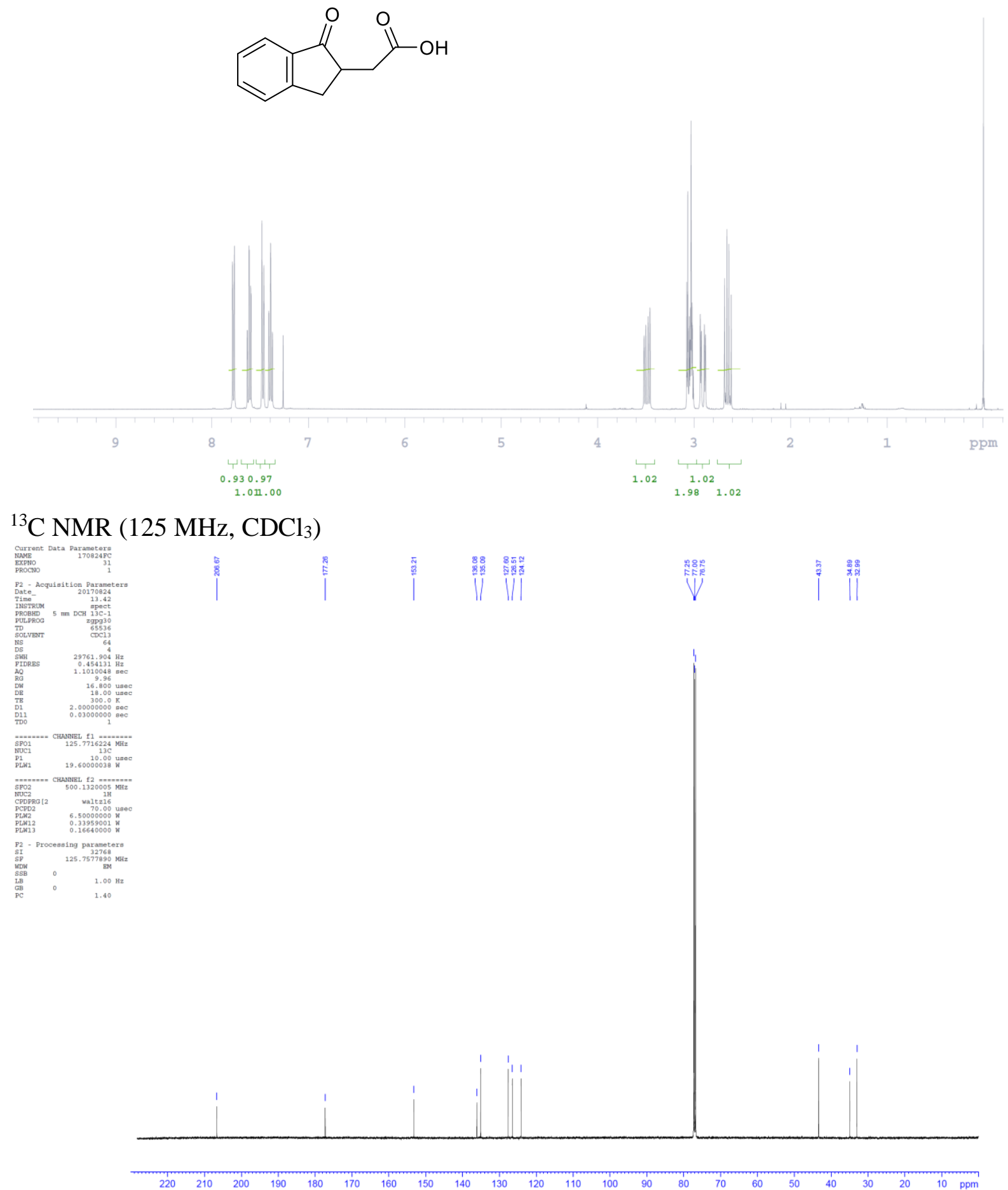
2-(5,6-Dimethoxy-1-oxo-2,3-dihydro-1H-inden-2-yl)acetic acid (11h)

${ }^{1} \mathrm{H}-\mathrm{NMR}\left(400 \mathrm{MHz}, \mathrm{CDCl}_{3}\right)$

$170830-5$

Sample Name $170830-5$

Pulse sequence PROTON
Solvent cdcl3

Temperature 25
Spectrometer

Agilent-NMR-vnmrs400

Study owner vnmr1

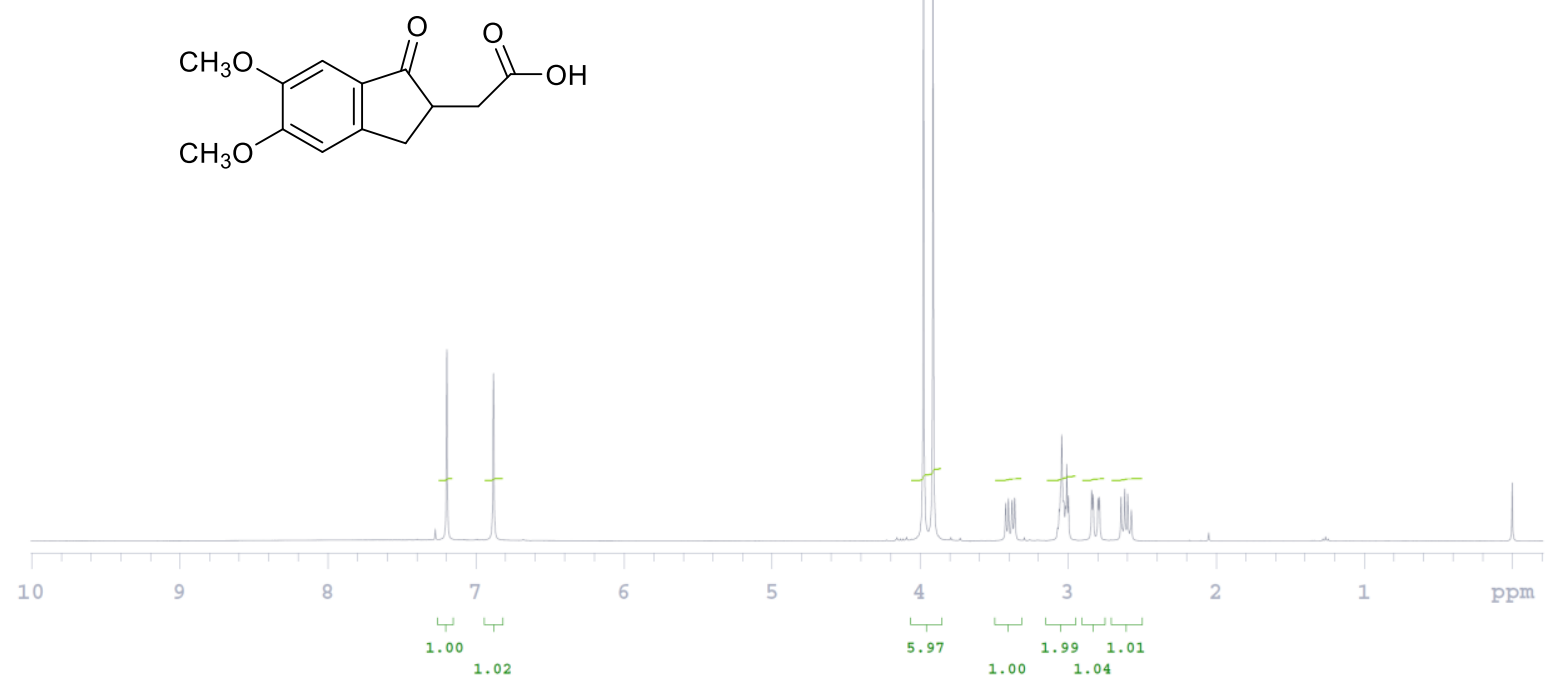

${ }^{13} \mathrm{C} \mathrm{NMR}\left(125 \mathrm{MHz}, \mathrm{CDCl}_{3}\right)$
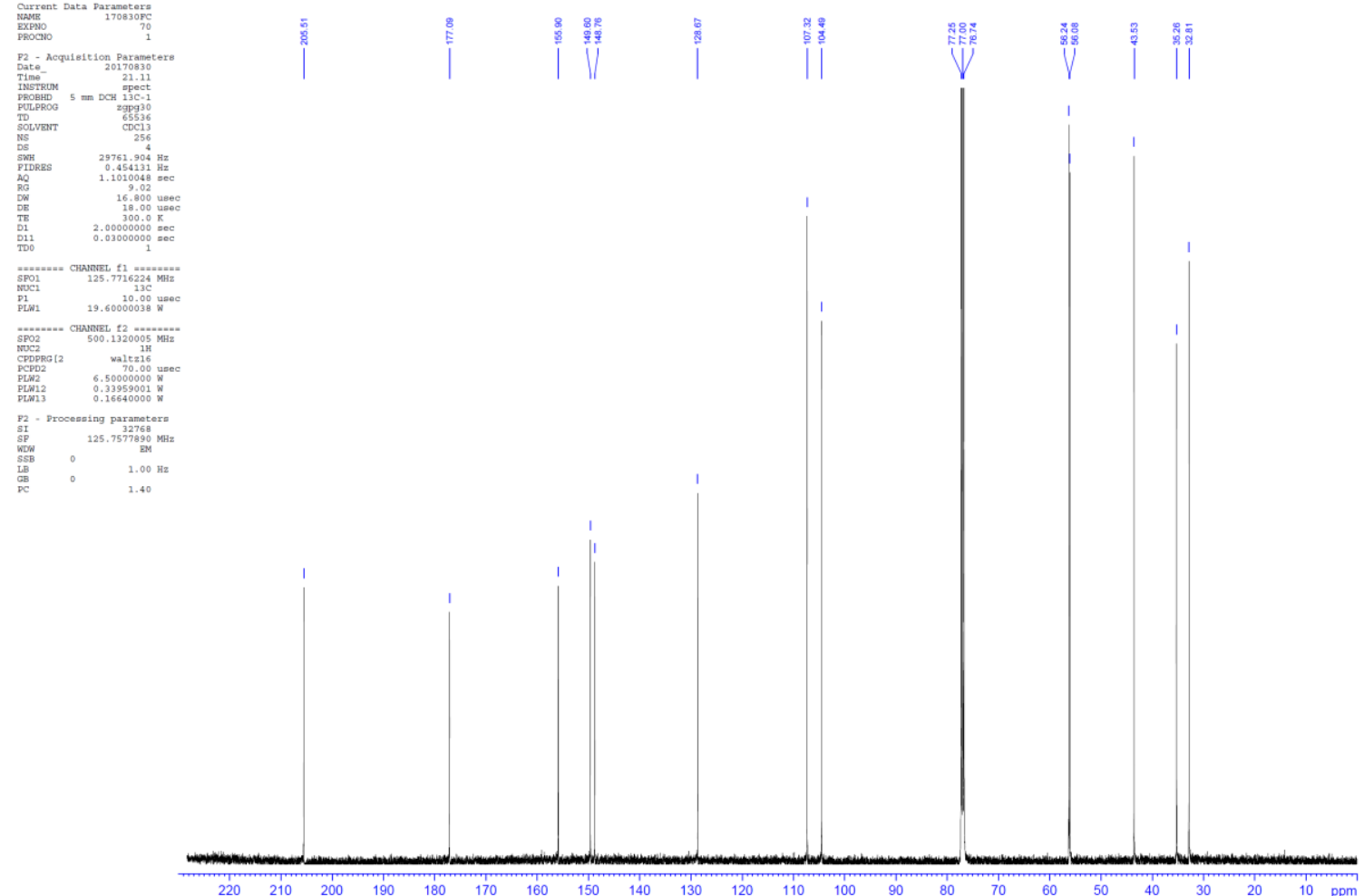
2-(6-Fluoro-1-oxo-2,3-dihydro-1H-inden-2-yl)acetic acid (11i)

${ }^{1} \mathrm{H}-\mathrm{NMR}\left(400 \mathrm{MHz}, \mathrm{DMSO}-d_{6}\right)$

170904-1

Sample Name 170904-1

Pulse sequence PROTON
Solvent dmso

Temperature 25
Spectrometer $\mathbf{A}$

Agllent-NMR-vnmrs400

Study owner vnmr-1
Operator vnmr1<smiles>O=C(O)CC1Cc2ccc(F)cc2C1=O</smiles>

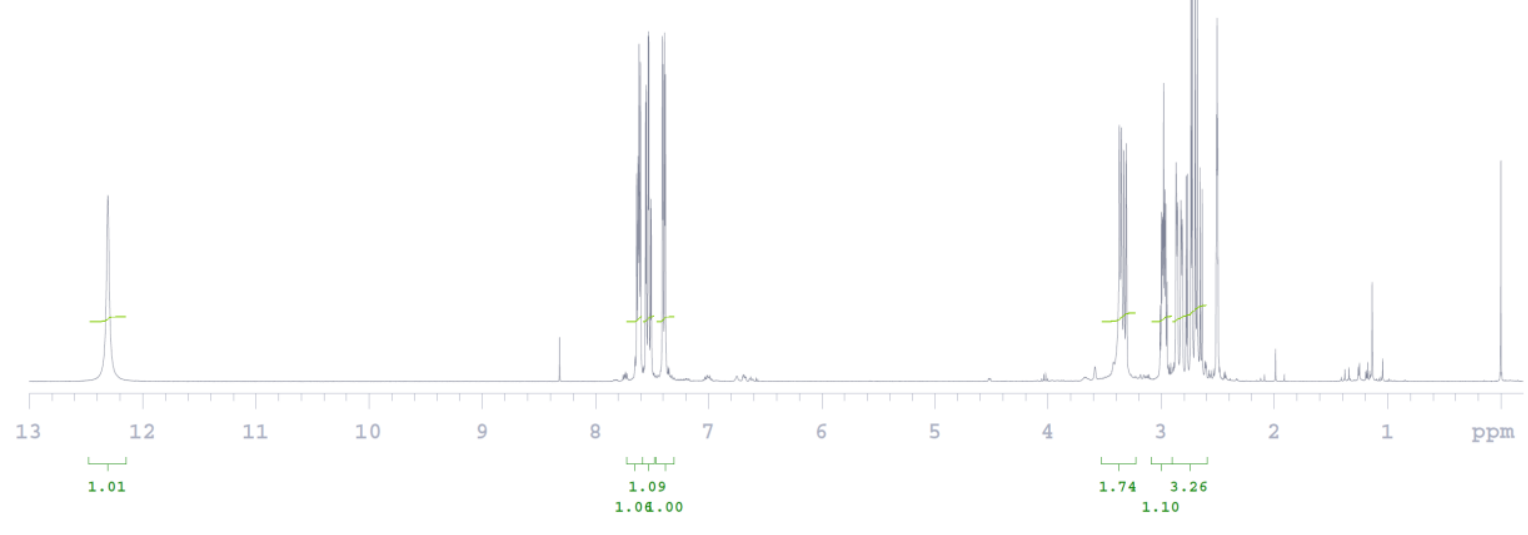

${ }^{13} \mathrm{C}$ NMR (125 MHz, DMSO-d6)
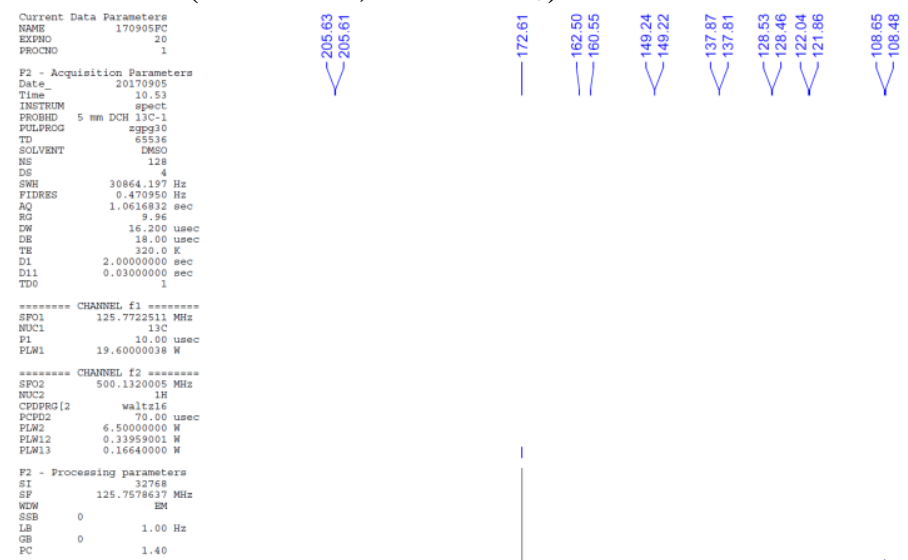

$\sum^{\circ}$

5ำ.

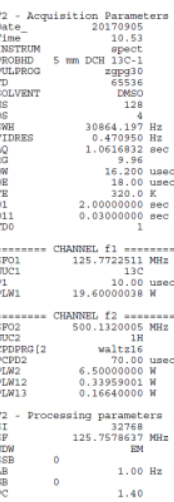


2-(6-Chloro-1-oxo-2,3-dihydro-1H-inden-2-yl)acetic acid (11j)

${ }^{1} \mathrm{H}-\mathrm{NMR}\left(400 \mathrm{MHz}, \mathrm{DMSO}-d_{6}\right)$

$171115-2$

\begin{tabular}{llll}
\hline Sample Name 171115-2 & Pulse sequence PROTON & Temperature 25 & 25 \\
Date collected 2017-11-15 & Solvent dmso & Spectrometer Agllent-NMR-vnmrs400 & $\begin{array}{l}\text { Study owner vnmr1 } \\
\text { Operator vnmr1 }\end{array}$
\end{tabular}<smiles>O=C(O)CC1Cc2ccc(Cl)cc2C1=O</smiles>

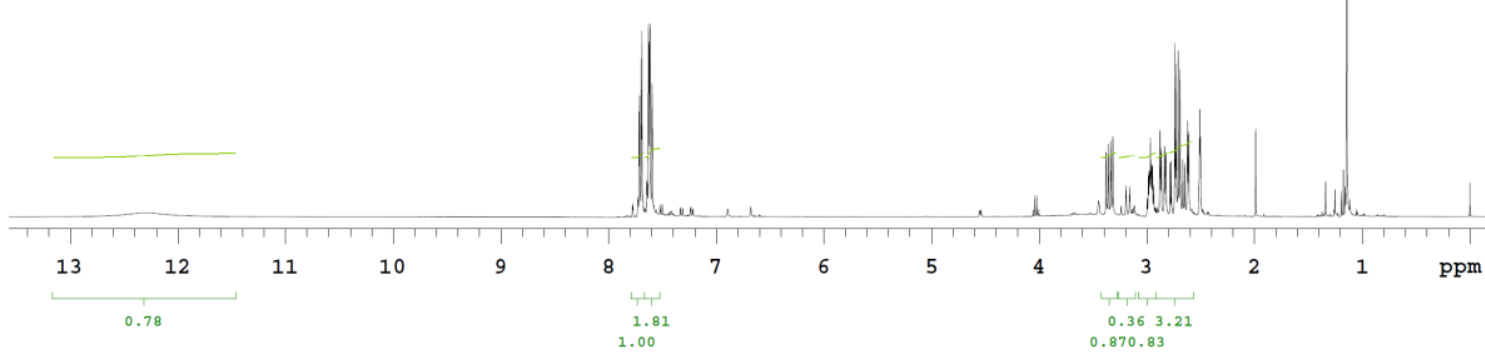

${ }^{13} \mathrm{C}$ NMR (125 MHz, DMSO- $\left.d_{6}\right)$
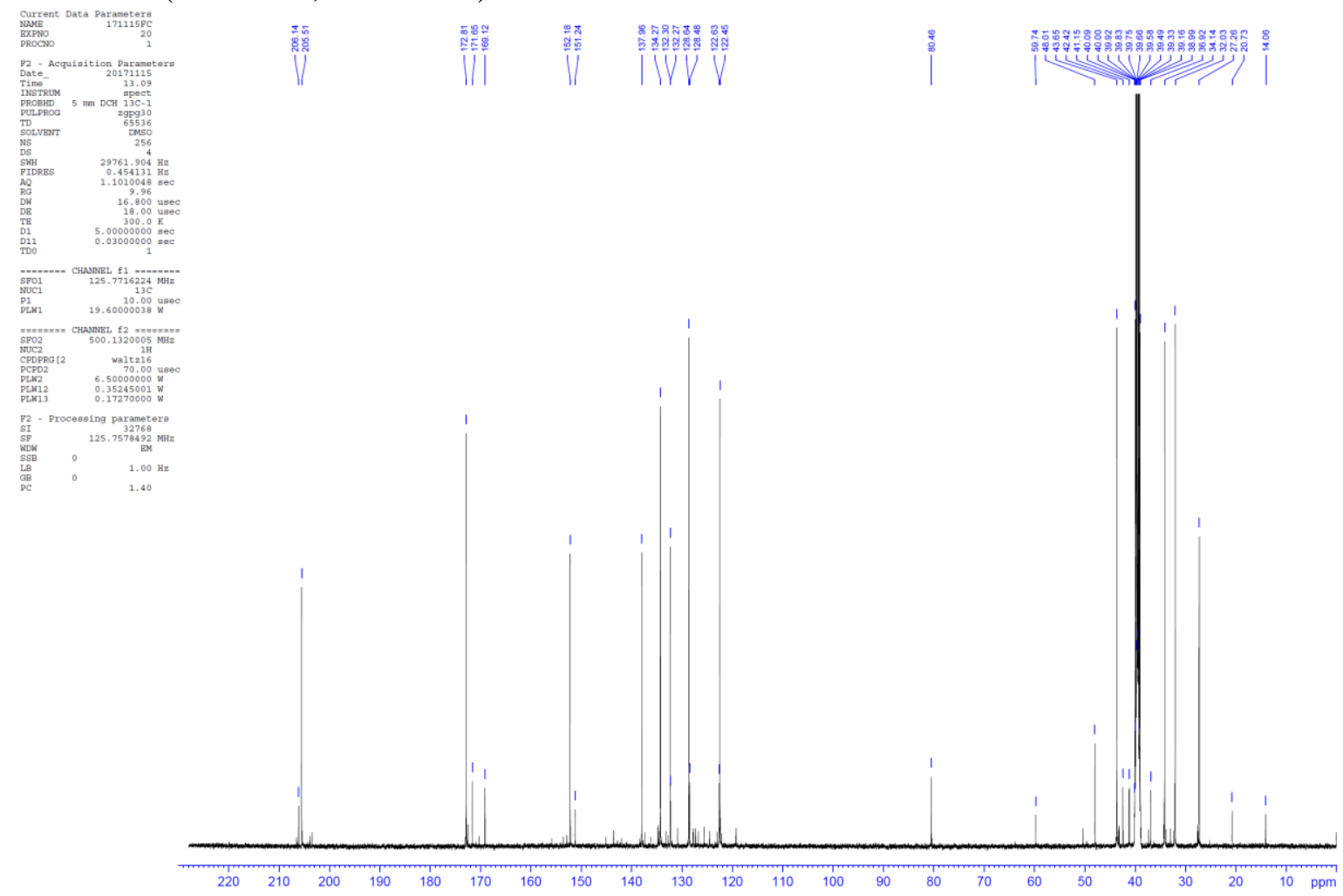
2-(5-Oxo-6,7,8,9-tetrahydro-5H-benzo[7]annulen-6-yl)acetic acid (11k)

${ }^{1} \mathrm{H}-\mathrm{NMR}\left(400 \mathrm{MHz}, \mathrm{CDCl}_{3}\right.$ )

$170824-6$

Sample Name $170824-6$

Pulse sequence PROTON
Solvent cdcl3

Temperature 25

Study owner vnmr1
Operator vnmri<smiles>O=C(O)CC1CCCc2ccccc2C1=O</smiles>
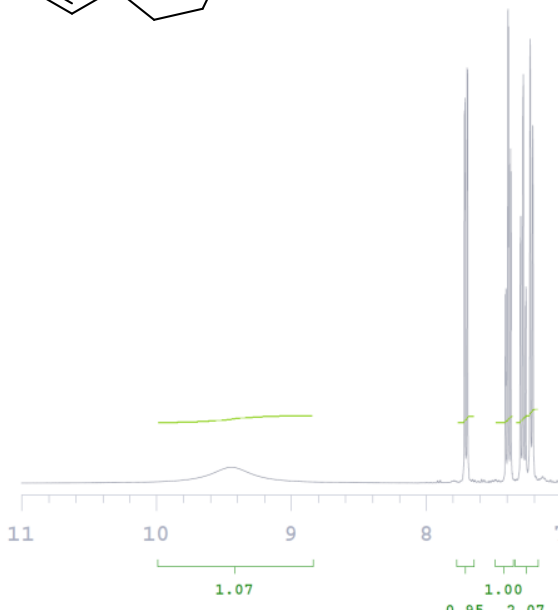

8

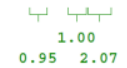

${ }^{13} \mathrm{C} \mathrm{NMR}\left(125 \mathrm{MHz}, \mathrm{CDCl}_{3}\right)$
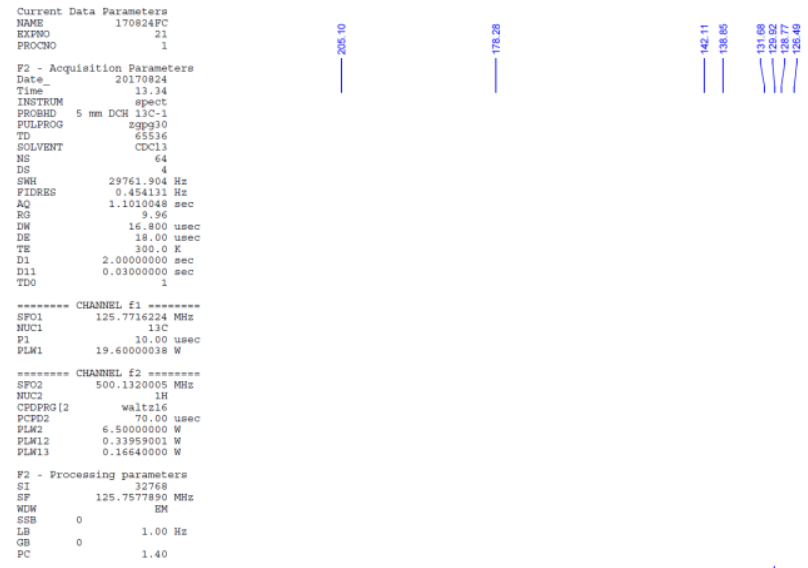

V
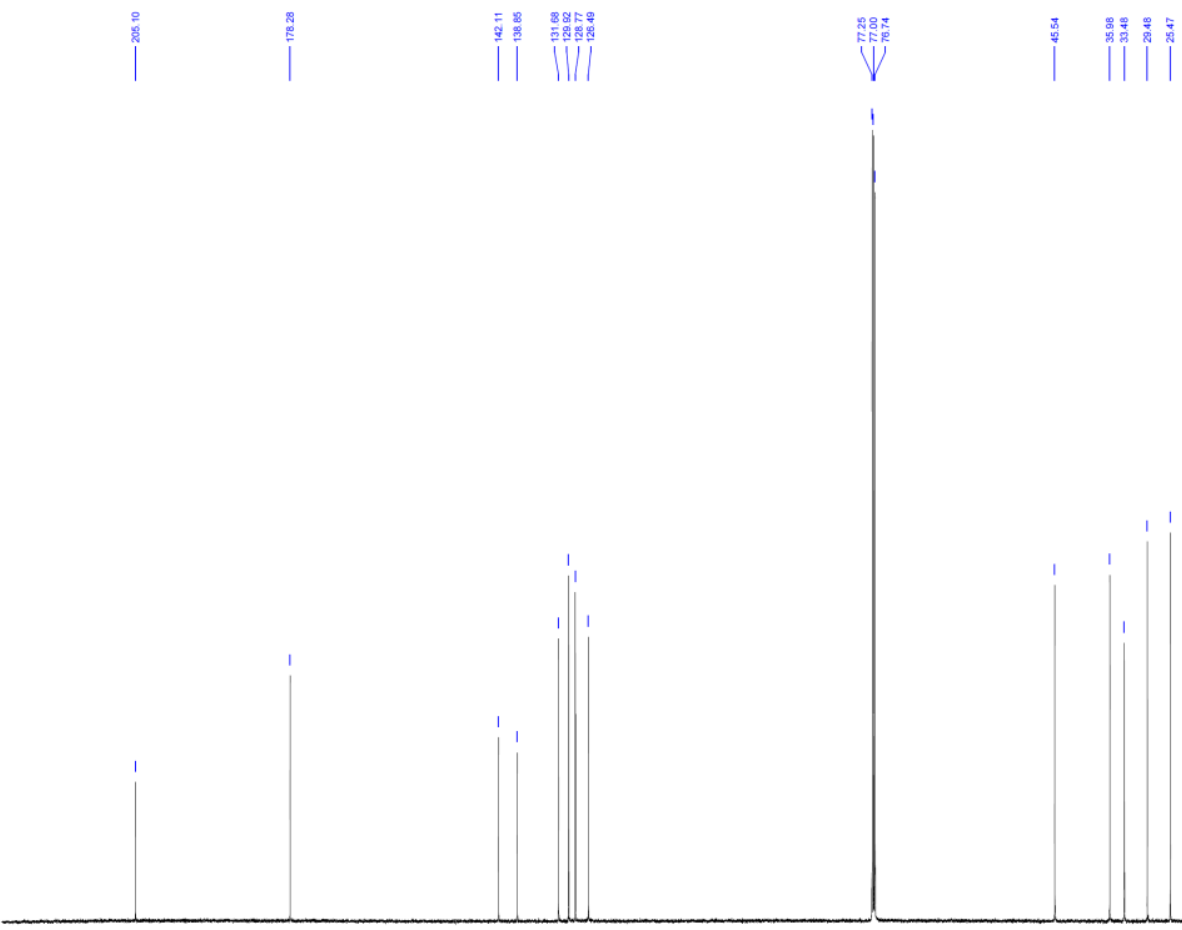

$\begin{array}{llllllllll}220 & 210 & 200 & 190 & 180 & 170 & 160 & 150 & 1\end{array}$ 
3-(1-Oxo-1,2,3,4-tetrahydronaphthalen-2-yl)propanoic acid (11I)

${ }^{1} \mathrm{H}-\mathrm{NMR}\left(400 \mathrm{MHz}, \mathrm{CDCl}_{3}\right)$

$170822-2$

\begin{tabular}{|c|c|c|}
\hline $\begin{array}{ll}\text { Sample Name } & 170822-2 \\
\text { Date collected } & 2017-08-22\end{array}$ & $\begin{array}{l}\text { Pulse sequence PROTON } \\
\text { Solvent cdcl3 }\end{array}$ & $\begin{array}{l}\text { Temperature } 25 \\
\text { Spectrometer } \\
\text { Agilent-NMR-vnmrs } 400\end{array}$ \\
\hline
\end{tabular}

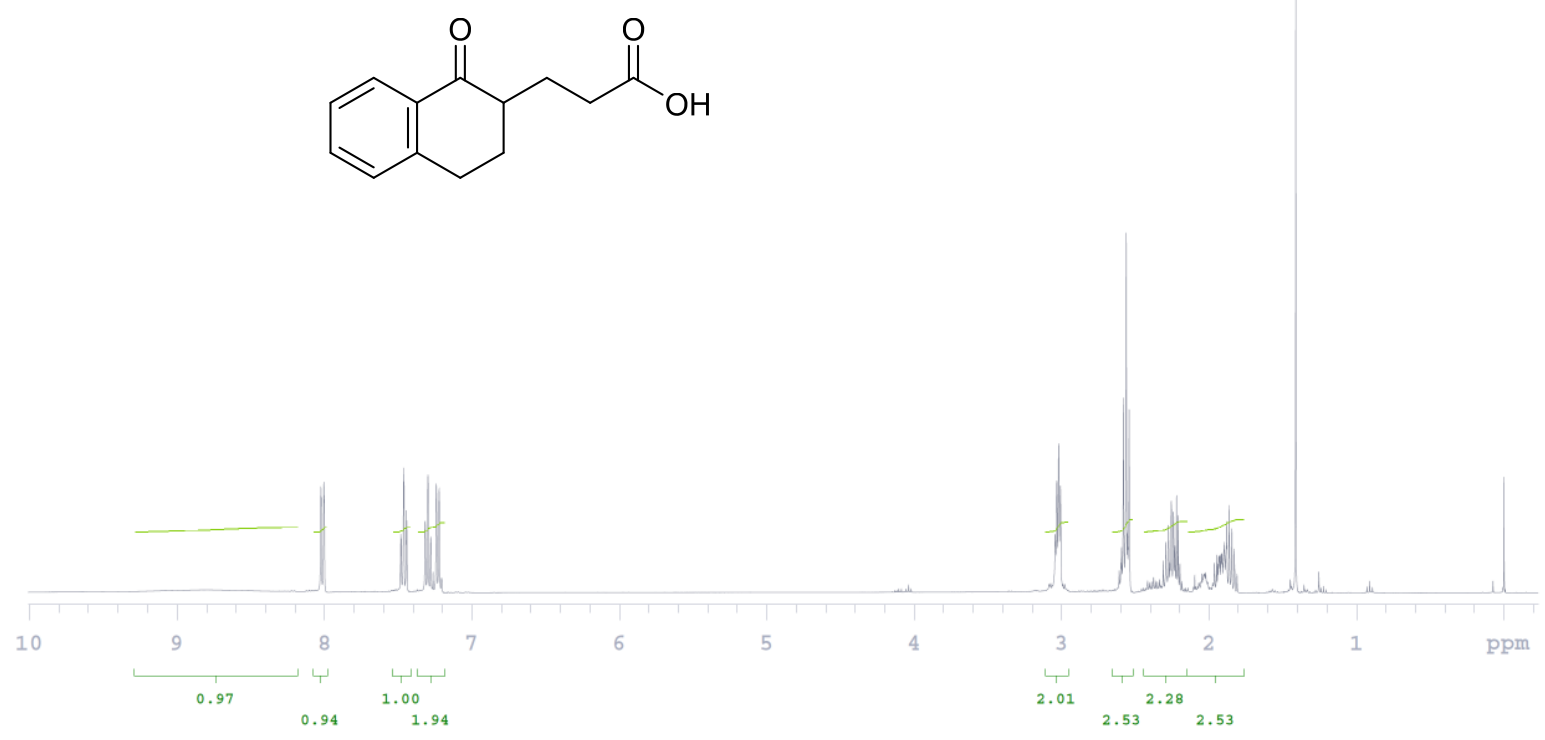

${ }^{13} \mathrm{C} \mathrm{NMR}\left(125 \mathrm{MHz}, \mathrm{CDCl}_{3}\right)$
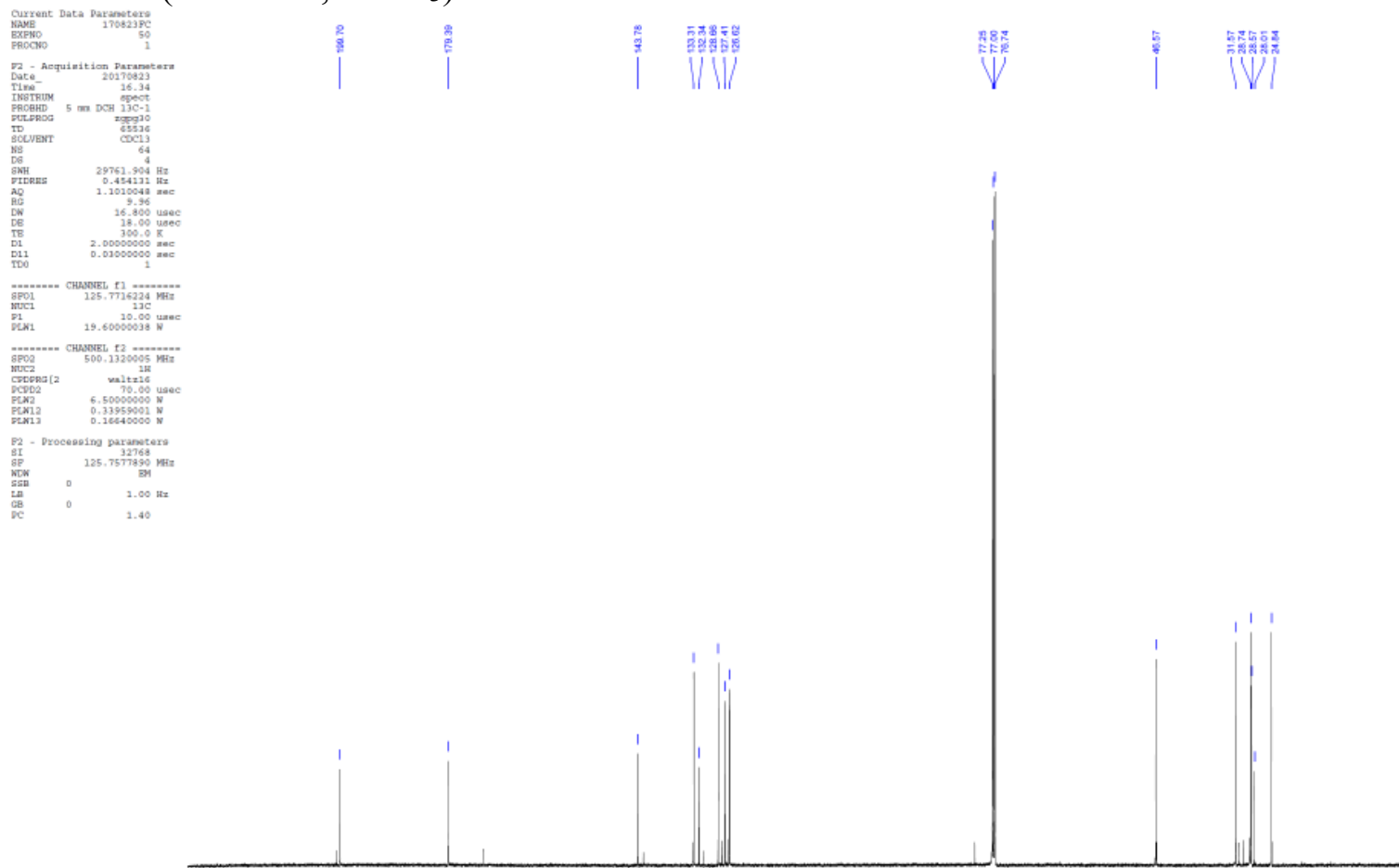

$\begin{array}{llllllll}220 & 210 & 200 & 190 & 180 & 170 & 160 & 150\end{array}$

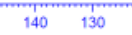


3-(7-Methoxy-1-oxo-1,2,3,4-tetrahydronaphthalen-2-yl)propanoic acid (11 m)

${ }^{1} \mathrm{H}-\mathrm{NMR}\left(400 \mathrm{MHz}, \mathrm{CDCl}_{3}\right)$

170904-5

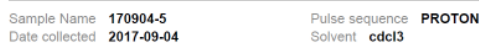

$\begin{array}{ll}\text { Temperature } 25 & \text { Study owner vnmr } \\ \text { Spectrometer Agilent-NMR-vnmrs400 } & \text { Operator vnmri }\end{array}$<smiles>COc1ccc2c(c1)C(=O)C(CCCC(=O)O)CC2</smiles>
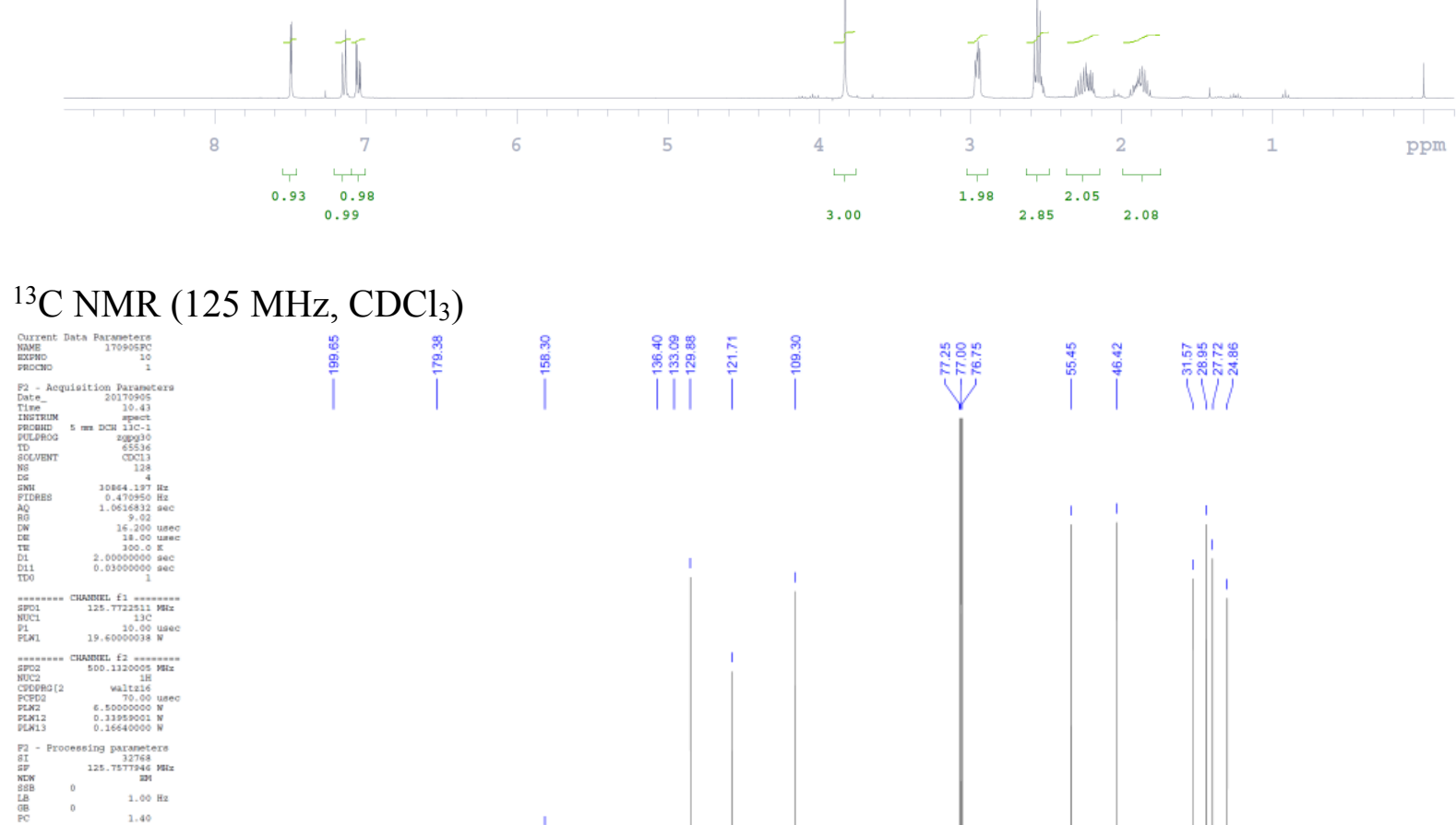
3-(7-Chloro-1-oxo-1,2,3,4-tetrahydronaphthalen-2-yl)propanoic acid (11n)

${ }^{1} \mathrm{H}-\mathrm{NMR}$ (400 MHz, DMSO-d6)

\begin{tabular}{|c|c|c|c|}
\hline $\begin{array}{ll}\text { Sample Name } & 170914-2 \\
\text { Date collected } & 2017-09-14\end{array}$ & $\begin{array}{l}\text { Pulse sequence PROTON } \\
\text { Solvent dmso }\end{array}$ & $\begin{array}{l}\text { Temperature } 25 \\
\text { Spectrometer Agilent-NMR-vnmrs400 }\end{array}$ & $\begin{array}{l}\text { Study owner vnmr1 } \\
\text { Operator vnmr1 }\end{array}$ \\
\hline
\end{tabular}

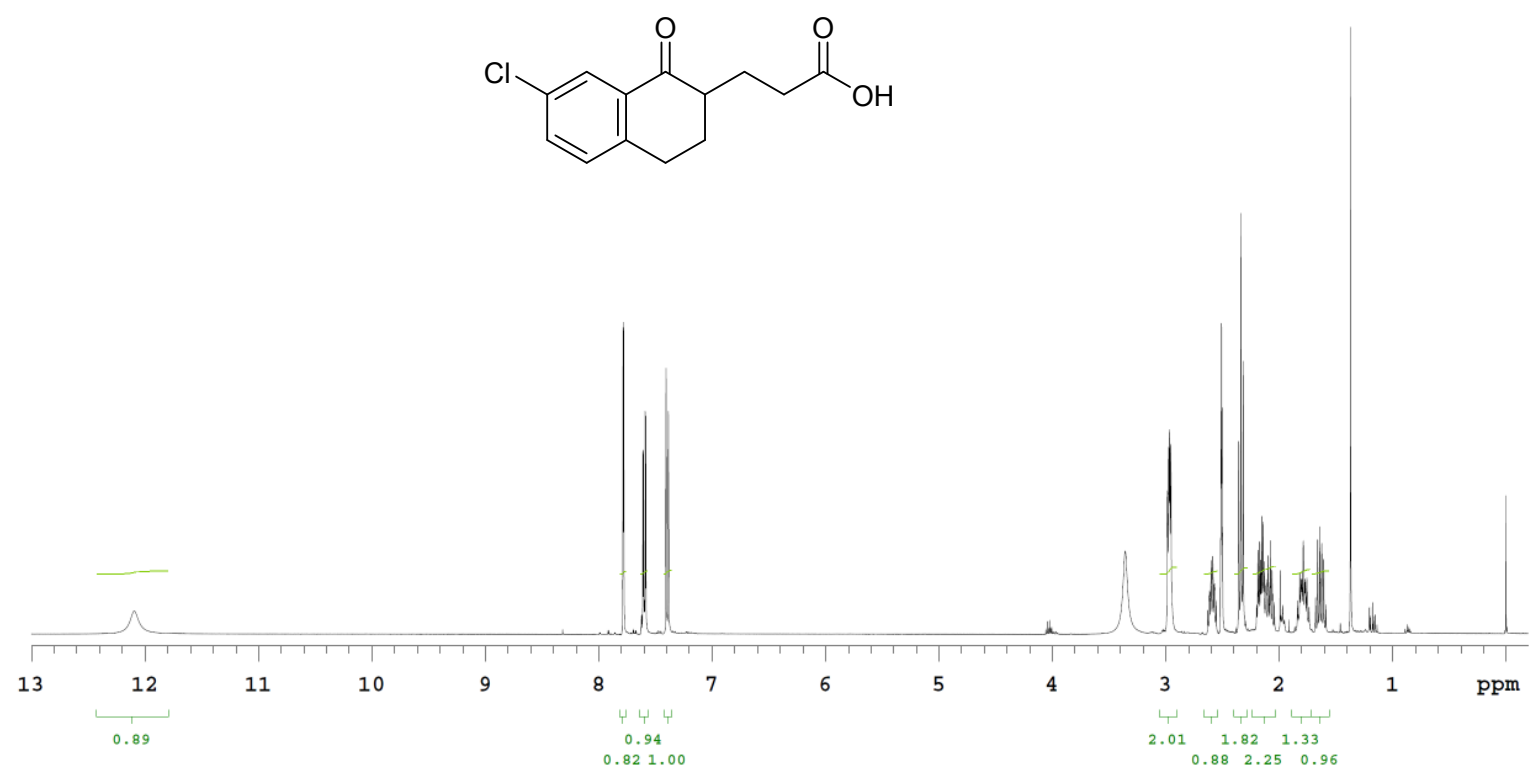

${ }^{13} \mathrm{C}$ NMR (125 MHz, DMSO- $\left.d_{6}\right)$
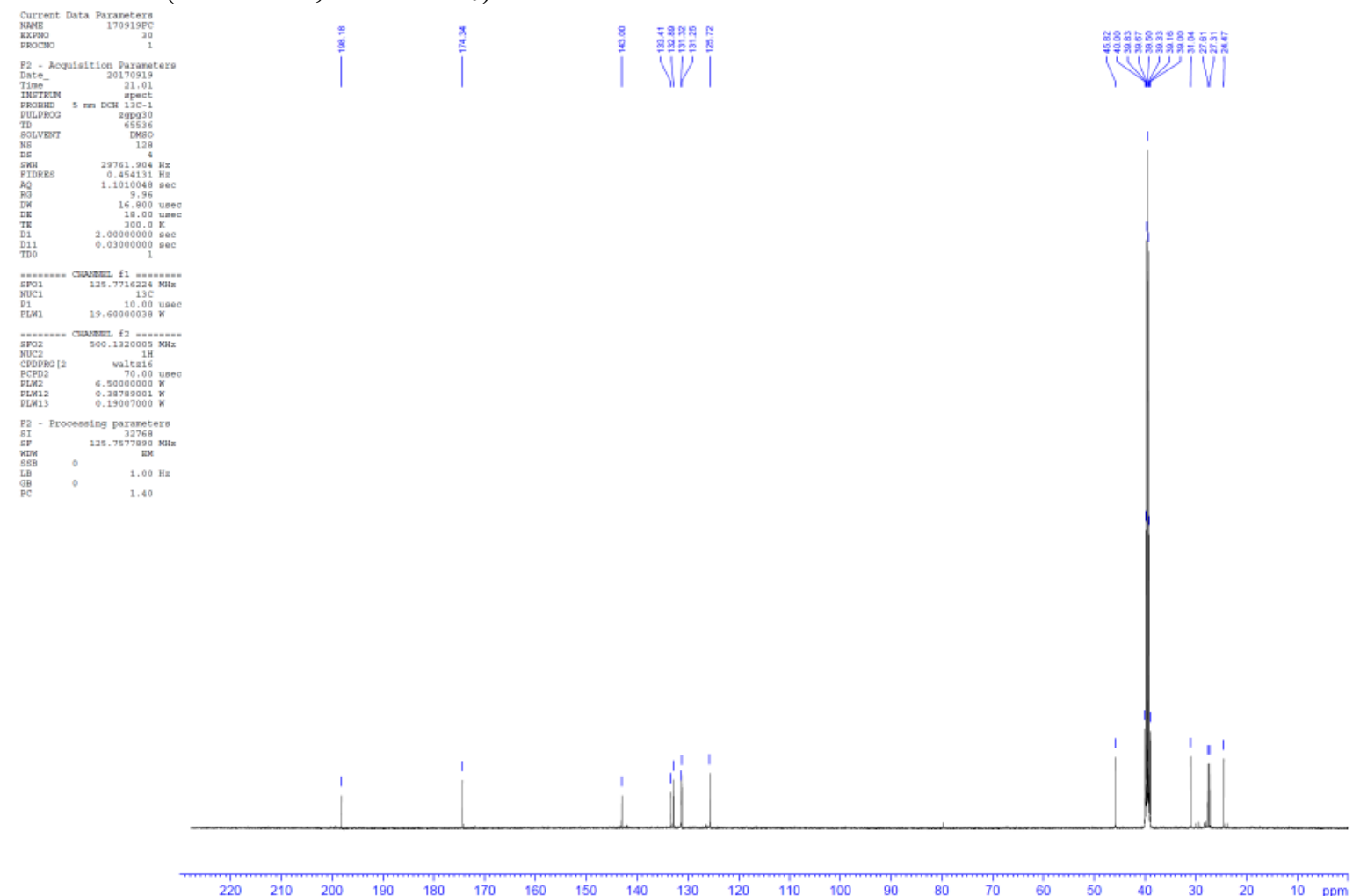
2-(2-Oxo-1,2,3,4-tetrahydronaphthalen-1-yl)acetic acid (110)

${ }^{1} \mathrm{H}-\mathrm{NMR}\left(400 \mathrm{MHz}, \mathrm{DMSO}-d_{6}\right)$

$171006-2$

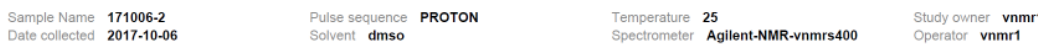<smiles>O=C(O)CC1C(=O)CCc2ccccc21</smiles>

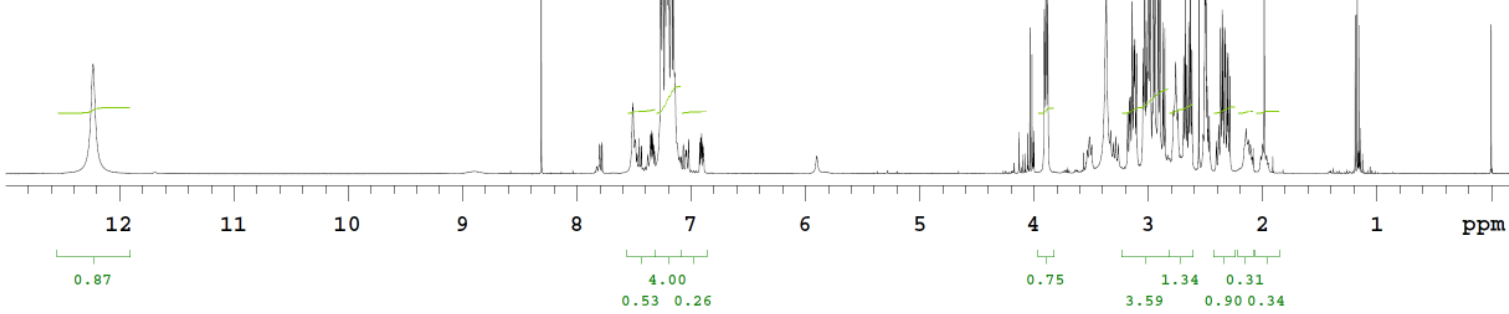

${ }^{13} \mathrm{C}$ NMR (125 MHz, DMSO- $\left.d_{6}\right)$
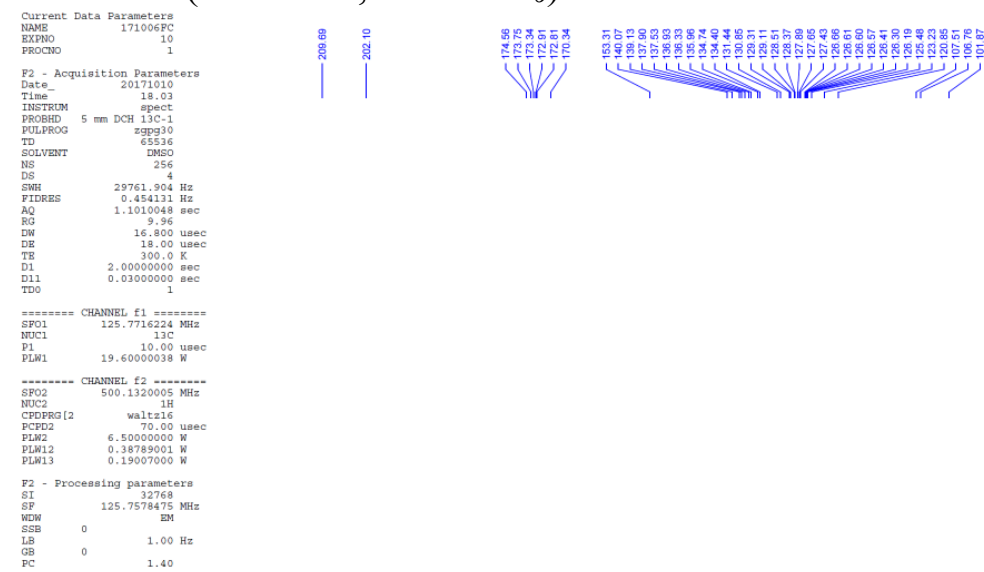

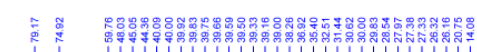
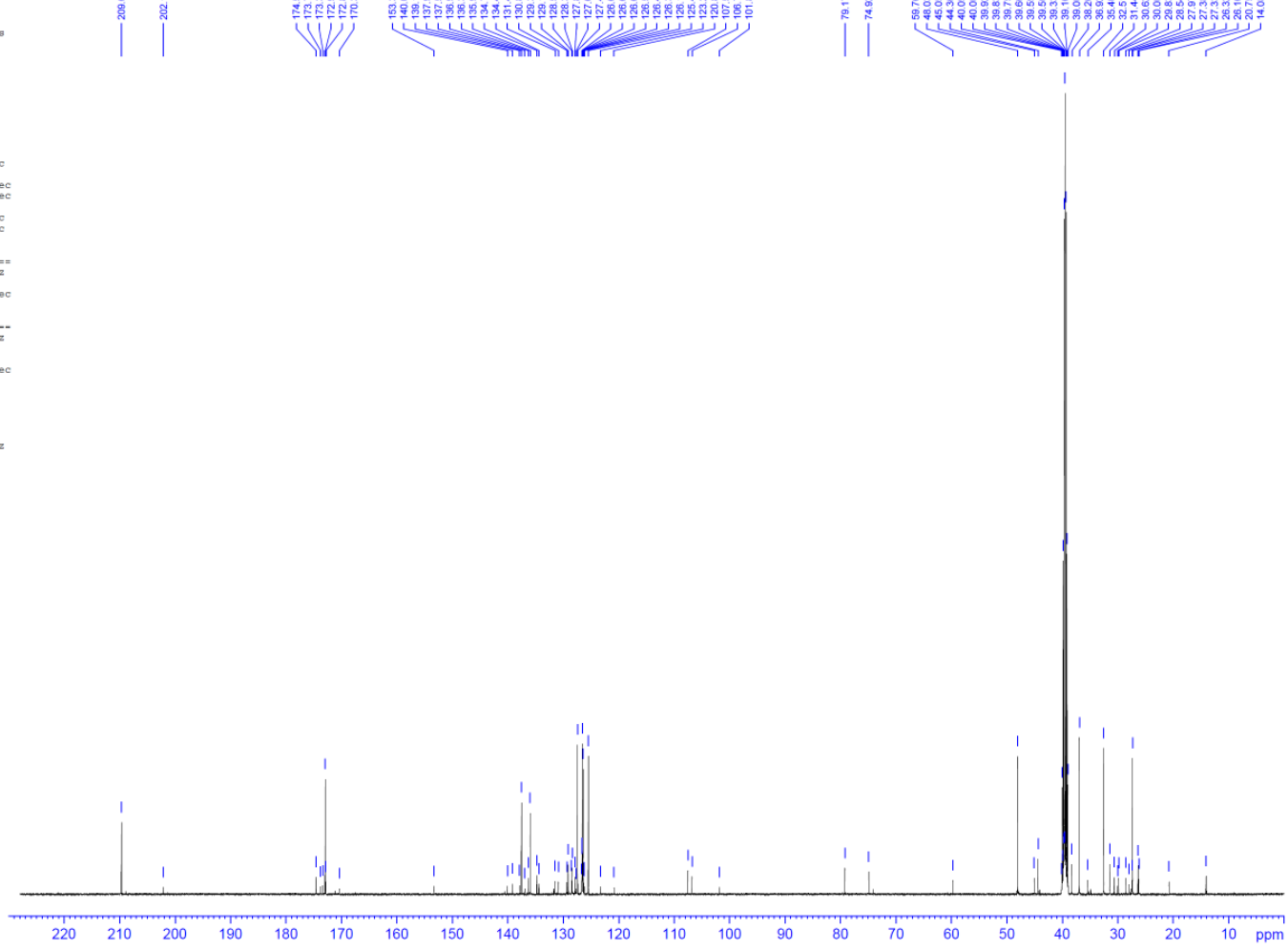
2-(5-Methoxy-2-oxo-1,2,3,4-tetrahydronaphthalen-1-yl)acetic acid (11p)

${ }^{1} \mathrm{H}-\mathrm{NMR}\left(400 \mathrm{MHz}, \mathrm{DMSO}-d_{6}\right)$

170904-3

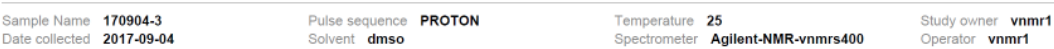<smiles>COc1cccc2c1CCC(=O)C2CC(=O)O</smiles>
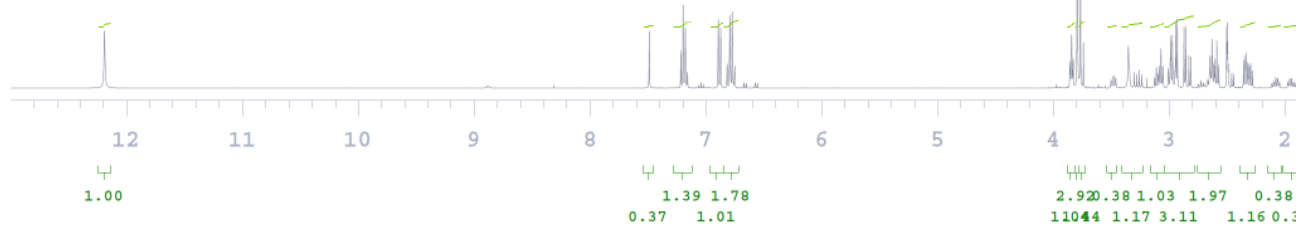

${ }^{13} \mathrm{C}$ NMR $\left(125 \mathrm{MHz}, \mathrm{DMSO}-d_{6}\right)$
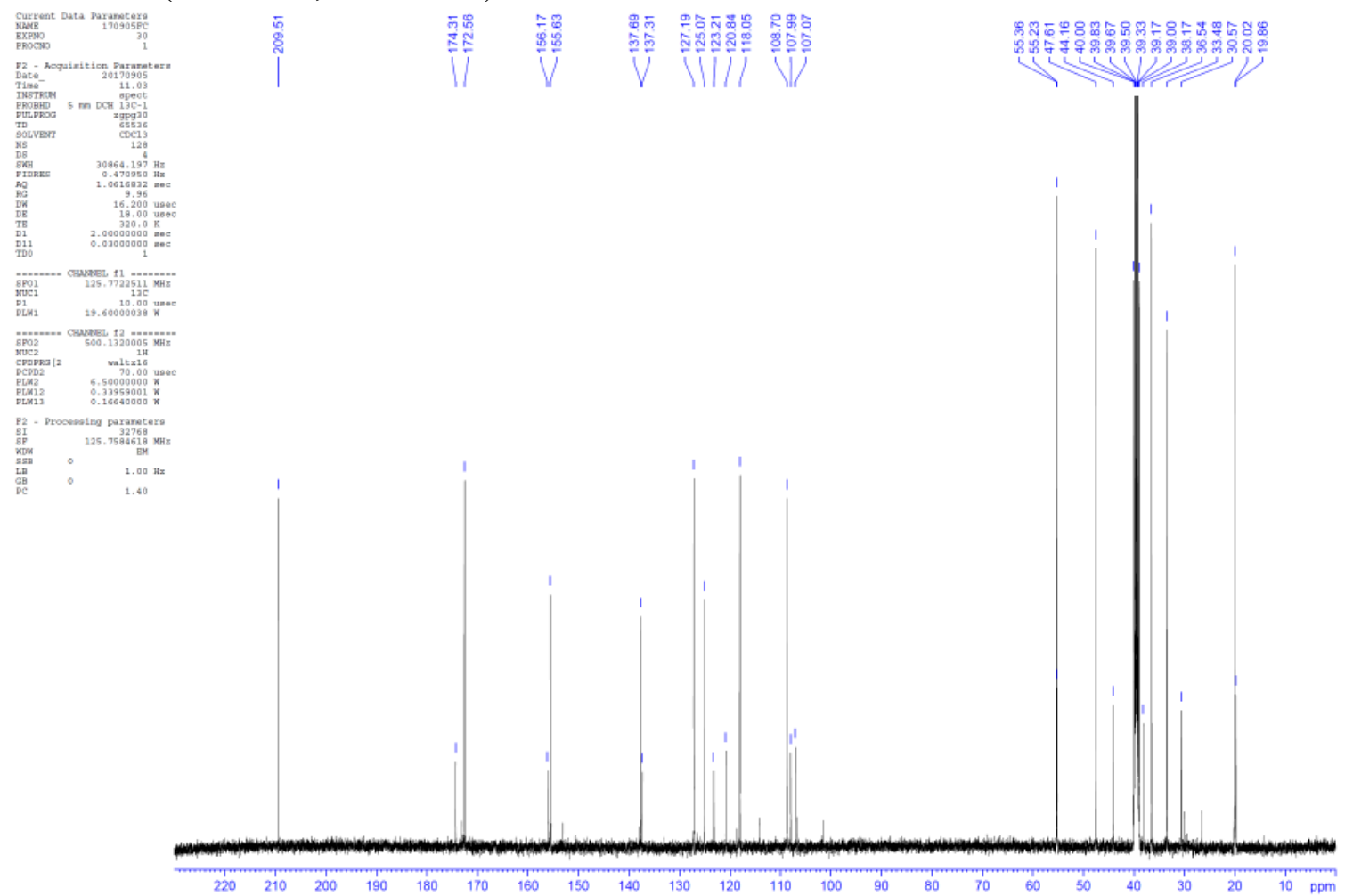
2-(6-Bromo-2-oxo-1,2,3,4-tetrahydronaphthalen-1-yl)acetic acid (11q)

${ }^{1} \mathrm{H}-\mathrm{NMR}\left(400 \mathrm{MHz}, \mathrm{DMSO}-d_{6}\right)$

$171115-3$

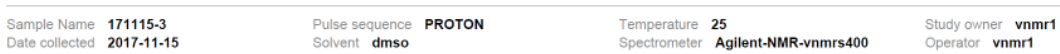
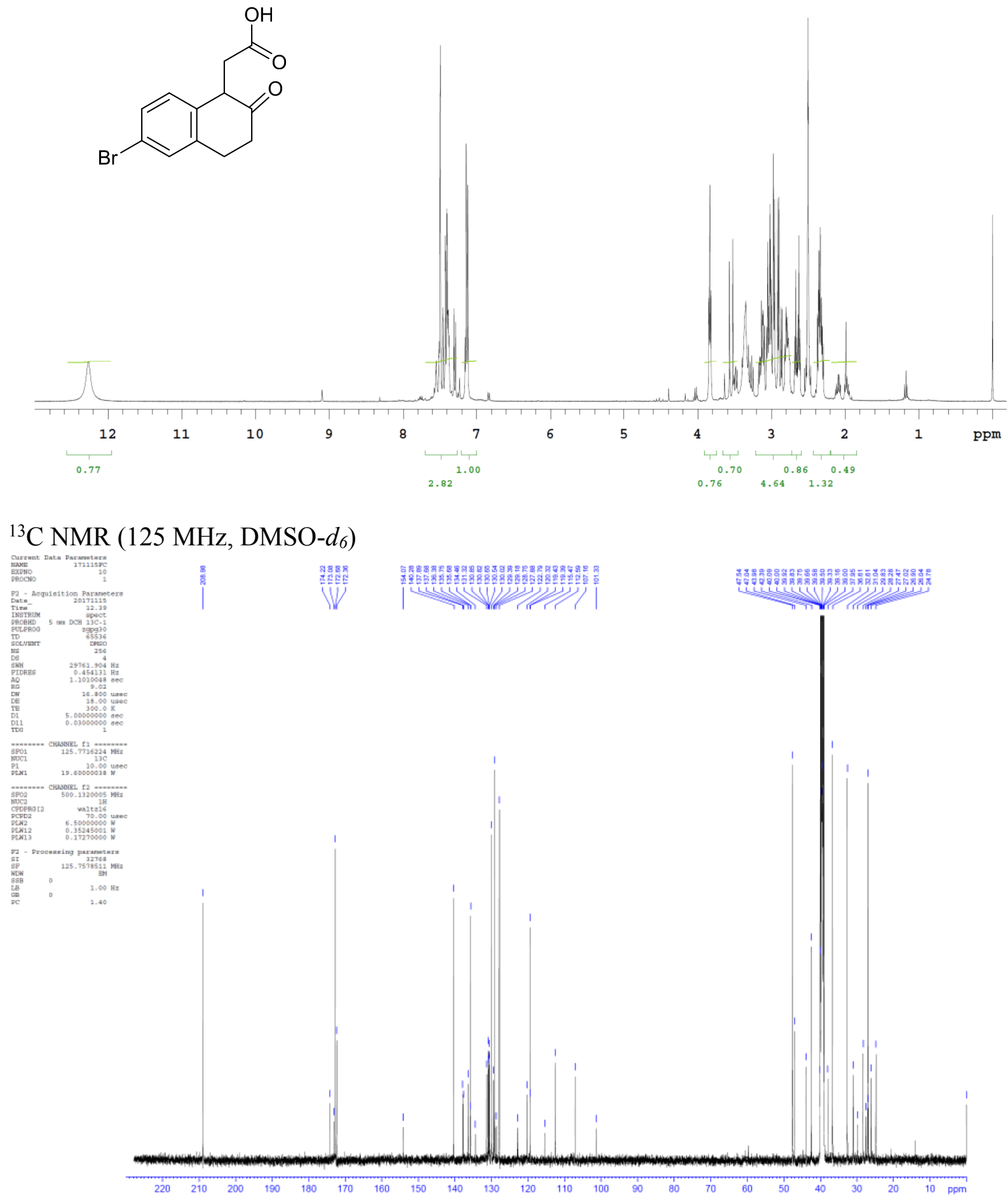
2-(6-Chloro-2-oxo-1,2,3,4-tetrahydronaphthalen-1-yl)acetic acid (11r)

${ }^{1} \mathrm{H}-\mathrm{NMR}\left(400 \mathrm{MHz}, \mathrm{DMSO}-d_{6}\right)$

Sample Name $170912-3$

Pulse sequence PROTON
Solvent dmso

Temperature 25

Agilent-NMR-vnmrs400

Study owner vnmr1
Operator vnmr1

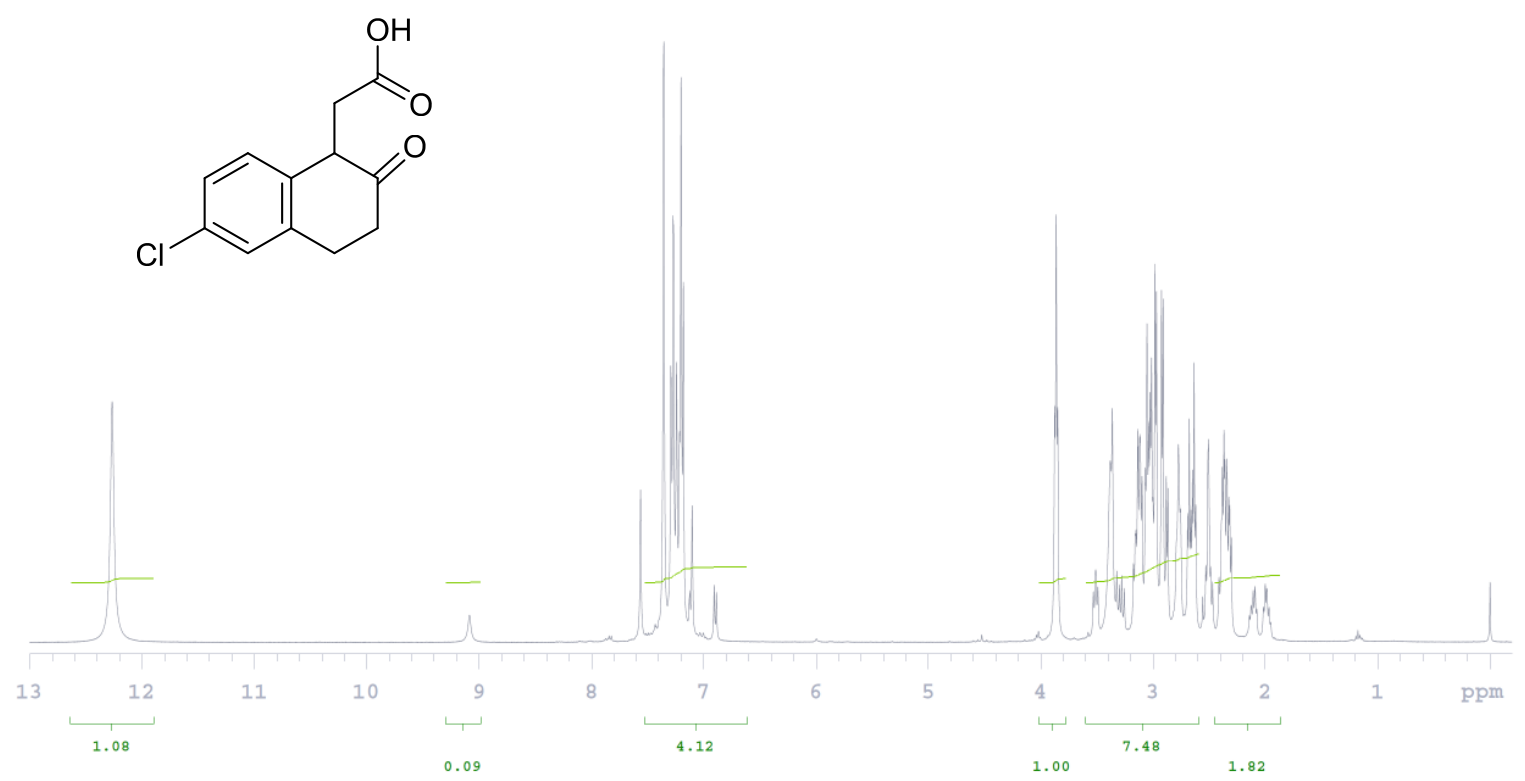

${ }^{13} \mathrm{C}$ NMR (125 MHz, DMSO- $\left.d_{6}\right)$
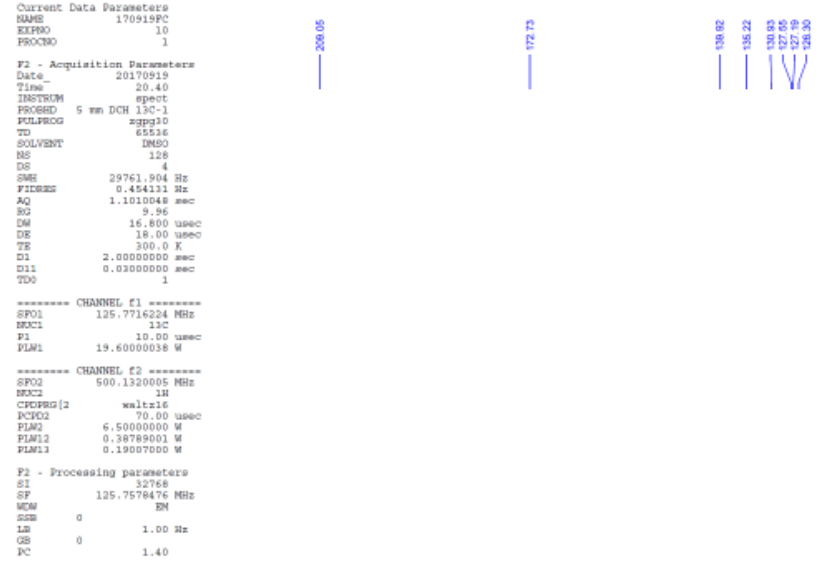

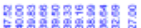
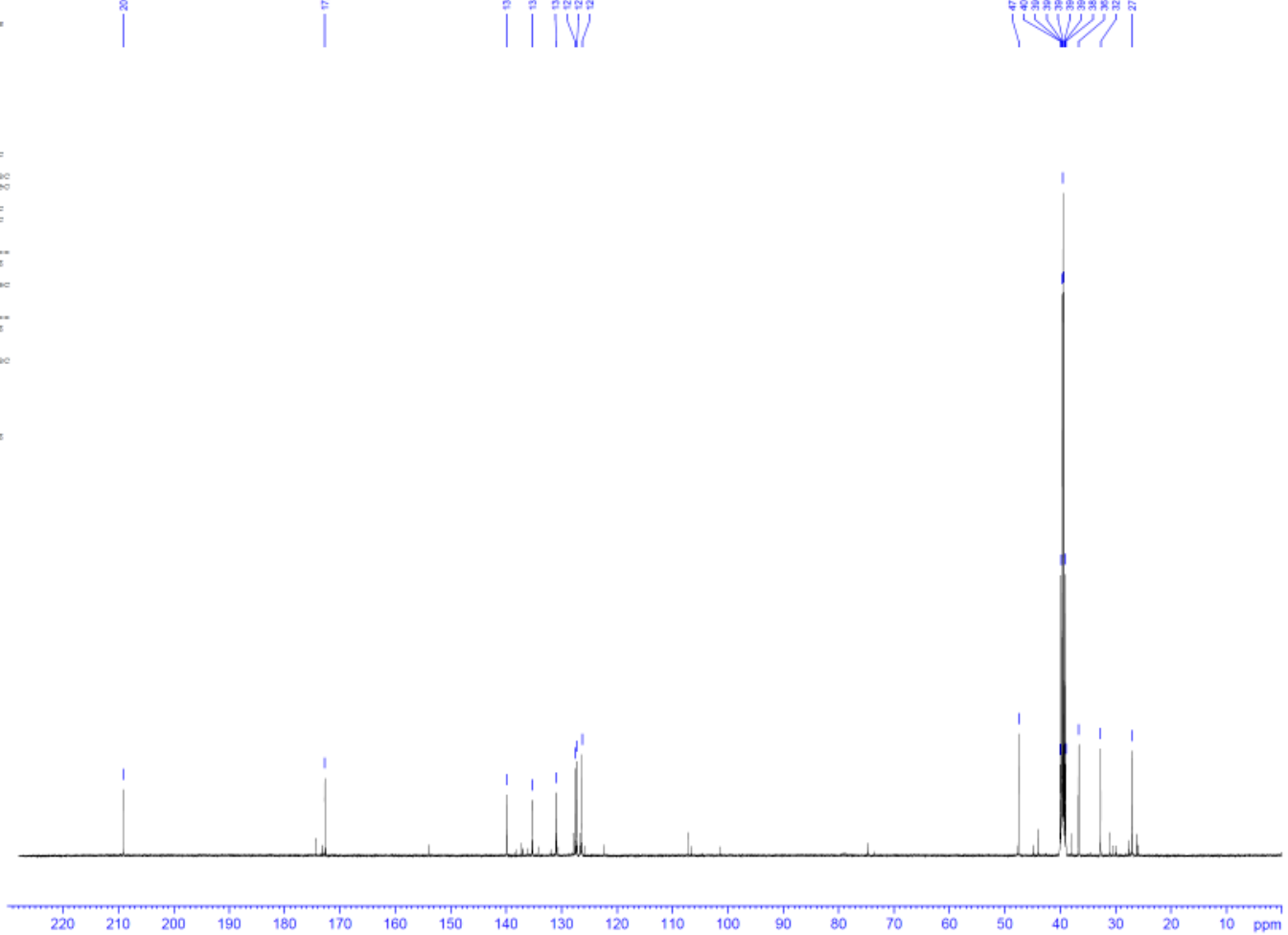
2-(2-Oxo-1,2,3,4-tetrahydronaphthalen-1-yl)propanoic acid (14a)

${ }^{1} \mathrm{H}-\mathrm{NMR}\left(500 \mathrm{MHz}, \mathrm{DMSO}-d_{6}\right)$
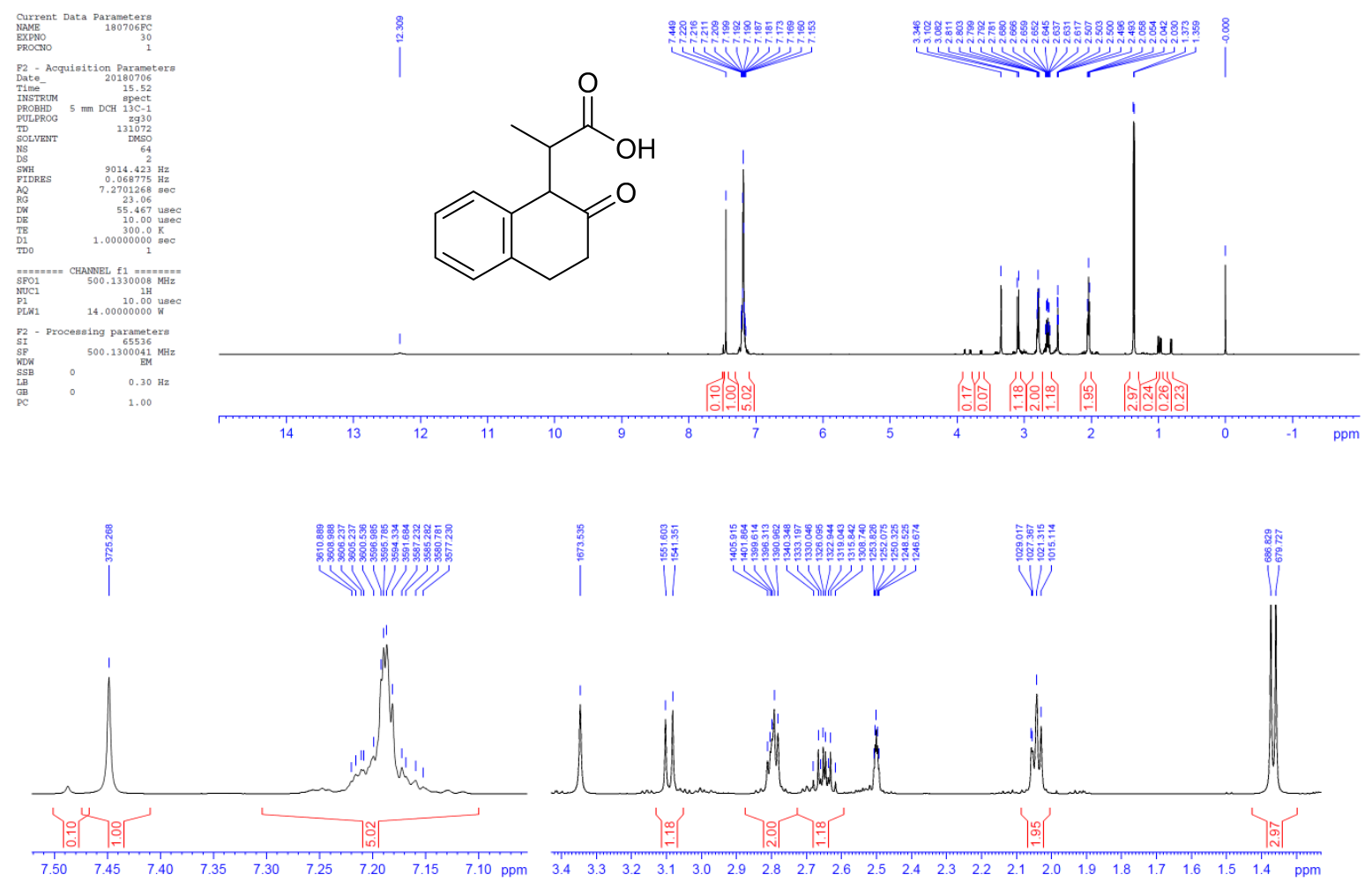

${ }^{13} \mathrm{C}$ NMR (125 MHz, DMSO- $\left.d_{6}\right)$
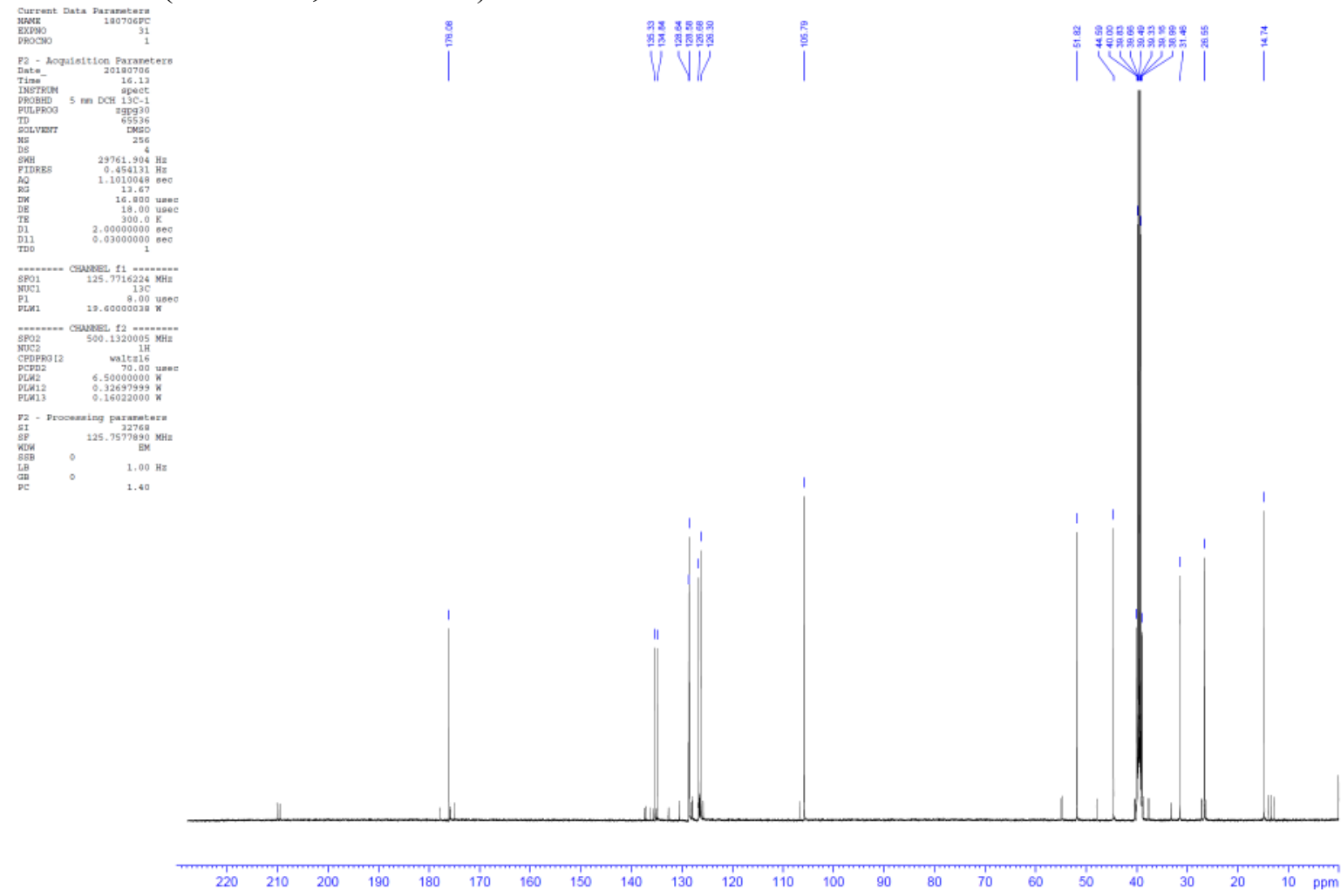
2-(2-Oxo-1,2,3,4-tetrahydronaphthalen-1-yl)butanoic acid (14b)

${ }^{1} \mathrm{H}-\mathrm{NMR}\left(500 \mathrm{MHz}, \mathrm{DMSO}-d_{6}\right)$
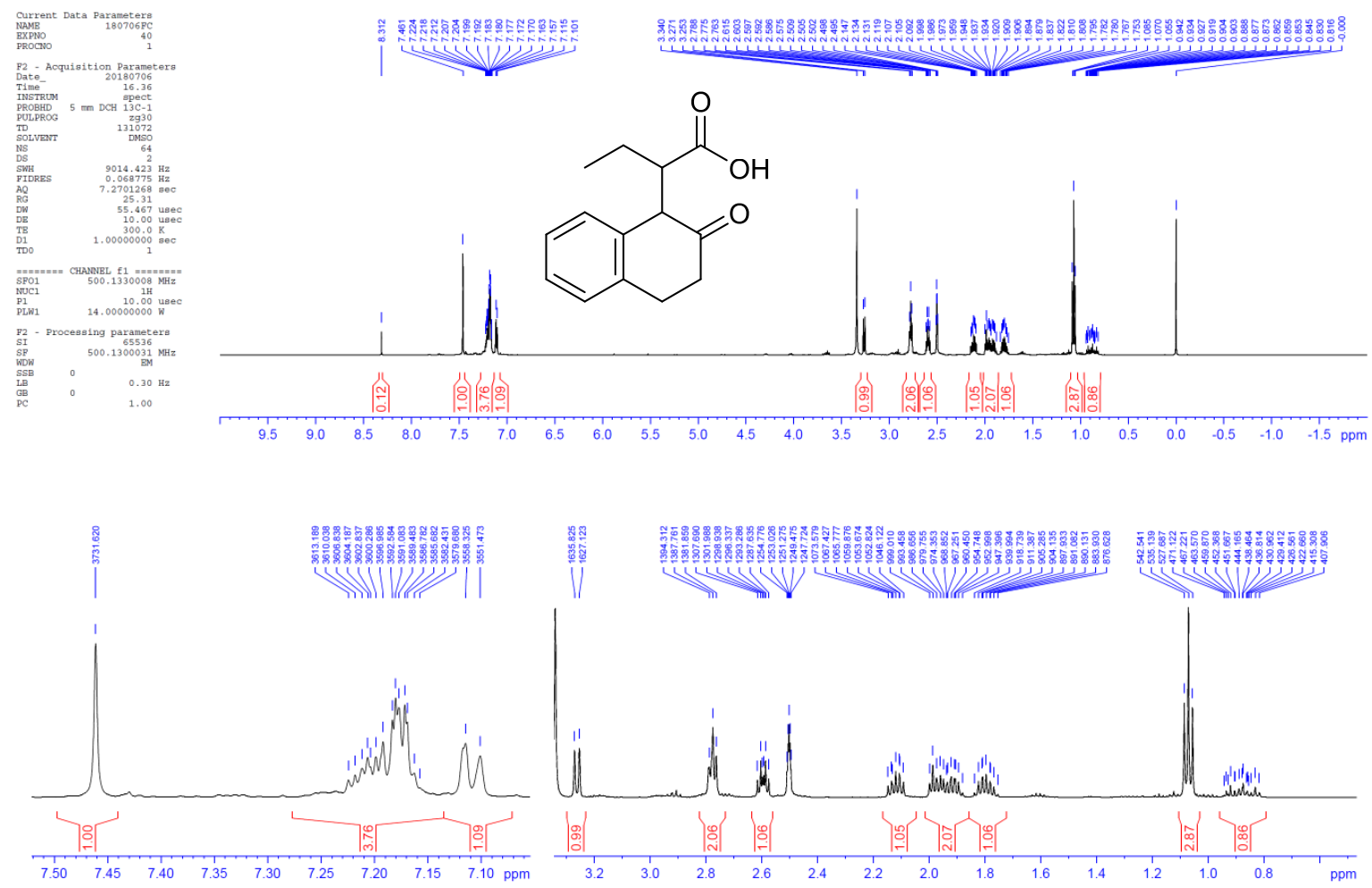

${ }^{13} \mathrm{C}$ NMR (125 MHz, DMSO- $\left.d_{6}\right)$
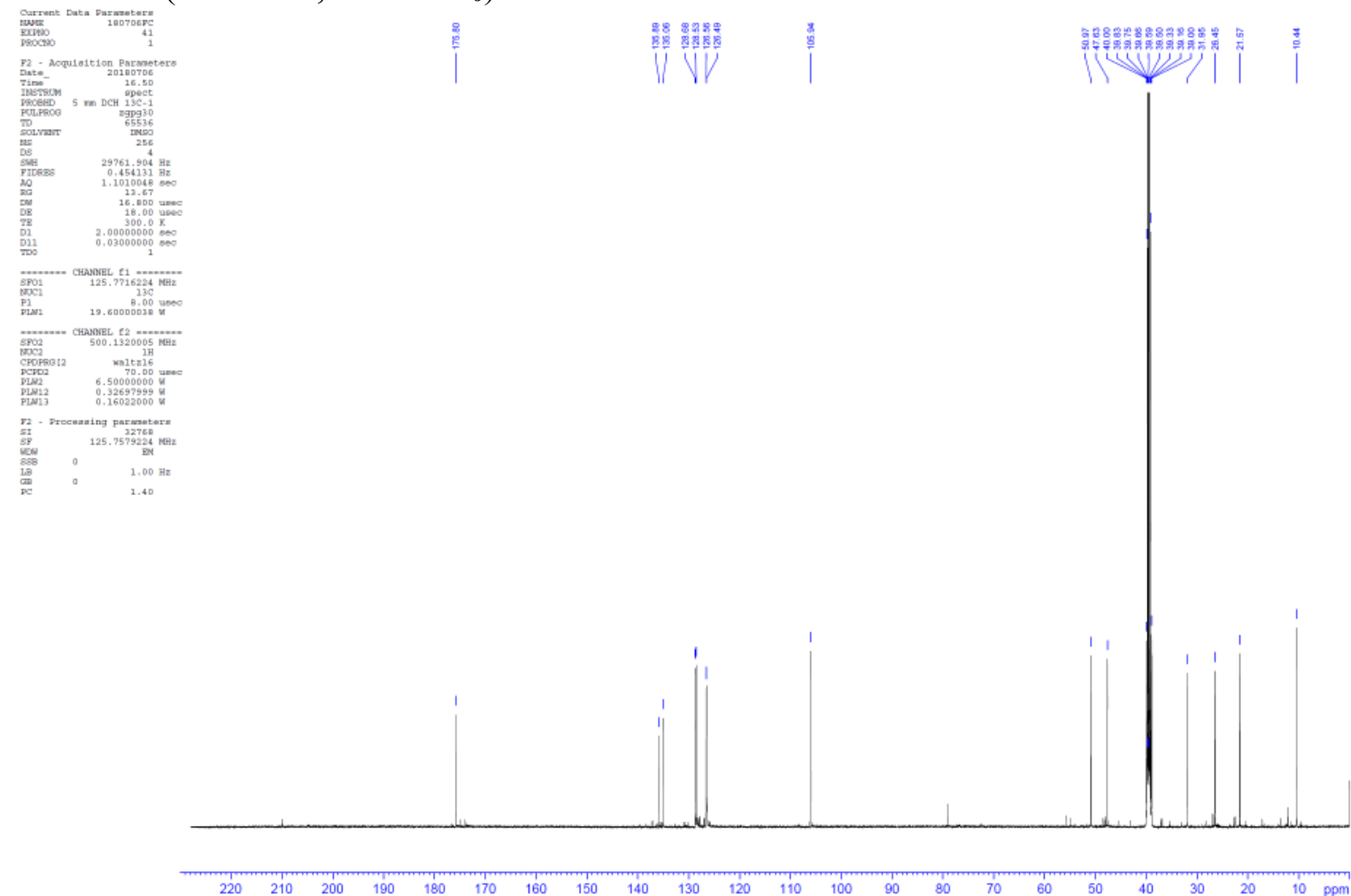
2-(2-Oxo-1,2,3,4-tetrahydronaphthalen-1-yl)-3-phenylpropanoic acid (14c)

${ }^{1} \mathrm{H}-\mathrm{NMR}\left(500 \mathrm{MHz}, \mathrm{DMSO}-d_{6}\right)$
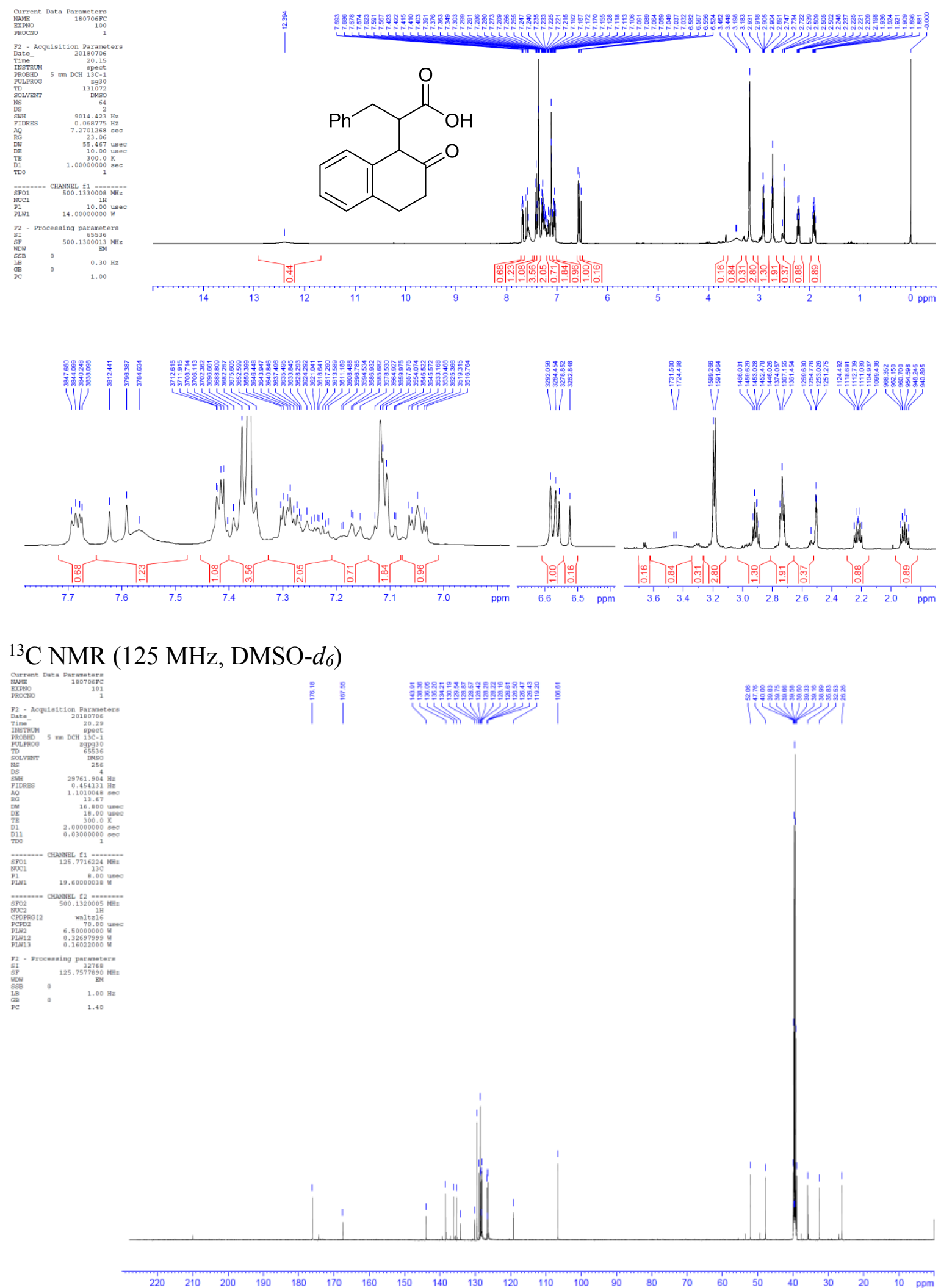
2-(2-Oxo-1,2,3,4-tetrahydronaphthalen-1-yl)-2-phenylacetic acid (14d)

${ }^{1} \mathrm{H}-\mathrm{NMR}\left(500 \mathrm{MHz}, \mathrm{DMSO}-d_{6}\right)$
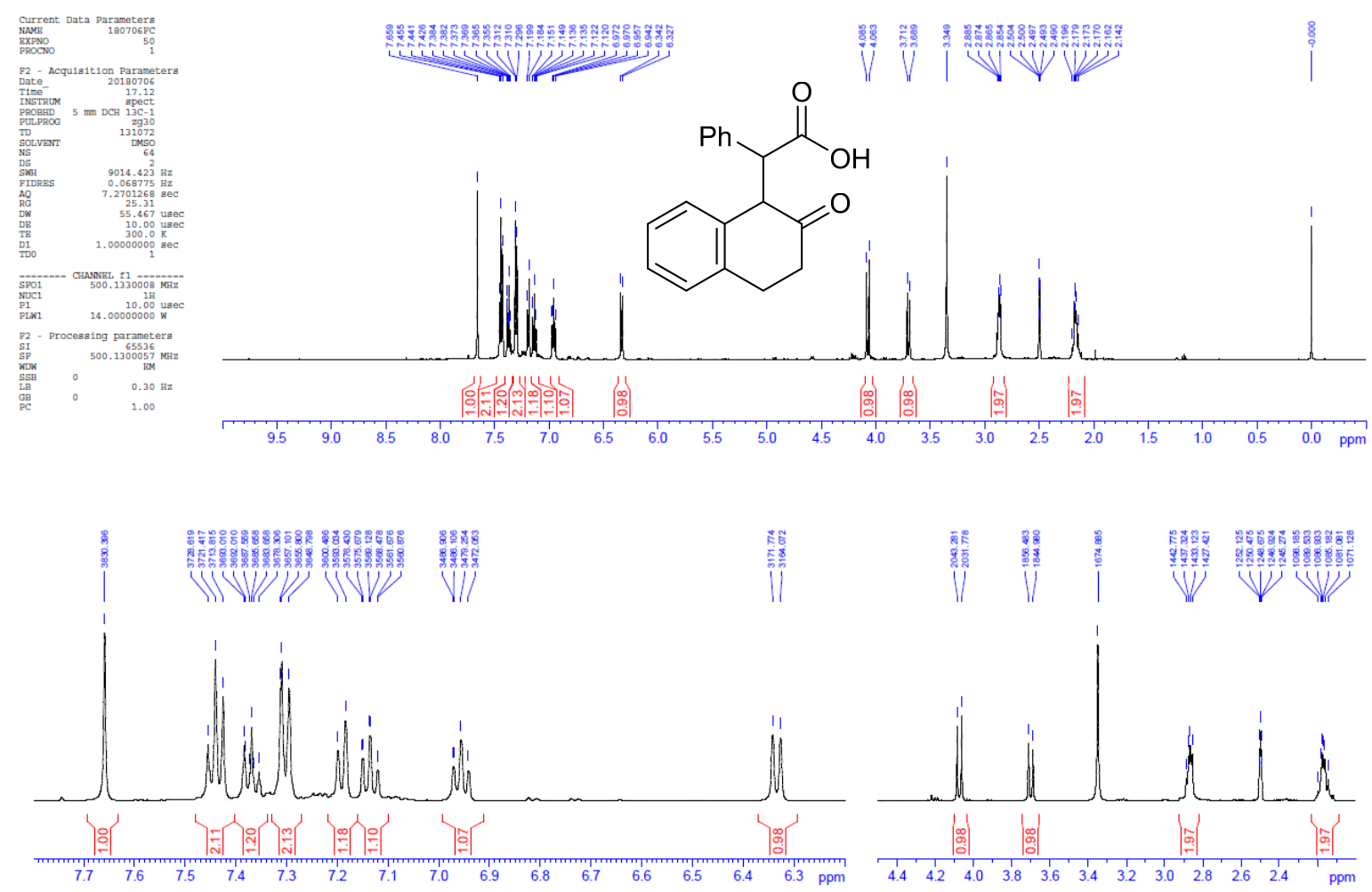

${ }^{13} \mathrm{C}$ NMR (125 MHz, DMSO- $\left.d_{6}\right)$
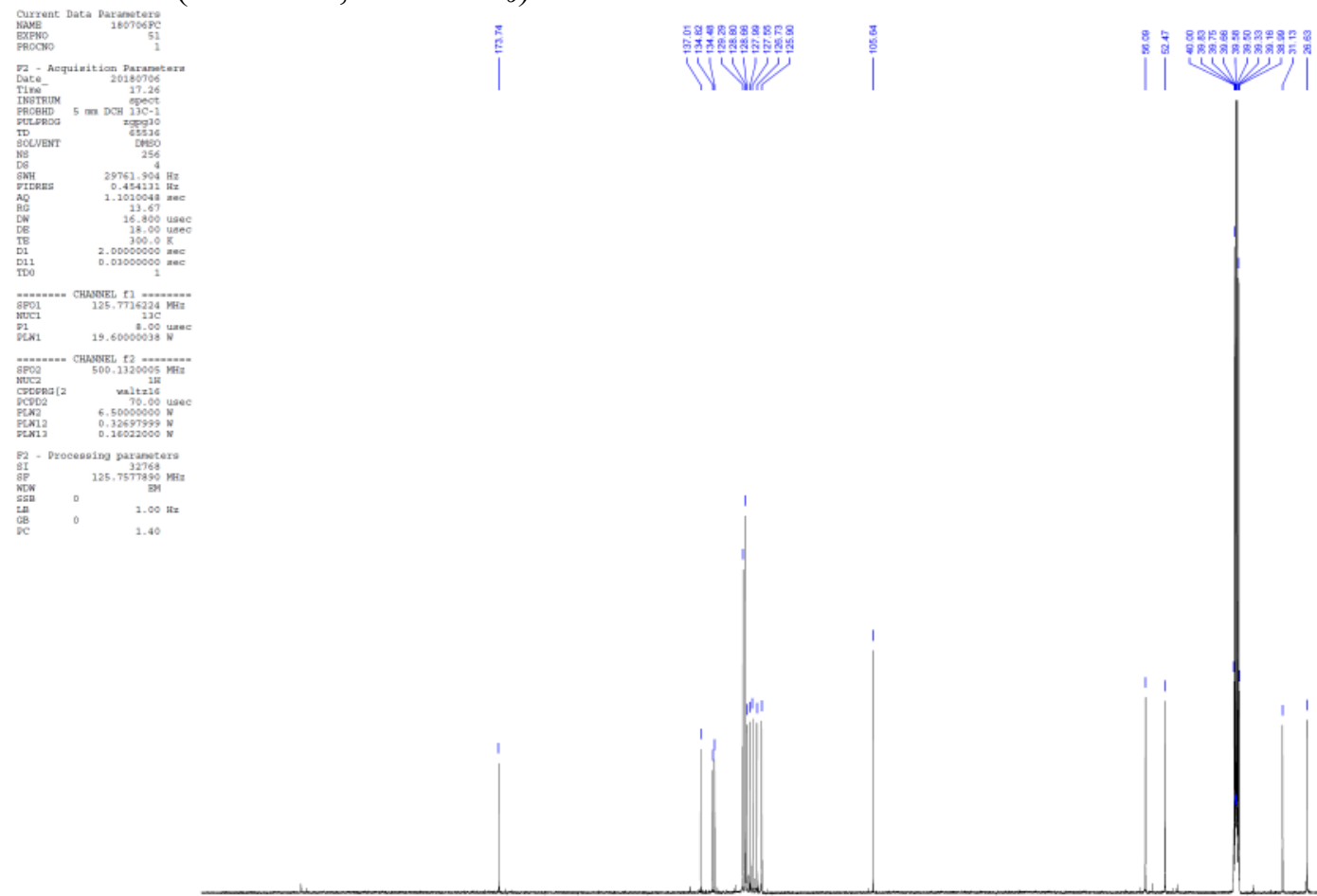

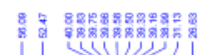
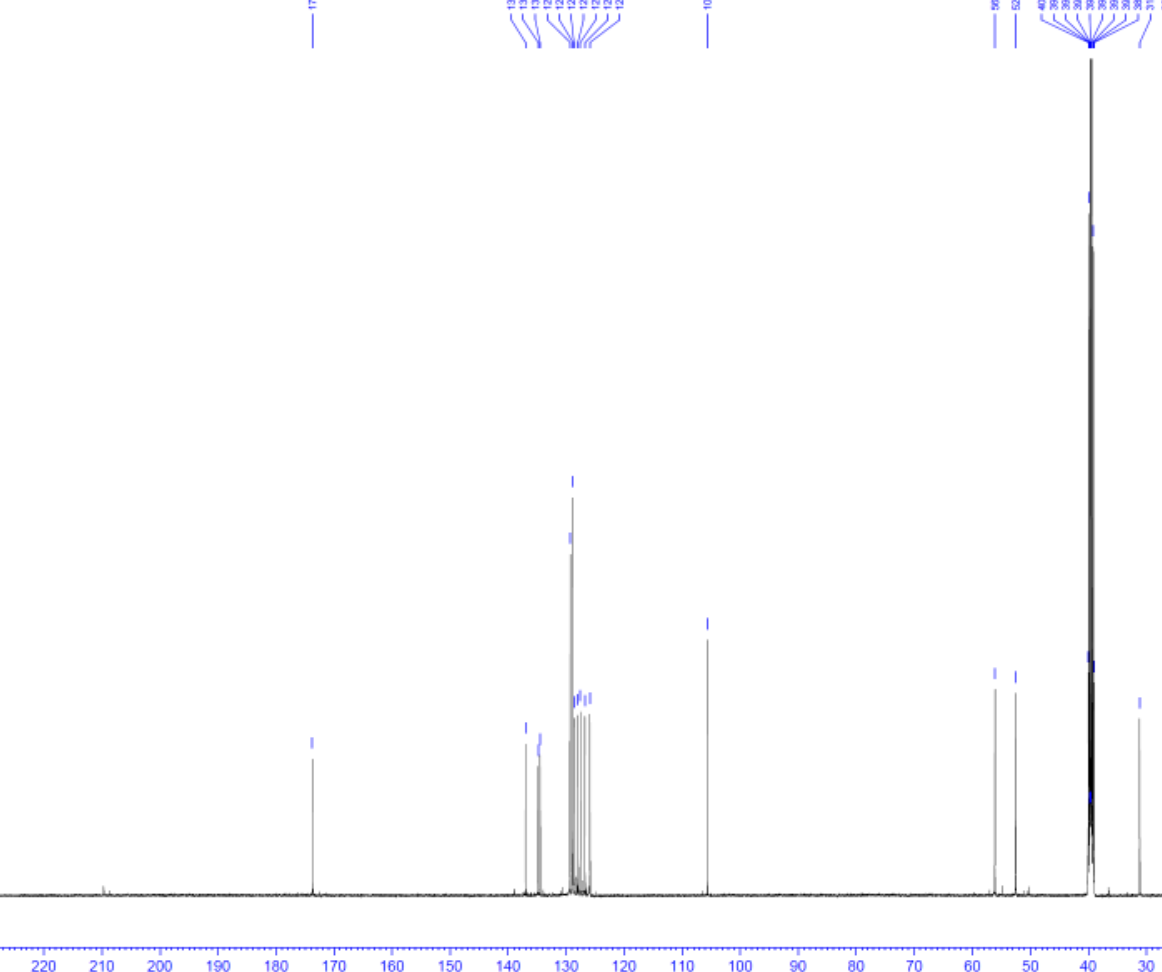

$140 \quad 130$

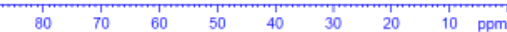


2-(6-Bromo-2-oxo-1,2,3,4-tetrahydronaphthalen-1-yl)-2-phenylacetic acid (14e)

${ }^{1} \mathrm{H}-\mathrm{NMR}\left(500 \mathrm{MHz}, \mathrm{DMSO}-d_{6}\right)$
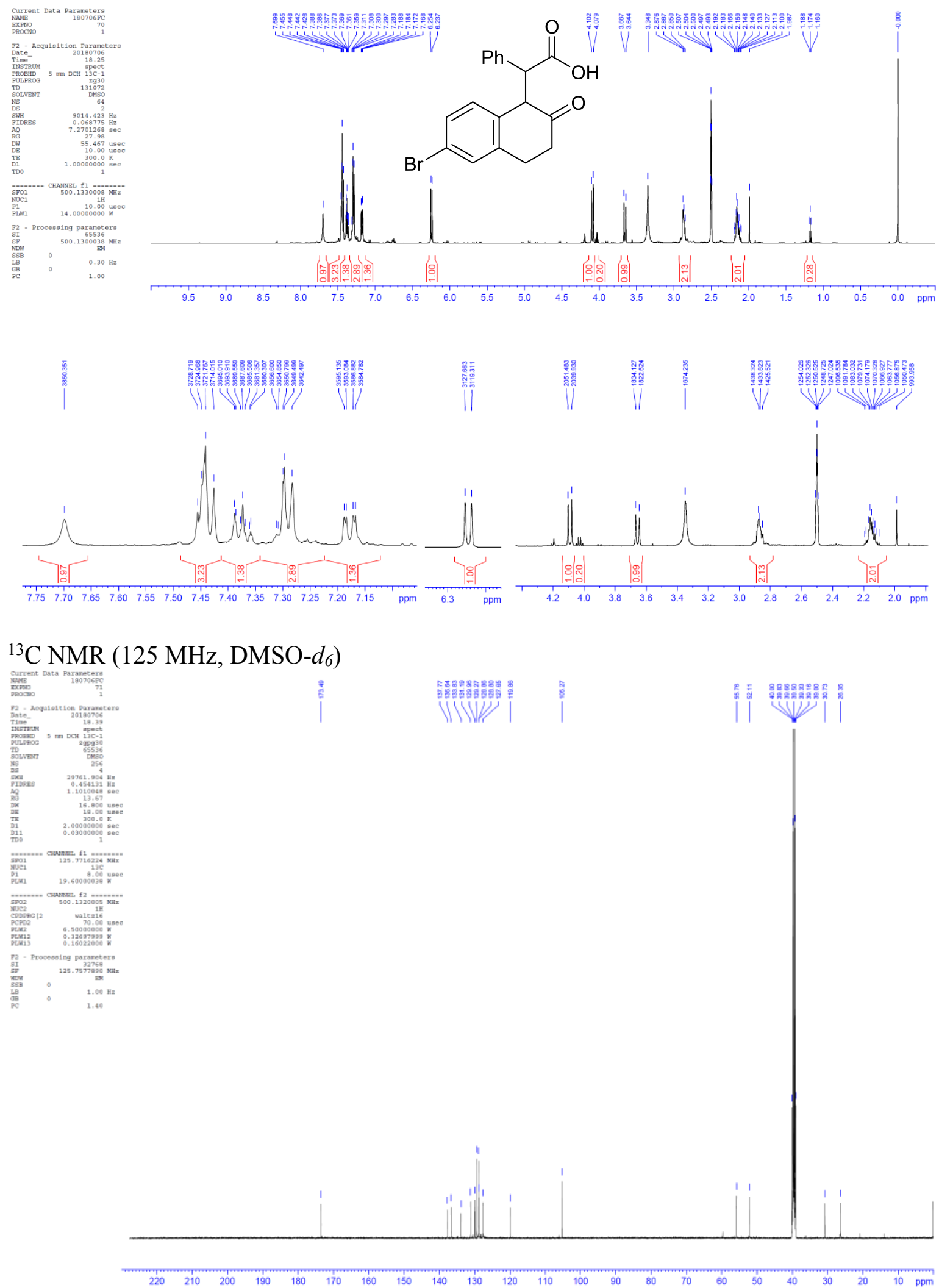
2-(6-Bromo-2-oxo-1,2,3,4-tetrahydronaphthalen-1-yl)-2-phenylacetic acid (14f)

${ }^{1} \mathrm{H}-\mathrm{NMR}\left(500 \mathrm{MHz}, \mathrm{DMSO}-d_{6}\right)$
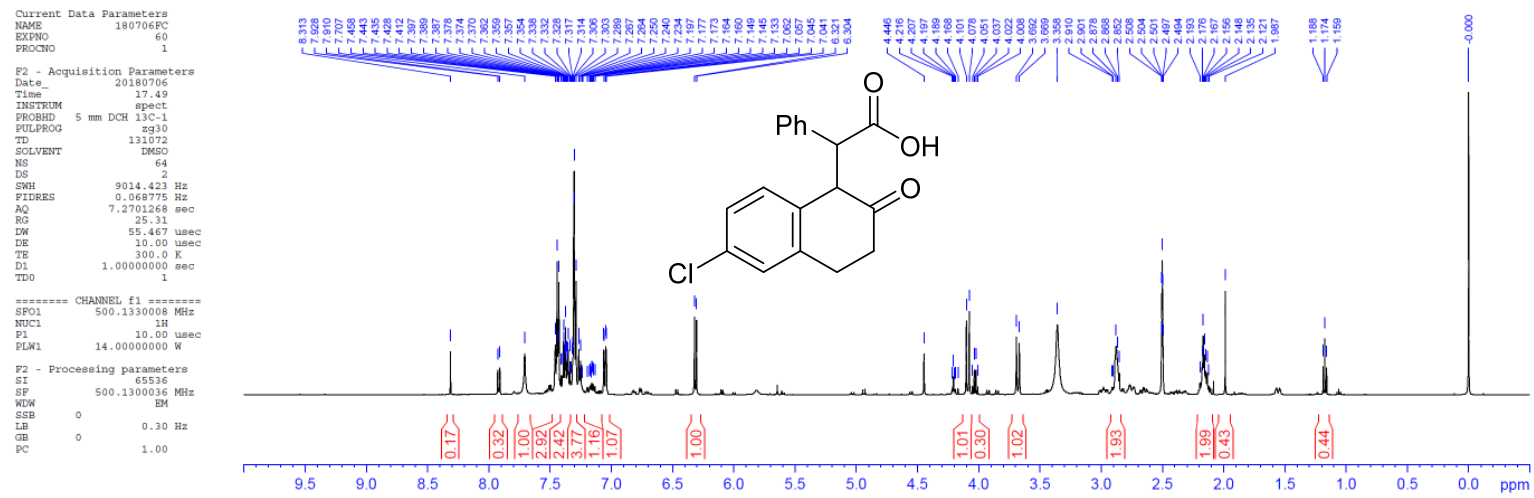

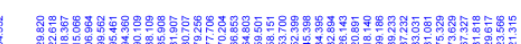

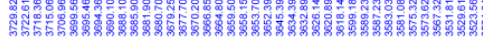
(1)
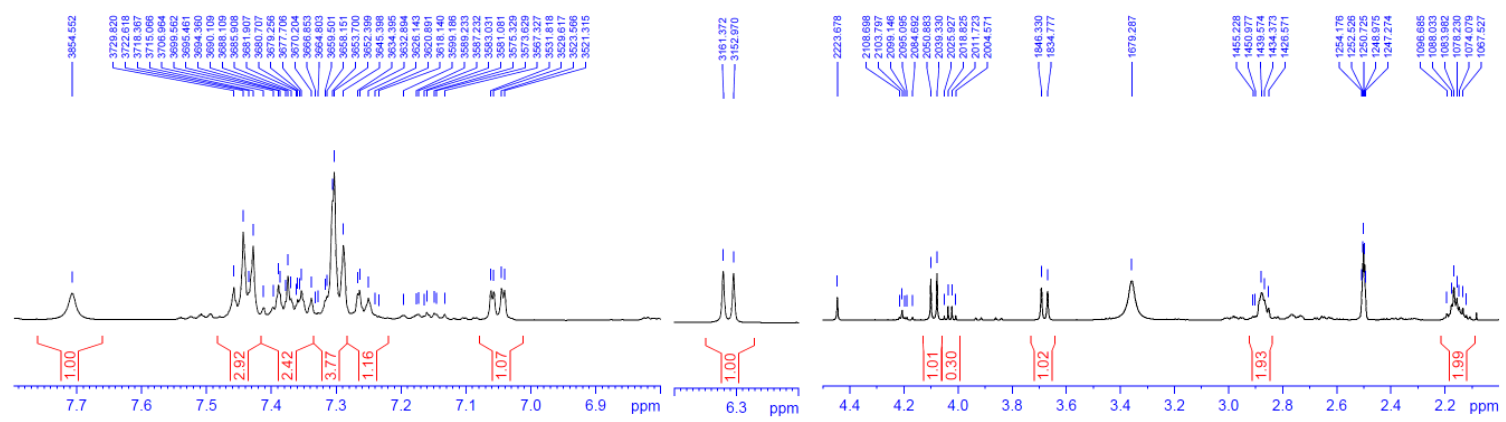

${ }^{13} \mathrm{C}$ NMR (125 MHz, DMSO- $\left.d_{6}\right)$
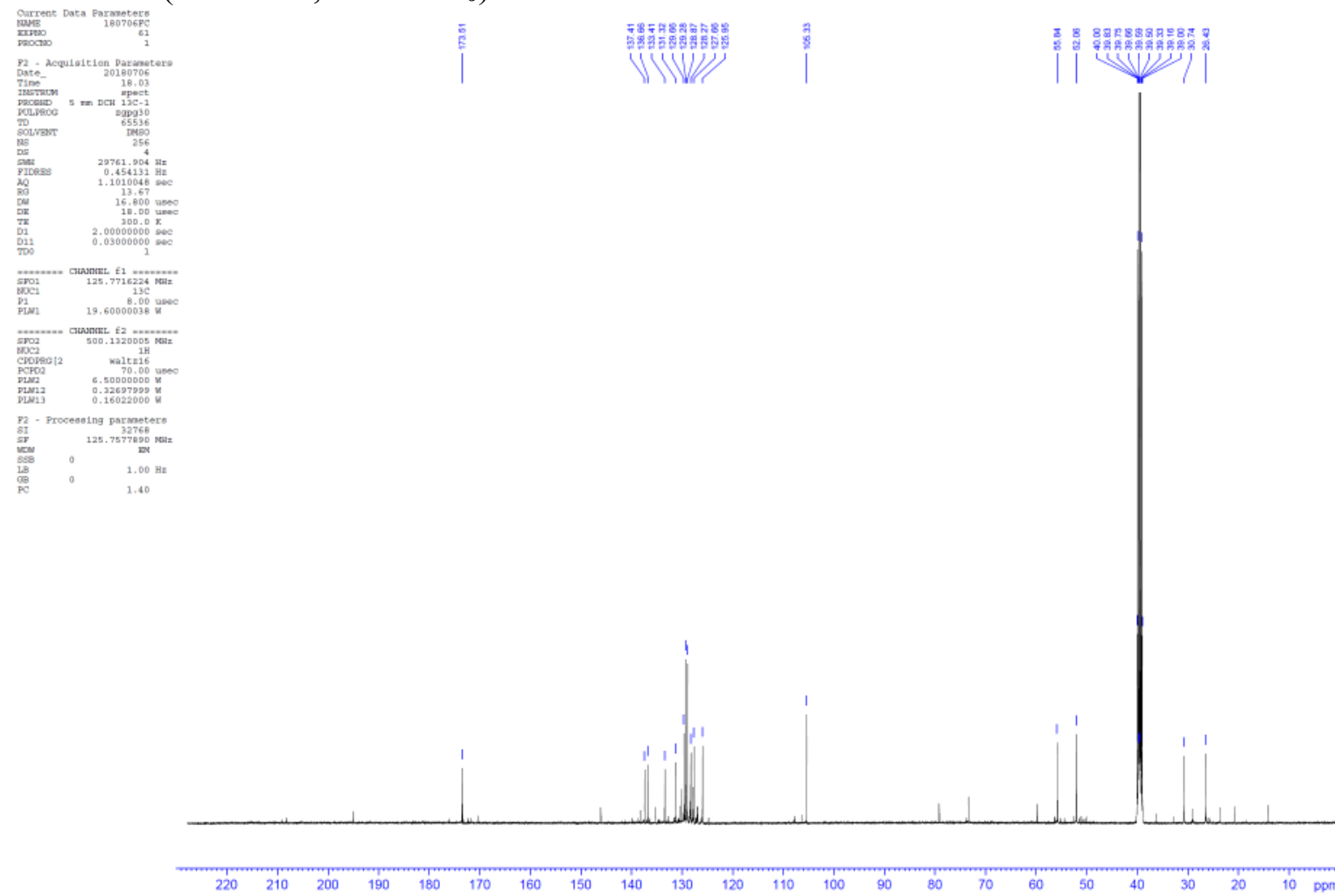
2-(8-Methoxy-2-oxo-1,2,3,4-tetrahydronaphthalen-1-yl)-2-phenylacetic acid (14g)

${ }^{1} \mathrm{H}-\mathrm{NMR}\left(500 \mathrm{MHz}, \mathrm{DMSO}-d_{6}\right)$
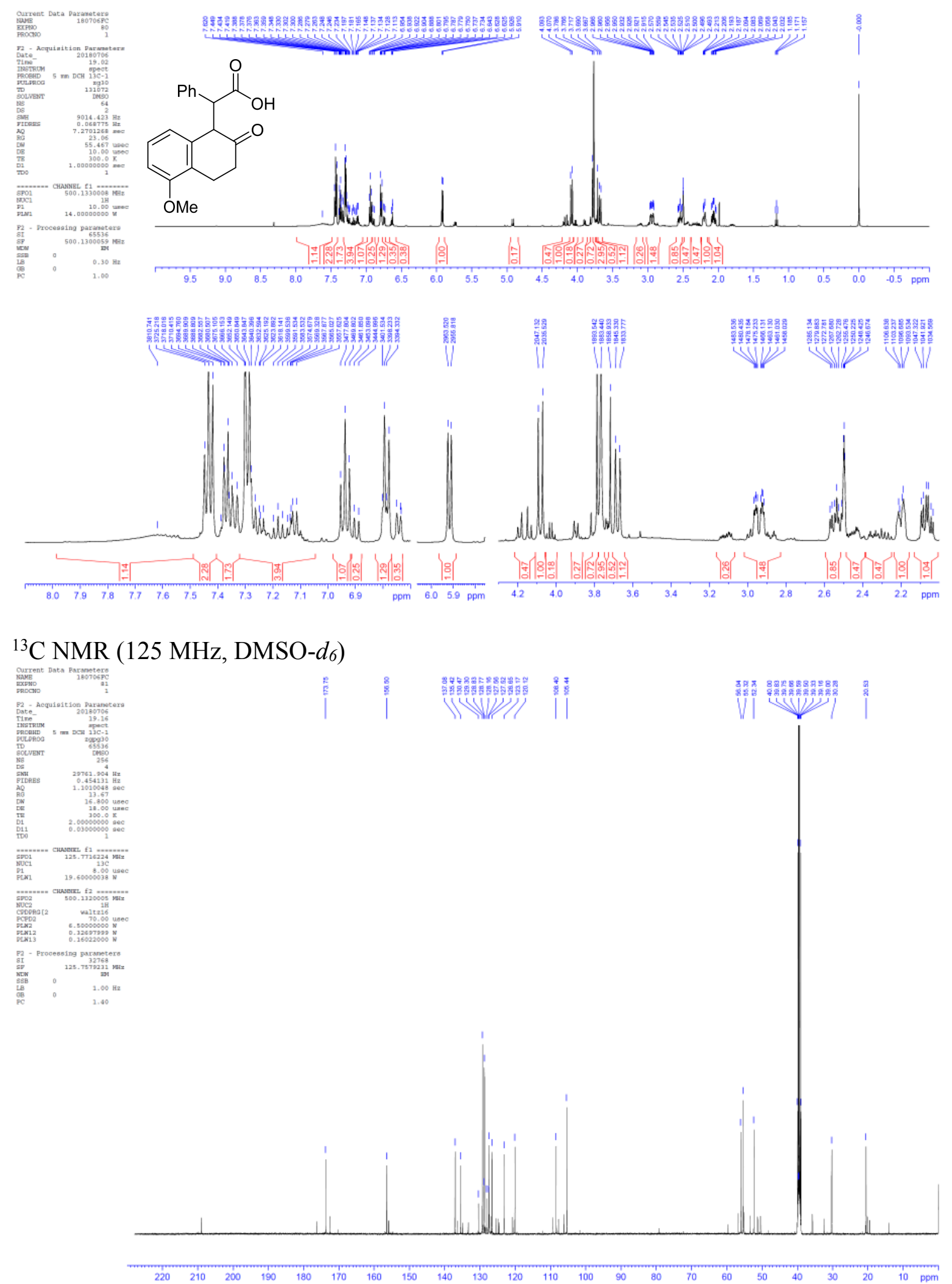
2-(2-Chlorophenyl)-2-(2-oxo-1,2,3,4-tetrahydronaphthalen-1-yl)acetic acid (14h)

${ }^{1} \mathrm{H}-\mathrm{NMR}\left(500 \mathrm{MHz}, \mathrm{DMSO}-d_{6}\right)$
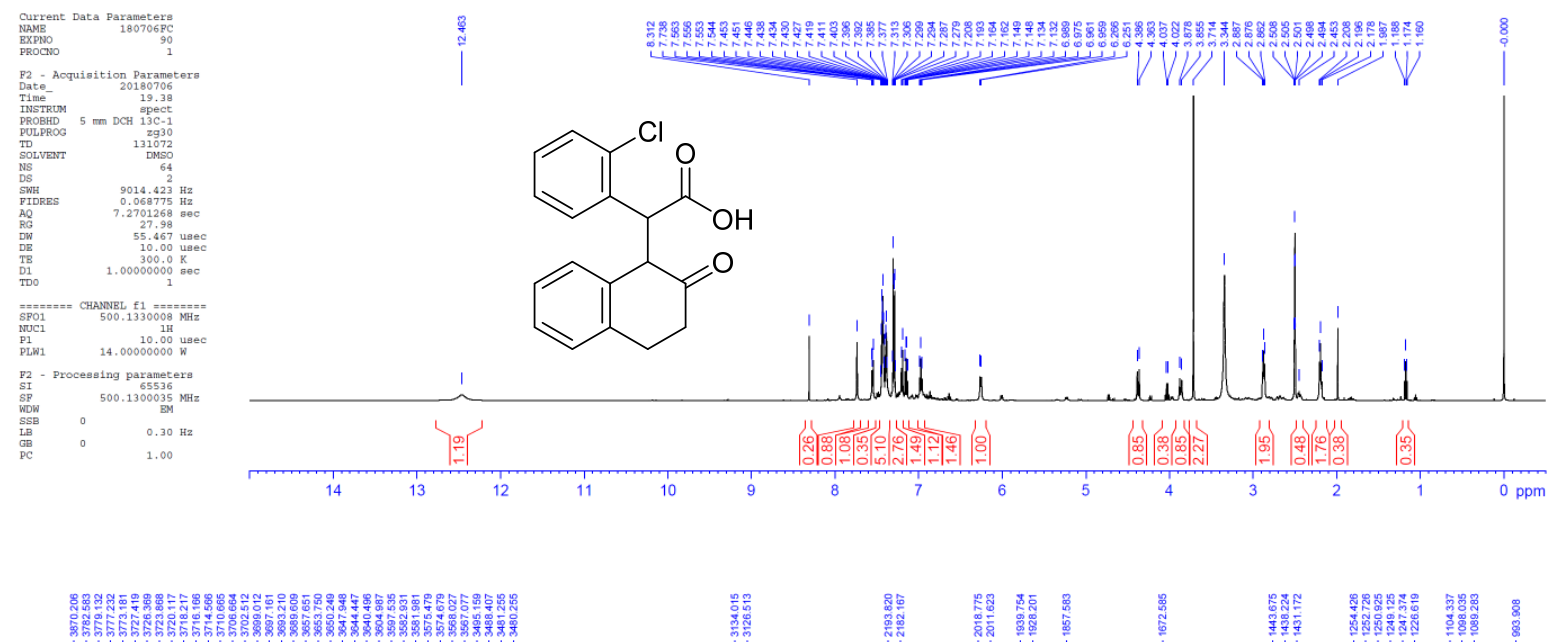

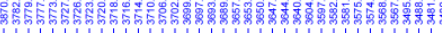

ahan WW IIIIIIIIIIII

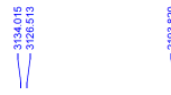
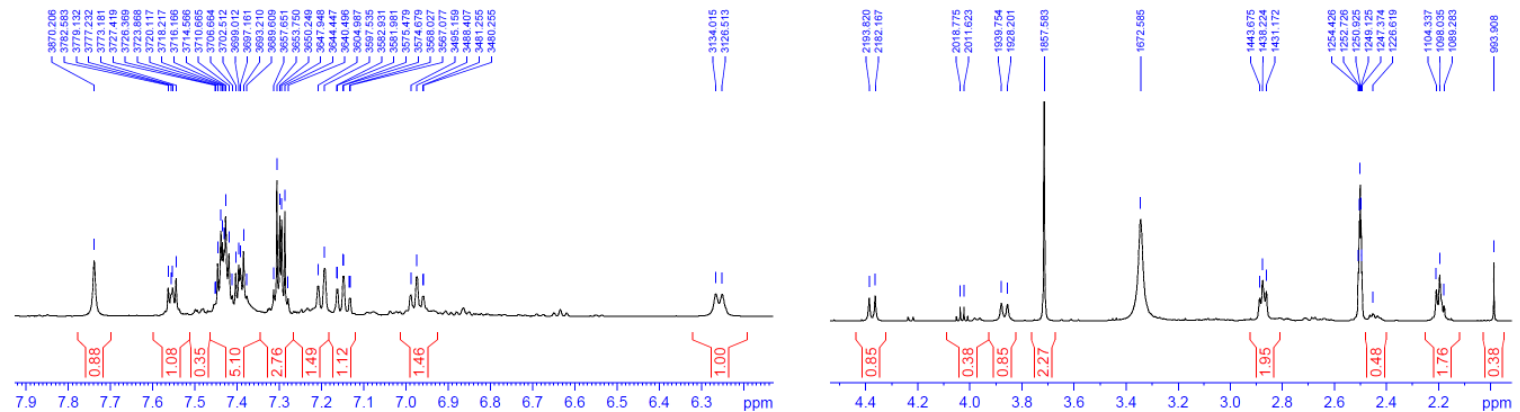

${ }^{13} \mathrm{C}$ NMR (125 MHz, DMSO- $\left.d_{6}\right)$
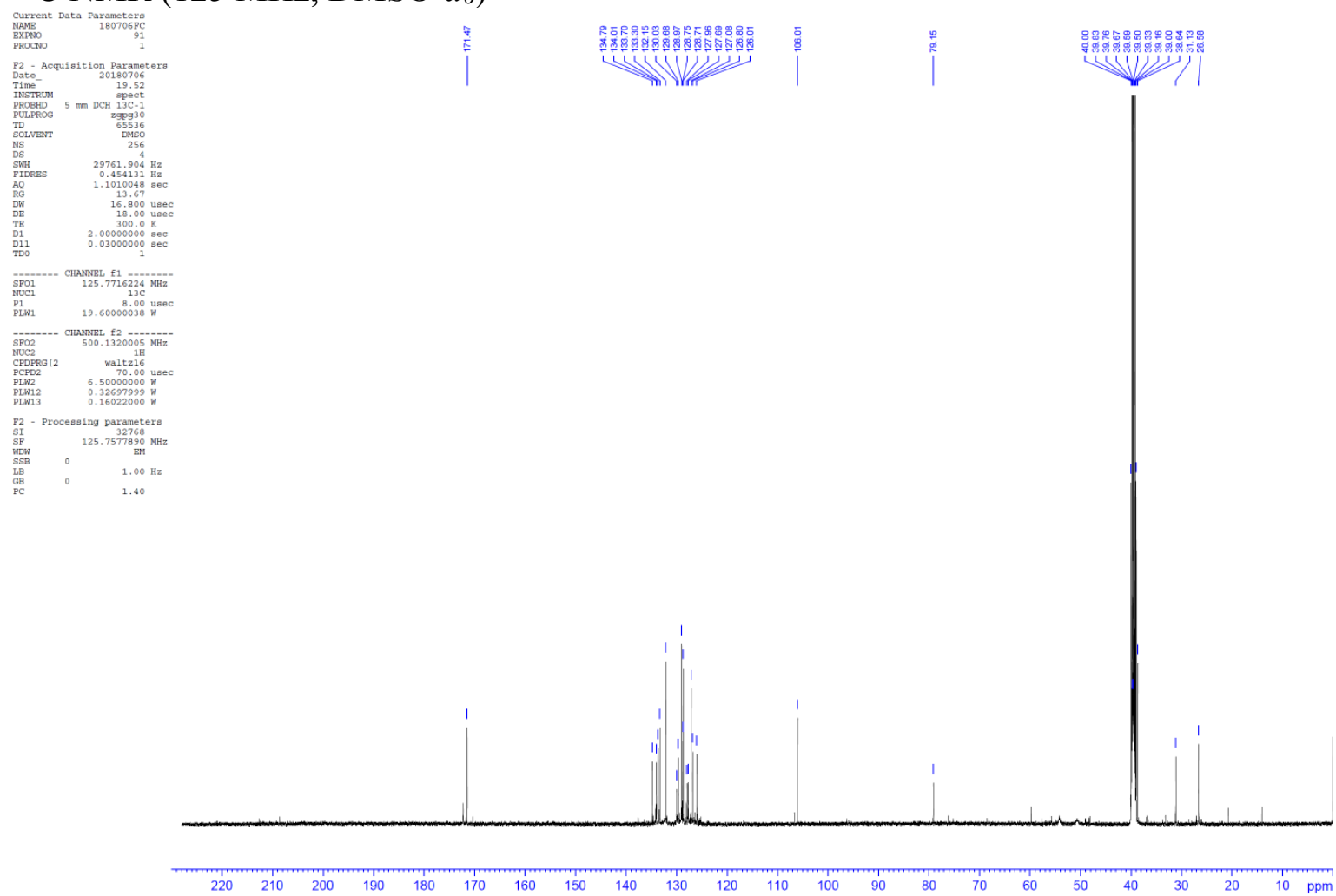
2-(7-Methoxy-2-oxo-1,2,3,4-tetrahydronaphthalen-1-yl)-2-phenylacetic acid (14i)

${ }^{1} \mathrm{H}-\mathrm{NMR}\left(500 \mathrm{MHz}, \mathrm{DMSO}-d_{6}\right)$
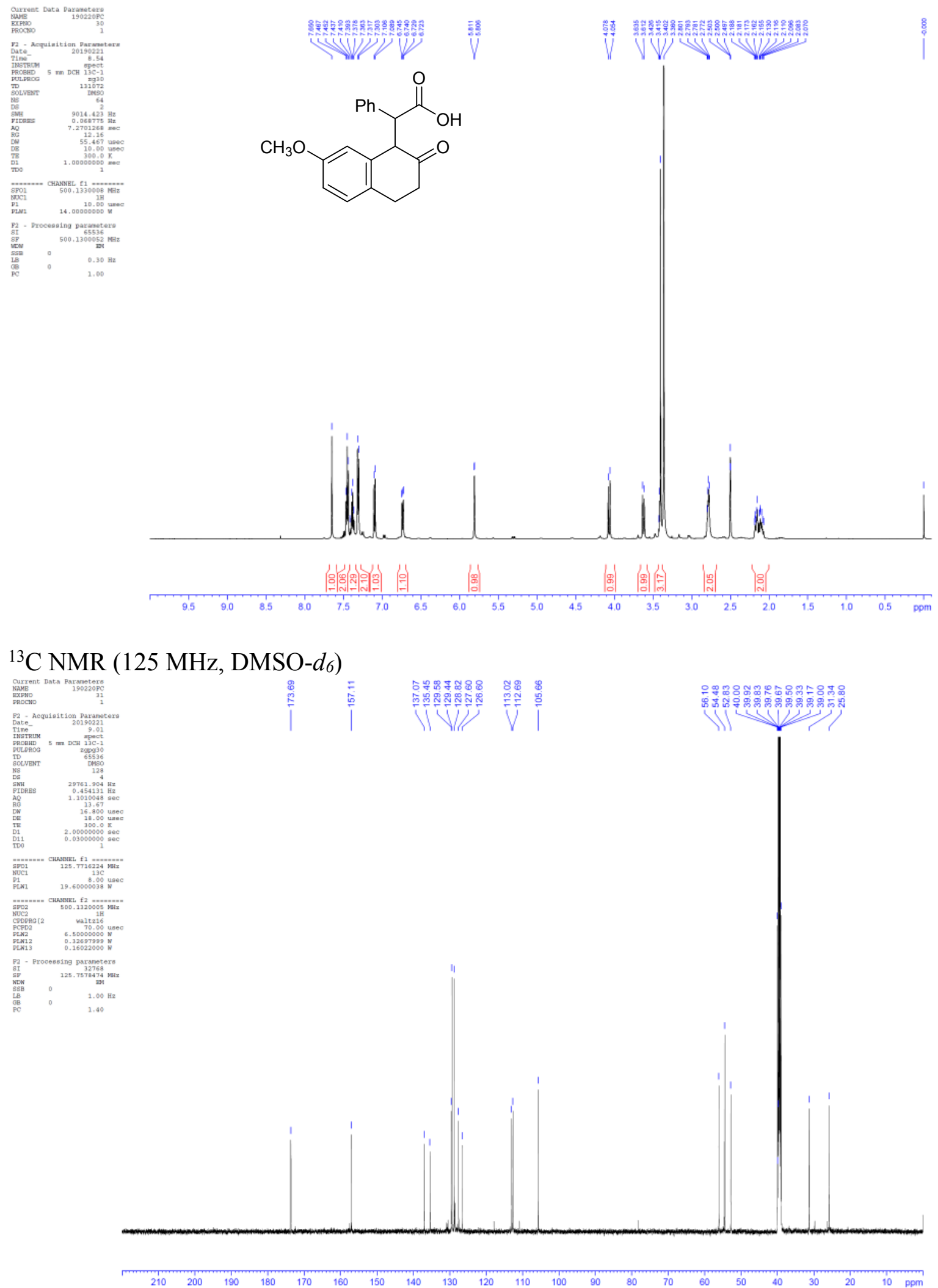
2-(1-Oxo-1,2,3,4-tetrahydronaphthalen-2-yl)-2-phenylacetic acid (17a)
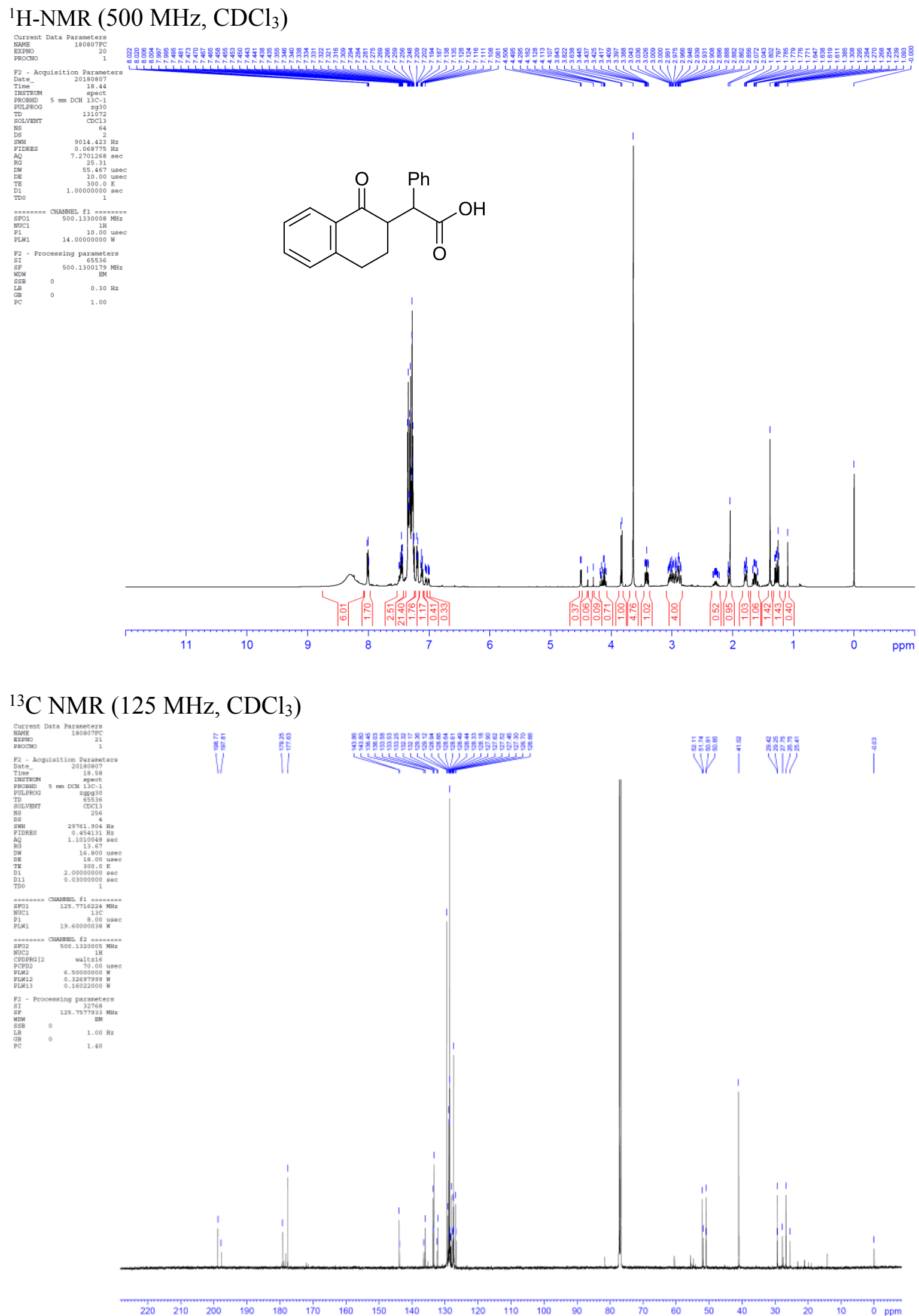
2-(7-Chloro-1-oxo-1,2,3,4-tetrahydronaphthalen-2-yl)-2-phenylacetic acid (17b)
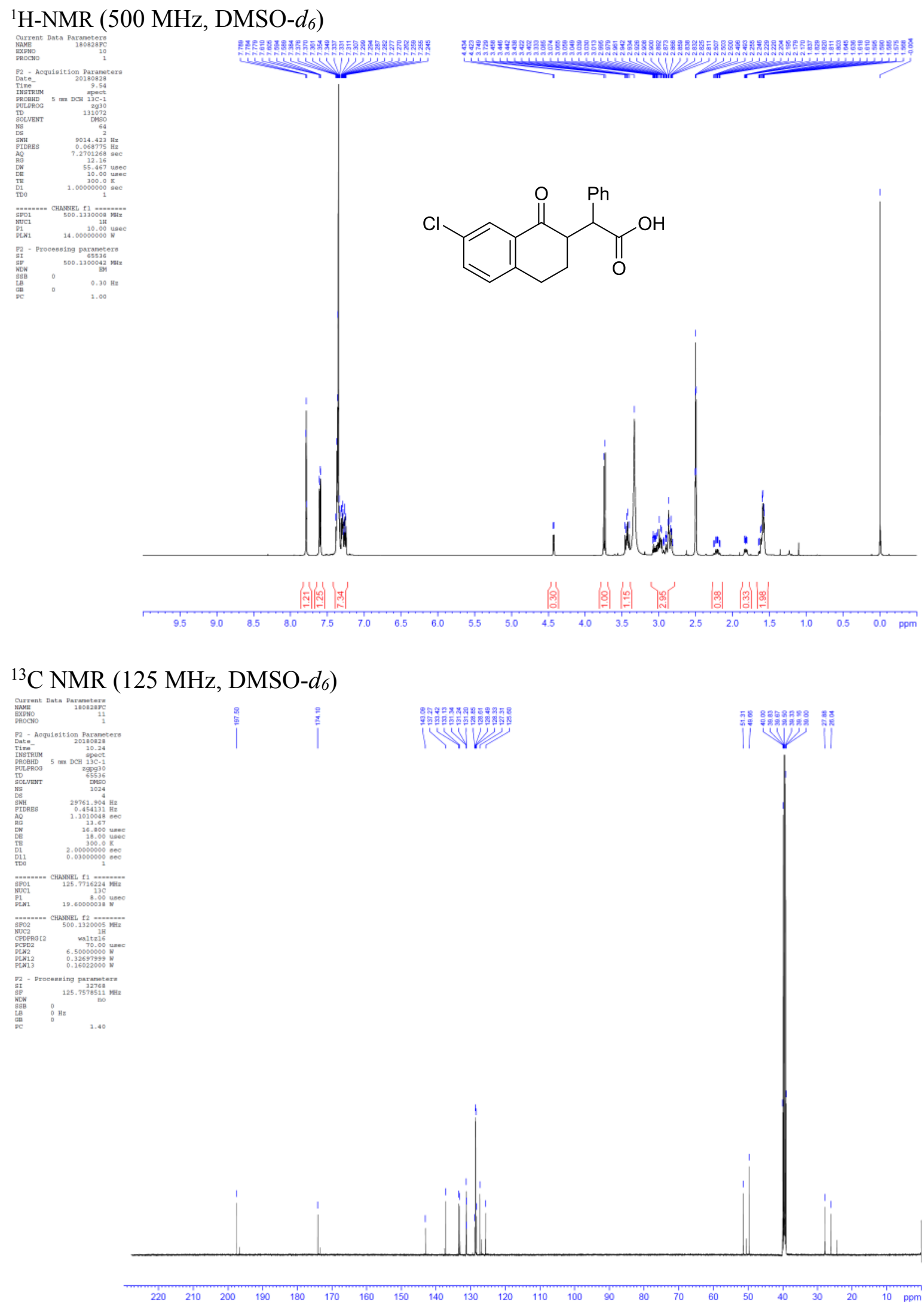
2-(1-Oxo-1,2,3,4-tetrahydronaphthalen-2-yl)propanoic acid (17c)

${ }^{1} \mathrm{H}-\mathrm{NMR}\left(500 \mathrm{MHz}, \mathrm{DMSO}-d_{6}\right)$
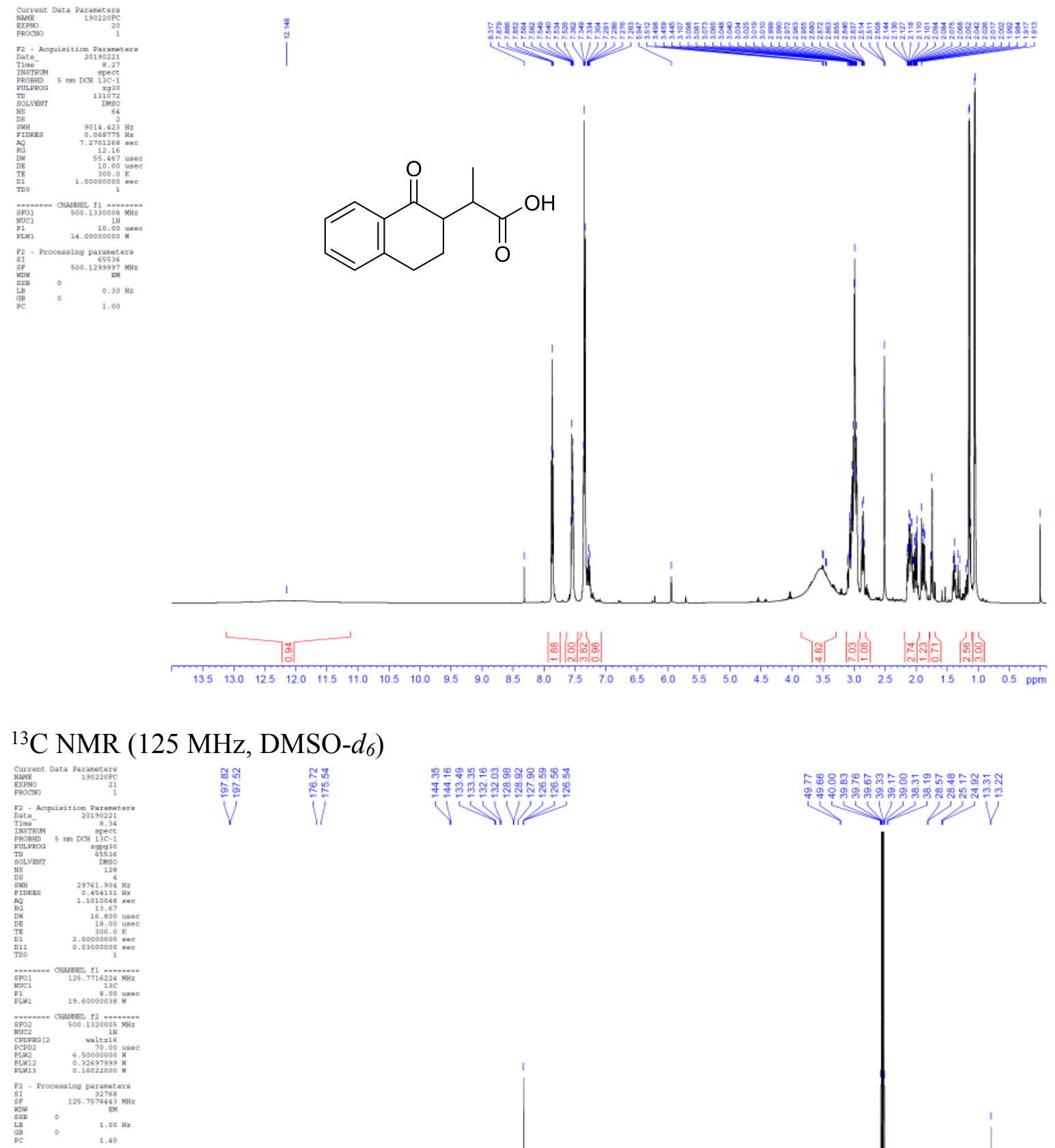

$\left.5 \mathrm{MHz}, \mathrm{DMSO}-d_{6}\right)$

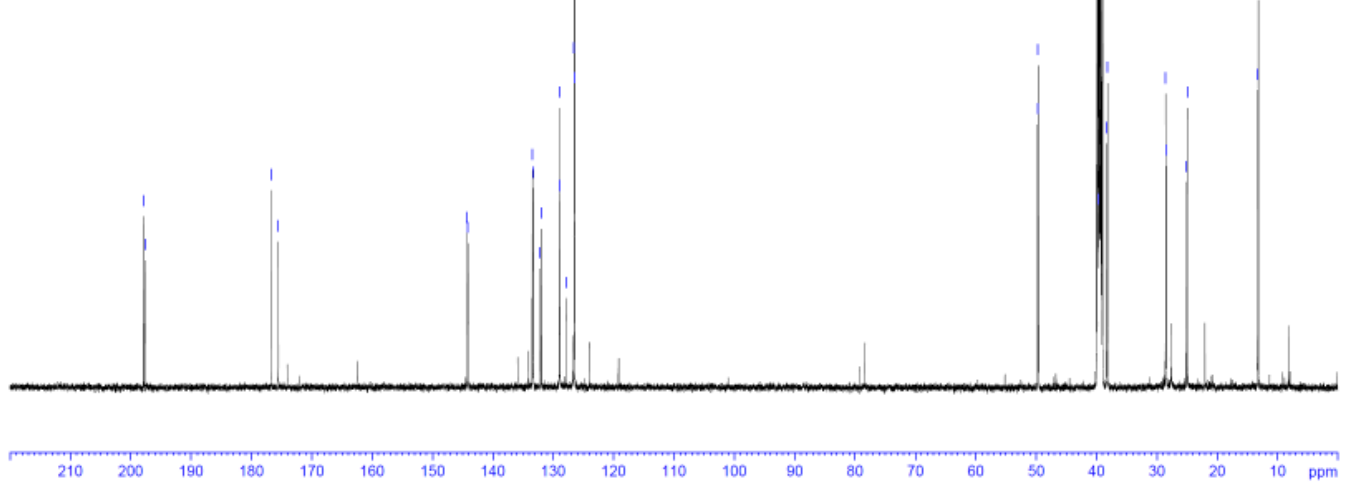


$(1 R, 2 R)$-2-Methyl-1,2,3,4-tetrahydronaphthalen-1-ol (9a)

${ }^{1} \mathrm{H}-\mathrm{NMR}\left(400 \mathrm{MHz}, \mathrm{CDCl}_{3}\right)$

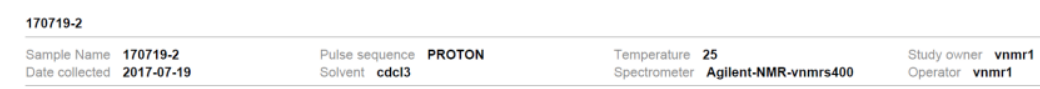
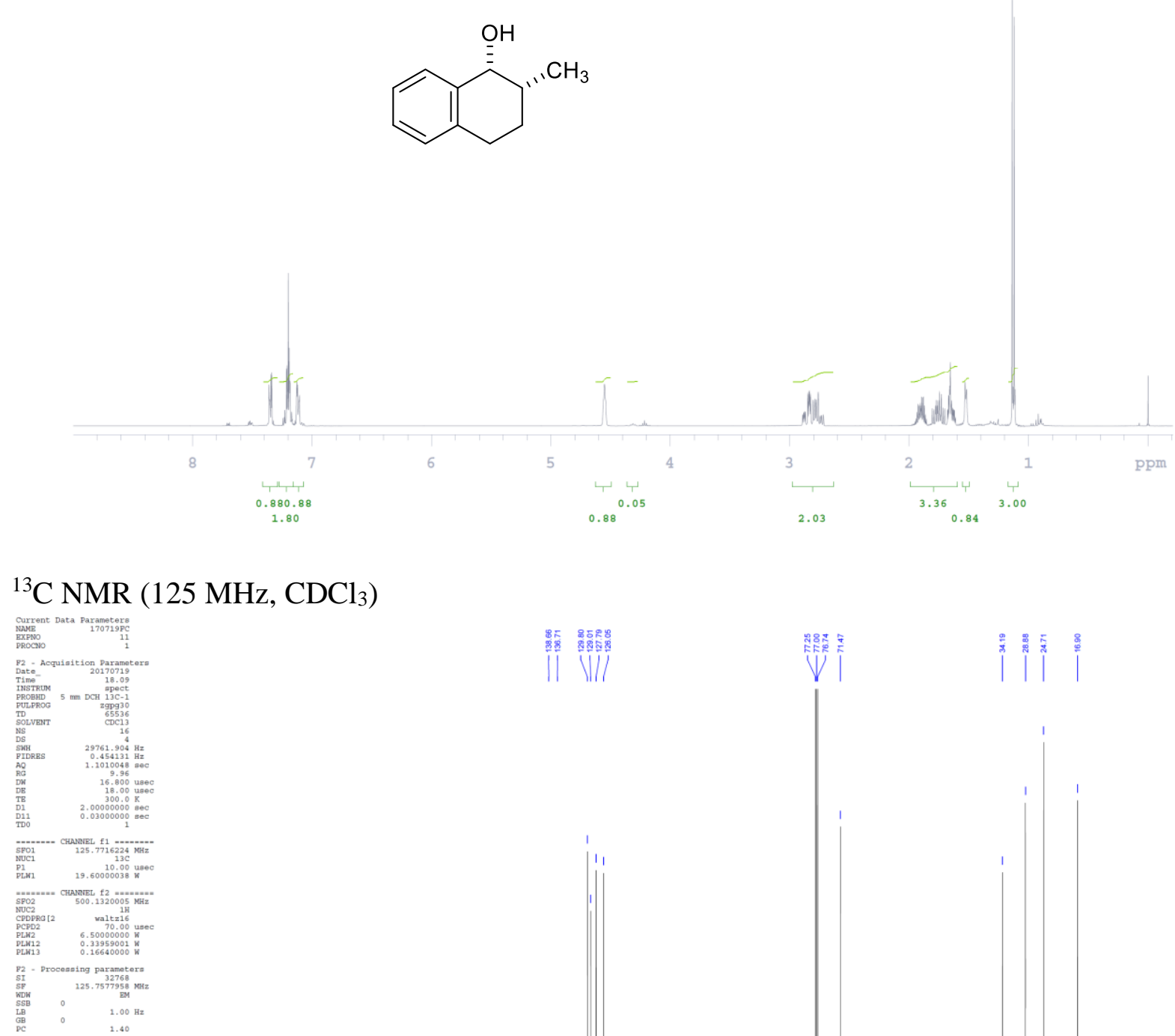

$\left.25 \mathrm{MHz}, \mathrm{CDCl}_{3}\right)$

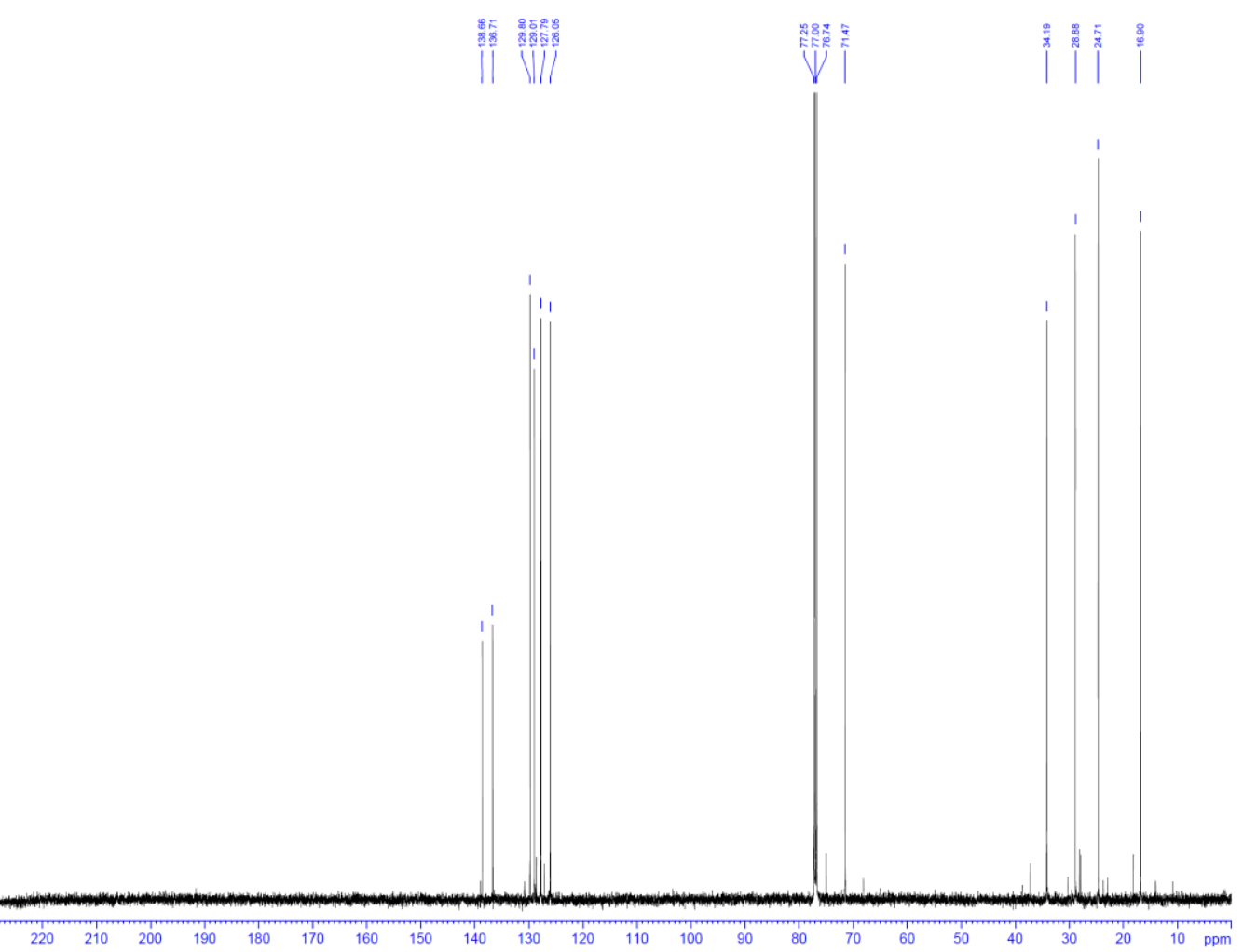


(1R,2R)-2-Methyl-2,3-dihydro-1H-inden-1-ol (9b)

${ }^{1} \mathrm{H}-\mathrm{NMR}\left(400 \mathrm{MHz}, \mathrm{CDCl}_{3}\right)$

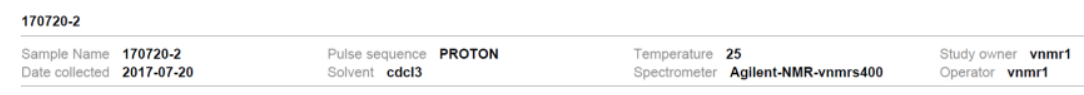

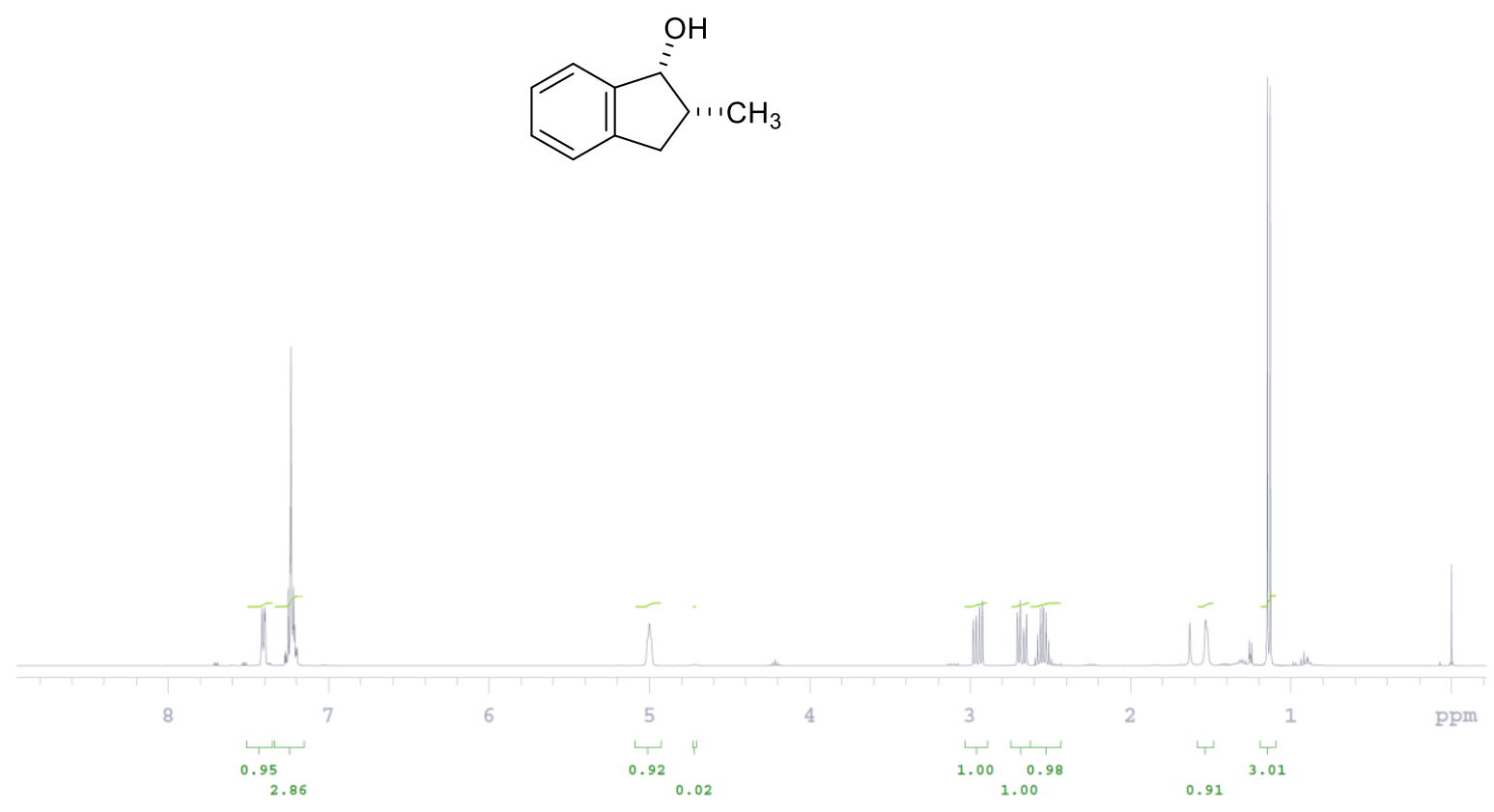

${ }^{13} \mathrm{C} \mathrm{NMR}\left(125 \mathrm{MHz}, \mathrm{CDCl}_{3}\right)$

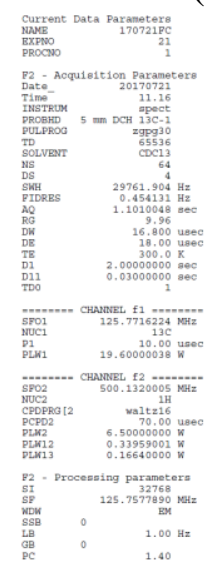

S111 
(5R,6R)-6-Methyl-6,7,8,9-tetrahydro-5H-benzo[7]annulen-5-ol (9c)

${ }^{1} \mathrm{H}-\mathrm{NMR}\left(400 \mathrm{MHz}, \mathrm{CDCl}_{3}\right.$ )

Sample Name 170720-3

Date collected 2017-07-20

Pulse sequence PROTON

Temperature 25

Agilent-NMR-vnmrs400

Study owner vnmr1
Operator vnmr1

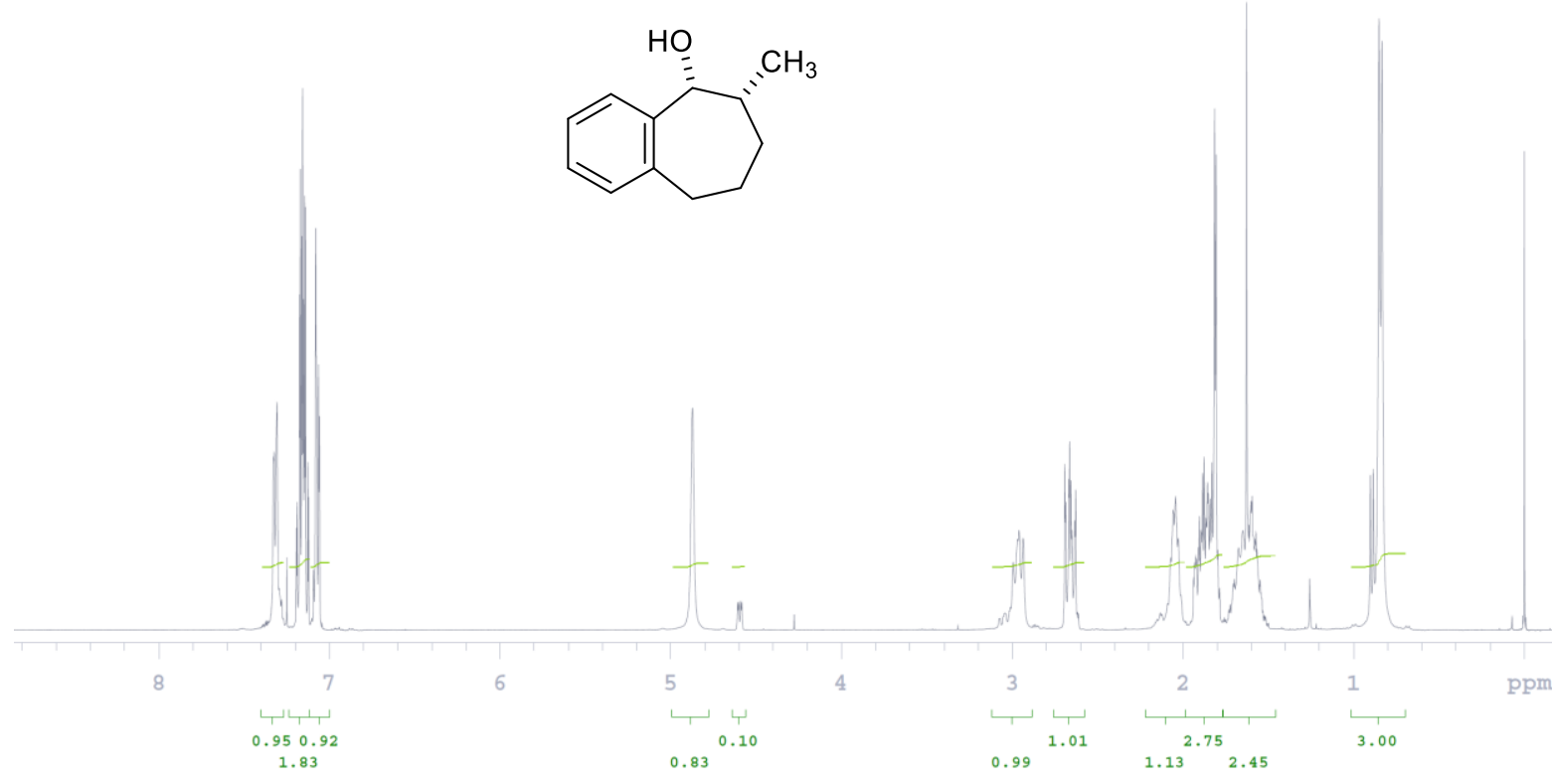

${ }^{13} \mathrm{C}$ NMR $\left(125 \mathrm{MHz}, \mathrm{CDCl}_{3}\right)$

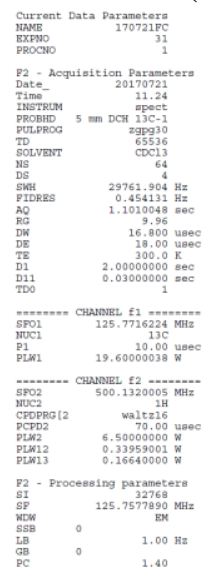


Methyl (1R,2R)-1-hydroxy-1,2,3,4-tetrahydronaphthalene-2-carboxylate (9d)

${ }^{1} \mathrm{H}-\mathrm{NMR}\left(400 \mathrm{MHz}, \mathrm{CDCl}_{3}\right)$

\begin{tabular}{|c|c|c|c|c|c|c|}
\hline $\begin{array}{l}\text { Sample Name } \\
\text { Date collected }\end{array}$ & $\begin{array}{l}\text { 170724-1 } \\
2017-07-24\end{array}$ & $\begin{array}{l}\text { Pulse sequence } \\
\text { Solvent cdcl3 }\end{array}$ & PROTON & $\begin{array}{l}\text { Temperature } \\
\text { Spectrometer }\end{array}$ & $\begin{array}{l}25 \\
\text { Agilent-NMR-vnmrs400 }\end{array}$ & $\begin{array}{l}\text { Study owner vnmr1 } \\
\text { Operator vnmr1 }\end{array}$ \\
\hline
\end{tabular}

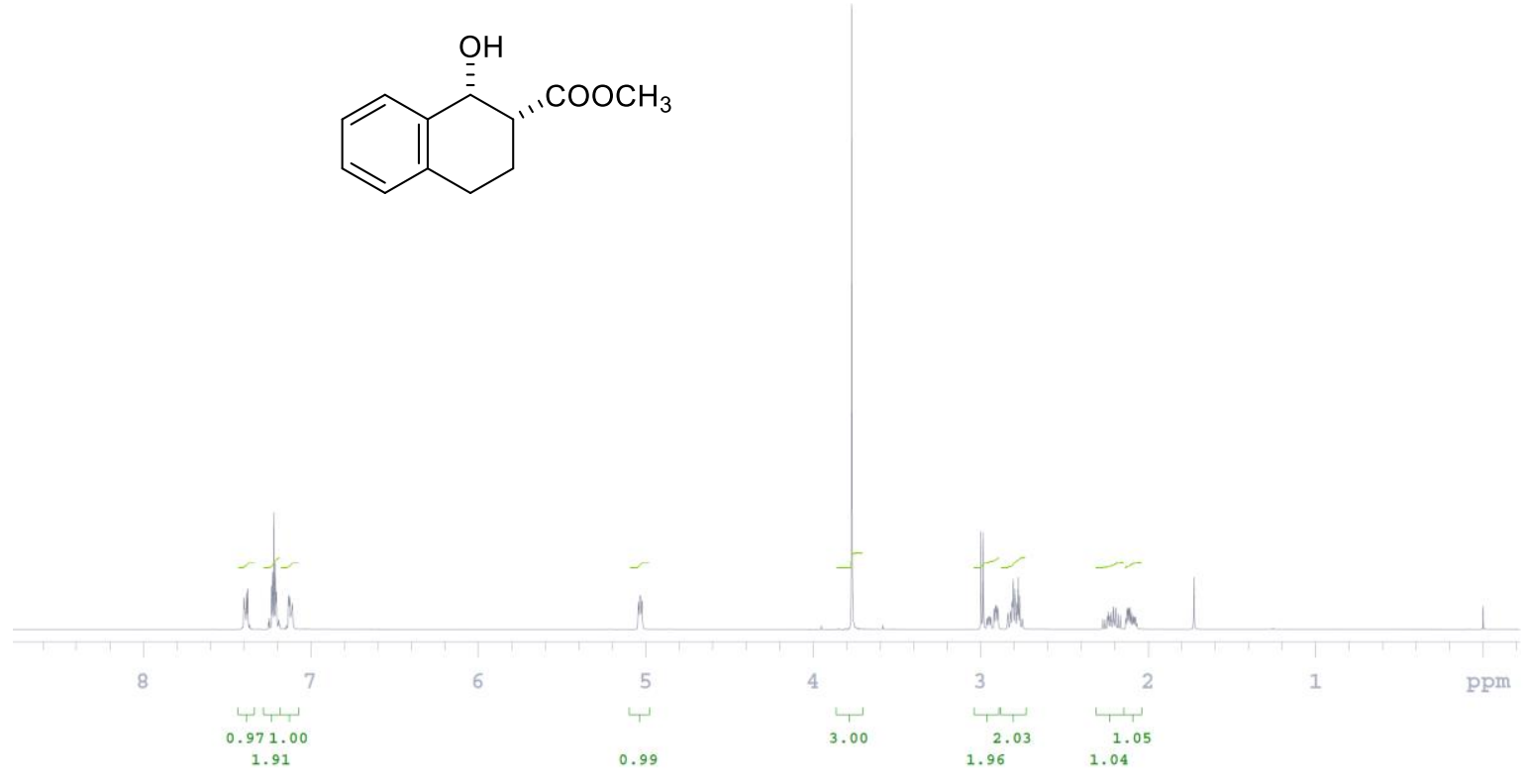

${ }^{13} \mathrm{C}$ NMR $\left(125 \mathrm{MHz}, \mathrm{CDCl}_{3}\right)$
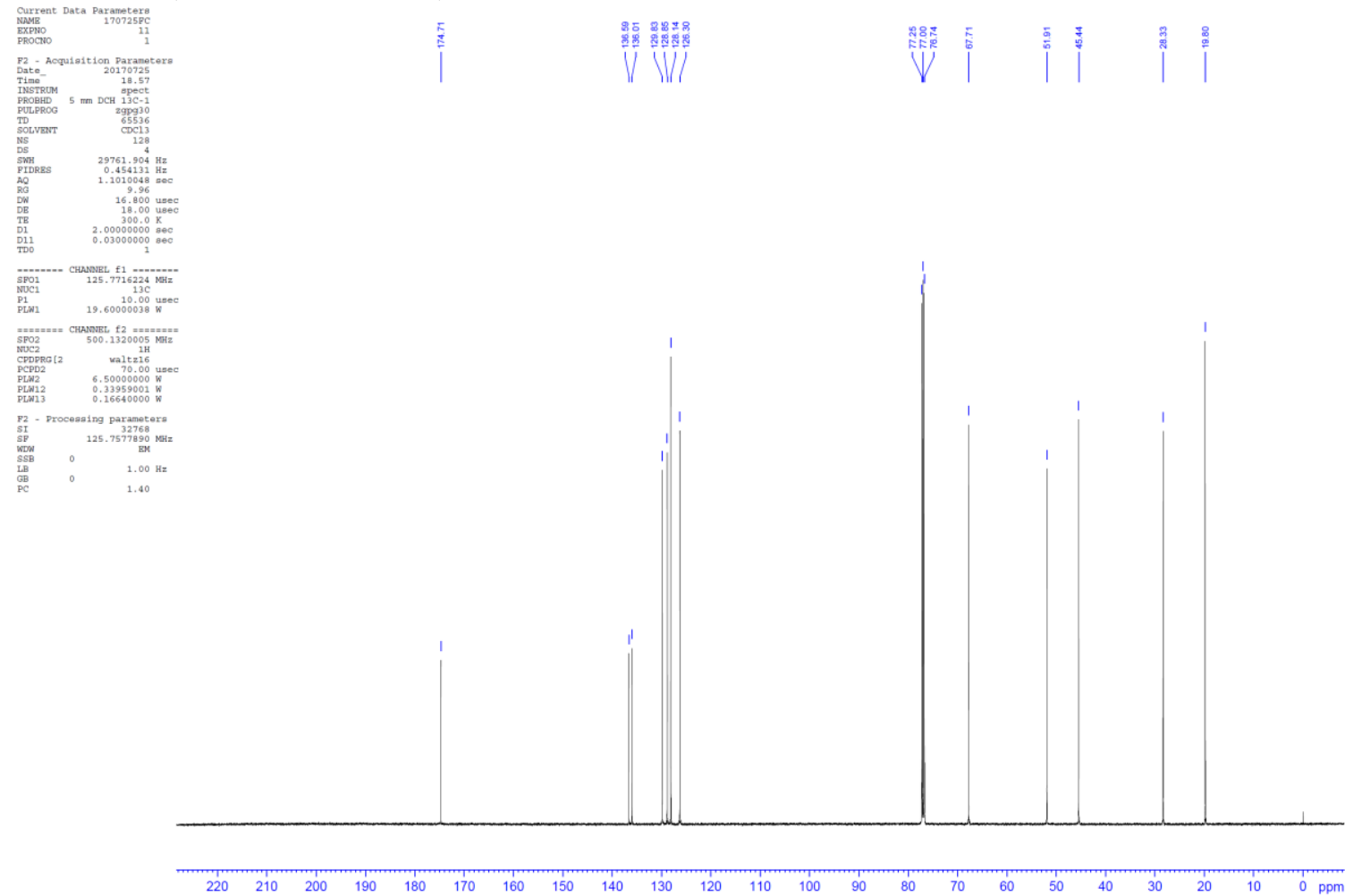
$(3 \mathrm{a}, 9 \mathrm{~b} R)-3 \mathrm{a}, 4,5,9 \mathrm{~b}-$-Tetrahydronaphtho[1,2-b]furan-2(3H)-one (13a)

${ }^{1} \mathrm{H}-\mathrm{NMR}\left(400 \mathrm{MHz}, \mathrm{CDCl}_{3}\right)$

Sample Name $170823-4$

Pulse sequence PROTON
Solvent cdc13

Temperature 25
Spectrometer Agilent-NMR-vnmrs400

Study owner vnmr1
Operator vnmr1

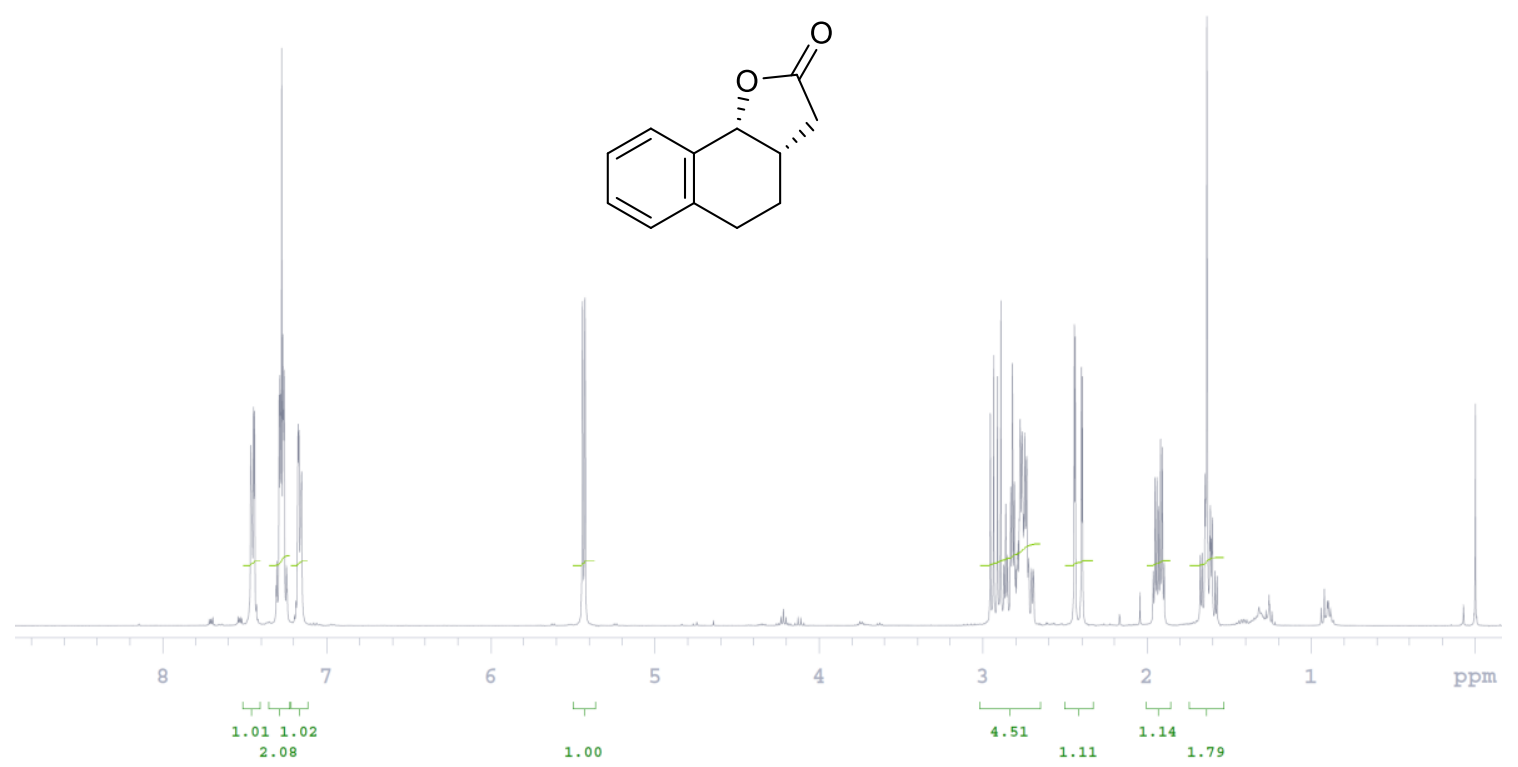

${ }^{13} \mathrm{C}$ NMR $\left(125 \mathrm{MHz}, \mathrm{CDCl}_{3}\right)$
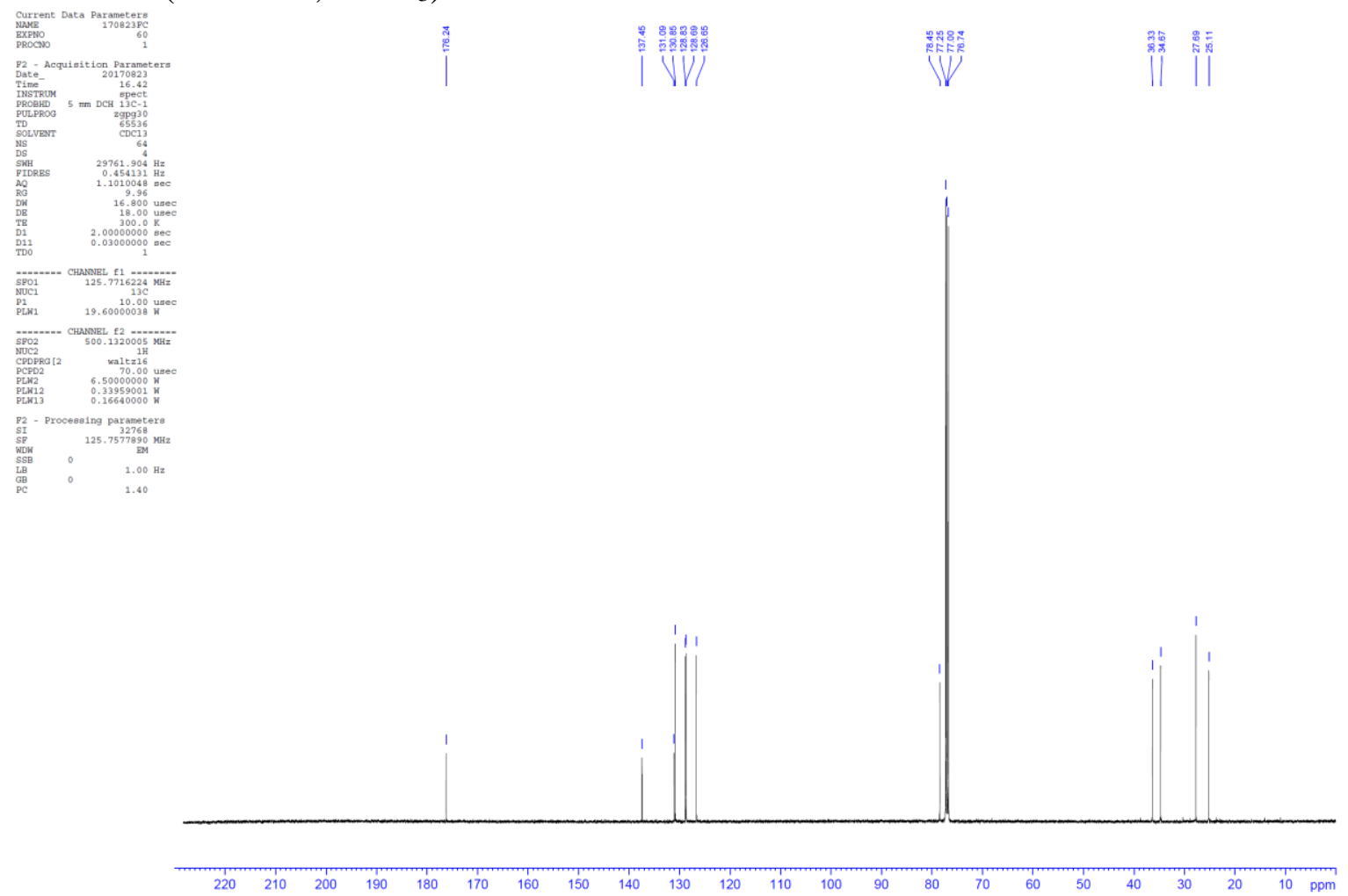
(3aS,9bR)-8-Methoxy-3a,4,5,9b-tetrahydronaphtho[1,2-b]furan-2(3H)-one (13b)

${ }^{1} \mathrm{H}-\mathrm{NMR}\left(400 \mathrm{MHz}, \mathrm{CDCl}_{3}\right)$

170908-2

Sample Name 170908-2

Pulse sequence PROTON
Solvent cdc13

Temperature 25

Agilent-NMR-vnmrs400

Study owner vnmr1
Operator vnmr1

(N)

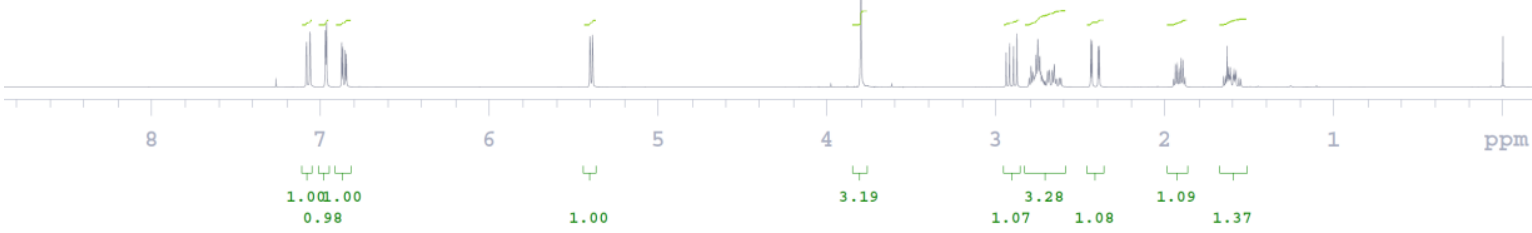

${ }^{13} \mathrm{C} \mathrm{NMR}\left(125 \mathrm{MHz}, \mathrm{CDCl}_{3}\right)$
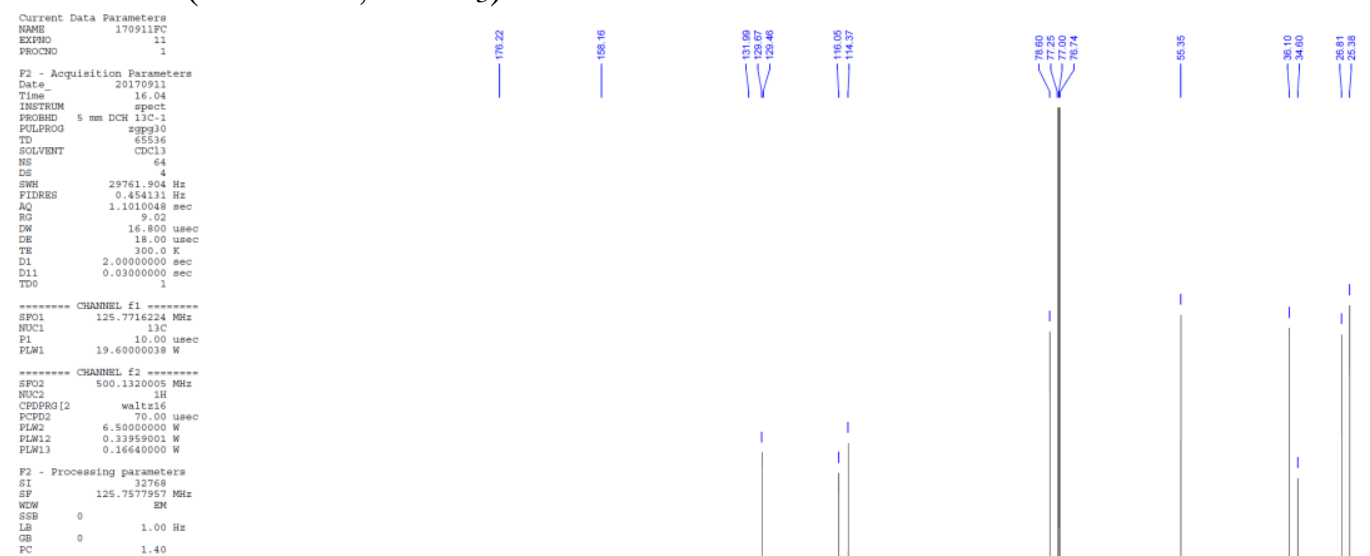

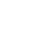

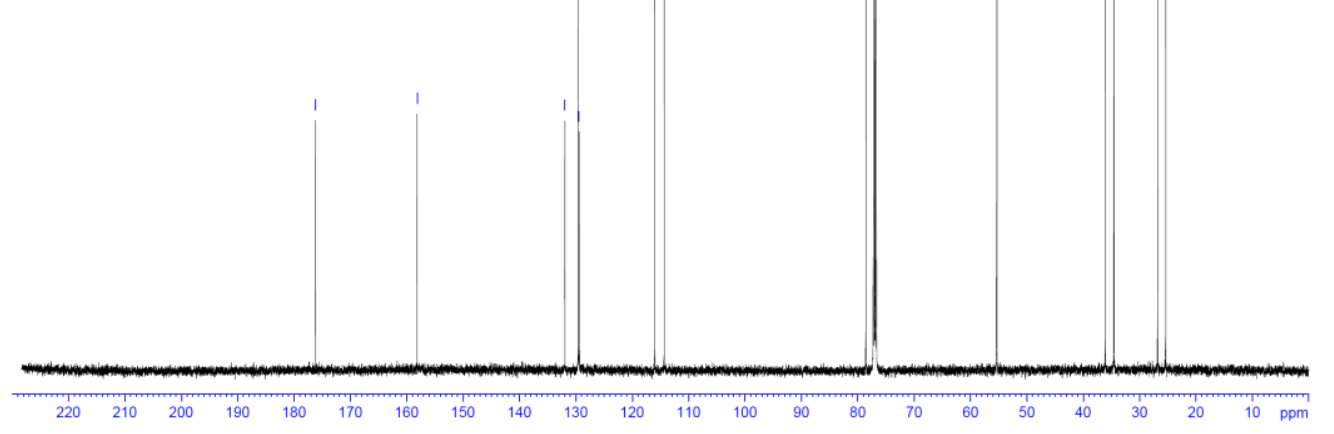


(3aS,9bR)-8-Chloro-3a,4,5,9b-tetrahydronaphtho[1,2-b]furan-2(3H)-one (13c)

${ }^{1} \mathrm{H}-\mathrm{NMR}\left(400 \mathrm{MHz}, \mathrm{CDCl}_{3}\right)$

\begin{tabular}{|c|c|c|c|c|}
\hline $\begin{array}{ll}\text { Sample Name } & 170908-1 \\
\text { Date collected } & \mathbf{2 0 1 7 - 0 9 - 0 8}\end{array}$ & $\begin{array}{l}\text { Pulse sequence PROTON } \\
\text { Solvent cdcl3 }\end{array}$ & $\begin{array}{l}\text { Temperature } \\
\text { Spectrometer }\end{array}$ & $\begin{array}{l}25 \\
\text { Agilent-NMR-vnmrs400 }\end{array}$ & $\begin{array}{l}\text { Study owner vnmr1 } \\
\text { Operator vnmr1 }\end{array}$ \\
\hline
\end{tabular}

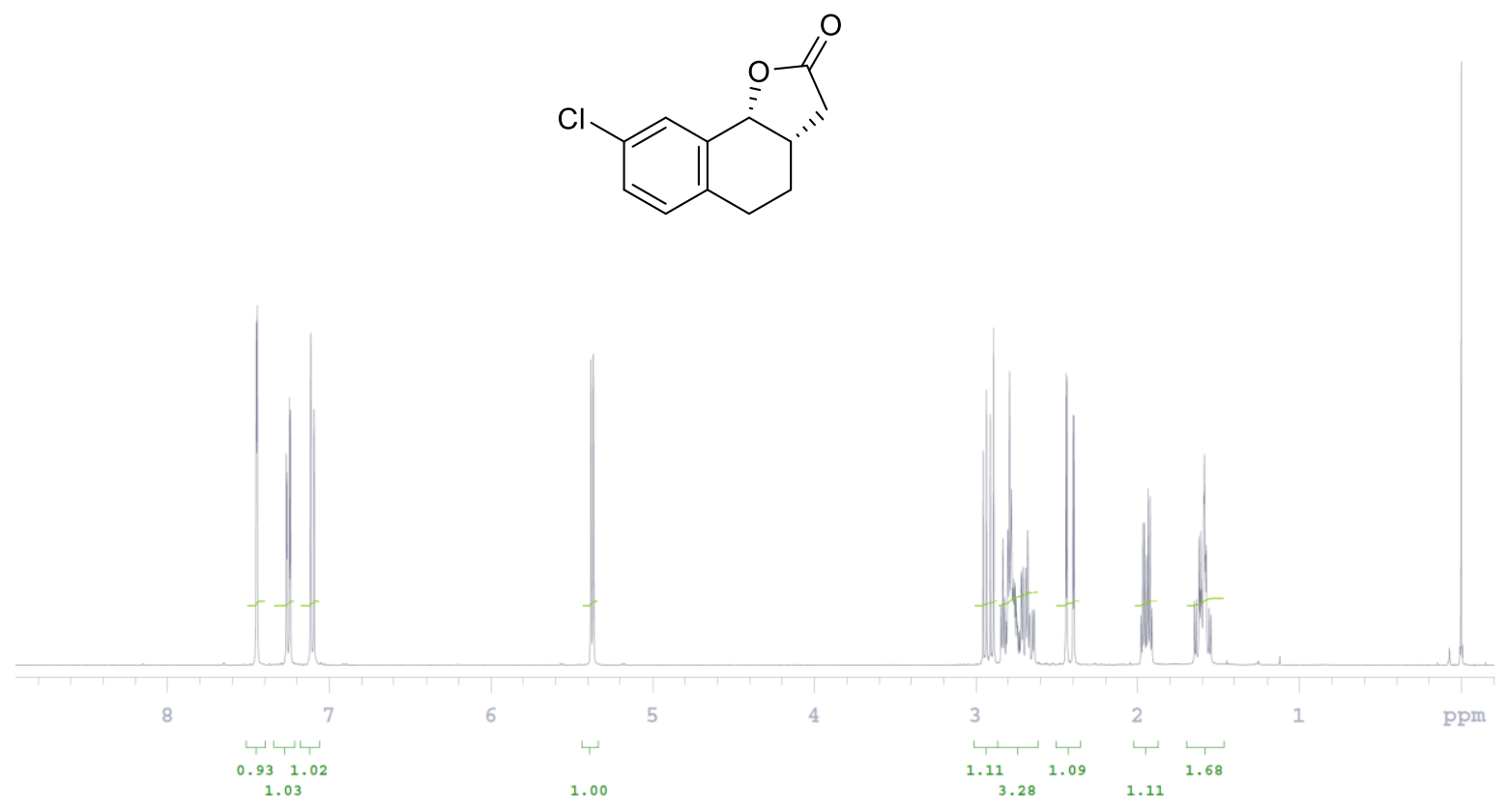

${ }^{13} \mathrm{C}$ NMR $\left(125 \mathrm{MHz}, \mathrm{CDCl}_{3}\right)$
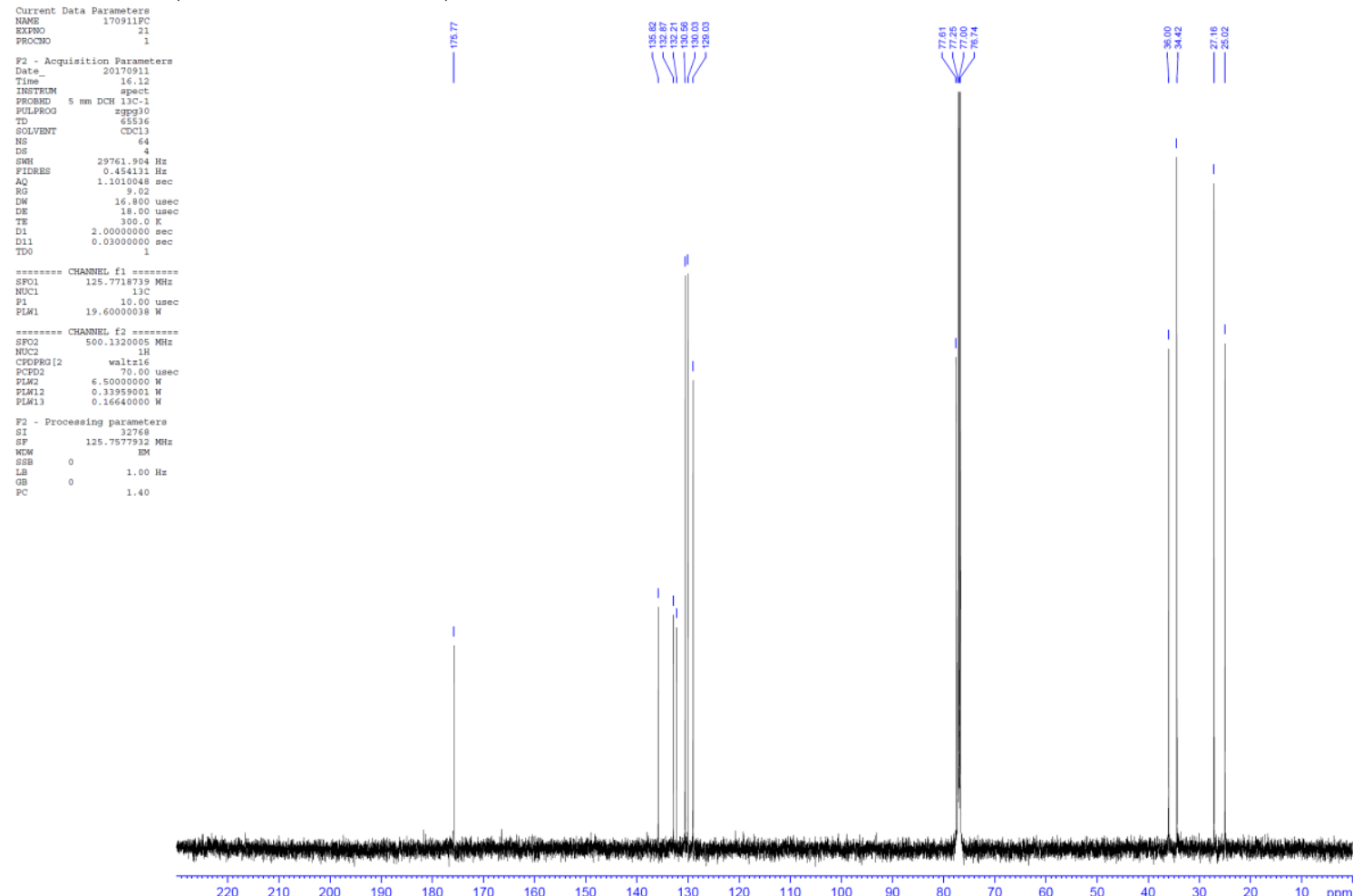
(3aS,9bR)-8-Bromo-3a,4,5,9b-tetrahydronaphtho[1,2-b]furan-2(3H)-one (13d)

${ }^{1} \mathrm{H}-\mathrm{NMR}\left(400 \mathrm{MHz}, \mathrm{CDCl}_{3}\right)$
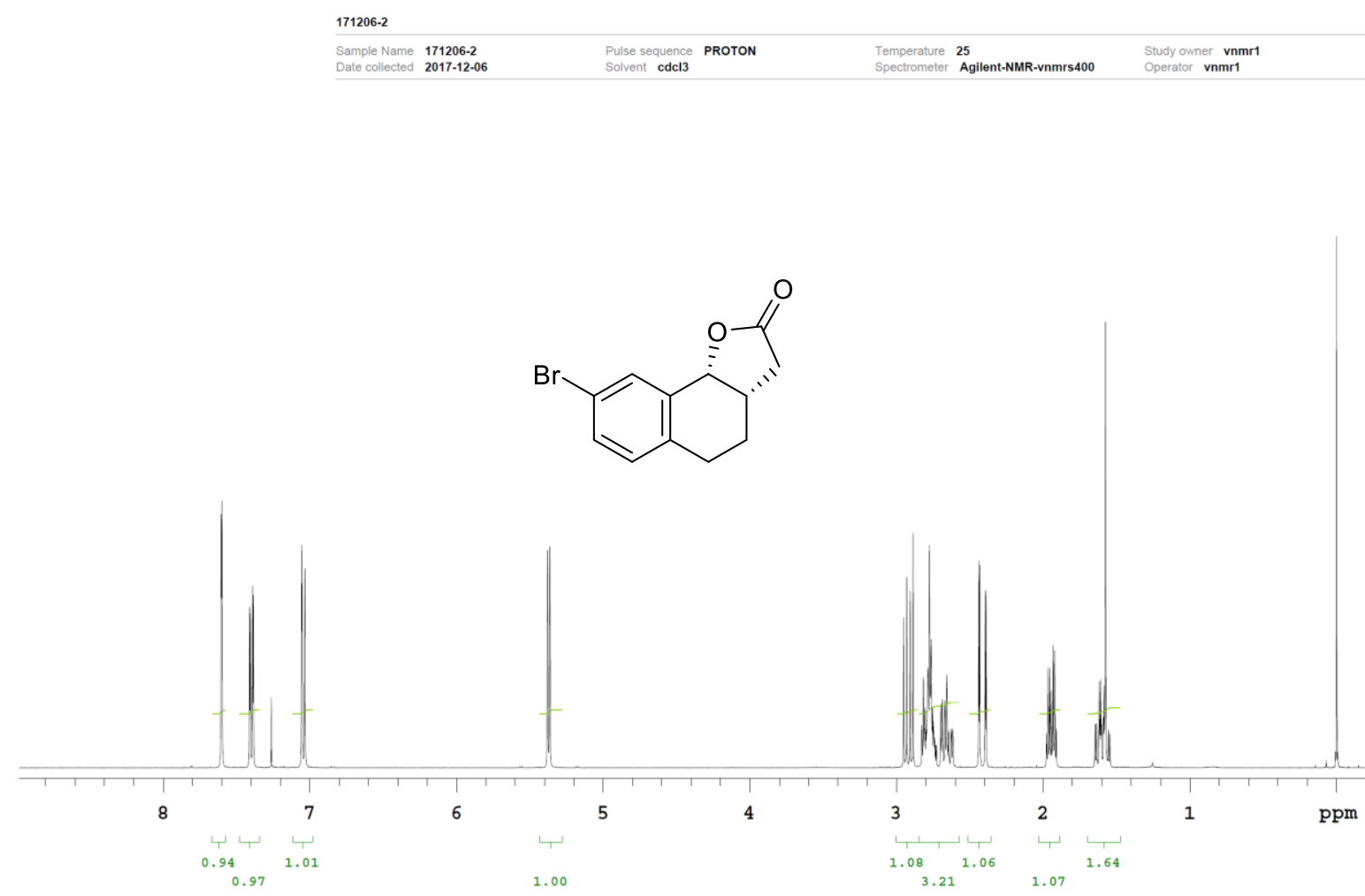

${ }^{13} \mathrm{C}$ NMR $\left(125 \mathrm{MHz}, \mathrm{CDCl}_{3}\right)$
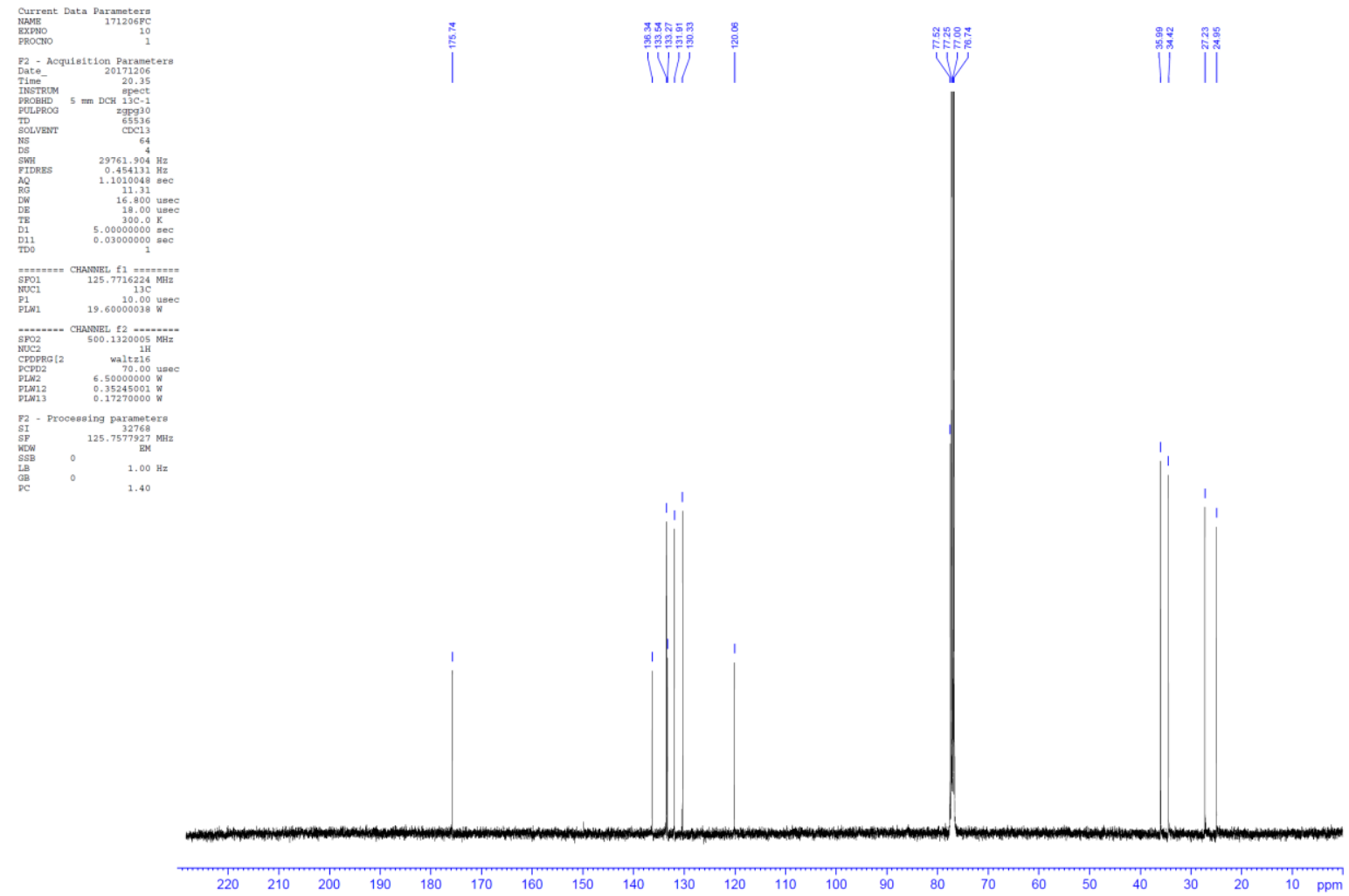
(3aS,9bR)-9-Bromo-8-methoxy-3a,4,5,9b-tetrahydronaphtho[1,2-b]furan-2(3H)-one (13e)
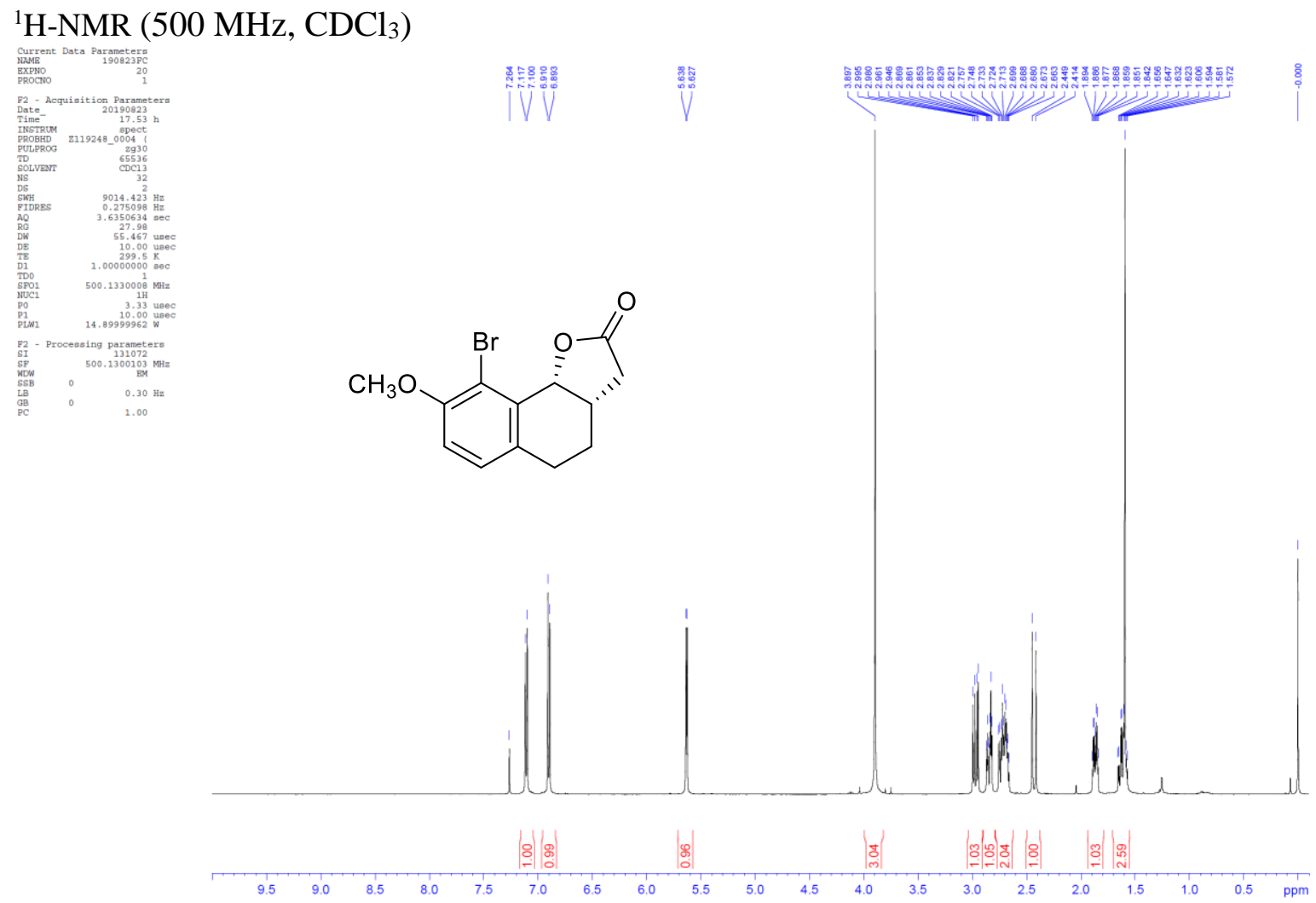

${ }^{13} \mathrm{C}$ NMR $\left(125 \mathrm{MHz}, \mathrm{CDCl}_{3}\right)$
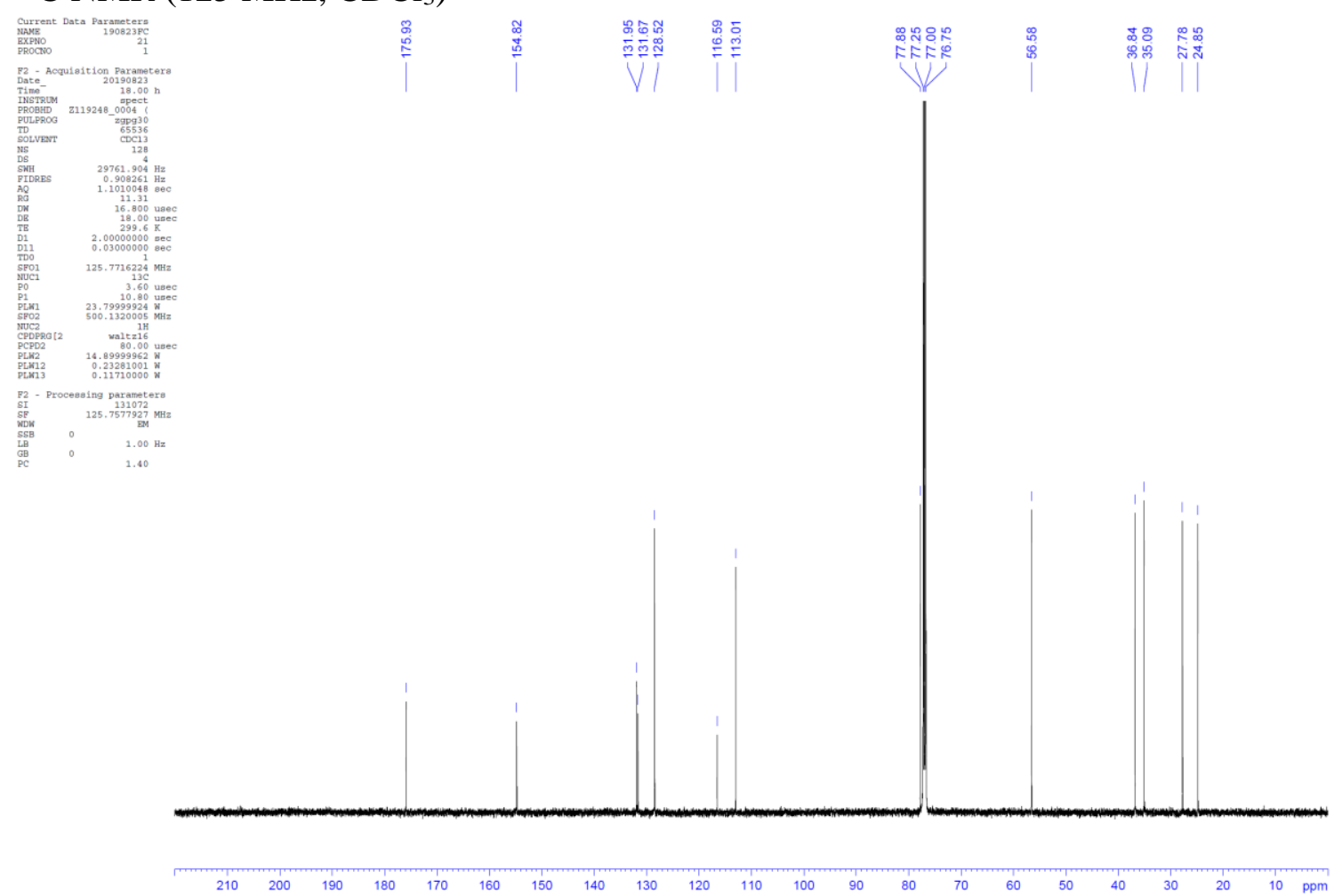
(3aS,9bR)-7-Hydroxy-3a,4,5,9b-tetrahydronaphtho[1,2-b]furan-2(3H)-one (13f)

${ }^{1} \mathrm{H}-\mathrm{NMR}\left(400 \mathrm{MHz}, \mathrm{CD}_{3} \mathrm{OD}\right)$

170919-1

$\begin{array}{ll}\text { Sample Name } & 170919-1 \\ \text { Date collected } 2017-09-19\end{array}$

Pulse sequence PROTON
Solvent cd3od

Temperature 25

$\begin{array}{ll}\text { Study Owner vnmr1 } & \\ \text { Opilent-NMR-vnmrs400 } & \end{array}$

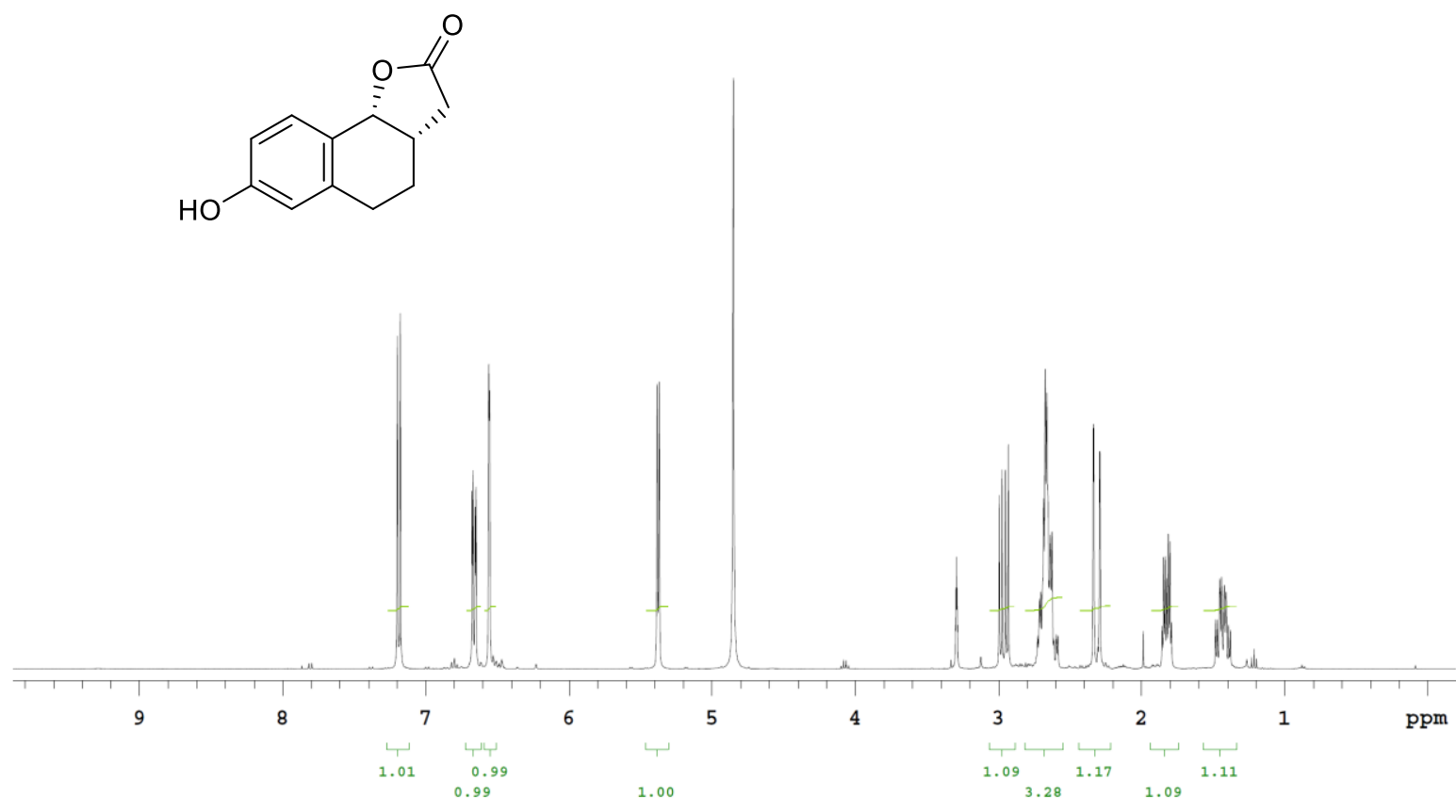

${ }^{13} \mathrm{C}$ NMR $\left(125 \mathrm{MHz}, \mathrm{CD}_{3} \mathrm{OD}\right)$
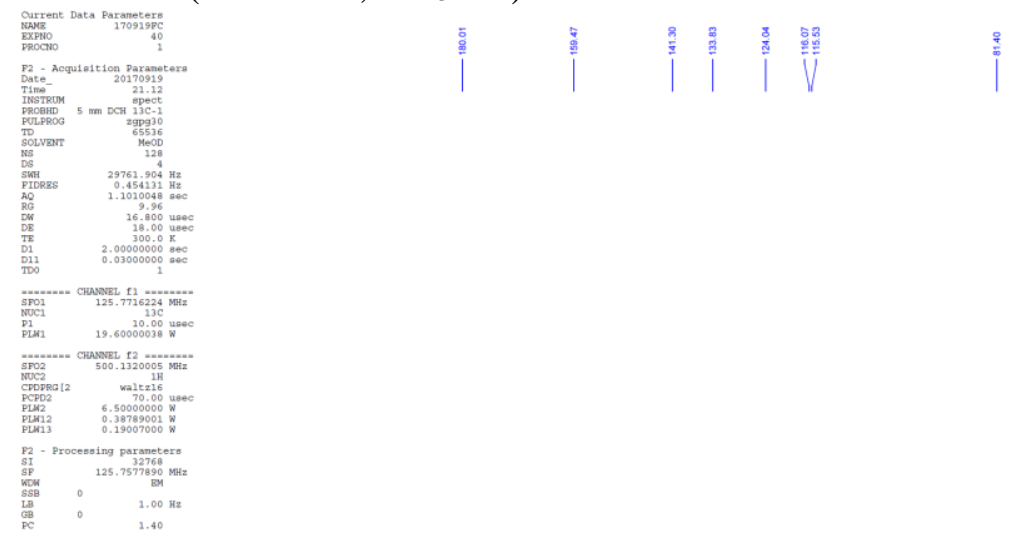

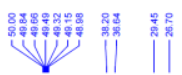

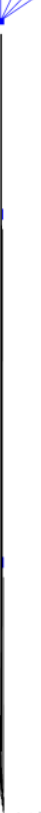

$\begin{array}{llllllll}220 & 210 & 200 & 190 & 180 & 170 & 160 & 150\end{array}$

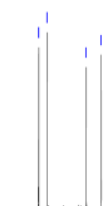


(3aS,8bR)-3,3a,4,8b-Tetrahydro-2H-indeno[1,2-b]furan-2-one (13g)

${ }^{1} \mathrm{H}-\mathrm{NMR}\left(400 \mathrm{MHz}, \mathrm{CDCl}_{3}\right)$

$170821-2$

Sample Name 170821-2

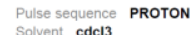

Temperature 25
Spectrometer Agilent-NMR-vnmrs400

Study owner vnmr1
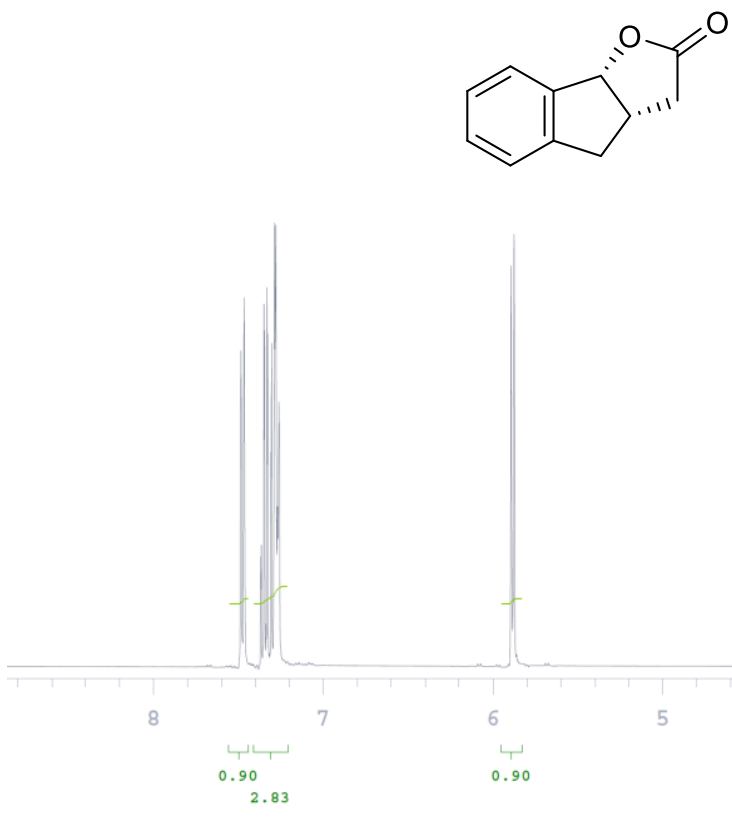

${ }^{13} \mathrm{C} \mathrm{NMR}\left(125 \mathrm{MHz}, \mathrm{CDCl}_{3}\right)$
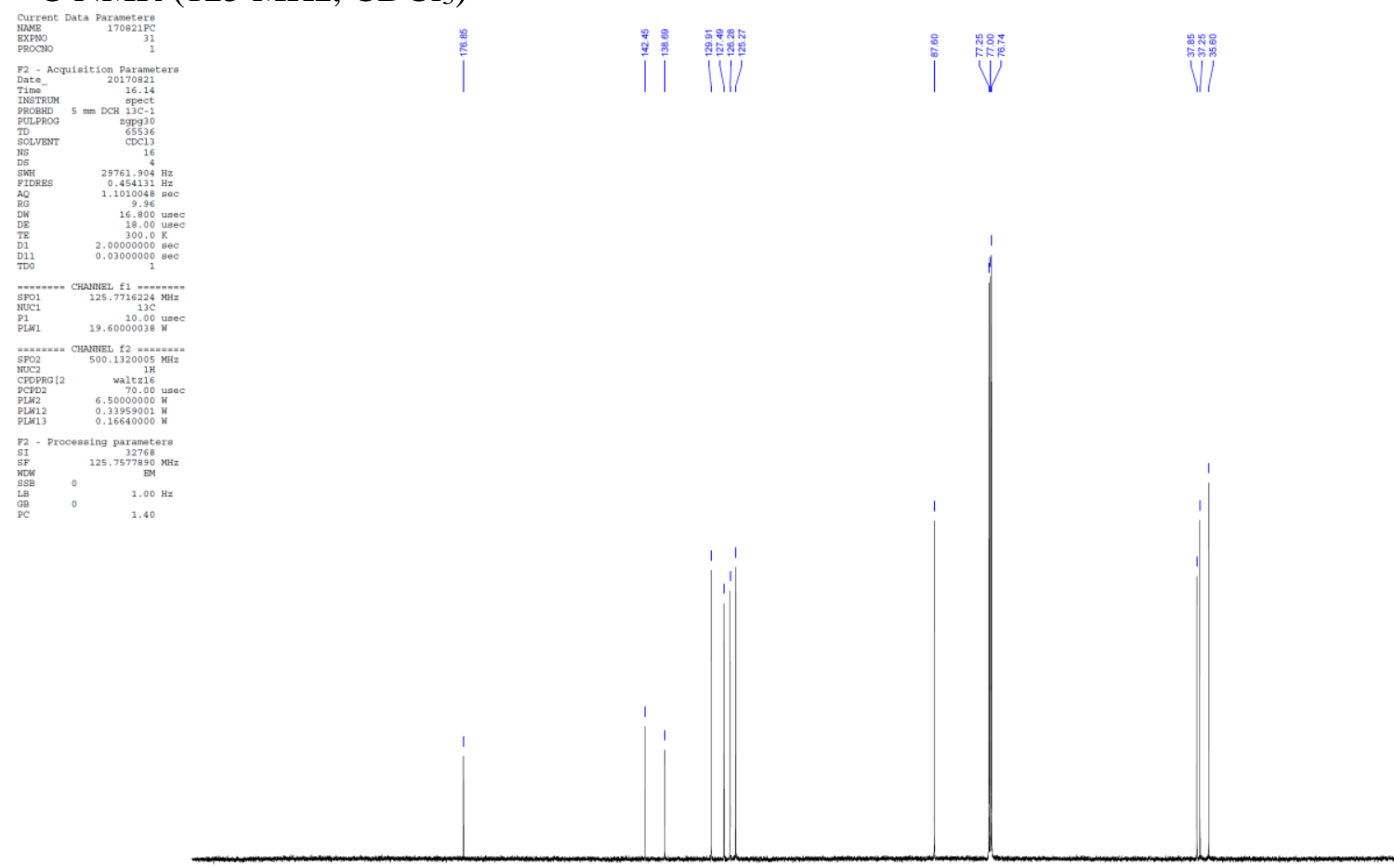

$\begin{array}{llllllll}220 & 210 & 200 & 190 & 180 & 170 & 160 & 150\end{array}$ 
(3aS,8bR)-6,7-Dimethoxy-3,3a,4,8b-tetrahydro-2H-indeno[1,2-b]furan-2-one (13h)

${ }^{1} \mathrm{H}-\mathrm{NMR}\left(400 \mathrm{MHz}, \mathrm{CDCl}_{3}\right)$

$170907-2$
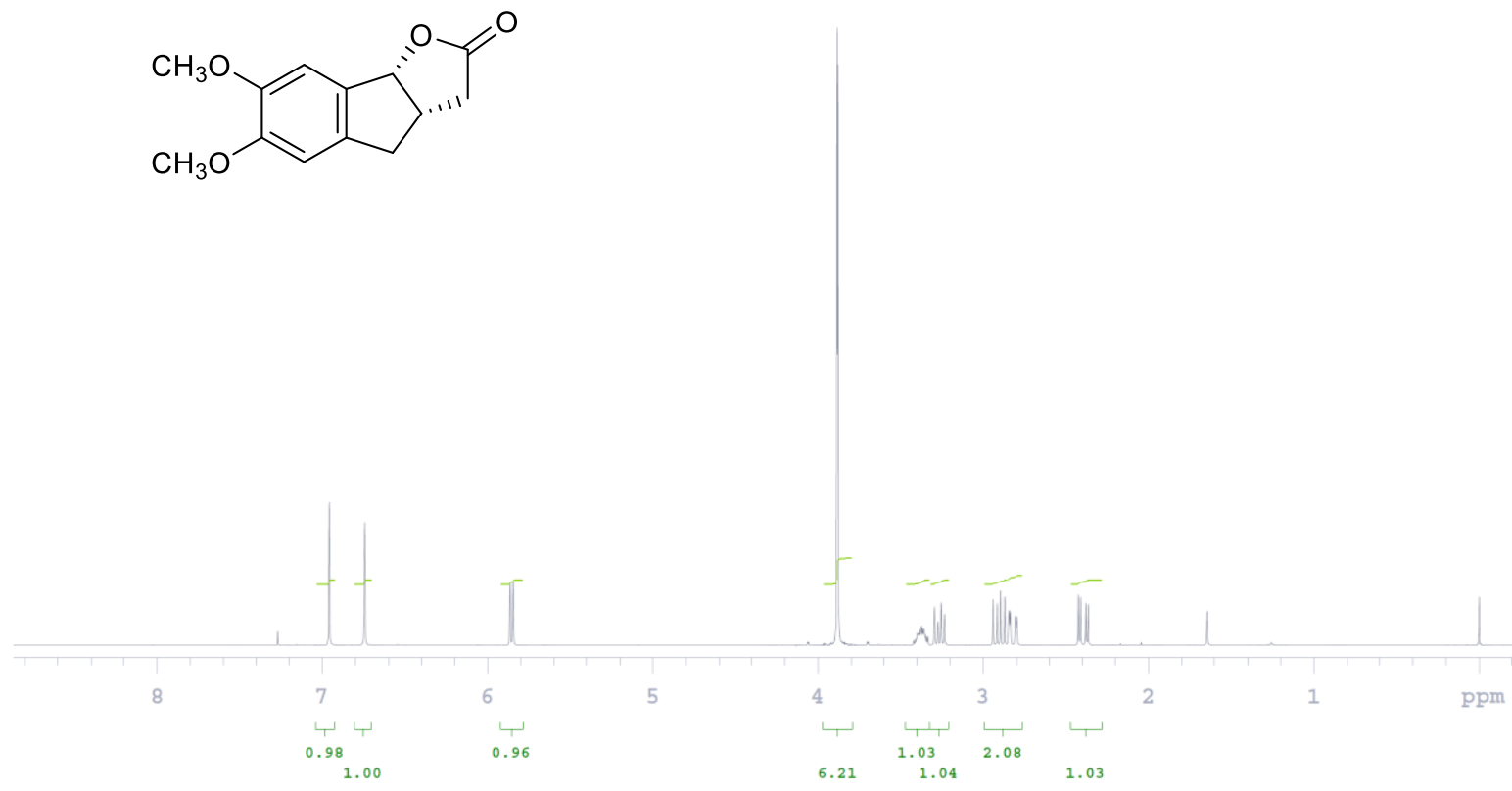

${ }^{13} \mathrm{C} \mathrm{NMR}\left(125 \mathrm{MHz}, \mathrm{CDCl}_{3}\right)$
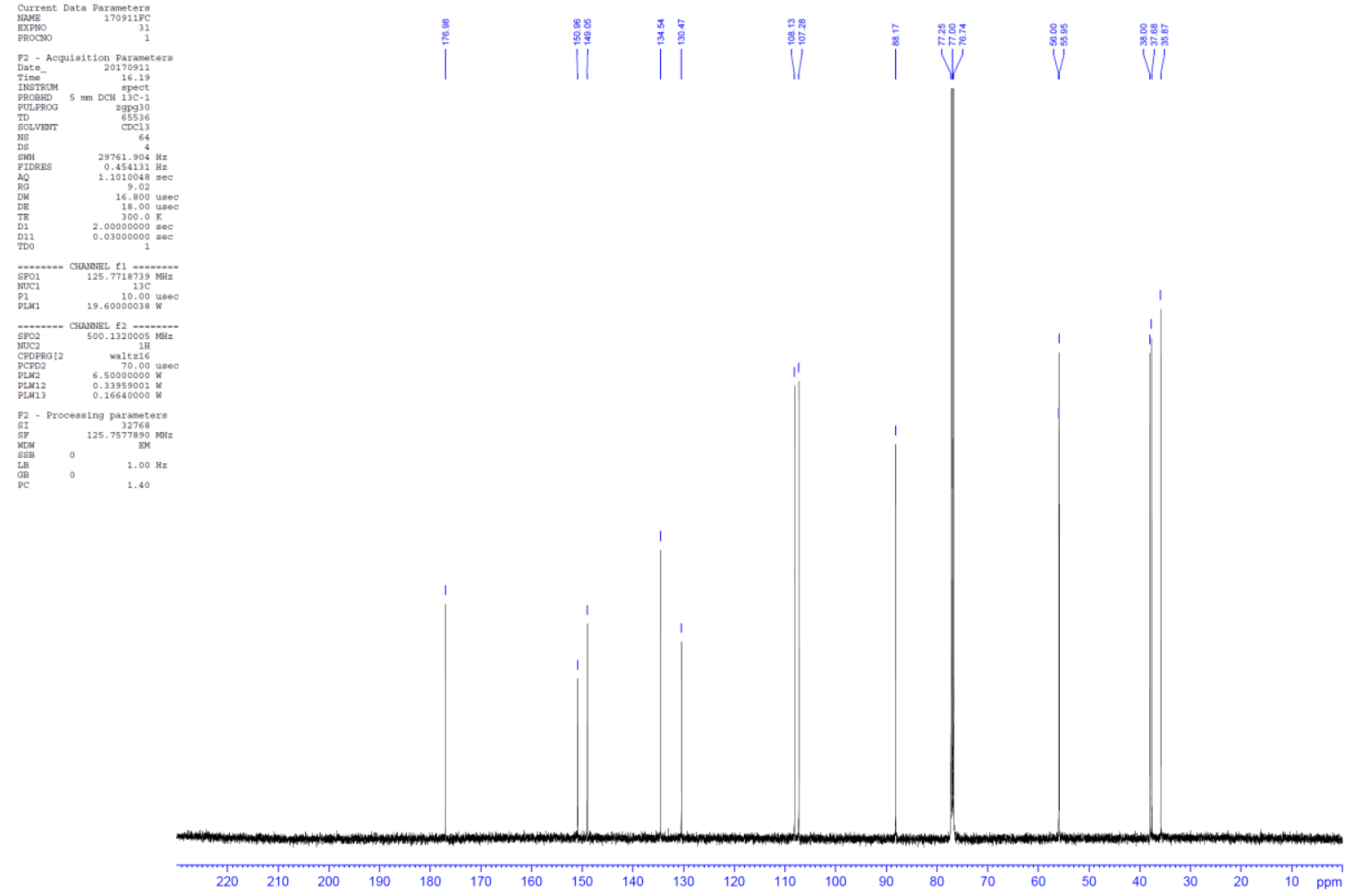
(3aS,8bR)-7-Fluoro-3,3a,4,8b-tetrahydro-2H-indeno[1,2-b]furan-2-one (13i)

${ }^{1} \mathrm{H}-\mathrm{NMR}\left(400 \mathrm{MHz}, \mathrm{CDCl}_{3}\right)$

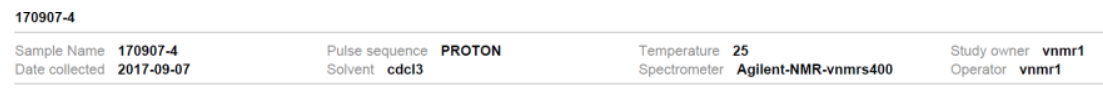

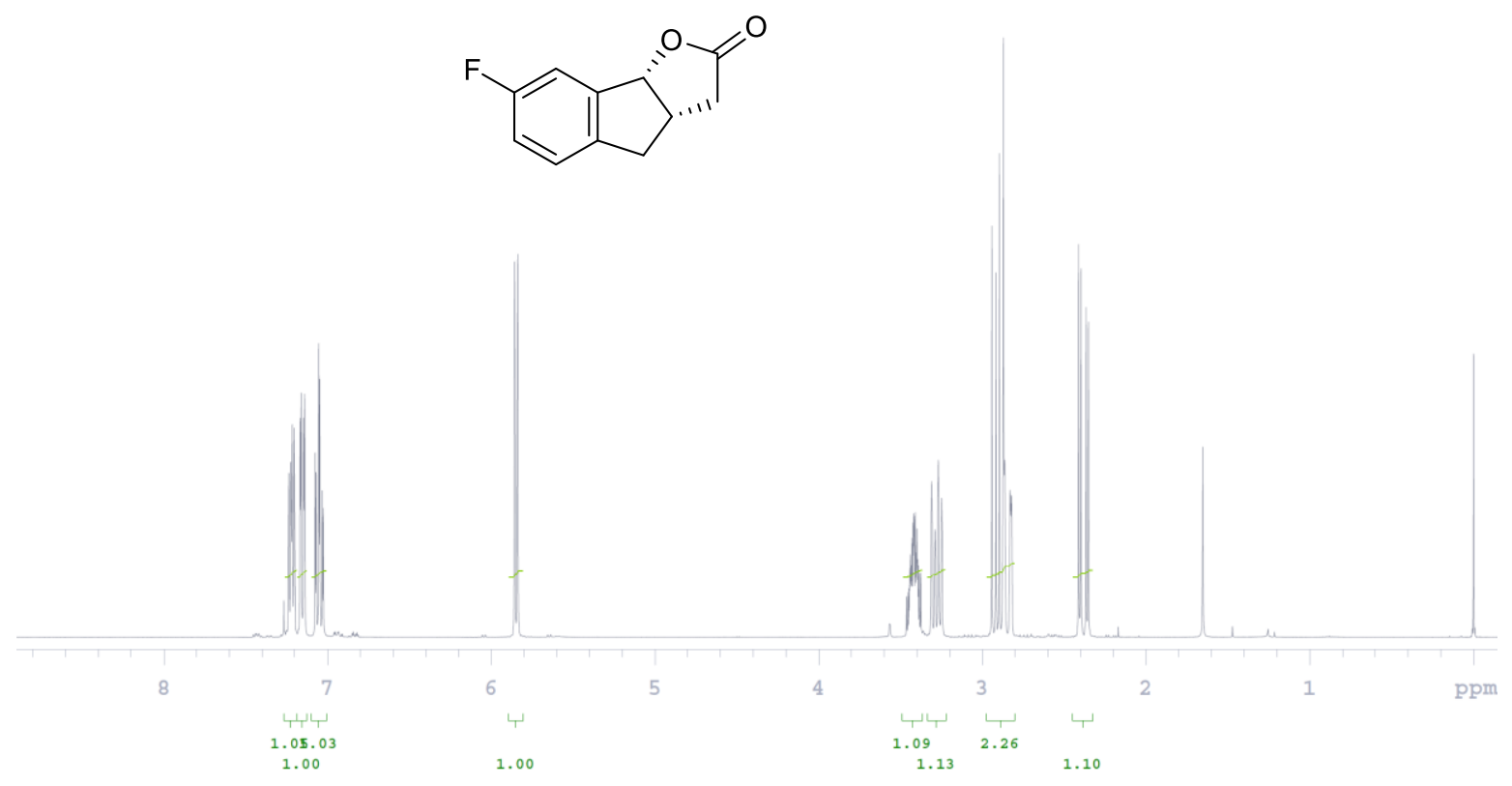

${ }^{13} \mathrm{C}$ NMR (125 MHz, $\left.\mathrm{CDCl}_{3}\right)$
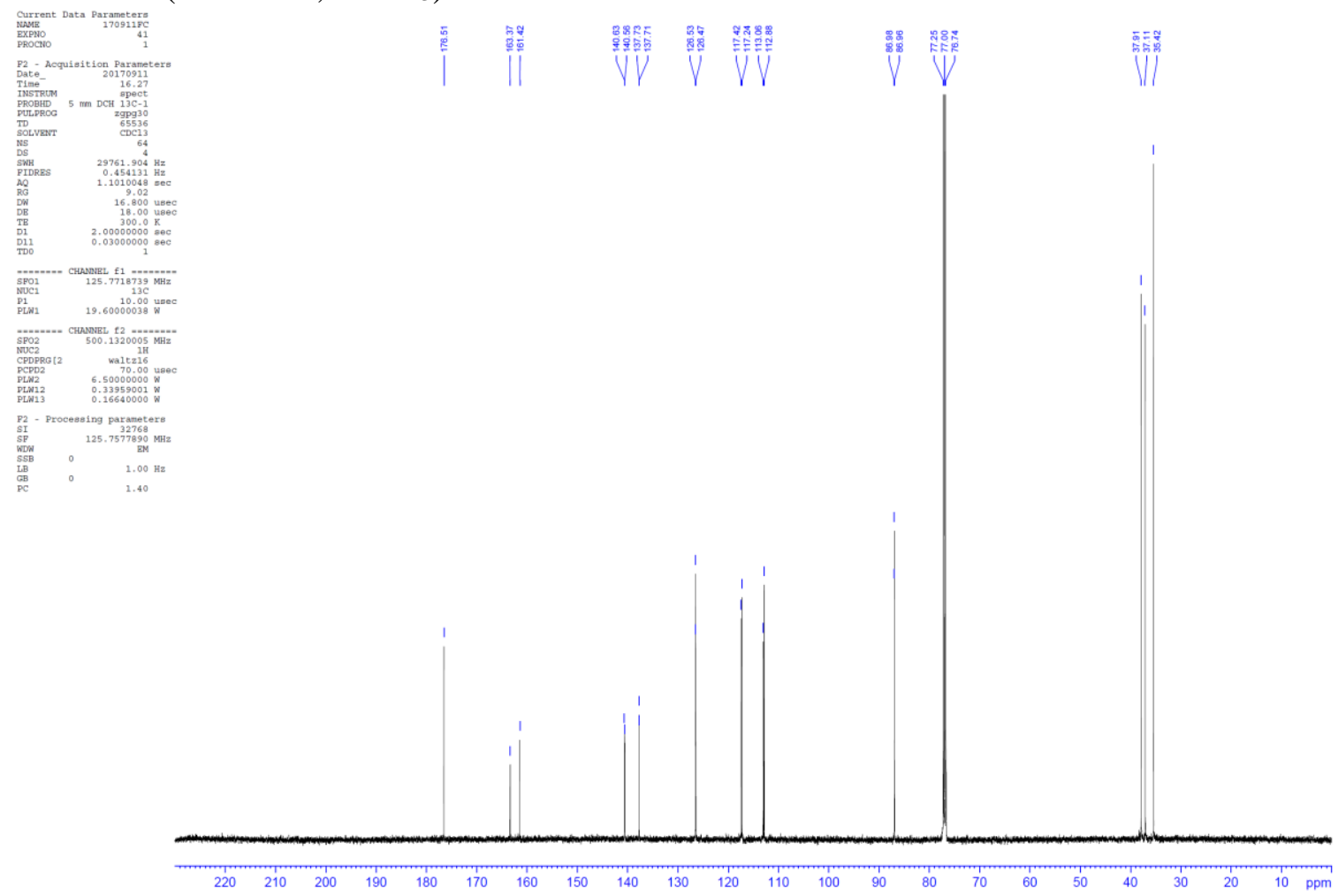

S122 
(3aS,8bR)-7-Chloro-3,3a,4,8b-tetrahydro-2H-indeno[1,2-b]furan-2-one (13j)

${ }^{1} \mathrm{H}-\mathrm{NMR}\left(400 \mathrm{MHz}, \mathrm{CDCl}_{3}\right)$

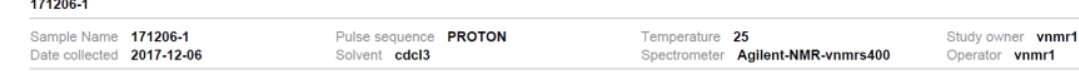
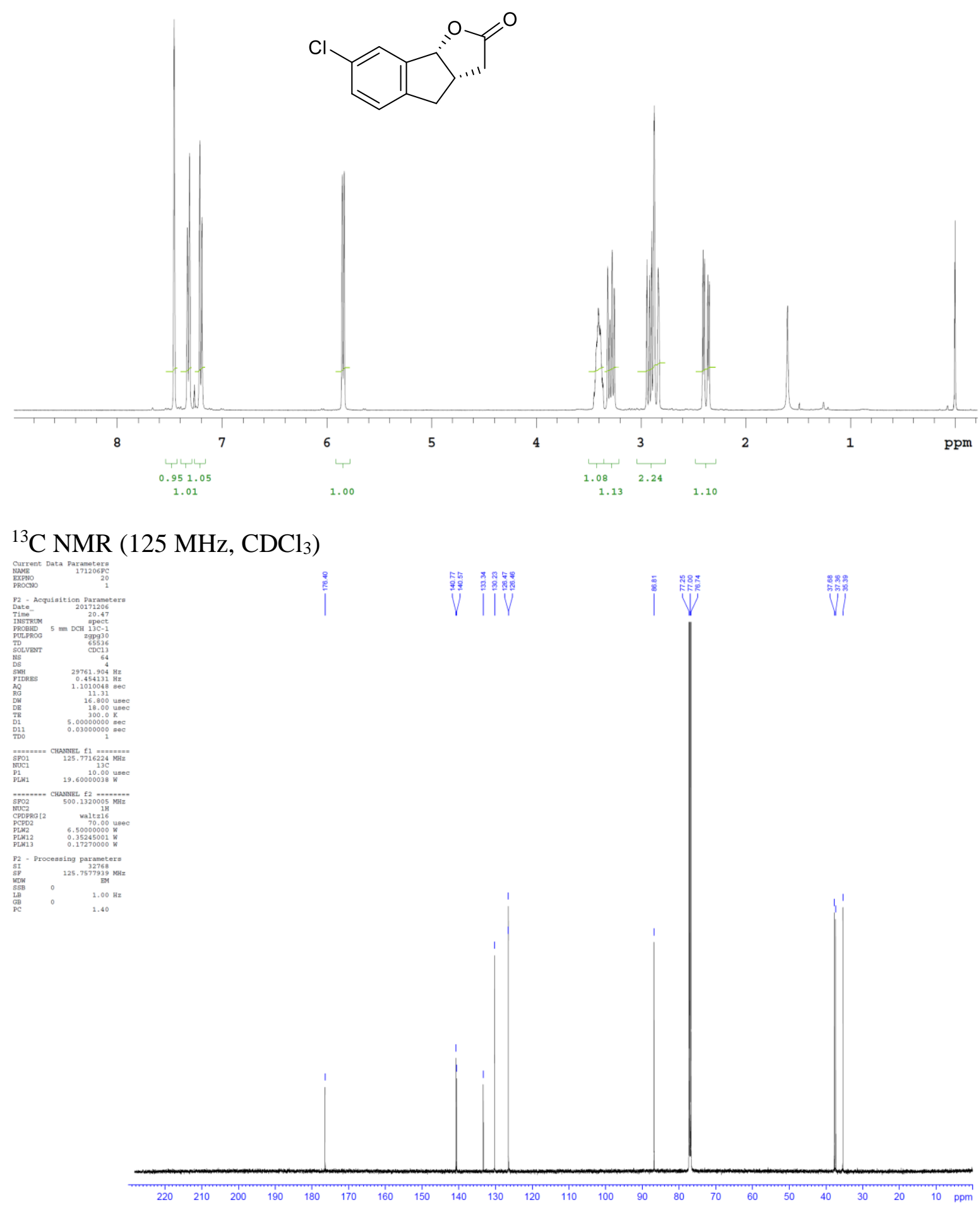

S123 
$(3 \mathrm{a} S, 10 \mathrm{~b} R)-3,3 \mathrm{a}, 4,5,6,10 \mathrm{~b}-H e x a h y d r o-2 H$-benzo[6,7]cyclohepta[1,2-b]furan-2-one (13k)

${ }^{1} \mathrm{H}-\mathrm{NMR}\left(400 \mathrm{MHz}, \mathrm{CDCl}_{3}\right)$

Sample Name $170810-3$

Pulse sequence PROTON
Solvent cdc13

Temperature 25

Study owner vnmr

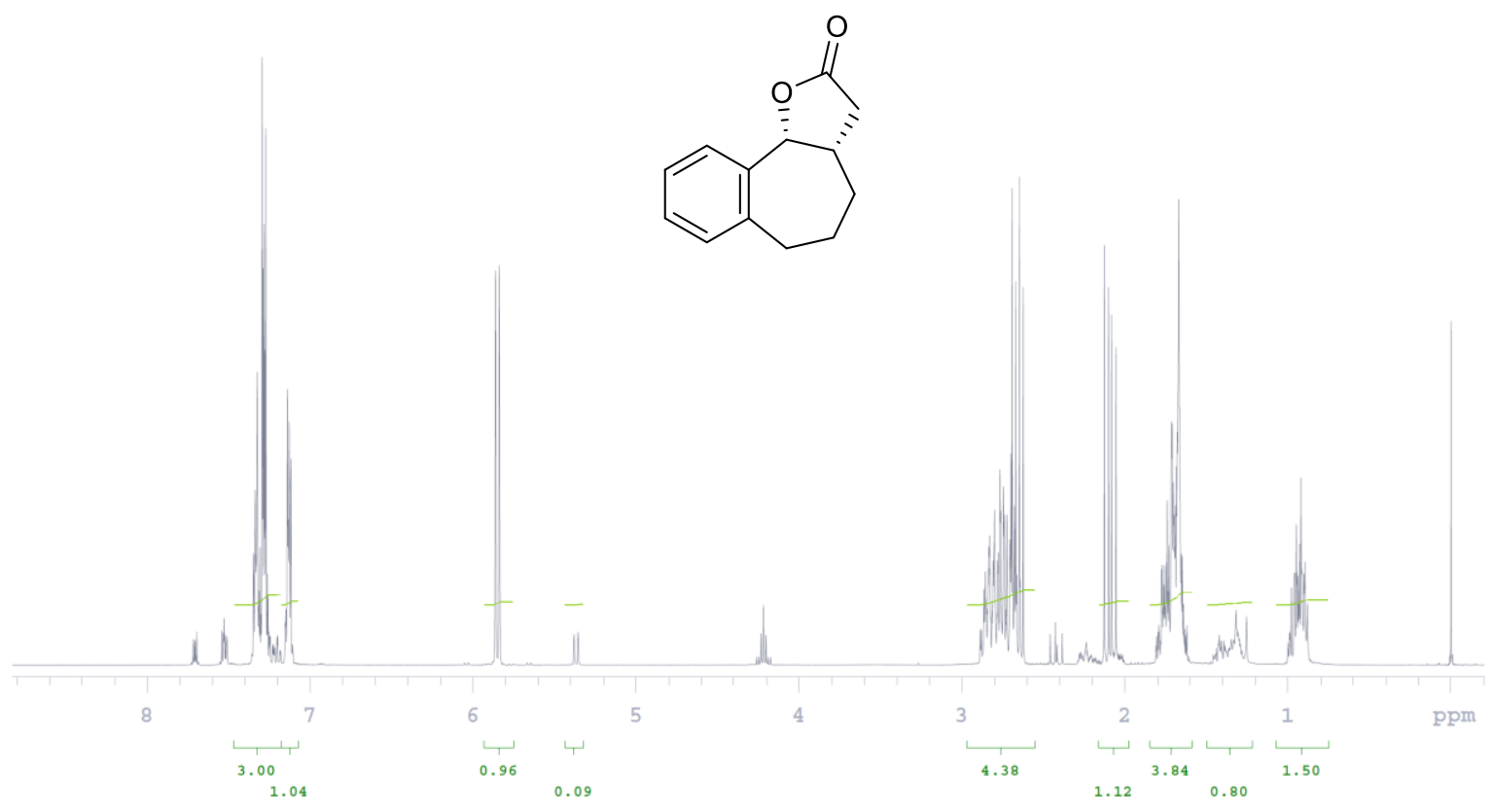

${ }^{13} \mathrm{C} \mathrm{NMR}\left(125 \mathrm{MHz}, \mathrm{CDCl}_{3}\right)$
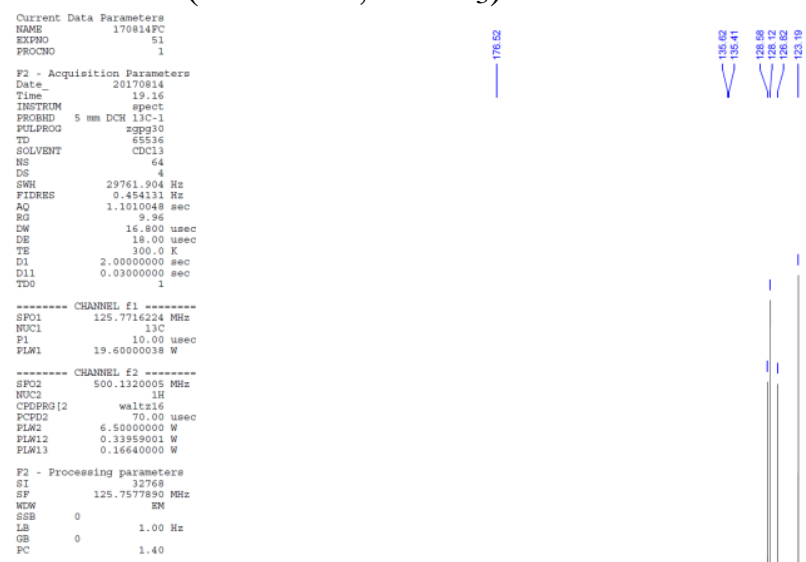

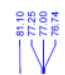

||$^{\circ}$

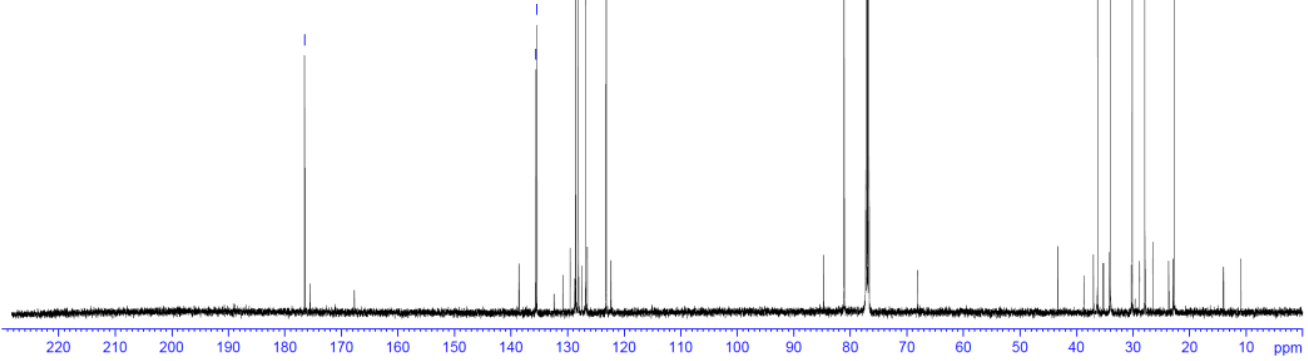

S124 
(4aS,10bR)-3,4,4a,5,6,10b-Hexahydro-2H-benzo[ $h]$ chromen-2-one (13I)

${ }^{1} \mathrm{H}-\mathrm{NMR}\left(400 \mathrm{MHz}, \mathrm{CDCl}_{3}\right)$

170823-2

$\begin{array}{ll}\text { Sample Name } & \text { 170823-2 } \\ \text { Date collected } & 2017-08-23\end{array}$

Pulse sequence PROTON
Solvent cdcl3

Temperature 25
Spectrometer
Agilent-NMR-vnmrs400

Study owner vnmr1
Operator vnmr1

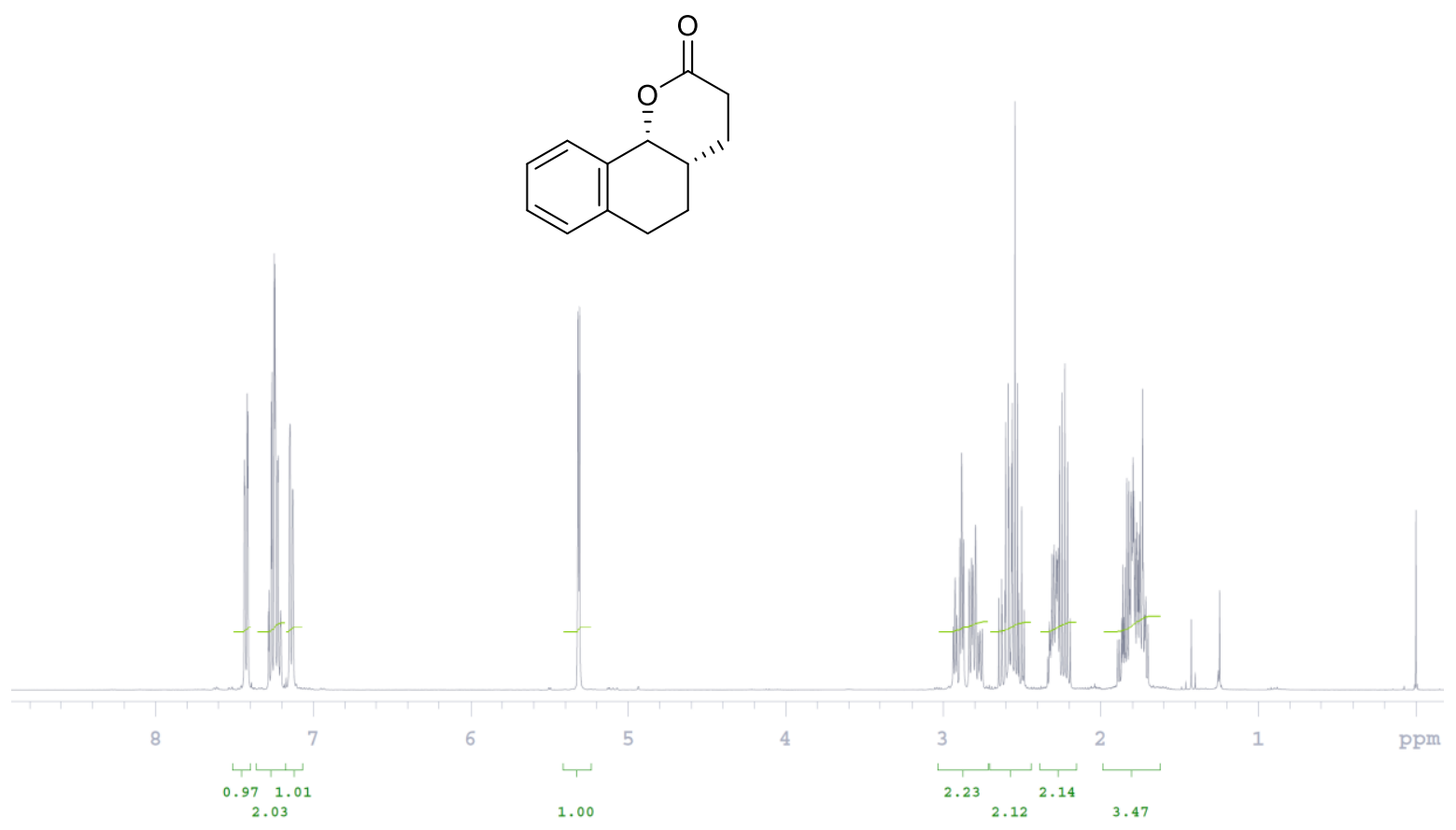

${ }^{13} \mathrm{C}$ NMR $\left(125 \mathrm{MHz}, \mathrm{CDCl}_{3}\right)$
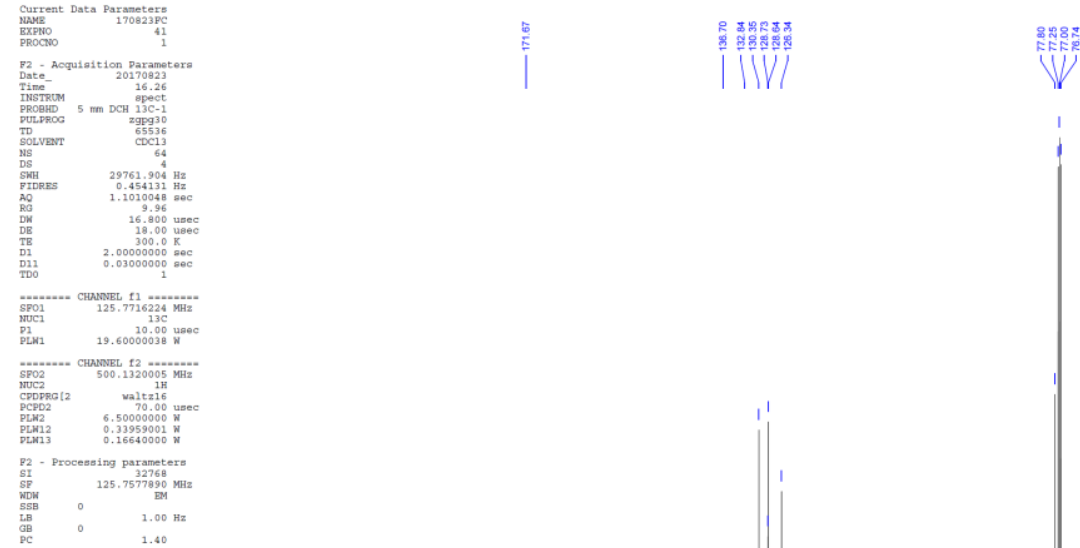

|Vi
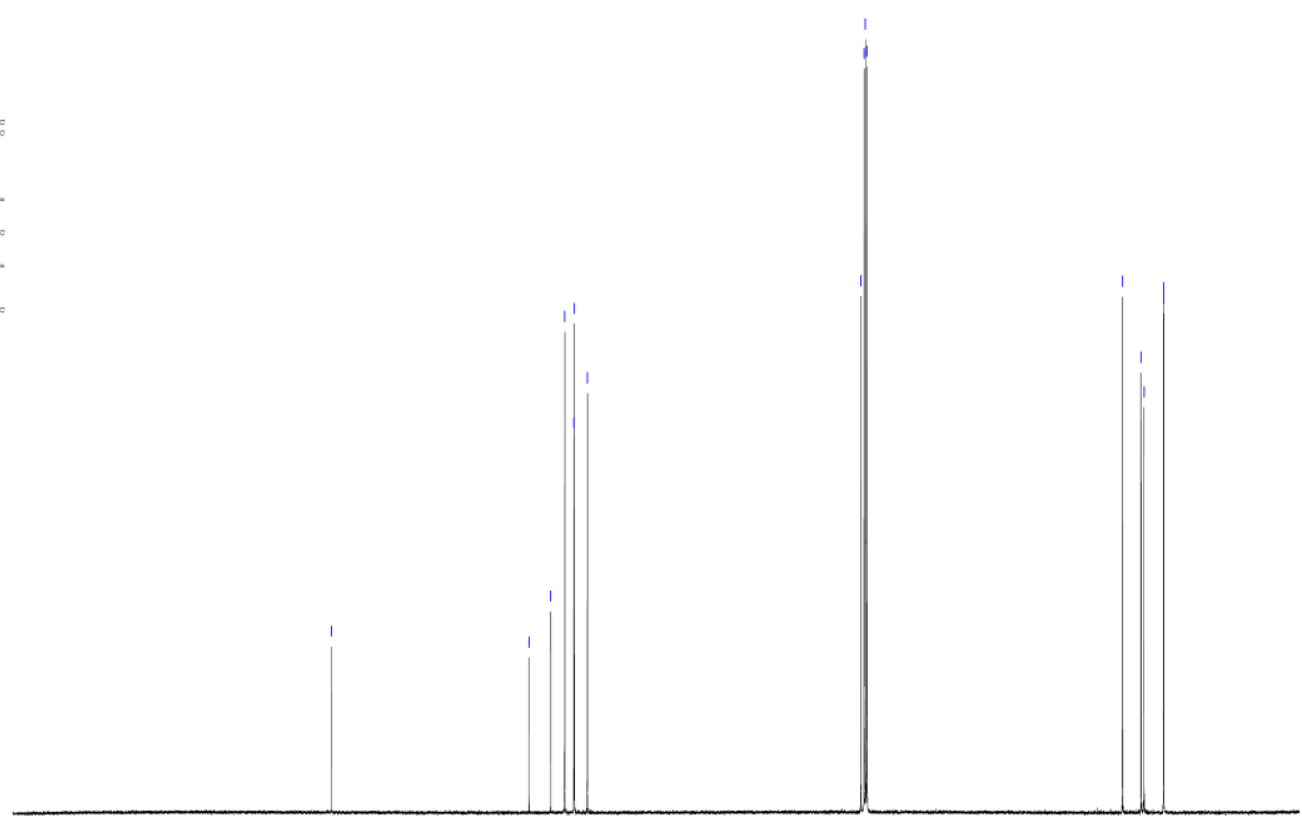

$\begin{array}{llllllll}220 & 210 & 200 & 190 & 180 & 170 & 160 & 150\end{array}$ 
(4aS,10bR)-9-Methoxy-3,4,4a,5,6,10b-hexahydro-2H-benzo[ $h]$ chromen-2-one (13m)

${ }^{1} \mathrm{H}-\mathrm{NMR}\left(400 \mathrm{MHz}, \mathrm{CDCl}_{3}\right)$

$170907-3$

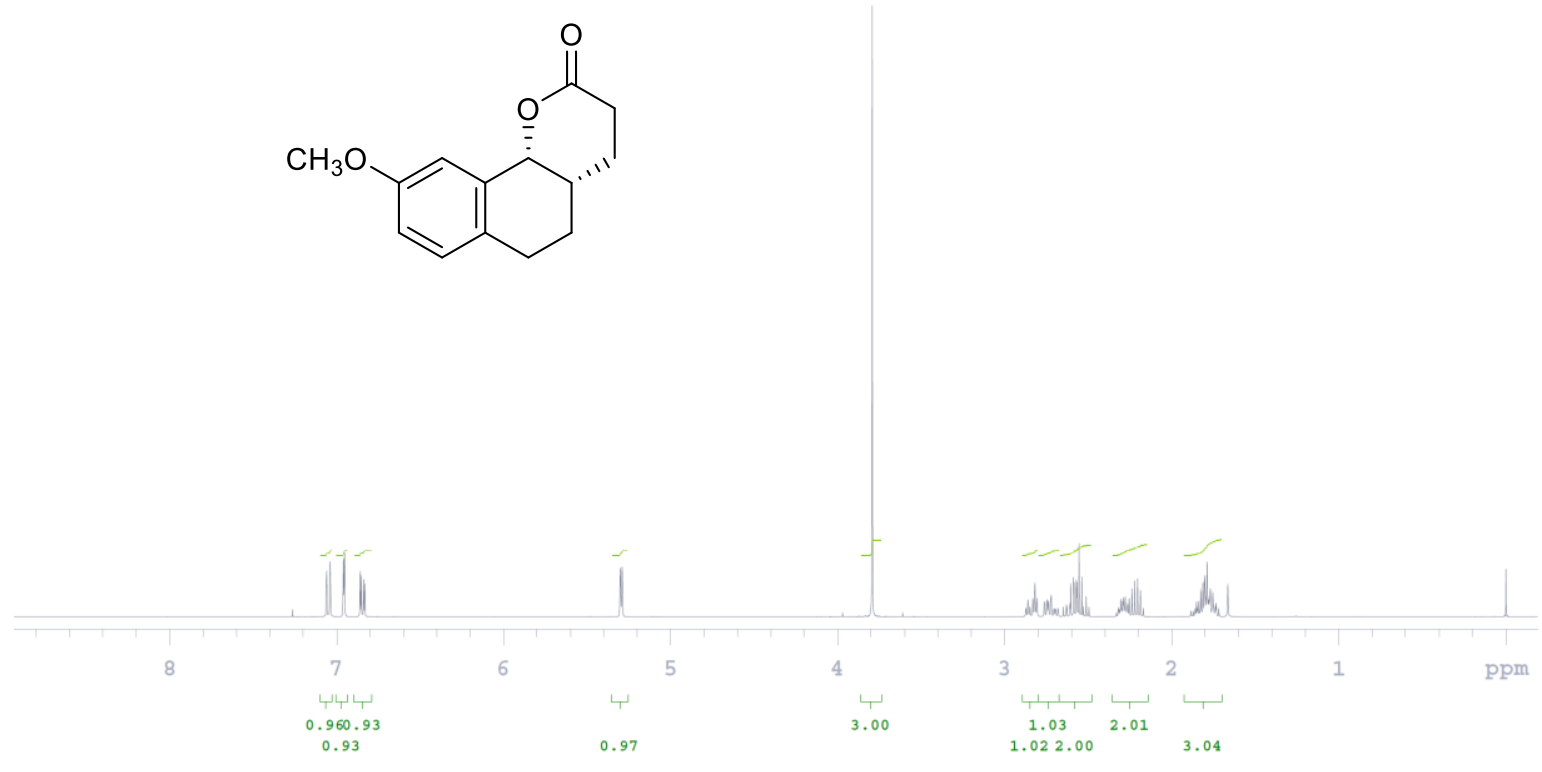

\section{${ }^{13} \mathrm{C} \mathrm{NMR}\left(125 \mathrm{MHz}, \mathrm{CDCl}_{3}\right)$}
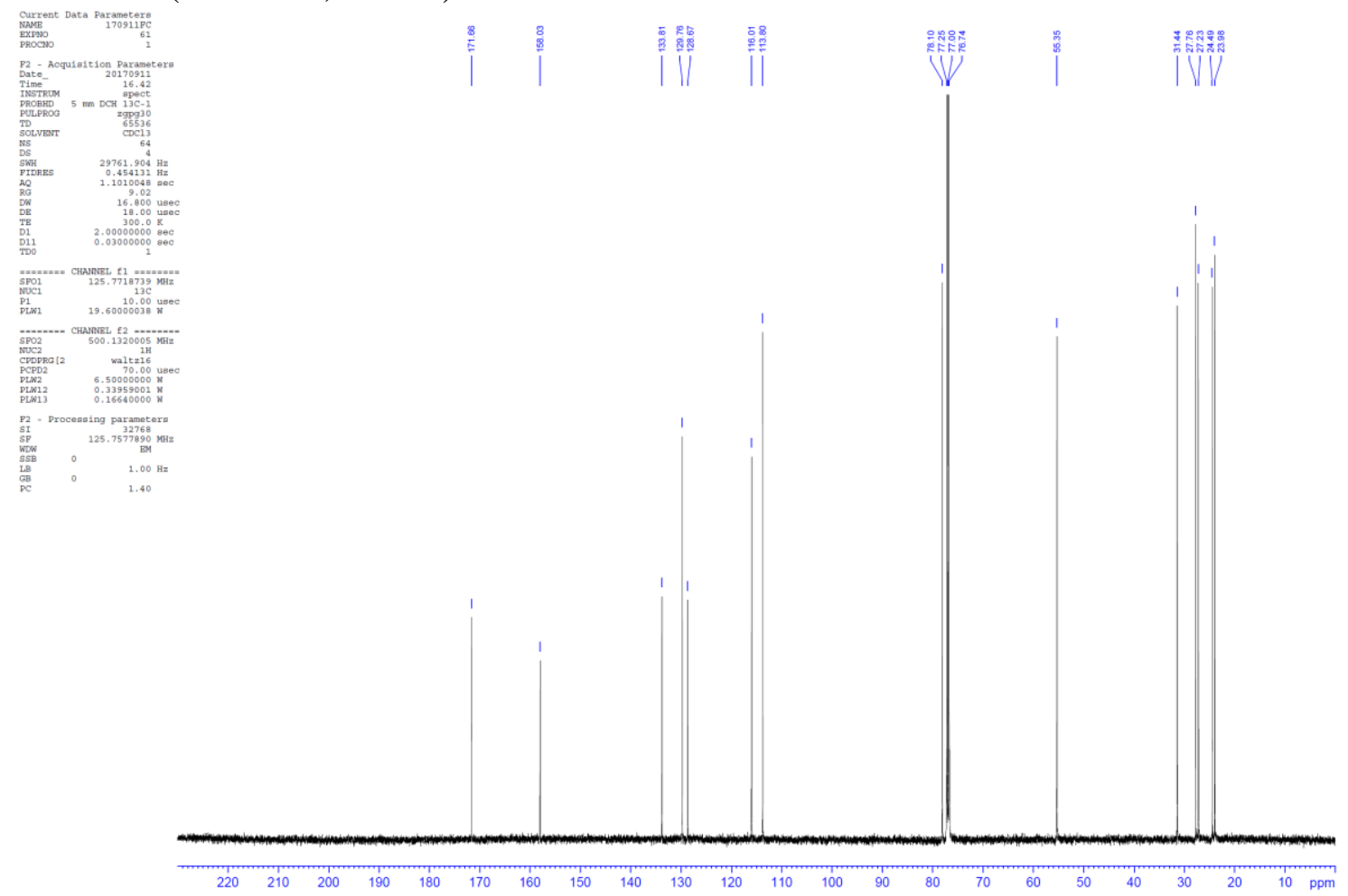
(4aS,10bR)-9-Chloro-3,4,4a,5,6,10b-hexahydro-2H-benzo[ $[h]$ chromen-2-one (13n)

${ }^{1} \mathrm{H}-\mathrm{NMR}\left(400 \mathrm{MHz}, \mathrm{CDCl}_{3}\right)$

170920-1

$\begin{array}{lll}\text { Sample Name } & \text { 170920-1 } & \text { Pulse sequence } \\ \text { Date collected } & \text { 2017-09-20 } & \text { Solvent cdcl3 }\end{array}$

Temperature
Spectrometer

Agilent-NMR-vnmrs400

Study owner vnmr1
Operator vnmr1

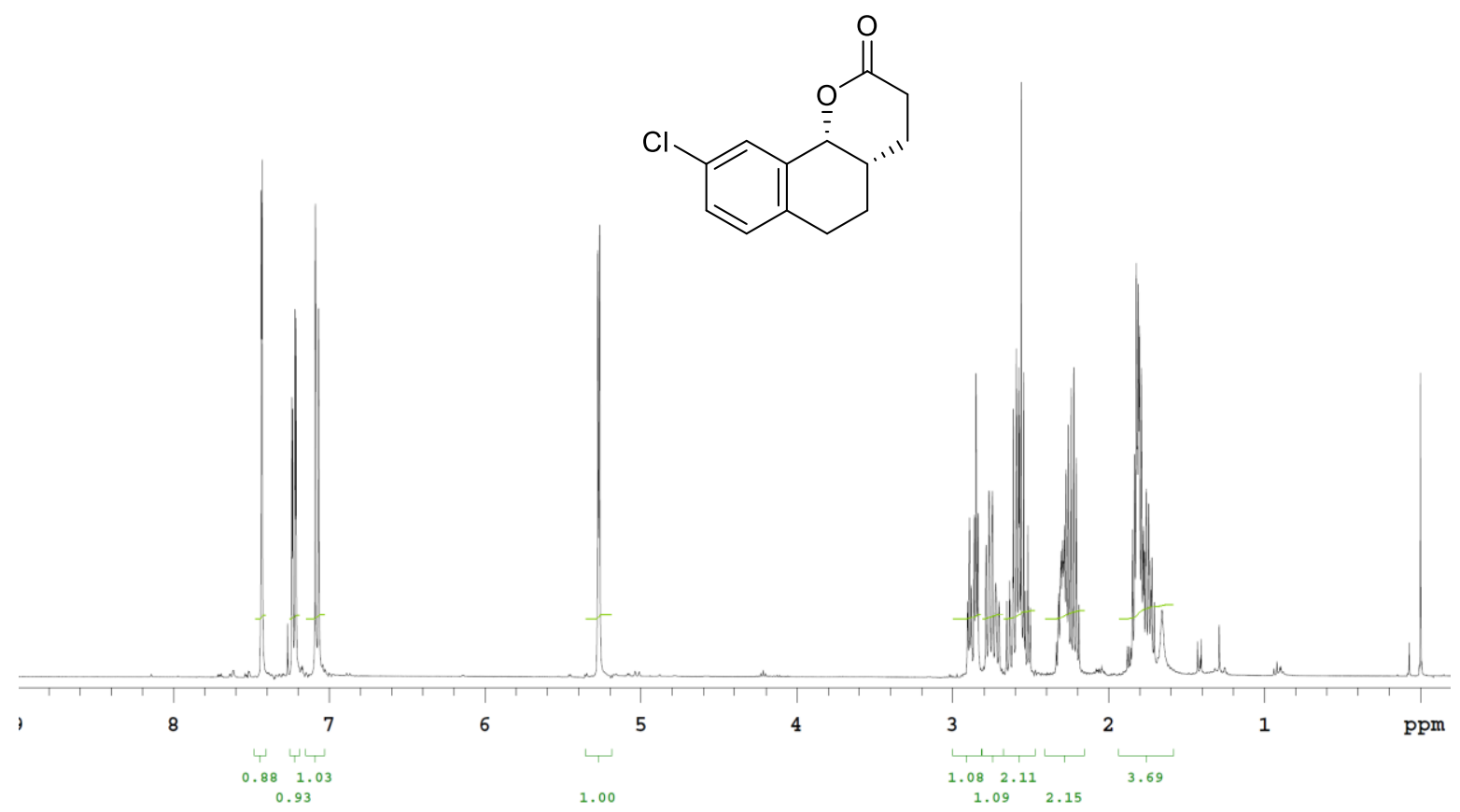

${ }^{13} \mathrm{C}$ NMR $\left(125 \mathrm{MHz}, \mathrm{CDCl}_{3}\right)$
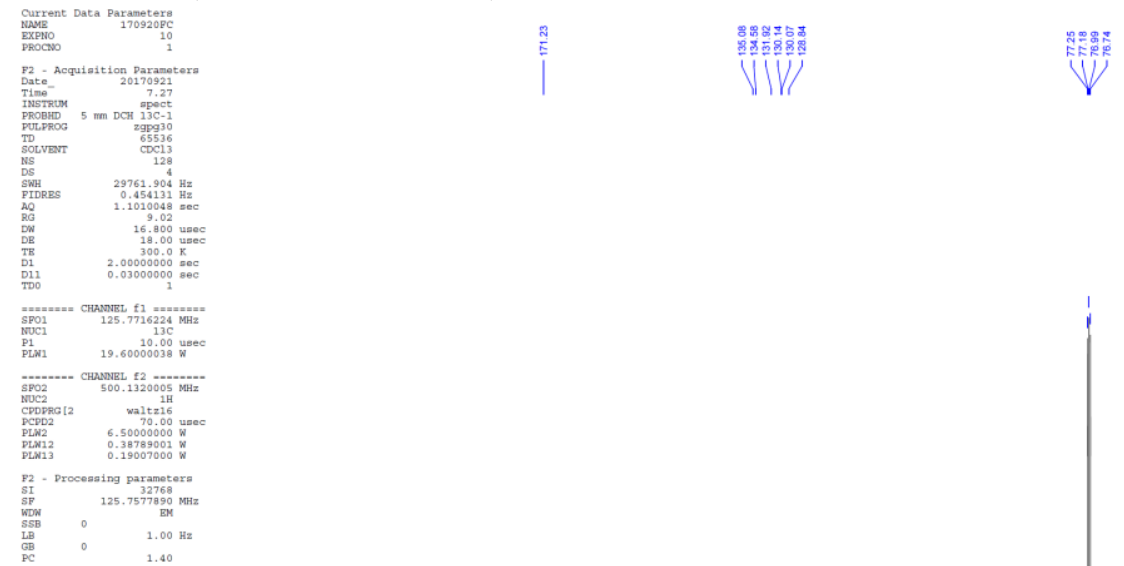

|Vบ

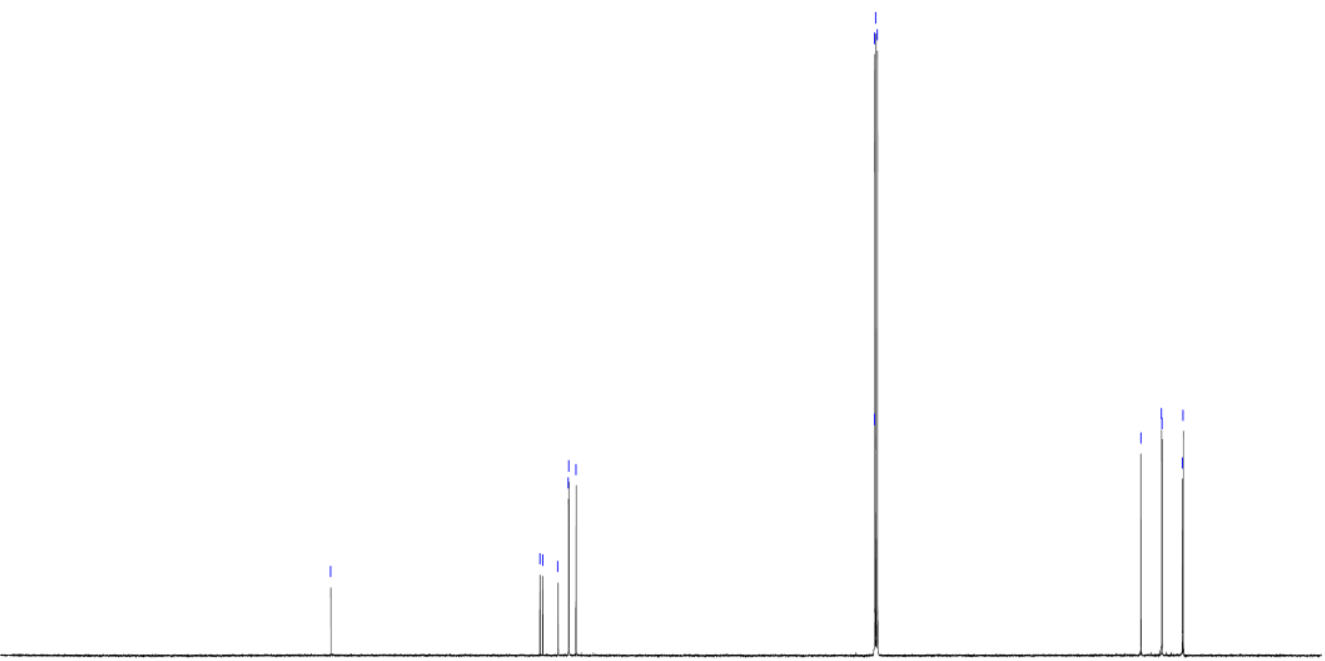


$(3 \mathrm{a}, 9 \mathrm{~b} R)-3 \mathrm{a}, 4,5,9 \mathrm{~b}-$-Tetrahydronaphtho[1,2-b]furan-2(3H)-one (13o)

${ }^{1} \mathrm{H}-\mathrm{NMR}\left(400 \mathrm{MHz}, \mathrm{CDCl}_{3}\right)$

Sample Name $170823-4$

Pulse sequence PROTON
Solvent cdc13

Temperature 25
Spectrometer Agilent-NMR-vnmrs400

Study owner vnmr1
Operator vnmr1

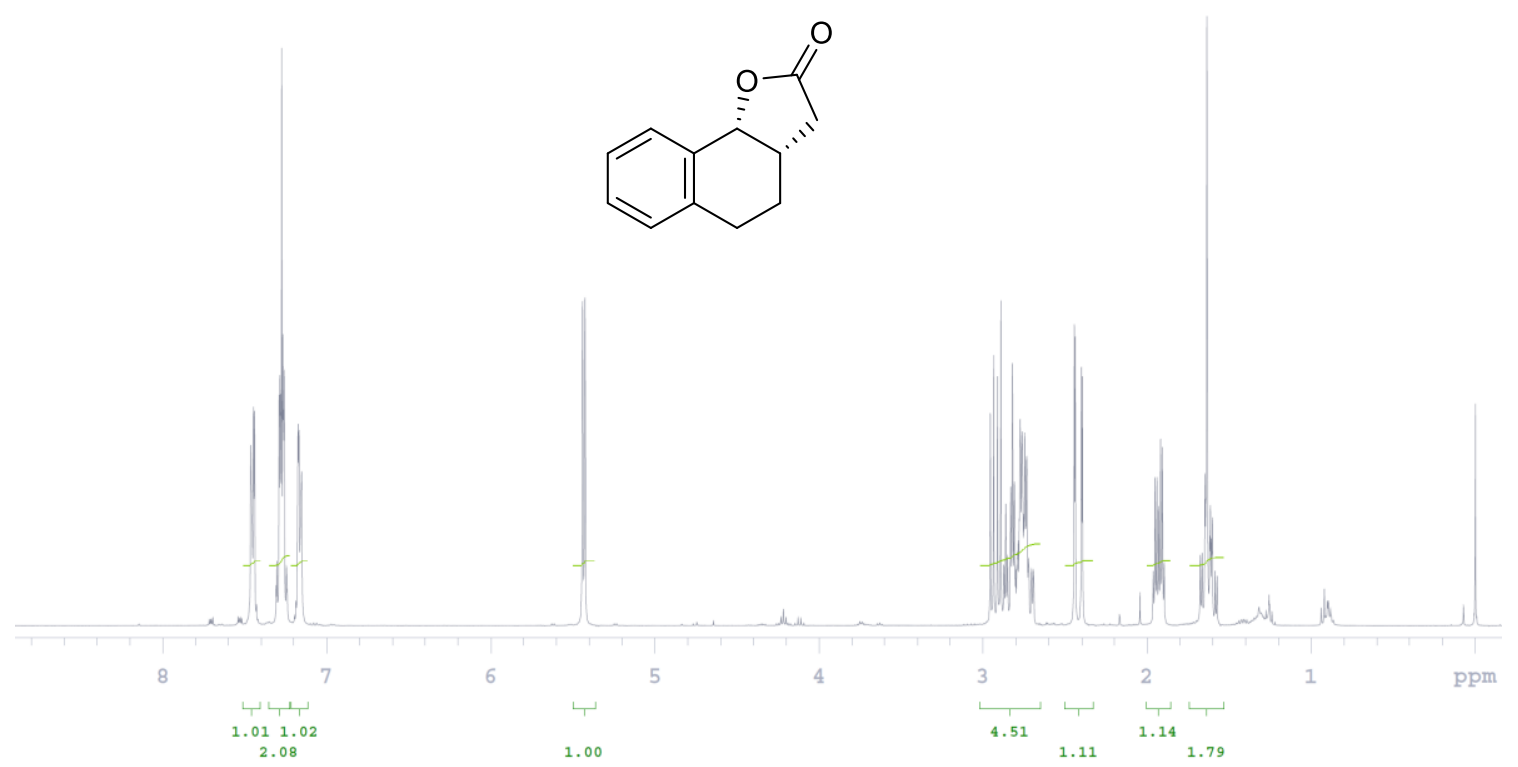

${ }^{13} \mathrm{C}$ NMR $\left(125 \mathrm{MHz}, \mathrm{CDCl}_{3}\right)$
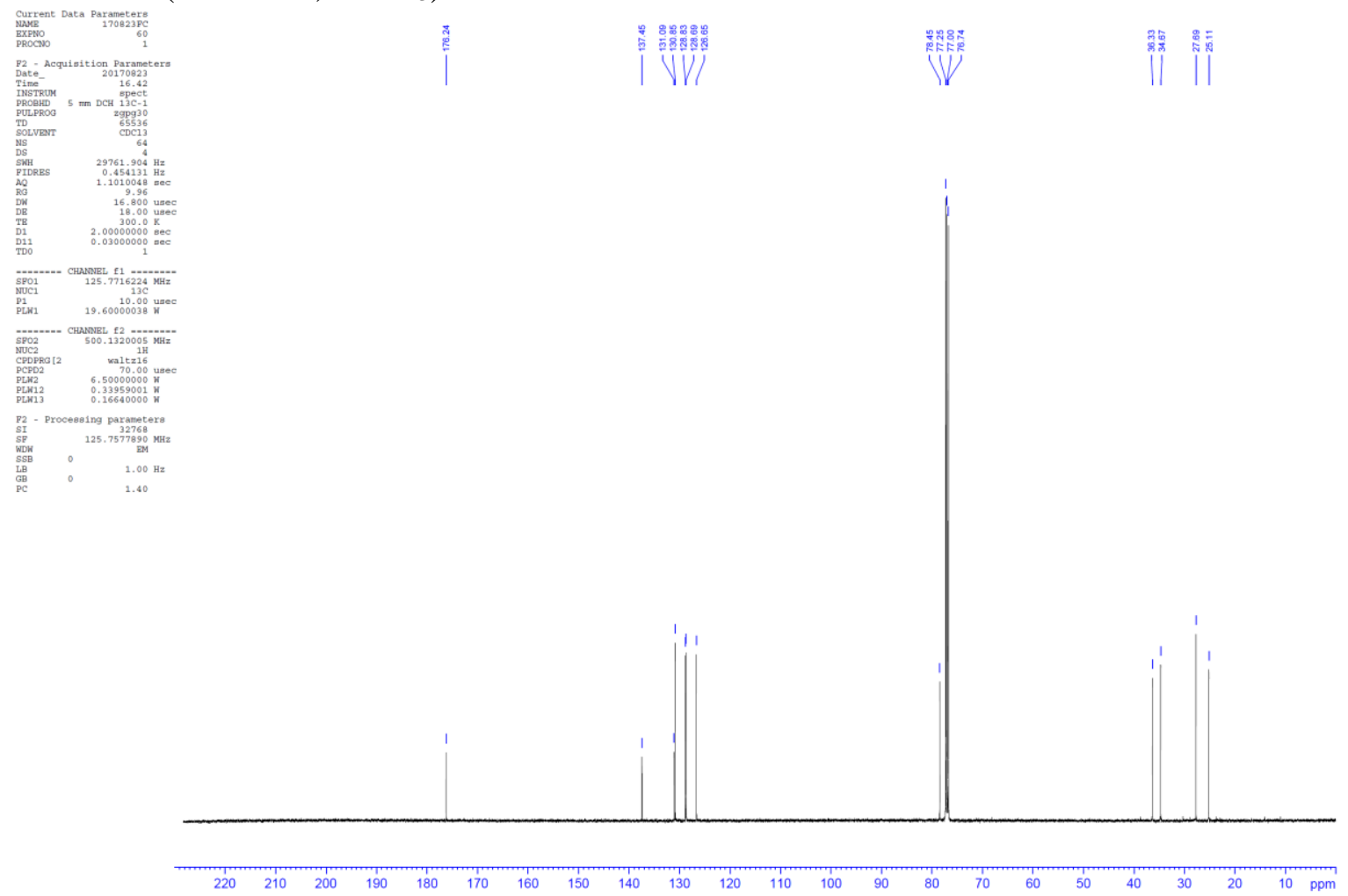

S128 
(3aR,9bS)-6-Methoxy-3a,4,5,9b-tetrahydronaphtho[2,1-b]furan-2(1H)-one (13p)

${ }^{1} \mathrm{H}-\mathrm{NMR}\left(400 \mathrm{MHz}, \mathrm{CDCl}_{3}\right)$

170908-3<smiles>COc1cccc2c1CC[C@@H]1OC(=O)C[C@H]21</smiles>
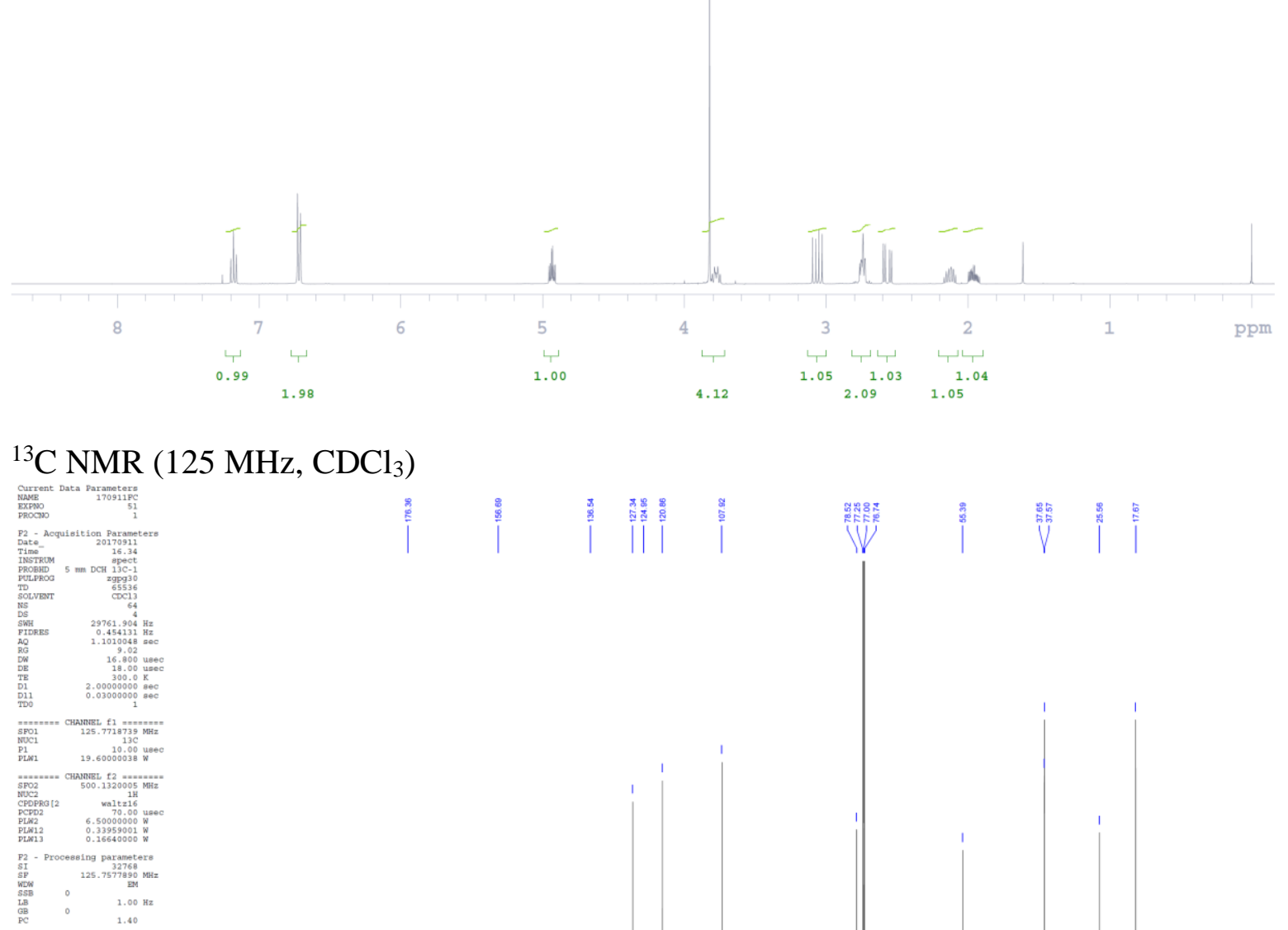

${ }^{13} \mathrm{C} \mathrm{NMR}\left(125 \mathrm{MHz}, \mathrm{CDCl}_{3}\right)$
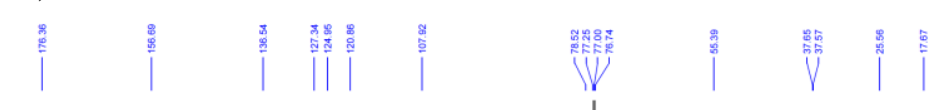

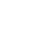

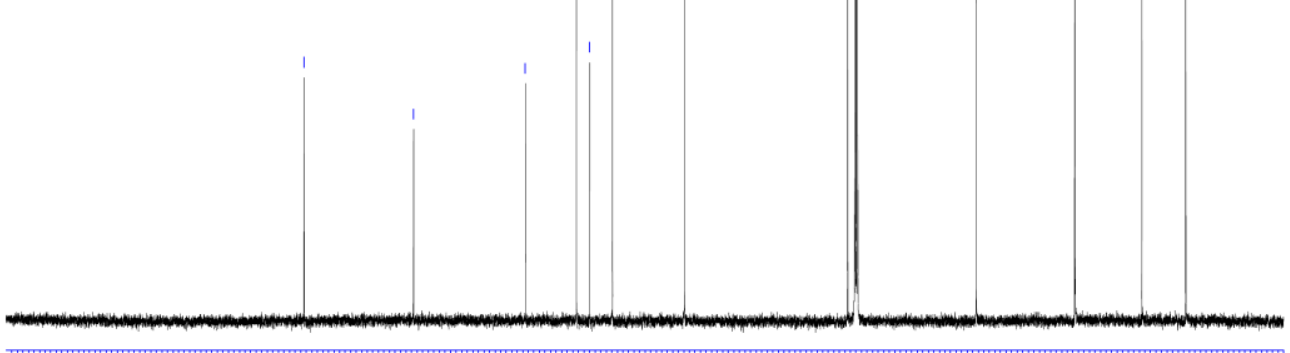


(3aR,9bS)-7-Bromo-3a,4,5,9b-tetrahydronaphtho[2,1-b]furan-2(1H)-one (13q)

${ }^{1} \mathrm{H}-\mathrm{NMR}\left(400 \mathrm{MHz}, \mathrm{CDCl}_{3}\right)$

171206-3

$\begin{array}{ll}\text { Sample Name } & \text { 171206-3 } \\ \text { Date collected } & \text { 2017-12-06 }\end{array}$

Pulse sequence PROTON
Solvent cdcl3

Temperature 25
Spectrometer

Agilent-NMR-vnmrs400

Study owner vnmr1
Operator vnmr1

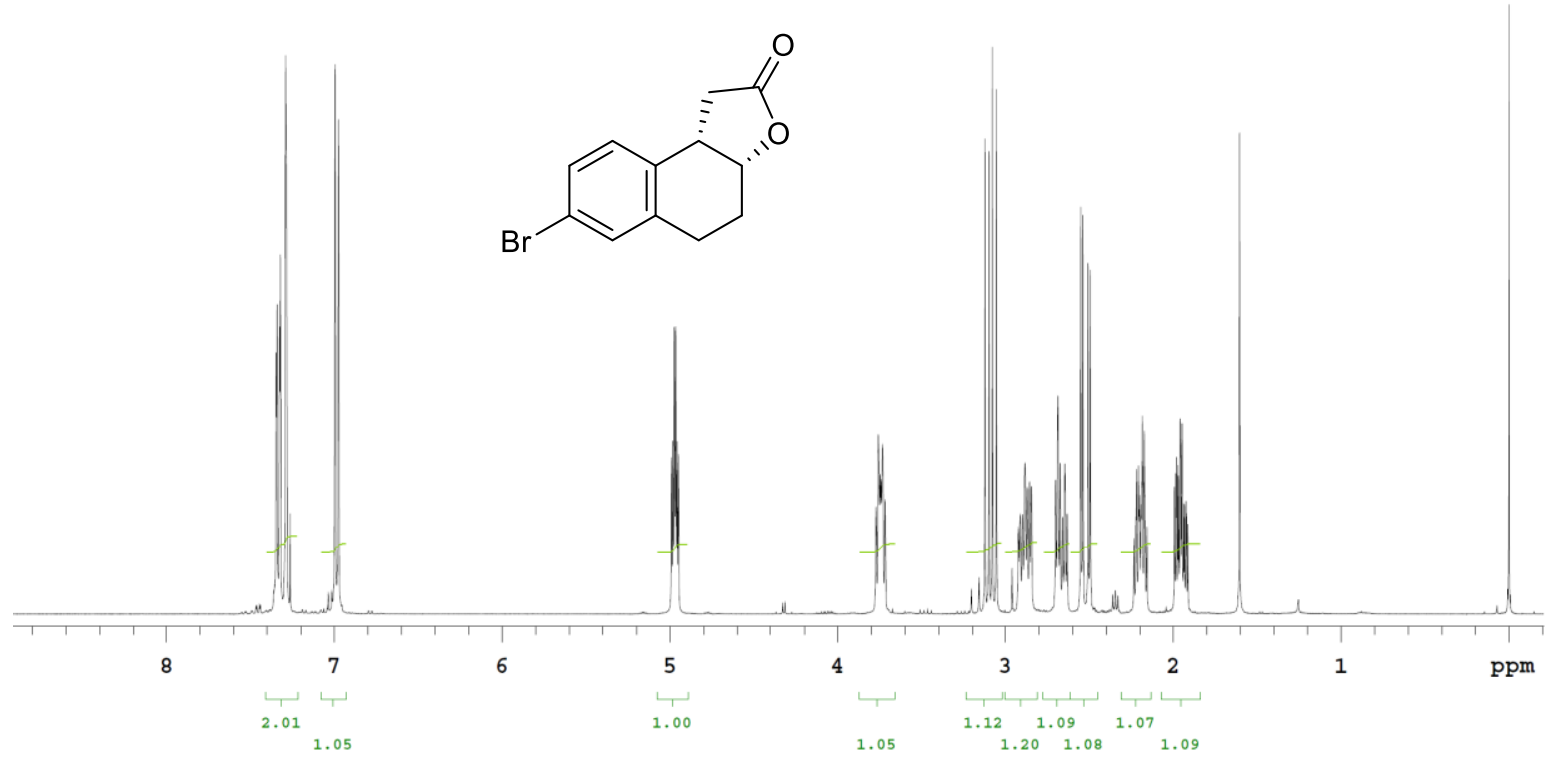

${ }^{13} \mathrm{C}$ NMR $\left(125 \mathrm{MHz}, \mathrm{CDCl}_{3}\right)$
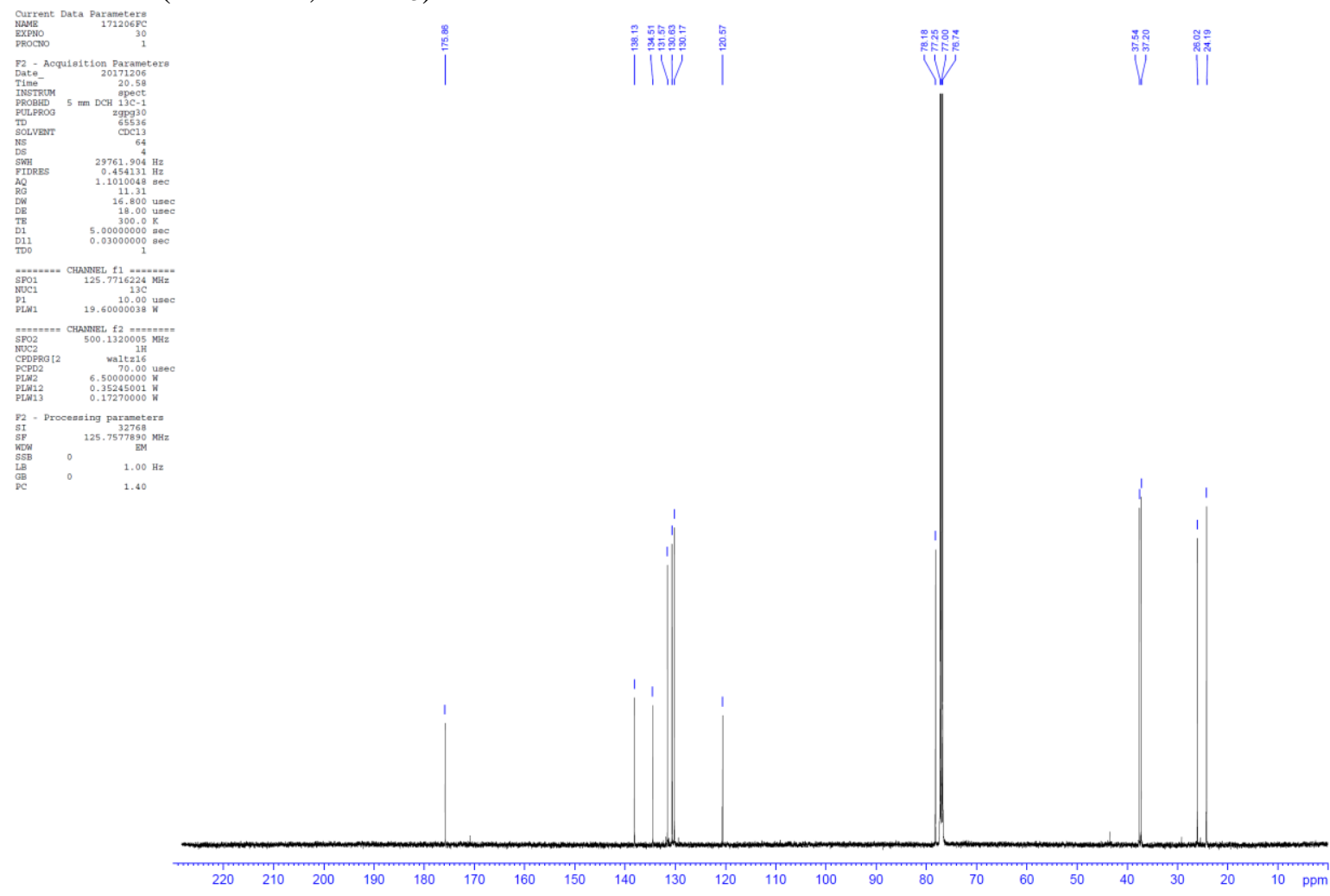
(3aR,9bS)-7-Chloro-3a,4,5,9b-tetrahydronaphtho[2,1-b]furan-2(1H)-one (13r)

${ }^{1} \mathrm{H}-\mathrm{NMR}\left(400 \mathrm{MHz}, \mathrm{CDCl}_{3}\right)$

170921-1

$\begin{array}{ll}\text { Sample Name } & 170921-1 \\ \text { Date collected } 2017-09-21\end{array}$

Pulse sequence PROTON
Solvent cdcl3

Temperature 25
Spectrometer Agilent-NMR-vnmrs400

Study owner vnmri
Operator vnmr1

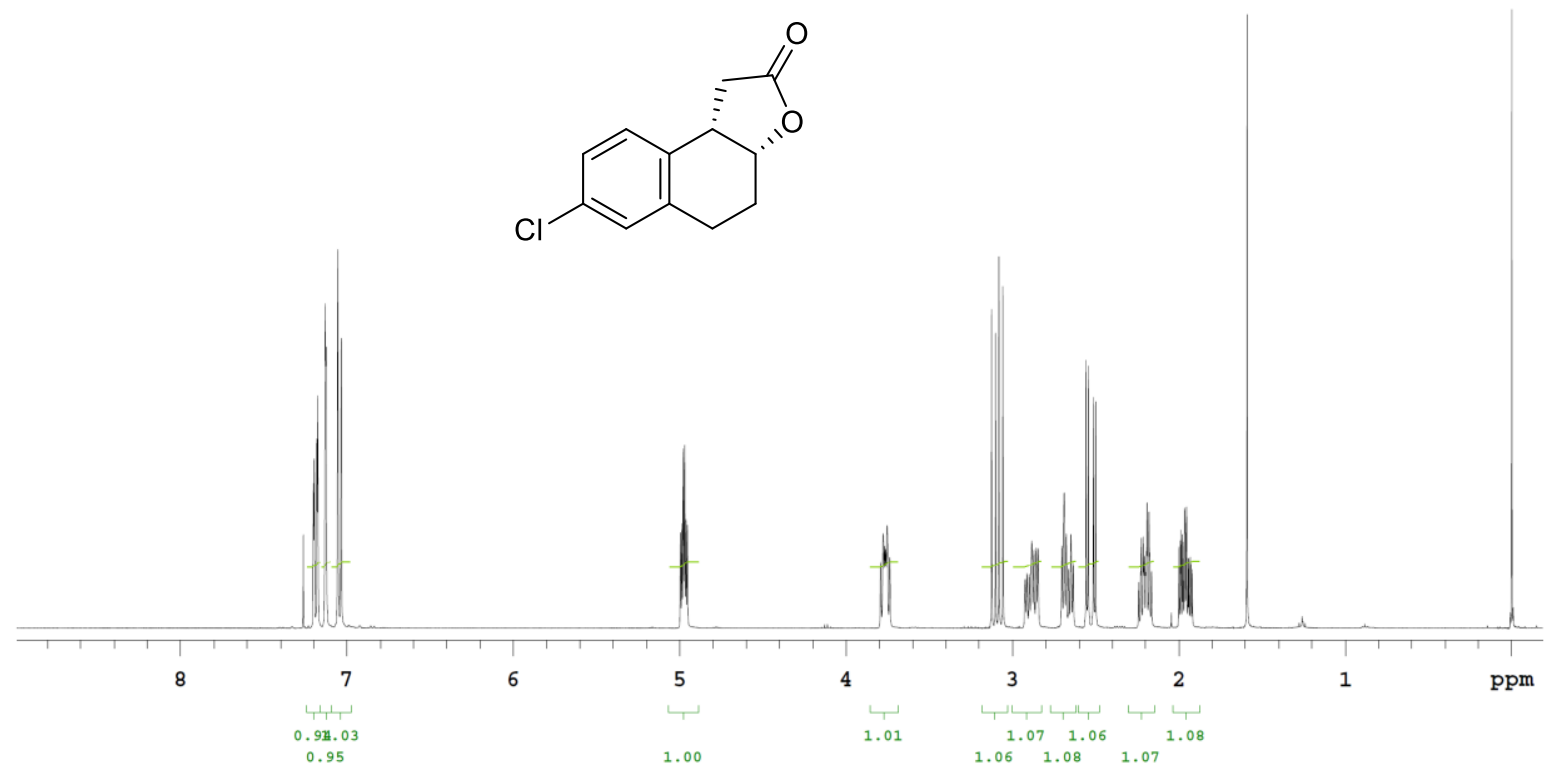

${ }^{13} \mathrm{C}$ NMR $\left(125 \mathrm{MHz}, \mathrm{CDCl}_{3}\right)$
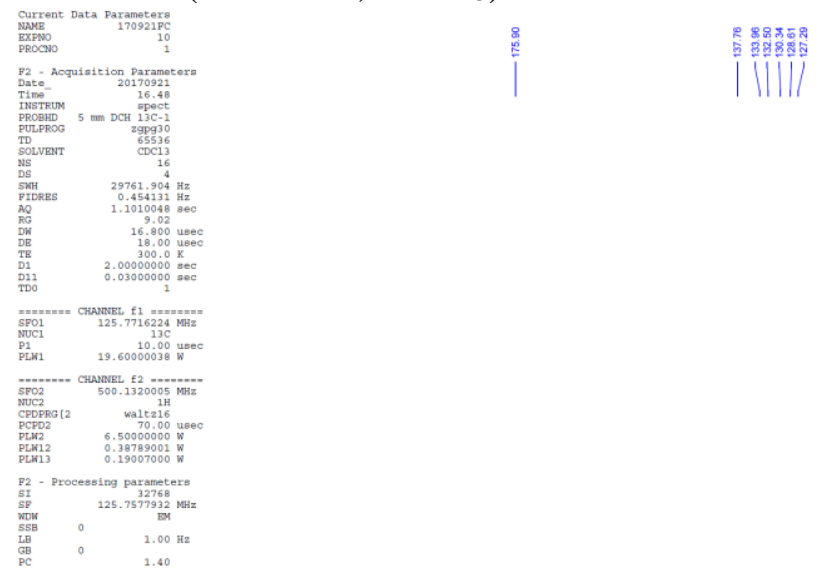

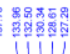

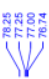

บ
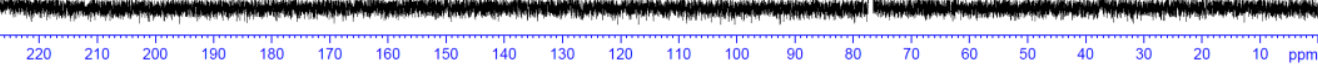


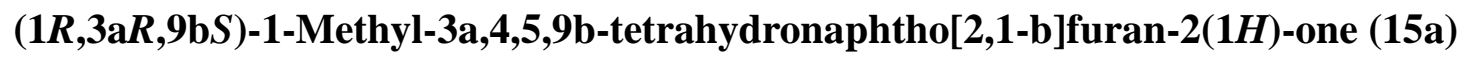

${ }^{1} \mathrm{H}-\mathrm{NMR}\left(500 \mathrm{MHz}, \mathrm{CDCl}_{3}\right)$
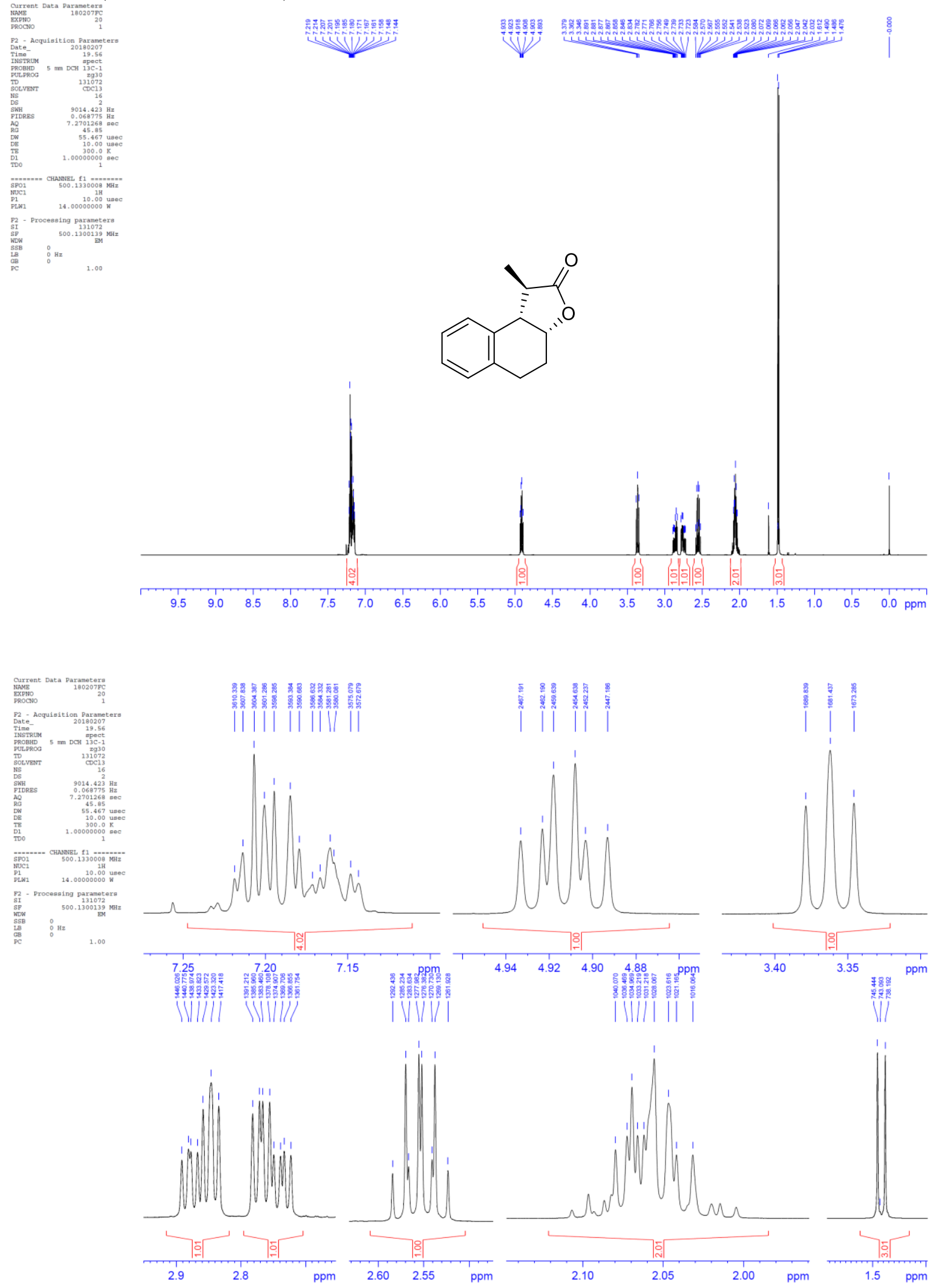

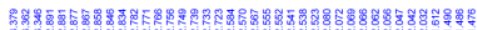

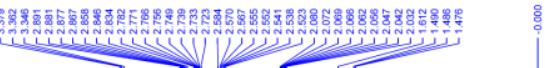


$\left.{ }^{13} \mathrm{C} \mathrm{NMR} \mathrm{(125} \mathrm{MHz,} \mathrm{CDCl}_{3}\right)$
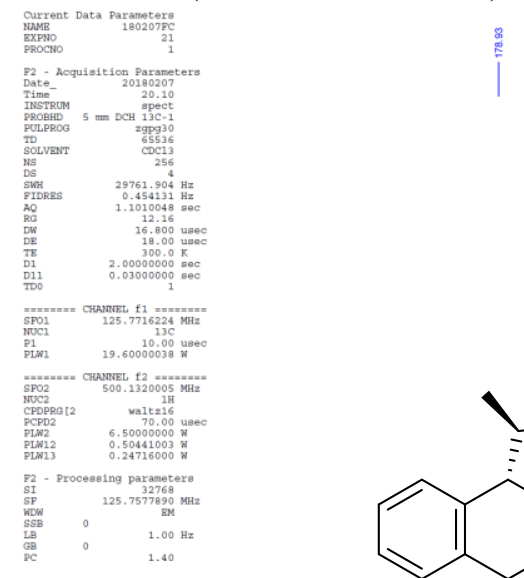

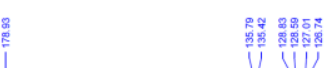

V V V

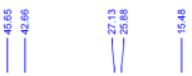<smiles>C[C@H]1C(=O)O[C@H]2CCc3ccccc3[C@H]21</smiles>
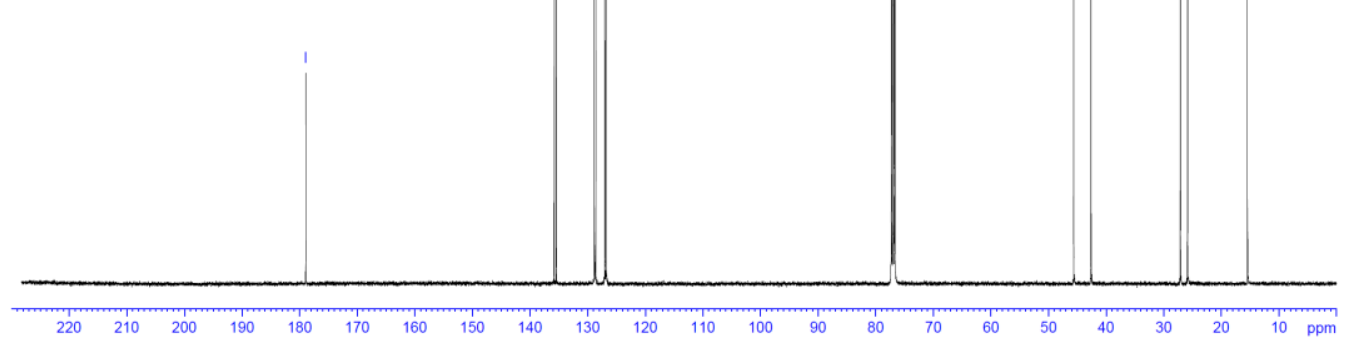
$(1 R, 3 a R, 9 b S)-1-E t h y l-3 a, 4,5,9 b-t e t r a h y d r o n a p h t h o[2,1-b] f u r a n-2(1 H)-o n e ~(15 b)$

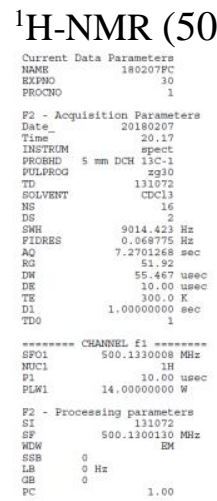

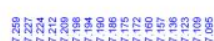

LWWIIIII

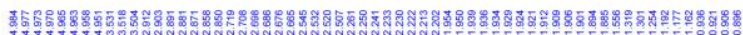

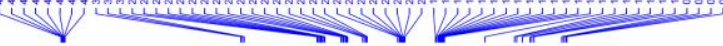<smiles>CC[C@H]1C(=O)O[C@H]2CCc3ccccc3[C@H]21</smiles>
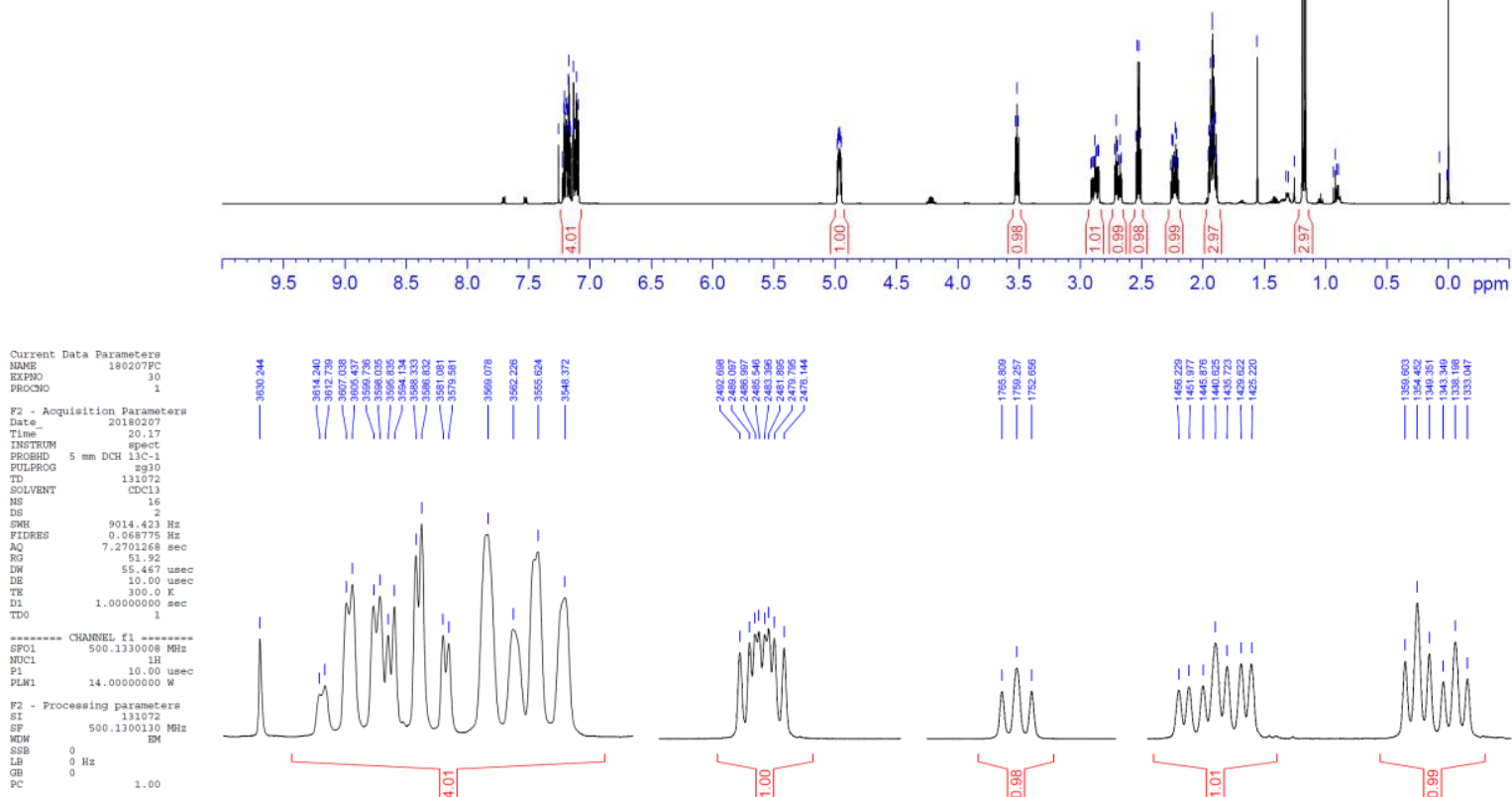

|

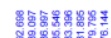

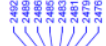

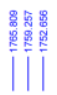

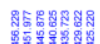

U110

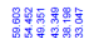

"III
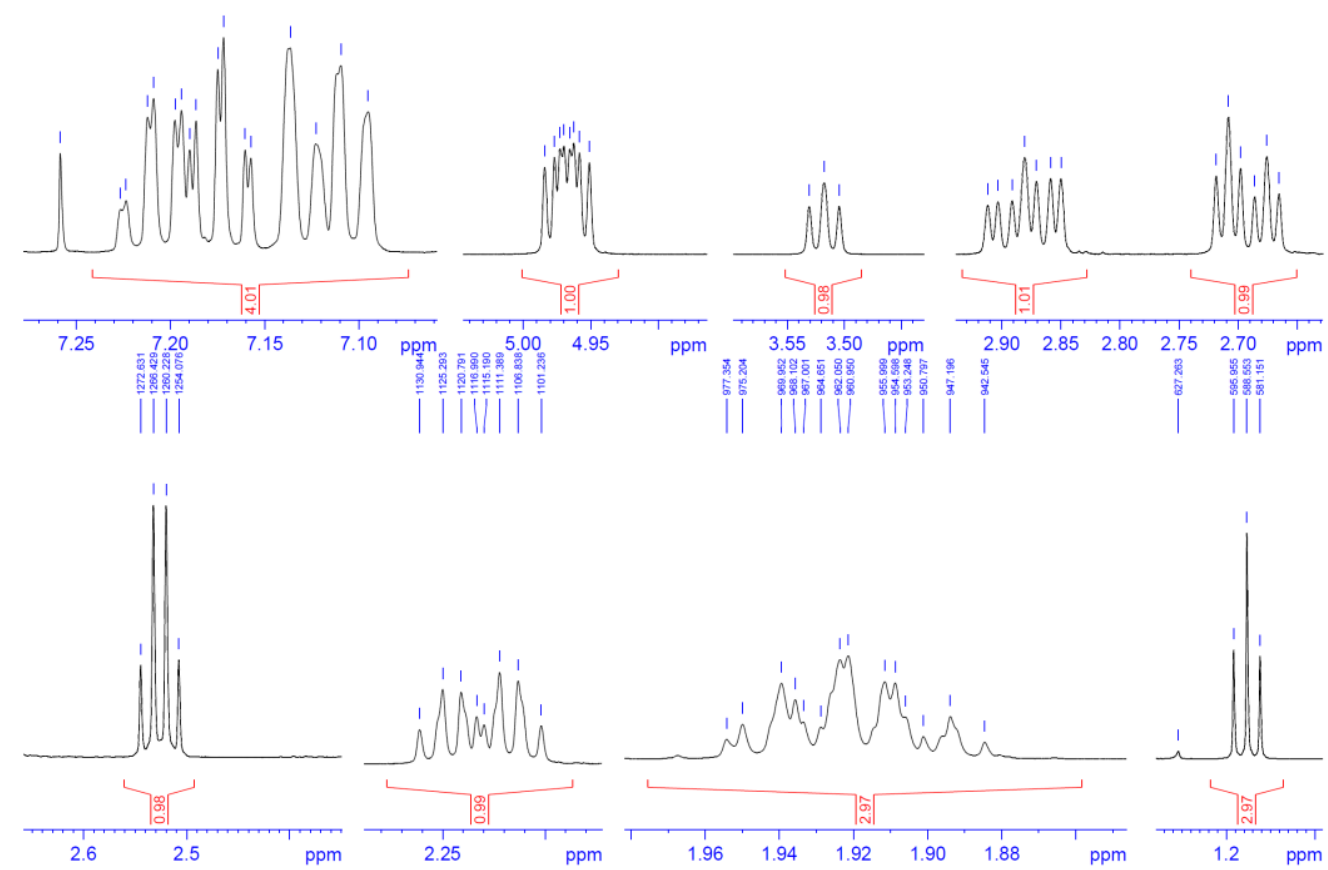
$\left.{ }^{13} \mathrm{C} \mathrm{NMR} \mathrm{(125} \mathrm{MHz,} \mathrm{CDCl}_{3}\right)$
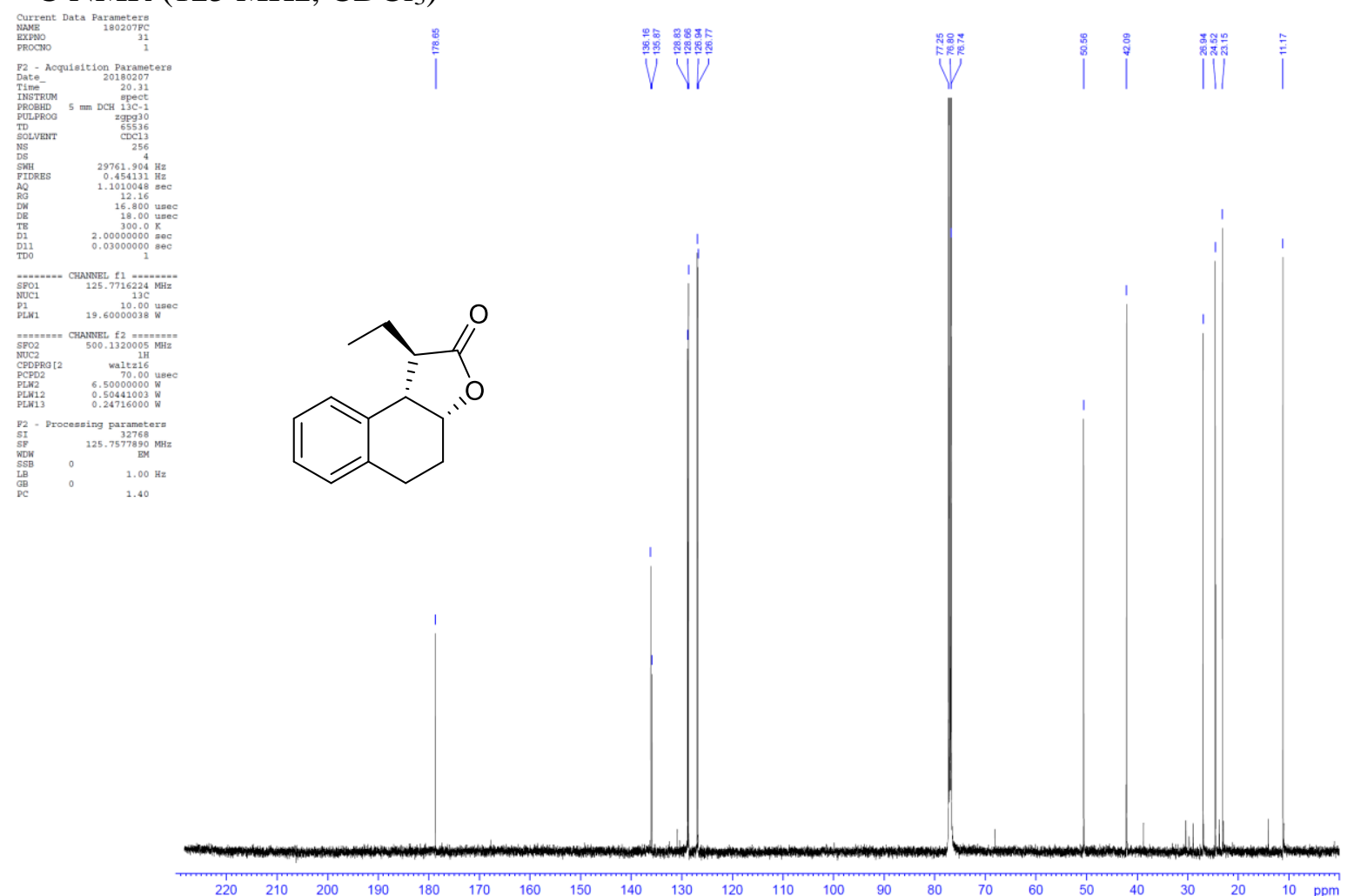


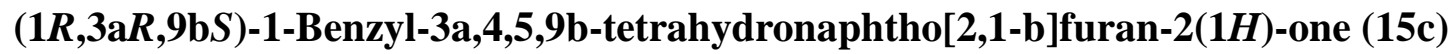

${ }^{1} \mathrm{H}-\mathrm{NMR}\left(500 \mathrm{MHz}, \mathrm{CDCl}_{3}\right)$

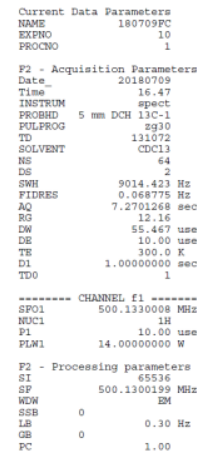

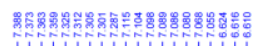

Nivili

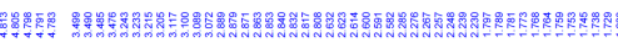
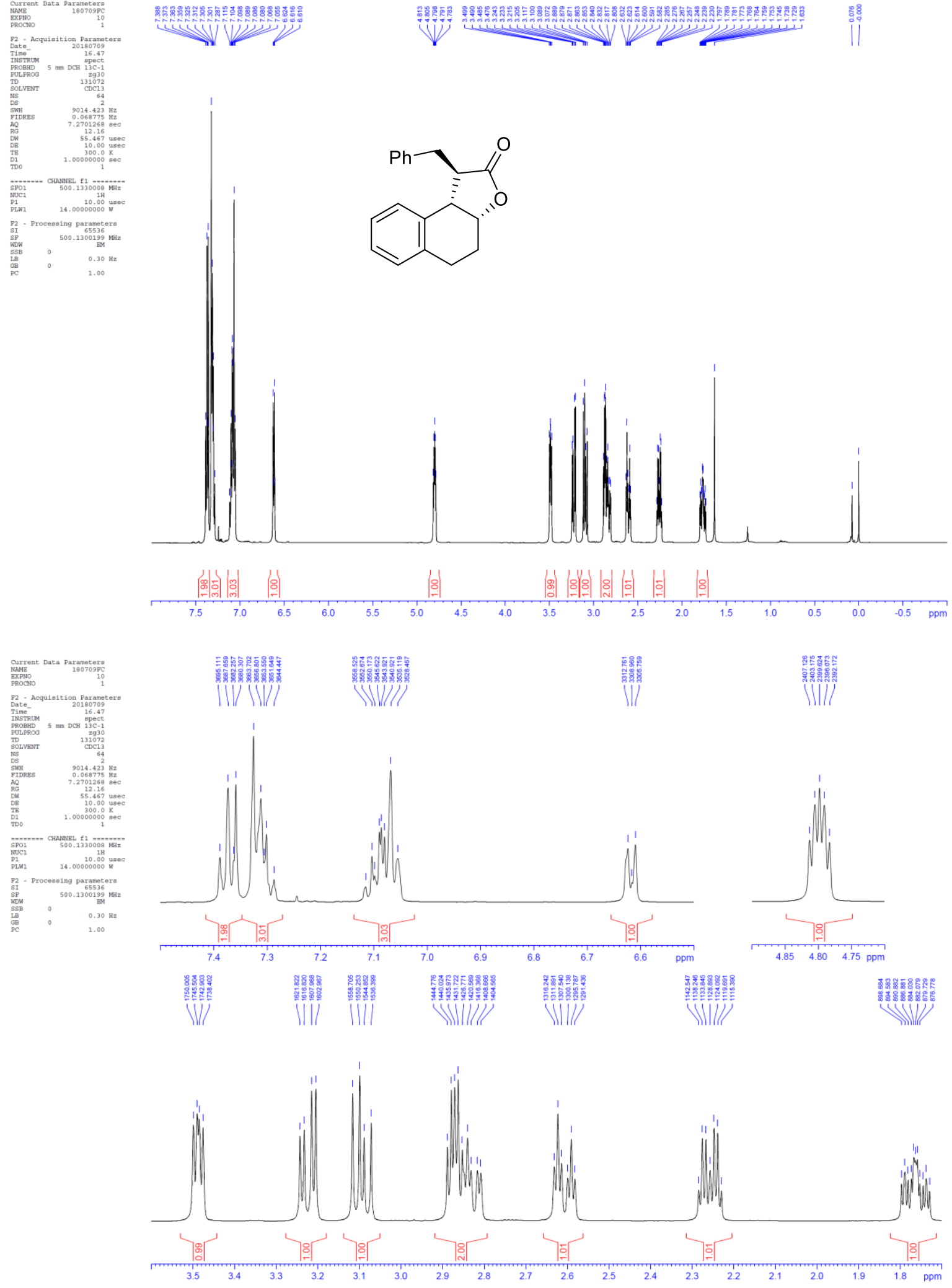
${ }^{13} \mathrm{C} \mathrm{NMR}\left(125 \mathrm{MHz}, \mathrm{CDCl}_{3}\right)$
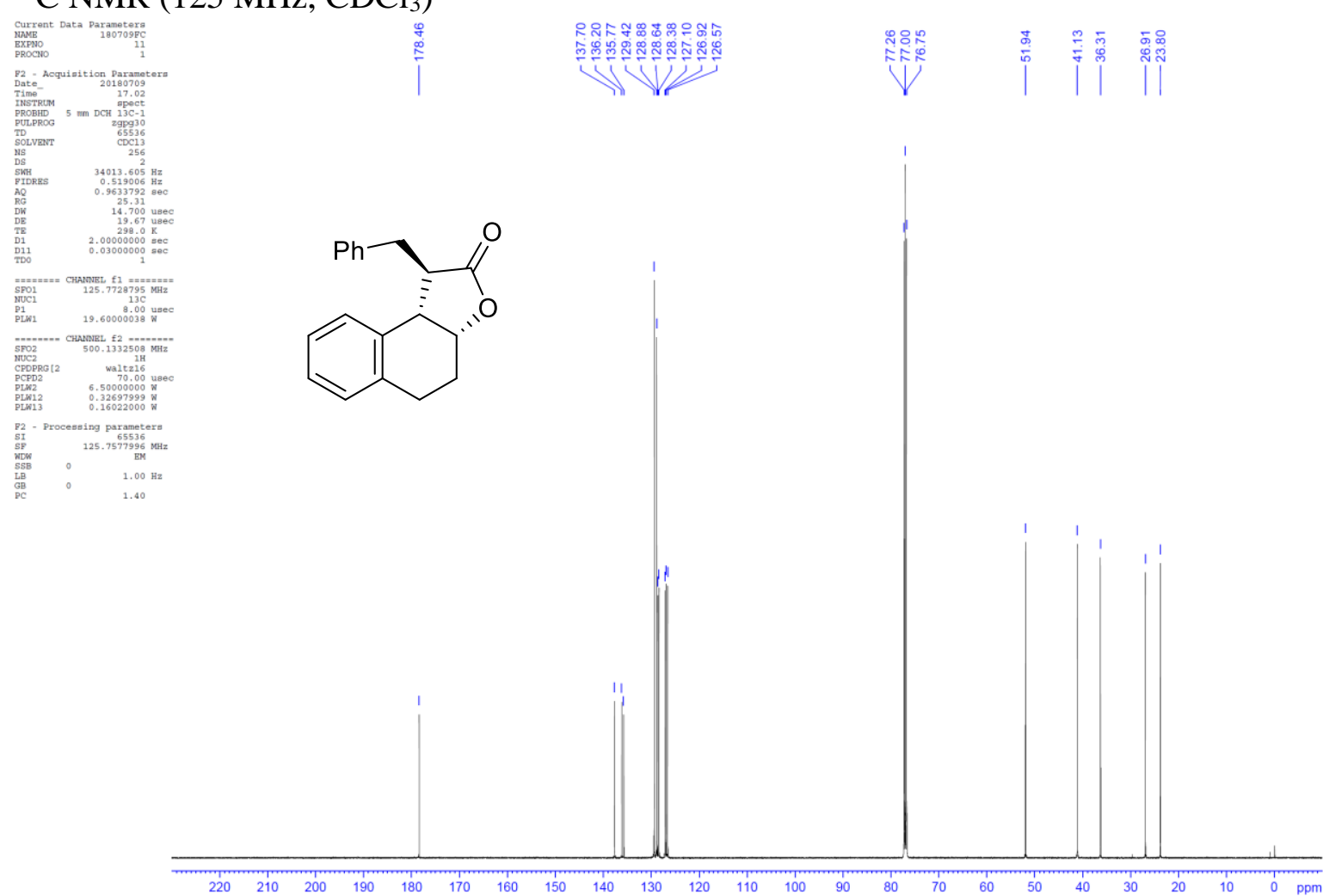

\section{HSQC}

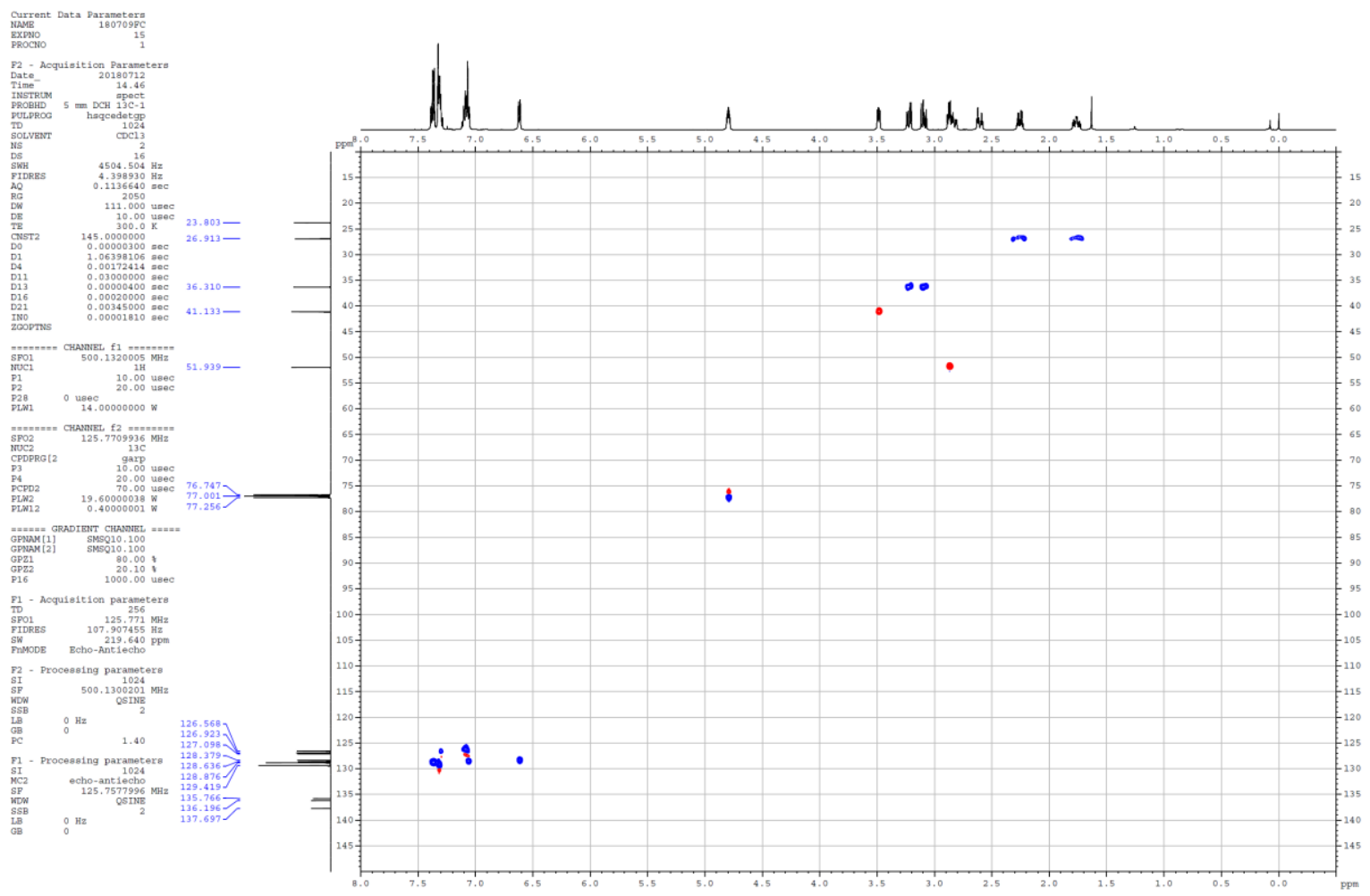


$(1 S, 3 a R, 9 b R)$-1-Phenyl-3a,4,5,9b-tetrahydronaphtho[2,1-b]furan-2(1H)-one (15d)

${ }^{1} \mathrm{H}-\mathrm{NMR}\left(500 \mathrm{MHz}, \mathrm{CDCl}_{3}\right)$
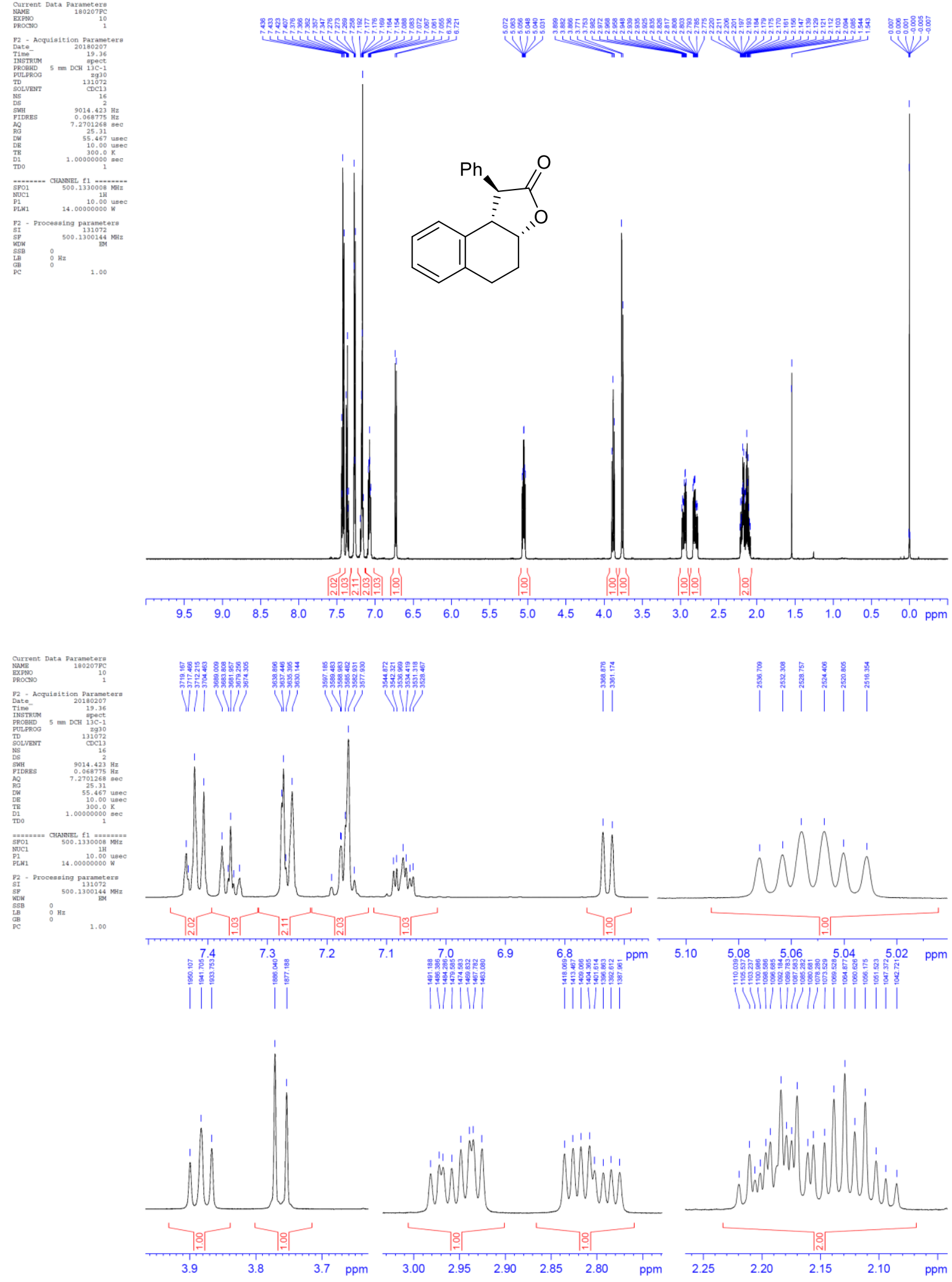
${ }^{13} \mathrm{C} \mathrm{NMR}\left(125 \mathrm{MHz}, \mathrm{CDCl}_{3}\right)$
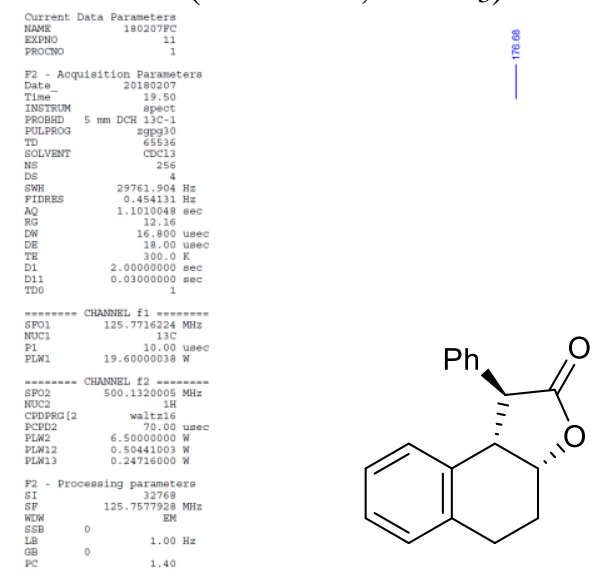

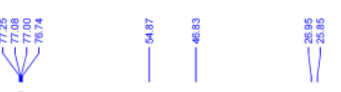

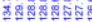

UWVI

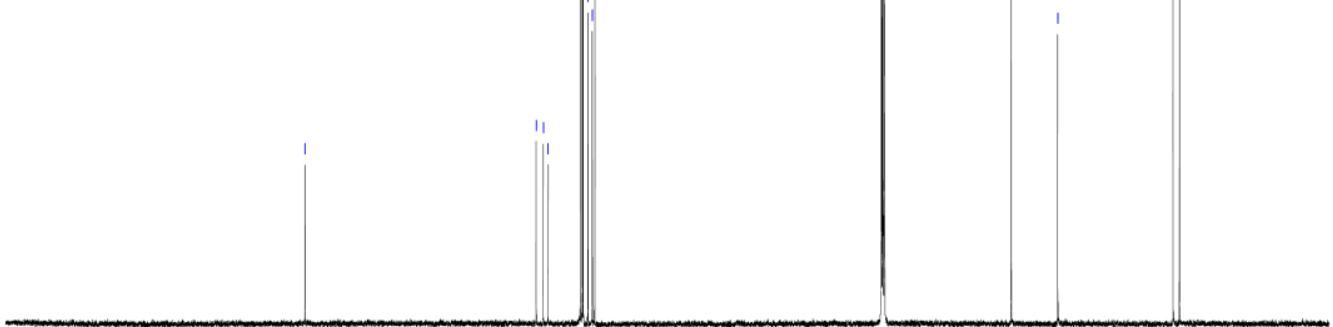

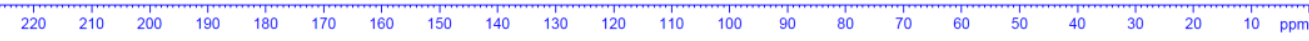




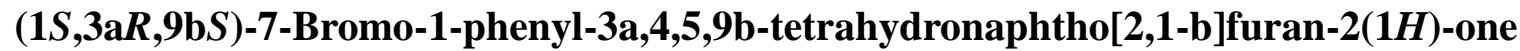
(15e)

${ }^{1} \mathrm{H}-\mathrm{NMR}\left(500 \mathrm{MHz}, \mathrm{CDCl}_{3}\right.$ )
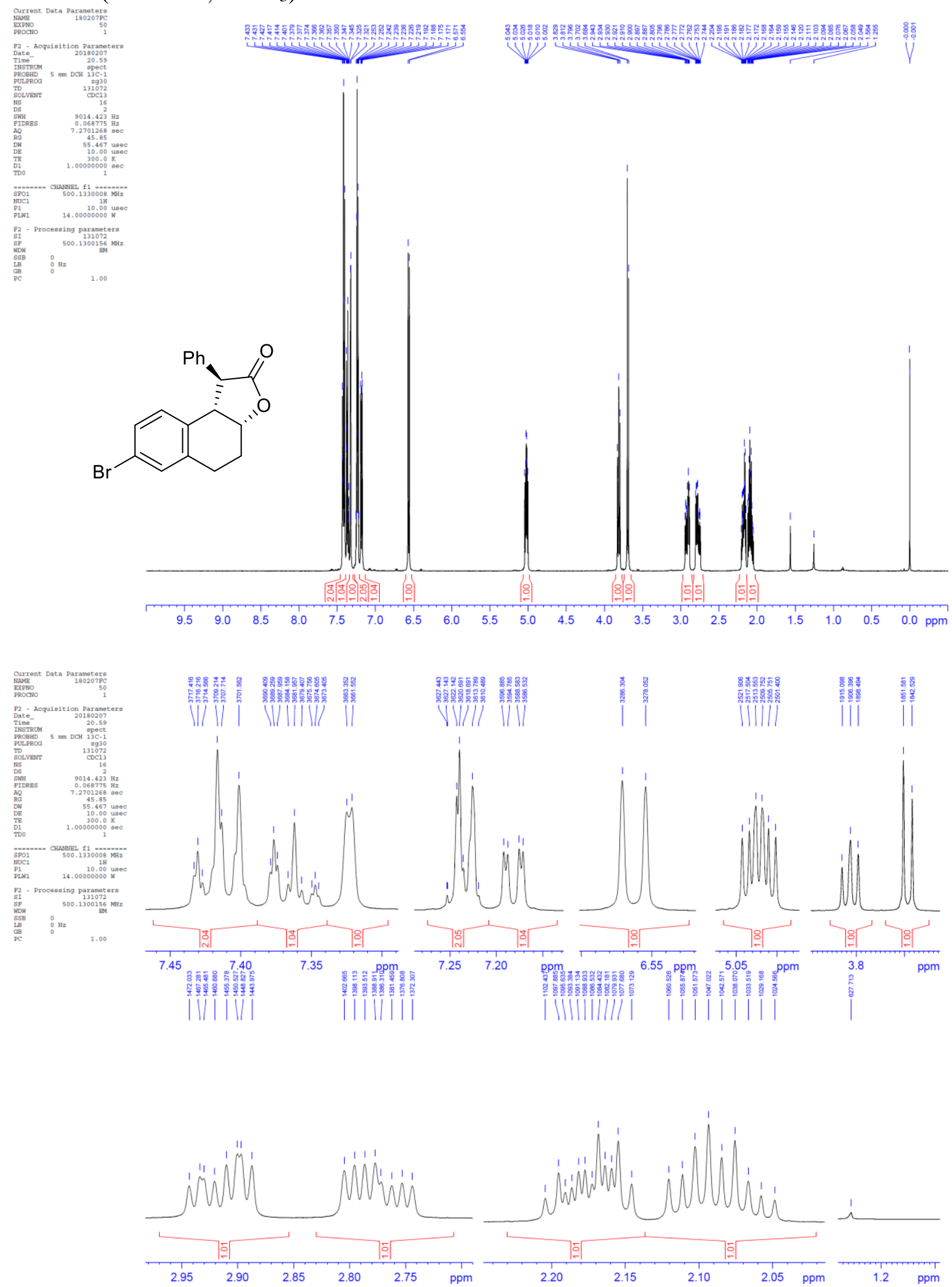
${ }^{13} \mathrm{C} \mathrm{NMR}\left(125 \mathrm{MHz}, \mathrm{CDCl}_{3}\right)$
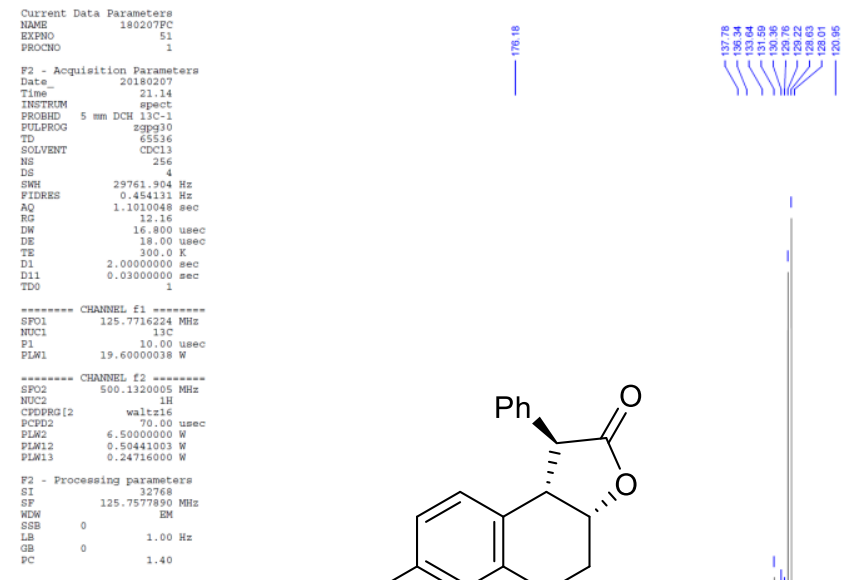

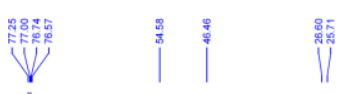

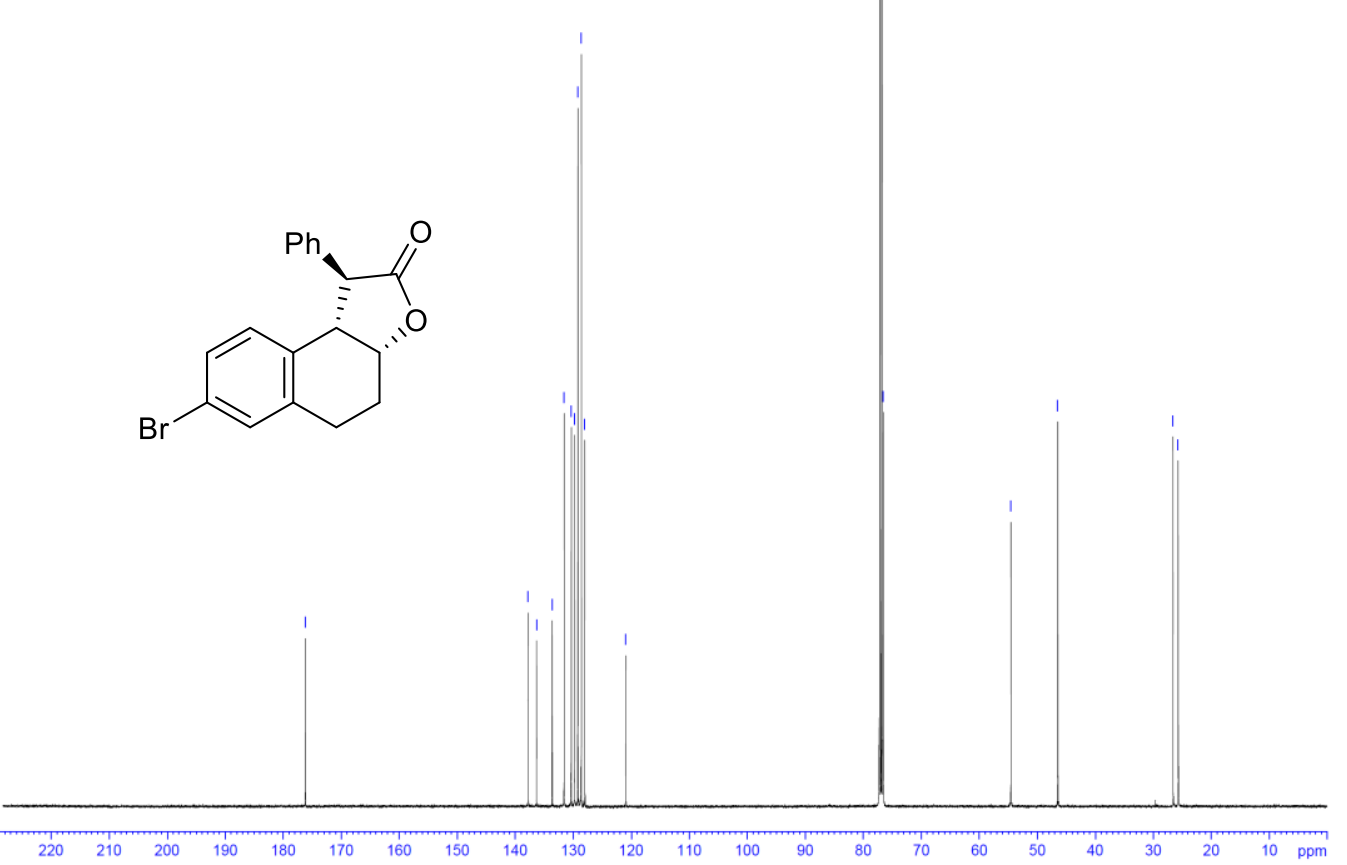


(1S,3aR,9bS)-7-Chloro-1-phenyl-3a,4,5,9b-tetrahydronaphtho[2,1-b]furan-2(1H)-one (15f)
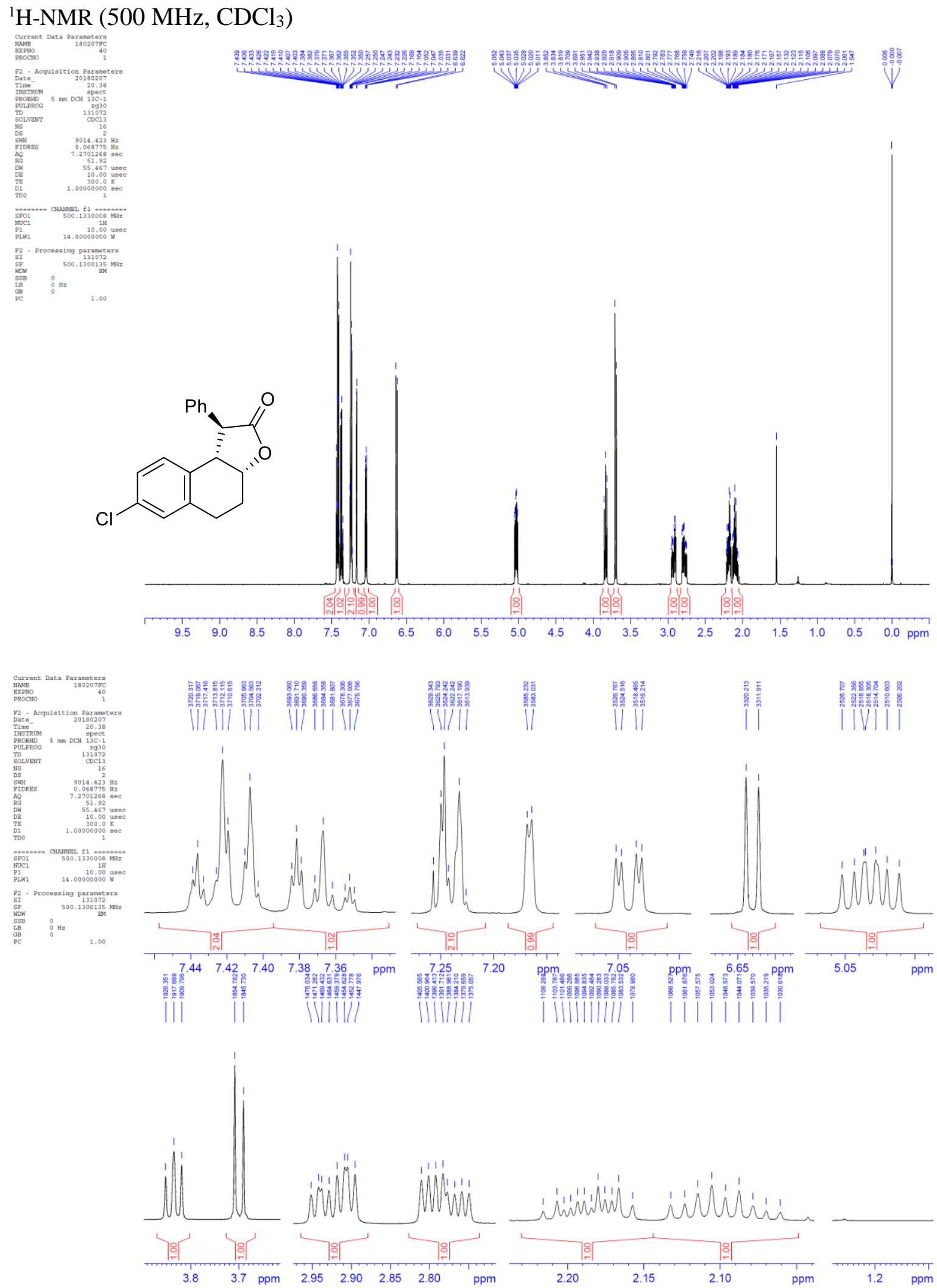
${ }^{13} \mathrm{C} \mathrm{NMR}\left(125 \mathrm{MHz}, \mathrm{CDCl}_{3}\right)$
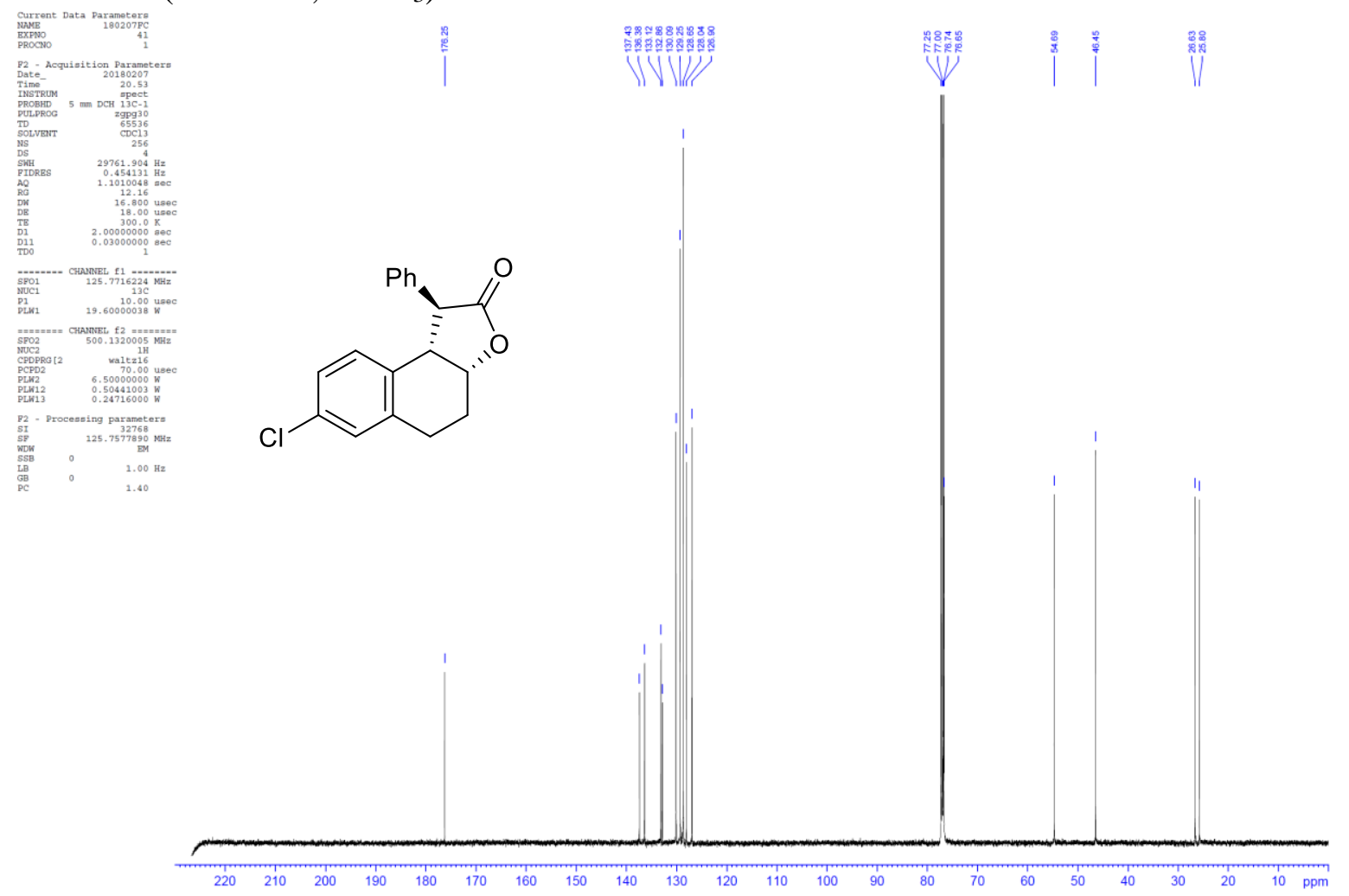
$(1 S, 3 a R, 9 b S)-9-M e t h o x y-1-p h e n y l-3 a, 4,5,9 b-t e t r a h y d r o n a p h t h o[2,1-b] f u r a n-2(1 H)$-on e (15g)

${ }^{1} \mathrm{H}-\mathrm{NMR}\left(500 \mathrm{MHz}, \mathrm{CDCl}_{3}\right)$

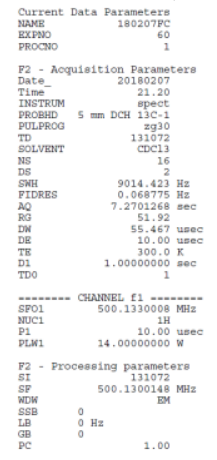

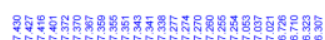

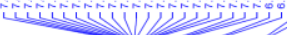

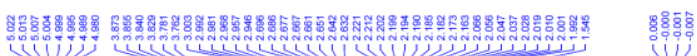<smiles>COc1cccc2c1CC[C@@H]1OC(=O)[C@H](c3ccccc3)[C@H]21</smiles>
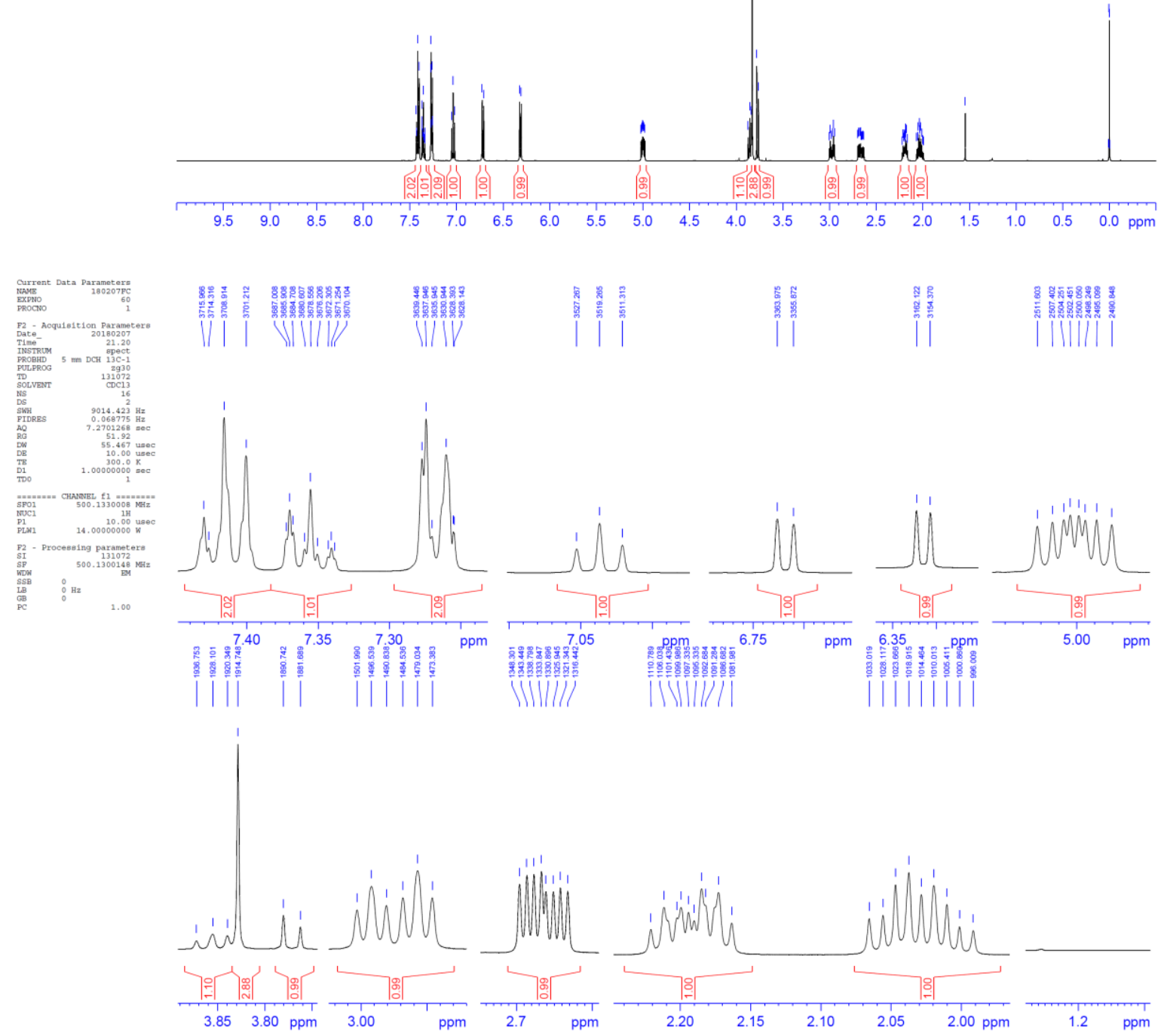
${ }^{13} \mathrm{C}$ NMR (125 MHz, $\left.\mathrm{CDCl}_{3}\right)$
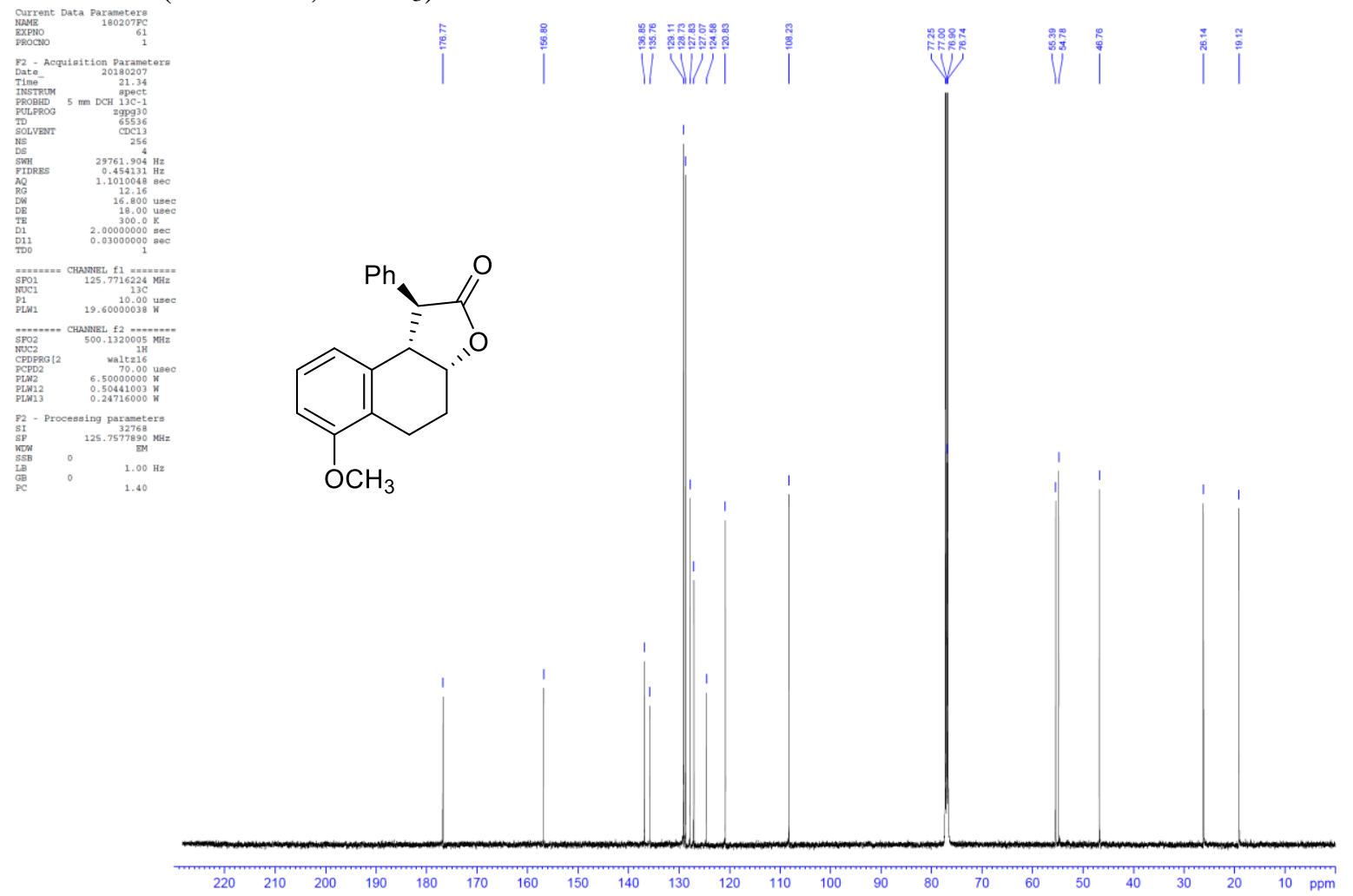
$(1 S, 3 \mathrm{a} R, 9 \mathrm{bS})$-1-(2-Chlorophenyl)-3a,4,5,9b-tetrahydronaphtho[2,1-b]furan-2(1H)-one (15h)
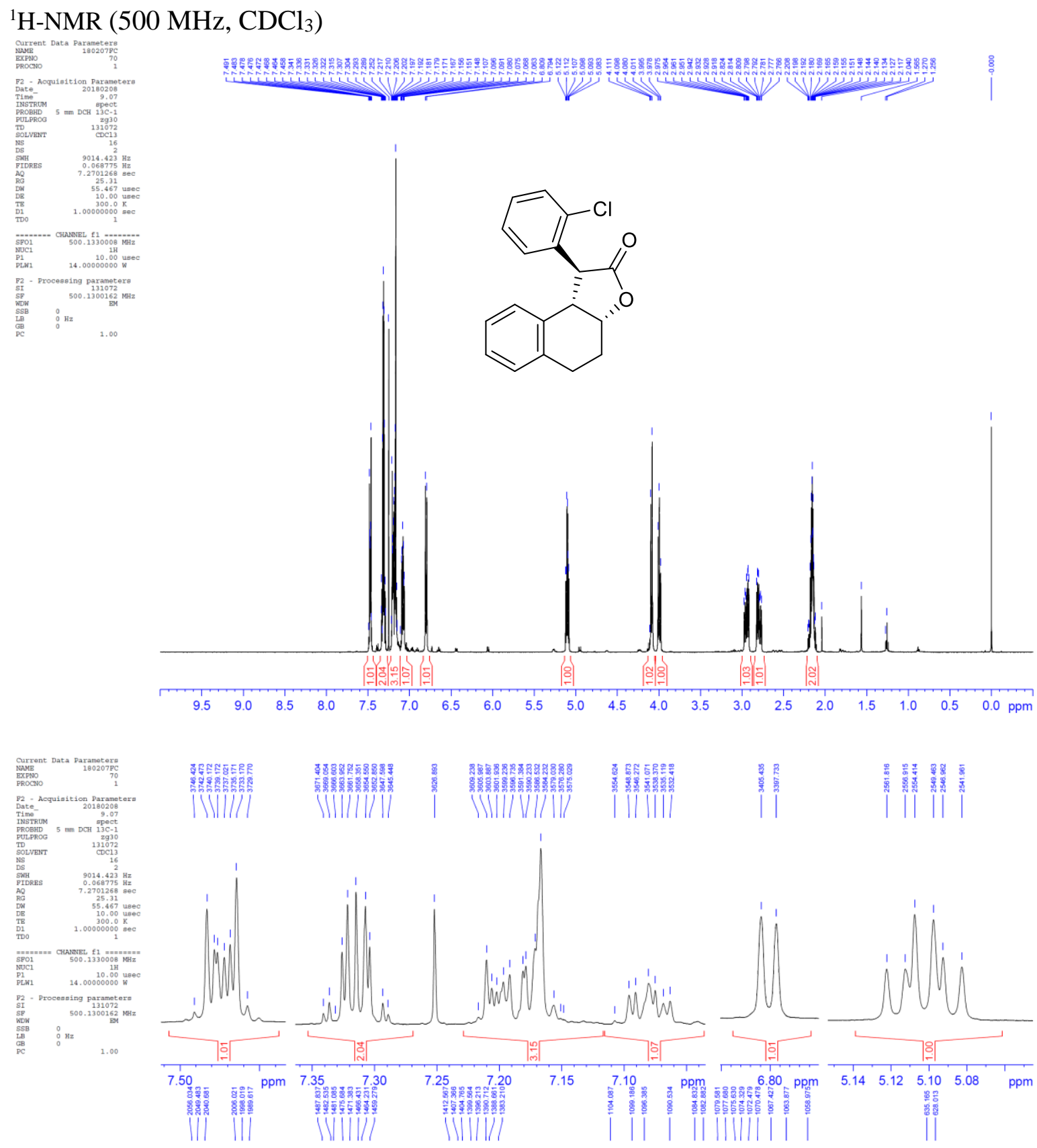

$\mid$
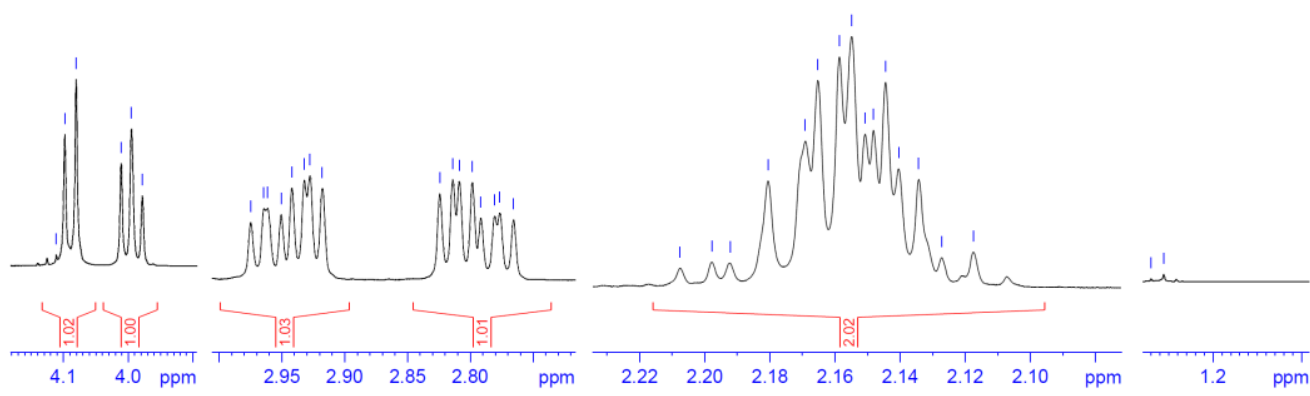
$\left.{ }^{13} \mathrm{C} \mathrm{NMR} \mathrm{(125} \mathrm{MHz,} \mathrm{CDCl}_{3}\right)$
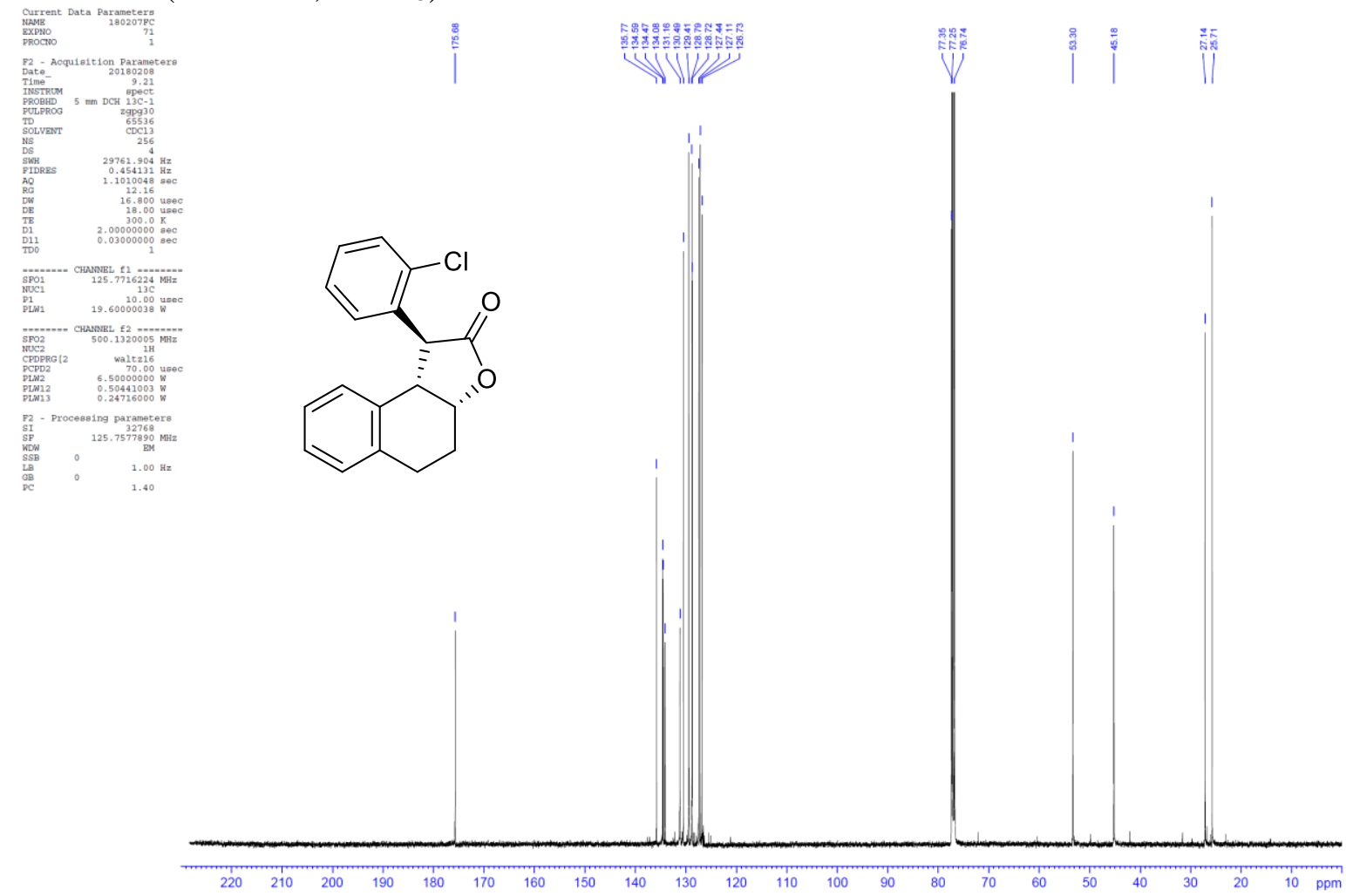
$(1 R, 3 a S, 9 b R)-8-M e t h o x y-1-p h e n y l-3 a, 4,5,9 b$-tetrahydronaphtho[2,1-b]furan-2(1H)-on e (7)

${ }^{1} \mathrm{H}-\mathrm{NMR}\left(500 \mathrm{MHz}, \mathrm{CDCl}_{3}\right)$
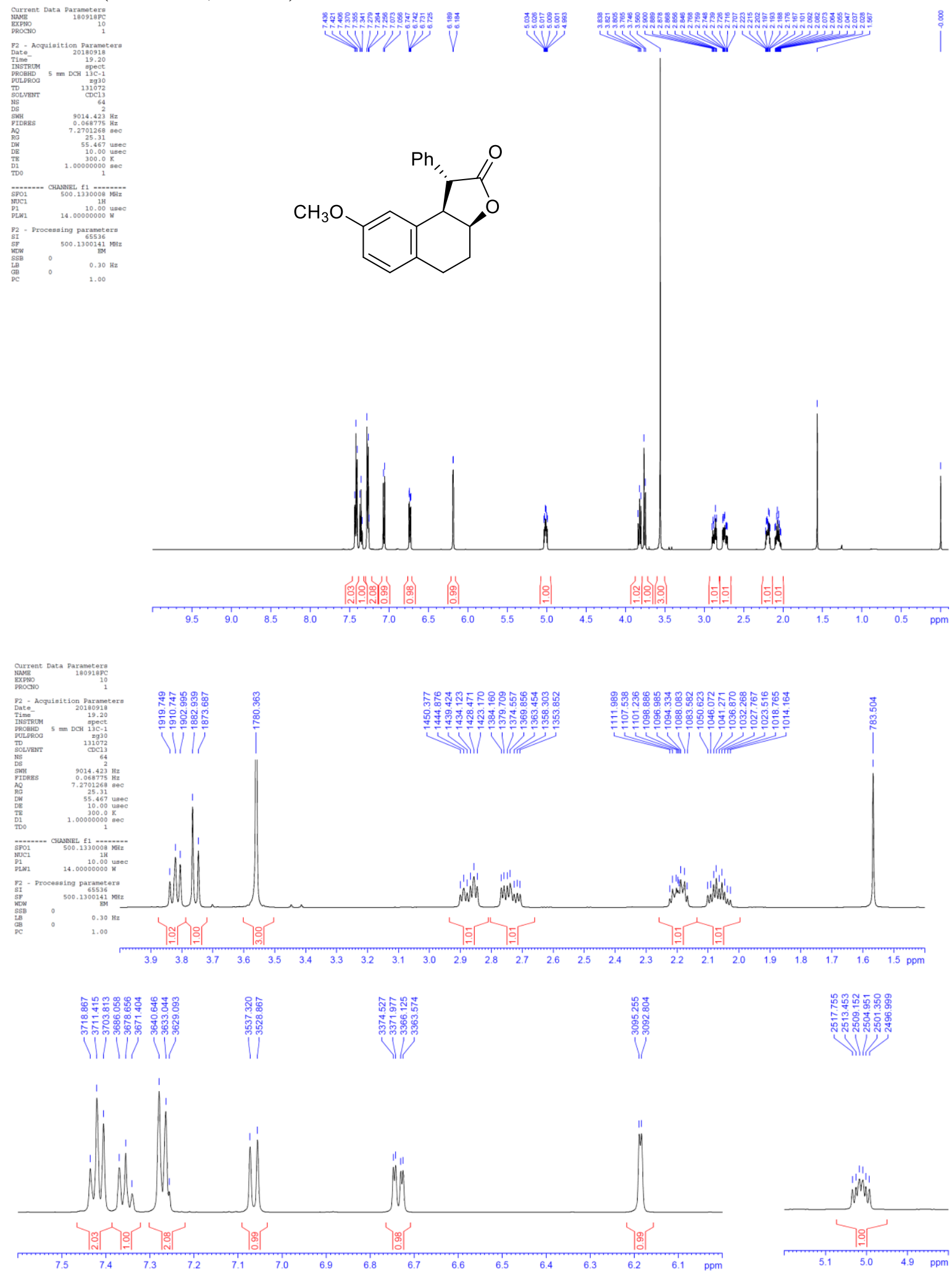
${ }^{13} \mathrm{C} \mathrm{NMR}\left(125 \mathrm{MHz}, \mathrm{CDCl}_{3}\right)$
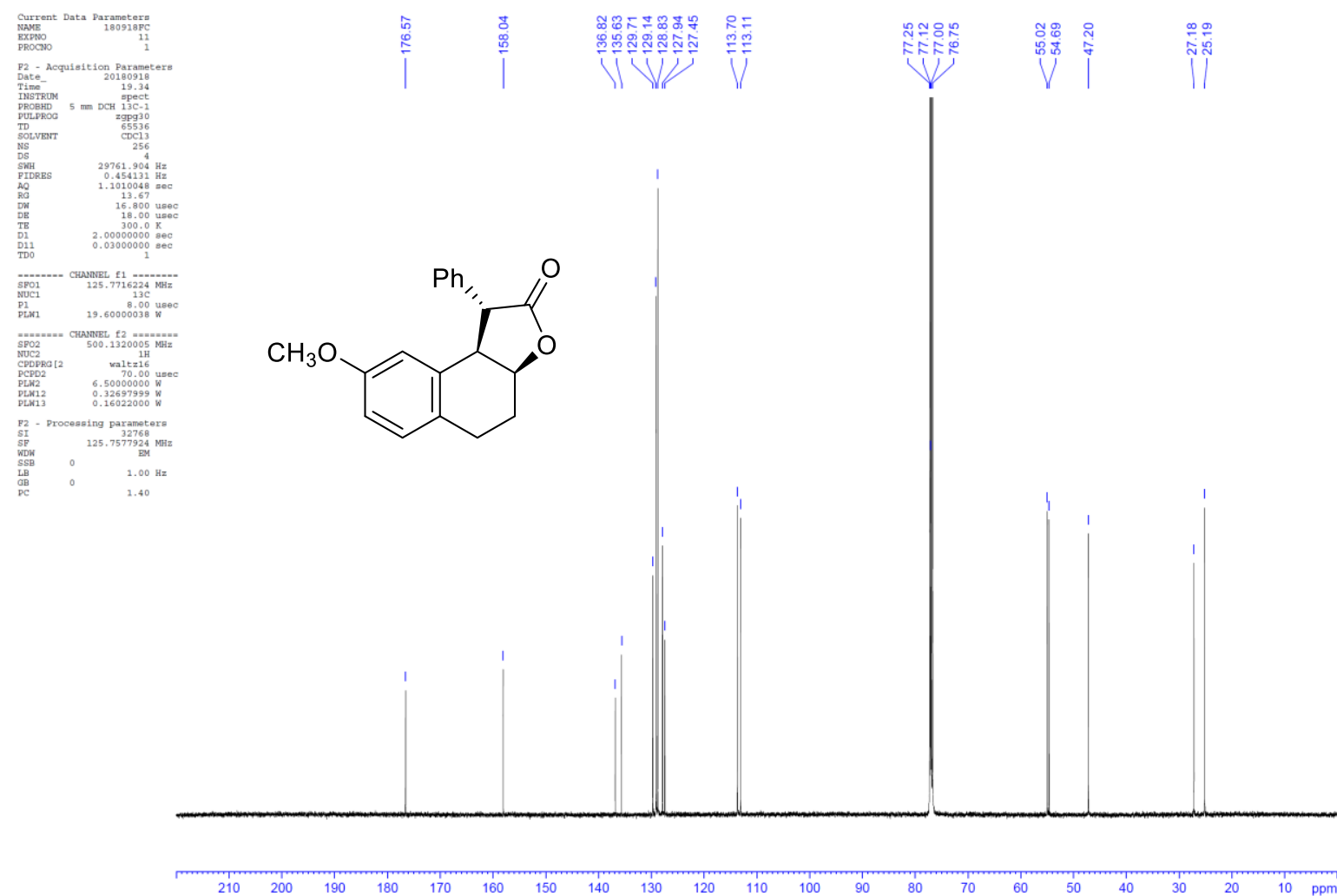
$(3 R, 3 a S, 9 b R)-3-P h e n y l-3 a, 4,5,9 b-t e t r a h y d r o n a p h t h o[1,2-b] f u r a n-2(3 H)$-one (18a)

${ }^{1} \mathrm{H}-\mathrm{NMR}\left(500 \mathrm{MHz}, \mathrm{CDCl}_{3}\right)$
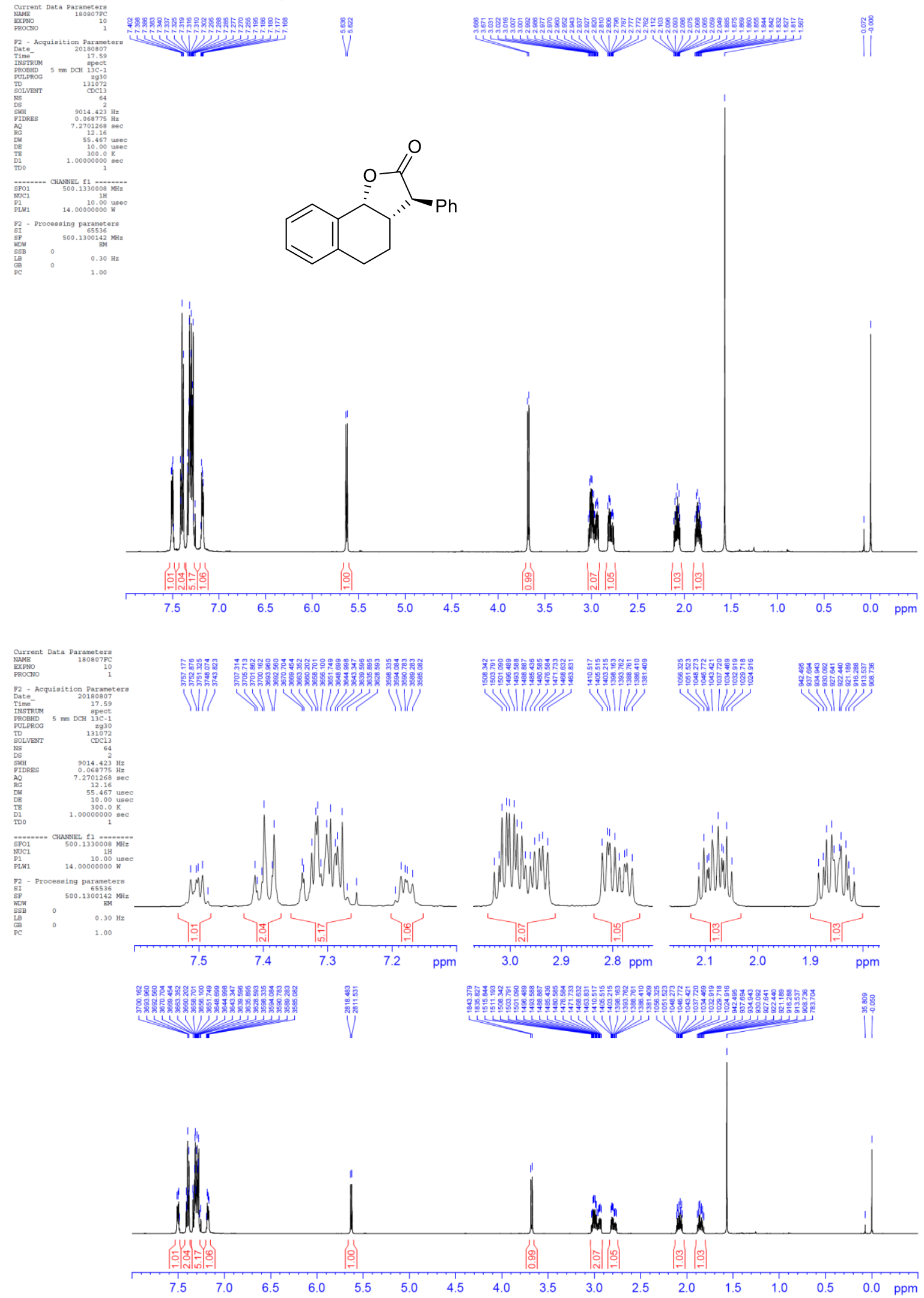
${ }^{13} \mathrm{C} \mathrm{NMR}\left(125 \mathrm{MHz}, \mathrm{CDCl}_{3}\right)$
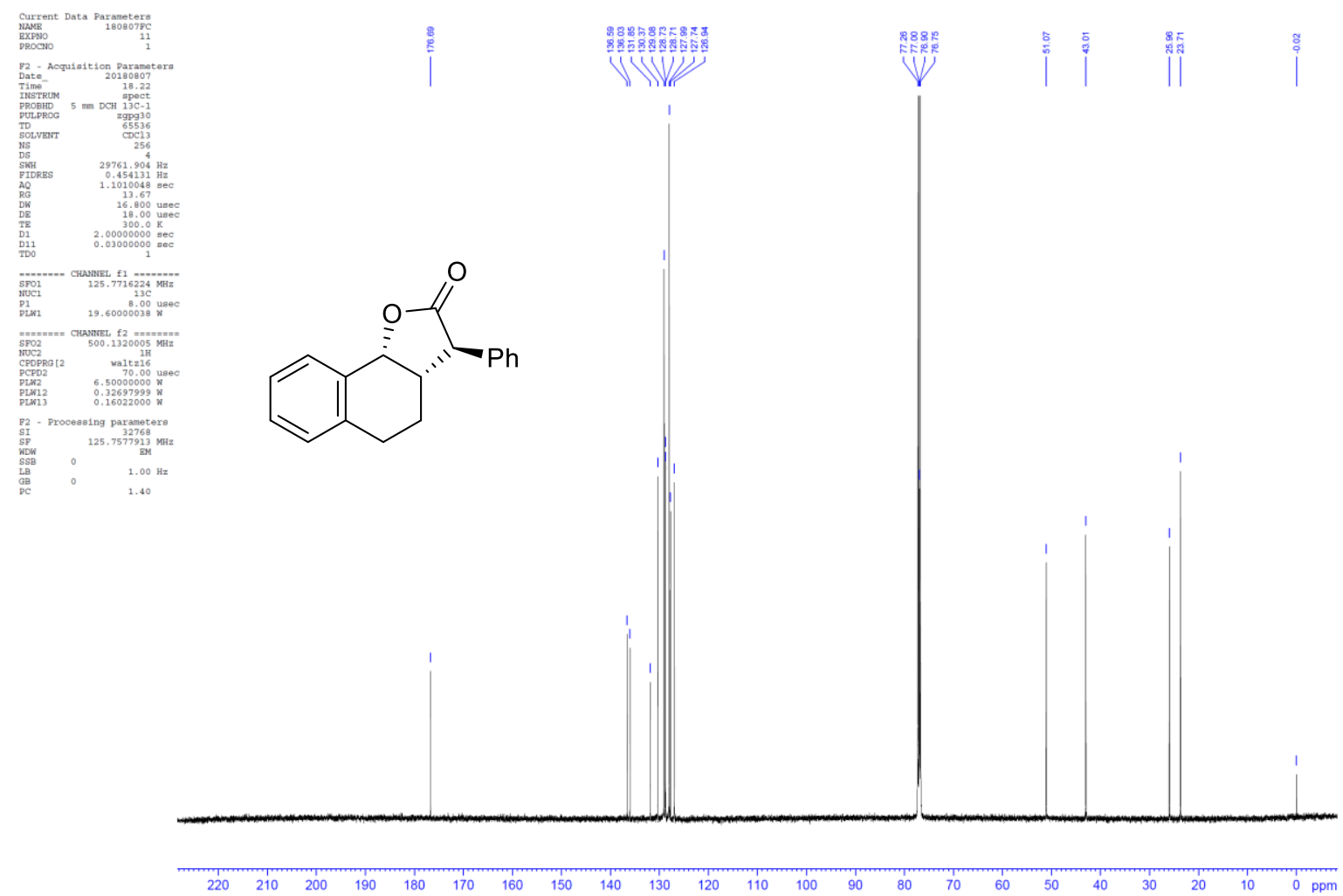
$(3 R, 3 \mathrm{a} S, 9 \mathrm{~b} R)-8$-Chloro-3-phenyl-3a,4,5,9b-tetrahydronaphtho[1,2-b]furan-2(3H)-one (18b)

\section{${ }^{1} \mathrm{H}-\mathrm{NMR}\left(500 \mathrm{MHz}, \mathrm{CDCl}_{3}\right)$}
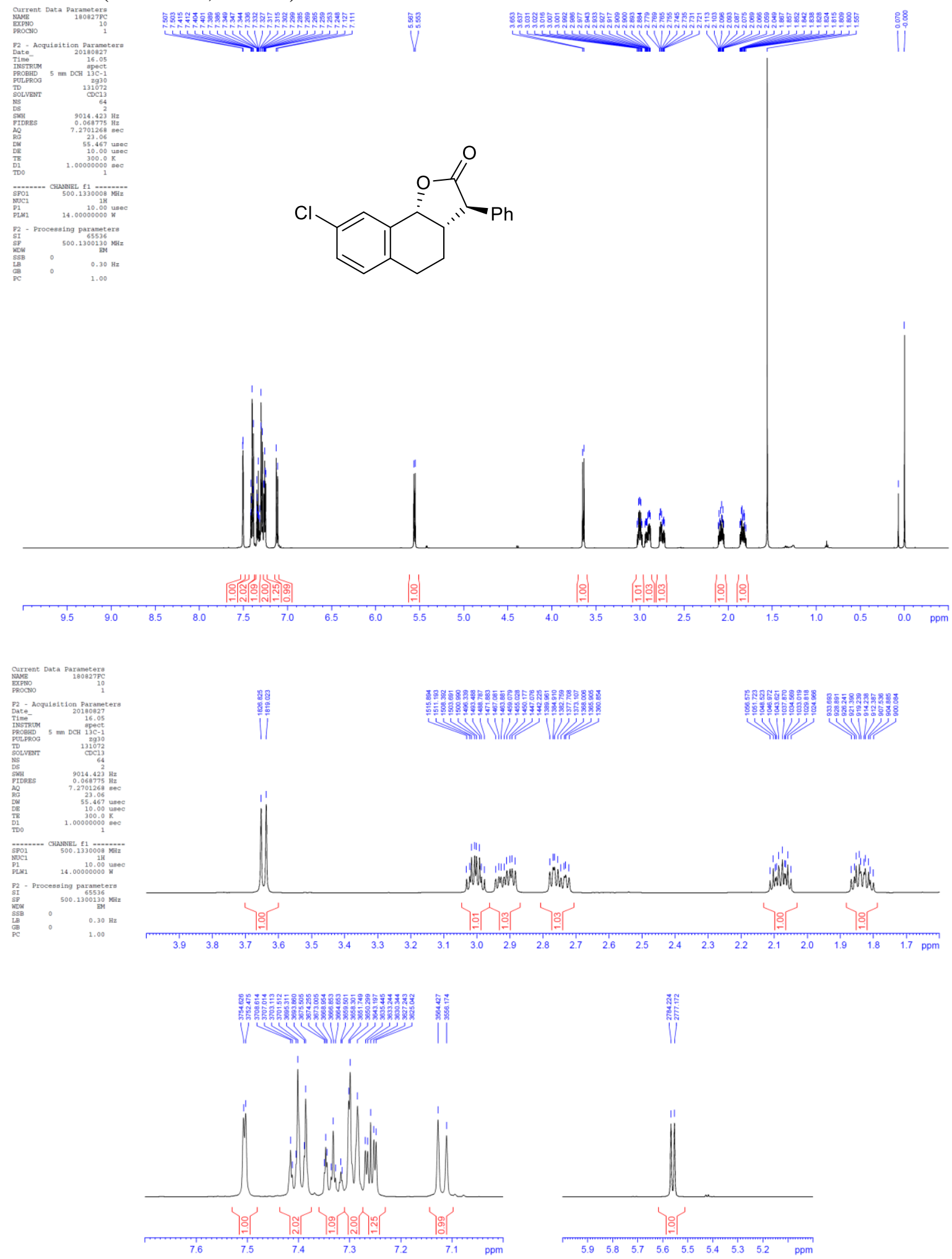

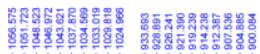
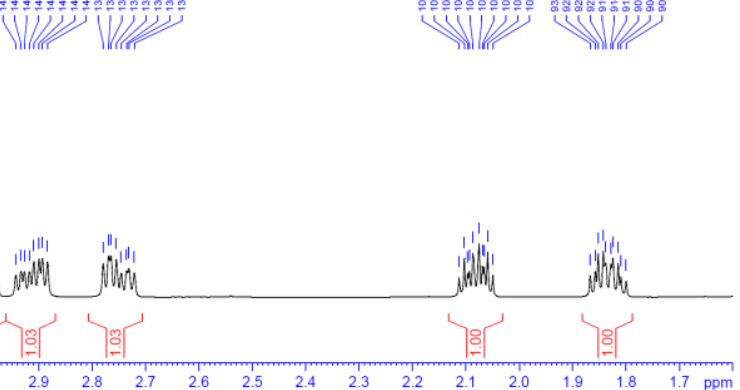
${ }^{13} \mathrm{C}$ NMR (125 MHz, $\left.\mathrm{CDCl}_{3}\right)$
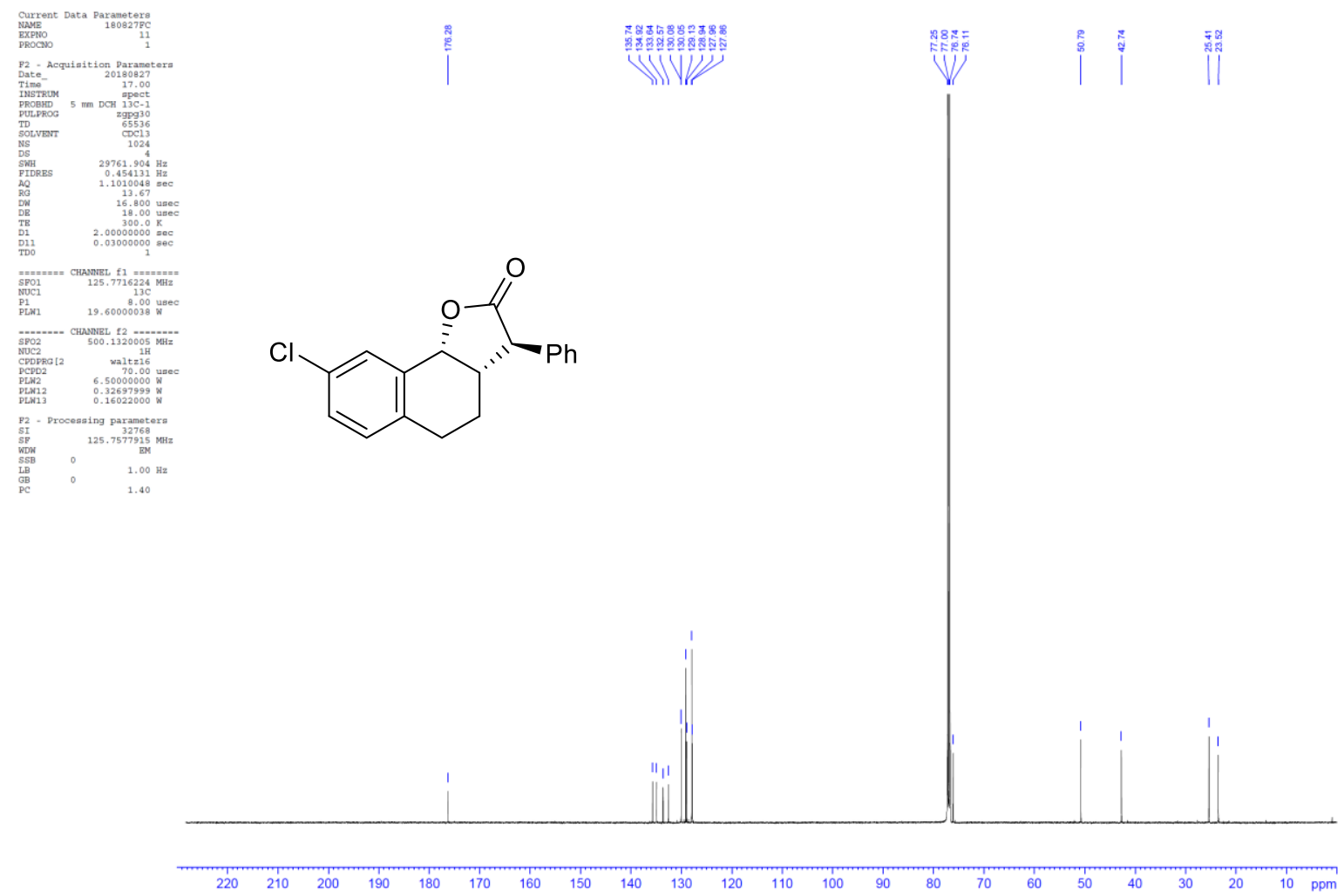
(3S,3aS,9bR)-3-Methyl-3a,4,5,9b-tetrahydronaphtho[1,2-b]furan-2(3H)-one (18c)

${ }^{1} \mathrm{H}-\mathrm{NMR}\left(500 \mathrm{MHz}, \mathrm{CDCl}_{3}\right)$
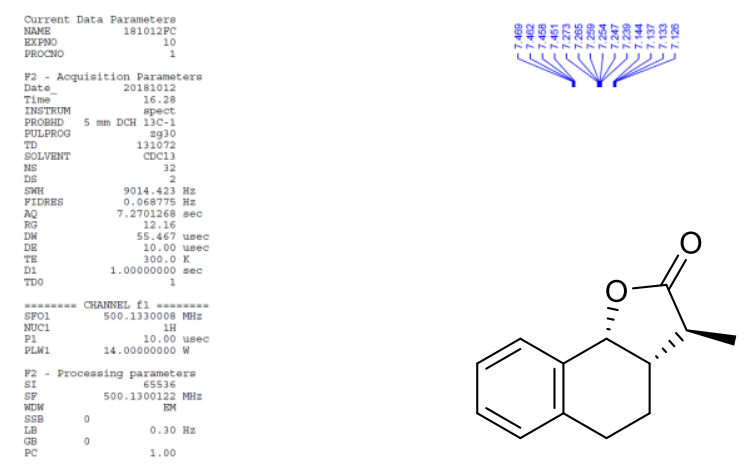

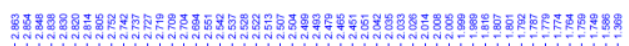
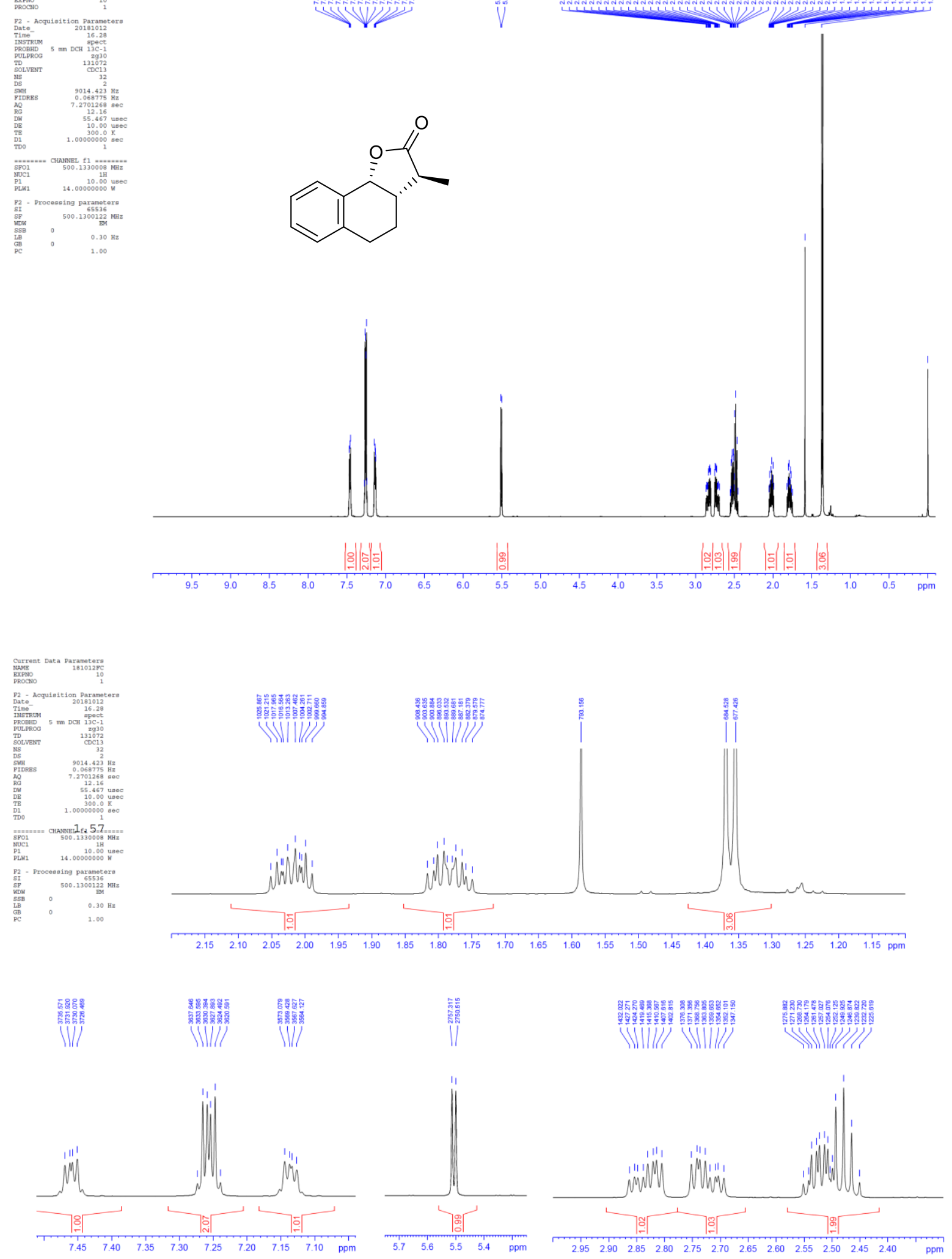
${ }^{13} \mathrm{C} \mathrm{NMR}\left(125 \mathrm{MHz}, \mathrm{CDCl}_{3}\right)$
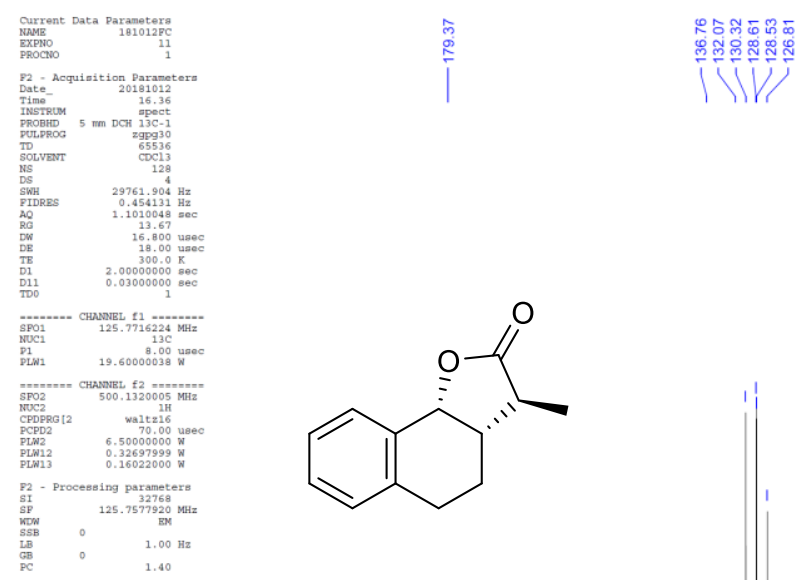

IVW

공ำ

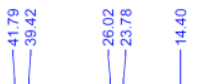
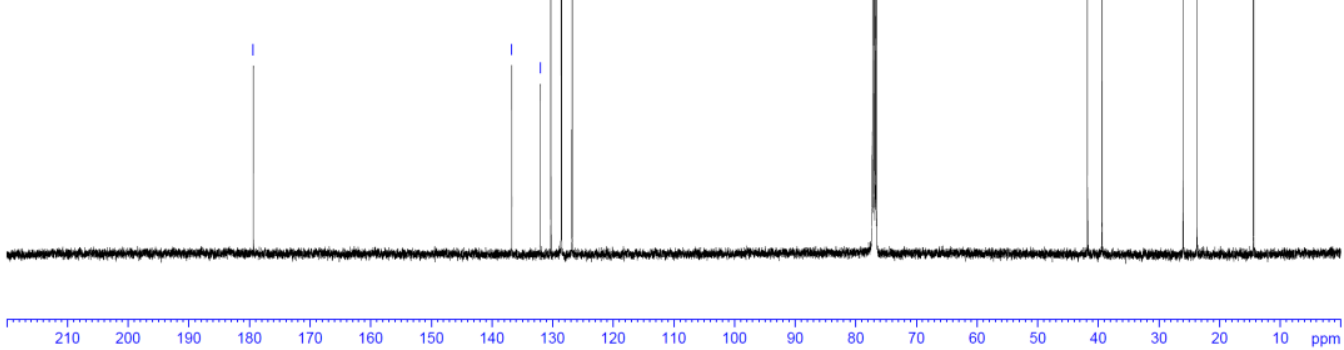
$(3 R, 3 \mathrm{a} S, 9 \mathrm{~b} R)-3-M e t h y l-3 \mathrm{a}, 4,5,9 \mathrm{~b}-$ tetrahydronaphtho[1,2-b]furan-2(3H)-one (19c)

${ }^{1} \mathrm{H}-\mathrm{NMR}\left(500 \mathrm{MHz}, \mathrm{CDCl}_{3}\right)$
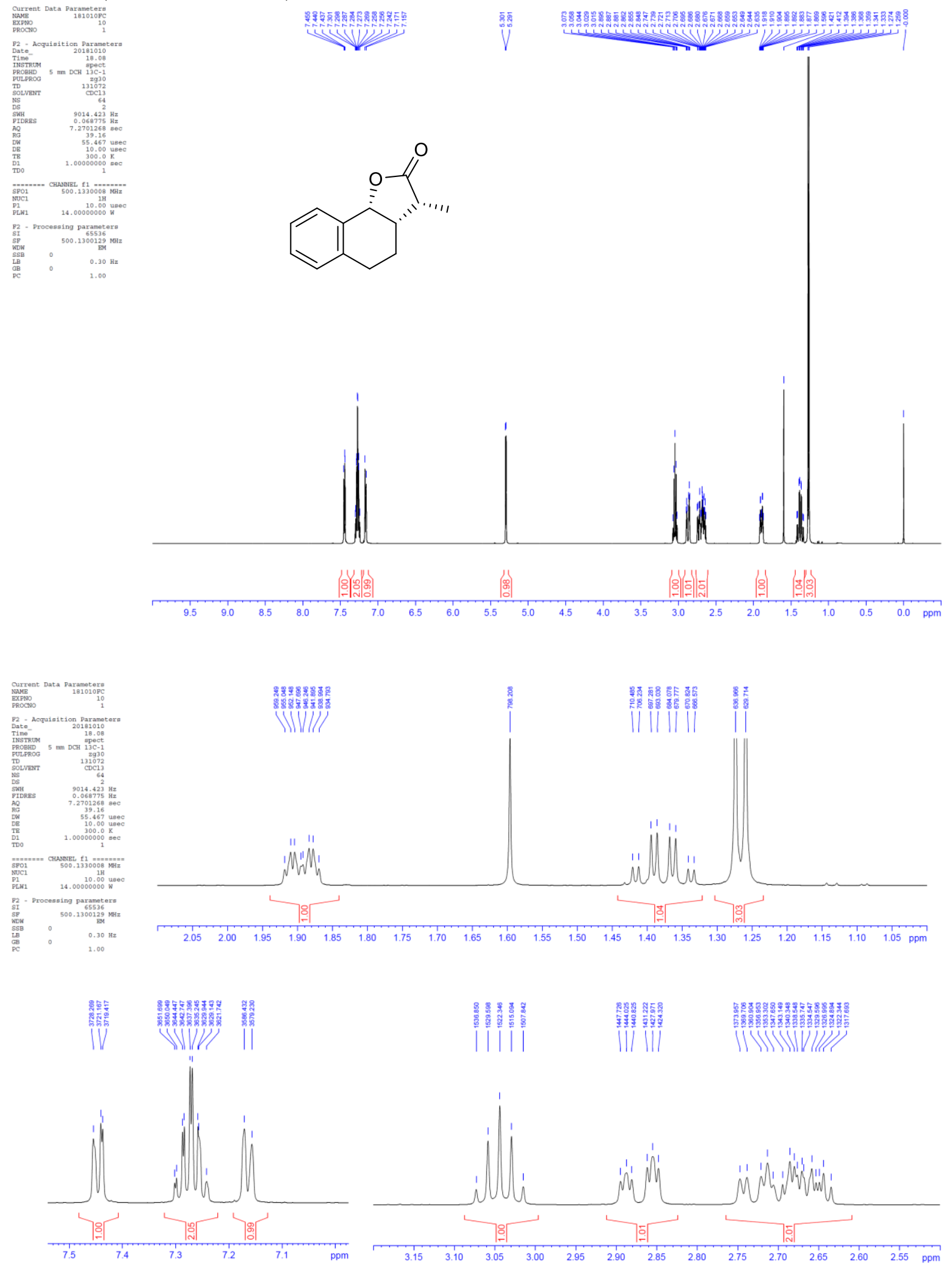
${ }^{13} \mathrm{C}$ NMR (125 MHz, $\left.\mathrm{CDCl}_{3}\right)$
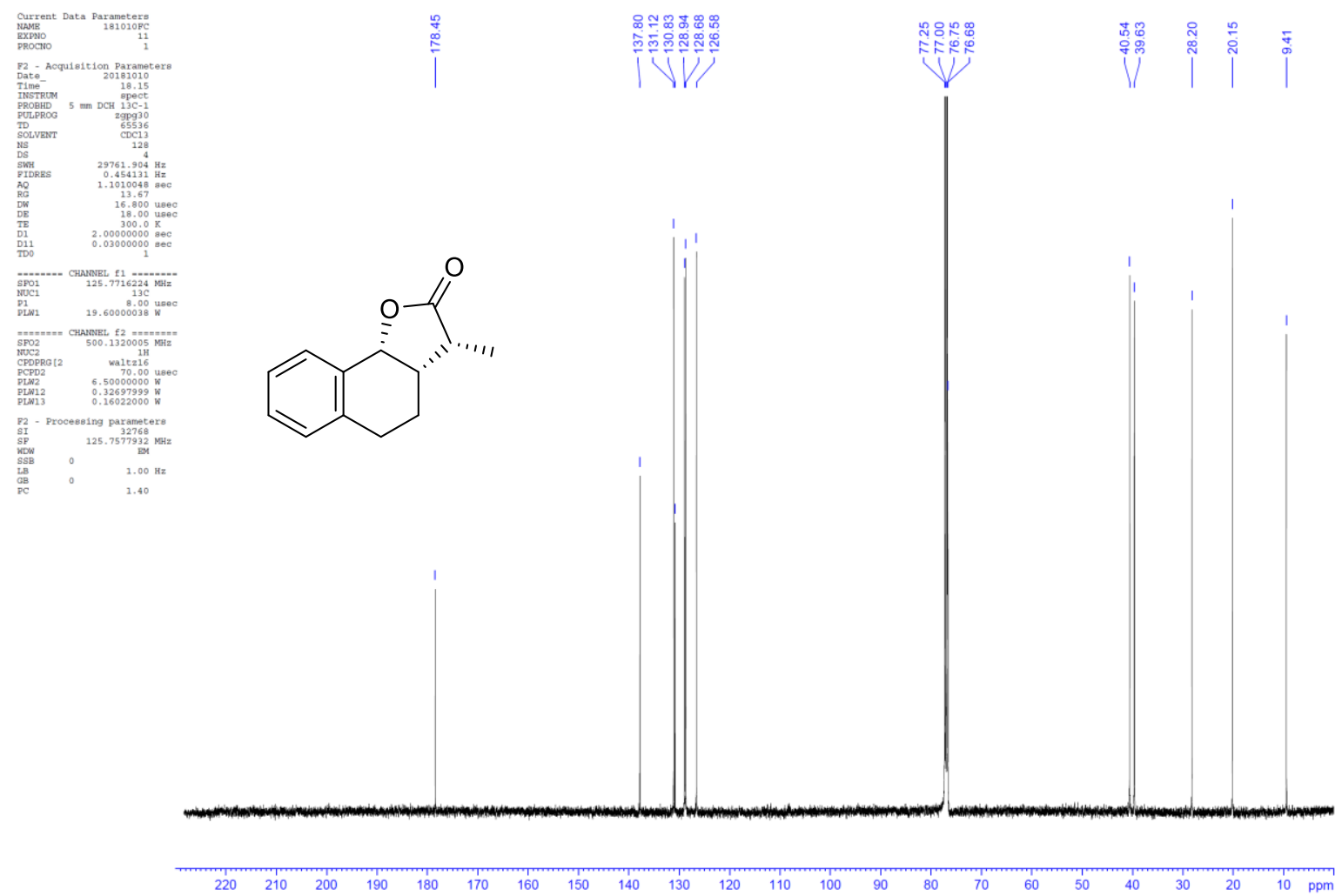
(3aS,9bR)-3-Methylene-3a,4,5,9b-tetrahydronaphtho[1,2-b]furan-2(3H)-one (20)

${ }^{1} \mathrm{H}-\mathrm{NMR}\left(500 \mathrm{MHz}, \mathrm{CDCl}_{3}\right)$
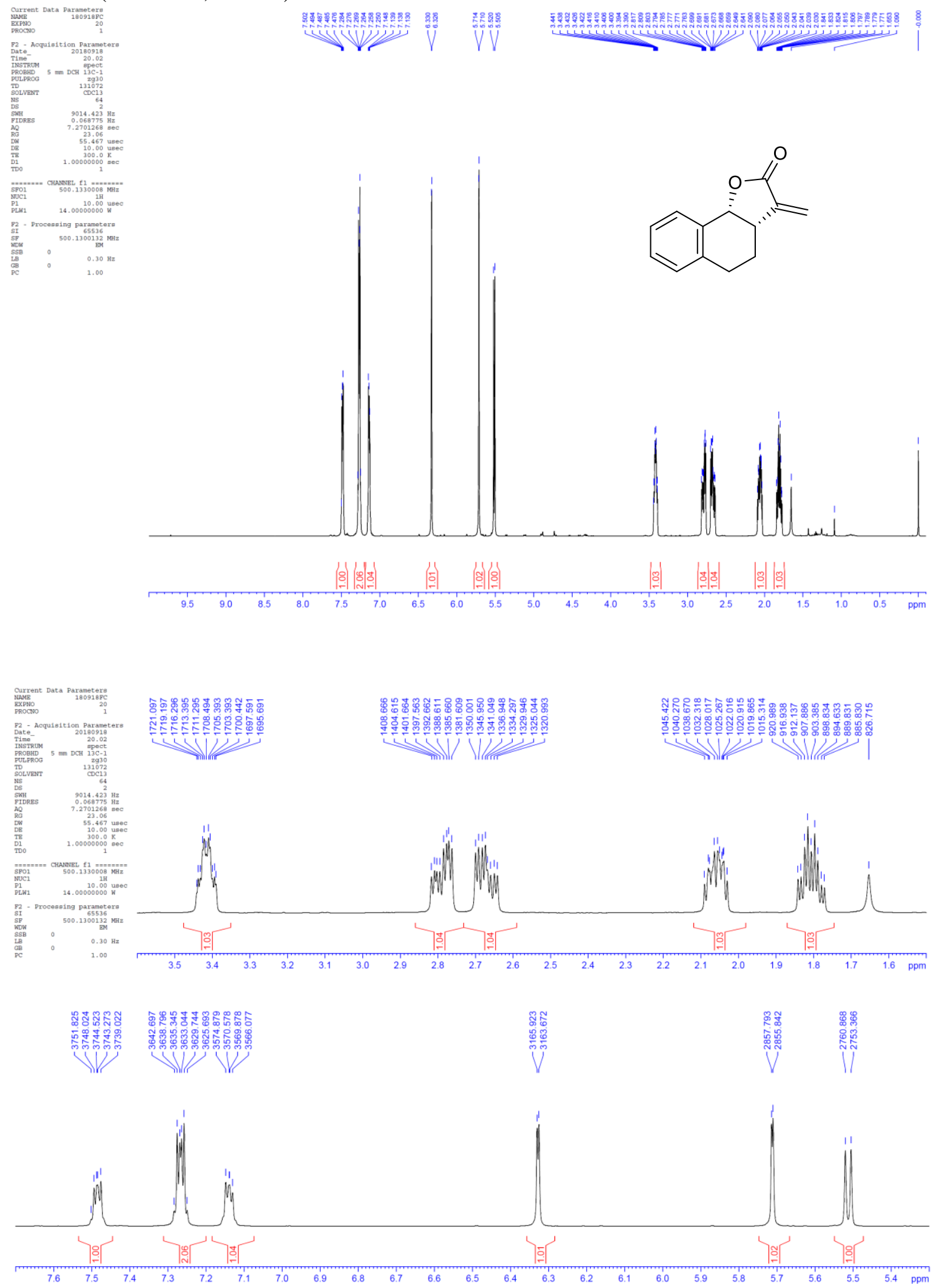
${ }^{13} \mathrm{C}$ NMR (125 MHz, $\left.\mathrm{CDCl}_{3}\right)$
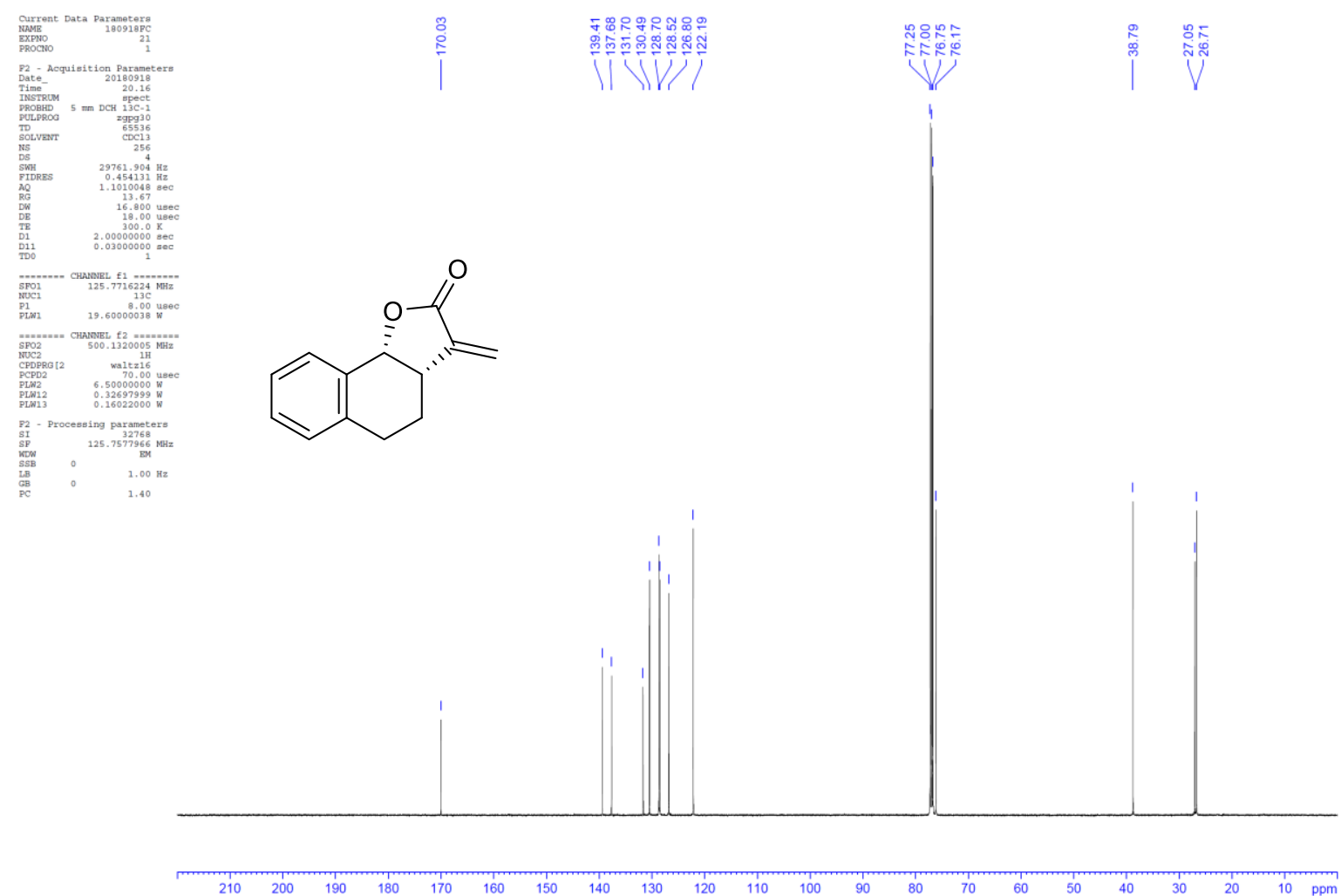
2-(4-Methyl-2-oxocyclohex-3-en-1-yl)acetic acid (23)

${ }^{1} \mathrm{H}-\mathrm{NMR}\left(500 \mathrm{MHz}, \mathrm{CDCl}_{3}\right)$
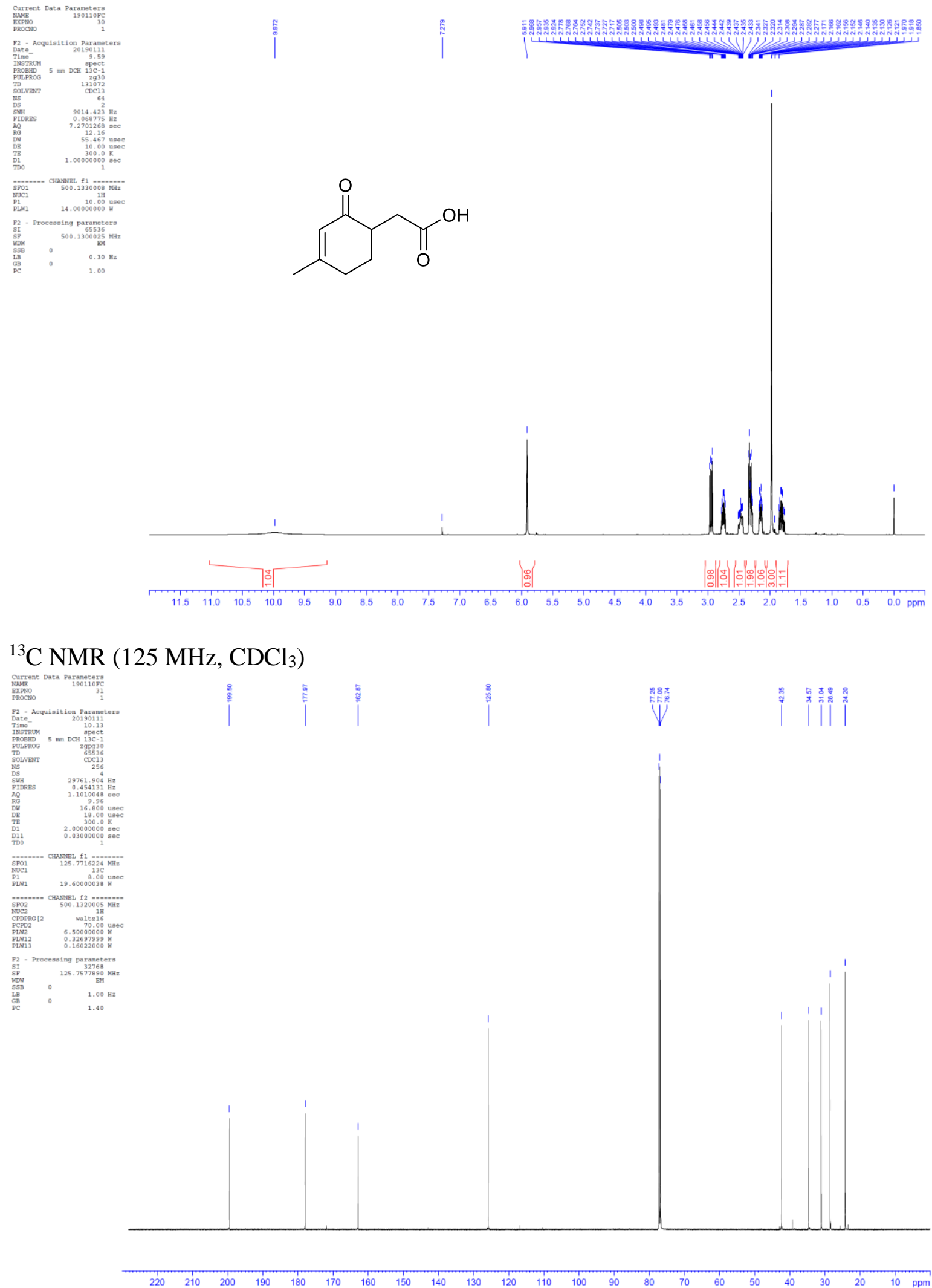
(3aS,7aR)-6-Methyl-3a,4,5,7a-tetrahydrobenzofuran-2(3H)-one (24)

${ }^{1} \mathrm{H}-\mathrm{NMR}\left(500 \mathrm{MHz}, \mathrm{CDCl}_{3}\right)$
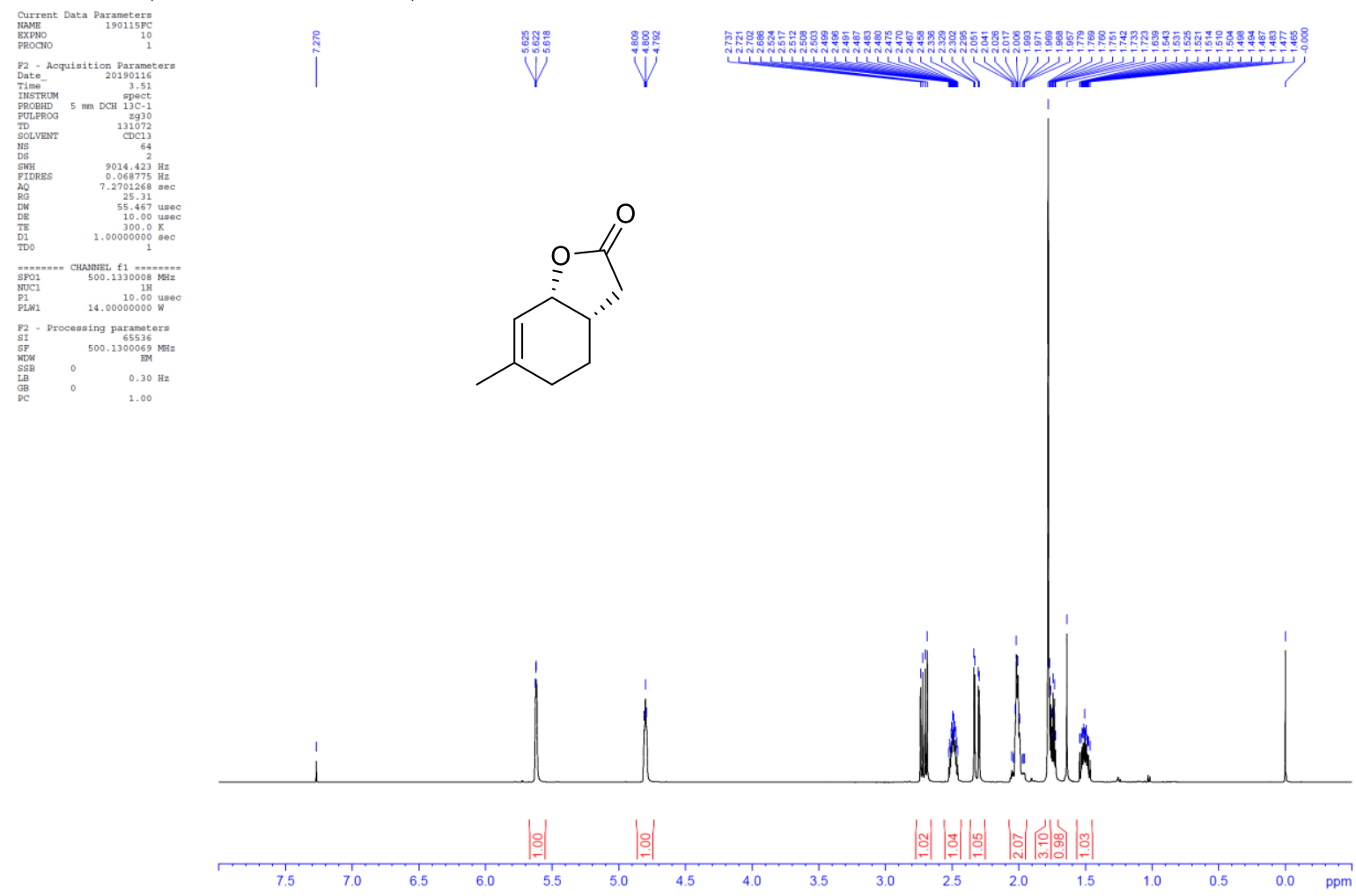

${ }^{13} \mathrm{C} \mathrm{NMR}\left(125 \mathrm{MHz}, \mathrm{CDCl}_{3}\right)$
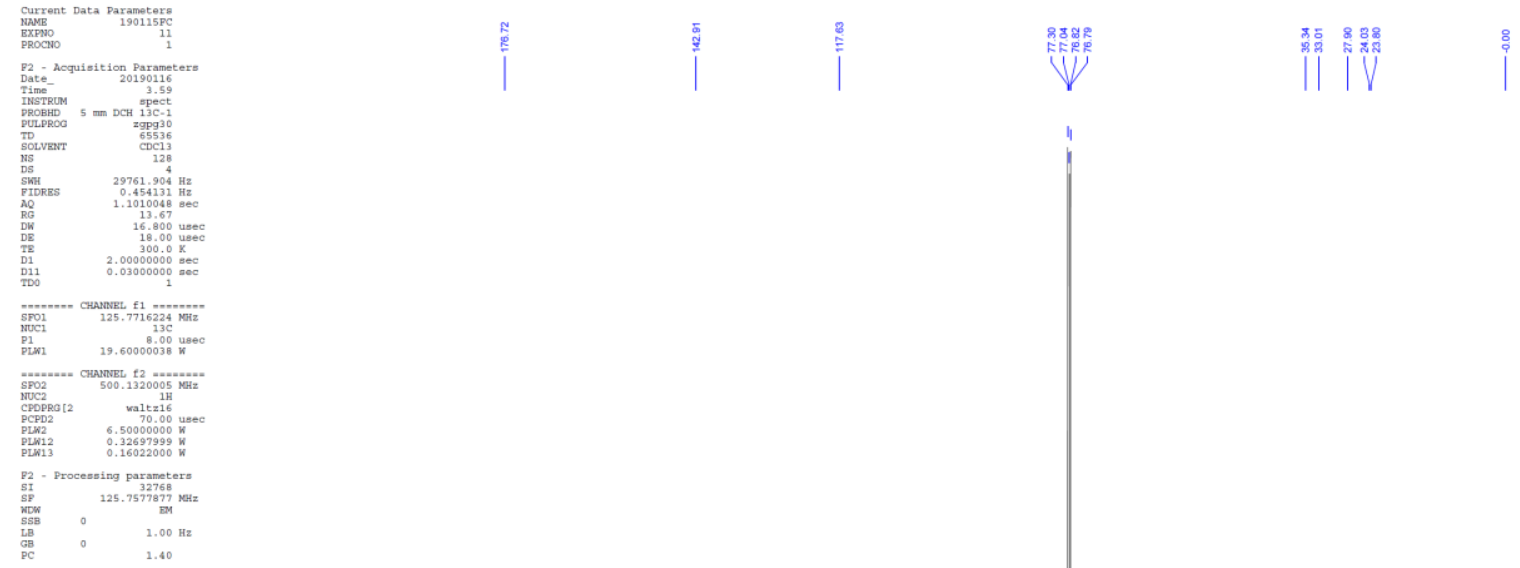

$$
\text { (1) }
$$

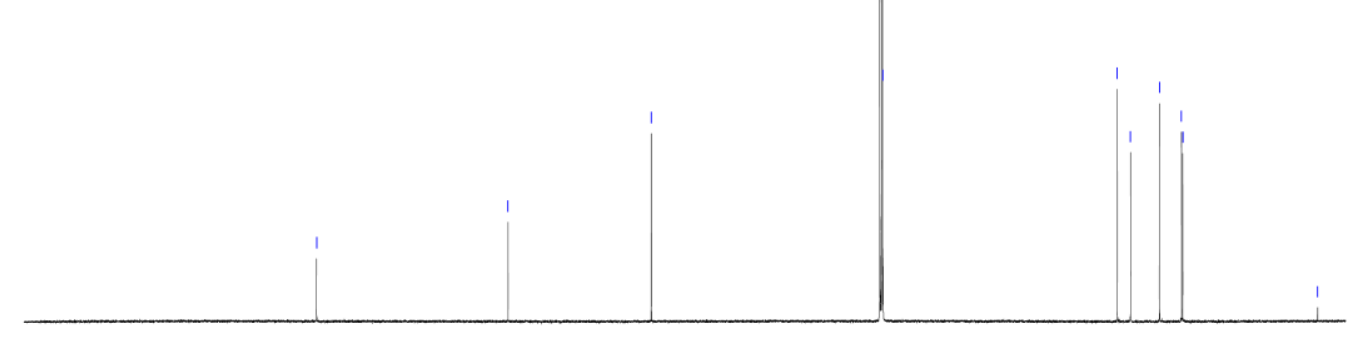

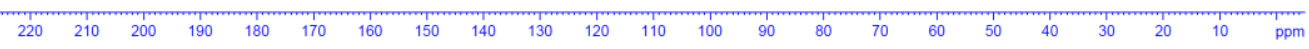


(3aS,7aS)-6-Methyl-3-methylene-3a,4,5,7a-tetrahydrobenzofuran-2(3H)-one (25)

${ }^{1} \mathrm{H}-\mathrm{NMR}\left(500 \mathrm{MHz}, \mathrm{CDCl}_{3}\right)$
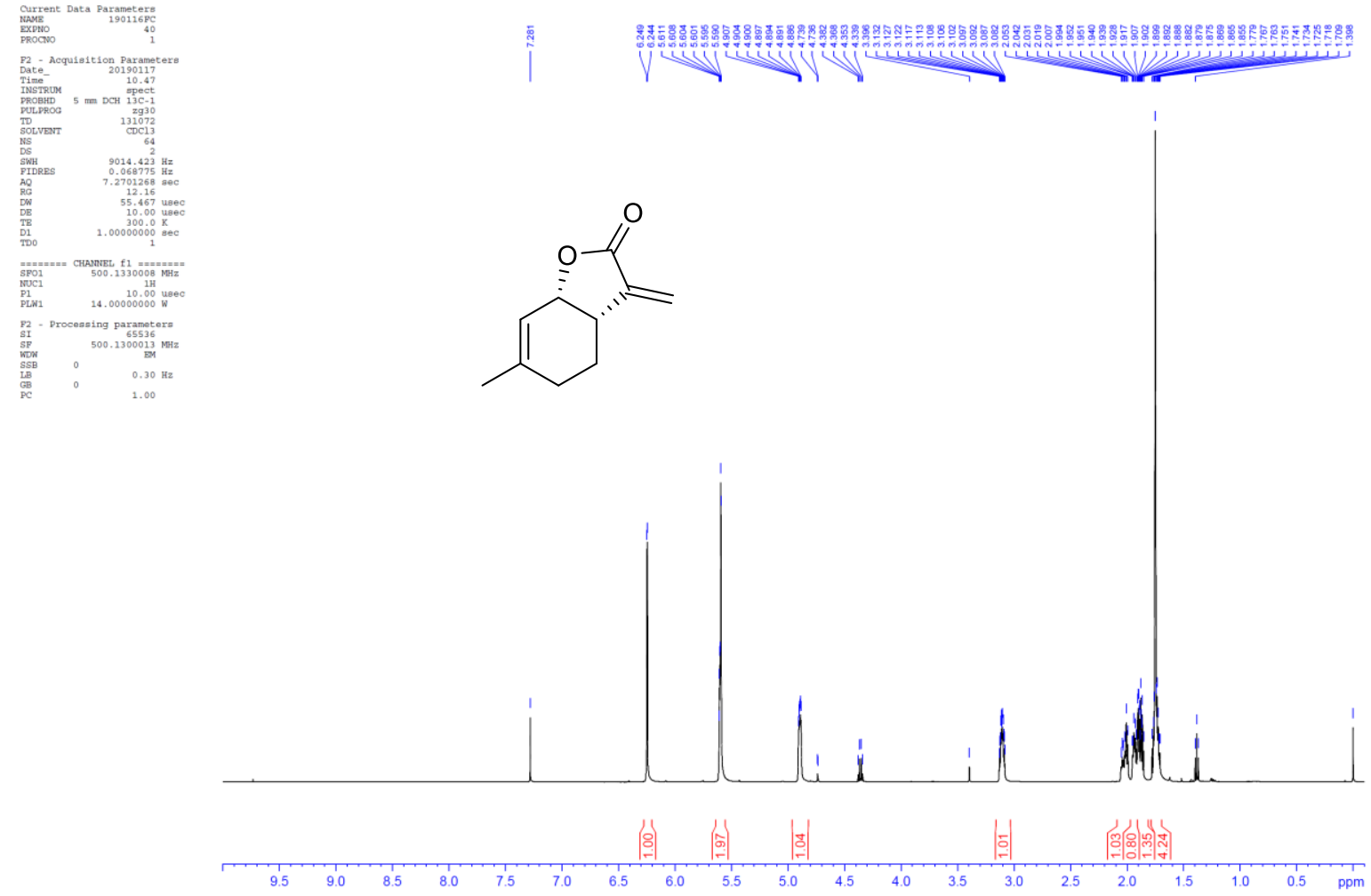

${ }^{13} \mathrm{C}$ NMR $\left(125 \mathrm{MHz}, \mathrm{CDCl}_{3}\right)$
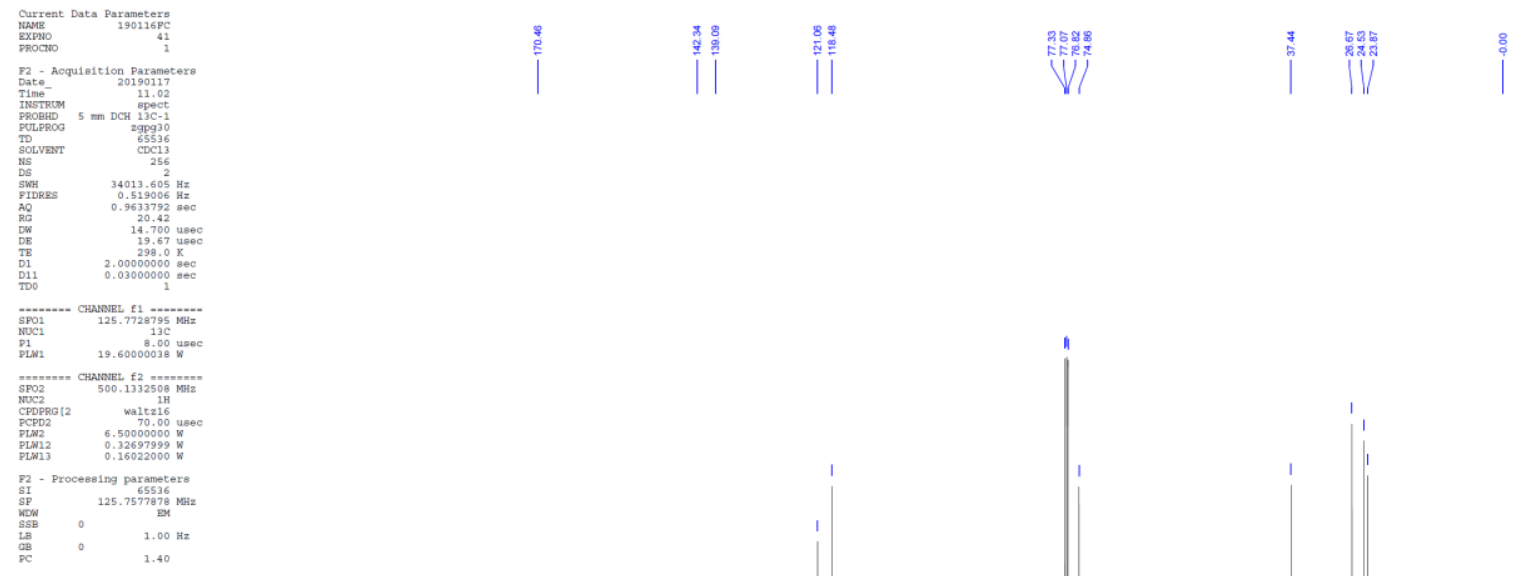

$$
\text { . }
$$

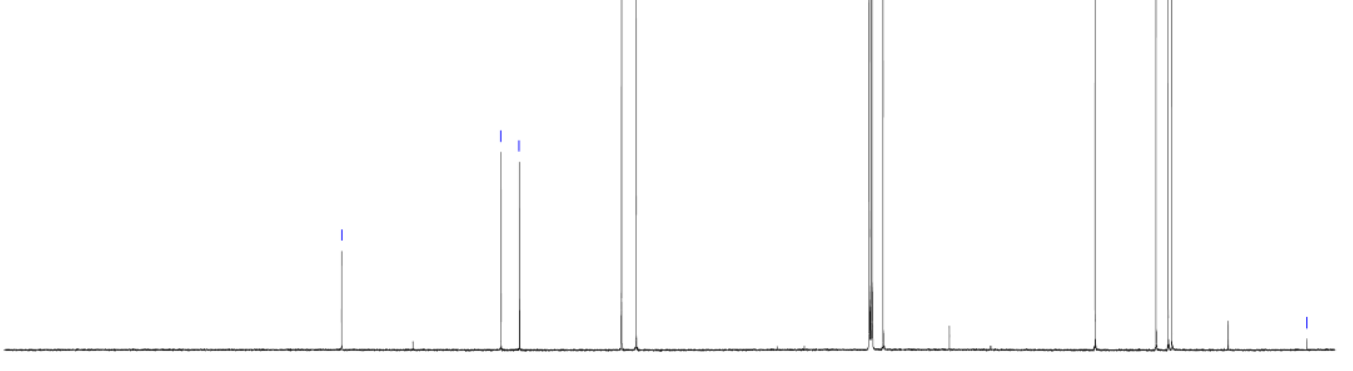


Wine Lactone ((3S-6))

$(3 S, 3 \mathrm{a} S, 7 \mathrm{a} R)$-3,6-Dimethyl-3a,4,5,7a-tetrahydrobenzofuran-2(3H)-one

${ }^{1} \mathrm{H}-\mathrm{NMR}\left(500 \mathrm{MHz}, \mathrm{CDCl}_{3}\right)$
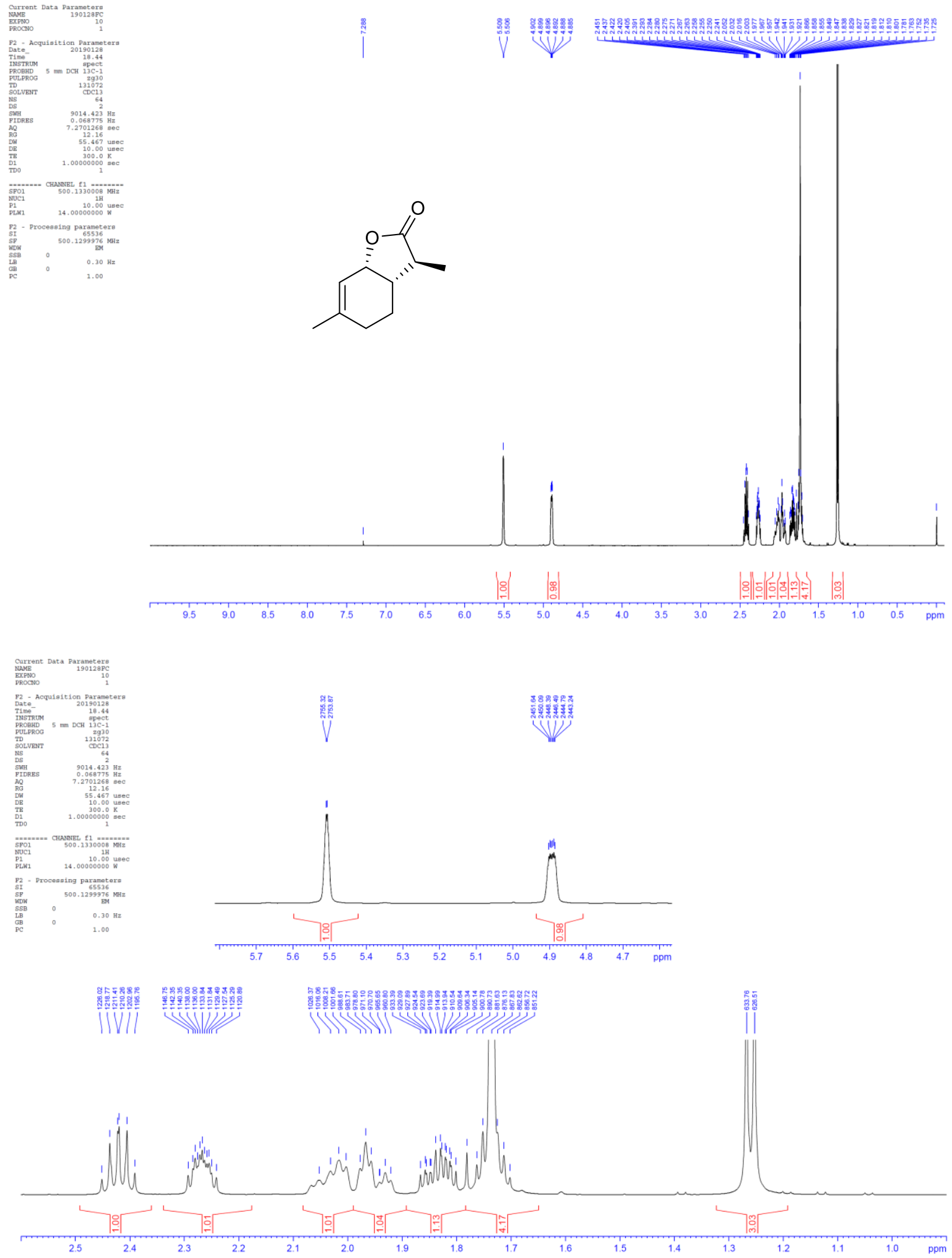
${ }^{13} \mathrm{C}$ NMR (125 MHz, $\left.\mathrm{CDCl}_{3}\right)$
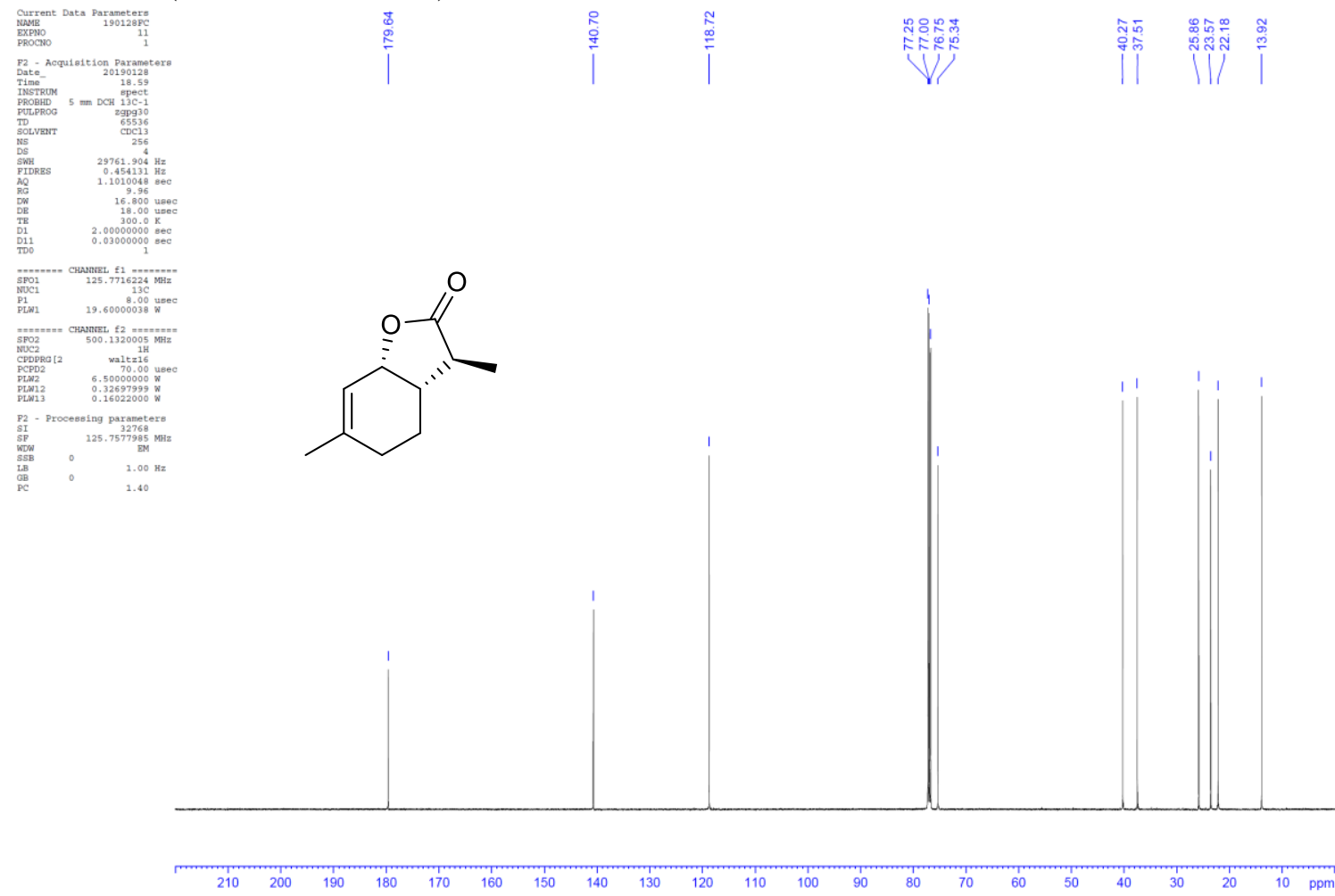
(3R,3aS,7aR)-3,6-Dimethyl-3a,4,5,7a-tetrahydrobenzofuran-2(3H)-one ((3R-6))

${ }^{1} \mathrm{H}-\mathrm{NMR}\left(500 \mathrm{MHz}, \mathrm{CDCl}_{3}\right)$
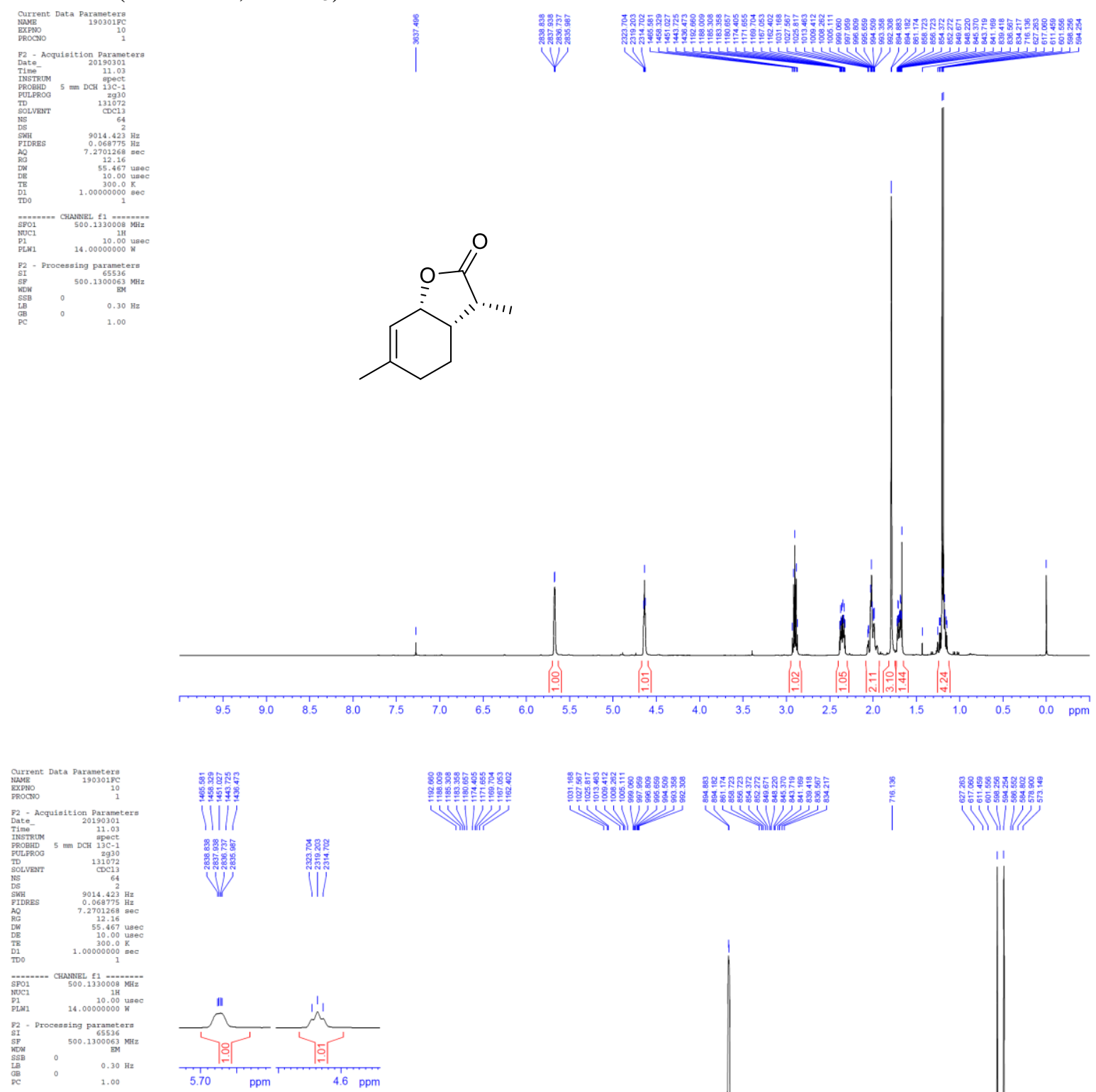

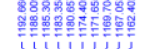

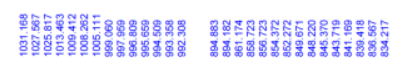

vive

岤
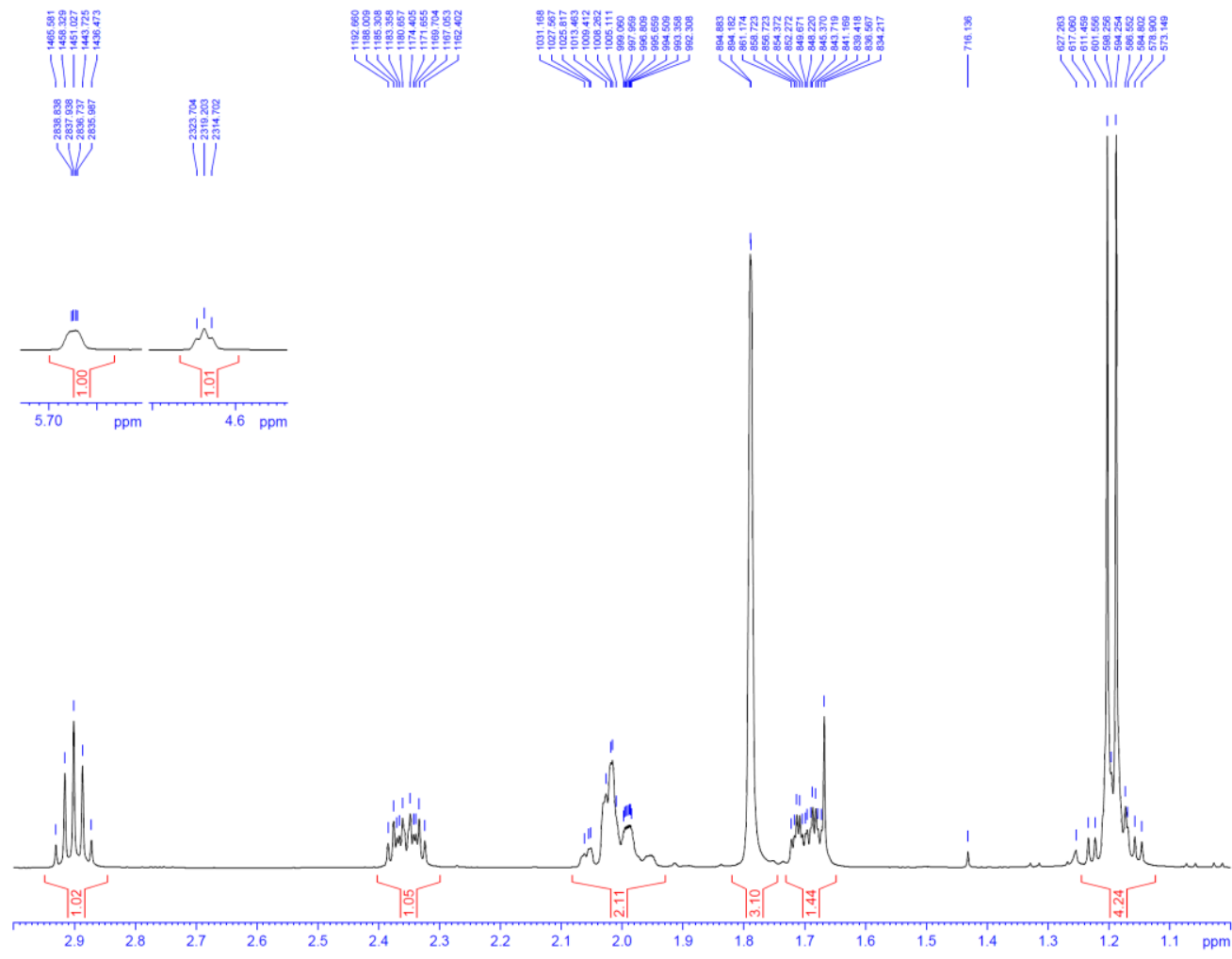
${ }^{13} \mathrm{C}$ NMR (125 MHz, $\left.\mathrm{CDCl}_{3}\right)$
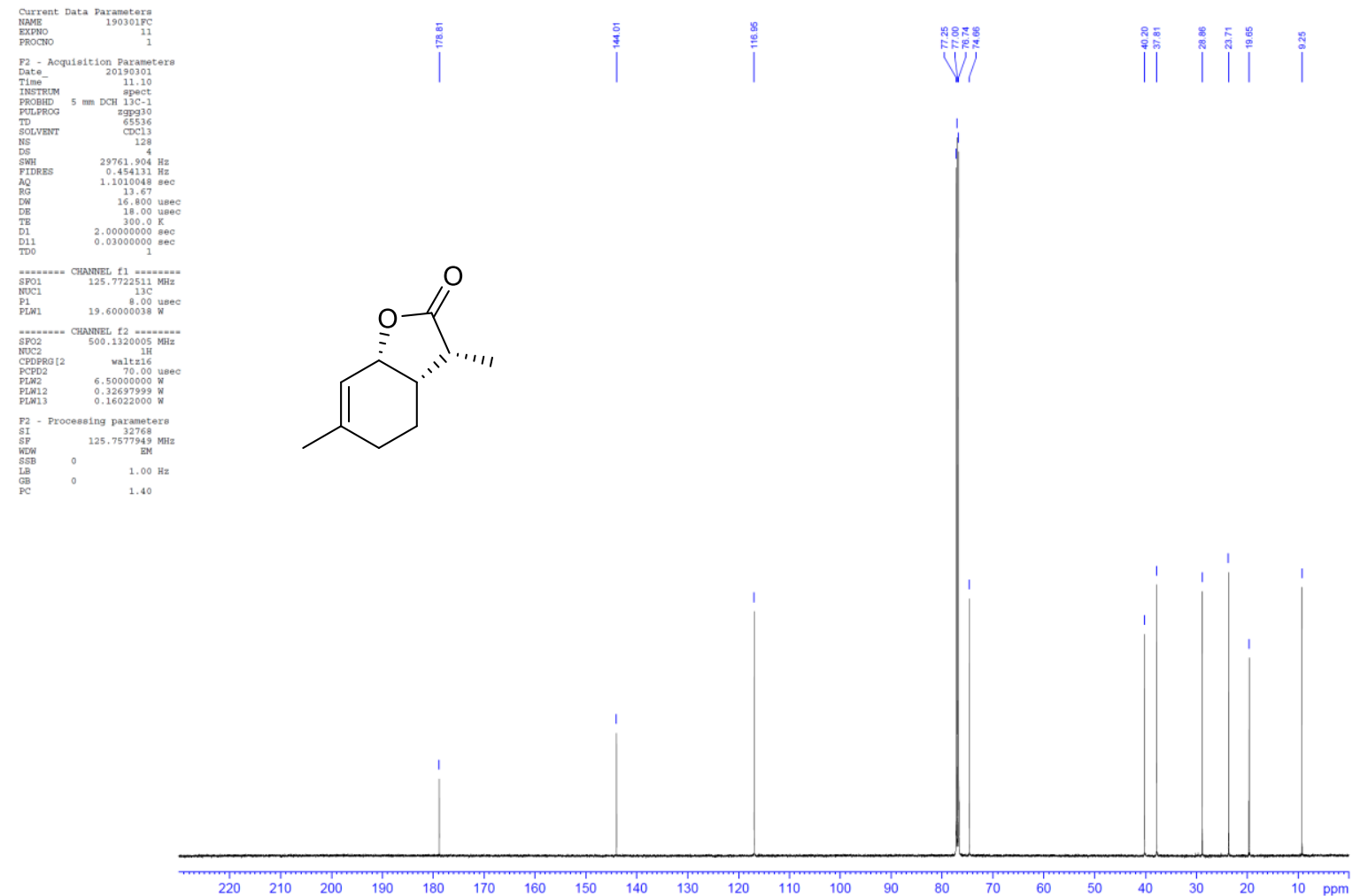
$N$-((1R,2R)-2-Amino-1,2-diphenylethyl)-2,4,6-triisopropylbenzenesulfonamide

${ }^{1} \mathrm{H}-\mathrm{NMR}\left(500 \mathrm{MHz}, \mathrm{CDCl}_{3}\right.$ )
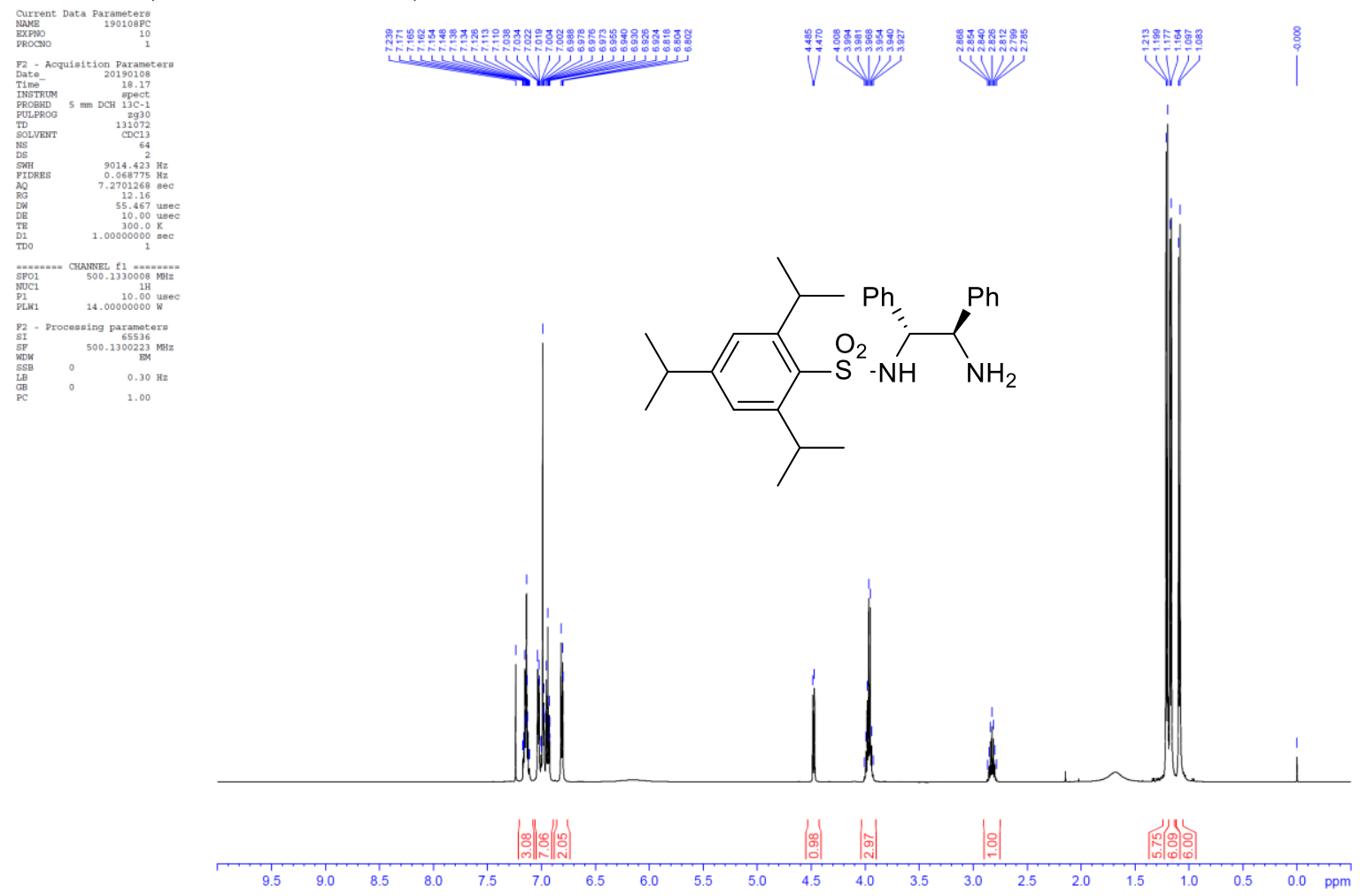

${ }^{13} \mathrm{C} \mathrm{NMR}\left(125 \mathrm{MHz}, \mathrm{CDCl}_{3}\right)$
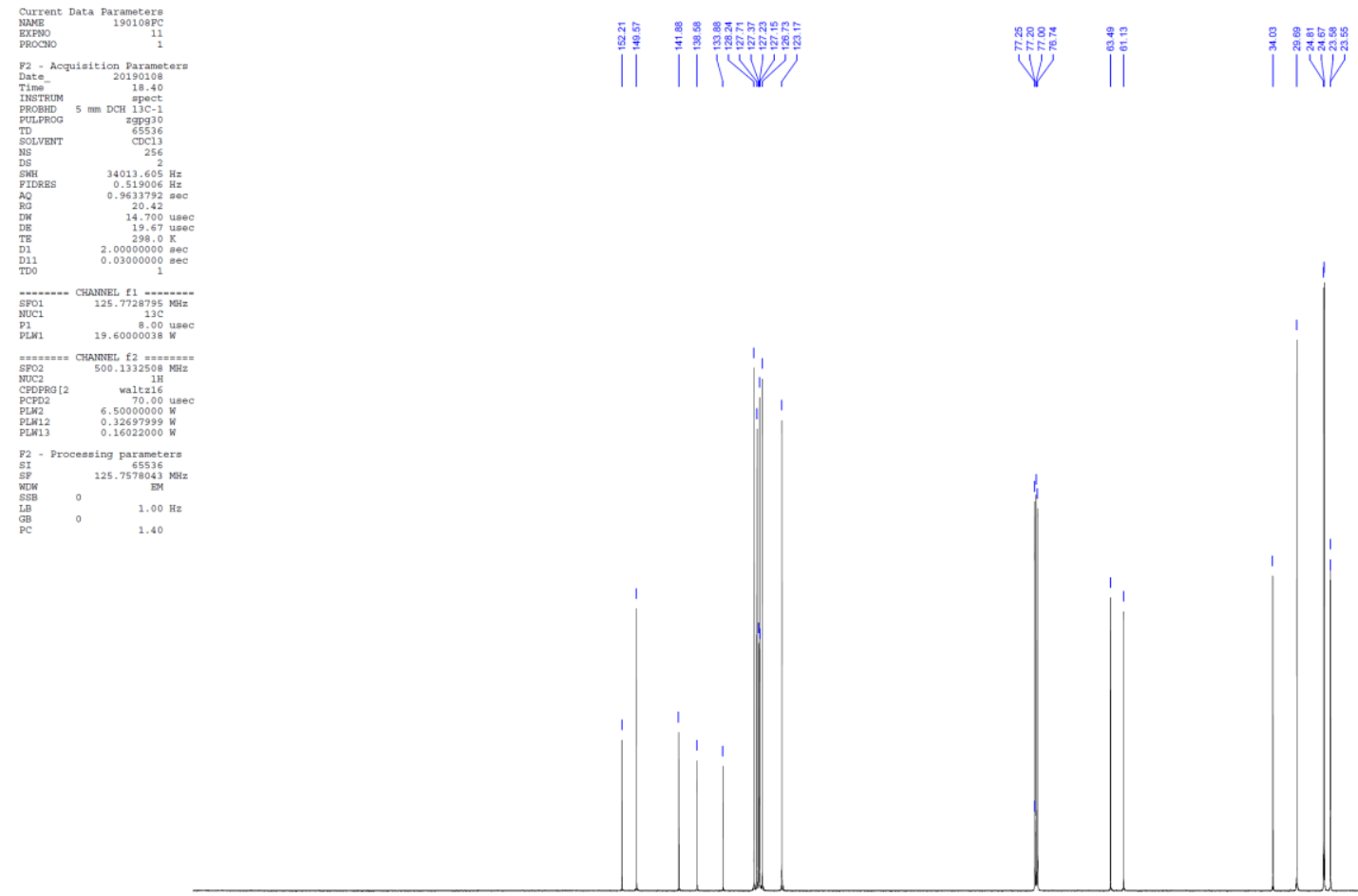

$\begin{array}{llllllll}220 & 210 & 200 & 190 & 180 & 170 & 160 & 150\end{array}$ 
2,4,6-Triisopropyl- $N$-((1R,2R)-2-((2-((4-methylcyclohexa-1,4-dien-1-yl)methoxy)ethyl) amino)-1,2-diphenylethyl)benzenesulfonamide

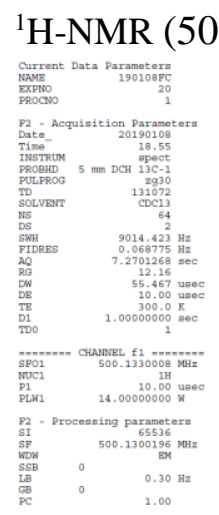

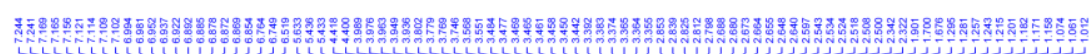
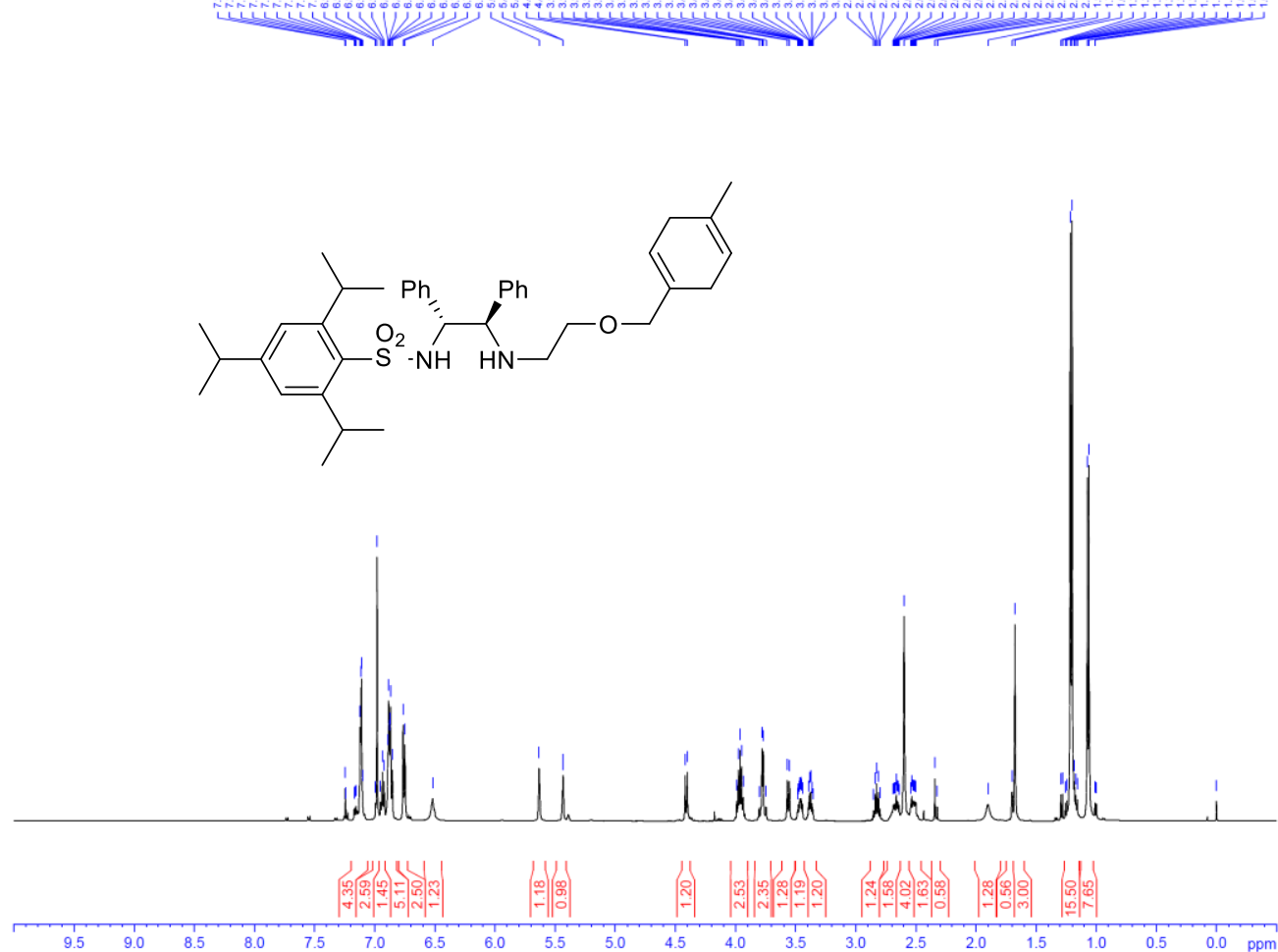

${ }^{13} \mathrm{C} \mathrm{NMR}\left(125 \mathrm{MHz}, \mathrm{CDCl}_{3}\right)$
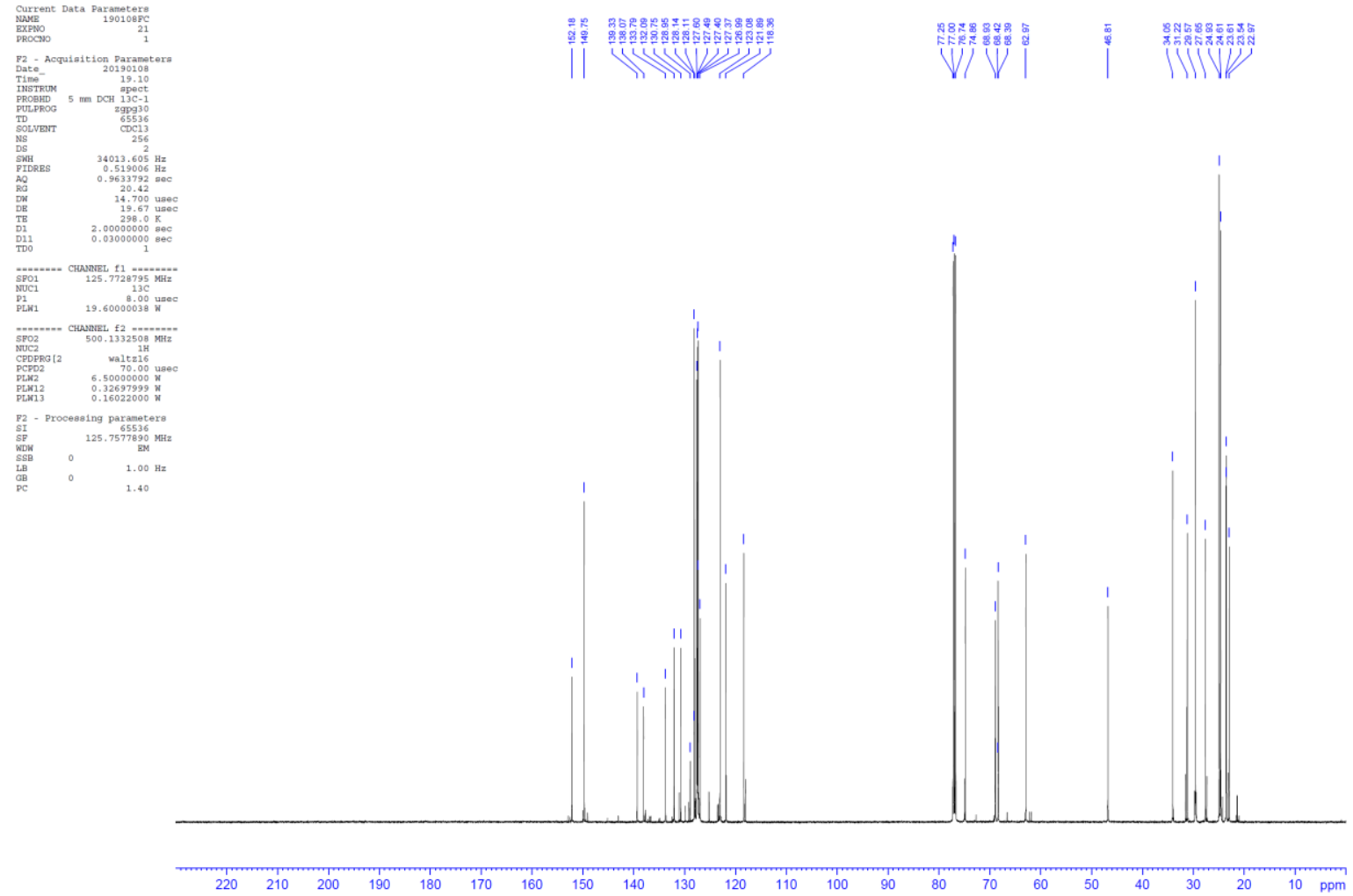


\section{$(R, R)$-TIPPs-DENEB $((R, R)-5)$}

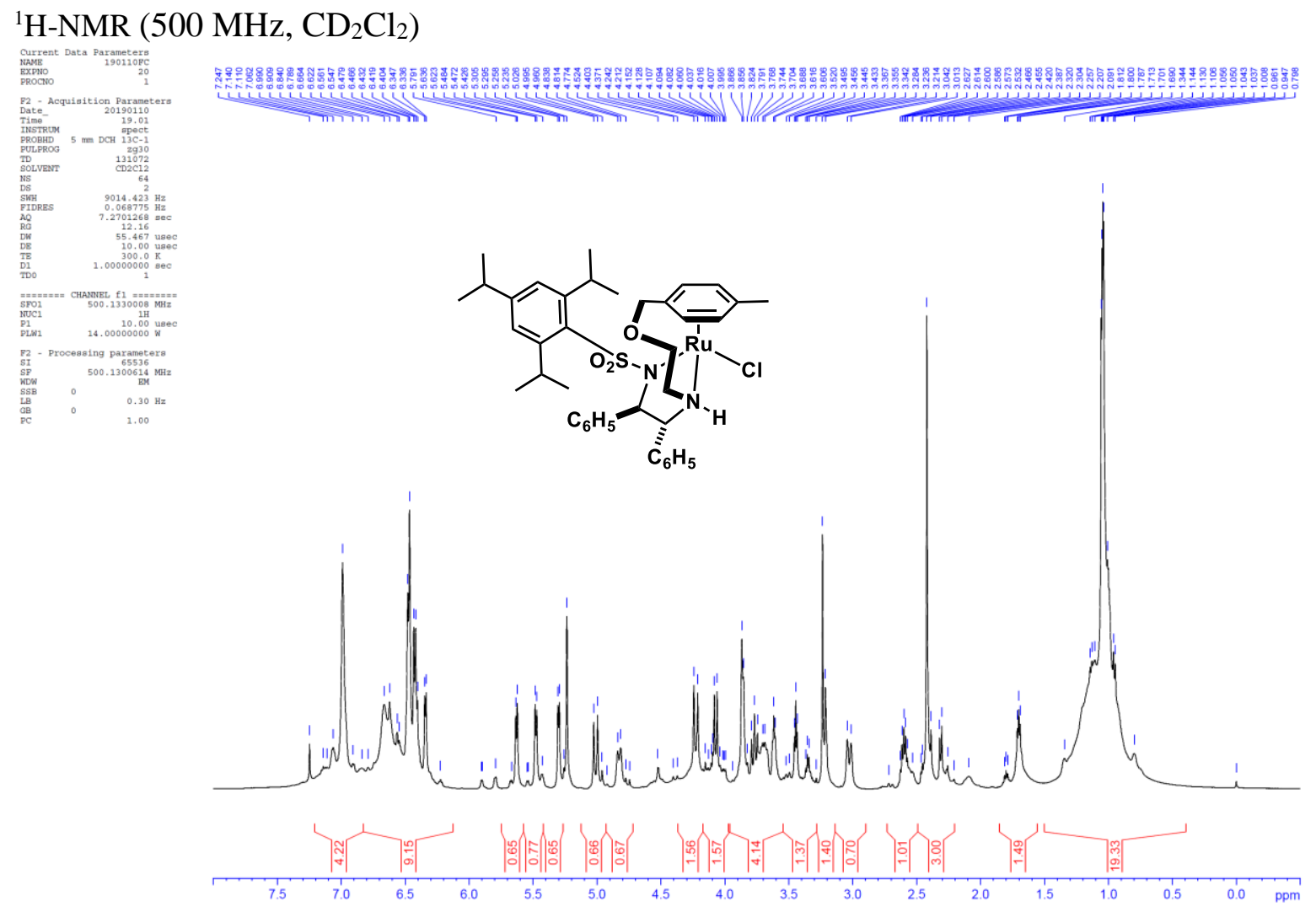

${ }^{13} \mathrm{C}$ NMR $\left(125 \mathrm{MHz}, \mathrm{CD}_{2} \mathrm{Cl}_{2}\right.$ )
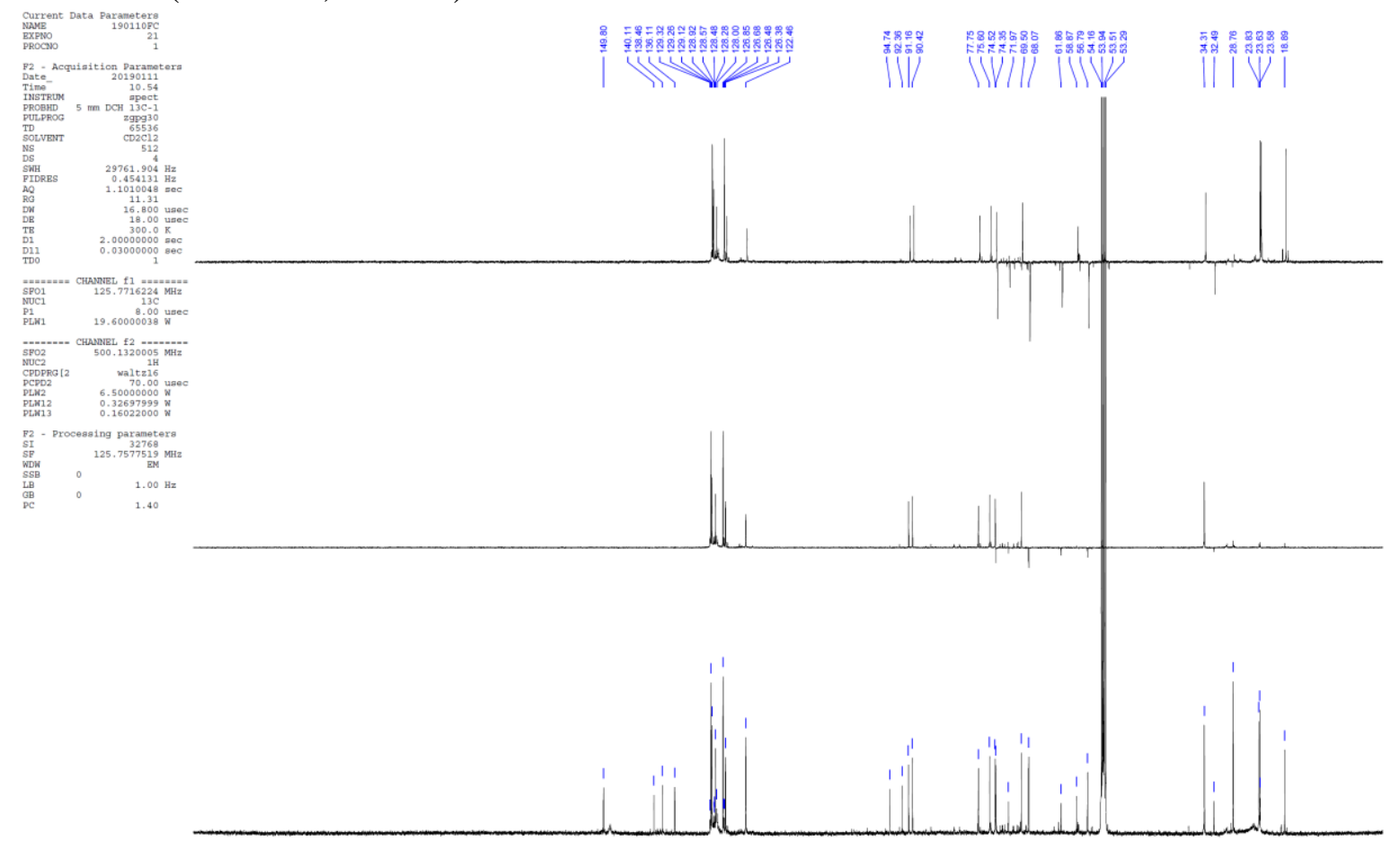
${ }^{1} \mathrm{H}-{ }^{1} \mathrm{H}-\mathrm{COSY}\left(\mathrm{CD}_{2} \mathrm{Cl}_{2}\right)$

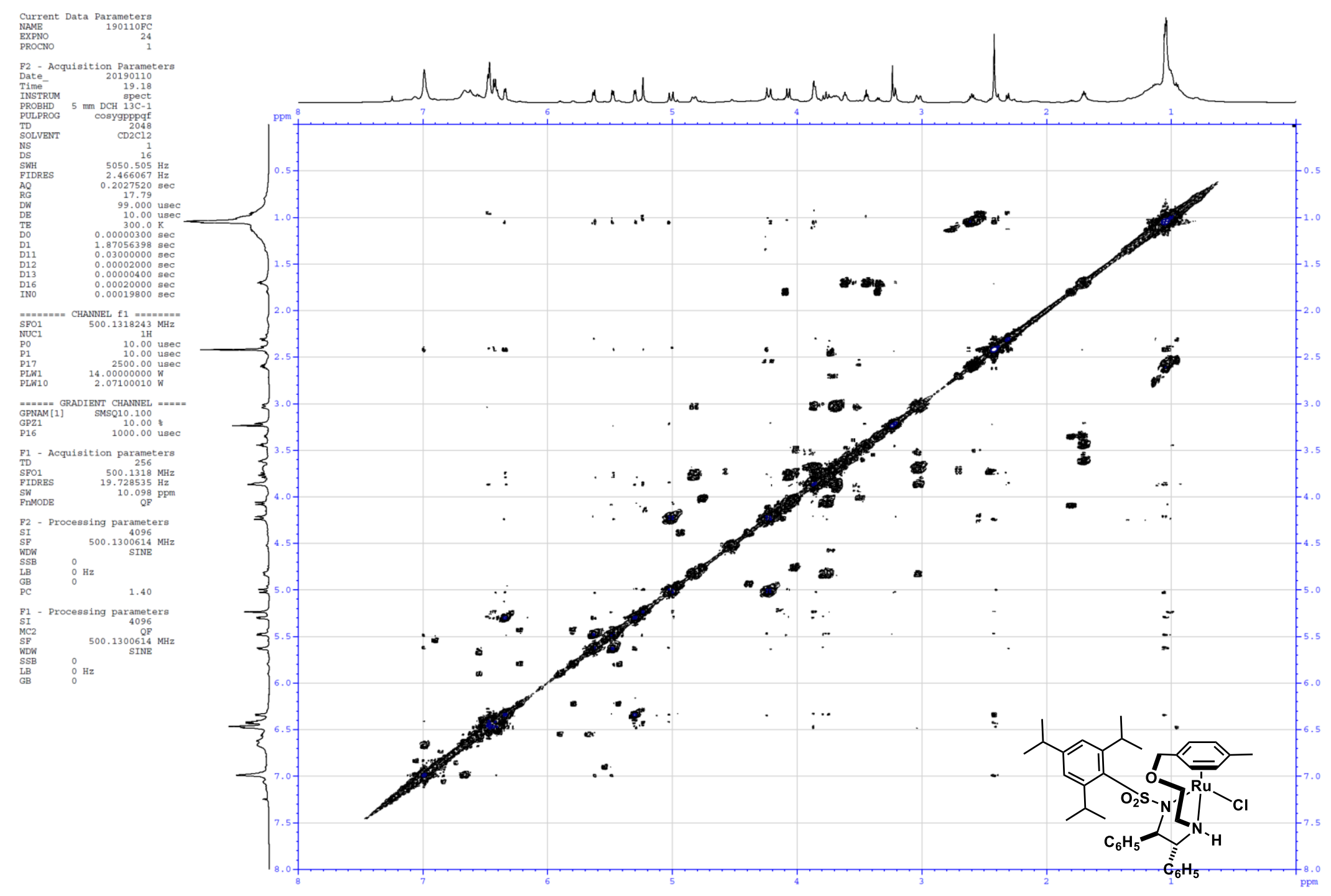

S170 
$\operatorname{HSQC}\left(\mathrm{CD}_{2} \mathrm{Cl}_{2}\right)$

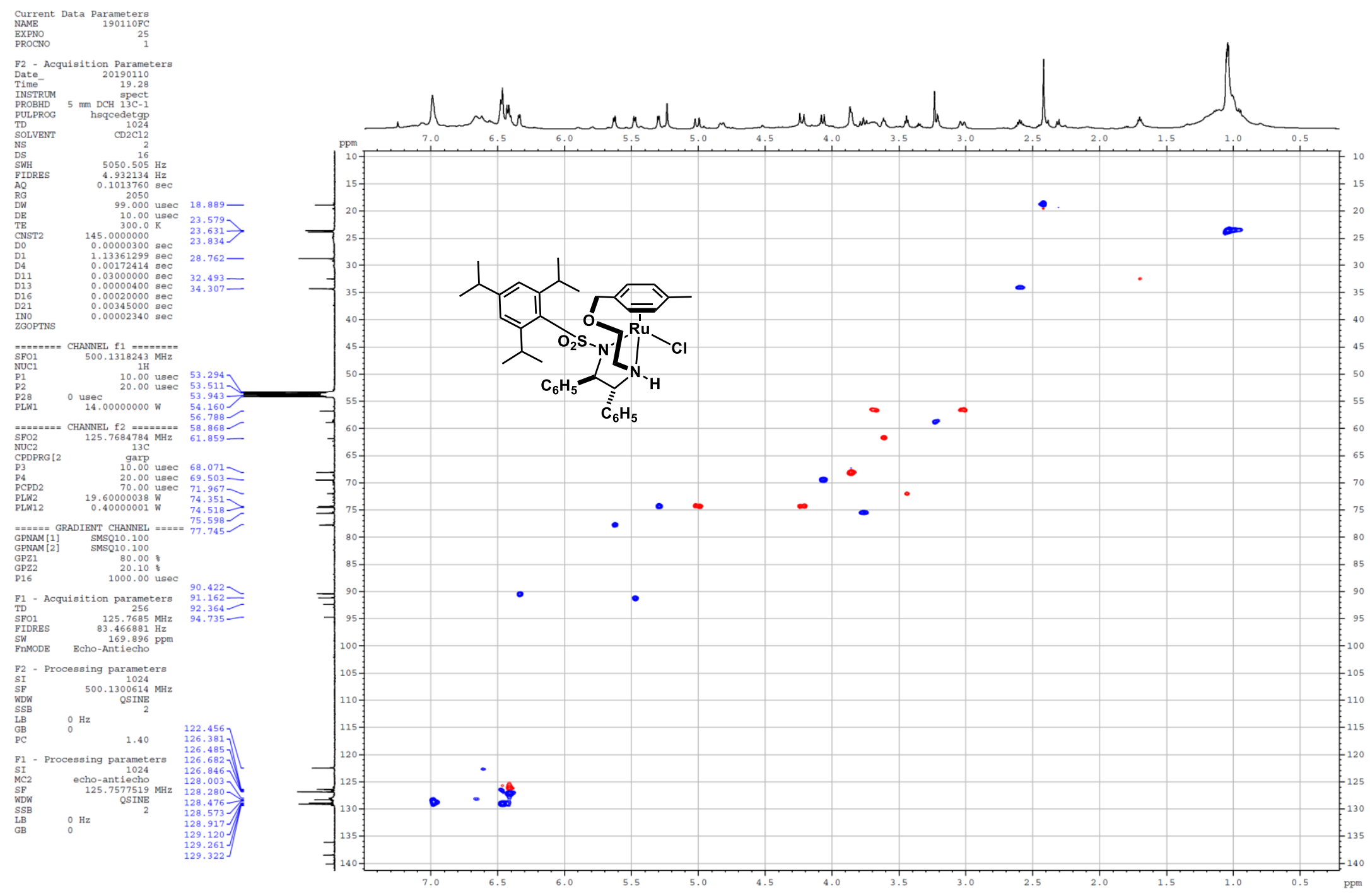




\section{$\operatorname{HMBC}\left(\mathrm{CD}_{2} \mathrm{Cl}_{2}\right)$}

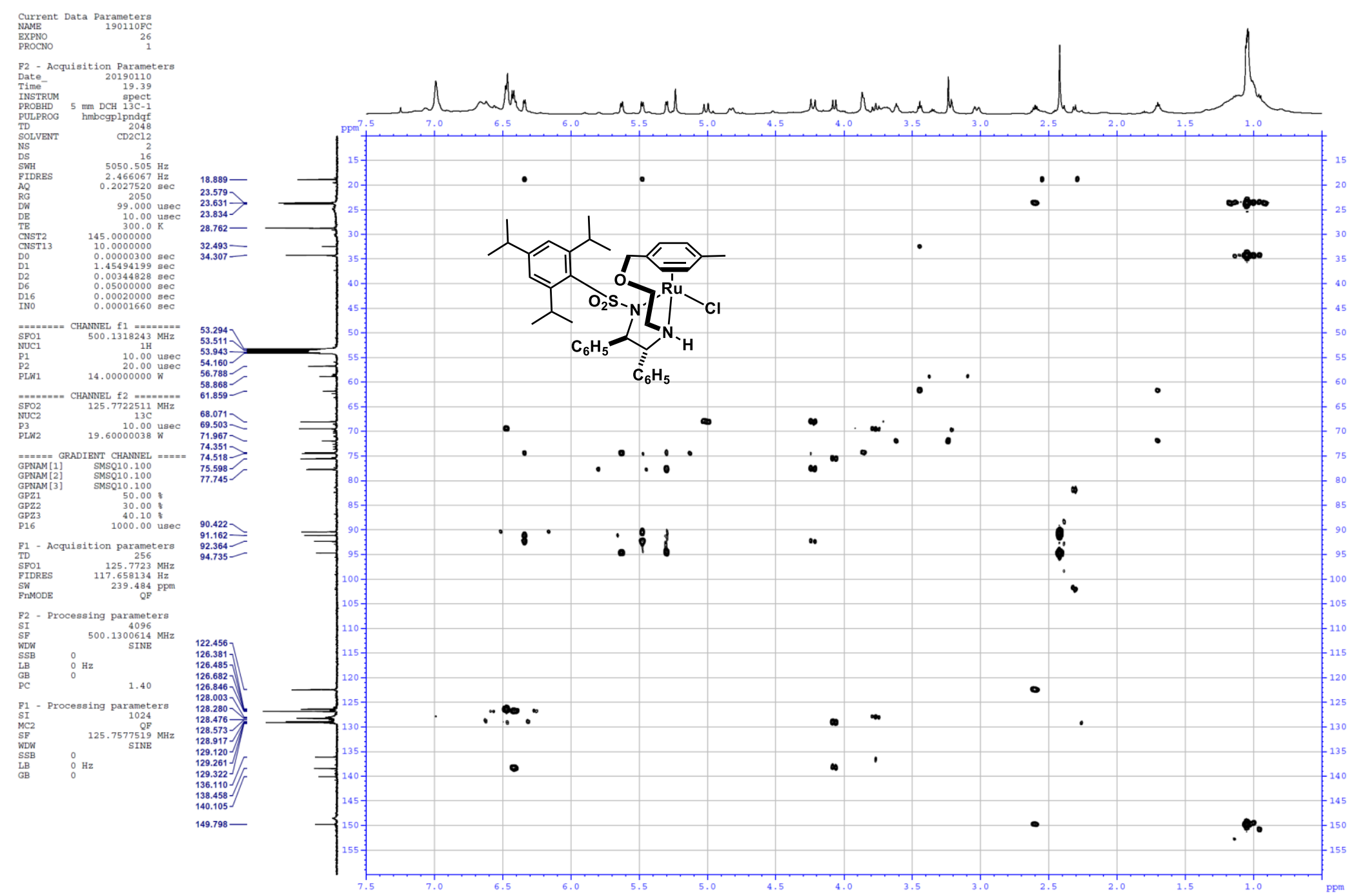




\section{Chiral HPLC Charts for Reduction Products.}

\section{$(1 R, 2 R)-2-M e t h y l-1,2,3,4-t e t r a h y d r o n a p h t h a l e n-1-o l ~(9 a)$}

YMC Alcyon SFC CPS Cellulose-SC, $250 \times 4.6 \mathrm{~mm}$ column, hexane $/ 2$-propanol 99:1, $1.0 \mathrm{~mL} / \mathrm{min}$, $220 \mathrm{~nm}, 40^{\circ} \mathrm{C}$

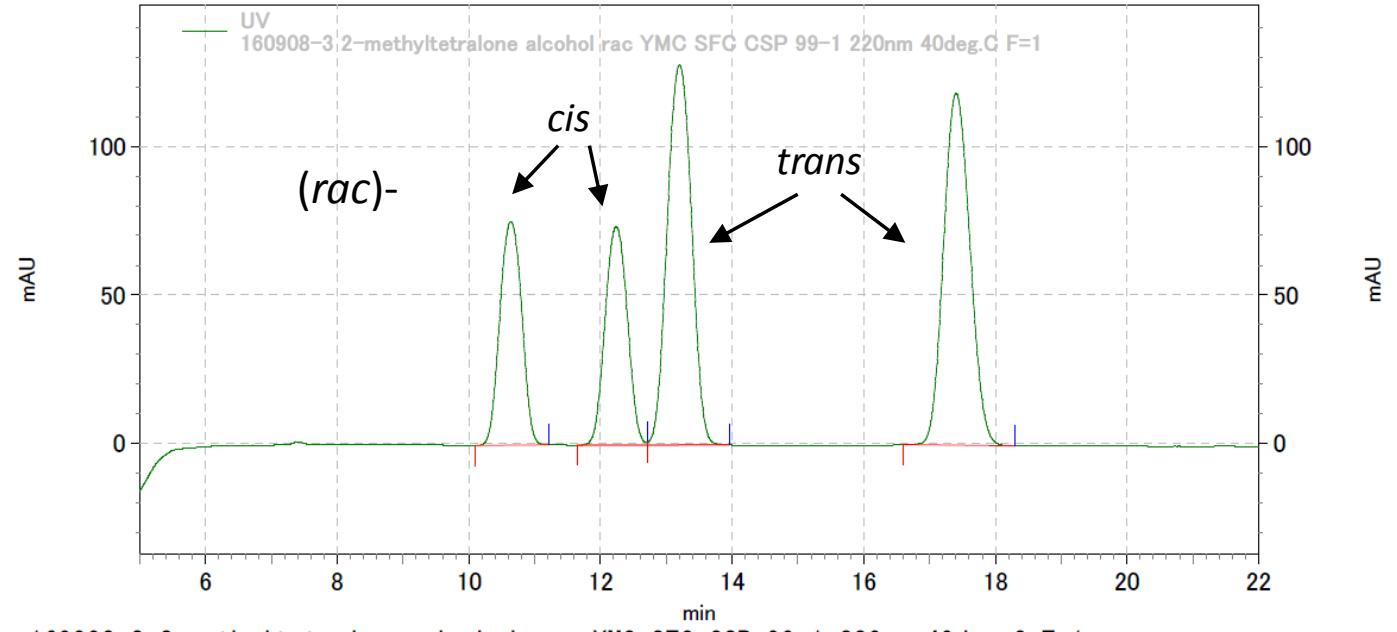

160908-3 2-methyltetralone alcohol rac YMC SFC CSP 99-1 220nm 40deg. C F=1

\begin{tabular}{rlrrrr}
$\begin{array}{c}\text { Pk } \\
\text { No. }\end{array}$ & R. T. & Area & Area\% & B. C. & Symmetry \\
\hline 1 & 10.637 & 6963586 & 17.270 & $\mathrm{MM}$ & 0.00000 \\
2 & 12.237 & 6934310 & 17.198 & $\mathrm{Mx}$ & 0.00000 \\
3 & 13.200 & 13197586 & 32.731 & $\mathrm{XM}$ & 0.00000 \\
4 & 17.403 & 13225704 & 32.801 & $\mathrm{MM}$ & 0.00000 \\
\hline & & & & & \\
& & & & & \\
\hline
\end{tabular}

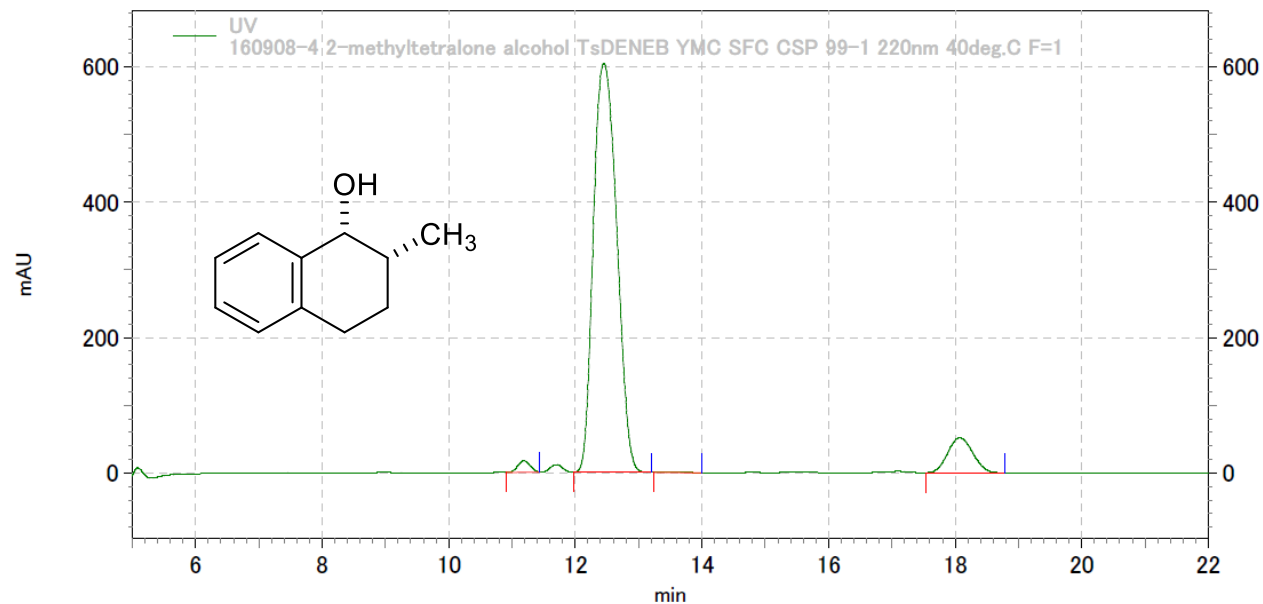

160908-4 2-methyltetralone alcohol TsDENEB YMC SFC CSP 99-1 220nm 40deg. C F=1

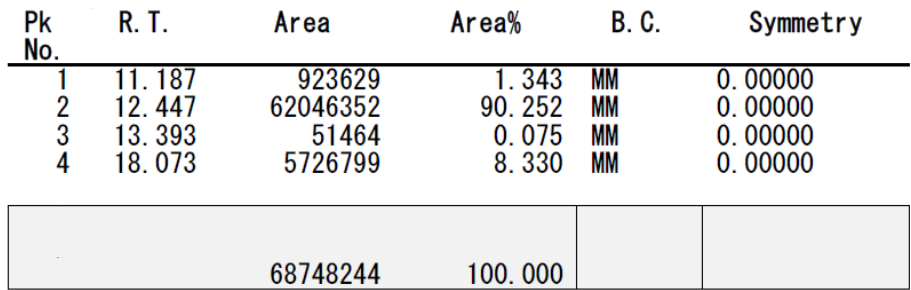




\section{(1R,2R)-2-Methyl-2,3-dihydro-1H-inden-1-ol (9b)}

Chiralcel OJ-H, $250 \times 4.6 \mathrm{~mm}$ column, hexane/2-propanol 98:2, $1.0 \mathrm{~mL} / \mathrm{min}, 220 \mathrm{~nm}, 30^{\circ} \mathrm{C}$

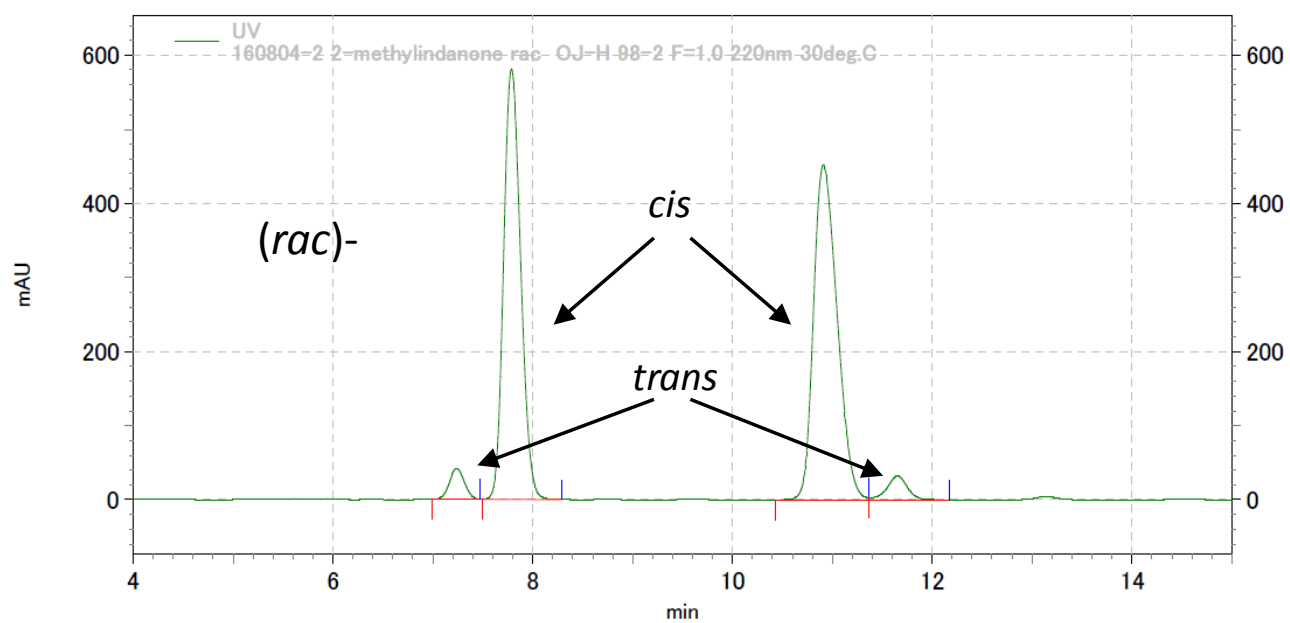

160804-2 2-methylindanone rac 0J-H 98-2 F=1.0 220nm 30deg. C

\begin{tabular}{rrrrrr}
$\begin{array}{r}\text { Pk } \\
\text { No. }\end{array}$ & \multicolumn{1}{l}{ R. T. } & \multicolumn{1}{c}{ Area } & Area\% & B. C. & Symmetry \\
\hline 1 & 7.240 & 1748277 & 2.875 & $\mathrm{MM}$ & 0.00000 \\
2 & 7.787 & 27542910 & 45.300 & $\mathrm{MM}$ & 0.00000 \\
3 & 10.910 & 29668460 & 48.796 & $\mathrm{Mx}$ & 0.00000 \\
4 & 11.653 & 1841400 & 3.029 & $\mathrm{xM}$ & 0.00000 \\
\hline & & & & & \\
& & & & & \\
\hline
\end{tabular}

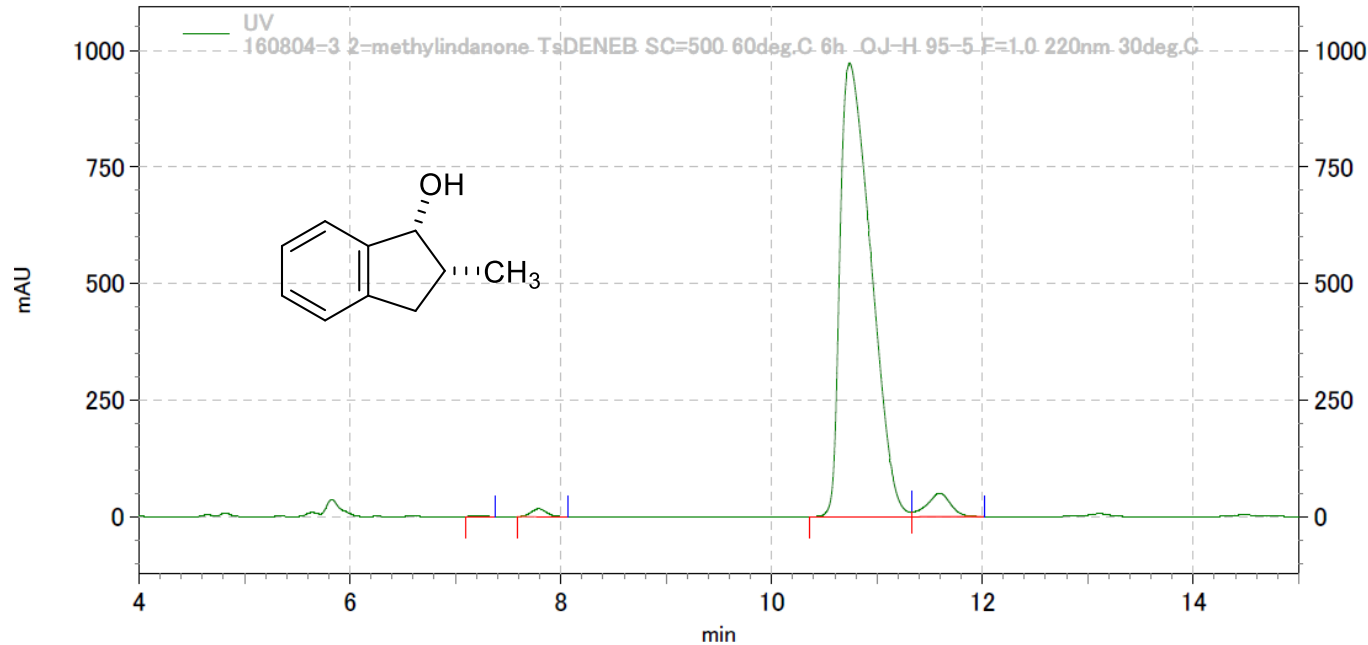

160804-3 2-methyl indanone TsDENEB SC=500 60deg. C 6h 0J-H 95-5 F=1.0 220nm 30deg. C

\begin{tabular}{rrrrrr}
$\begin{array}{r}\text { Pk } \\
\text { No. }\end{array}$ & \multicolumn{1}{l}{ R. T. } & \multicolumn{1}{c}{ Area } & \multicolumn{1}{l}{ Area\% } & B. C. & Symmetry \\
\hline 1 & 7.220 & 70373 & 0.085 & $\mathrm{MM}$ & 0.00000 \\
2 & 7.787 & 728530 & 0.876 & $\mathrm{MM}$ & 0.00000 \\
3 & 10.740 & 79130313 & 95.172 & $\mathrm{Mx}$ & 0.00000 \\
4 & 11.597 & 3215585 & 3.867 & $\mathrm{xM}$ & 0.00000 \\
\hline & \multicolumn{5}{c}{} \\
\end{tabular}


(5R,6R)-6-Methyl-6,7,8,9-tetrahydro-5H-benzo[7]annulen-5-ol (9c)

Chiralcel OJ-H, $250 \times 4.6 \mathrm{~mm}$ column, hexane/2-propanol 98:2, $1.0 \mathrm{~mL} / \mathrm{min}, 220 \mathrm{~nm}, 30^{\circ} \mathrm{C}$

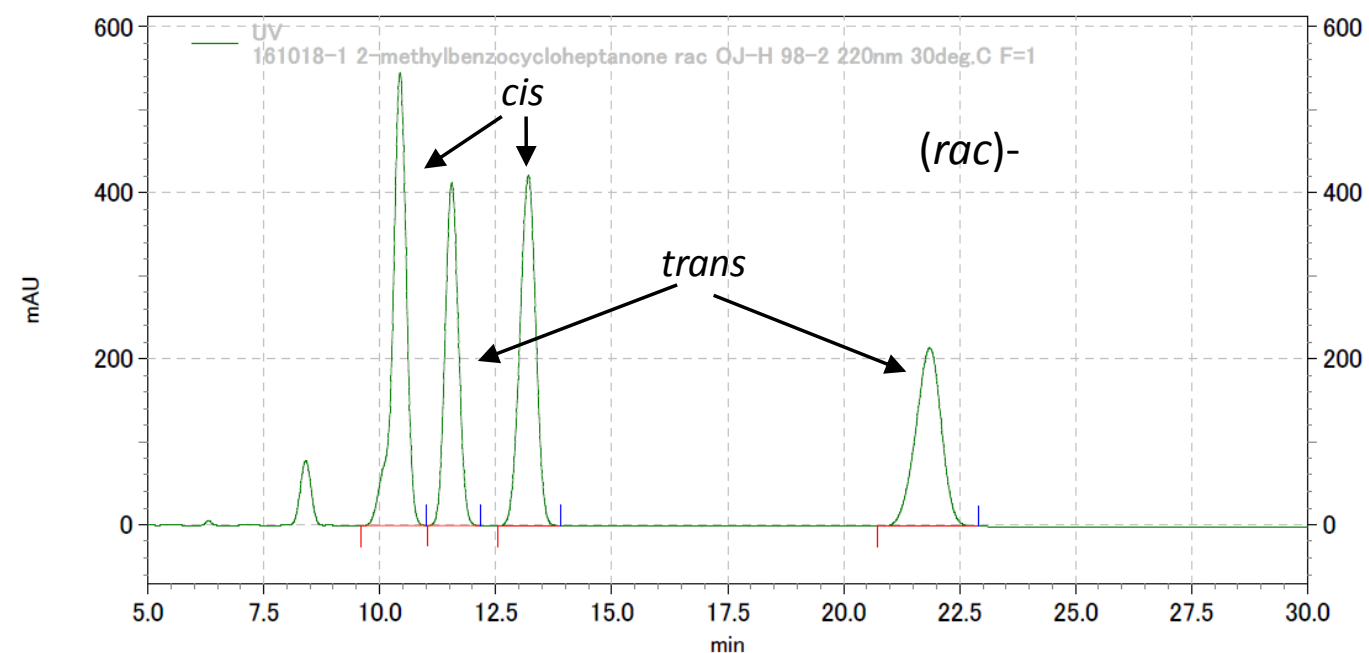

161018-1 2-methylbenzocycloheptanone rac 0J-H 98-2 220nm 30deg. C F=1

\begin{tabular}{rlllll}
$\begin{array}{c}\text { Pk } \\
\text { No. }\end{array}$ & R. T. & Area & Area\% & B. C. & Symmetry \\
\hline 1 & 10.437 & 45281823 & 29.821 & MM & 0.00000 \\
2 & 11.550 & 32850103 & 21.634 & MM & 0.00000 \\
3 & 13.203 & 40453964 & 26.642 & MM & 0.00000 \\
4 & 21.857 & 33259176 & 21.903 & MM & 0.00000
\end{tabular}
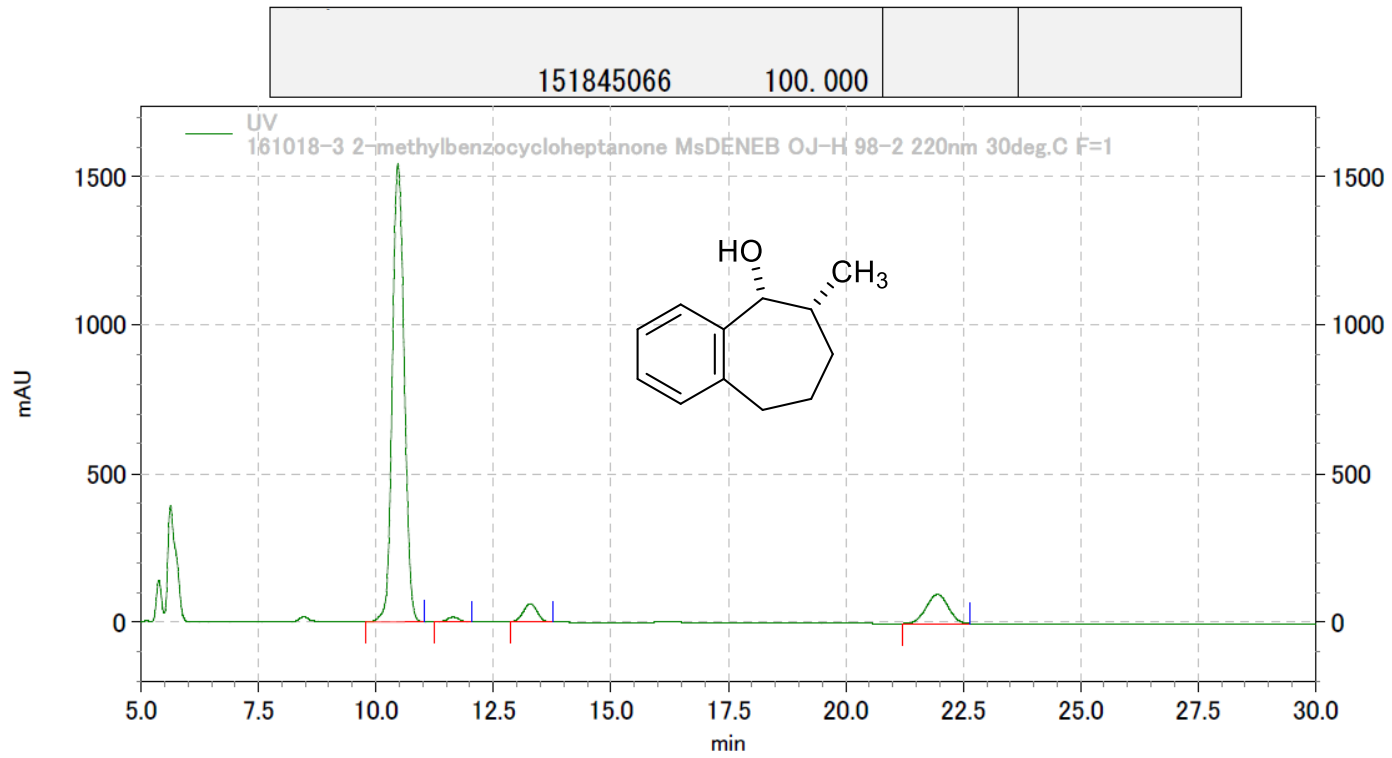

161018-3 2-methylbenzocycloheptanone MsDENEB 0J-H 98-2 220nm 30deg. C F=1

\begin{tabular}{|c|c|c|c|c|c|}
\hline $\mathrm{Pk}$ & R. T. & Area & Area\% & B. C. & Symmetry \\
\hline $\begin{array}{l}1 \\
2 \\
3 \\
4\end{array}$ & $\begin{array}{l}10.470 \\
11.647 \\
13.287 \\
21.957\end{array}$ & $\begin{array}{r}108942366 \\
1207180 \\
5146825 \\
12985733\end{array}$ & $\begin{array}{r}84.924 \\
0.941 \\
4.012 \\
10.123\end{array}$ & $\begin{array}{l}\text { MM } \\
\text { MM } \\
\text { MM } \\
\text { MM }\end{array}$ & $\begin{array}{l}0.00000 \\
0.00000 \\
0.00000 \\
0.00000\end{array}$ \\
\hline & & 128282104 & 100.000 & & \\
\hline
\end{tabular}


Methyl (1R,2R)-1-hydroxy-1,2,3,4-tetrahydronaphthalene-2-carboxylate (9d)

Chiralcel OJ-H, $250 \times 4.6 \mathrm{~mm}$ column, hexane/2-propanol 96:4, $1.0 \mathrm{~mL} / \mathrm{min}, 220 \mathrm{~nm}, 30^{\circ} \mathrm{C}$

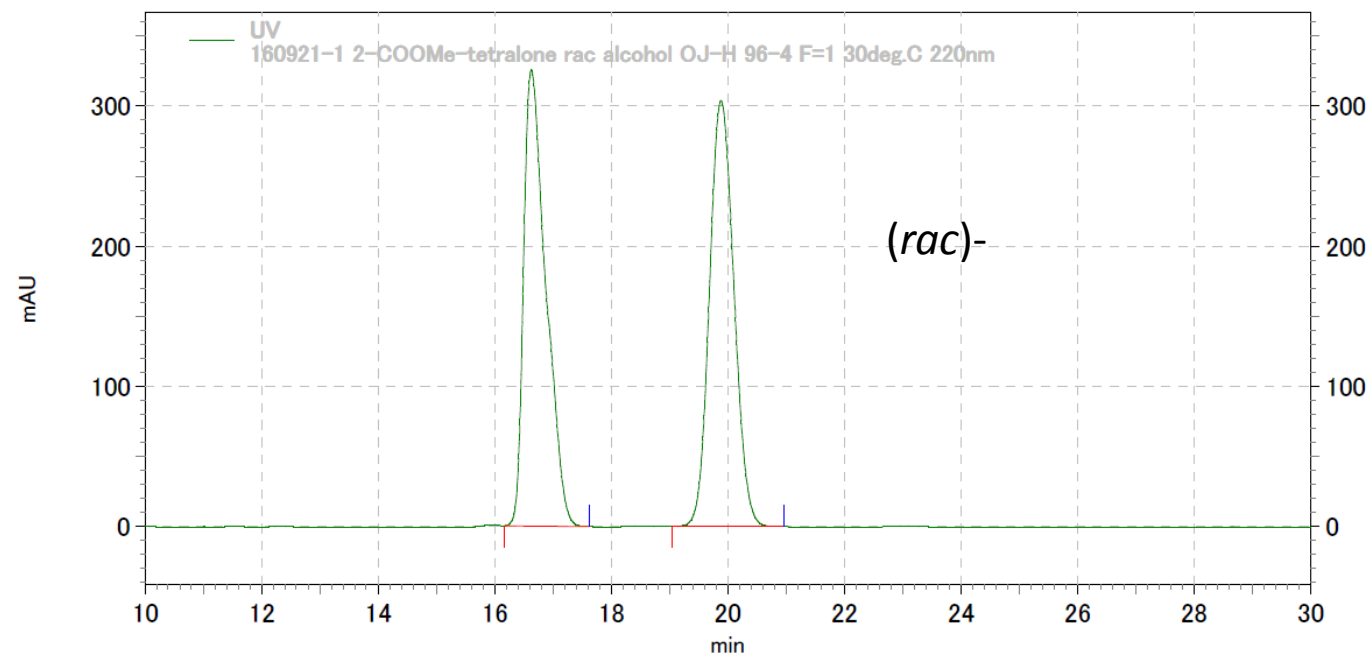

160921-1 2-C00Me-tetralone rac alcohol 0J-H 96-4 F=1 30deg. C 220nm

\begin{tabular}{rlllll}
$\begin{array}{l}\text { Pk } \\
\text { No. }\end{array}$ & R. T. & Area & Area\% & B. C. & Symmetry \\
\hline 1 & 16.630 & 34478059 & 49.284 & MM & 1.61942 \\
2 & 19.887 & 35479440 & 50.716 & MM & 1.10259
\end{tabular}
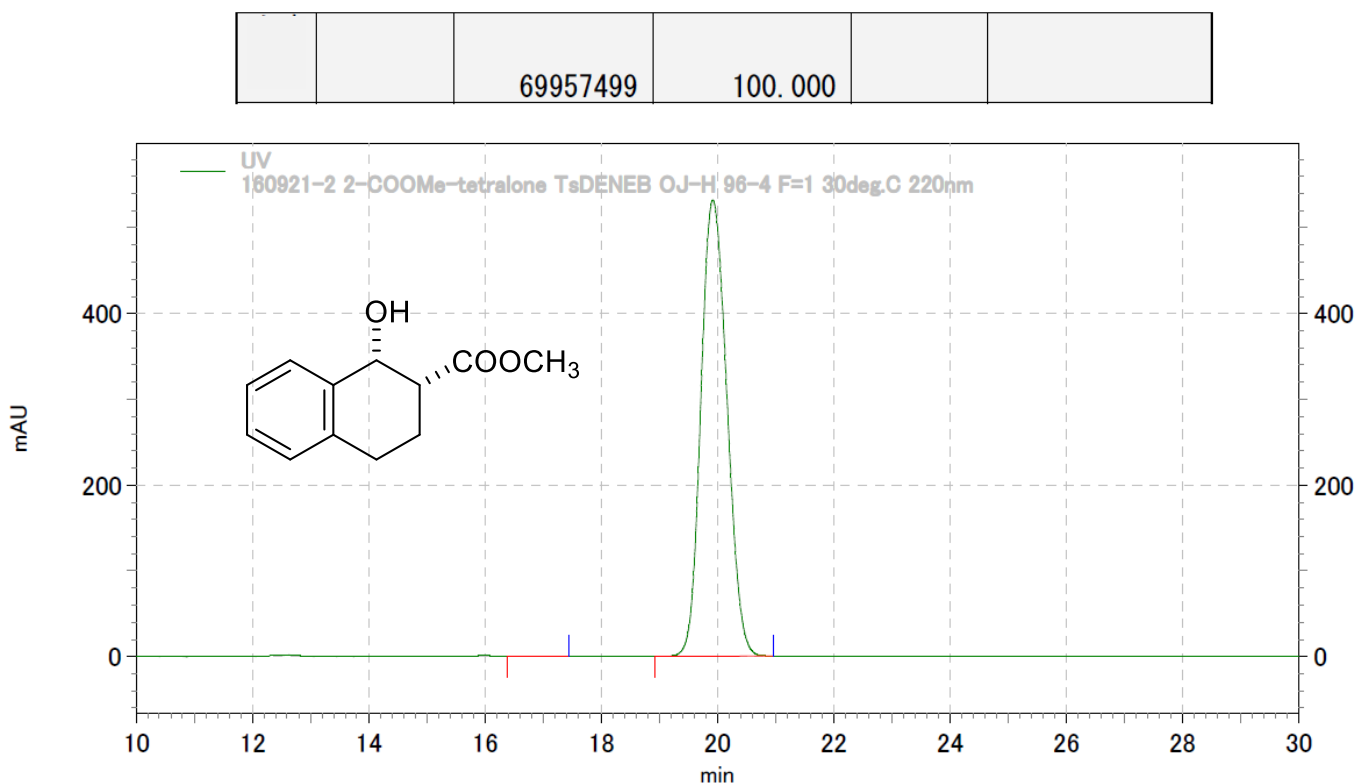

160921-2 2-COOMe-tetralone TsDENEB 0J-H 96-4 F=1 30deg. C 220nm

\begin{tabular}{rl|r|r|r|r}
$\begin{array}{l}\text { Pk } \\
\text { No. }\end{array}$ & R. T. & Area & Area\% & B. C. & Symmetry \\
\hline 1 & 16.880 & 32152 & 0.049 & MM & 0.00000 \\
2 & 19.923 & 66106576 & 99.951 & MM & 0.00000 \\
\hline & & & & & \\
\hline & & 66138728 & 100.000 & & \\
\hline
\end{tabular}




\section{(3aS,9bR)-3a,4,5,9b-Tetrahydronaphtho[1,2-b]furan-2(3H)-one (13a)}

Chiralcel OD-H, $250 \times 4.6 \mathrm{~mm}$ column, hexane/2-propanol 95:5, $1.0 \mathrm{~mL} / \mathrm{min}, 220 \mathrm{~nm}, 30^{\circ} \mathrm{C}$

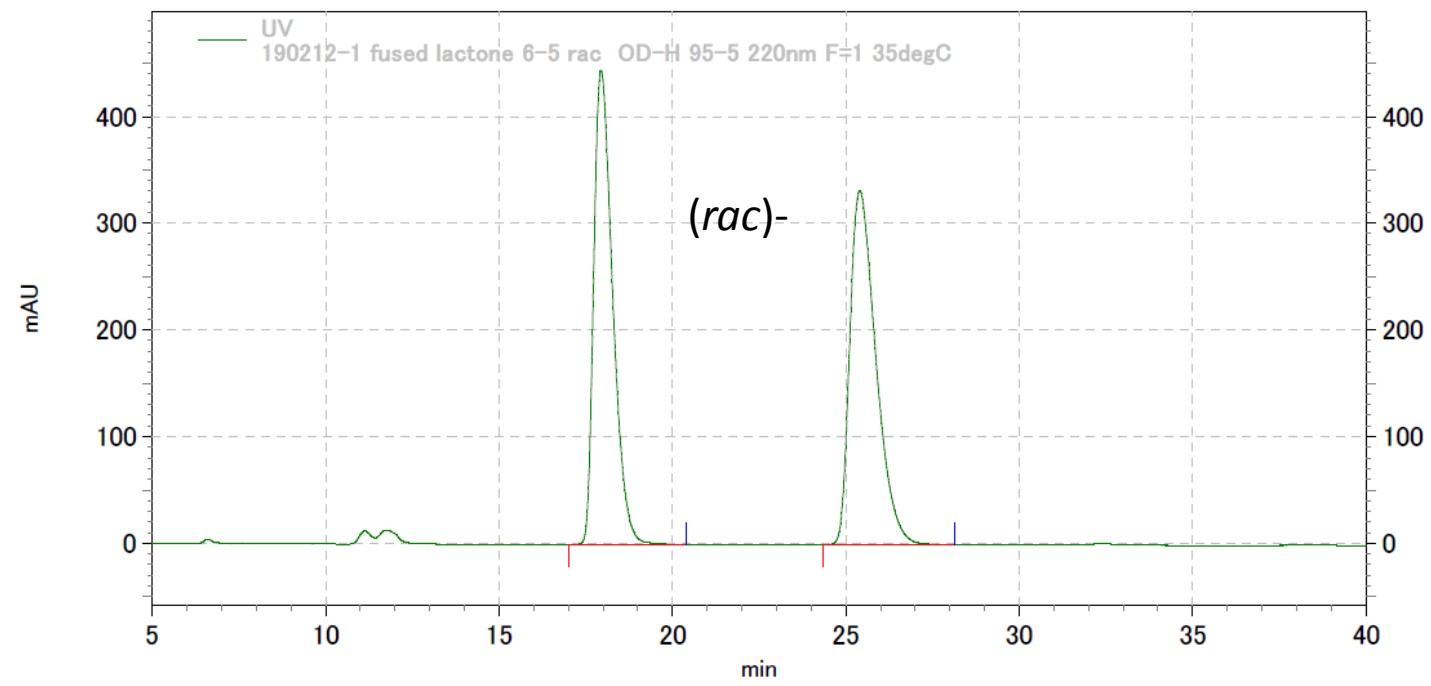

190212-1 fused lactone 6-5 rac OD-H 95-5 220 nm F=1 35degC

\begin{tabular}{rlllll}
$\begin{array}{r}\text { Pk } \\
\text { No. }\end{array}$ & R. T. & Area & Area\% & B. C. & Symmetry \\
\hline 1 & 17.943 & 67337585 & 49.320 & MM & 0.00000 \\
2 & 25.400 & 69194892 & 50.680 & MM & 0.00000
\end{tabular}

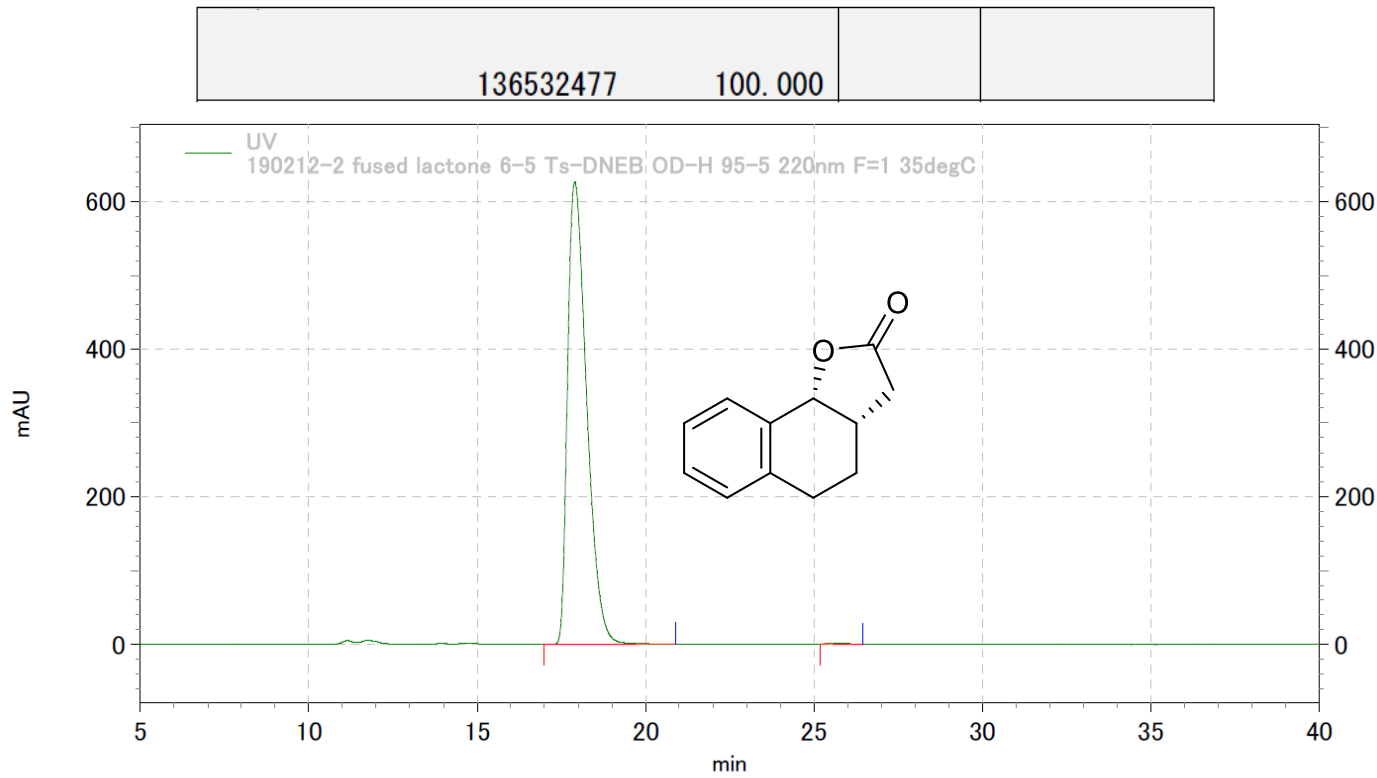

190212-2 fused lactone 6-5 Ts-DNEB OD-H 95-5 220nm F=1 35degC

\begin{tabular}{|c|c|c|c|c|c|}
\hline Pk & R. T. & Area & Area\% & B. C. & Symmetry \\
\hline $\begin{array}{l}1 \\
2\end{array}$ & $\begin{array}{l}17.897 \\
25.680\end{array}$ & $\begin{array}{r}98552435 \\
152288\end{array}$ & $\begin{array}{r}99.846 \\
0.154\end{array}$ & $\begin{array}{l}\text { MM } \\
\text { MM }\end{array}$ & $\begin{array}{l}0.00000 \\
0.00000\end{array}$ \\
\hline & & 98704723 & 100.000 & & \\
\hline
\end{tabular}




\section{(3aS,9bR)-8-Methoxy-3a,4,5,9b-tetrahydronaphtho[1,2-b]furan-2(3H)-one (13b)}

Chiralcel OD-H, $250 \times 4.6 \mathrm{~mm}$ column, hexane/2-propanol 95:5, $0.8 \mathrm{~mL} / \mathrm{min}, 220 \mathrm{~nm}, 30^{\circ} \mathrm{C}$

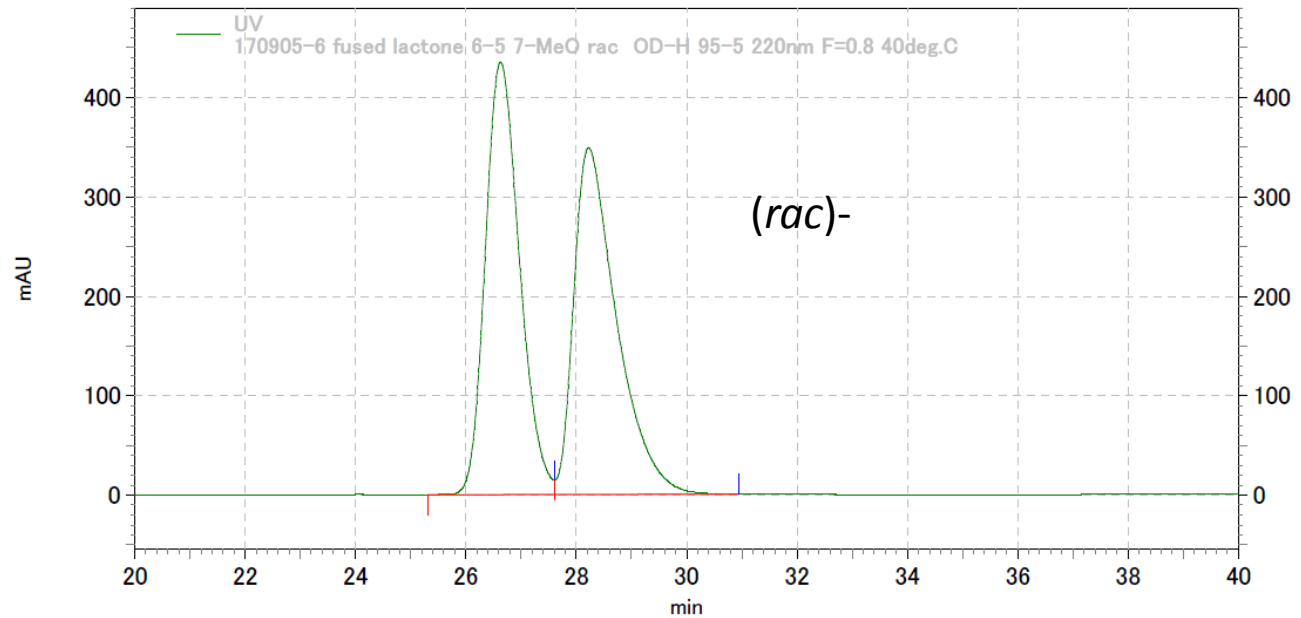

170905-6 fused lactone 6-5 7-Me0 rac OD-H 95-5 220nm F=0. 8 40deg. C

\begin{tabular}{rllrll}
$\begin{array}{c}\text { Pk } \\
\text { No. }\end{array}$ & R. T. & Area & Area\% & B. C. & Symmetry \\
\hline 1 & 26.627 & 75006310 & 49.955 & Mx & 0.00000 \\
2 & 28.223 & 75140007 & 50.045 & xM & 0.00000 \\
\hline & & & & & \\
& & & & & \\
\hline
\end{tabular}

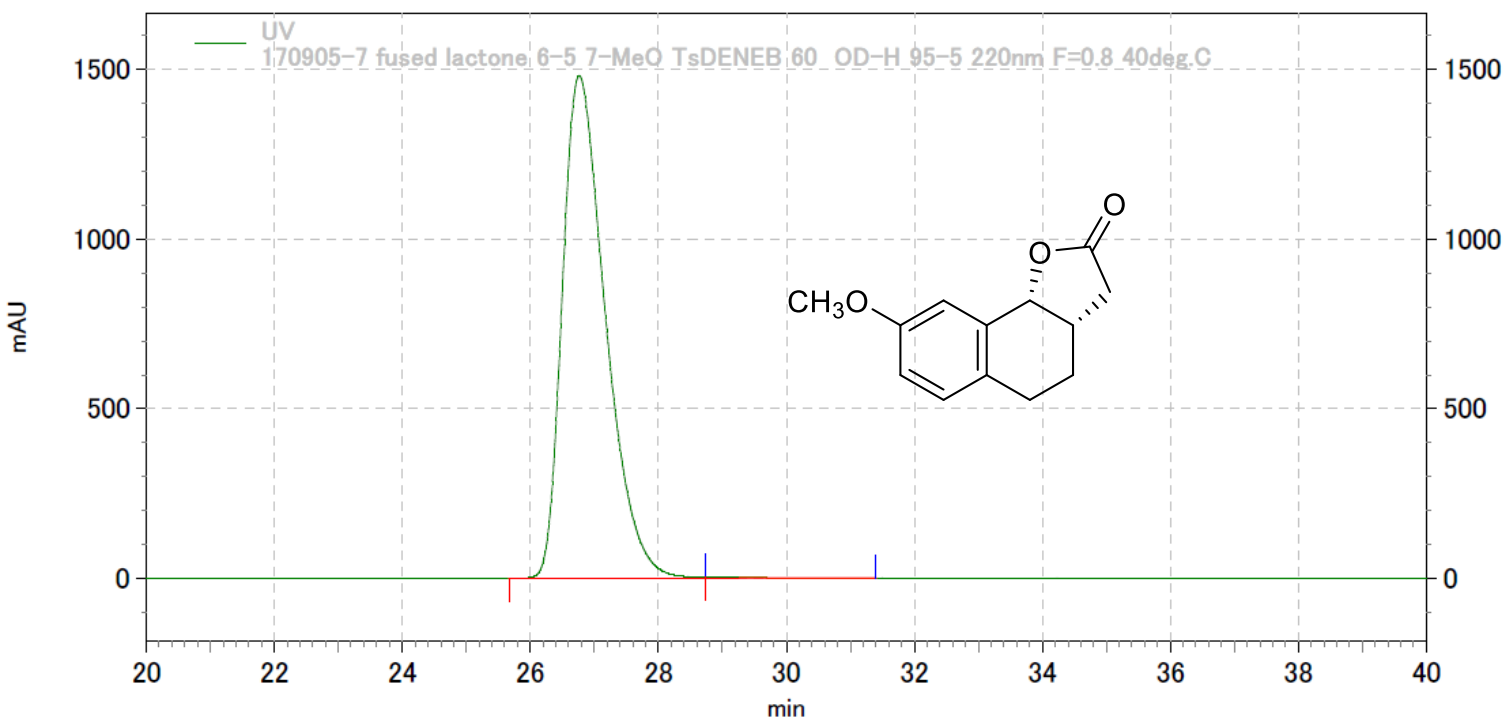

170905-7 fused lactone 6-5 7-Me0 TsDENEB 60 OD-H 95-5 220nm F=0. 8 40deg. C

\begin{tabular}{|c|c|c|c|c|c|}
\hline $\begin{array}{l}\mathrm{Pk} \\
\text { No. }\end{array}$ & R. T. & Area & Area\% & B. C. & Symmetry \\
\hline $\begin{array}{l}1 \\
2\end{array}$ & $\begin{array}{l}26.763 \\
28.743\end{array}$ & $\begin{array}{r}276789087 \\
849857\end{array}$ & $\begin{array}{r}99.694 \\
0.306\end{array}$ & $\begin{array}{l}\mathrm{Mx} \\
\mathrm{XM}\end{array}$ & $\begin{array}{l}0.00000 \\
0.00000\end{array}$ \\
\hline & & 277638944 & 100.000 & & \\
\hline
\end{tabular}




\section{(3aS,9bR)-8-Chloro-3a,4,5,9b-tetrahydronaphtho[1,2-b]furan-2(3H)-one (13c)}

Chiralcel OD-H, $250 \times 4.6 \mathrm{~mm}$ column, hexane/2-propanol 93:7, $1.0 \mathrm{~mL} / \mathrm{min}, 220 \mathrm{~nm}, 30^{\circ} \mathrm{C}$

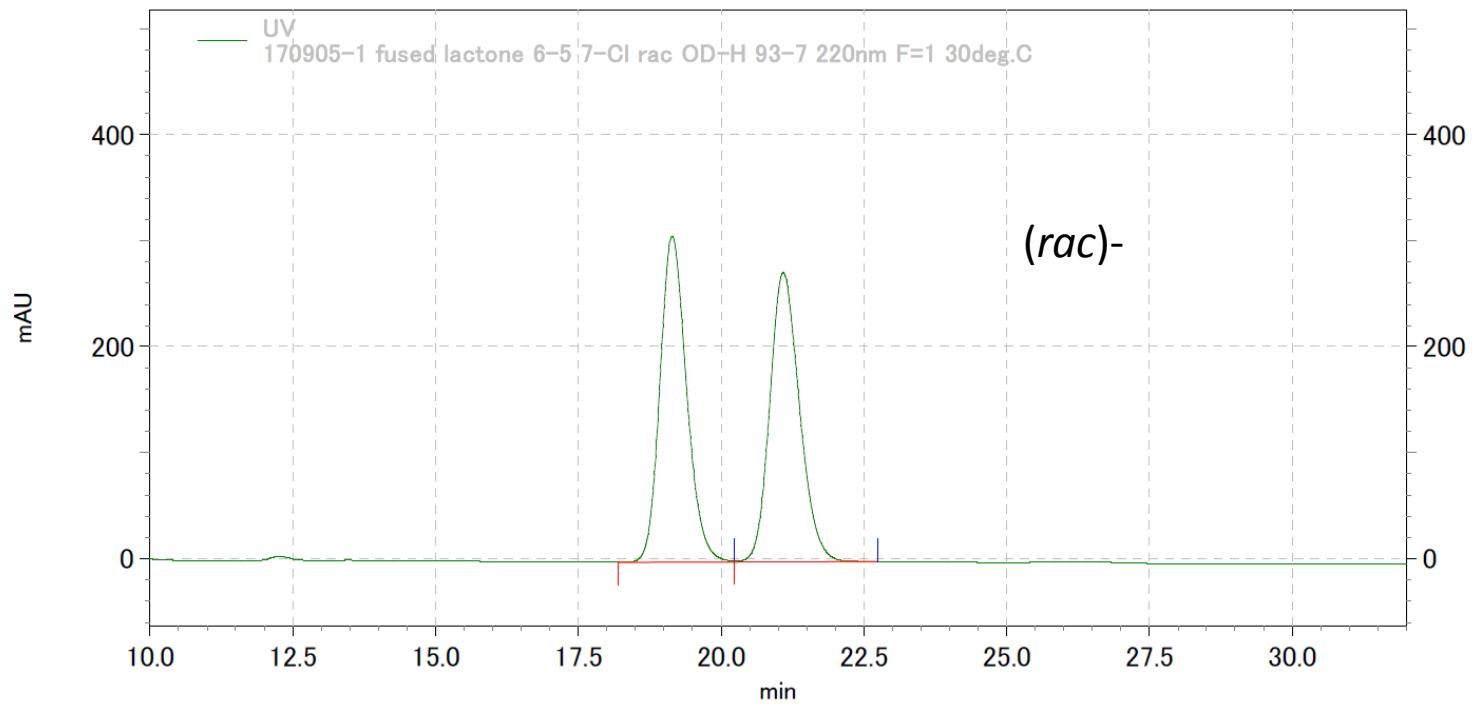

170905-1 fused lactone 6-5 7-Cl rac 0D-H 93-7 220nm F=1 30deg. C

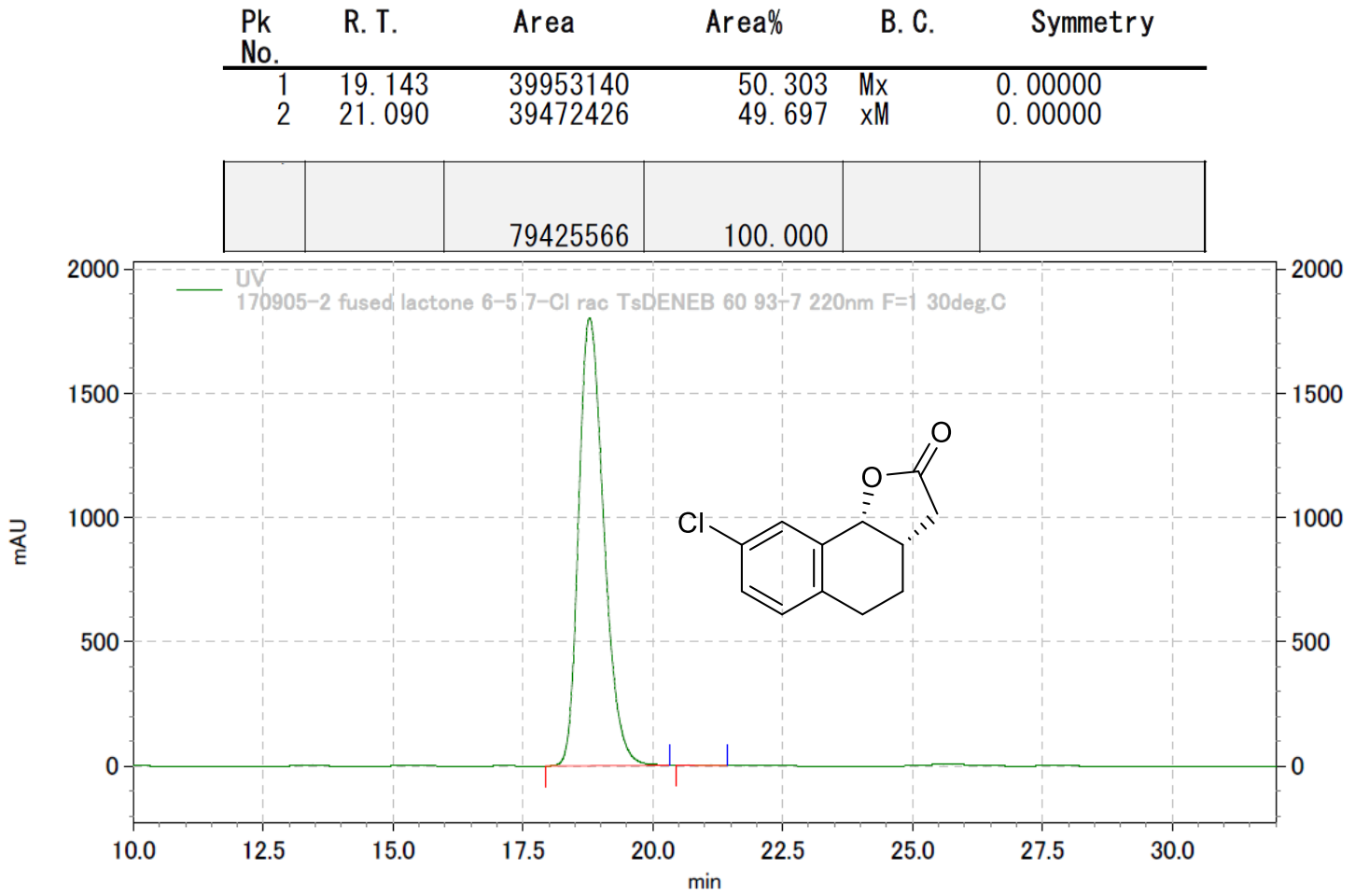

170905-2 fused lactone 6-5 7-CI rac TsDENEB 60 93-7 220nm F=1 30deg. C

\begin{tabular}{rlrrrr}
$\begin{array}{rlr}\text { Pk } \\
\text { No. }\end{array}$ & \multicolumn{1}{l}{ R. T. } & \multicolumn{1}{c}{ Area } & \multicolumn{1}{c}{ Area\% } & B. C. & Symmetry \\
\hline 1 & 18.783 & 242813475 & 99.894 & MM & 0.00000 \\
2 & 20.927 & 258831 & 0.106 & MM & 0.00000 \\
\hline & & & & & \\
\hline
\end{tabular}




\section{(3aS,9bR)-8-Bromo-3a,4,5,9b-tetrahydronaphtho[1,2-b]furan-2(3H)-one (13d)}

Chiralcel OD-H, $250 \times 4.6 \mathrm{~mm}$ column, hexane/2-propanol 94:6, $1.0 \mathrm{~mL} / \mathrm{min}, 220 \mathrm{~nm}, 35^{\circ} \mathrm{C}$

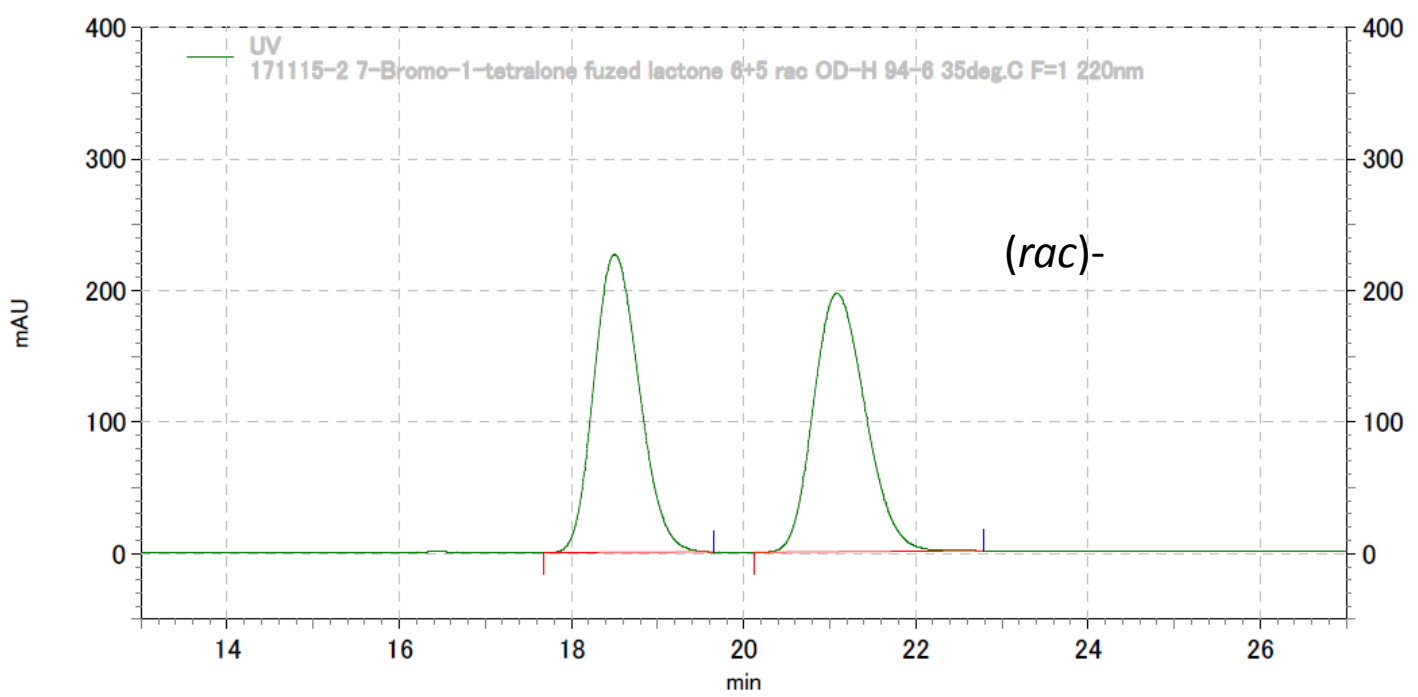

171115-2 7-Bromo-1-tetralone fuzed lactone 6+5 rac 0D-H 94-6 35deg. C F=1 220nm

\begin{tabular}{rllrrl}
$\begin{array}{l}\text { Pk } \\
\text { No. }\end{array}$ & R. T. & Area & \multicolumn{1}{l}{ Area\% } & B.C. & Symmetry \\
\hline 1 & 18.500 & 33079615 & 50.196 & MM & 0.00000 \\
2 & 21.083 & 32821205 & 49.804 & MM & 0.00000
\end{tabular}

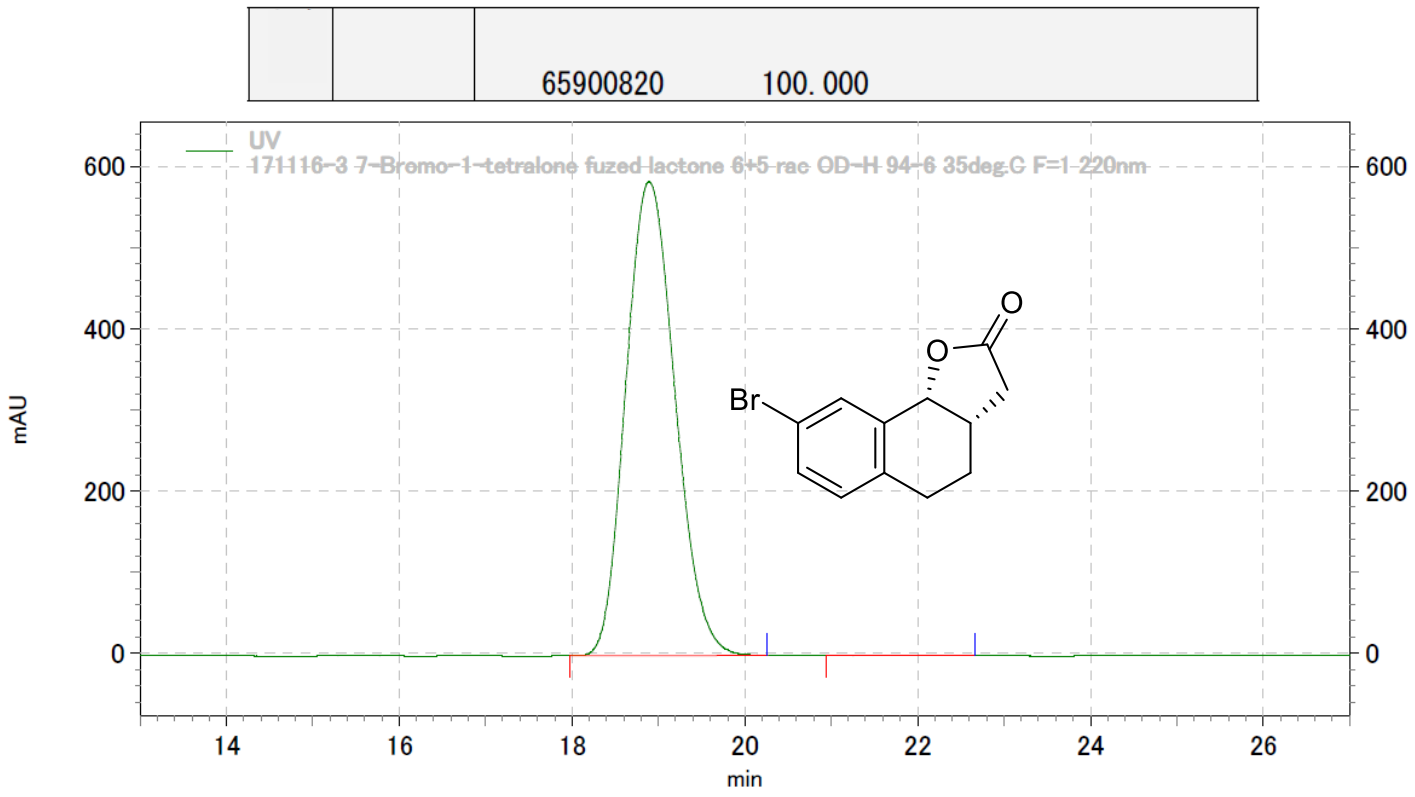

171116-3 7-Bromo-1-tetralone fuzed lactone 6+5 rac OD-H 94-6 35deg. C F=1 220nm

\begin{tabular}{rl|l|r|r|r}
$\begin{array}{r}\text { Pk } \\
\text { No. }\end{array}$ & \multicolumn{1}{l}{ R. T. } & \multicolumn{1}{l}{ Area } & \multicolumn{1}{l}{ Area\% } & B. C. & \multicolumn{1}{l}{ Symmetry } \\
\hline 1 & 18.893 & 92548224 & 99.866 & MM & 0.00000 \\
2 & 21.620 & 123894 & 0.134 & MM & 0.00000 \\
\hline & & & & & \\
\hline & & 92672118 & 100.000 & & \\
\hline
\end{tabular}


(3aS,9bR)-9-Bromo-8-methoxy-3a,4,5,9b-tetrahydronaphtho[1,2-b]furan-2(3H)-one (13e)

Chiralcel OD-H, $250 \times 4.6 \mathrm{~mm}$ column, hexane/2-propanol 88:12, $1.0 \mathrm{~mL} / \mathrm{min}, 220 \mathrm{~nm}, 35^{\circ} \mathrm{C}$

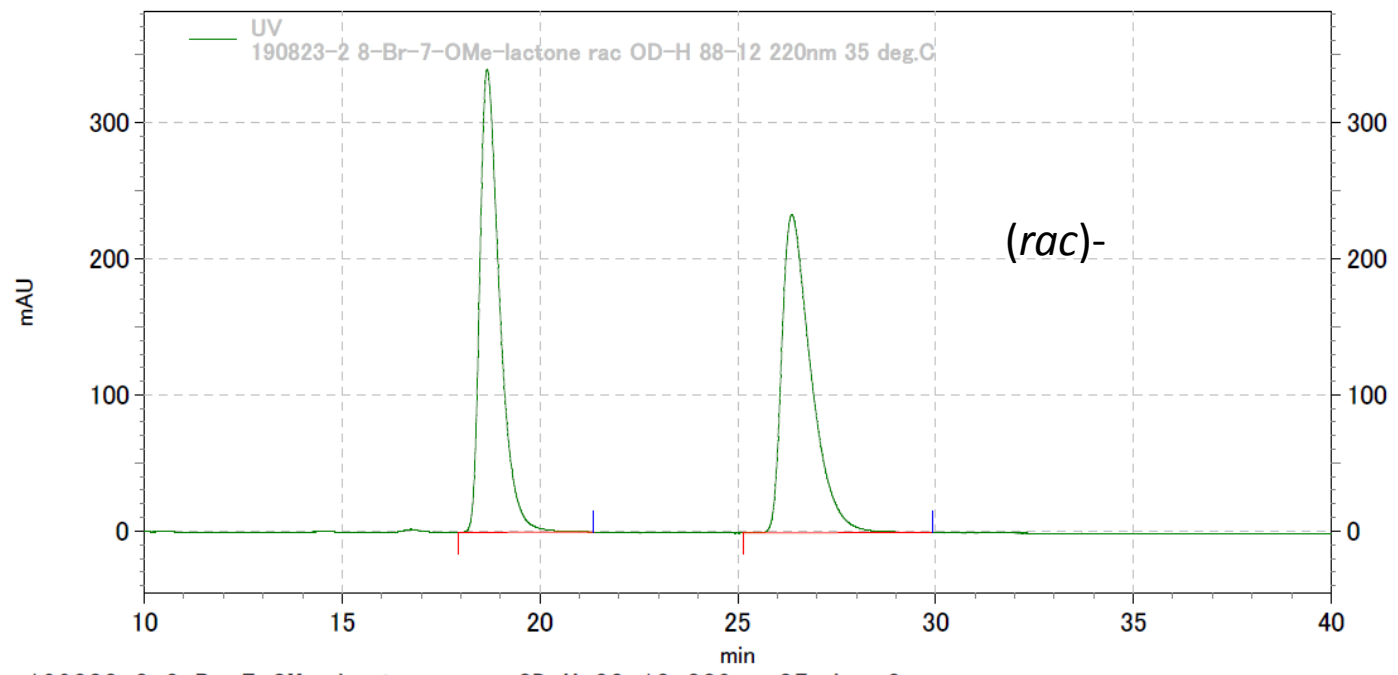

190823-2 8-Br-7-0Me-lactone rac OD-H 88-12 220nm 35 deg. C

\begin{tabular}{rlllll}
$\begin{array}{c}\text { Pk } \\
\text { No. }\end{array}$ & R. T. & Area & Area\% & B. C. & Symmetry \\
\hline 1 & 18.670 & 47293000 & 49.674 & MM & 0.00000 \\
2 & 26.370 & 47914248 & 50.326 & MM & 0.00000
\end{tabular}
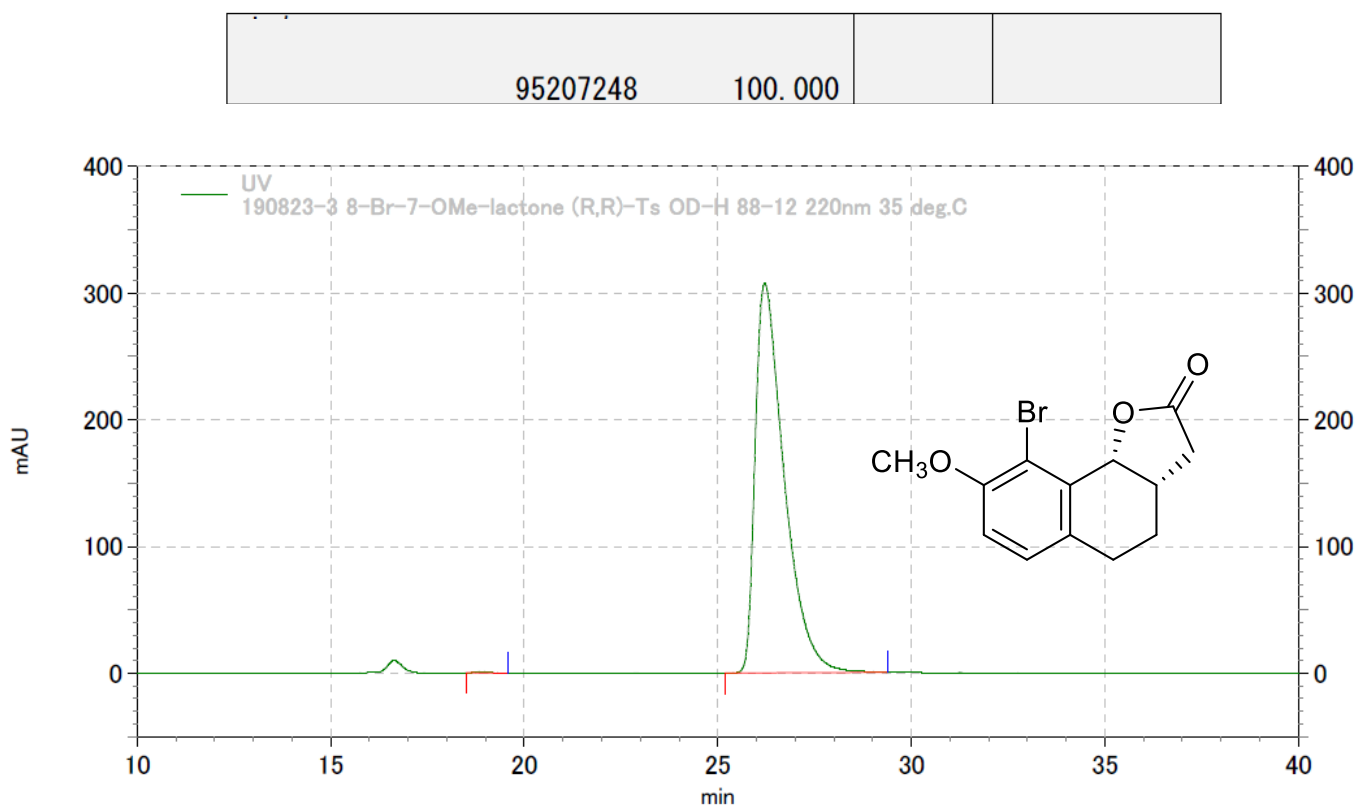

190823-3 8-Br-7-0Me-lactone (R, R) -Ts OD-H 88-12 220nm 35 deg. C

\begin{tabular}{rrrrrr}
$\begin{array}{lllll}\text { Pk } \\
\text { No. }\end{array}$ & \multicolumn{1}{l}{ R. T. } & \multicolumn{1}{l}{ Area } & Area\% & B. C. & Symmetry \\
\hline 1 & 18.920 & 123942 & 0.193 & MM & 0.00000 \\
2 & 26.213 & 63947488 & 99.807 & MM & 0.00000 \\
\hline & & & & & \\
\hline & & 64071430 & 100.000 & \\
\hline
\end{tabular}




\section{(3aS,9bR)-7-Hydroxy-3a,4,5,9b-tetrahydronaphtho[1,2-b]furan-2(3H)-one (13f)}

Chiralpak AD-H, $250 \times 4.6 \mathrm{~mm}$ column, hexane/2-propanol 85:15, $1.0 \mathrm{~mL} / \mathrm{min}, 220 \mathrm{~nm}, 40{ }^{\circ} \mathrm{C}$

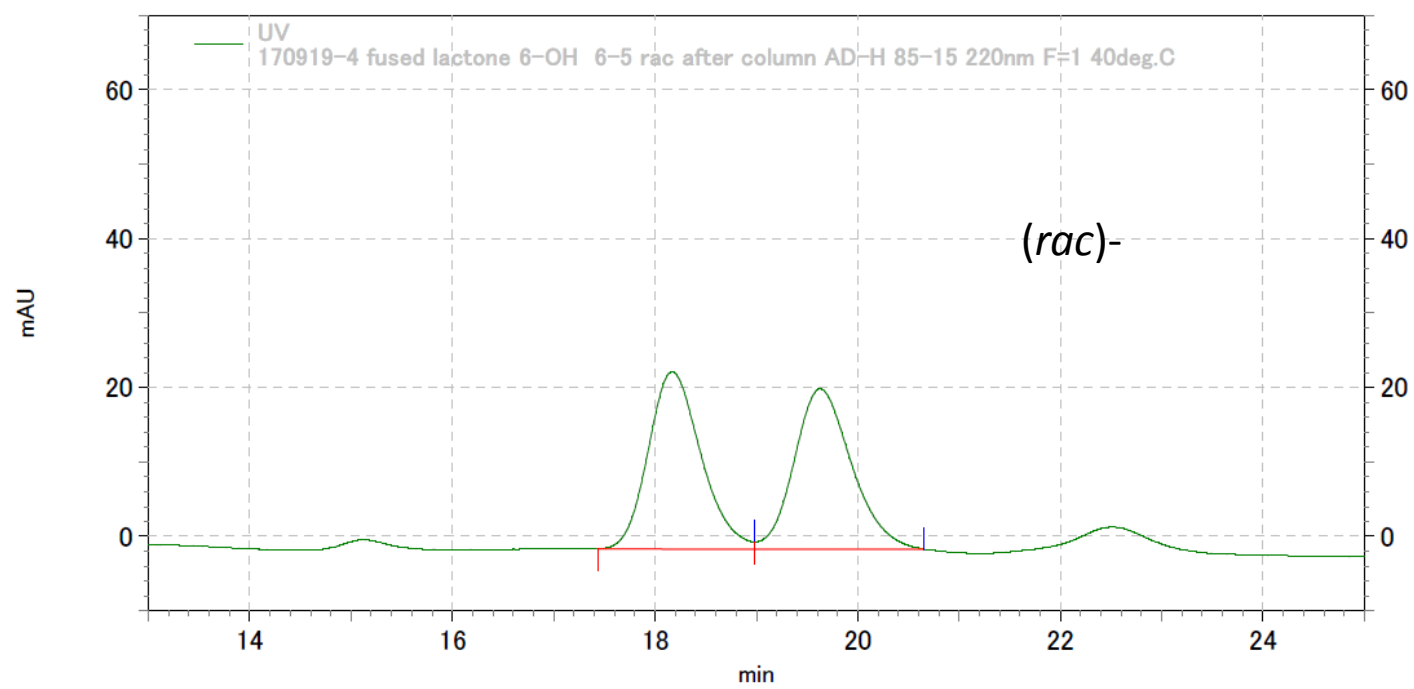

170919-4 fused lactone 6-OH 6-5 rac after column AD-H 85-15 220nm F=1 40deg. C

\begin{tabular}{rllrll}
$\begin{array}{c}\text { Pk } \\
\text { No. }\end{array}$ & R. T. & Area & Area\% & B.C. & Symmetry \\
\hline 1 & 18.170 & 3443063 & 50.432 & $\mathrm{Mx}$ & 0.00000 \\
2 & 19.627 & 3384114 & 49.568 & $\mathrm{xM}$ & 0.00000
\end{tabular}

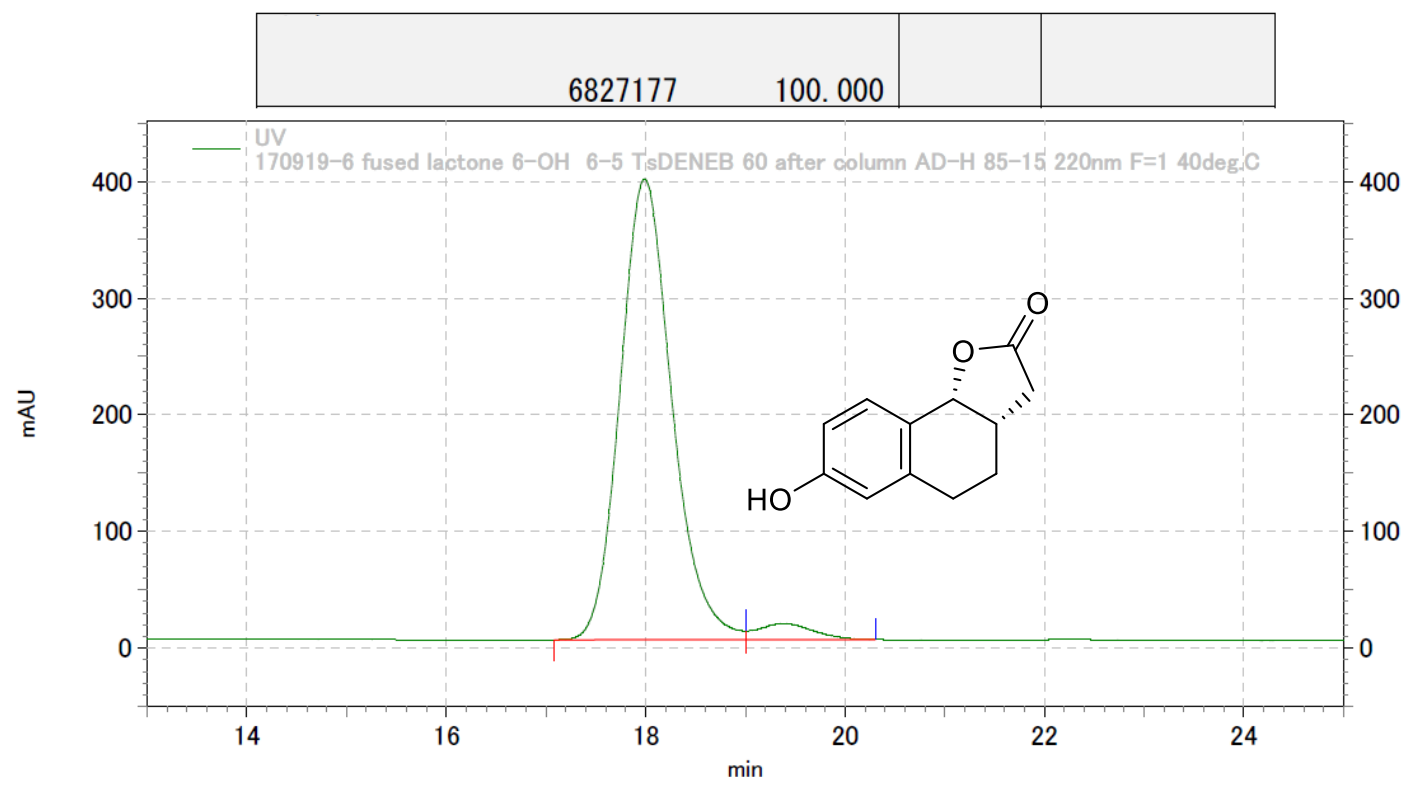

170919-6 fused lactone 6-0H 6-5 TsDENEB 60 after column AD-H 85-15 220nm F=1 40deg. C

\begin{tabular}{rlrrrr}
$\begin{array}{rlr}\text { Pk } \\
\text { No. }\end{array}$ & R. T. & \multicolumn{1}{c}{ Area } & \multicolumn{1}{l}{ Area\% } & \multicolumn{1}{l}{ B. C. } & \multicolumn{1}{c}{ Symmetry } \\
\hline 1 & 17.990 & 56957682 & 96.356 & Mx & 0.00000 \\
2 & 19.387 & 2154285 & 3.644 & xM & 0.00000 \\
\hline & & & & & \\
\hline & & & & & \\
\hline
\end{tabular}




\section{(3aS,8bR)-3,3a,4,8b-Tetrahydro-2H-indeno[1,2-b]furan-2-one (13g)}

Chiralcel OJ-H, $250 \times 4.6 \mathrm{~mm}$ column, hexane/2-propanol 95:5, $1.0 \mathrm{~mL} / \mathrm{min}, 220 \mathrm{~nm}, 30^{\circ} \mathrm{C}$

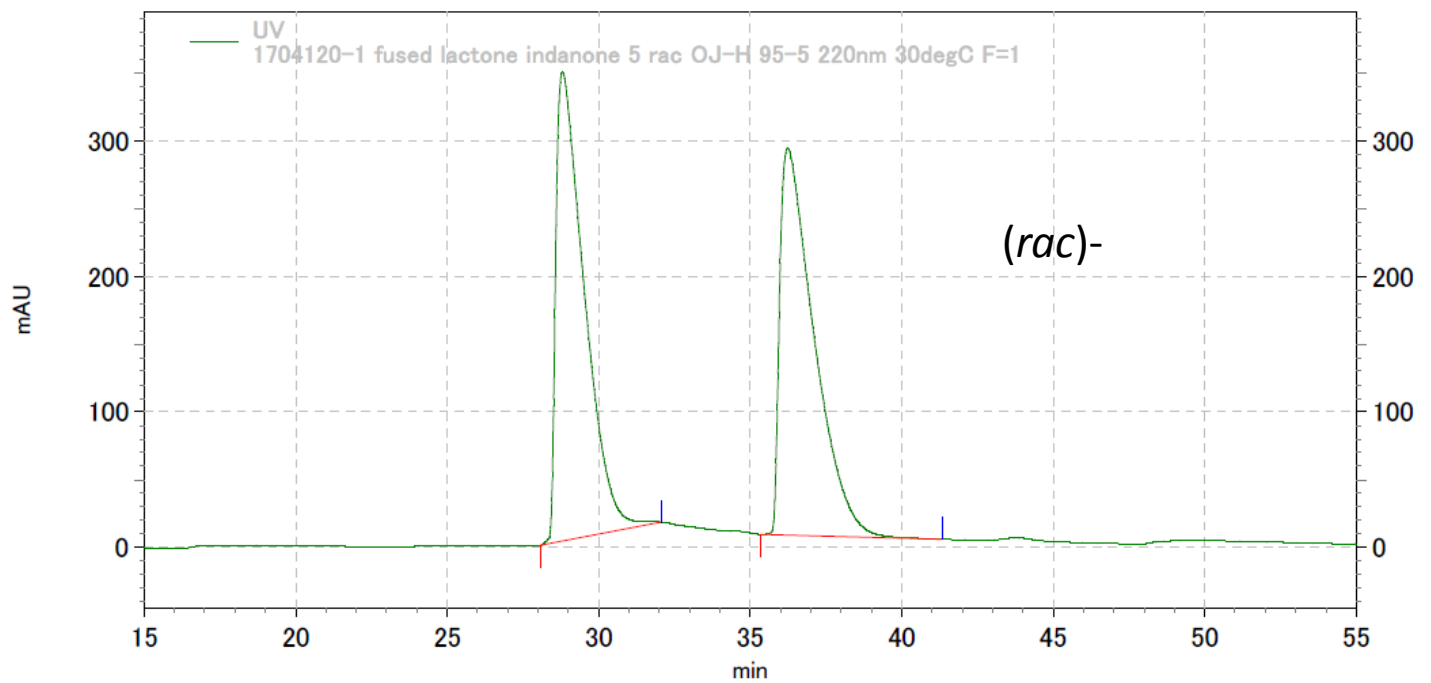

1704120-1 fused lactone indanone 5 rac 0J-H 95-5 220nm 30degC F=1

\begin{tabular}{rlllll}
$\begin{array}{l}\text { Pk } \\
\text { No. }\end{array}$ & R. T. & Area & Area\% & B. C. & Symmetry \\
\hline 1 & 28.807 & 90251151 & 50.164 & MM & 0.00000 \\
2 & 36.240 & 89661849 & 49.836 & MM & 0.00000 \\
\hline & & & & & \\
\hline & & 179913000 & 100.000 & \\
\hline
\end{tabular}

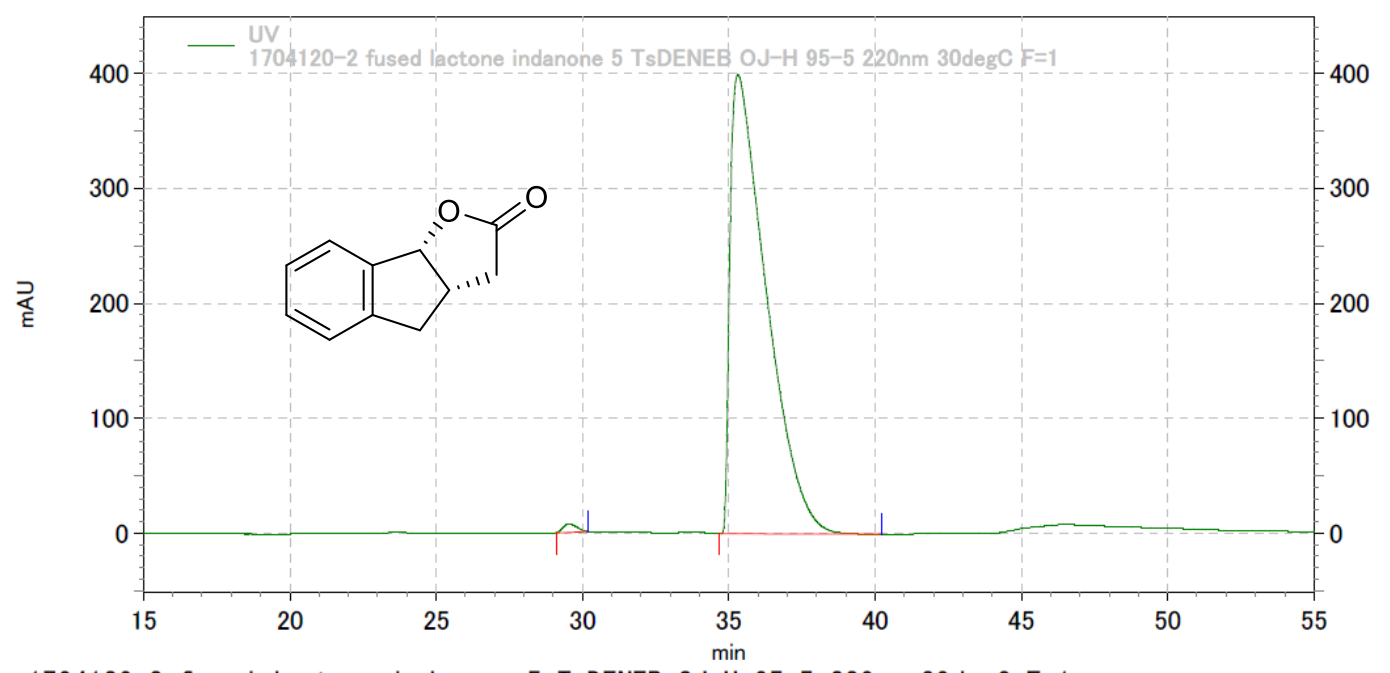

1704120-2 fused lactone indanone 5 TsDENEB 0J-H 95-5 220nm 30degC F=1

\begin{tabular}{rrrrrr}
$\begin{array}{ll}\text { Pk } \\
\text { No. }\end{array}$ & R. T. & \multicolumn{1}{c}{ Area } & Area\% & B. C. & Symmetry \\
\hline 1 & 29.553 & 961349 & 0.695 & MM & 0.00000 \\
2 & 35.313 & 137424154 & 99.305 & MM & 0.00000 \\
\hline & & & & & \\
\hline & & 138385503 & 100.000 & \\
\hline
\end{tabular}


(3aS,8bR)-6,7-Dimethoxy-3,3a,4,8b-tetrahydro-2H-indeno[1,2-b]furan-2-one (13h)

Chiralcel OD-H, $250 \times 4.6 \mathrm{~mm}$ column, hexane/2-propanol 90:10, $1.0 \mathrm{~mL} / \mathrm{min}, 220 \mathrm{~nm}, 30^{\circ} \mathrm{C}$

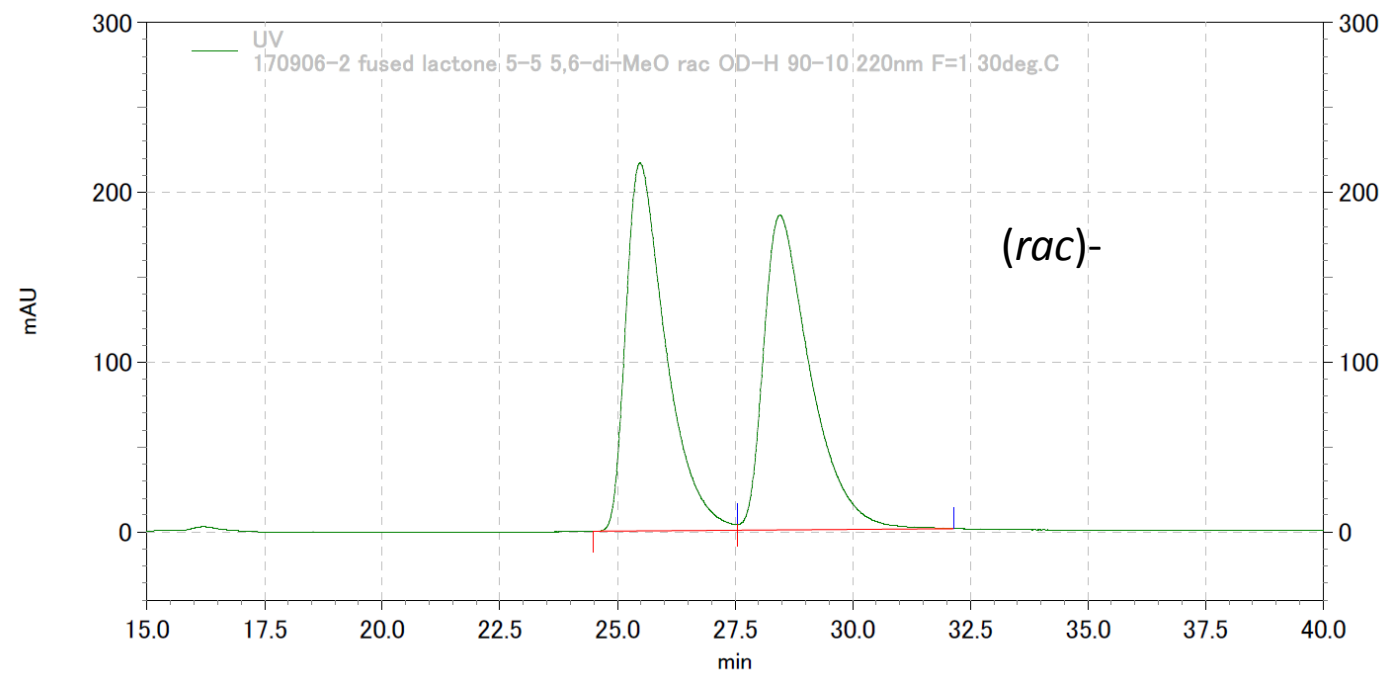

170906-2 fused lactone 5-5 5, 6-di-Me0 rac OD-H 90-10 220nm F=1 30deg. C

\begin{tabular}{rlllll}
$\begin{aligned} \text { Pk } \\
\text { No. }\end{aligned}$ & R. T. & Area & Area\% & B. C. & Symmetry \\
\hline 1 & 25.480 & 51273829 & 49.967 & Mx & 0.00000 \\
2 & 28.453 & 51340613 & 50.033 & xM & 0.00000
\end{tabular}
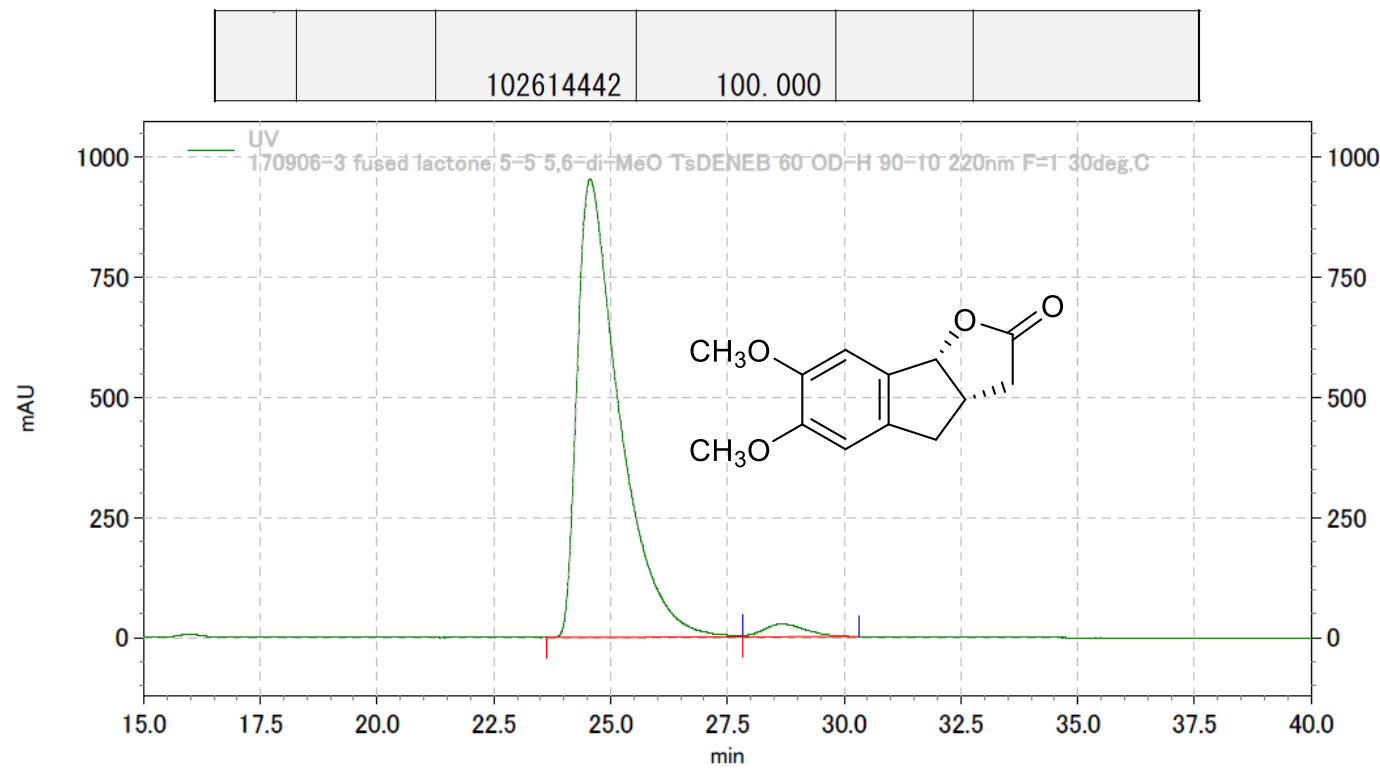

170906-3 fused lactone 5-5 5, 6-di-Me0 TsDENEB 60 OD-H 90-10 220nm F=1 30deg. C

\begin{tabular}{rlrrrr}
$\begin{aligned} \text { Pk } \\
\text { No. }\end{aligned}$ & R. T. & \multicolumn{1}{c}{ Area } & \multicolumn{1}{c}{ Area\% } & B. C. & Symmetry \\
\hline 1 & 24.560 & 239961033 & 97.194 & Mx & 0.00000 \\
2 & 28.670 & 6926697 & 2.806 & XM & 0.00000
\end{tabular}

\begin{tabular}{|l|l|l|}
\hline & & \\
& 246887730 & 100.000 \\
\hline
\end{tabular}


$(3 \mathrm{a} S, 8 \mathrm{~b} R)$-7-Fluoro-3,3a,4,8b-tetrahydro- $2 H$-indeno[1,2-b]furan-2-one (13i)

Chiralcel OJ-H, $250 \times 4.6 \mathrm{~mm}$ column, hexane/2-propanol 93:7, $1.0 \mathrm{~mL} / \mathrm{min}, 220 \mathrm{~nm}, 30^{\circ} \mathrm{C}$

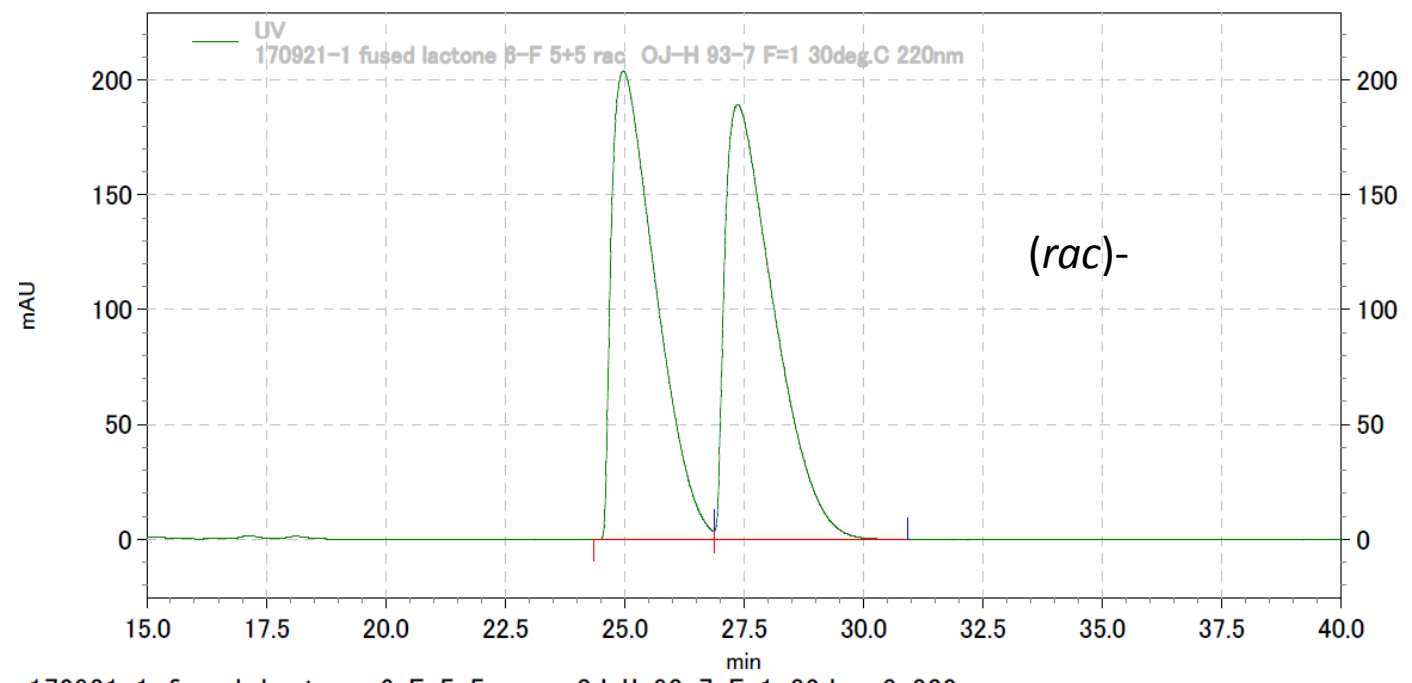

170921-1 fused lactone 6-F 5+5 rac 0J-H 93-7 F=1 30deg. C 220nm

\begin{tabular}{rlllll}
$\begin{array}{r}\text { Pk } \\
\text { No. }\end{array}$ & R. T. & Area & Area\% & B. C. & Symmetry \\
\hline 1 & 24.977 & 52238541 & 49.101 & $\mathrm{Mx}$ & 0.00000 \\
2 & 27.370 & 54150624 & 50.899 & $\mathrm{xM}$ & 0.00000
\end{tabular}
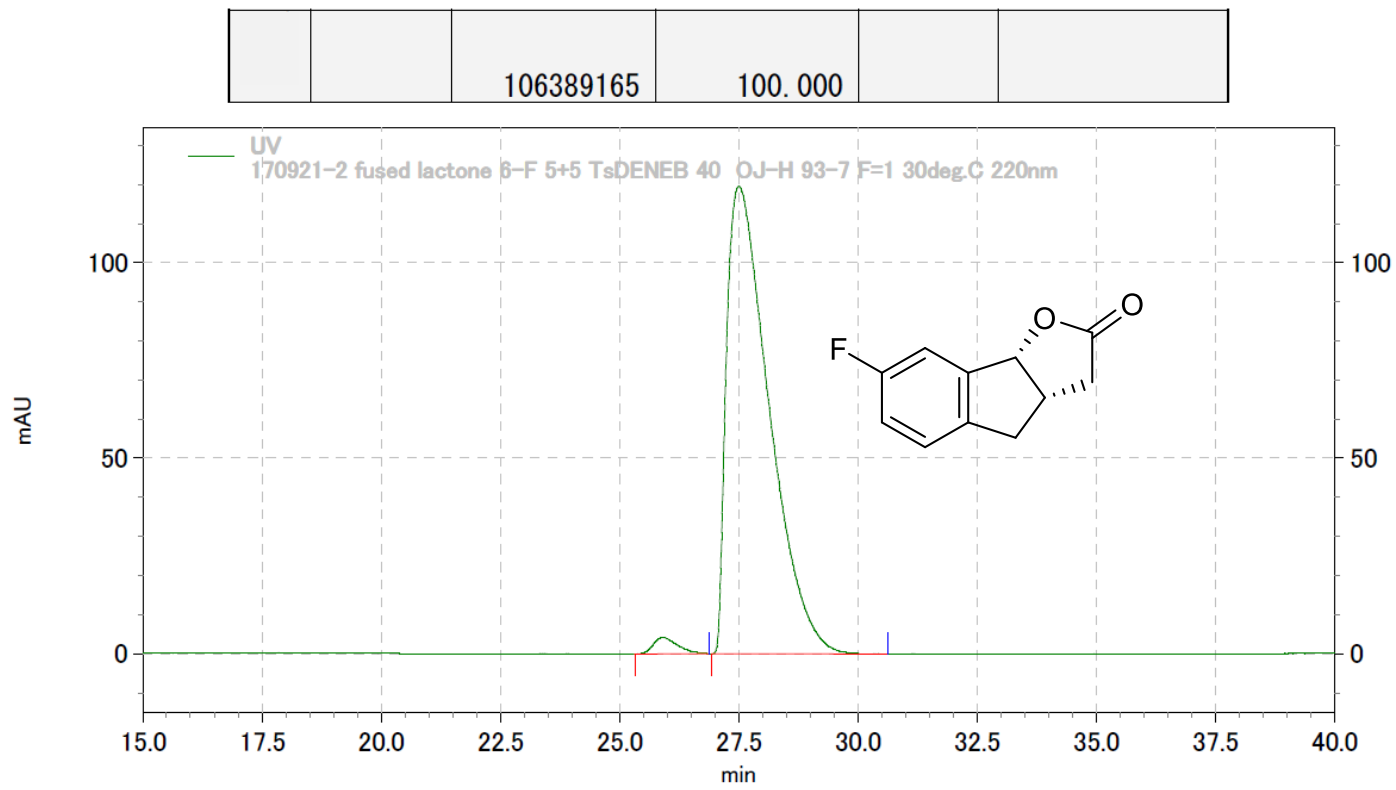

170921-2 fused lactone 6-F 5+5 TsDENEB 40 0J-H 93-7 F=1 30deg. C 220nm

\begin{tabular}{rlrrrrr}
\multicolumn{1}{l}{$\begin{array}{l}\text { Pk } \\
\text { No. }\end{array}$} & \multicolumn{1}{l}{ R. T. } & \multicolumn{1}{l}{ Area } & \multicolumn{1}{l}{ Area\% } & \multicolumn{1}{l}{ B. C. } & Symmetry \\
\hline 1 & 25.910 & 593946 & 1.957 & MM & 0.00000 \\
2 & 27.510 & 29748288 & 98.043 & MM & 0.00000 \\
\hline & & & & & \\
\hline
\end{tabular}




\section{(3aS,8bR)-7-Chloro-3,3a,4,8b-tetrahydro-2H-indeno[1,2-b]furan-2-one (13j)}

Chiralpak AD-H, $250 \times 4.6 \mathrm{~mm}$ column, hexane/2-propanol 94:6, $1.0 \mathrm{~mL} / \mathrm{min}, 220 \mathrm{~nm}, 35^{\circ} \mathrm{C}$

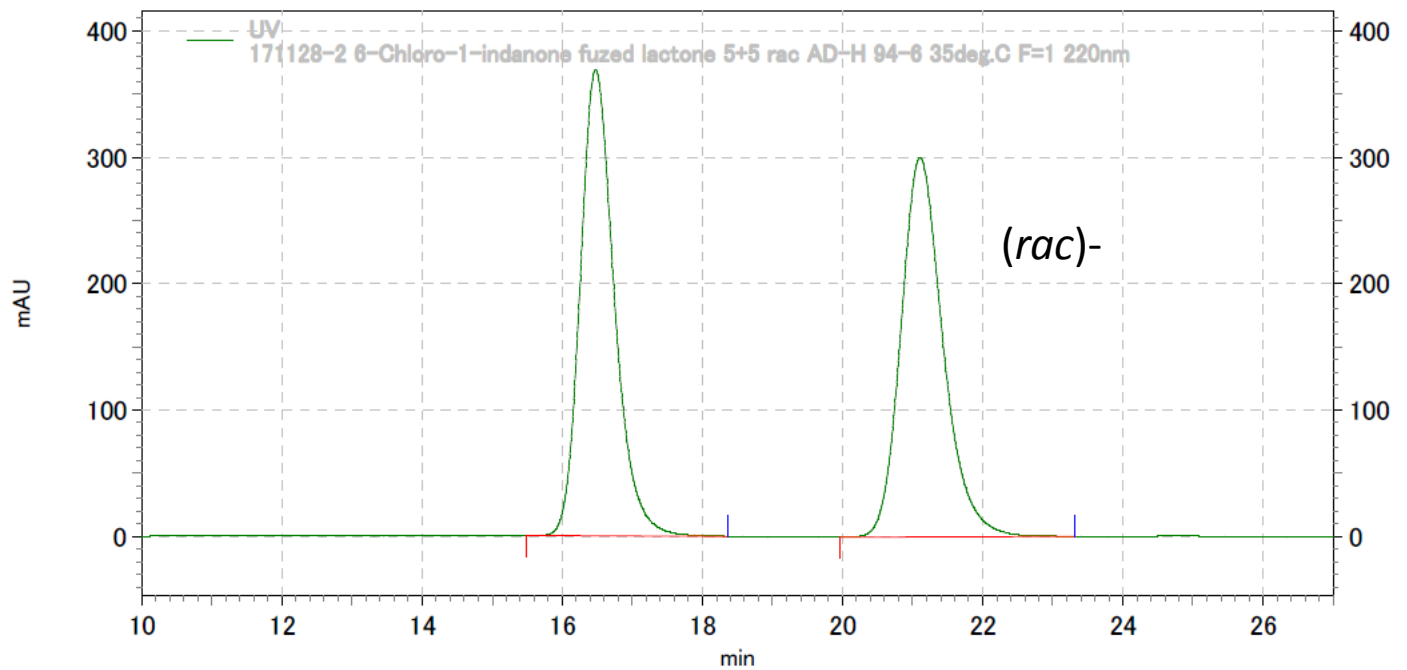

171128-2 6-Chloro-1-indanone fuzed lactone 5+5 rac AD-H 94-6 35deg. C F=1 220nm

\begin{tabular}{rlllll}
$\begin{array}{l}\text { Pk } \\
\text { No. }\end{array}$ & R. T. & Area & Area\% & B. C. & Symmetry \\
\hline 1 & 16.477 & 49824713 & 49.840 & MM & 0.00000 \\
2 & 21.107 & 50144635 & 50.160 & MM & 0.00000
\end{tabular}
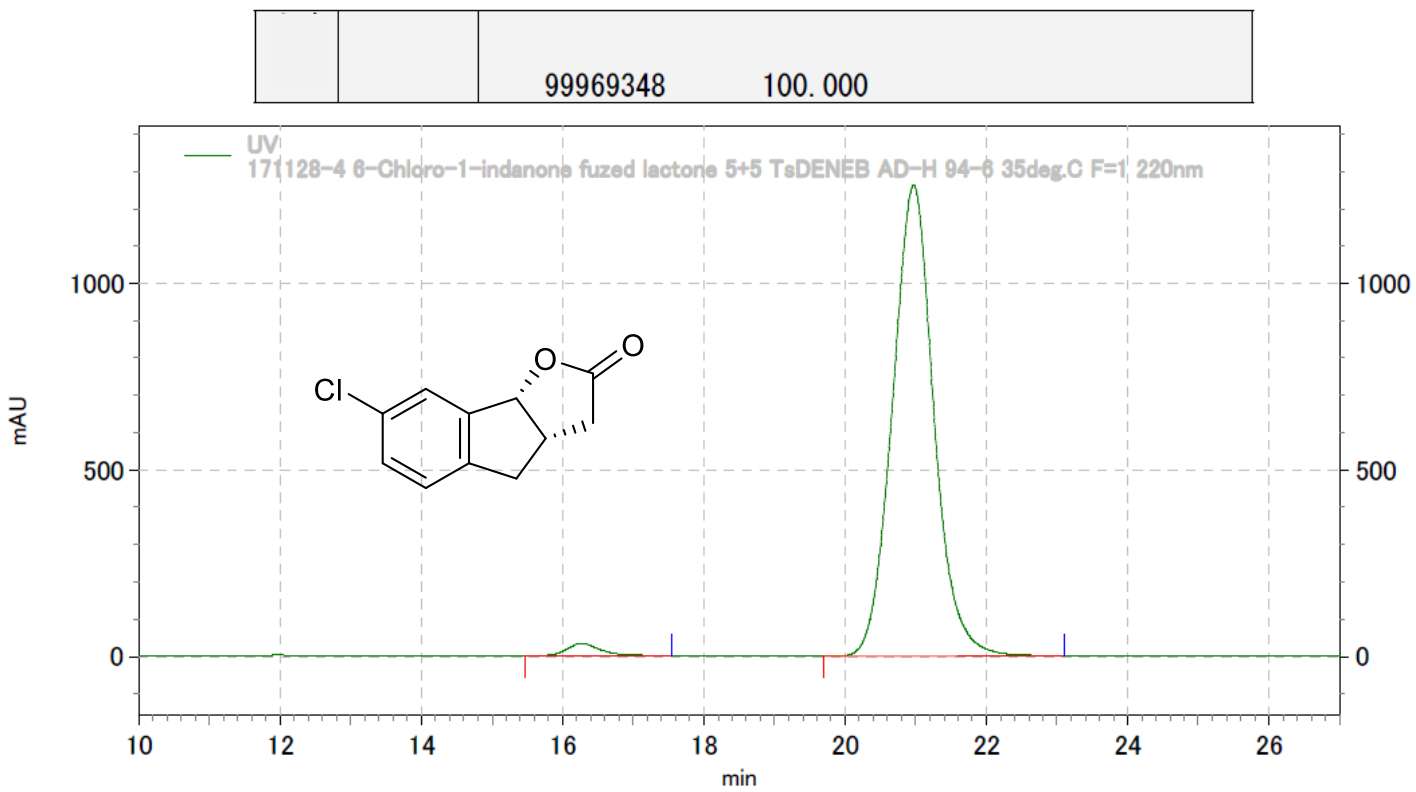

171128-4 6-Chloro-1-indanone fuzed lactone 5+5 TsDENEB AD-H 94-6 35deg. C F=1 220nm

\begin{tabular}{rl|rrrr}
$\begin{array}{r}\text { Pk } \\
\text { No. }\end{array}$ & R. T. & \multicolumn{1}{c}{ Area } & \multicolumn{1}{l}{ Area\% } & B. C. & Symmetry \\
\hline 1 & 16.267 & 4561456 & 2.168 & MM & 0.00000 \\
2 & 20.970 & 205813212 & 97.832 & MM & 0.00000 \\
\hline & & & & & \\
\hline & & 210374668 & 100.000 & & \\
\hline
\end{tabular}


$(3 \mathrm{a} S, 10 \mathrm{~b} R)-3,3 \mathrm{a}, 4,5,6,10 \mathrm{~b}-H e x a h y d r o-2 H$-benzo[6,7]cyclohepta[1,2-b]furan-2-one (13k)

Chiralcel OD-H, $250 \times 4.6 \mathrm{~mm}$ column, hexane/2-propanol 90:10, $1.0 \mathrm{~mL} / \mathrm{min}, 220 \mathrm{~nm}, 40^{\circ} \mathrm{C}$

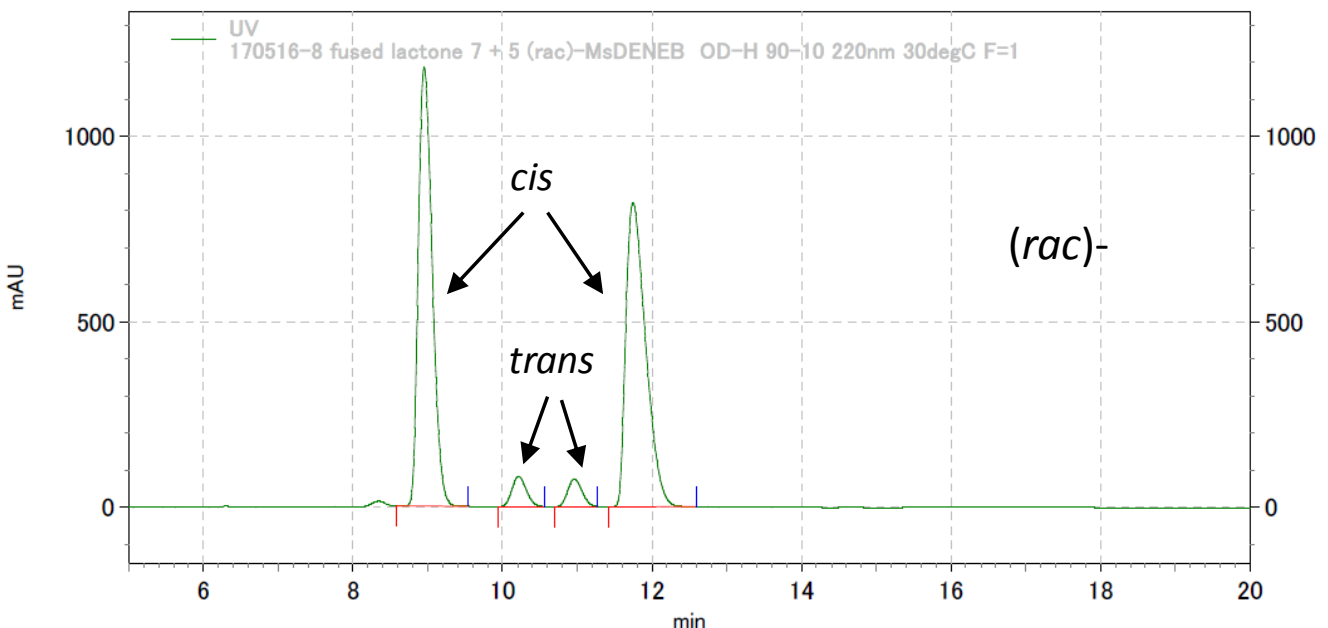

170516-8 fused lactone $7+5$ ( $\mathrm{rac}$-MsDENEB OD-H 90-10 220nm 30degC F=1

\begin{tabular}{rrrrrl}
$\begin{array}{c}\text { Pk } \\
\text { No. }\end{array}$ & \multicolumn{1}{l}{ R. T. } & \multicolumn{1}{c}{ Area } & Area\% & B. C. & Symmetry \\
\hline 1 & 8.953 & 61208525 & 47.143 & MM & 0.00000 \\
2 & 10.217 & 4397896 & 3.387 & MM & 0.00000 \\
3 & 10.960 & 4015952 & 3.093 & MM & 0.00000 \\
4 & 11.747 & 60212201 & 46.376 & MM & 0.00000
\end{tabular}
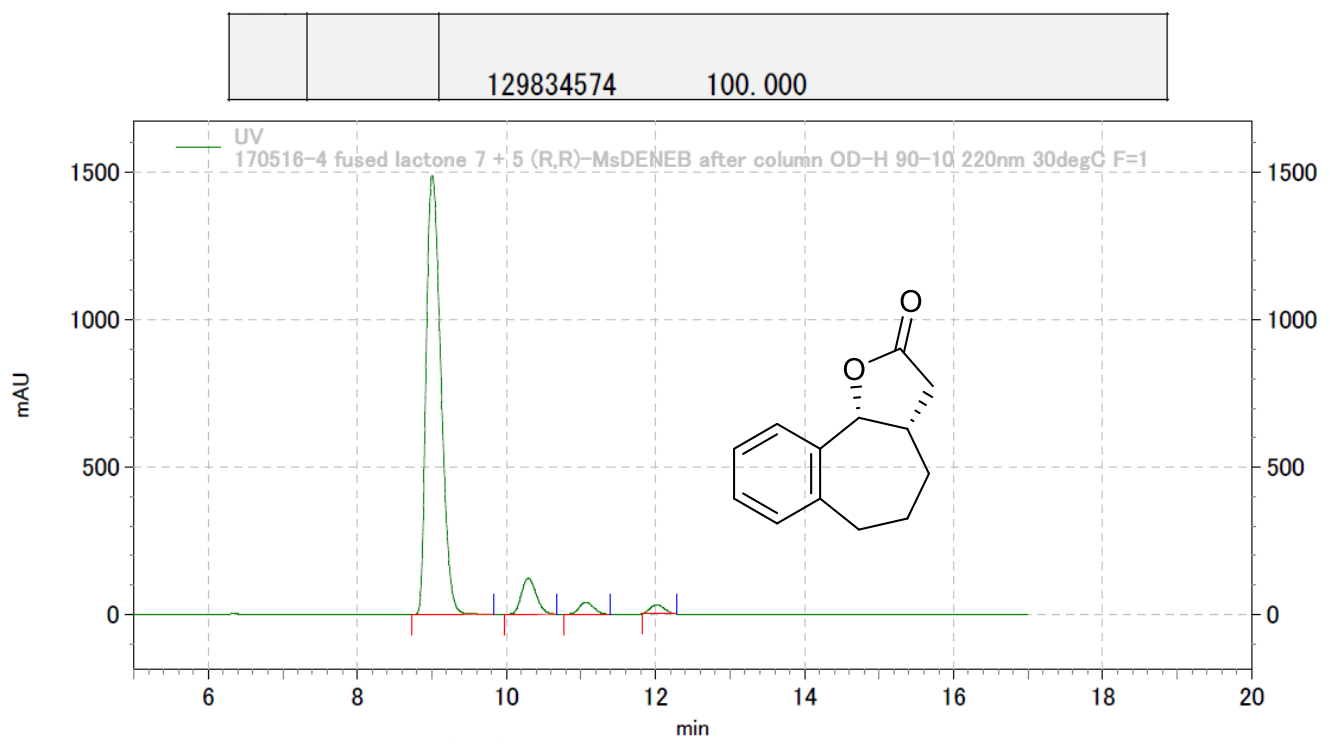

170516-4 fused lactone $7+5$ (R, R) -MsDENEB after column OD-H 90-10 220nm 30degC F=1

\begin{tabular}{rrrrrr}
$\begin{array}{r}\text { Pk } \\
\text { No. }\end{array}$ & \multicolumn{1}{l}{ R. T. } & \multicolumn{1}{l}{ Area } & Area\% & B. C. & Symmetry \\
\hline 1 & 9.003 & 81333505 & 88.267 & MM & 0.00000 \\
2 & 10.293 & 6818352 & 7.400 & MM & 0.00000 \\
3 & 11.070 & 2292114 & 2.487 & MM & 0.00000 \\
4 & 12.017 & 1701320 & 1.846 & MM & 0.00000 \\
\hline & & & & \\
\hline
\end{tabular}


$(4 \mathrm{a} S, 10 \mathrm{~b} R)-3,4,4 \mathrm{a}, 5,6,10 \mathrm{~b}-H e x a h y d r o-2 H$-benzo[ $h]$ chromen-2-one (131)

Chiralcel OD-H, $250 \times 4.6 \mathrm{~mm}$ column, hexane/2-propanol 95:5, $1.0 \mathrm{~mL} / \mathrm{min}, 220 \mathrm{~nm}, 35^{\circ} \mathrm{C}$

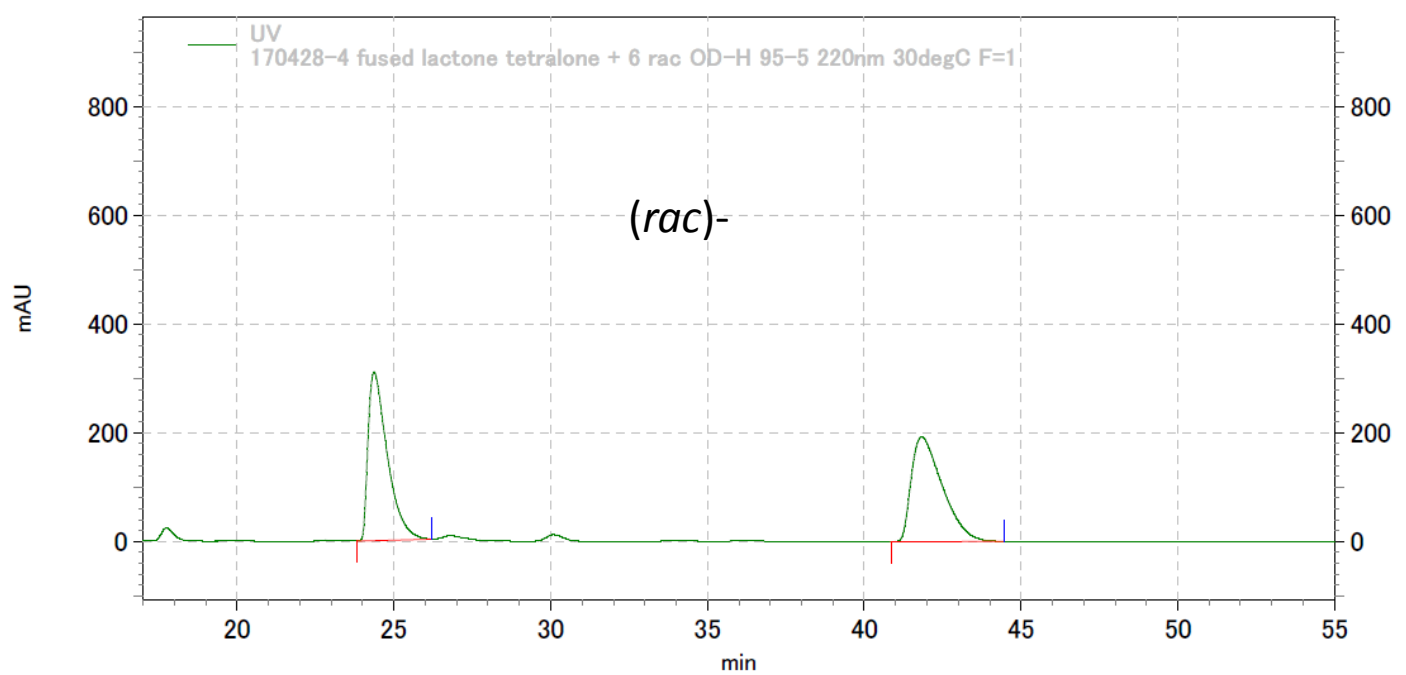

170428-4 fused lactone tetralone +6 rac 0D-H 95-5 220nm 30degC F=1

\begin{tabular}{rlllll}
$\begin{array}{l}\text { Pk } \\
\text { No. }\end{array}$ & R. T. & Area & Area\% & B. C. & Symmetry \\
\hline 1 & 24.370 & 53140928 & 49.981 & MM & 0.00000 \\
2 & 41.837 & 53181090 & 50.019 & MM & 0.00000
\end{tabular}

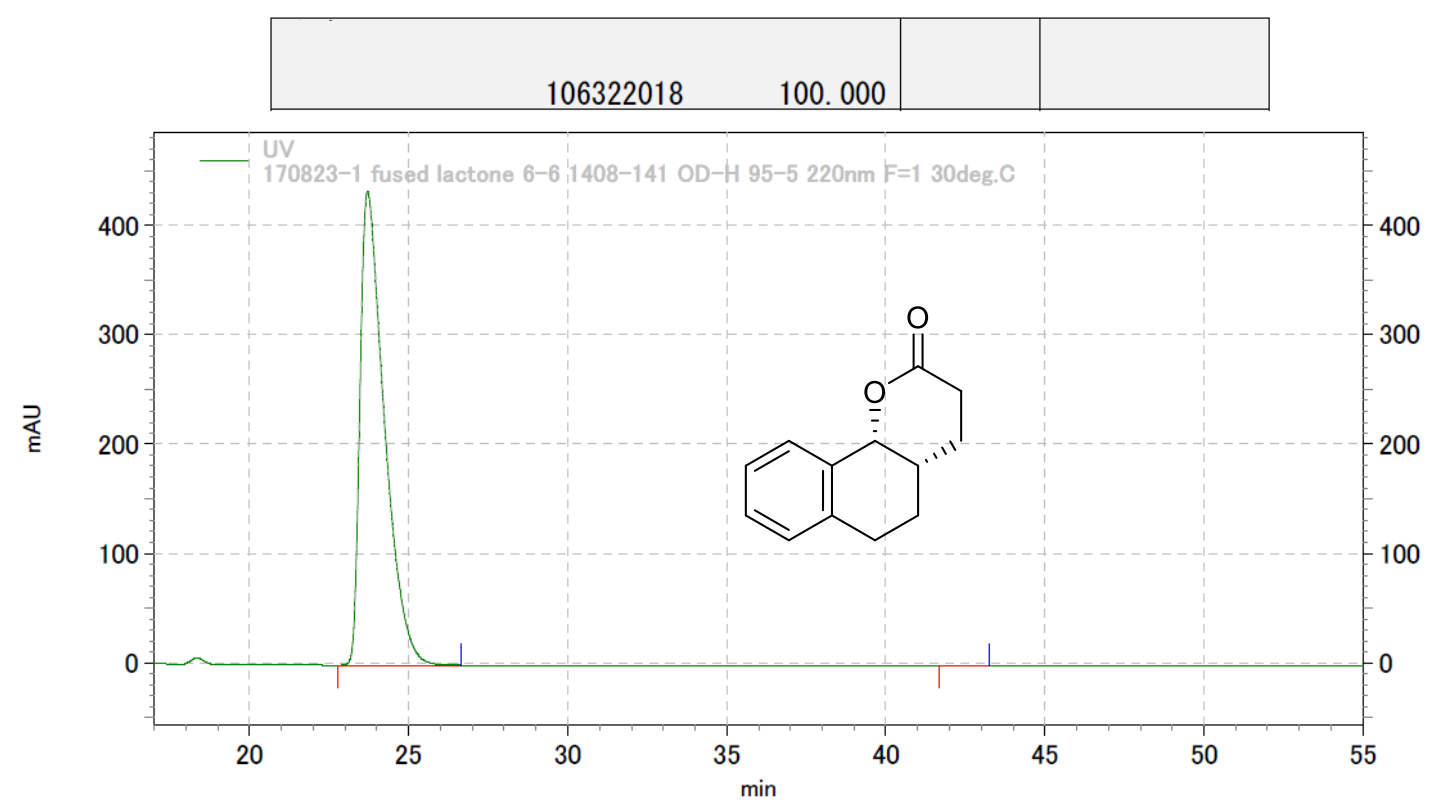

170823-1 fused lactone 6-6 1408-141 OD-H 95-5 220nm F=1 30deg. C

\begin{tabular}{|c|c|c|c|c|c|}
\hline Pk & R. T. & Area & Area\% & B. C. & Symmetry \\
\hline 1 & $\begin{array}{l}23.710 \\
42.653\end{array}$ & $\begin{array}{r}92216484 \\
29174\end{array}$ & $\begin{array}{r}99.968 \\
0.032\end{array}$ & $\begin{array}{l}\text { MM } \\
M M\end{array}$ & $\begin{array}{l}0.00000 \\
0.00000\end{array}$ \\
\hline & & 92245658 & 100.000 & & \\
\hline
\end{tabular}


(4aS,10bR)-9-Methoxy-3,4,4a,5,6,10b-hexahydro-2H-benzo[h]chromen-2-one (13m)

Chiralcel OJ-3, $250 \times 4.6 \mathrm{~mm}$ column, hexane/2-propanol 94:6, $1.0 \mathrm{~mL} / \mathrm{min}, 220 \mathrm{~nm}, 40^{\circ} \mathrm{C}$

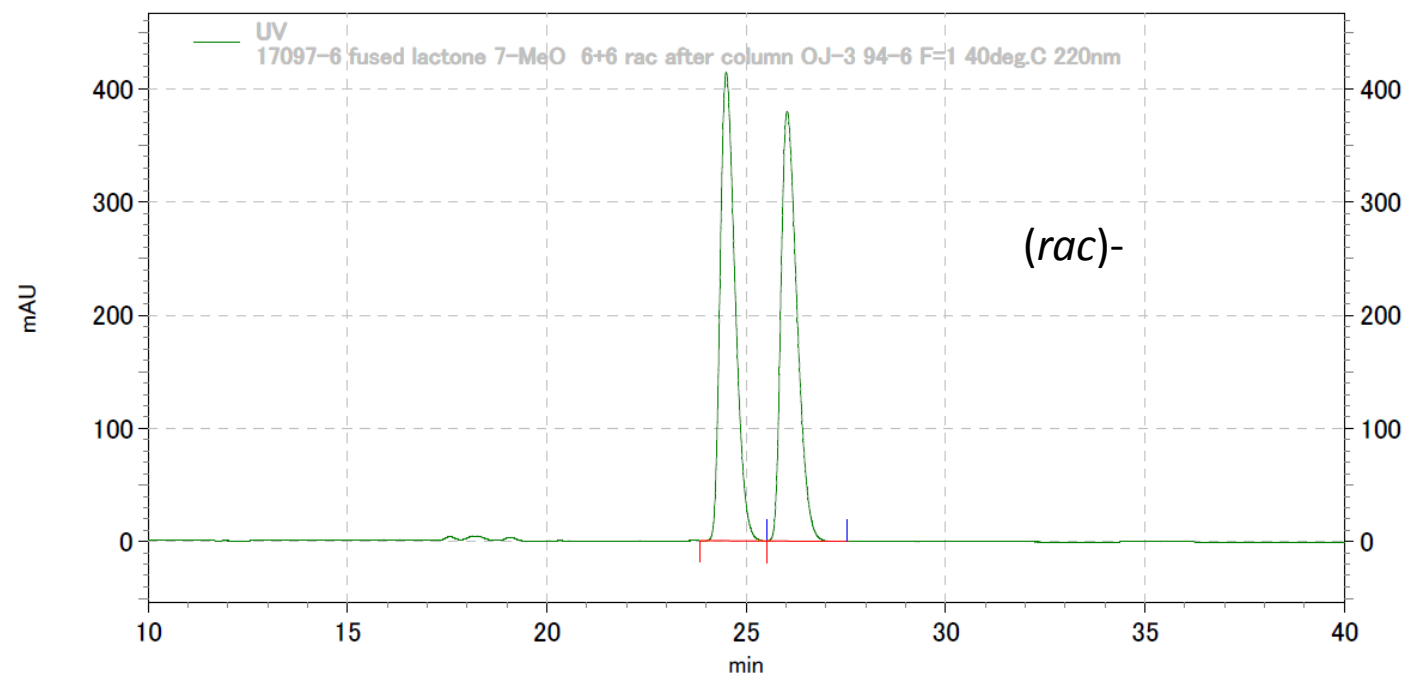

17097-6 fused lactone 7-Me0 6+6 rac after column 0J-3 94-6 F=1 40deg. C 220nm

\begin{tabular}{rlllll}
$\begin{aligned} \text { Pk } \\
\text { No. }\end{aligned}$ & R. T. & Area & Area\% & B. C. & Symmetry \\
\hline 1 & 24.500 & 43120231 & 50.277 & Mx & 0.00000 \\
2 & 26.030 & 42645481 & 49.723 & xM & 0.00000
\end{tabular}
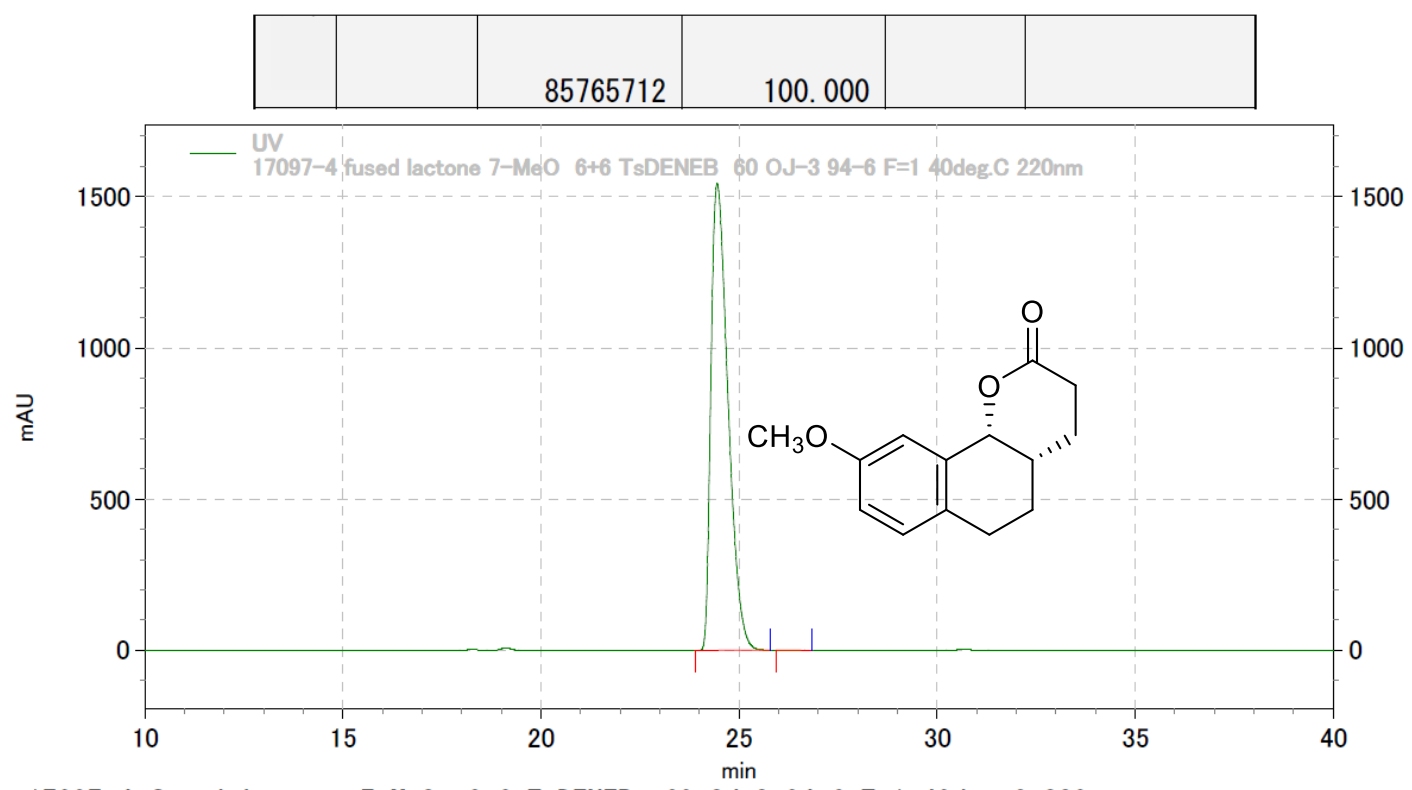

17097-4 fused lactone 7-Me0 6+6 TsDENEB 60 0J-3 94-6 F=1 40deg. C 220nm

\begin{tabular}{|c|c|c|c|c|c|}
\hline Pk & R. T. & Area & Area\% & B. C. & Symmetry \\
\hline $\begin{array}{l}1 \\
2\end{array}$ & $\begin{array}{l}24.450 \\
26.337\end{array}$ & $\begin{array}{r}185286985 \\
107952\end{array}$ & $\begin{array}{r}99.942 \\
0.058\end{array}$ & $\begin{array}{l}\text { MM } \\
\text { MM }\end{array}$ & $\begin{array}{l}0.00000 \\
0.00000\end{array}$ \\
\hline & & 185394937 & 100.000 & & \\
\hline
\end{tabular}


(4aS,10bR)-9-Chloro-3,4,4a,5,6,10b-hexahydro-2H-benzo[ $h]$ chromen-2-one (13n)

Chiralcel OD-H, $250 \times 4.6 \mathrm{~mm}$ column, hexane/2-propanol 94:6, $1.0 \mathrm{~mL} / \mathrm{min}, 220 \mathrm{~nm}, 40^{\circ} \mathrm{C}$

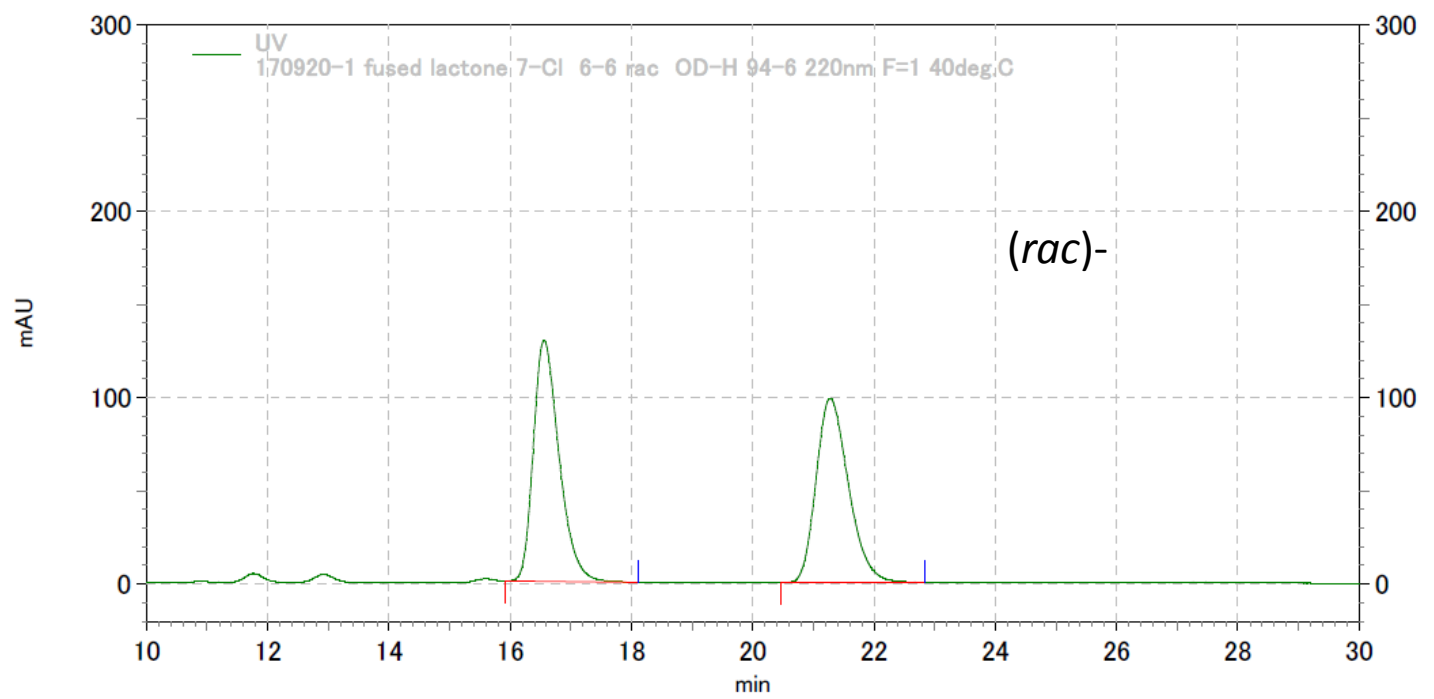

170920-1 fused lactone 7-Cl 6-6 rac OD-H 94-6 220nm F=1 40deg. C

\begin{tabular}{rlllll}
$\begin{array}{c}\text { Pk } \\
\text { No. }\end{array}$ & R. T. & Area & Area\% & B. C. & Symmetry \\
\hline 1 & 16.557 & 15349397 & 51.111 & MM & 0.00000 \\
2 & 21.277 & 14682048 & 48.889 & MM & 0.00000
\end{tabular}
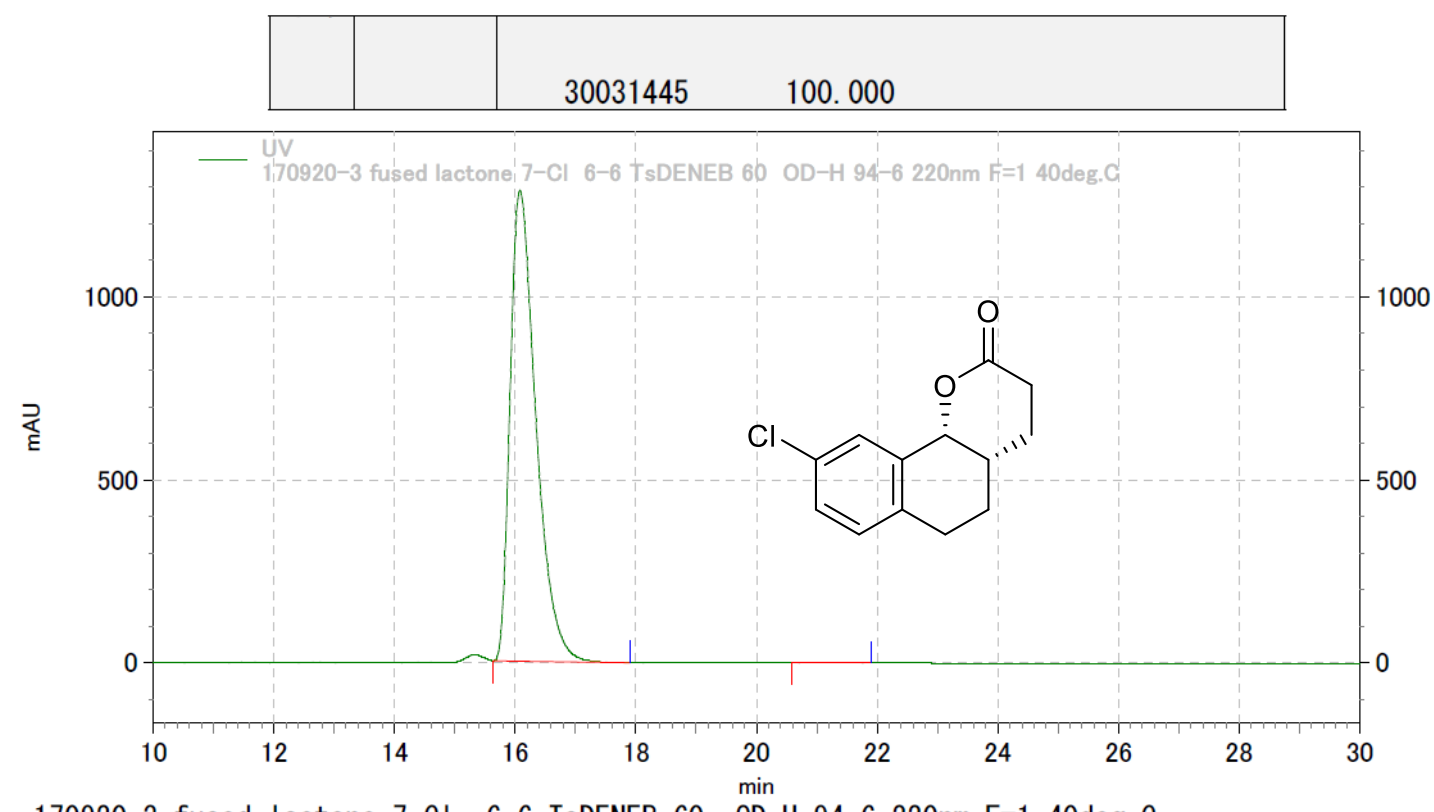

170920-3 fused lactone 7-Cl 6-6 TsDENEB 60 OD-H 94-6 220nm F=1 40deg. C

\begin{tabular}{rlrr|r|r}
$\begin{aligned} \text { Pk } \\
\text { No. }\end{aligned}$ & R. T. & \multicolumn{1}{c}{ Area } & \multicolumn{1}{l}{ Area\% } & \multicolumn{1}{l}{ B. C. } & Symmetry \\
\hline 1 & 16.080 & 152708433 & 99.844 & MM & 0.00000 \\
2 & 21.067 & 238220 & 0.156 & MM & 0.00000 \\
\hline & & & & & \\
\hline & & & & & \\
\hline
\end{tabular}




\section{$(3 \mathrm{a} R, 9 \mathrm{~b} S)-3 \mathrm{a}, 4,5,9 \mathrm{~b}-$ Tetrahydronaphtho[2,1-b]furan-2(1H)-one (13o)}

Chiralcel OJ-H, $250 \times 4.6 \mathrm{~mm}$ column, hexane/2-propanol 95:5, $1.0 \mathrm{~mL} / \mathrm{min}, 220 \mathrm{~nm}, 30^{\circ} \mathrm{C}$

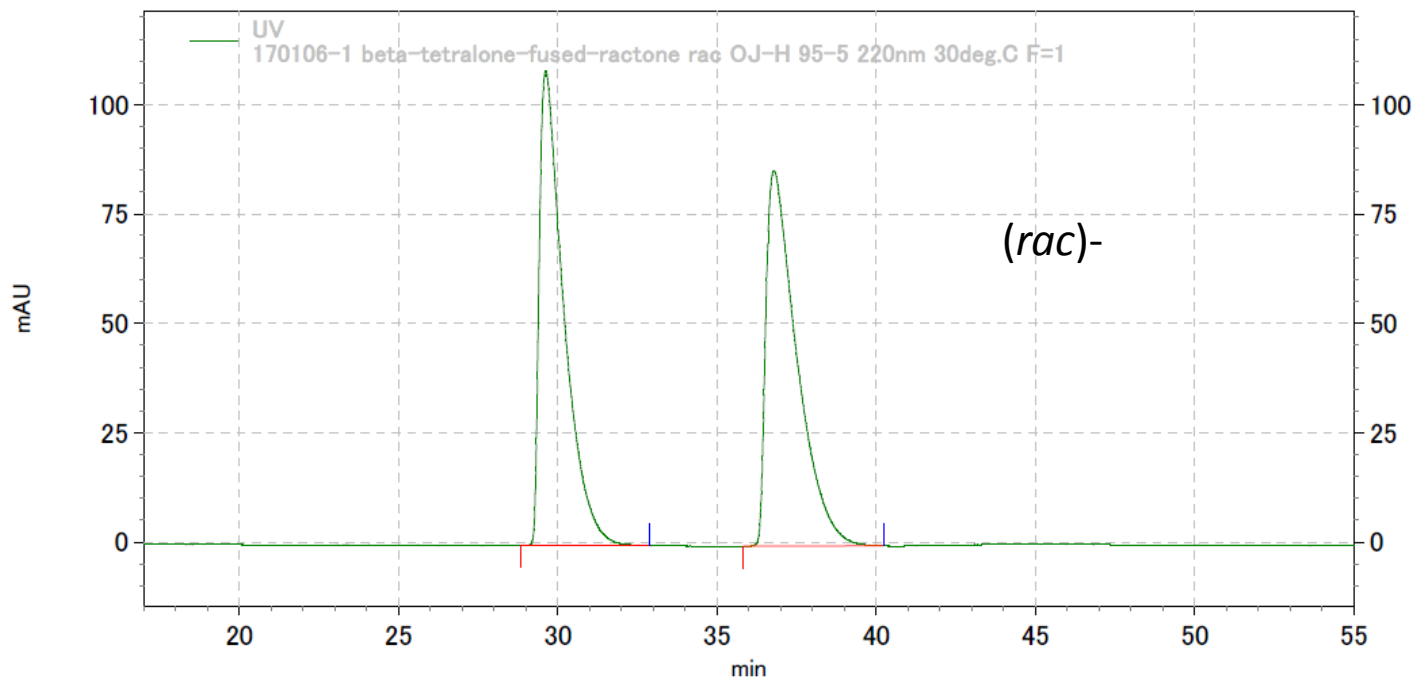

170106-1 beta-tetralone-fused-ractone rac 0J-H 95-5 220nm 30deg. C F=1

\begin{tabular}{|c|c|c|c|c|c|}
\hline $\begin{array}{l}\mathrm{Pk} \\
\text { No. }\end{array}$ & R. T. & Area & Area\% & B. C. & Symmetry \\
\hline $\begin{array}{l}1 \\
2\end{array}$ & $\begin{array}{l}29.617 \\
36.787\end{array}$ & $\begin{array}{l}23208167 \\
23097737\end{array}$ & $\begin{array}{l}50.119 \\
49.881\end{array}$ & $\begin{array}{l}\mathrm{MM} \\
\mathrm{MM}\end{array}$ & $\begin{array}{l}0.00000 \\
0.00000\end{array}$ \\
\hline & & 46305904 & 100.000 & & \\
\hline
\end{tabular}

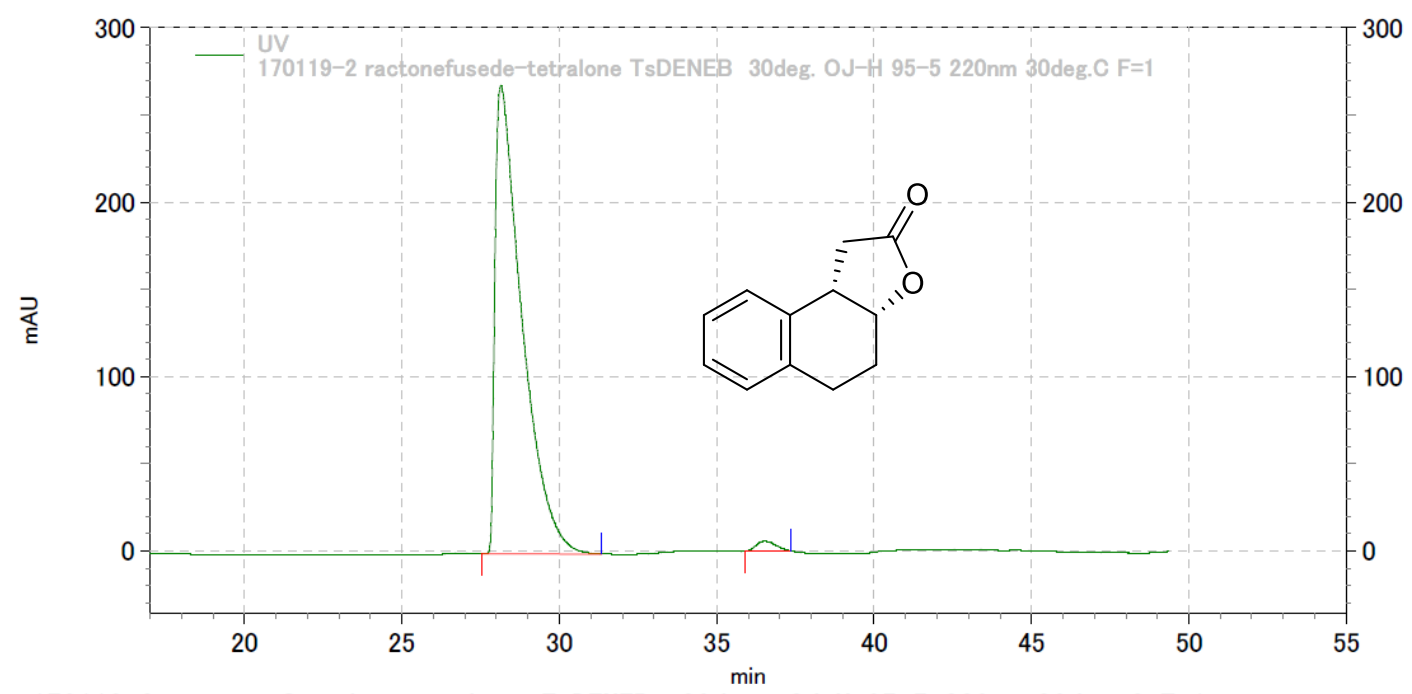

170119-2 ractonefusede-tetralone TsDENEB 30deg. OJ-H 95-5 220nm 30deg. C F=1

\begin{tabular}{|c|c|c|c|c|c|}
\hline $\begin{array}{l}\mathrm{Pk} \\
\text { No. }\end{array}$ & R. T. & Area & Area\% & B. C. & Symmetry \\
\hline $\begin{array}{l}1 \\
2\end{array}$ & $\begin{array}{l}28.147 \\
36.520\end{array}$ & $\begin{array}{r}63860226 \\
1007725\end{array}$ & $\begin{array}{r}98.446 \\
1.554\end{array}$ & $\begin{array}{l}\text { MM } \\
\text { MM }\end{array}$ & $\begin{array}{l}0.00000 \\
0.00000\end{array}$ \\
\hline & & 64867951 & 100.000 & & \\
\hline
\end{tabular}


$(3 \mathrm{a} R, 9 \mathrm{~b} S)-6-$ Methoxy-3a,4,5,9b-tetrahydronaphtho[2,1-b]furan-2(1H)-one (13p)

Chiralcel OJ-H, $250 \times 4.6 \mathrm{~mm}$ column, hexane/2-propanol 94:6, $1.0 \mathrm{~mL} / \mathrm{min}, 220 \mathrm{~nm}, 40{ }^{\circ} \mathrm{C}$

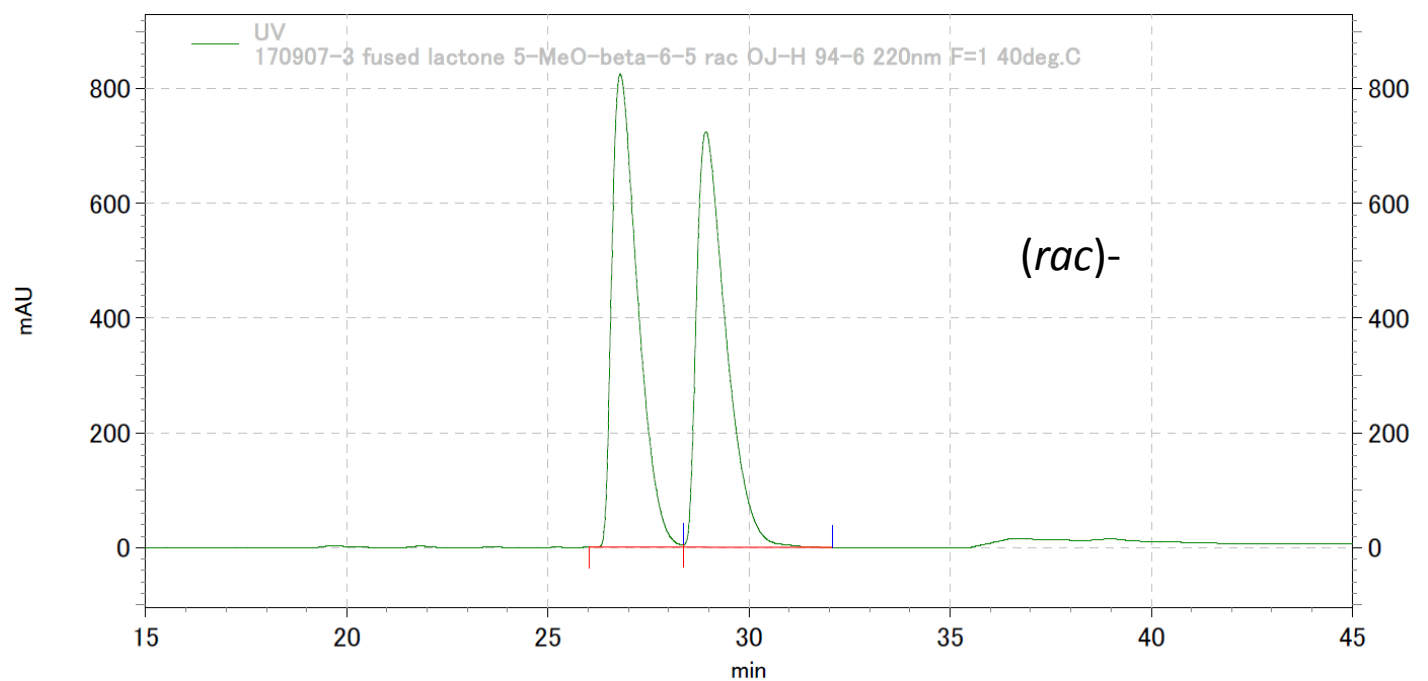

170907-3 fused lactone 5-Me0-beta-6-5 rac 0J-H 94-6 220nm F=1 40deg. C

\begin{tabular}{rlllll}
$\begin{array}{l}\text { Pk } \\
\text { No. }\end{array}$ & R. T. & Area & Area\% & B. C. & Symmetry \\
\hline 1 & 26.800 & 147198945 & 50.116 & Mx & 0.00000 \\
2 & 28.930 & 146518515 & 49.884 & xM & 0.00000
\end{tabular}
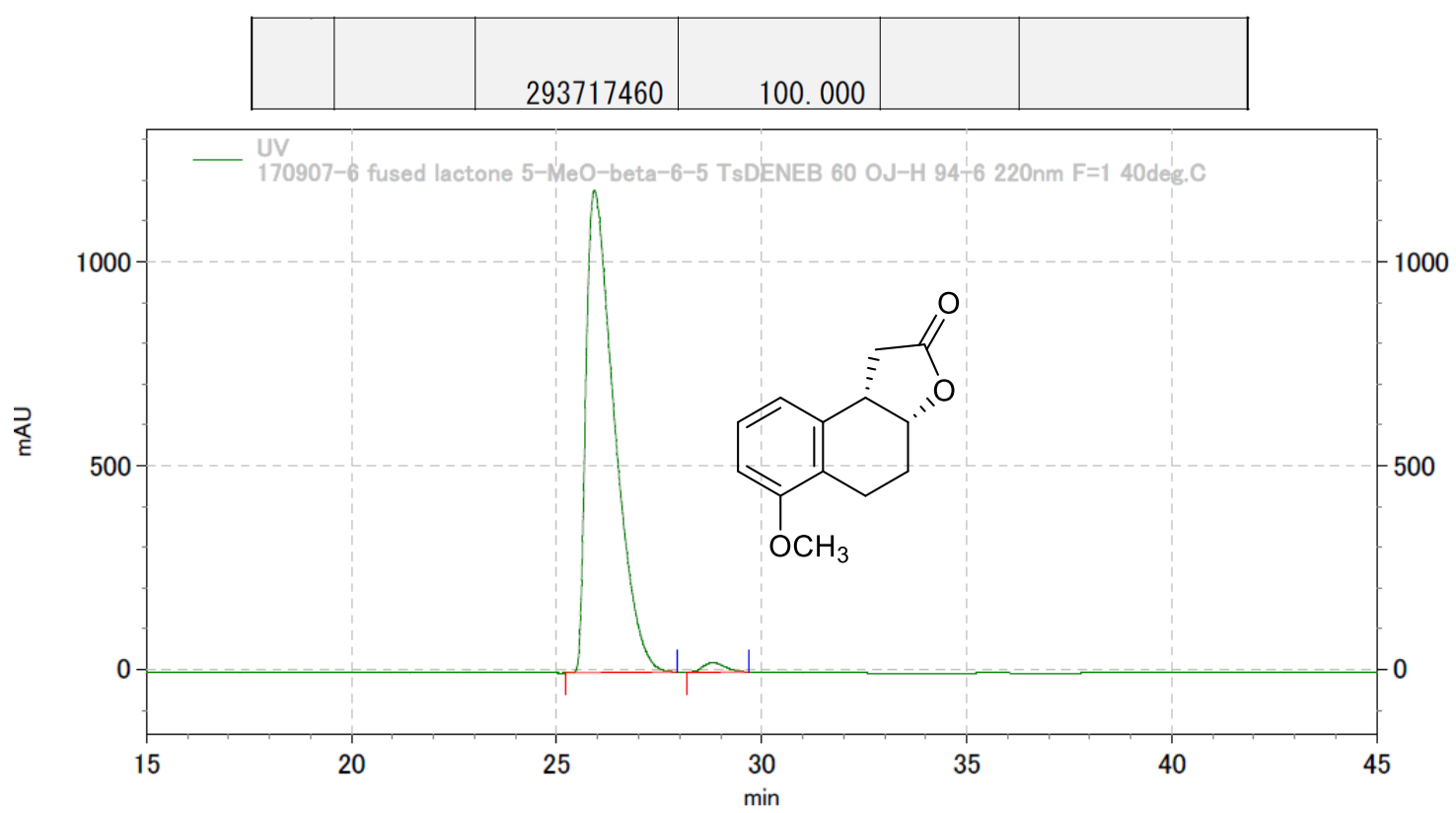

170907-6 fused Iactone 5-Me0-beta-6-5 TsDENEB 60 0J-H 94-6 220nm F=1 40deg. C

\begin{tabular}{rlrrrr}
$\begin{array}{r}\text { Pk } \\
\text { No. }\end{array}$ & R. T. & \multicolumn{1}{c}{ Area } & \multicolumn{1}{c}{ Area\% } & B. C. & Symmetry \\
\hline 1 & 25.923 & 227631682 & 98.484 & MM & 0.00000 \\
2 & 28.810 & 3504448 & 1.516 & MM & 0.00000 \\
\hline & & & & & \\
\hline & & 231136130 & 100.000 & \\
\hline
\end{tabular}




\section{(3aR,9bS)-7-Bromo-3a,4,5,9b-tetrahydronaphtho[2,1-b]furan-2(1H)-one (13q)}

Chiralcel OD-H, $250 \times 4.6 \mathrm{~mm}$ column, hexane/2-propanol 94:6, $1.0 \mathrm{~mL} / \mathrm{min}, 220 \mathrm{~nm}, 35^{\circ} \mathrm{C}$

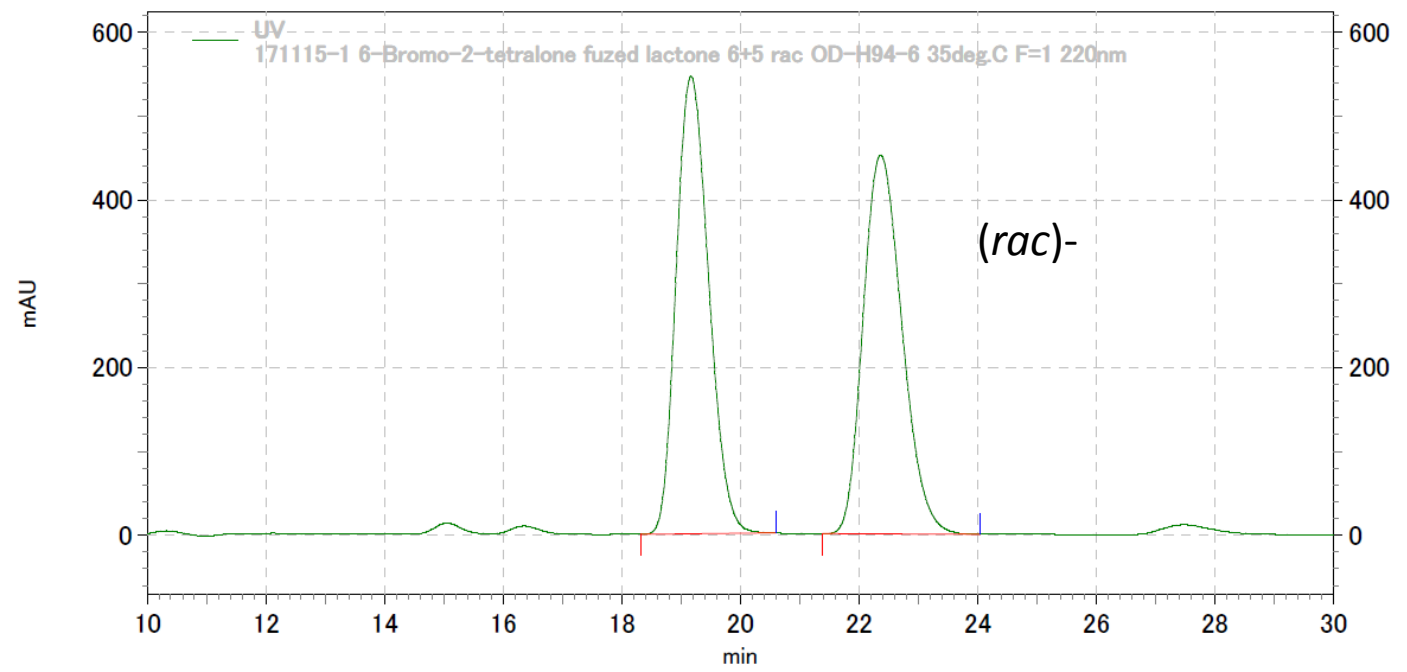

171115-1 6-Bromo-2-tetralone fuzed lactone 6+5 rac 0D-H94-6 35deg. C F=1 220nm

\begin{tabular}{rlllll}
$\begin{array}{l}\text { Pk } \\
\text { No. }\end{array}$ & R. T. & Area & Area\% & B. C. & Symmetry \\
\hline 1 & 19.170 & 83230803 & 50.202 & MM & 0.00000 \\
2 & 22. 370 & 82561450 & 49.798 & MM & 0.00000
\end{tabular}
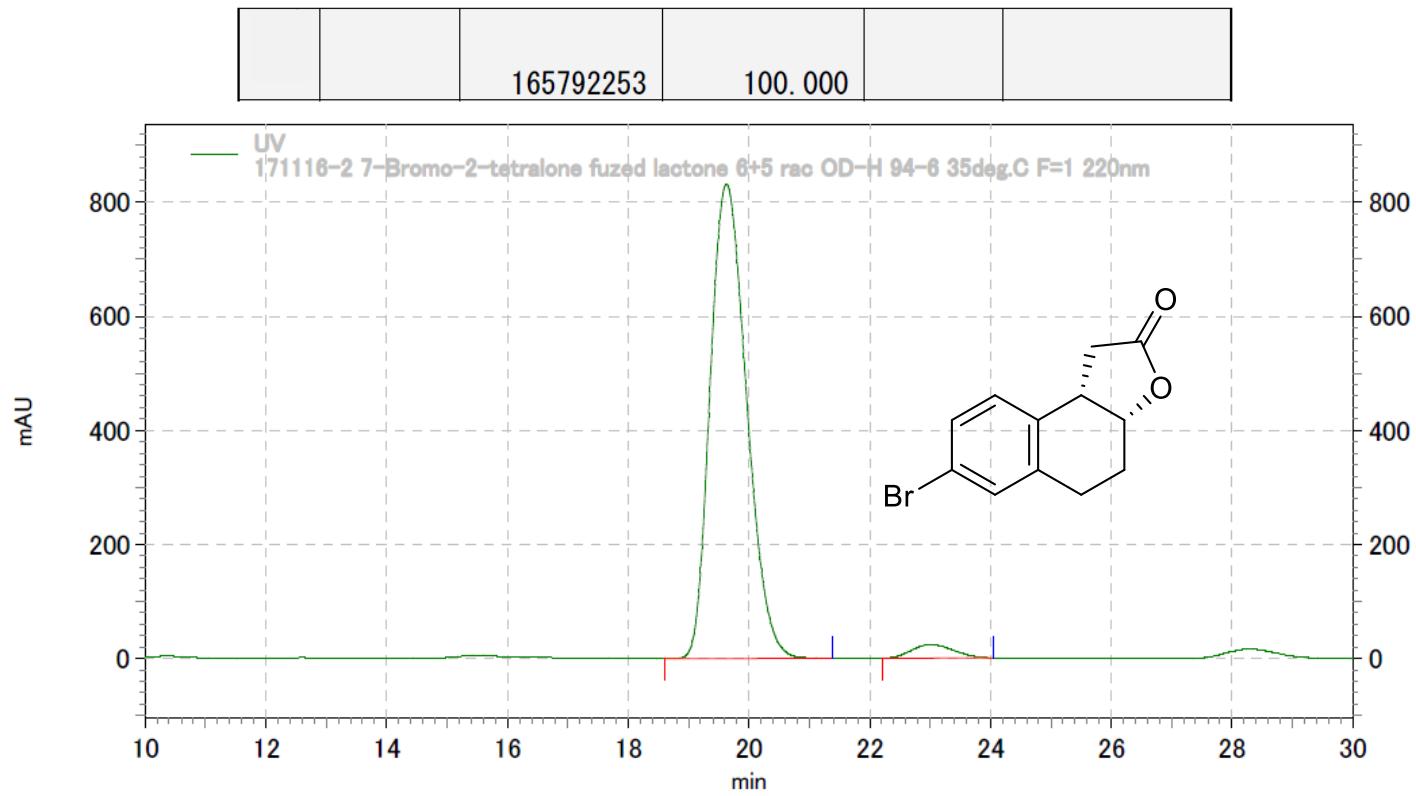

171116-2 7-Bromo-2-tetralone fuzed lactone 6+5 rac 0D-H 94-6 35deg. C F=1 220nm

\begin{tabular}{rlrrrr}
$\begin{array}{r}\text { Pk } \\
\text { No. }\end{array}$ & \multicolumn{1}{l}{ R. T. } & \multicolumn{1}{c}{ Area } & \multicolumn{1}{c}{ Area\% } & B.C. & Symmetry \\
\hline 1 & 19.630 & 139034093 & 96.750 & MM & 0.00000 \\
2 & 23.013 & 4670790 & 3.250 & MM & 0.00000 \\
\hline & & & & & \\
\hline & & 143704883 & 100.000 & \\
\hline
\end{tabular}




\section{(3aR,9bS)-7-Chloro-3a,4,5,9b-tetrahydronaphtho[2,1-b]furan-2(1H)-one (13r)}

Chiralcel OD-H, $250 \times 4.6 \mathrm{~mm}$ column, hexane/2-propanol 94:6, $1.0 \mathrm{~mL} / \mathrm{min}, 220 \mathrm{~nm}, 40{ }^{\circ} \mathrm{C}$

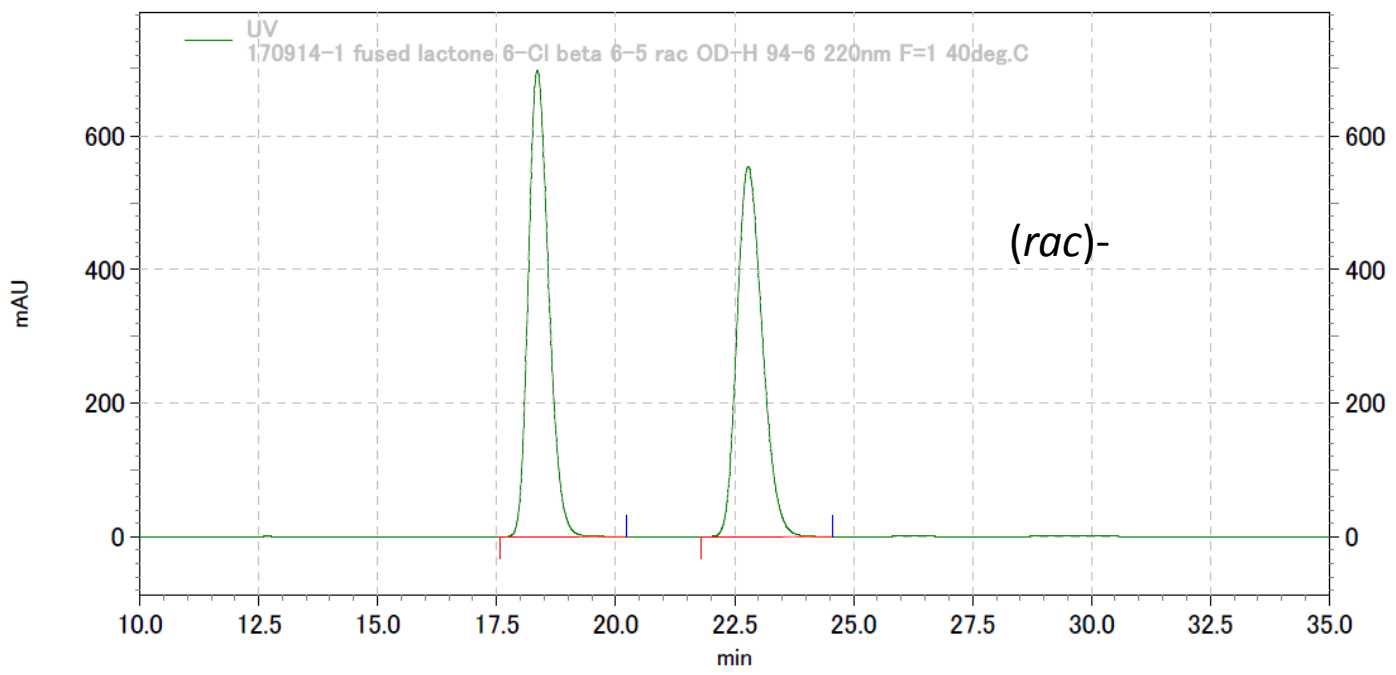

170914-1 fused lactone 6-Cl beta 6-5 rac 0D-H 94-6 220nm F=1 40deg. C

\begin{tabular}{rlllll}
$\begin{array}{l}\text { Pk } \\
\text { No. }\end{array}$ & R. T. & Area & Area\% & B. C. & Symmetry \\
\hline 1 & 18.357 & 83221590 & 50.315 & MM & 0.00000 \\
2 & 22.787 & 82180089 & 49.685 & MM & 0.00000
\end{tabular}
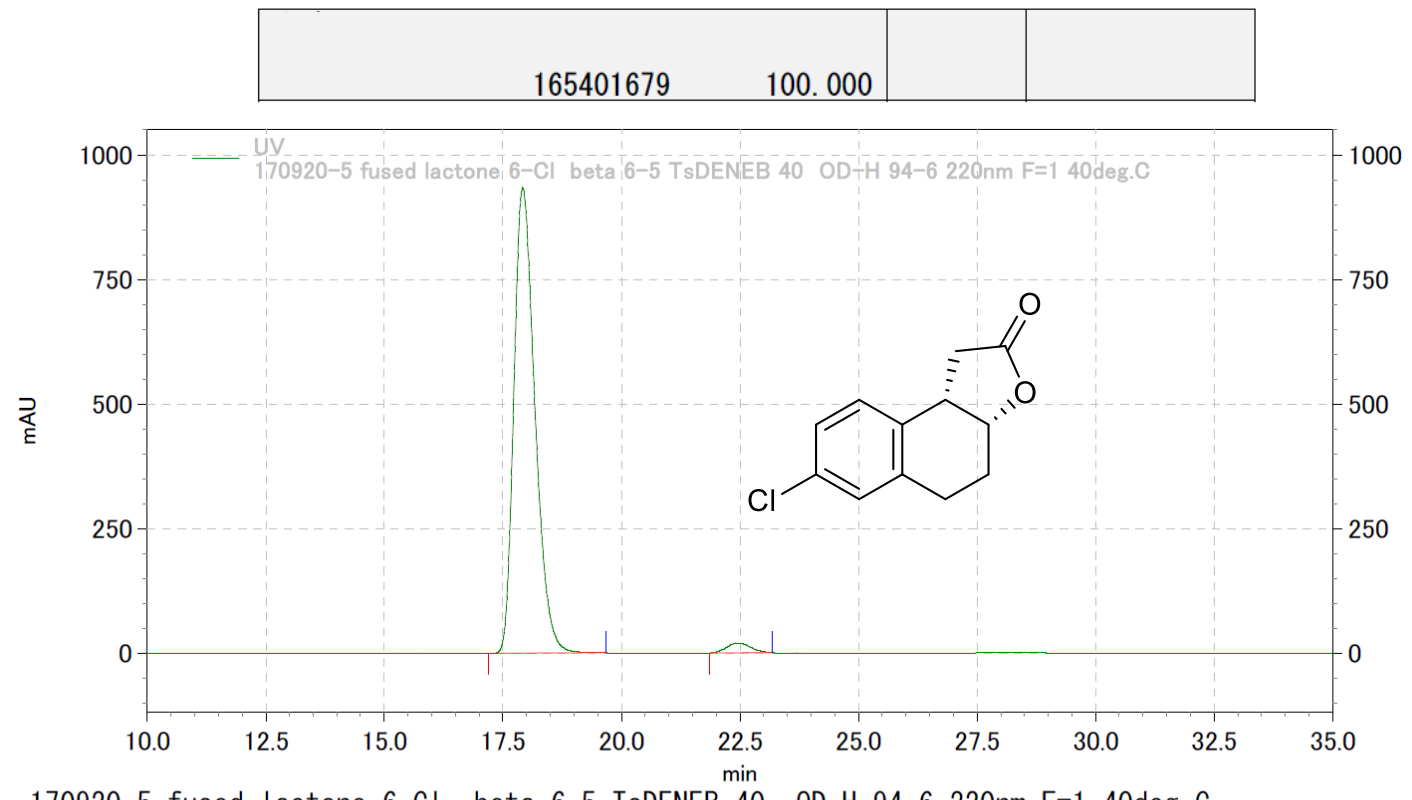

170920-5 fused lactone 6-Cl beta 6-5 TsDENEB 40 OD-H 94-6 220nm F=1 40deg. C

\begin{tabular}{rrrr|r|r}
$\begin{aligned} \text { Pk } \\
\text { No. }\end{aligned}$ & \multicolumn{1}{l}{ R. T. } & \multicolumn{1}{c}{ Area } & Area\% & B. C. & Symmetry \\
\hline 1 & 17.917 & 114900069 & 97.689 & MM & 0.00000 \\
2 & 22.460 & 2718620 & 2.311 & MM & 0.00000 \\
\hline & & & & & \\
& & 117618689 & 100.000 & & \\
\hline
\end{tabular}


Chiralpak IB, $250 \times 4.6 \mathrm{~mm}$ column, hexane/2-propanol 94:6, $1.0 \mathrm{~mL} / \mathrm{min}, 220 \mathrm{~nm}, 35^{\circ} \mathrm{C}$

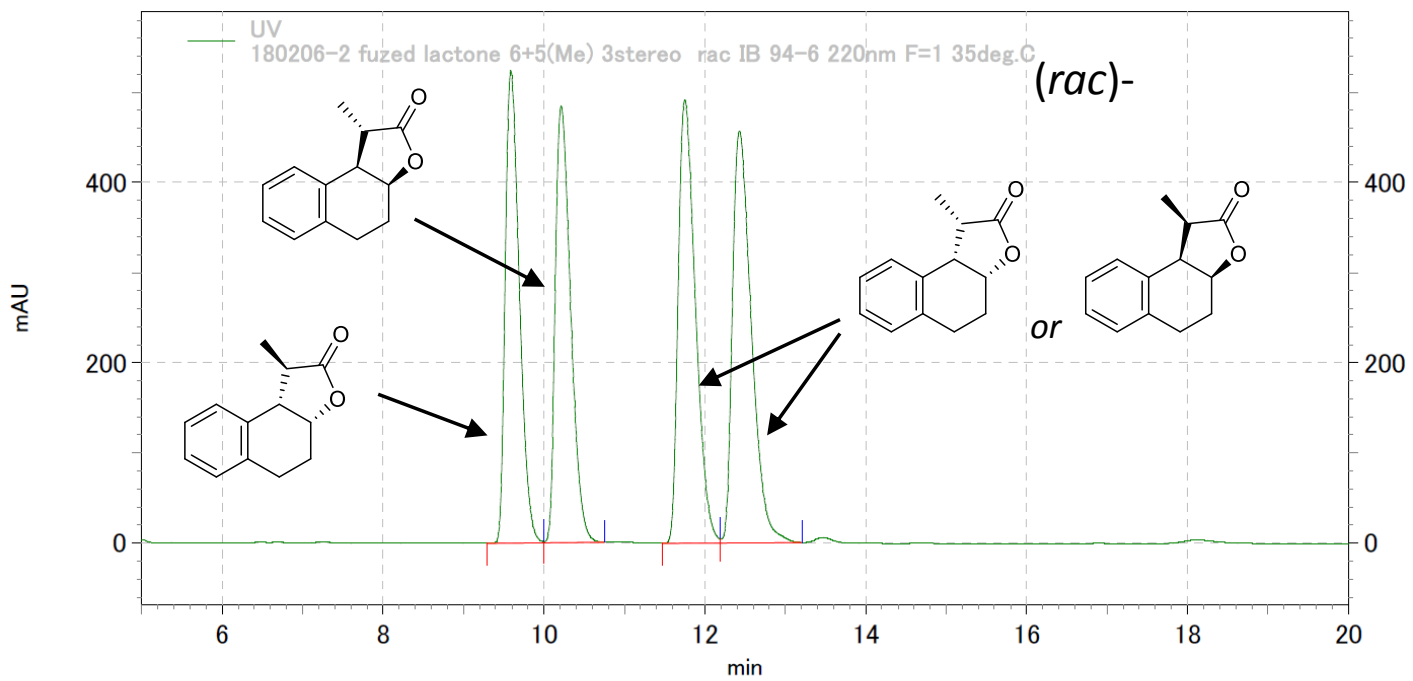

180206-2 fuzed lactone $6+5(\mathrm{Me}) 3$ stereo rac IB $94-6220 \mathrm{~nm} F=135 \mathrm{deg}$. C

\begin{tabular}{rrllll}
$\begin{array}{r}\text { Pk } \\
\text { No. }\end{array}$ & R. T. & Area & Area\% & B. C. & Symmetry \\
\hline 1 & 9.587 & 25630357 & 23.181 & Mx & 0.00000 \\
2 & 10.213 & 25701887 & 23.246 & XM & 0.00000 \\
3 & 11.750 & 29263880 & 26.468 & Mx & 0.00000 \\
4 & 12.430 & 29969183 & 27.105 & xM & 0.00000
\end{tabular}

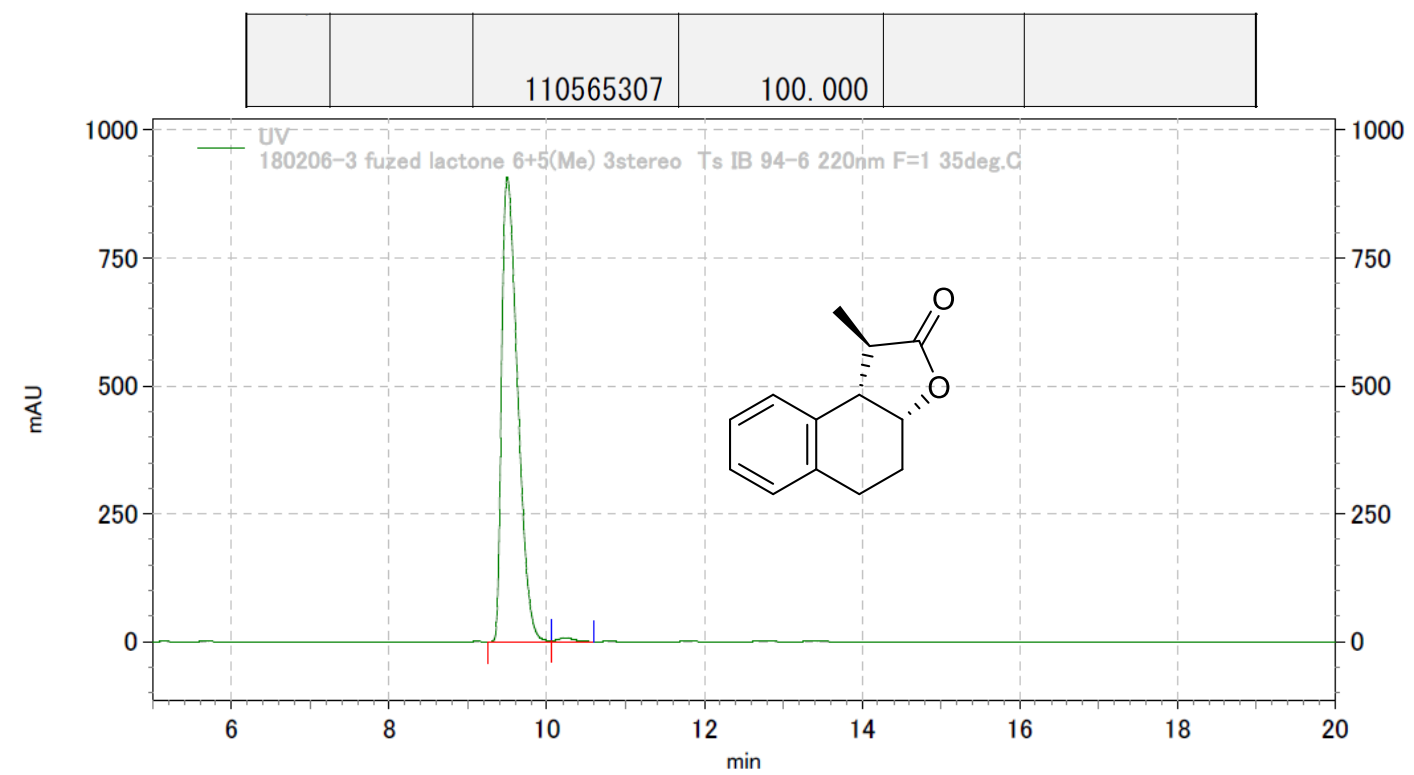

180206-3 fuzed lactone 6+5(Me) 3stereo Ts IB 94-6 220nm F=1 35deg. C

\begin{tabular}{rrrrrr}
$\begin{array}{r}\text { Pk } \\
\text { No. }\end{array}$ & \multicolumn{1}{l}{ R. T. } & \multicolumn{1}{l}{ Area } & \multicolumn{1}{l}{ Area\% } & B. C. & Symmetry \\
\hline 1 & 9.500 & 51135961 & 99.218 & $\mathrm{Mx}$ & 0.00000 \\
2 & 10.240 & 403061 & 0.782 & xM & 0.00000 \\
\hline & & & & & \\
\hline & & 51539022 & 100.000 & \\
\hline
\end{tabular}


$(1 R, 3 a R, 9 b S)-1-E t h y l-3 a, 4,5,9 b-t e t r a h y d r o n a p h t h o[2,1-b] f u r a n-2(1 H)-o n e ~(15 b)$

Chiralcel OZ-3, $250 \times 4.6 \mathrm{~mm}$ column, hexane/2-propanol 97:3, $1.0 \mathrm{~mL} / \mathrm{min}, 220 \mathrm{~nm}, 35^{\circ} \mathrm{C}$

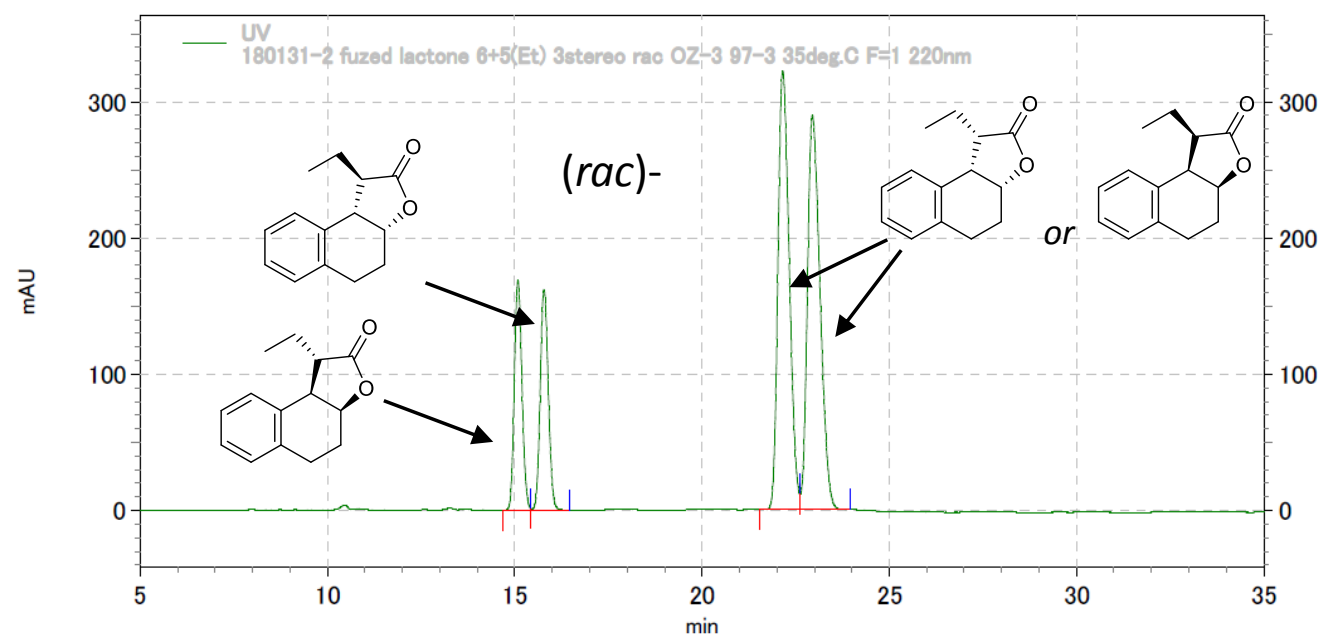

180131-2 fuzed lactone 6+5(Et) 3stereo rac 0Z-3 97-3 35deg. C F=1 220nm

\begin{tabular}{|c|c|c|c|c|c|}
\hline Pk & R. T. & Area & Area\% & B. C. & Symmetry \\
\hline $\begin{array}{l}1 \\
2\end{array}$ & $\begin{array}{l}15.087 \\
15.783 \\
22.157 \\
22.950\end{array}$ & $\begin{array}{r}9521975 \\
9669986 \\
26964067 \\
27210540\end{array}$ & $\begin{array}{l}12.979 \\
13.180 \\
36.753 \\
37.088\end{array}$ & $\begin{array}{l}M x \\
x M \\
M x \\
x M\end{array}$ & $\begin{array}{l}.00000 \\
0.00000 \\
0.00000 \\
0.00000\end{array}$ \\
\hline
\end{tabular}
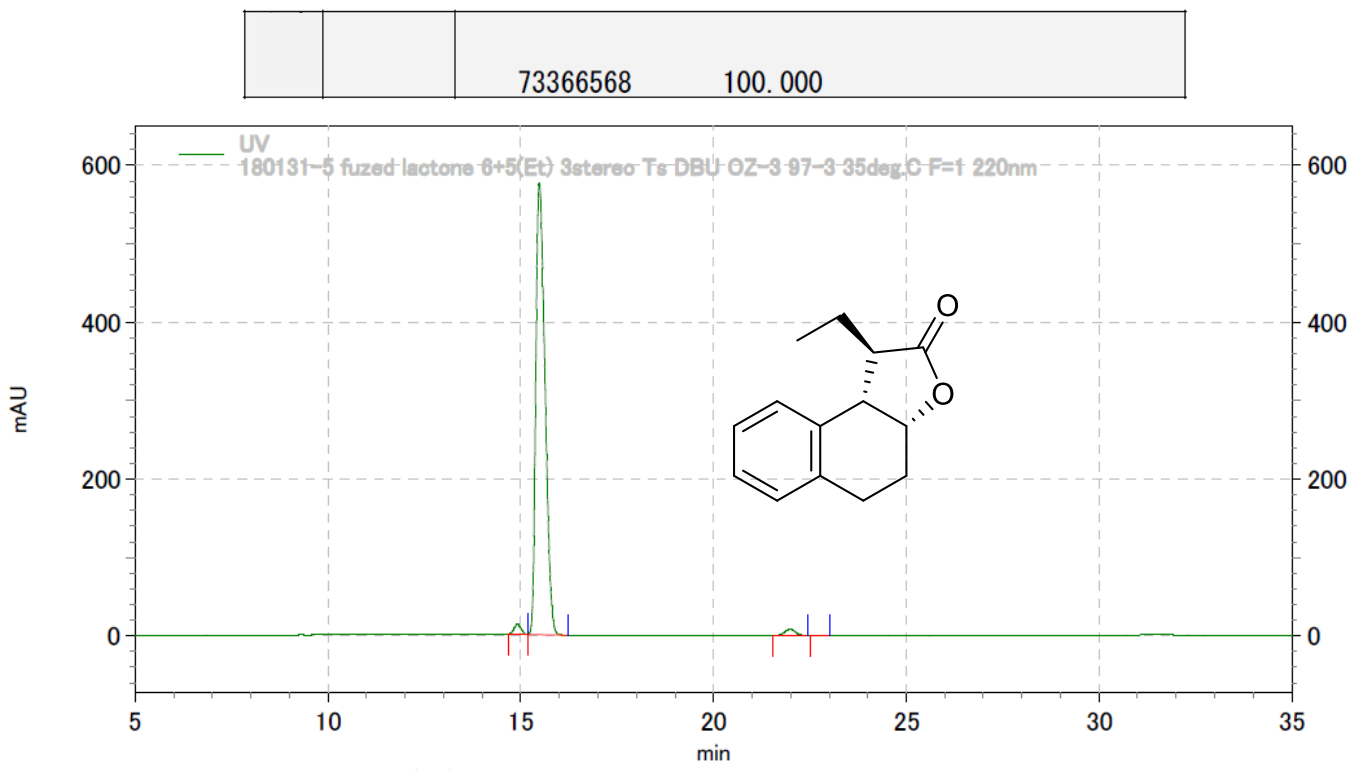

180131-5 fuzed lactone 6+5(Et) 3stereo Ts DBU 0Z-3 97-3 35deg. C F=1 220nm

\begin{tabular}{rlrrrr}
$\begin{array}{r}\text { Pk } \\
\text { No. }\end{array}$ & R. T. & \multicolumn{1}{c}{ Area } & \multicolumn{1}{c}{ Area\% } & B.C. & Symmetry \\
\hline 1 & 14.920 & 651821 & 1.645 & MM & 0.00000 \\
2 & 15.487 & 3831471 & 96.700 & MM & 0.00000 \\
3 & 21.983 & 648557 & 1.637 & MM & 0.00000 \\
4 & 22.830 & 7190 & 0.018 & MM & 0.00000 \\
\hline & & & & & \\
\hline & & 39622339 & 100.000 & \\
\hline
\end{tabular}




\section{$(1 R, 3 \mathrm{a} R, 9 \mathrm{bS})-1-B e n z y l-3 \mathrm{a}, 4,5,9 \mathrm{~b}-t e t r a h y d r o n a p h t h o[2,1-\mathrm{b}]$ furan-2(1H)-one $(15 \mathrm{c})$}

YMC Alcyon SFC CPS Cellulose-SC, $250 \times 4.6 \mathrm{~mm}$ column, hexane $/ 2$-propanol 94:6, $1.0 \mathrm{~mL} / \mathrm{min}$, $220 \mathrm{~nm}, 35^{\circ} \mathrm{C}$

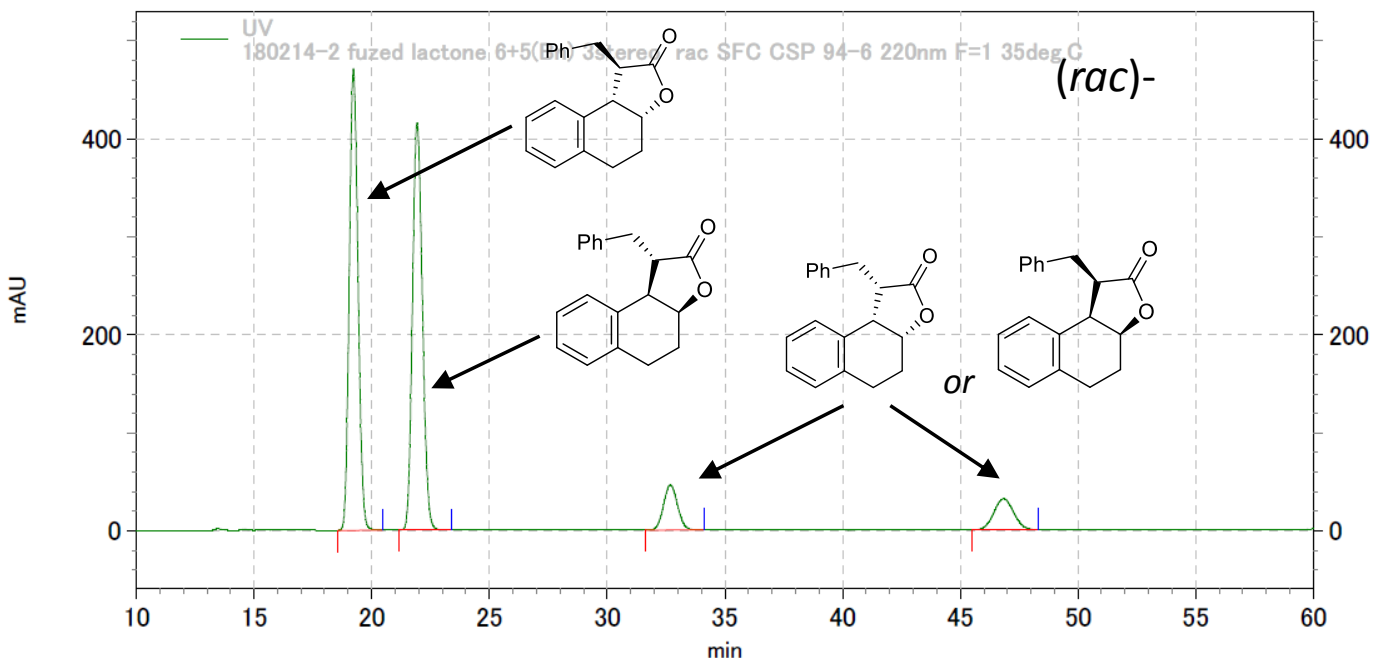

180214-2 fuzed lactone 6+5(Bn) 3stereo rac SFC CSP 94-6 220nm F=1 35deg. C

\begin{tabular}{rlrrrl}
$\begin{array}{r}\text { Pk } \\
\text { No. }\end{array}$ & R. T. & \multicolumn{1}{l}{ Area } & \multicolumn{1}{l}{ Area\% } & B. C. & Symmetry \\
\hline 1 & 19.220 & 48648578 & 43.214 & MM & 0.00000 \\
2 & 21.927 & 48822280 & 43.368 & MM & 0.00000 \\
3 & 32.683 & 7651027 & 6.796 & MM & 0.00000 \\
4 & 46.840 & 7453717 & 6.621 & MM & 0.00000
\end{tabular}

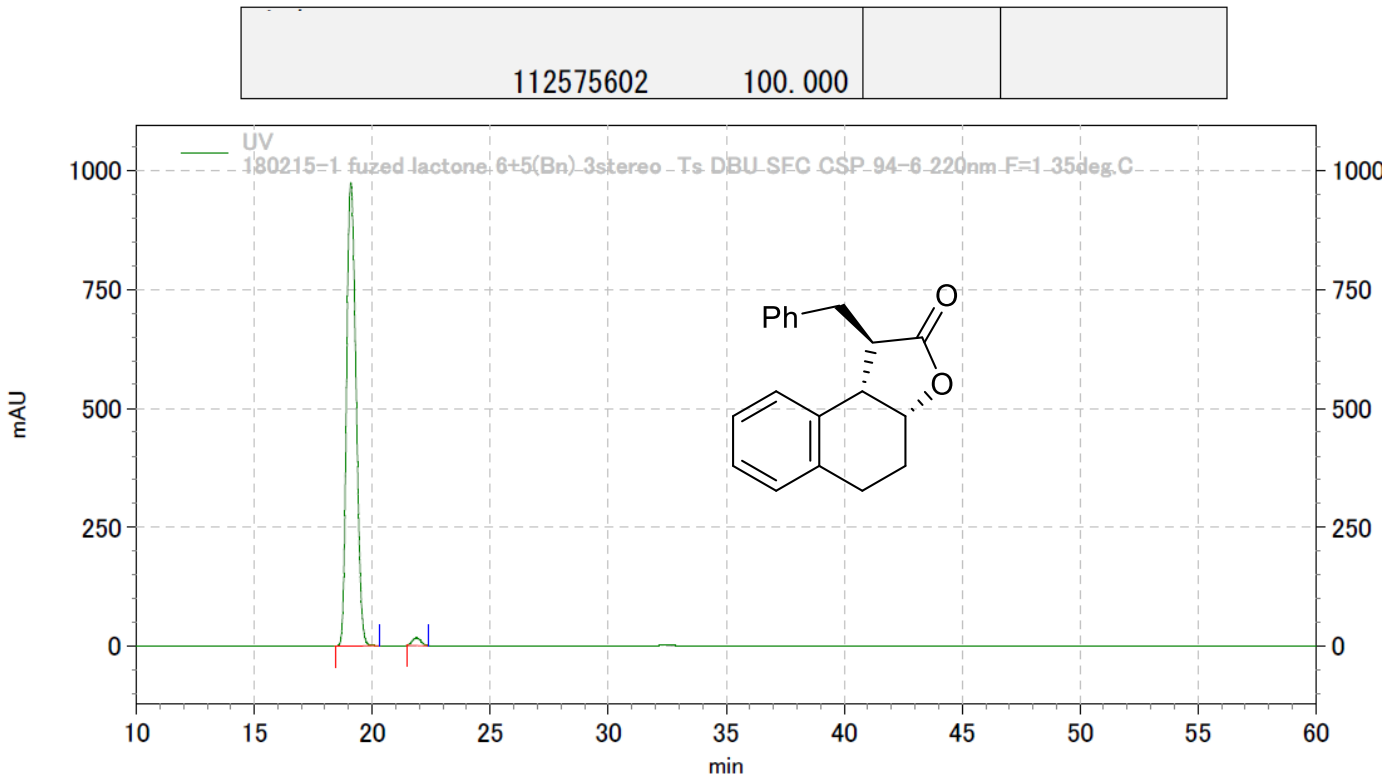

180215-1 fuzed lactone 6+5(Bn) 3stereo Ts DBU SFC CSP 94-6 220nm F=1 35deg. C

\begin{tabular}{rlrrrr}
$\begin{aligned} \text { Pk } \\
\text { No. }\end{aligned}$ & R. T. & \multicolumn{1}{c}{ Area } & \multicolumn{1}{c}{ Area\% } & B. C. & Symmetry \\
\hline 1 & 19.090 & 108371856 & 98.443 & MM & 0.00000 \\
2 & 21.873 & 1713696 & 1.557 & MM & 0.00000 \\
\hline & & & & & \\
\hline & & 110085552 & 100.000 & & \\
\hline
\end{tabular}


Chiralpak AD-H, $250 \times 4.6 \mathrm{~mm}$ column, hexane/2-propanol 94:6, $1.0 \mathrm{~mL} / \mathrm{min}, 220 \mathrm{~nm}, 35^{\circ} \mathrm{C}$

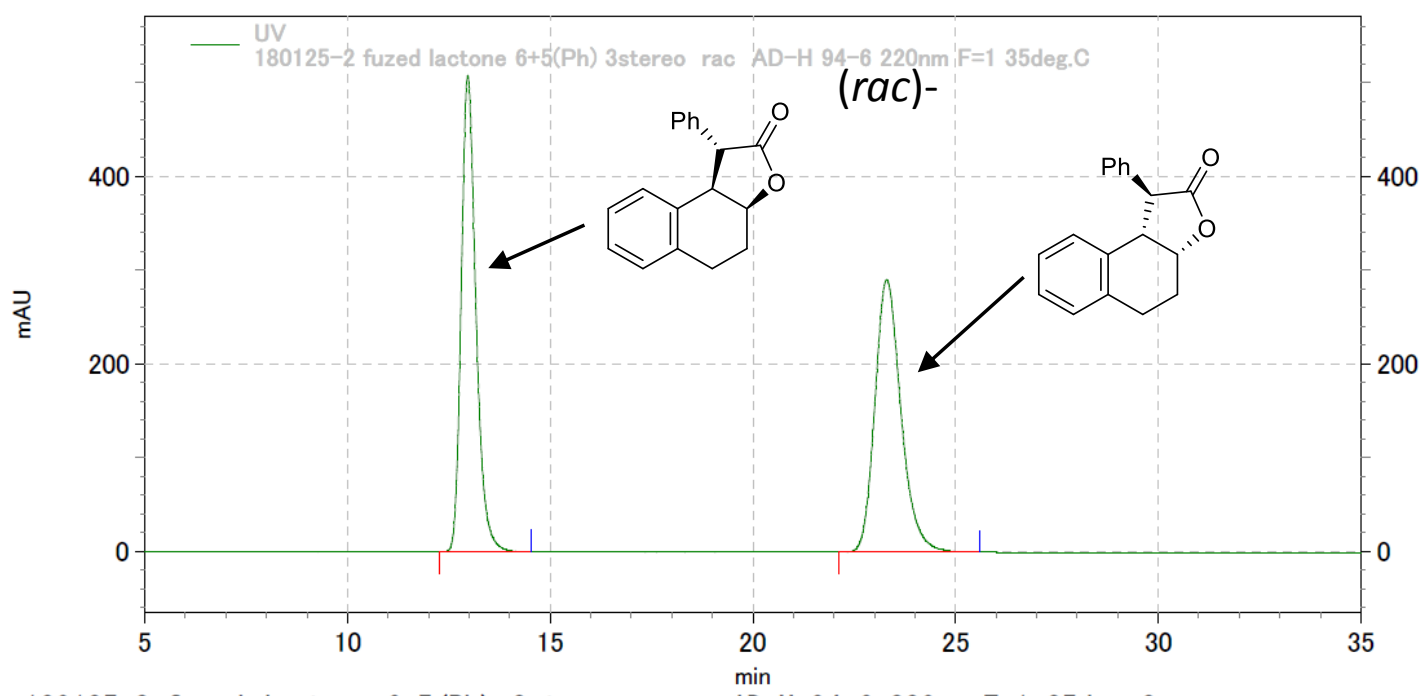

180125-2 fuzed lactone 6+5(Ph) 3stereo rac AD-H 94-6 220nm F=1 35deg. C

\begin{tabular}{rlllll}
$\begin{array}{l}\text { Pk } \\
\text { No. }\end{array}$ & R. T. & Area & Area\% & B. C. & Symmetry \\
\hline 1 & 12.967 & 51410872 & 49.628 & MM & 0.00000 \\
2 & 23.303 & 52182296 & 50.372 & MM & 0.00000
\end{tabular}

\begin{tabular}{ll|l}
103593168 & 100.000 & \\
\hline
\end{tabular}

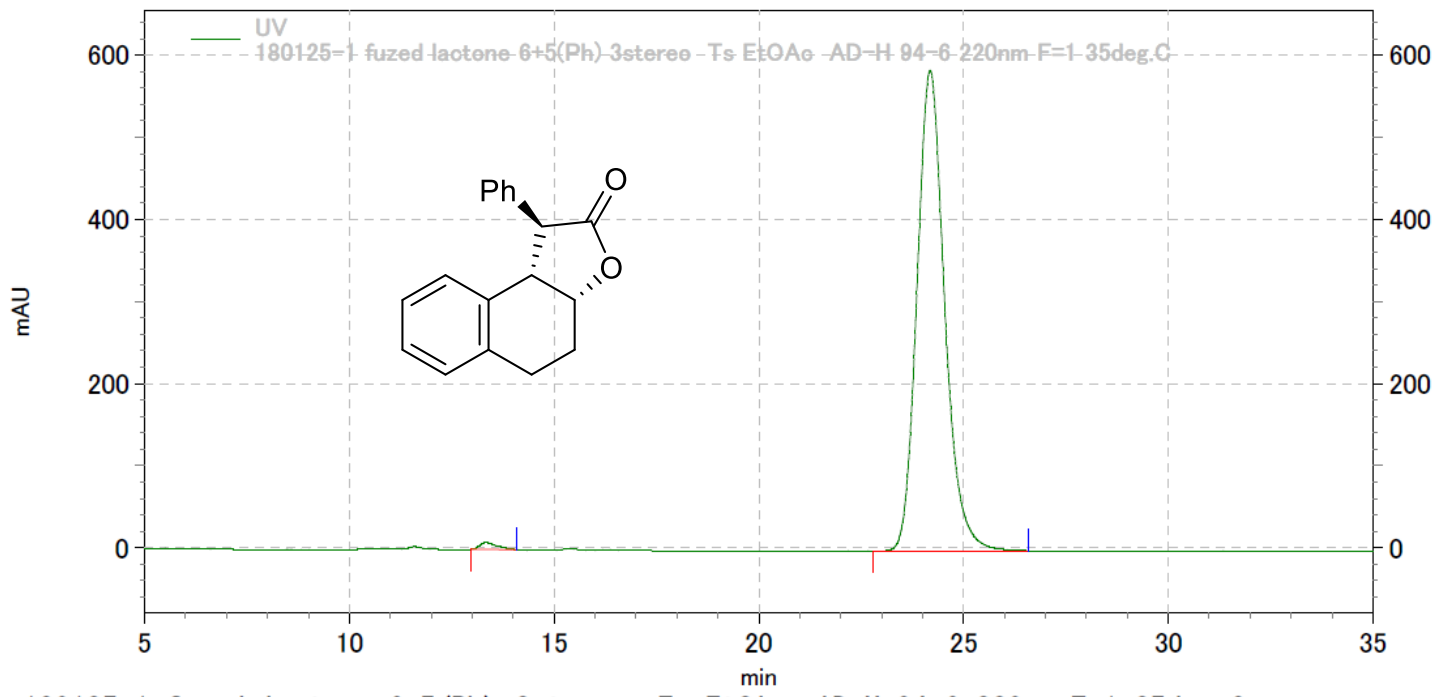

180125-1 fuzed lactone 6+5(Ph) 3stereo Ts EtOAc AD-H 94-6 220nm F=1 35deg. C

\begin{tabular}{rrrrrr}
$\begin{aligned} \text { Pk } \\
\text { No. }\end{aligned}$ & R. T. & \multicolumn{1}{c}{ Area } & \multicolumn{1}{l}{ Area\% } & \multicolumn{1}{c}{ B. C. } & Symmetry \\
\hline 1 & 13.343 & 1018056 & 0.919 & MM & 0.00000 \\
2 & 24.187 & 109751993 & 99.081 & MM & 0.00000 \\
\hline & & & & & \\
\hline & & & & & \\
\hline
\end{tabular}



(15e)

Chiralpak AD-H, $250 \times 4.6 \mathrm{~mm}$ column, hexane/2-propanol 94:6, $1.0 \mathrm{~mL} / \mathrm{min}, 220 \mathrm{~nm}, 35^{\circ} \mathrm{C}$

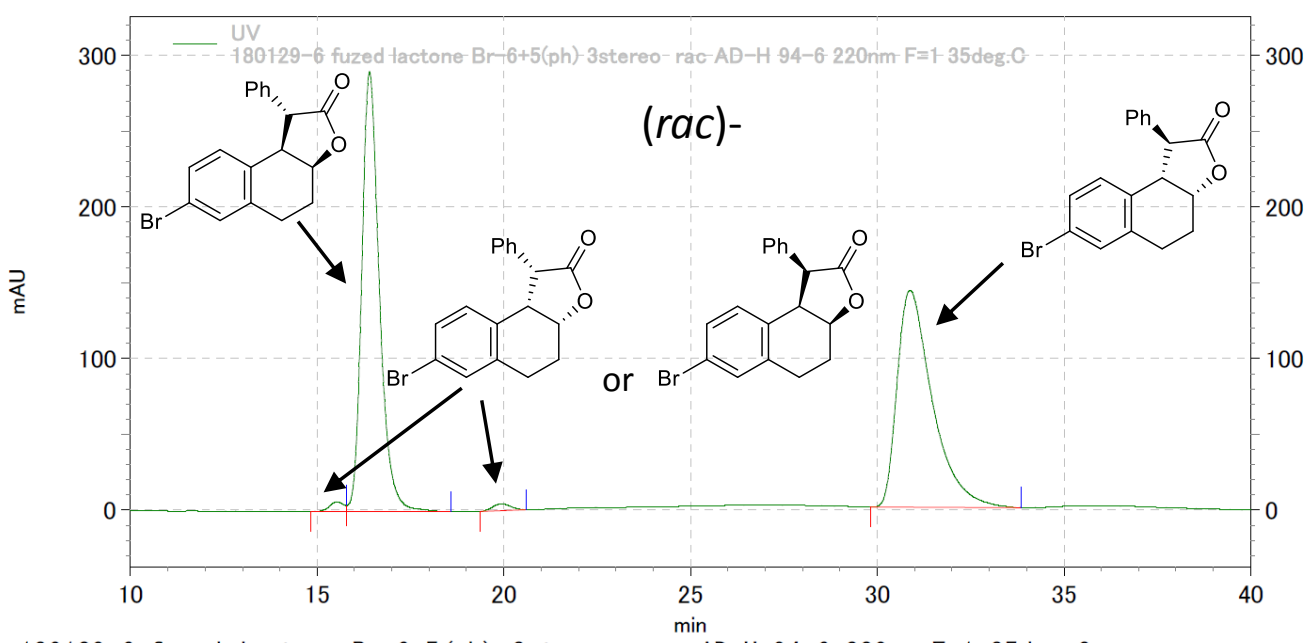

180129-6 fuzed lactone $\mathrm{Br}-6+5$ (ph) 3stereo rac AD-H 94-6 220nm F=1 35deg. C

\begin{tabular}{rlrrrr}
$\begin{array}{c}\text { Pk } \\
\text { No. }\end{array}$ & R. T. & \multicolumn{1}{c}{ Area } & Area\% & B. C. & Symmetry \\
\hline 1 & 15.530 & 653305 & 0.851 & Mx & 0.00000 \\
2 & 16.403 & 38044027 & 49.542 & xM & 0.00000 \\
3 & 19.943 & 601760 & 0.784 & MM & 0.00000 \\
4 & 30.880 & 37492912 & 48.824 & MM & 0.00000
\end{tabular}

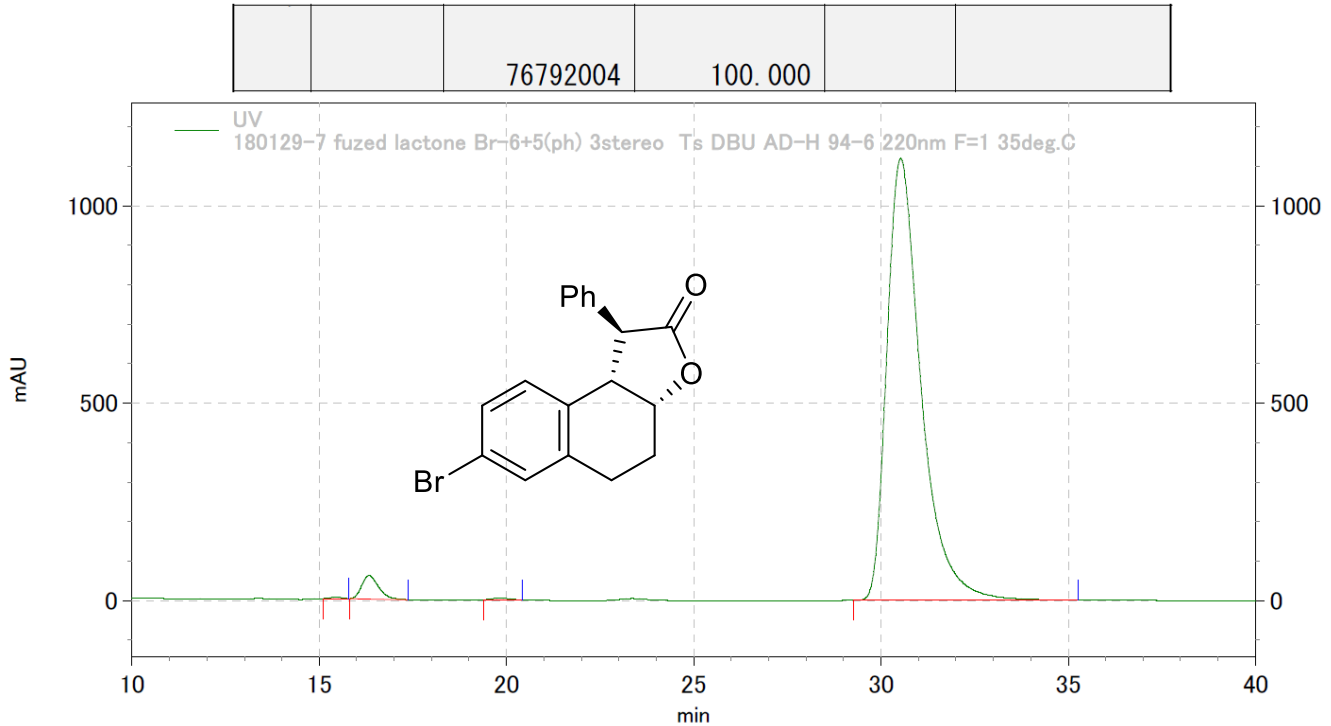

180129-7 fuzed lactone $\mathrm{Br}-6+5$ (ph) 3stereo Ts DBU AD-H 94-6 220nm F=1 35deg. C

\begin{tabular}{rlrrrr}
$\begin{array}{r}\text { Pk } \\
\text { No. }\end{array}$ & R. T. & \multicolumn{1}{c}{ Area } & \multicolumn{1}{l}{ Area\% } & B. C. & Symmetry \\
\hline 1 & 15.450 & 336224 & 0.115 & $\mathrm{MM}$ & 0.00000 \\
2 & 16.330 & 7487598 & 2.565 & $\mathrm{MM}$ & 0.00000 \\
3 & 19.830 & 576944 & 0.198 & $\mathrm{MM}$ & 0.00000 \\
4 & 30.527 & 283457936 & 97.122 & $\mathrm{MM}$ & 0.00000 \\
\hline & & & & & \\
\hline
\end{tabular}



(15f)

Chiralpak AD-H, $250 \times 4.6 \mathrm{~mm}$ column, hexane/2-propanol 94:6, $1.0 \mathrm{~mL} / \mathrm{min}, 220 \mathrm{~nm}, 35^{\circ} \mathrm{C}$

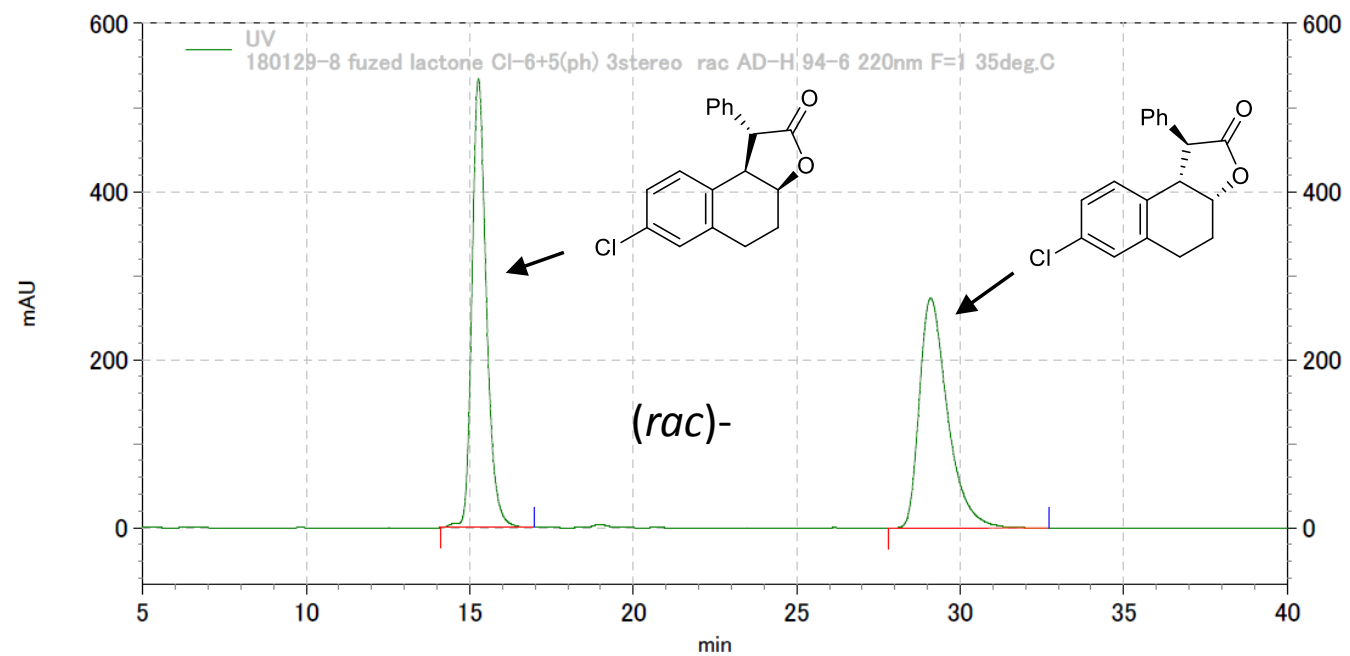

180129-8 fuzed lactone $\mathrm{Cl}-6+5$ (ph) 3stereo rac AD-H 94-6 220nm F=1 35deg. C

\begin{tabular}{rlllll}
$\begin{array}{l}\text { Pk } \\
\text { No. }\end{array}$ & R. T. & Area & Area\% & B.C. & Symmetry \\
\hline 1 & 15.267 & 65469665 & 49.909 & MM & 0.00000 \\
2 & 29.097 & 65709287 & 50.091 & MM & 0.00000
\end{tabular}

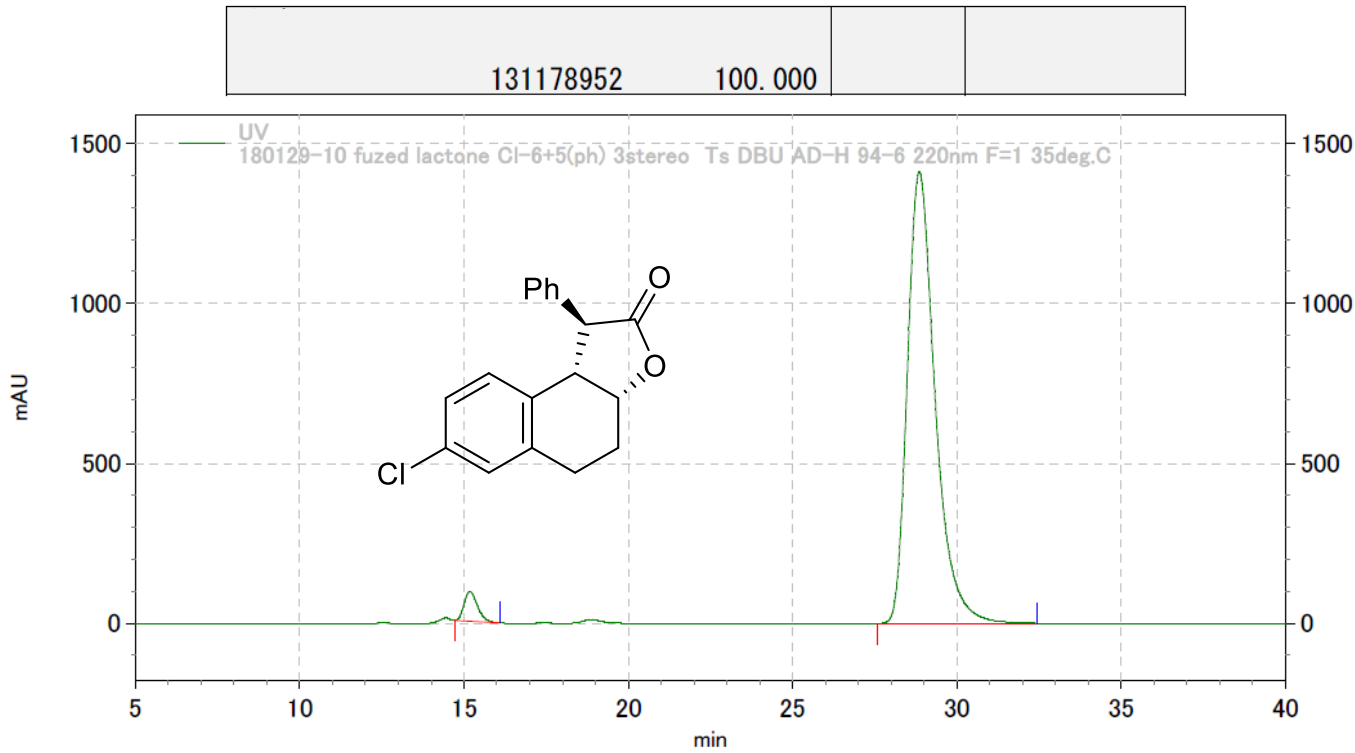

180129-10 fuzed Iactone $\mathrm{Cl}-6+5$ (ph) 3stereo Ts DBU AD-H 94-6 220nm F=1 35deg. C

\begin{tabular}{rlrrrr}
$\begin{array}{r}\text { Pk } \\
\text { No. }\end{array}$ & R. T. & \multicolumn{1}{c}{ Area } & Area\% & B. C. & Symmetry \\
\hline 1 & 15.180 & 10580113 & 3.095 & MM & 0.00000 \\
2 & 28.860 & 331310853 & 96.905 & MM & 0.00000 \\
\hline & & & & & \\
& & 341890966 & 100.000 & \\
\hline
\end{tabular}



e (15g)

Chiralpak AD-H, $250 \times 4.6 \mathrm{~mm}$ column, hexane/2-propanol 94:6, $1.0 \mathrm{~mL} / \mathrm{min}, 220 \mathrm{~nm}, 35^{\circ} \mathrm{C}$

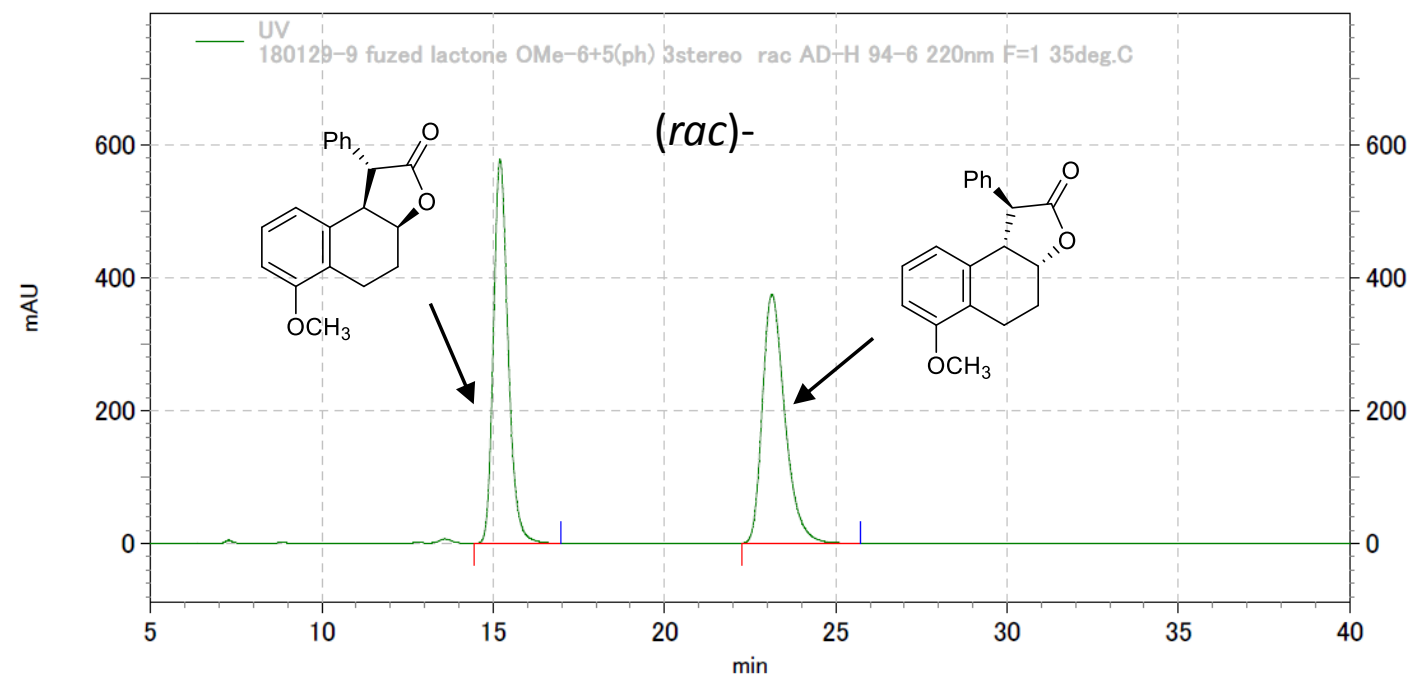

180129-9 fuzed lactone $0 M \mathrm{Me}-6+5(\mathrm{ph}) 3$ stereo rac $\mathrm{AD}-\mathrm{H}$ 94-6 220nm F=1 35deg. C

\begin{tabular}{rlll|l|l}
$\begin{array}{r}\text { Pk } \\
\text { No. }\end{array}$ & R. T. & Area & Area\% & B. C. & Symmetry \\
\hline 1 & 15.203 & 68803801 & 50.012 & MM & 0.00000 \\
2 & 23.137 & 68770973 & 49.988 & MM & 0.00000 \\
\hline & & & & & \\
& & & & & \\
\hline
\end{tabular}

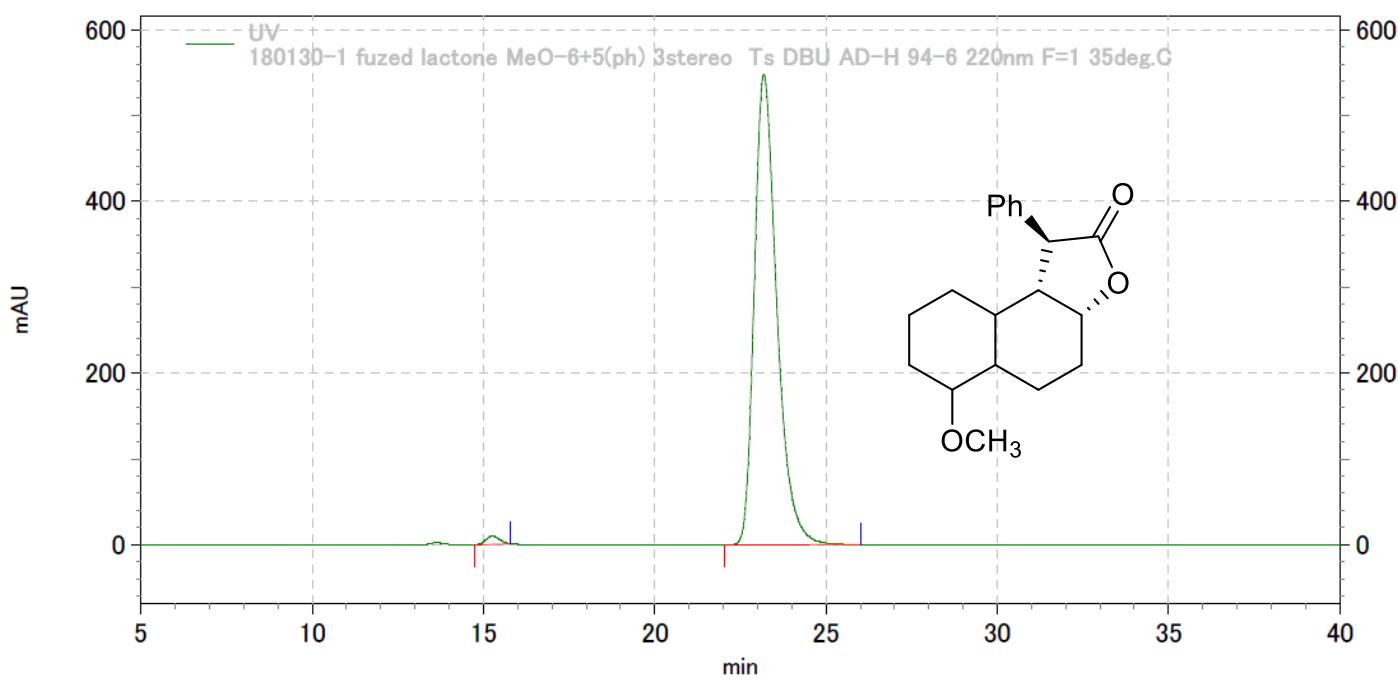

180130-1 fuzed lactone Me0-6+5(ph) 3stereo Ts DBU AD-H 94-6 220nm F=1 35deg. C

\begin{tabular}{rrrrrr}
$\begin{array}{r}\text { Pk } \\
\text { No. }\end{array}$ & R. T. & \multicolumn{1}{c}{ Area } & Area\% & \multicolumn{1}{l}{ B. C. } & Symmetry \\
\hline 1 & 15.263 & 1051630 & 1.038 & MM & 0.00000 \\
2 & 23.187 & 100218148 & 98.962 & MM & 0.00000 \\
\hline & & & & \\
& & & & & \\
\hline
\end{tabular}



(15h)

Chiralcel OD-H, $250 \times 4.6 \mathrm{~mm}$ column, hexane/2-propanol 94:6, $1.0 \mathrm{~mL} / \mathrm{min}, 220 \mathrm{~nm}, 35^{\circ} \mathrm{C}$

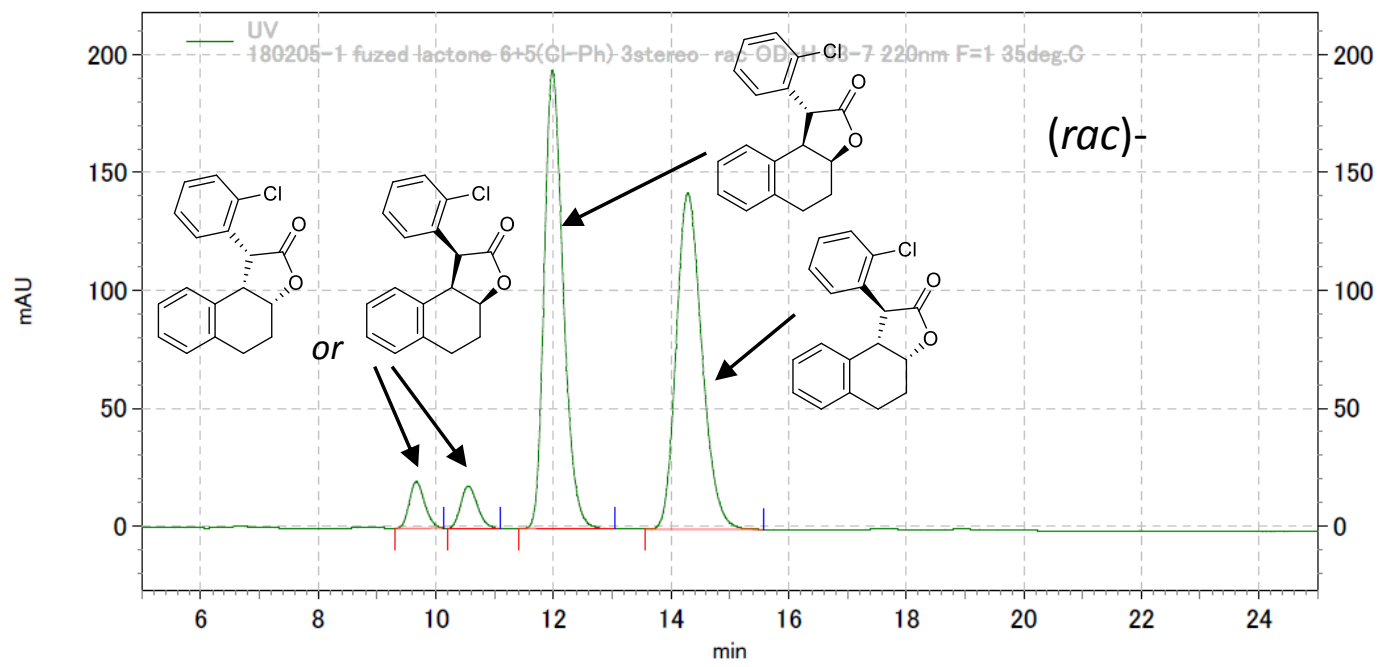

180205-1 fuzed lactone 6+5(Cl-Ph) 3stereo rac 0D-H 93-7 220nm F=1 35deg. C

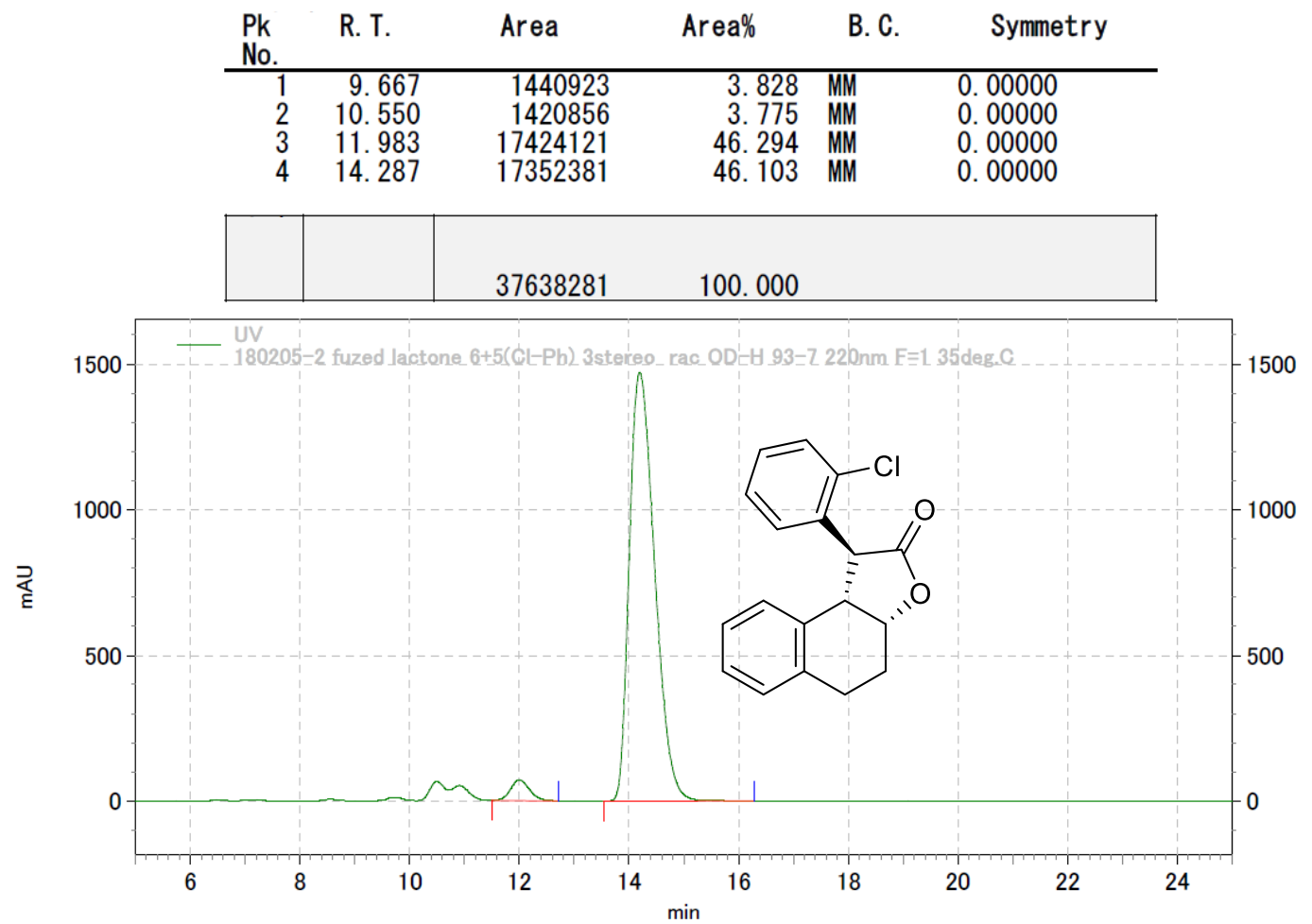

180205-2 fuzed lactone 6+5(Cl-Ph) 3stereo rac OD-H 93-7 220nm F=1 35deg. C

\begin{tabular}{|c|c|c|c|c|c|}
\hline $\begin{array}{l}\mathrm{Pk} \\
\text { No. }\end{array}$ & R. T. & Area & Area\% & B. C. & Symmetry \\
\hline $\begin{array}{l}1 \\
2\end{array}$ & $\begin{array}{l}12.000 \\
14.197\end{array}$ & $\begin{array}{r}6959168 \\
188667395\end{array}$ & $\begin{array}{r}3.557 \\
96.443\end{array}$ & $\begin{array}{l}\text { MM } \\
\text { MM }\end{array}$ & $\begin{array}{l}0.00000 \\
0.00000\end{array}$ \\
\hline & & 195626563 & 100.000 & & \\
\hline
\end{tabular}


$(1 R, 3 a S, 9 b R)-8-M e t h o x y-1-p h e n y l-3 a, 4,5,9 b$-tetrahydronaphtho[2,1-b]furan-2(1H)-on e (7)

Chiralcel OZ-3, $250 \times 4.6 \mathrm{~mm}$ column, hexane/2-propanol 95:5, $1.0 \mathrm{~mL} / \mathrm{min}, 220 \mathrm{~nm}, 35^{\circ} \mathrm{C}$

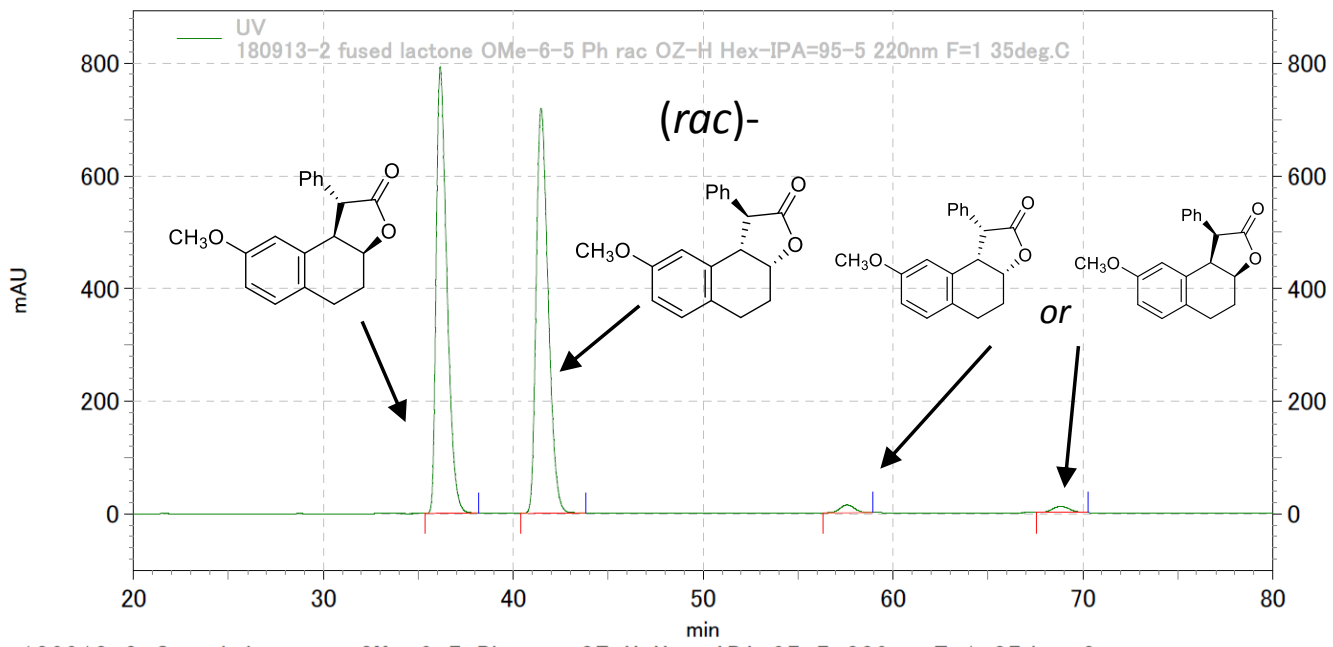

180913-2 fused lactone OMe-6-5 Ph rac 0Z-H Hex-IPA=95-5 220nm F=1 35deg. C

\begin{tabular}{rlrrrr} 
Pk & R. T. & \multicolumn{1}{c}{ Area } & Area\% & B. C. & Symmetry \\
No. & & & & & \\
\hline 1 & 36.150 & 124520545 & 48.537 & MM & 0.00000 \\
2 & 41.467 & 126090007 & 49.149 & MM & 0.00000 \\
3 & 57.590 & 2989451 & 1.165 & MM & 0.00000 \\
4 & 68.850 & 2948039 & 1.149 & MM & 0.00000
\end{tabular}
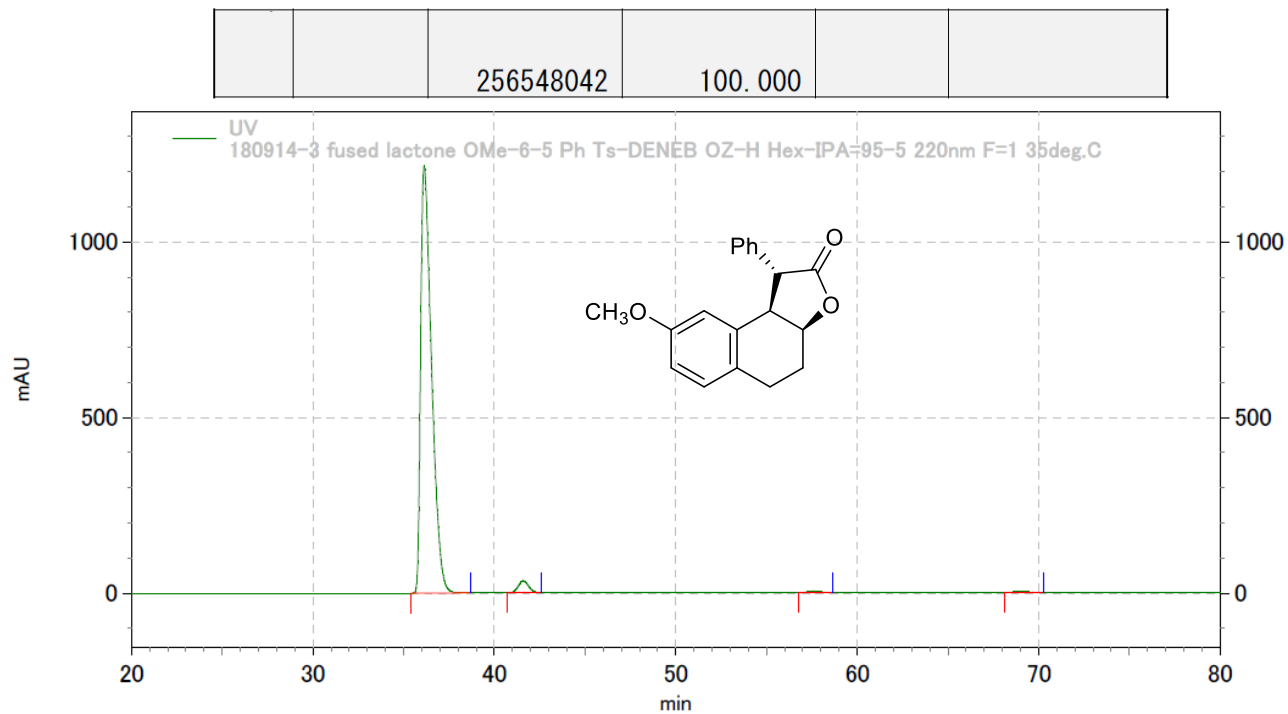

180914-3 fused Iactone 0Me-6-5 Ph Ts-DENEB 0Z-H Hex-IPA=95-5 220nm F=1 35deg. C

\begin{tabular}{rlrrrr}
$\begin{aligned} \text { Pk } \\
\text { No. }\end{aligned}$ & \multicolumn{1}{l}{ R. T. } & \multicolumn{1}{c}{ Area } & \multicolumn{1}{l}{ Area\% } & B.C. & Symmetry \\
\hline 1 & 36.127 & 203422592 & 96.411 & MM & 0.00000 \\
2 & 41.587 & 5523344 & 2.618 & MM & 0.00000 \\
3 & 57.657 & 1039026 & 0.492 & MM & 0.00000 \\
4 & 69.053 & 1010833 & 0.479 & MM & 0.00000 \\
\hline & & & & & \\
\hline & & 210995795 & 100.000 & \\
\hline
\end{tabular}


Chiralcel OZ-3, $250 \times 4.6 \mathrm{~mm}$ column, hexane/2-propanol 95:5, $1.0 \mathrm{~mL} / \mathrm{min}, 215 \mathrm{~nm}, 35^{\circ} \mathrm{C}$

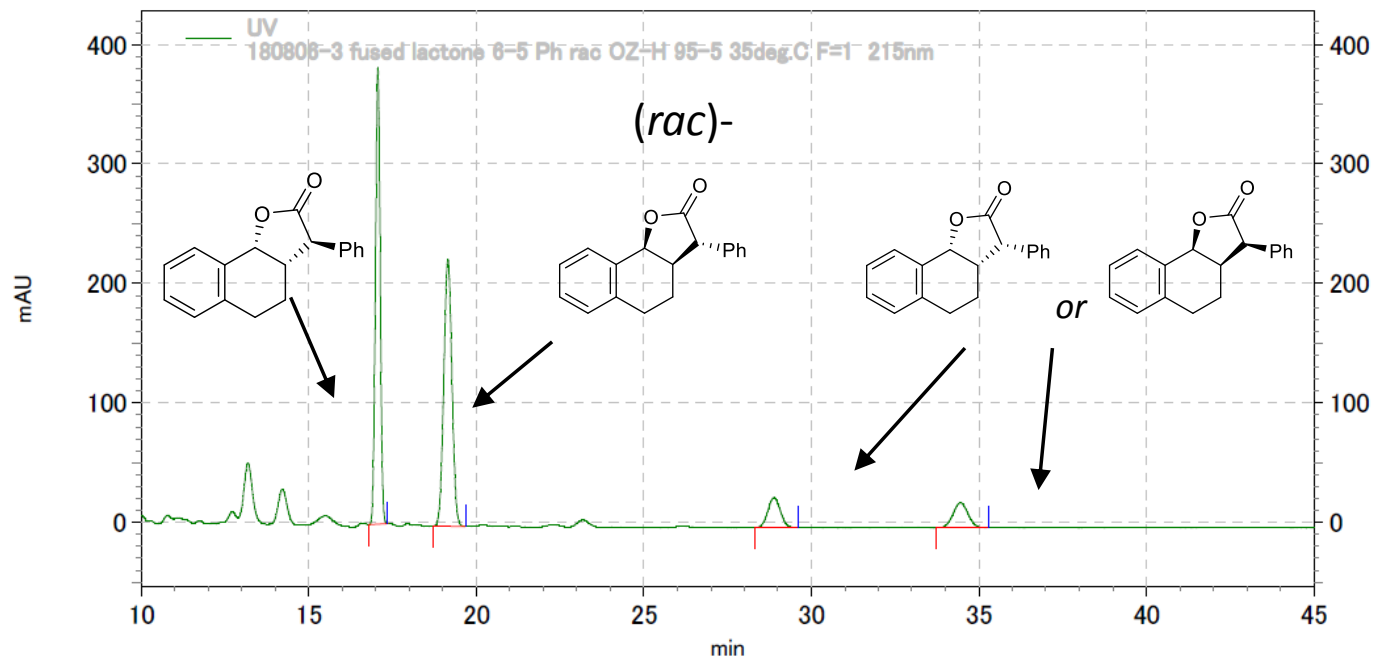

180806-3 fused lactone 6-5 Ph rac 0Z-H 95-5 35deg. C F=1 $215 \mathrm{~nm}$

\begin{tabular}{rlrrrr}
$\begin{array}{c}\text { Pk } \\
\text { No. }\end{array}$ & R. T. & \multicolumn{1}{c}{ Area } & Area\% & B. C. & Symmetry \\
\hline 1 & 17.067 & 15187575 & 42.676 & MM & 0.00000 \\
2 & 19.153 & 15172154 & 42.633 & MM & 0.00000 \\
3 & 28.887 & 2592795 & 7.286 & MM & 0.00000 \\
4 & 34.450 & 2635381 & 7.405 & MM & 0.00000
\end{tabular}
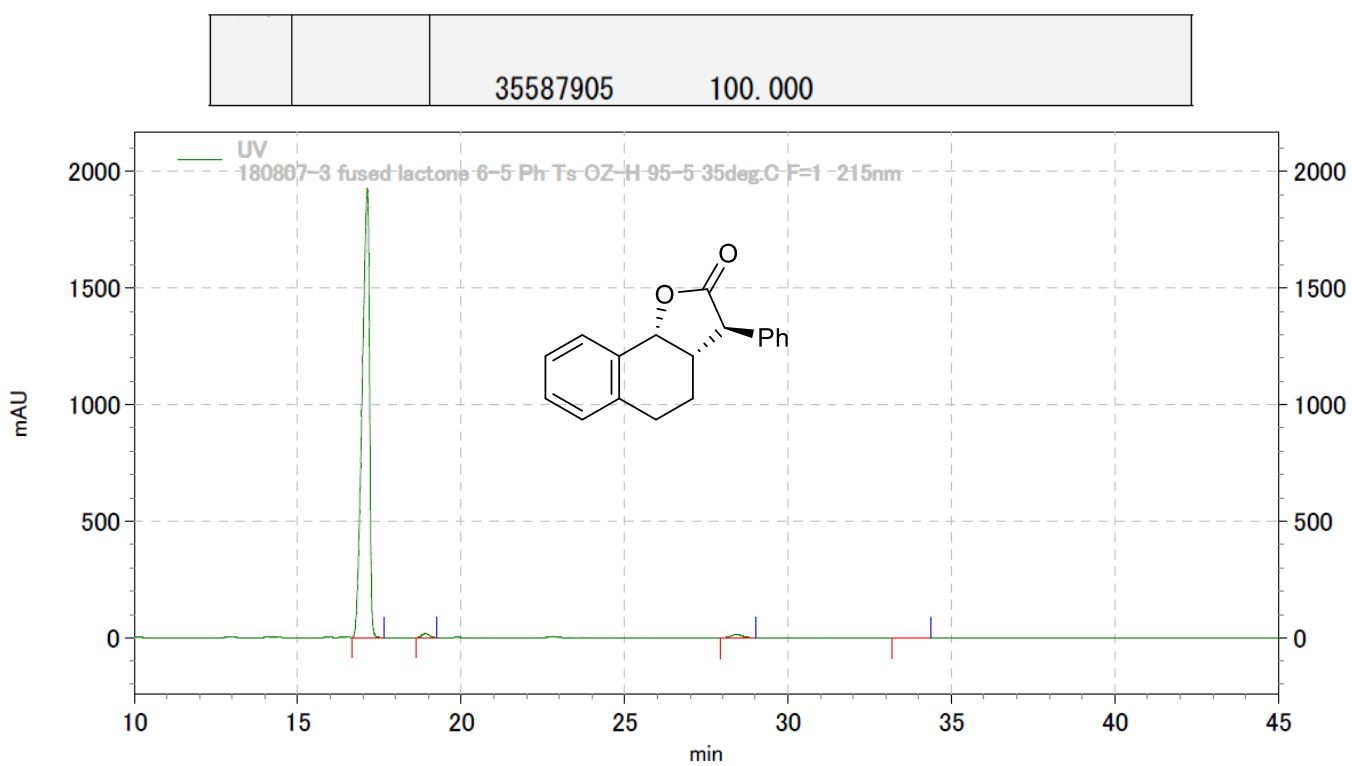

180807-3 fused lactone $6-5 \mathrm{Ph}$ Ts $0 \mathrm{Z}-\mathrm{H}$ 95-5 $35 \mathrm{deg}$. C F=1 $215 \mathrm{~nm}$

\begin{tabular}{rlrrrr}
$\begin{array}{r}\text { Pk } \\
\text { No. }\end{array}$ & R. T. & \multicolumn{1}{c}{ Area } & \multicolumn{1}{l}{ Area\% } & B. C. & Symmetry \\
\hline 1 & 17.133 & 119885147 & 98.082 & MM & 0.00000 \\
2 & 18.913 & 1036221 & 0.848 & MM & 0.00000 \\
3 & 28.427 & 1292687 & 1.058 & MM & 0.00000 \\
4 & 33.937 & 14942 & 0.012 & MM & 0.00000 \\
\hline & & & & & \\
\hline
\end{tabular}


$(3 R, 3 a S, 9 b R)-8-C h l o r o-3-p h e n y l-3 a, 4,5,9 b$-tetrahydronaphtho[1,2-b]furan-2(3H)-one (18b)

Chiralcel AD-H, $250 \times 4.6 \mathrm{~mm}$ column, hexane/2-propanol 95:5, $1.0 \mathrm{~mL} / \mathrm{min}, 220 \mathrm{~nm}, 35^{\circ} \mathrm{C}$

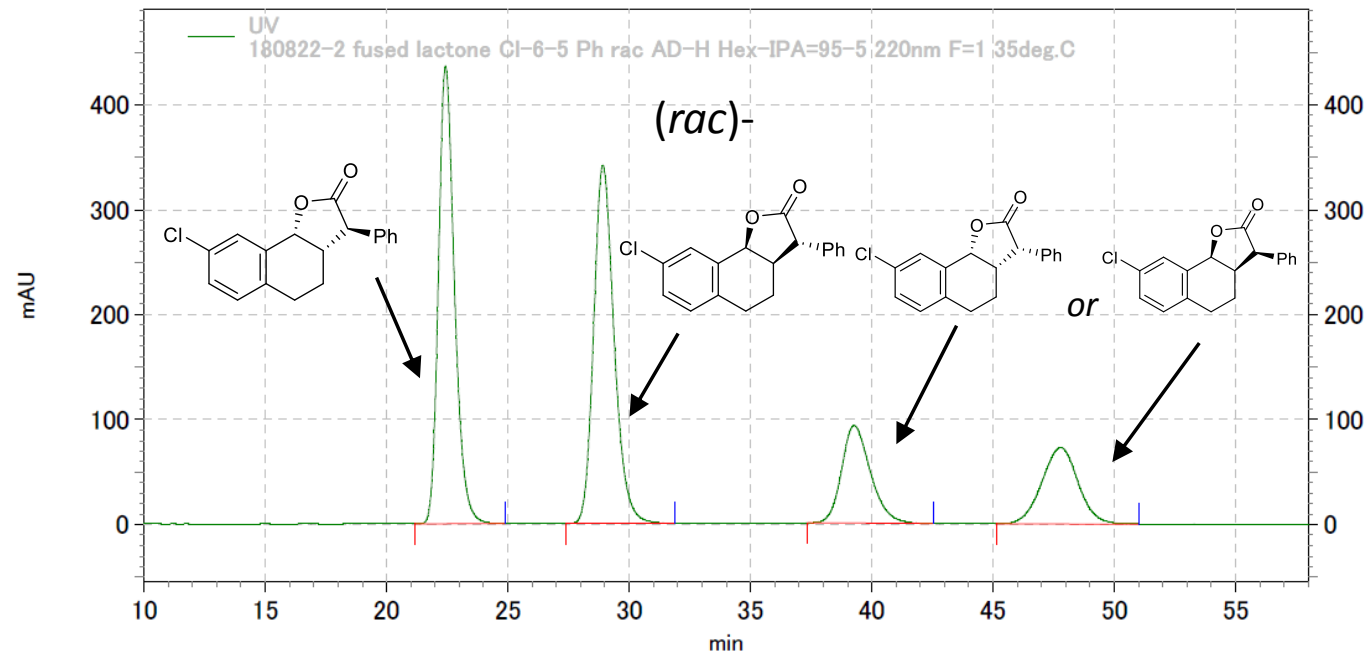

180822-2 fused lactone $\mathrm{Cl}-6-5 \mathrm{Ph}$ rac AD-H Hex-IPA=95-5 220nm F=1 35deg. C

\begin{tabular}{|c|c|c|c|c|c|}
\hline $\begin{array}{l}\mathrm{Pk} \\
\text { No. }\end{array}$ & R. T. & Area & Area\% & B. C. & Symmetry \\
\hline $\begin{array}{l}1 \\
2 \\
3 \\
4\end{array}$ & $\begin{array}{l}22.433 \\
28.920 \\
39.270 \\
47.787\end{array}$ & $\begin{array}{l}80306558 \\
80044806 \\
30863739 \\
30473993\end{array}$ & $\begin{array}{l}36.225 \\
36.107 \\
13.922 \\
13.746\end{array}$ & $\begin{array}{l}\text { MM } \\
\text { MM } \\
\text { MM } \\
\text { MM }\end{array}$ & $\begin{array}{l}0.00000 \\
0.00000 \\
0.00000 \\
0.00000\end{array}$ \\
\hline & & 221689096 & 100.000 & & \\
\hline
\end{tabular}

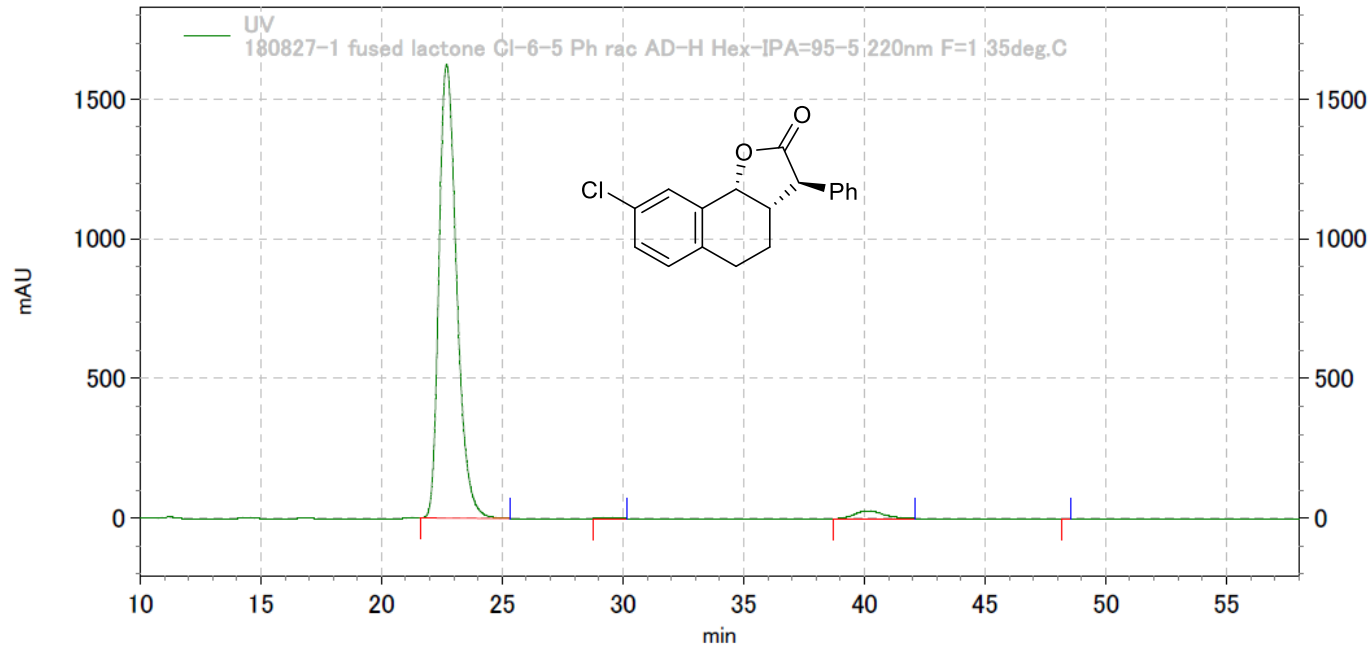

180827-1 fused lactone $\mathrm{Cl}-6-5 \mathrm{Ph}$ rac AD-H Hex-IPA=95-5 220nm F=1 35deg. C

\begin{tabular}{rrrrrr}
$\begin{array}{r}\text { Pk } \\
\text { No. }\end{array}$ & R. T. & \multicolumn{1}{c}{ Area } & \multicolumn{1}{l}{ Area\% } & B. C. & Symmetry \\
\hline 1 & 22.697 & 330617267 & 97.199 & MM & 0.00000 \\
2 & 29.363 & 545511 & 0.160 & MM & 0.00000 \\
3 & 40.137 & 8979896 & 2.640 & MM & 0.00000 \\
4 & 48.483 & 803 & 0.000 & MM & 0.00000 \\
\hline & & & & & \\
\hline & & 340143477 & 100.000 & \\
\hline
\end{tabular}


Chiralcel OJ-3, $250 \times 4.6 \mathrm{~mm}$ column, hexane/2-propanol 95:5, $1.0 \mathrm{~mL} / \mathrm{min}, 220 \mathrm{~nm}, 35^{\circ} \mathrm{C}$

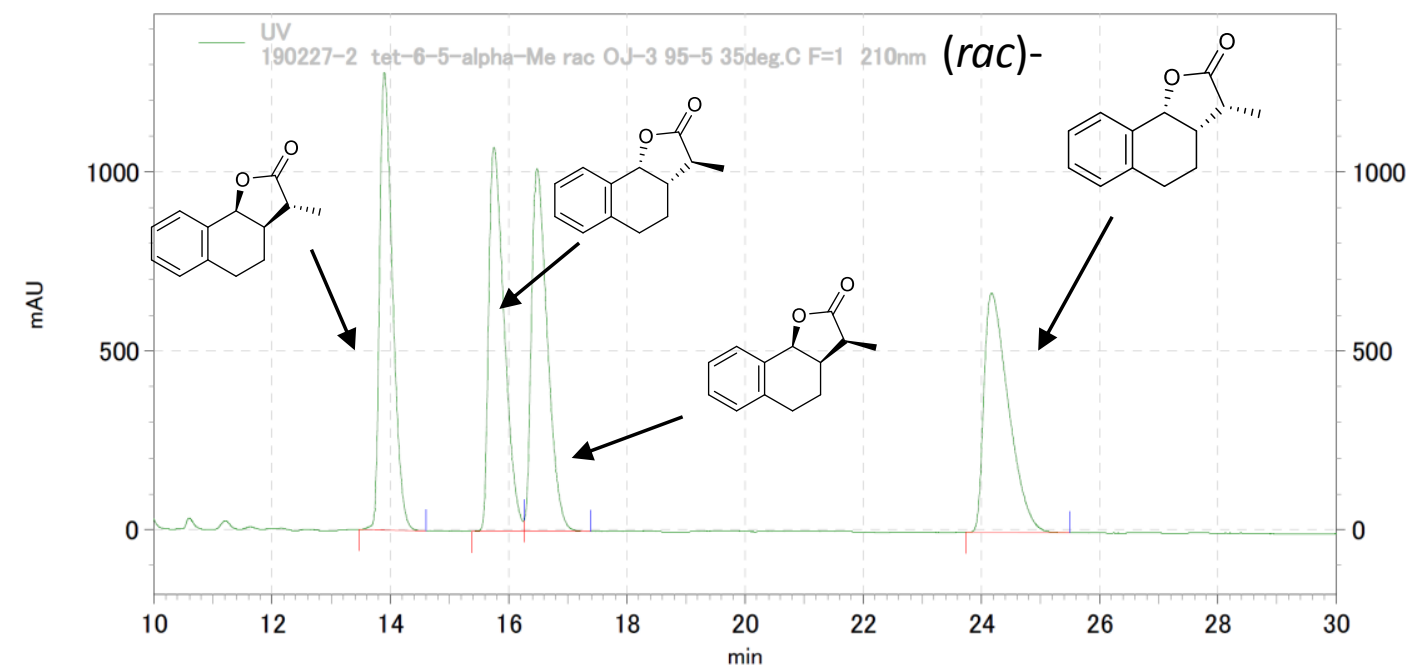

190227-2 tet-6-5-alpha-Me rac 0J-3 95-5 35deg. C F=1 210nm

\begin{tabular}{rlllll}
$\begin{array}{c}\text { Pk } \\
\text { No. }\end{array}$ & R. T. & Area & Area\% & B. C. & Symmetry \\
\hline 1 & 13.900 & 77887511 & 25.145 & MM & 0.00000 \\
2 & 15.753 & 78508109 & 25.346 & MX & 0.00000 \\
3 & 16.483 & 76166227 & 24.590 & XM & 0.00000 \\
4 & 24.170 & 77187682 & 24.919 & MM & 0.00000
\end{tabular}
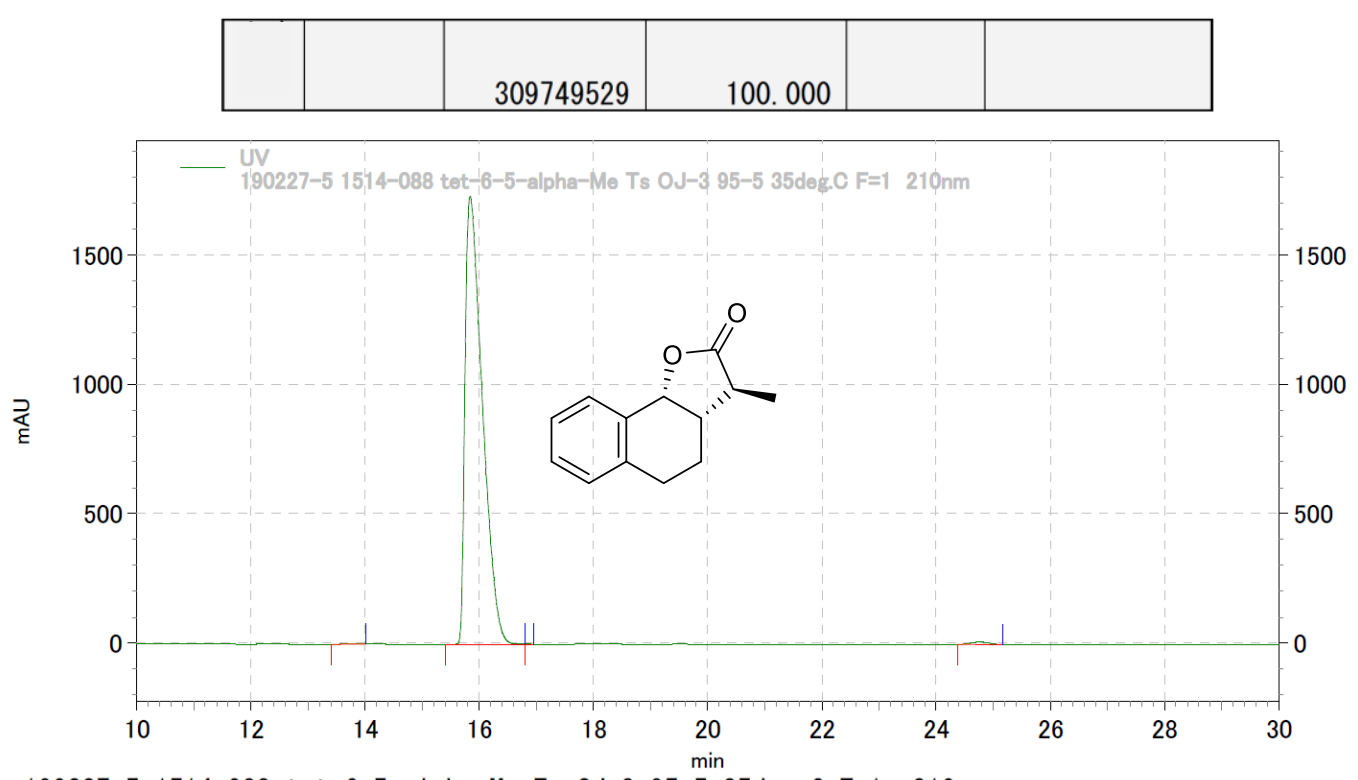

190227-5 1514-088 tet-6-5-alpha-Me Ts $0 J-3$ 95-5 35deg. C F=1 210nm

\begin{tabular}{rlrrrr}
$\begin{array}{r}\text { Pk } \\
\text { No. }\end{array}$ & R. T. & \multicolumn{1}{c}{ Area } & \multicolumn{1}{c}{ Area\% } & B. C. & Symmetry \\
\hline 1 & 13.883 & 39151 & 0.027 & MM & 0.00000 \\
2 & 15.840 & 145716809 & 99.343 & MM & 0.00000 \\
3 & 16.797 & 0 & 0.000 & MM & 0.00000 \\
4 & 24.760 & 924043 & 0.630 & MM & 0.00000 \\
\hline & & & & & \\
\hline
\end{tabular}


$(3 R, 3 \mathrm{a} S, 9 \mathrm{~b} R)-3-M e t h y l-3 \mathrm{a}, 4,5,9 \mathrm{~b}-$ tetrahydronaphtho[1,2-b]furan-2(3H)-one (19c)

Chiralcel OJ-3, $250 \times 4.6 \mathrm{~mm}$ column, hexane/2-propanol 95:5, $1.0 \mathrm{~mL} / \mathrm{min}, 220 \mathrm{~nm}, 35^{\circ} \mathrm{C}$

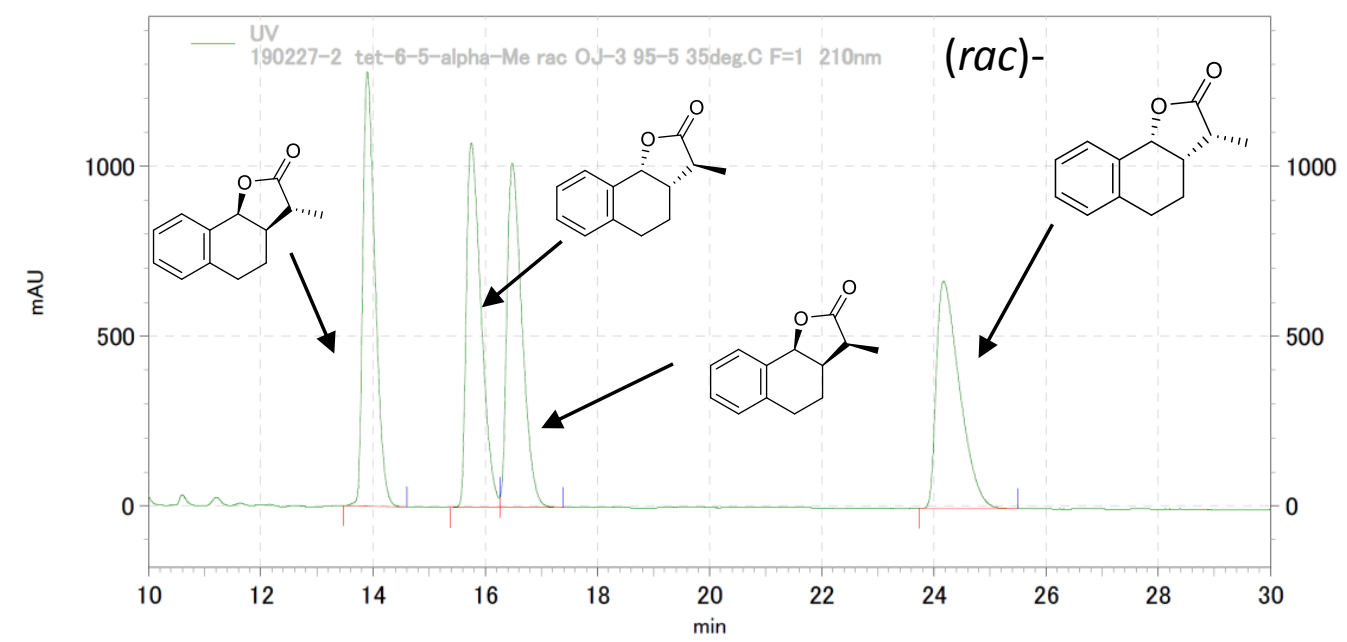

190227-2 tet-6-5-alpha-Me rac 0J-3 95-5 35deg. C F=1 210nm

\begin{tabular}{rlllll}
$\begin{array}{c}\text { Pk } \\
\text { No. }\end{array}$ & R. T. & Area & Area\% & B. C. & Symmetry \\
\hline 1 & 13.900 & 77887511 & 25.145 & $\mathrm{MM}$ & 0.00000 \\
2 & 15.753 & 78508109 & 25.346 & $\mathrm{Mx}$ & 0.00000 \\
3 & 16.483 & 76166227 & 24.590 & $\mathrm{XM}$ & 0.00000 \\
4 & 24.170 & 77187682 & 24.919 & $\mathrm{MM}$ & 0.00000
\end{tabular}
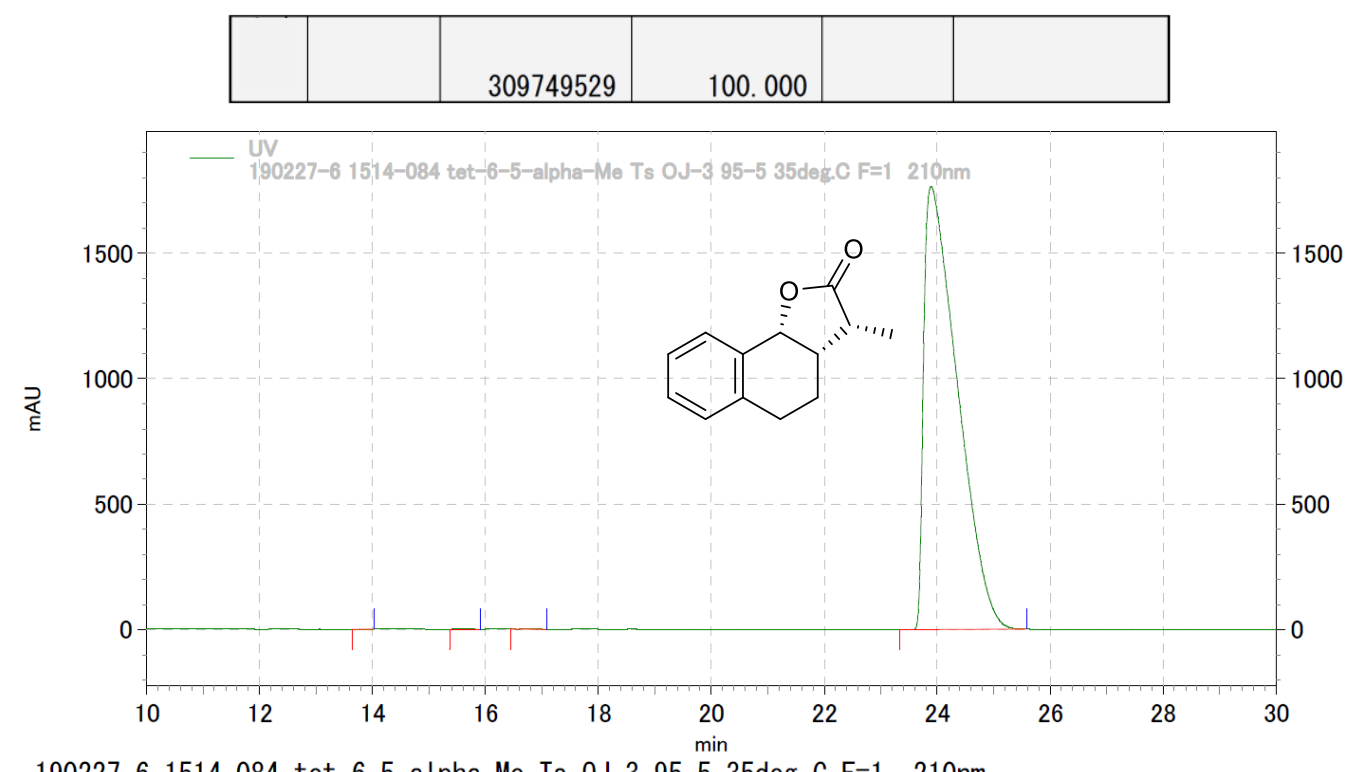

190227-6 1514-084 tet-6-5-alpha-Me Ts 0J-3 95-5 35deg. C F=1 210nm

\begin{tabular}{|c|c|c|c|c|c|}
\hline $\begin{array}{l}\text { Pk } \\
\text { No. }\end{array}$ & R. T. & Area & Area\% & B. C. & Symmetry \\
\hline $\begin{array}{l}1 \\
2 \\
3 \\
4\end{array}$ & $\begin{array}{l}13.637 \\
15.593 \\
16.833 \\
23.890\end{array}$ & $\begin{array}{r}8953 \\
73802 \\
154773 \\
291777927\end{array}$ & $\begin{array}{r}0.003 \\
0.025 \\
0.053 \\
99.919\end{array}$ & $\begin{array}{l}\text { MM } \\
\text { MM } \\
\text { MM } \\
\text { MM }\end{array}$ & $\begin{array}{l}0.00000 \\
0.00000 \\
0.00000 \\
0.00000\end{array}$ \\
\hline & & 292015455 & 100. 000 & & \\
\hline
\end{tabular}




\section{(3aS,7aR)-6-Methyl-3a,4,5,7a-tetrahydrobenzofuran-2(3H)-one (24)}

CHIRALSIL-DEX-CB, $0.25 \mathrm{~mm} \times 25 \mathrm{~m}$ column, $\mathrm{DF}=0.25$, Temp.$=130{ }^{\circ} \mathrm{C}$ const.

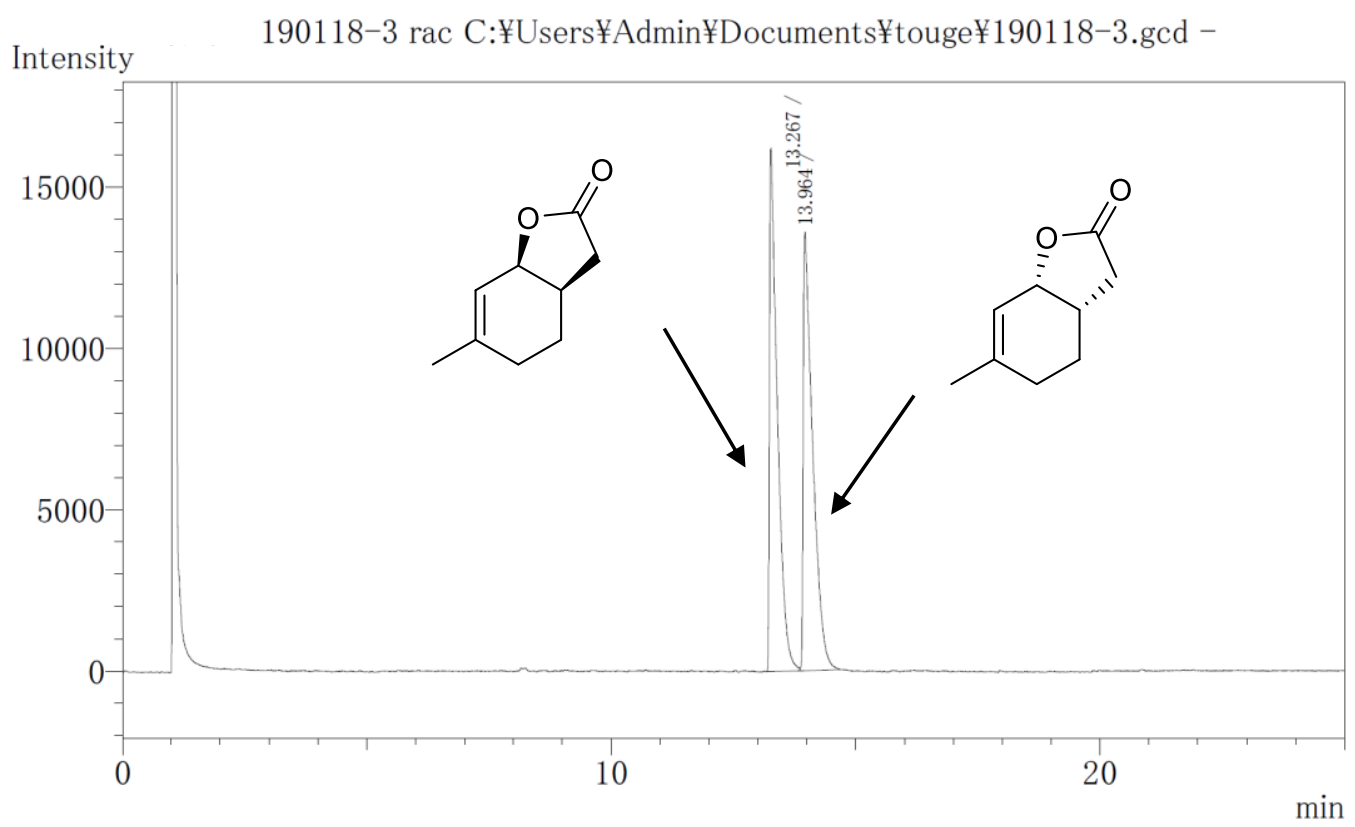

\begin{tabular}{|r|c|r|r|}
\hline Peak No. & RT & Area\% & Area \\
\hline 1 & 13.267 & 50.0192 & 182755 \\
\hline 2 & 13.964 & 49.9808 & 182615 \\
\hline & & 100.0000 & 365370 \\
\hline
\end{tabular}

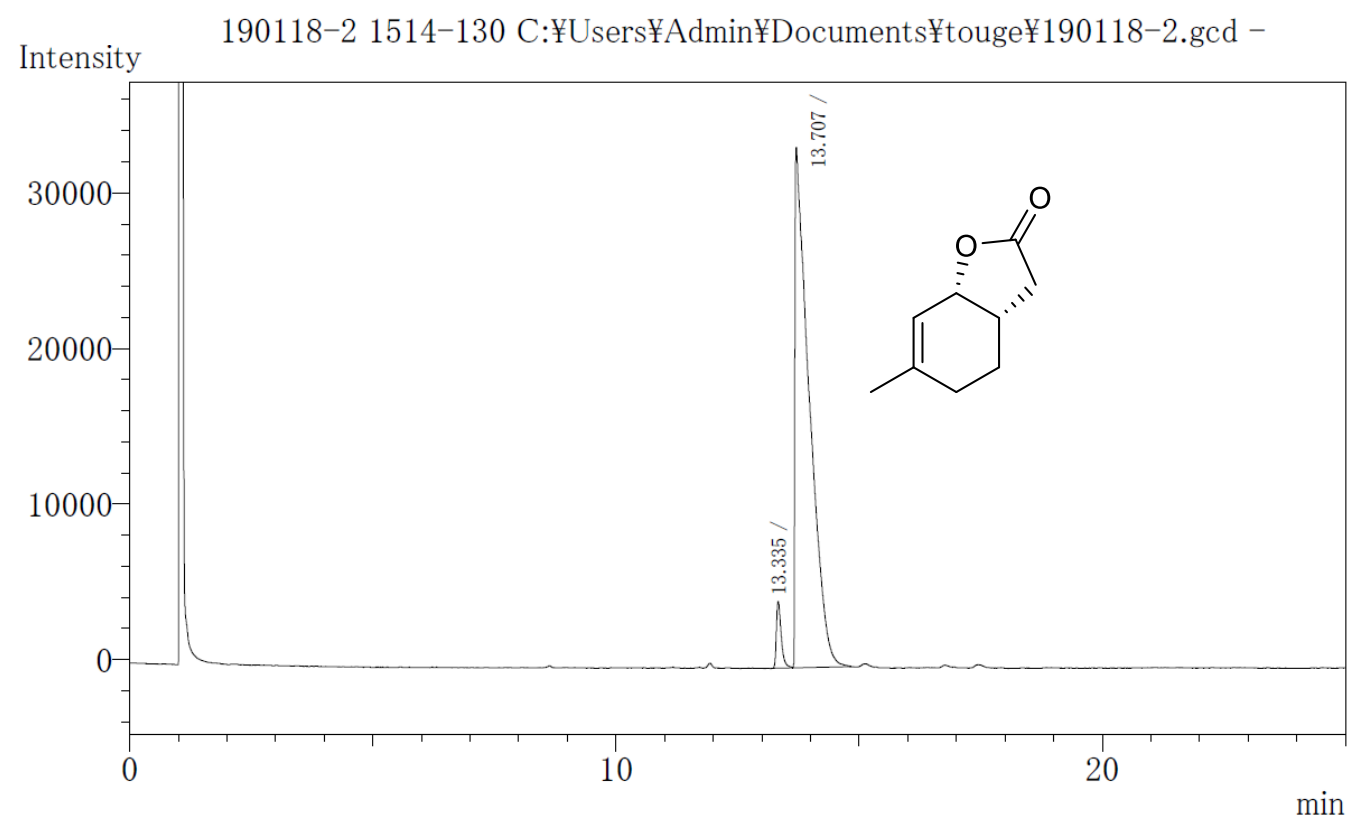

\begin{tabular}{|r|r|r|r|}
\hline Peak No. & RT & \multicolumn{1}{|c|}{ Area\% } & \multicolumn{1}{c|}{ Area } \\
\hline 1 & 13.335 & 4.2091 & 29828 \\
\hline 2 & 13.707 & 95.7909 & 678835 \\
\hline & & 100.0000 & 708663 \\
\hline
\end{tabular}


Wine Lactone ((3S-6))

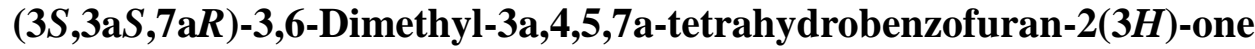

Chiralcel OZ-3, $250 \times 4.6 \mathrm{~mm}$ column, hexane/2-propanol 97:3, $1.0 \mathrm{~mL} / \mathrm{min}, 210 \mathrm{~nm}, 35^{\circ} \mathrm{C}$

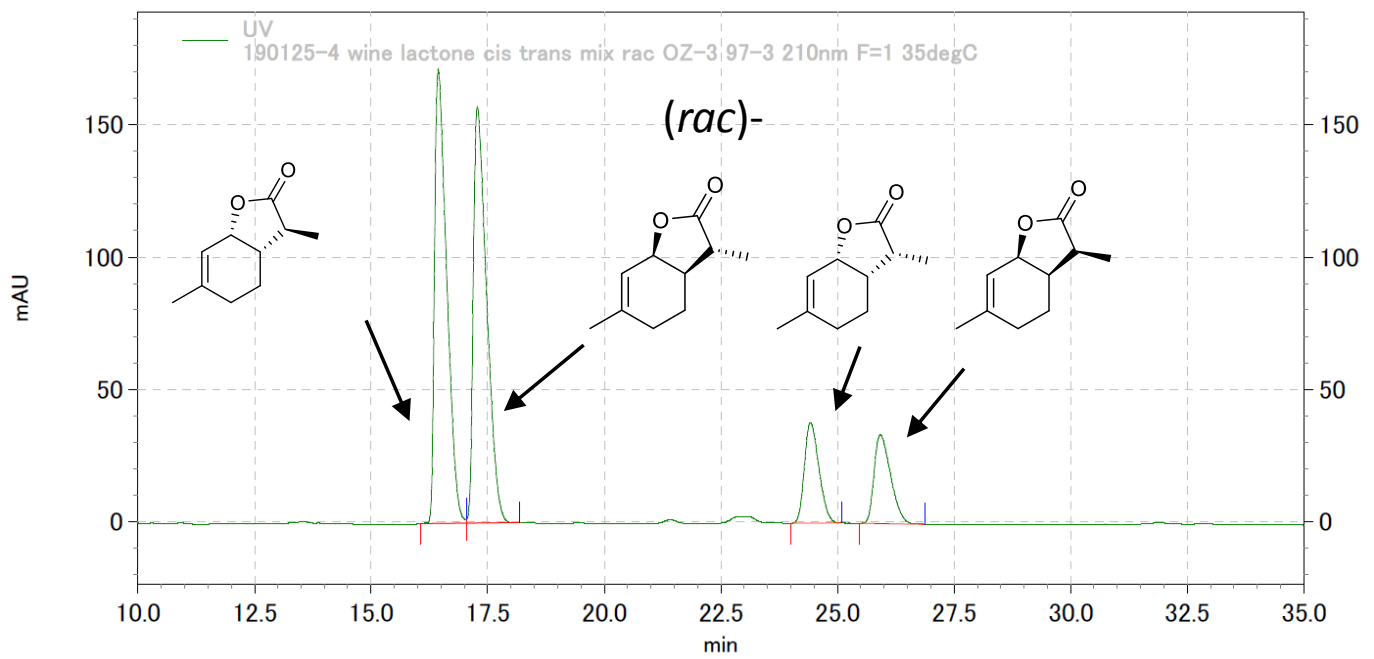

190125-4 wine lactone cis trans mix rac 0Z-3 97-3 210nm F=1 35degC

\begin{tabular}{rlrrrr}
$\begin{array}{c}\text { Pk } \\
\text { No. }\end{array}$ & R. T. & \multicolumn{1}{c}{ Area } & Area\% & B. C. & Symmetry \\
\hline 1 & 16. 440 & 12406479 & 39.383 & Mx & 0.00000 \\
2 & 17.280 & 12501842 & 39.685 & XM & 0.00000 \\
3 & 24.420 & 3290966 & 10.447 & MM & 0.00000 \\
4 & 25. 920 & 3303014 & 10.485 & MM & 0.00000
\end{tabular}

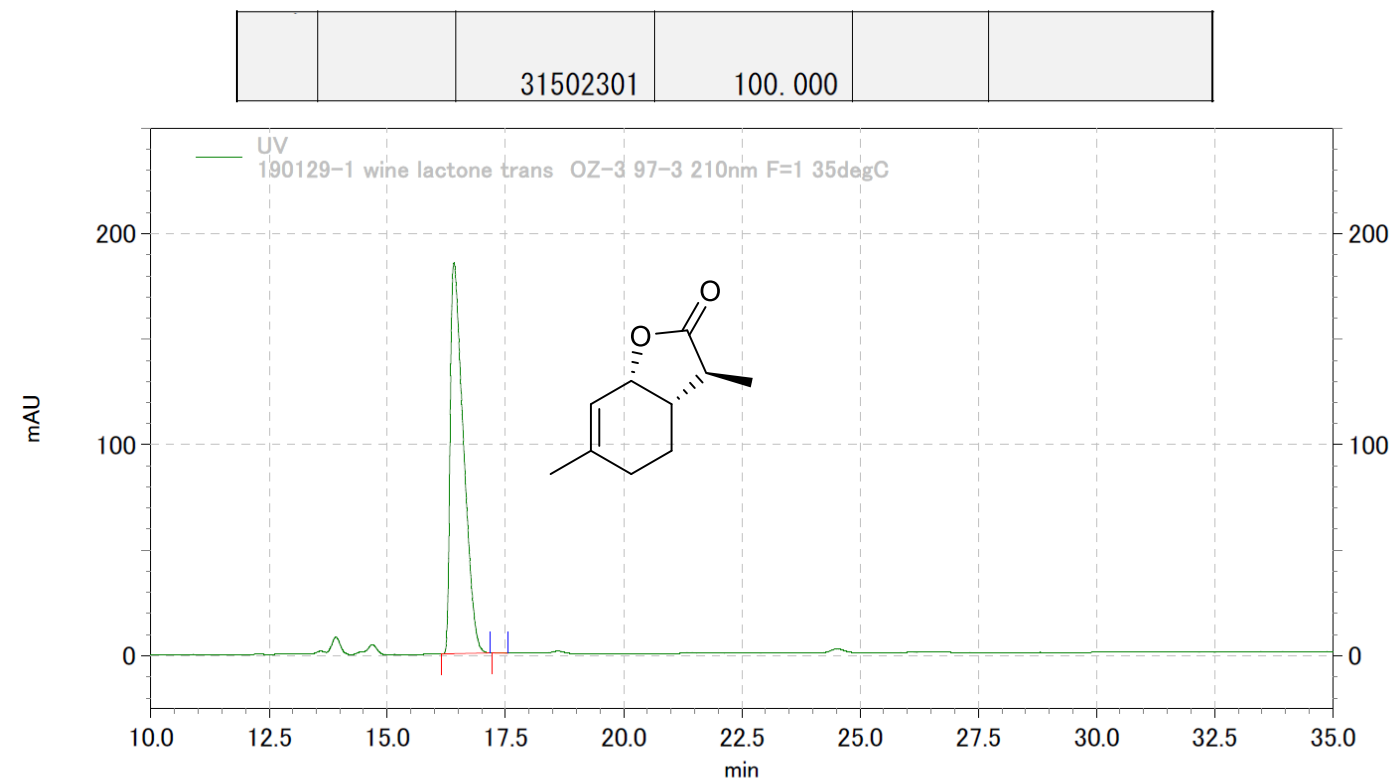

190129-1 wine lactone trans 0Z-3 97-3 210nm $\mathrm{F}=1$ 35degC

\begin{tabular}{rlrrrrr}
$\begin{aligned} \text { Pk } \\
\text { No. }\end{aligned}$ & \multicolumn{1}{l}{ R. T. } & \multicolumn{1}{c}{ Area } & \multicolumn{1}{l}{ Area\% } & \multicolumn{1}{l}{ B. C. } & Symmetry \\
\hline 1 & 16.410 & 14386829 & 99.961 & MM & 0.00000 \\
2 & 17.360 & 5664 & 0.039 & MM & 0.00000 \\
\hline & & & & & \\
\hline & & 14392493 & 100.000 & & \\
\hline
\end{tabular}




\section{$(3 R, 3 a S, 7 a R)-3,6-D i m e t h y l-3 a, 4,5,7 a-t e t r a h y d r o b e n z o f u r a n-2(3 H)-o n e ~((3 R-6))$}

Chiralcel OZ-3, $250 \times 4.6 \mathrm{~mm}$ column, hexane/2-propanol 97:3, $1.0 \mathrm{~mL} / \mathrm{min}, 210 \mathrm{~nm}, 35^{\circ} \mathrm{C}$

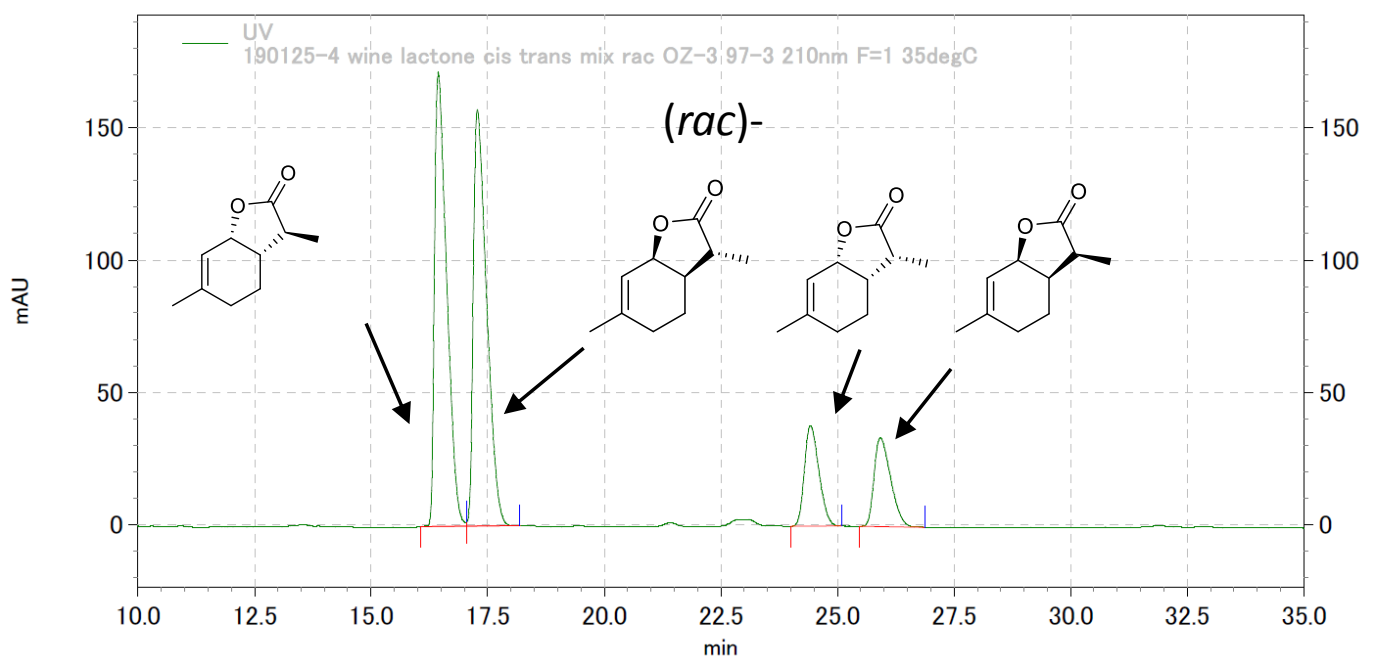

190125-4 wine lactone cis trans mix rac 0Z-3 97-3 210nm F=1 35degC

\begin{tabular}{|c|c|c|c|c|c|}
\hline $\begin{array}{l}\mathrm{Pk} \\
\text { No. }\end{array}$ & R. T. & Area & Area\% & B. C. & Symmetry \\
\hline $\begin{array}{l}1 \\
2 \\
3 \\
4\end{array}$ & $\begin{array}{l}16.440 \\
17.280 \\
24.420 \\
25.920\end{array}$ & $\begin{array}{r}12406479 \\
12501842 \\
3290966 \\
3303014\end{array}$ & $\begin{array}{l}39.383 \\
39.685 \\
10.447 \\
10.485\end{array}$ & $\begin{array}{l}M x \\
X M \\
M M \\
M M\end{array}$ & $\begin{array}{l}0.00000 \\
0.00000 \\
0.00000 \\
0.00000\end{array}$ \\
\hline & & 31502301 & 100.000 & & \\
\hline
\end{tabular}

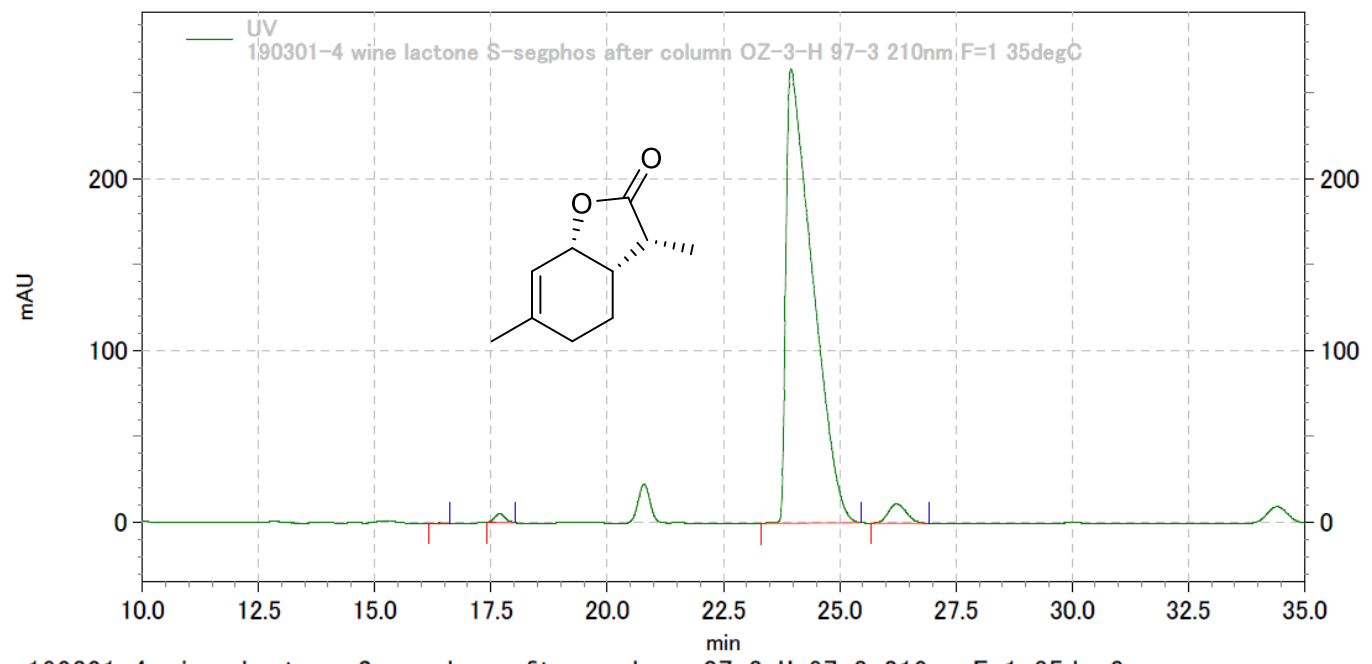

190301-4 wine lactone S-segphos after column 0Z-3-H 97-3 210nm F=1 35degC

\begin{tabular}{rlrrrr}
$\begin{array}{c}\text { Pk } \\
\text { No. }\end{array}$ & R. T. & \multicolumn{1}{c}{ Area } & Area\% & B. C. & Symmetry \\
\hline 1 & 16.393 & 10286 & 0.024 & MM & 0.00000 \\
2 & 17.683 & 328056 & 0.757 & MM & 0.00000 \\
3 & 23.953 & 41764049 & 96.414 & MM & 0.00000 \\
4 & 26.223 & 1214987 & 2.805 & MM & 0.00000 \\
\hline & \multicolumn{5}{c}{} \\
\end{tabular}


M. HR-MS Spectra for Substrates and Products.

6-Methyl-6,7,8,9-tetrahydro-5H-benzo[7]annulen-5-one (8c)

(ESI positive)

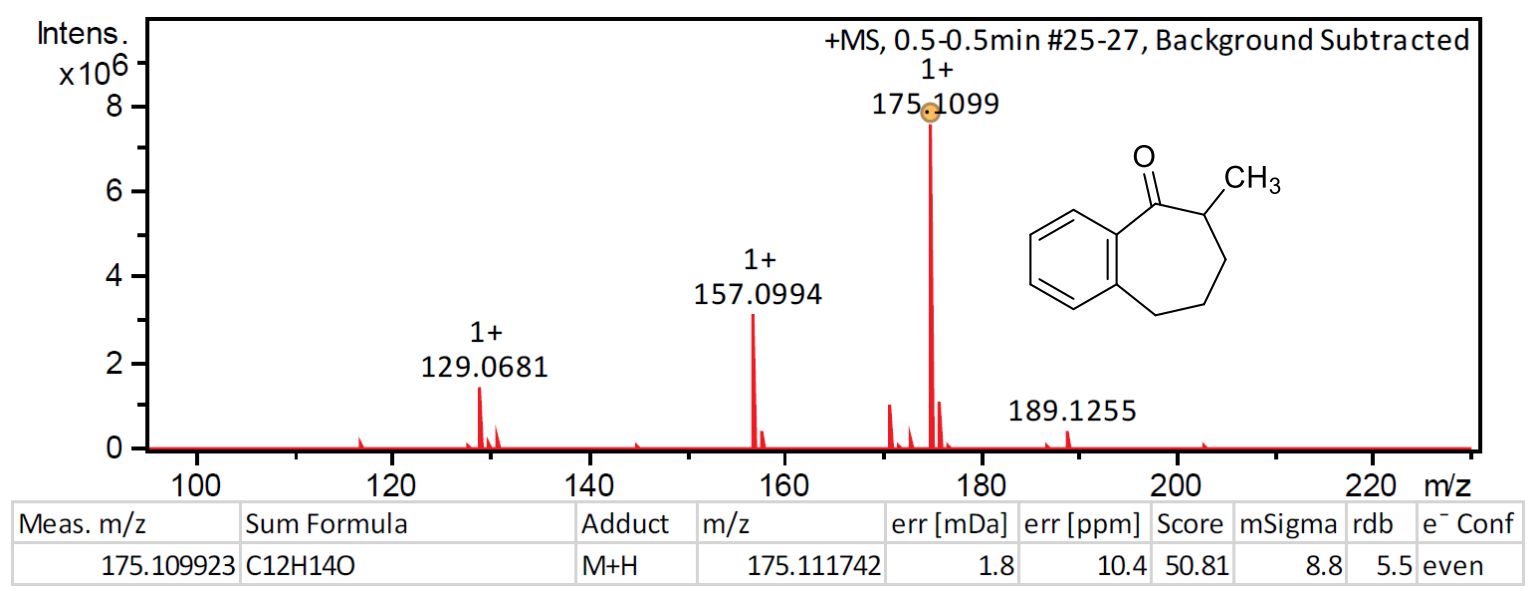

Methyl 1-oxo-1,2,3,4-tetrahydronaphthalene-2-carboxylate (8d)

(ESI positive)

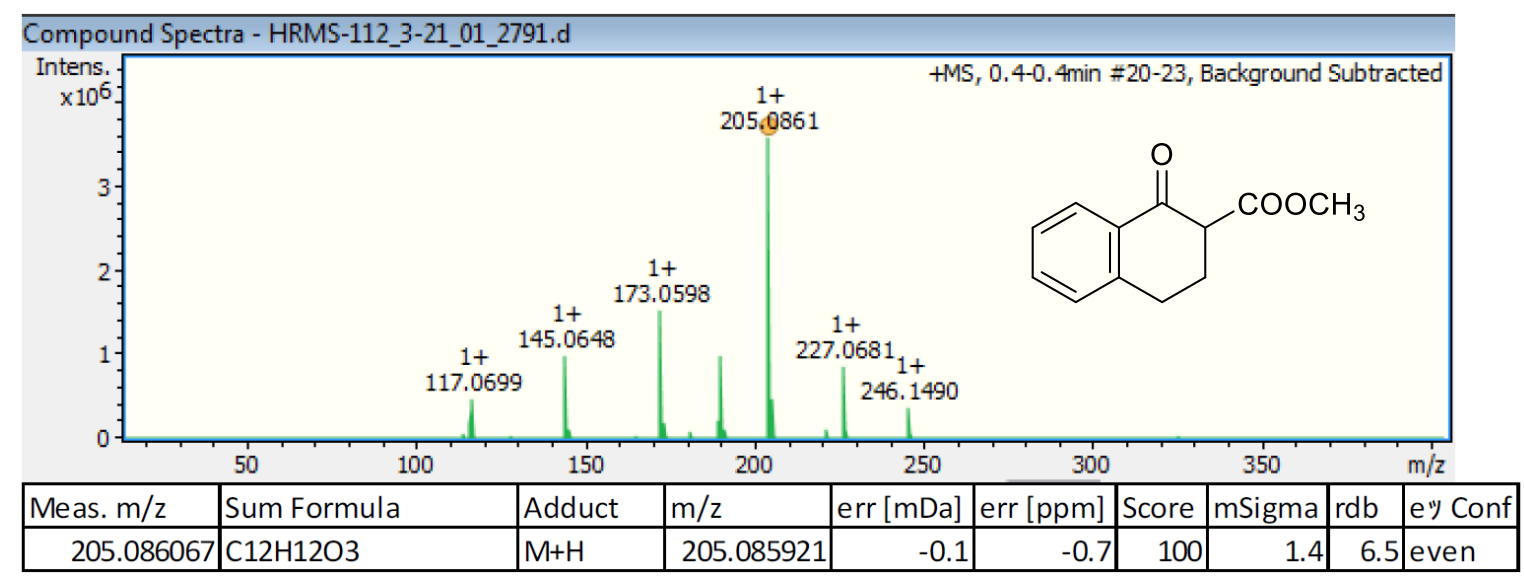


Methyl 2-(1-oxo-1,2,3,4-tetrahydronaphthalen-2-yl)acetate (8e)

(APPI positive)

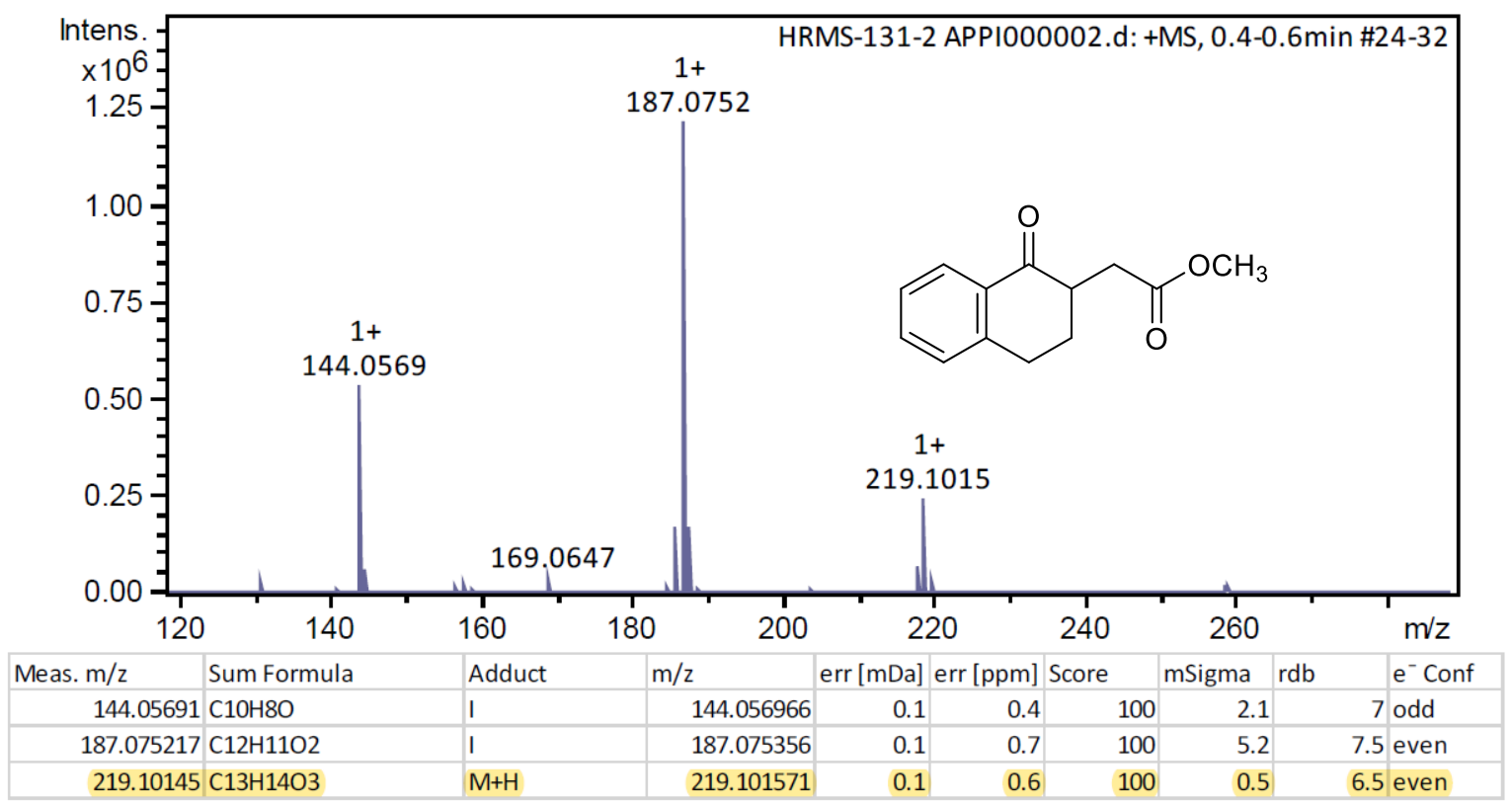

\section{8-Bromo-7-methoxy-3,4-dihydronaphthalen-1(2H)-one}

\section{(ESI positive)}

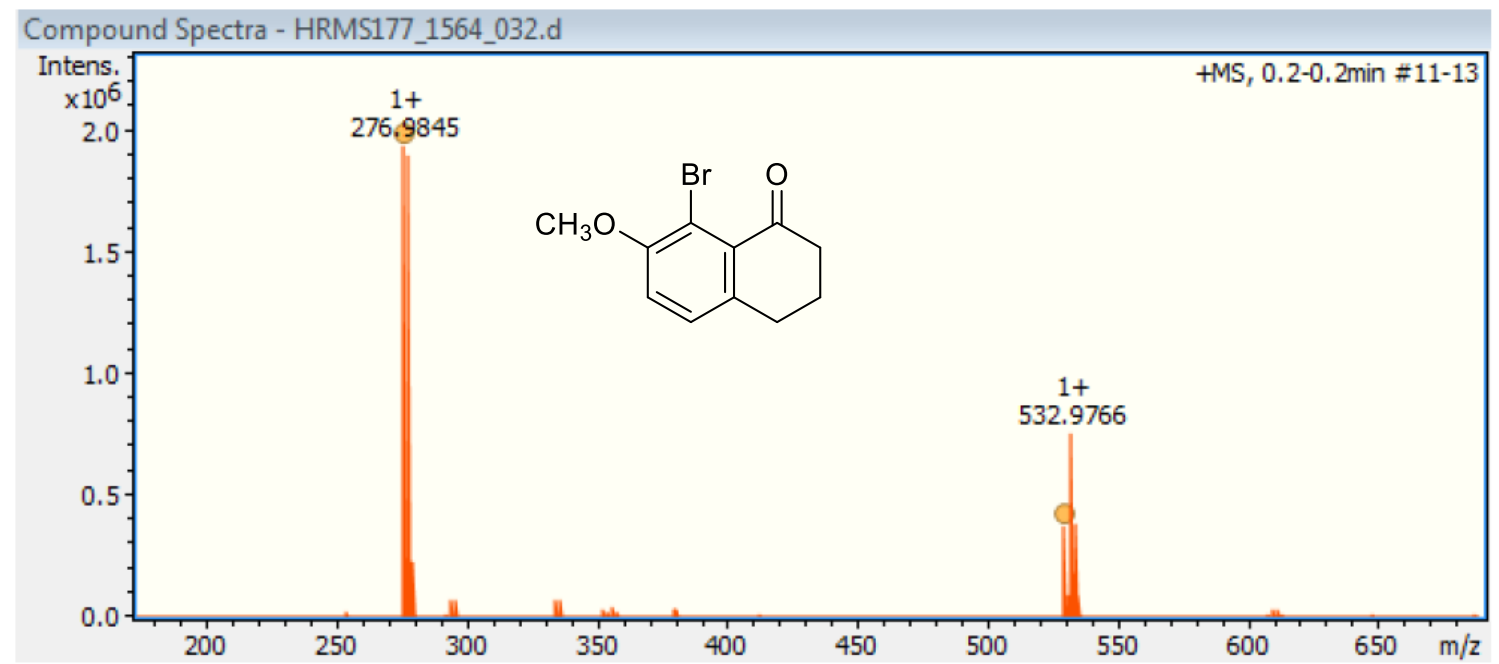

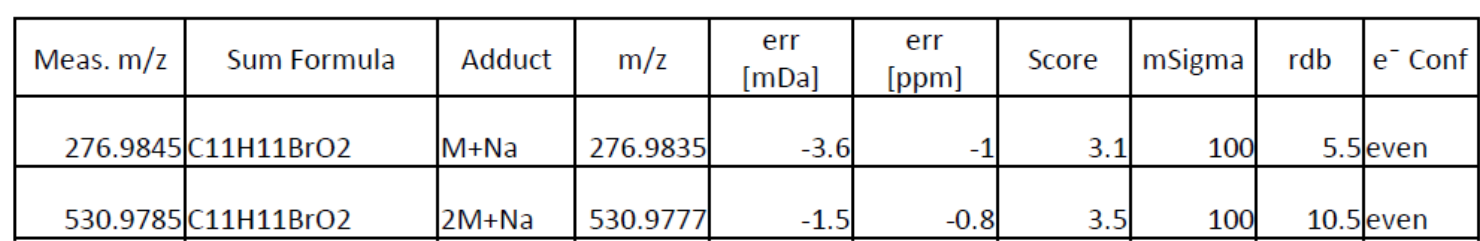




\section{2-(1-Oxo-1,2,3,4-tetrahydronaphthalen-2-yl)acetic acid (11a)}

(ESI positive)

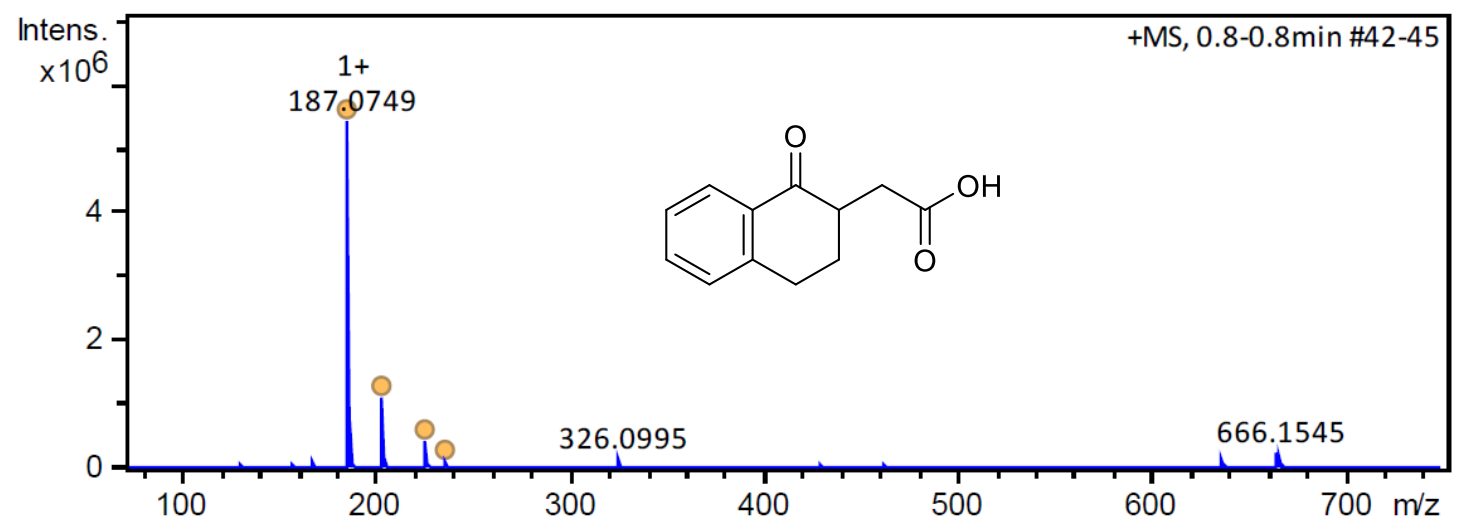

\begin{tabular}{|r|l|l|r|r|r|r|r|r|r|r|r|r|r|}
\hline Meas. $\mathrm{m} / \mathrm{z}$ & Sum Formula & Adduct & $\mathrm{m} / \mathrm{z}$ & err [mDa] & err [ppm] & Score & mSigma & rdb & $\mathrm{e}^{-}$Conf \\
\hline 237.090643 & $\mathrm{C} 16 \mathrm{H} 12 \mathrm{O} 2$ & $\mathrm{M}+\mathrm{H}$ & 237.091006 & 0.4 & 1.5 & 100 & 11.1 & 10.5 & even \\
\hline 227.067525 & $\mathrm{C} 12 \mathrm{H} 12 \mathrm{O} 3$ & $\mathrm{M}+\mathrm{Na}$ & 227.067865 & 0.3 & 1.5 & 100 & 1.3 & 6.5 even \\
\hline 205.08554 & $\mathrm{M}+\mathrm{H}$ & 205.085921 & 0.4 & 1.9 & 100 & 3.2 & 6.5 even \\
\hline $187.074918 \mathrm{C} 12 \mathrm{H} 10 \mathrm{O} 2$ & $\mathrm{M}+\mathrm{H}$ & 187.075356 & 0.4 & 2.3 & 100 & 3.3 & 7.5 even \\
\hline
\end{tabular}

\section{2-(7-Methoxy-1-oxo-1,2,3,4-tetrahydronaphthalen-2-yl)acetic acid (11b)}

(ESI positive)

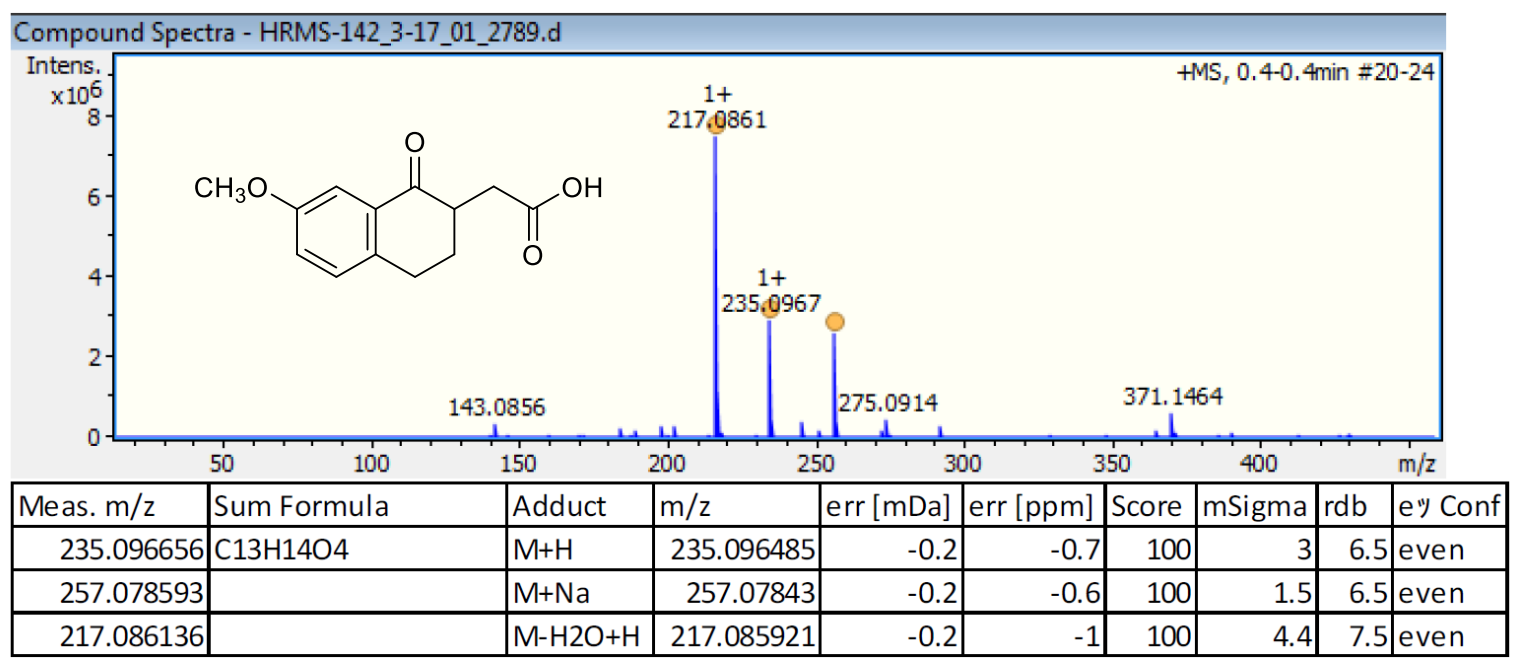




\section{2-(7-Chloro-1-oxo-1,2,3,4-tetrahydronaphthalen-2-yl)acetic acid (11c)}

(ESI negative)

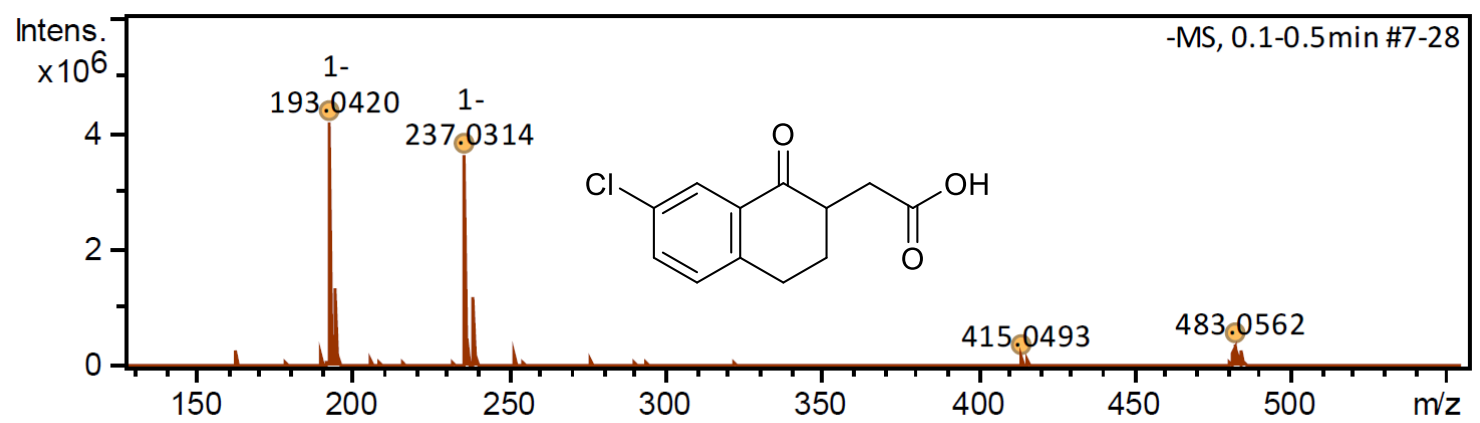

\begin{tabular}{|c|l|l|l|r|r|r|r|r|r|}
\hline Meas. $\mathrm{m} / \mathrm{z}$ & Sum Formula & Adduct & $\mathrm{m} / \mathrm{z}$ & $\operatorname{err}[\mathrm{mDa}]$ & $\operatorname{err}[\mathrm{ppm}]$ & Score & $\mathrm{mSigma}$ & $\mathrm{rdb}$ & $\mathrm{e}^{-}$Conf \\
\hline 193.041977 & $\mathrm{C} 11 \mathrm{H} 11 \mathrm{ClO}$ & $\mathrm{M}-\mathrm{H}$ & 193.042566 & 0.6 & 3 & 100 & 3.7 & 6.5 even \\
\hline 237.031449 & $\mathrm{C} 12 \mathrm{H} 11 \mathrm{ClO} 3$ & $\mathrm{M}-\mathrm{H}$ & 237.032395 & 0.9 & 4 & 79.79 & 5.1 & 7.5 even \\
\hline $415.049326 \mathrm{C} 22 \mathrm{H} 18 \mathrm{Cl} 2 \mathrm{O} 4$ & $\mathrm{M}-\mathrm{H}$ & 415.050938 & 1.6 & 3.9 & 32.44 & 15.4 & 13.5 even \\
\hline C11H9ClO2 & $2 \mathrm{M}-\mathrm{H}$ & 415.050938 & 1.6 & 3.9 & 32.44 & 15.4 & 13.5 even \\
\hline
\end{tabular}

\section{2-(7-Bromo-1-oxo-1,2,3,4-tetrahydronaphthalen-2-yl)acetic acid (11d)}

(APPI negative)

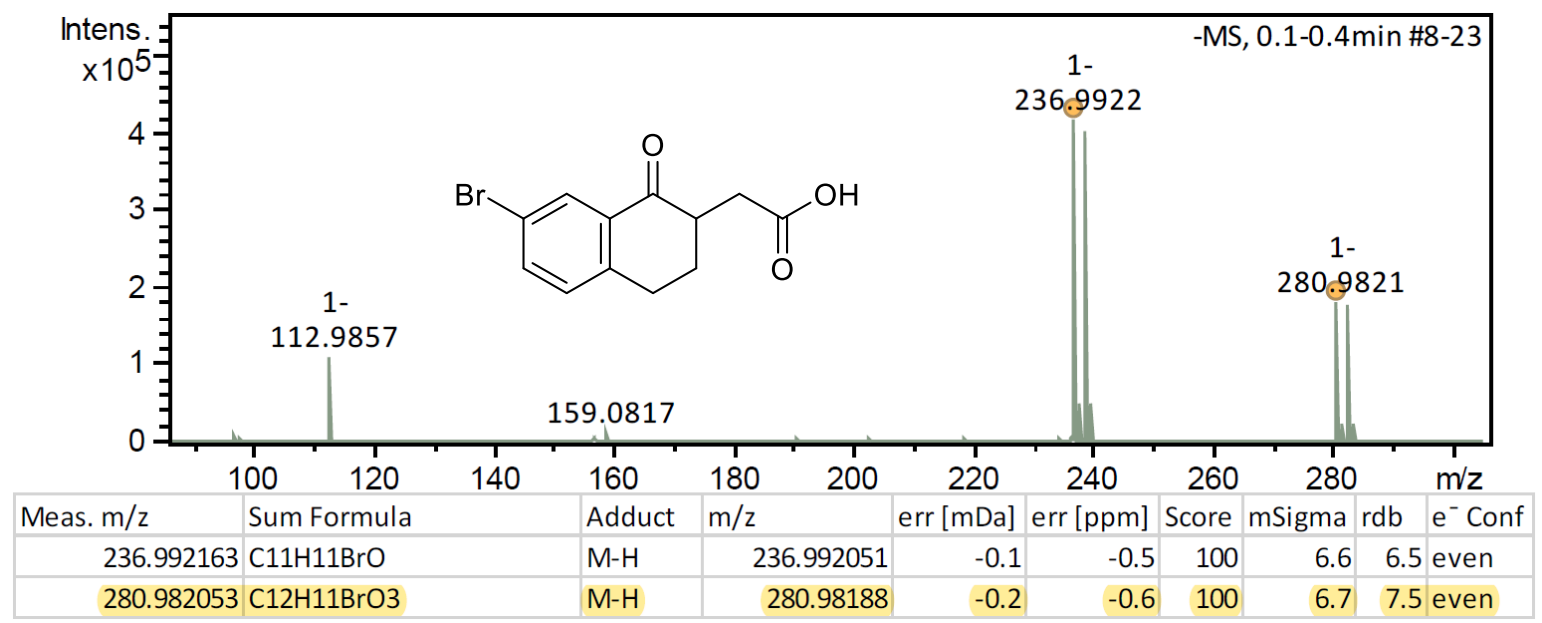




\section{2-(8-Bromo-7-methoxy-1-oxo-1,2,3,4-tetrahydronaphthalen-2-yl)acetic acid (11e)}

(ESI positive)

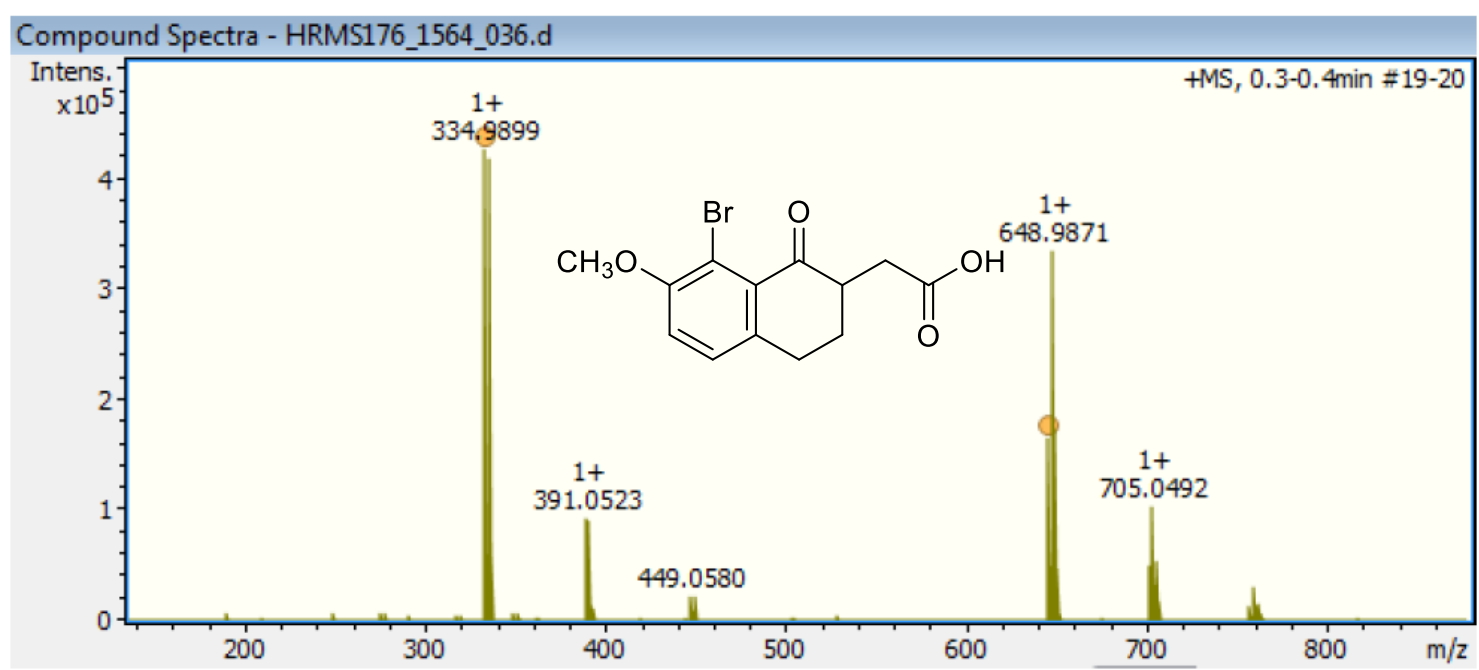

\begin{tabular}{|c|c|c|r|r|r|r|r|r|c|}
\hline Meas. $\mathrm{m} / \mathrm{z}$ & Sum Formula & Adduct & $\mathrm{m} / \mathrm{z}$ & $\begin{array}{c}\text { err } \\
{[\mathrm{mDa}]}\end{array}$ & $\begin{array}{c}\text { err } \\
{[\mathrm{ppm}]}\end{array}$ & Score & mSigma & $\mathrm{rdb}^{-}$Conf \\
\hline 334.9899 & $\mathrm{C} 13 \mathrm{H} 13 \mathrm{BrO} 4$ & $\mathrm{M}+\mathrm{Na}$ & 334.9889 & -0.9 & -2.8 & 100 & 5 & 6.5 & $\mathrm{e}_{\text {even }}$ \\
\hline 646.9889 & $\mathrm{C} 13 \mathrm{H} 13 \mathrm{BrO} 4$ & $2 \mathrm{M}+\mathrm{Na}$ & 646.9887 & -0.2 & -0.3 & 100 & 4.1 & 12.5 & even \\
\hline
\end{tabular}

\section{2-(6-Hydroxy-1-oxo-1,2,3,4-tetrahydronaphthalen-2-yl)acetic acid (11f)}

(APCI negative)

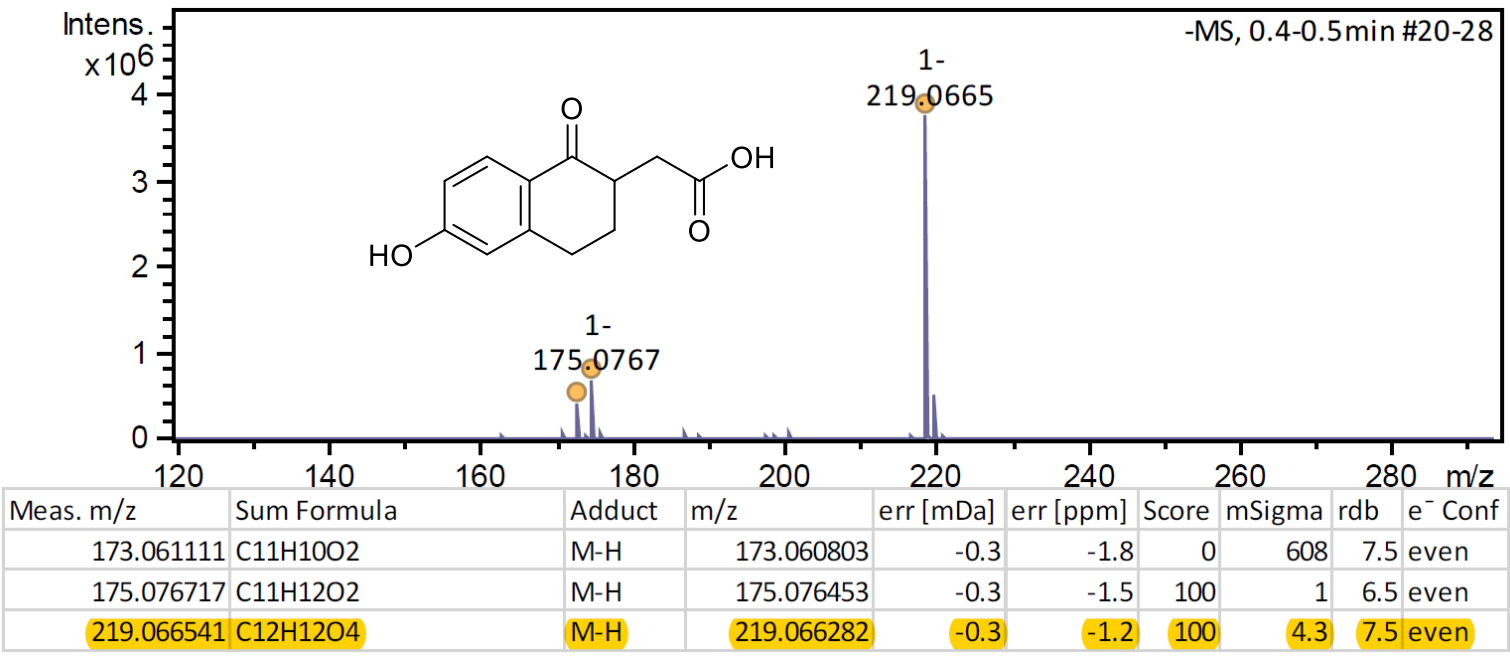




\section{2-(1-Oxo-2,3-dihydro-1H-inden-2-yl)acetic acid (11g)}

(ESI positive)

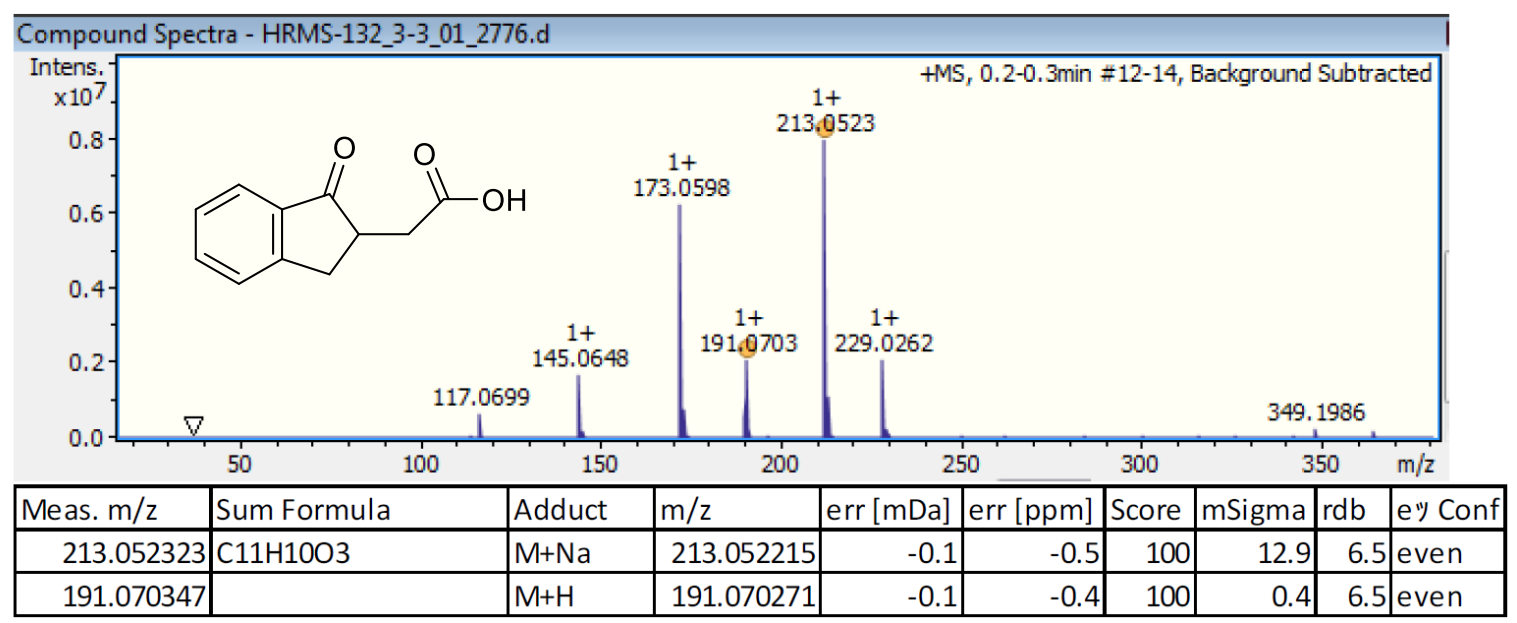

\section{2-(5,6-Dimethoxy-1-oxo-2,3-dihydro-1H-inden-2-yl)acetic acid (11h)}

(ESI positive)

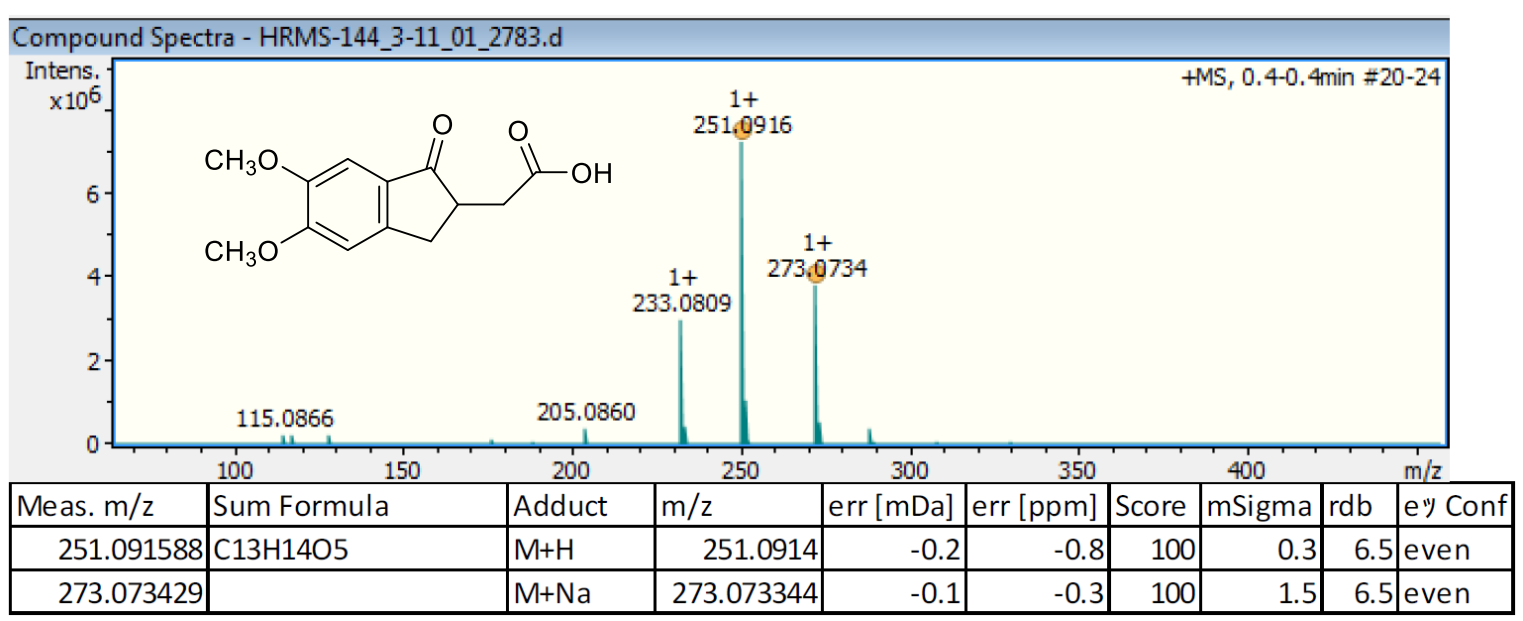




\section{2-(6-Fluoro-1-oxo-2,3-dihydro-1H-inden-2-yl)acetic acid (11i)}

(ESI negative)

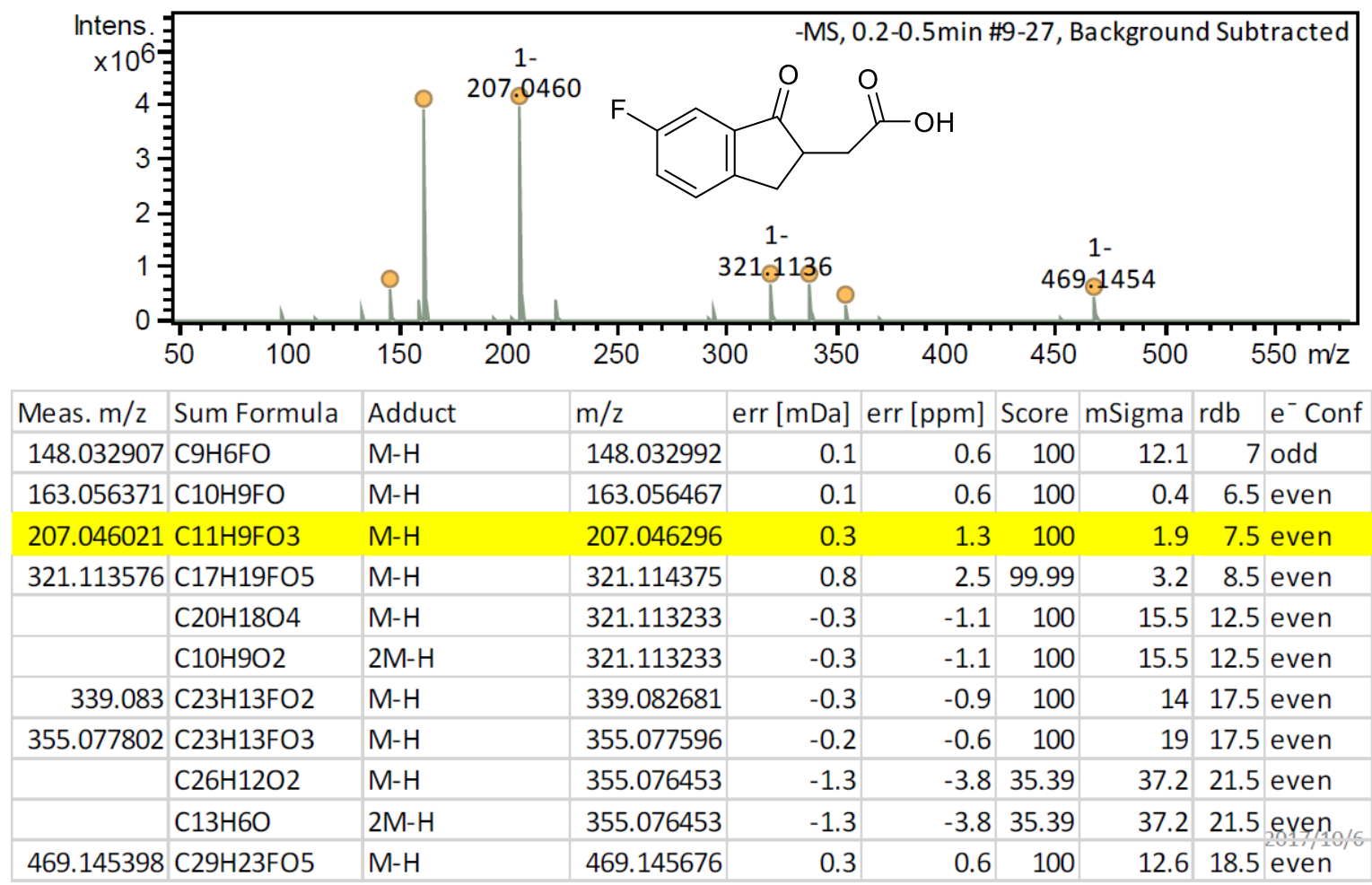

\section{2-(6-Chloro-1-oxo-2,3-dihydro-1H-inden-2-yl)acetic acid (11j)}

(APPI negative)

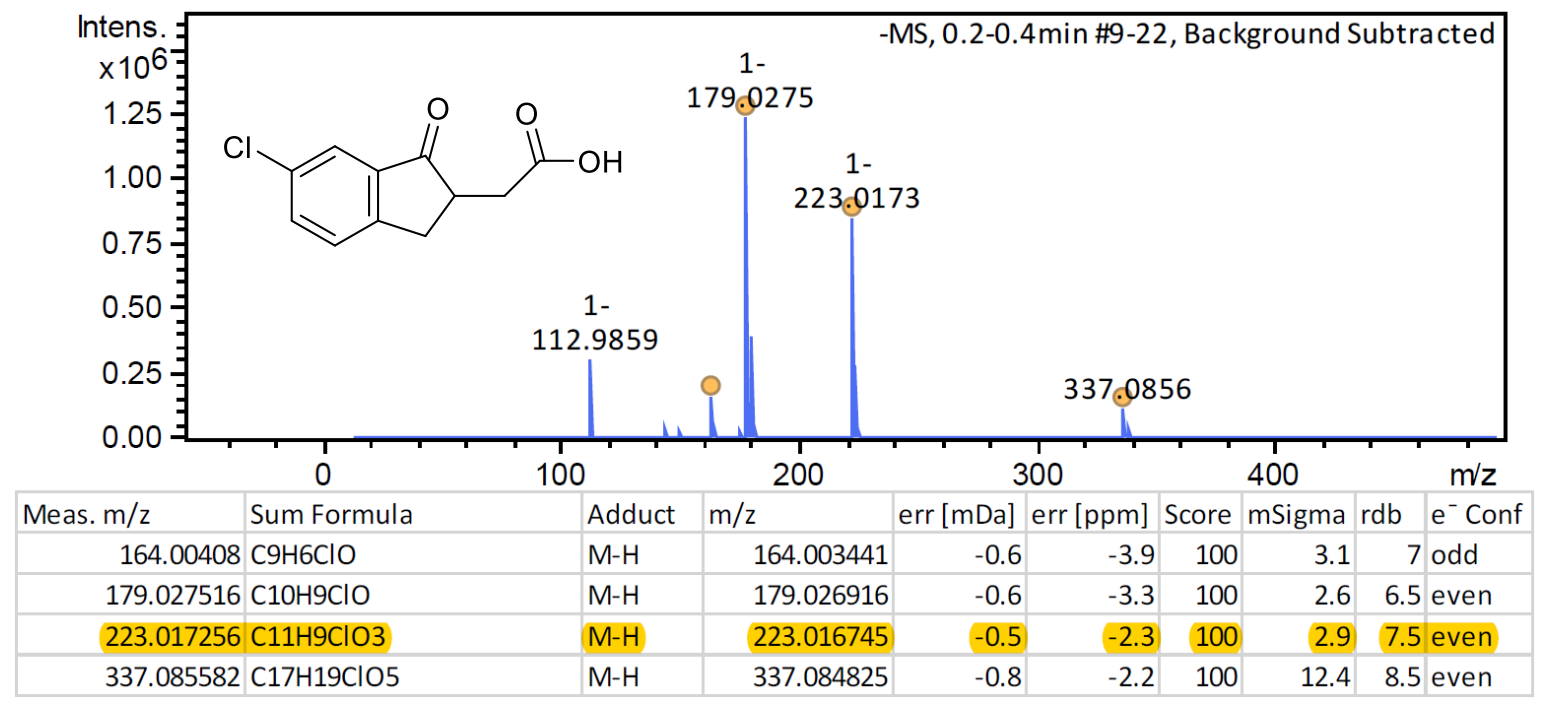




\section{2-(5-Oxo-6,7,8,9-tetrahydro-5H-benzo[7]annulen-6-yl)acetic acid (11k)}

(ESI positive)

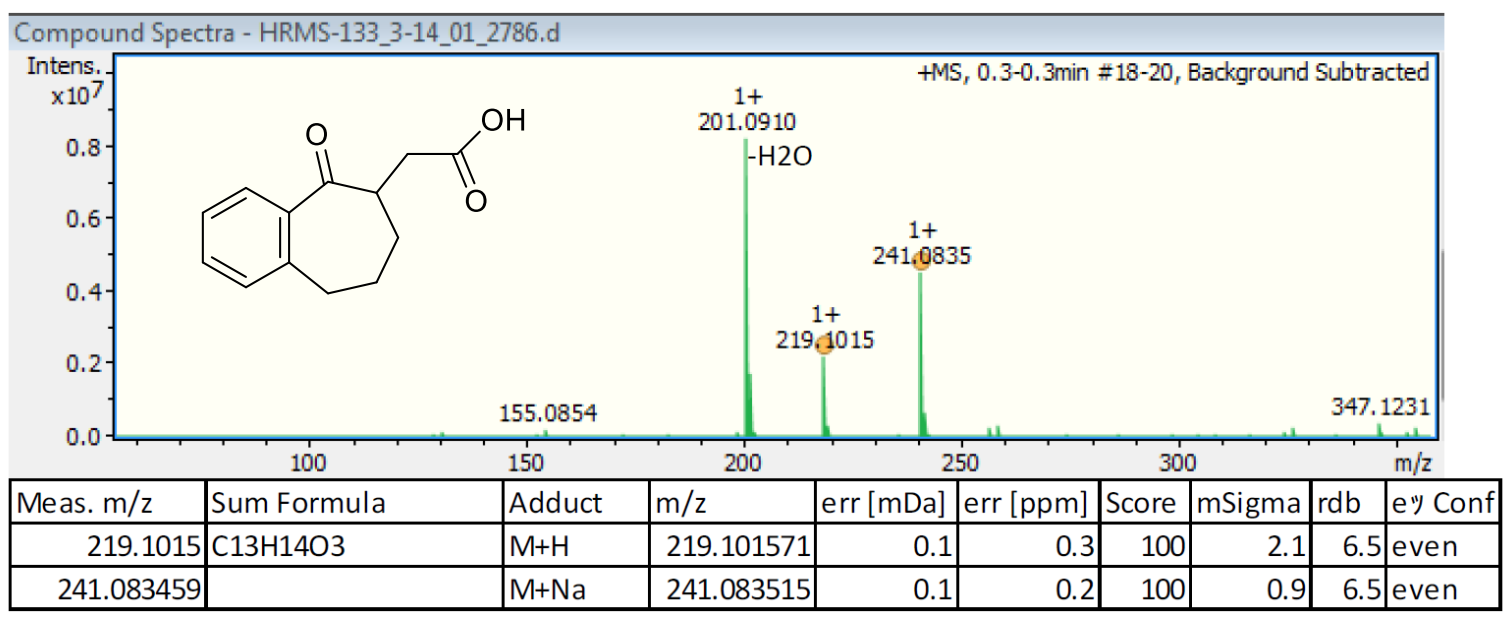

\section{3-(1-Oxo-1,2,3,4-tetrahydronaphthalen-2-yl)propanoic acid (11l)}

(ESI negative)

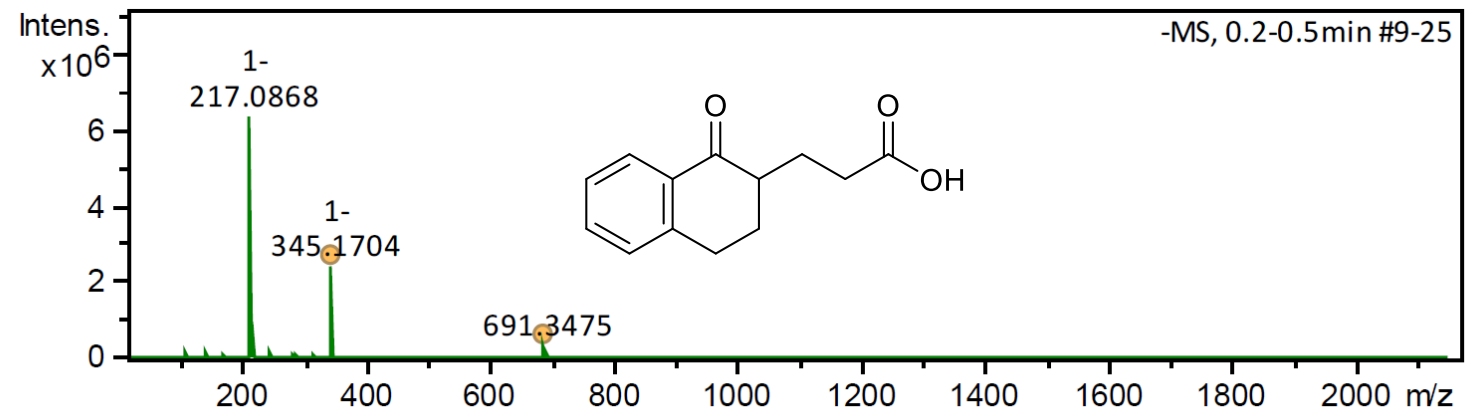

\begin{tabular}{|c|c|c|c|c|c|c|c|c|c|}
\hline Meas. m/z & Sum Formula & Adduct & $\mathrm{m} / \mathrm{z}$ & err [mDa] & err [ppm] & Score & mSigma & $\mathrm{rdb}$ & $\mathrm{e}^{-}$Conf \\
\hline 217.086842 & $\mathrm{C} 13 \mathrm{H} 14 \mathrm{O} 3$ & $\mathrm{M}-\mathrm{H}$ & 217.087018 & 0.2 & 0.8 & 100 & 2.7 & 7.5 & even \\
\hline 345.17036 & $\mathrm{C} 2 \mathrm{OH} 26 \mathrm{O} 5$ & $\mathrm{M}-\mathrm{H}$ & 345.170747 & 0.4 & 1.1 & 100 & 2.4 & 8.5 & even \\
\hline 691.347493 & $\mathrm{C} 40 \mathrm{H} 52 \mathrm{O} 10$ & $\mathrm{M}-\mathrm{H}$ & 691.348771 & 1.3 & 1.8 & 100 & 6.5 & 15.5 & even \\
\hline & $\mathrm{C} 2 \mathrm{OH} 26 \mathrm{O} 5$ & $2 \mathrm{M}-\mathrm{H}$ & 691.348771 & 1.3 & 1.8 & 100 & 6.5 & 15.5 & even \\
\hline
\end{tabular}




\section{3-(7-Methoxy-1-oxo-1,2,3,4-tetrahydronaphthalen-2-yl)propanoic acid (11m)}

(ESI negative)

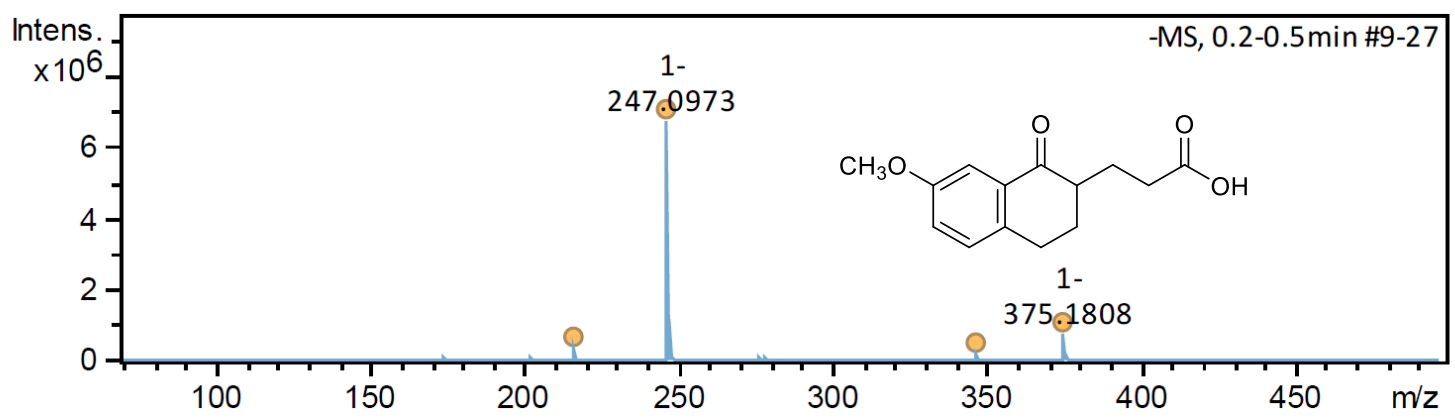

\begin{tabular}{|l|l|l|l|r|r|r|r|r|r|}
\hline Meas. $\mathrm{m} / \mathrm{z}$ & Sum Formula & Adduct & $\mathrm{m} / \mathrm{z}$ & err [mDa] & err [ppm] & Score & mSigma & rdb & $\mathrm{e}^{-}$Conf \\
\hline 217.086851 & $\mathrm{C} 13 \mathrm{H} 14 \mathrm{O} 3$ & $\mathrm{M}-\mathrm{H}$ & 217.087018 & 0.2 & 0.8 & 100 & 9 & 7.5 even \\
\hline $247.097307 \mathrm{C} 14 \mathrm{H} 1604$ & $\mathrm{M}-\mathrm{H}$ & 247.097583 & 0.3 & 1.1 & 100 & 9.2 & 7.5 even \\
\hline 347.149561 & $\mathrm{C} 19 \mathrm{H} 24 \mathrm{6}$ & $\mathrm{M}-\mathrm{H}$ & 347.150012 & 0.5 & 1.3 & 100 & 1.2 & 8.5 even \\
\hline 375.180776 & $\mathrm{C} 21 \mathrm{H} 2806$ & $\mathrm{M}-\mathrm{H}$ & 375.181312 & 0.5 & 1.4 & 100 & 5.7 & 8.5 even \\
\hline
\end{tabular}

\section{3-(7-Chloro-1-oxo-1,2,3,4-tetrahydronaphthalen-2-yl)propanoic acid (11n)}

(ESI negative)

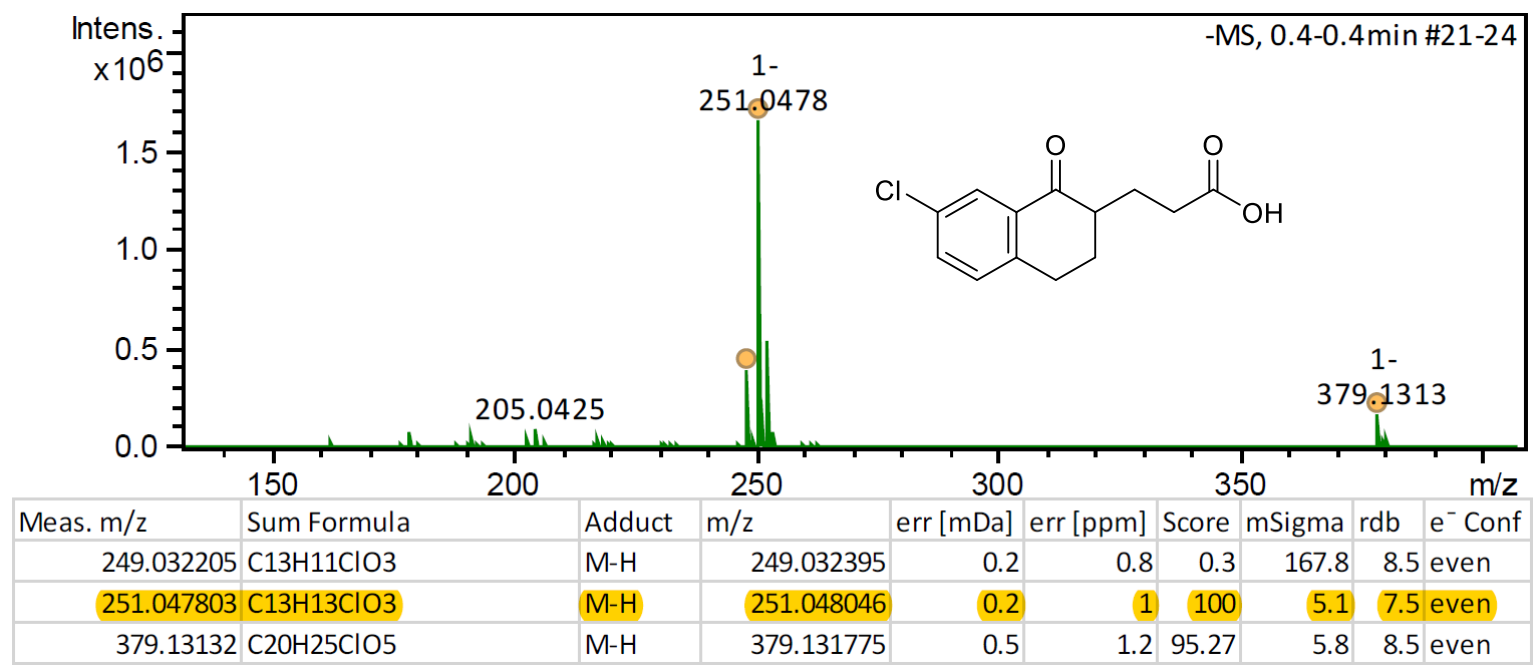




\section{2-(2-Oxo-1,2,3,4-tetrahydronaphthalen-1-yl)acetic acid (11o)}

(APPI negative)

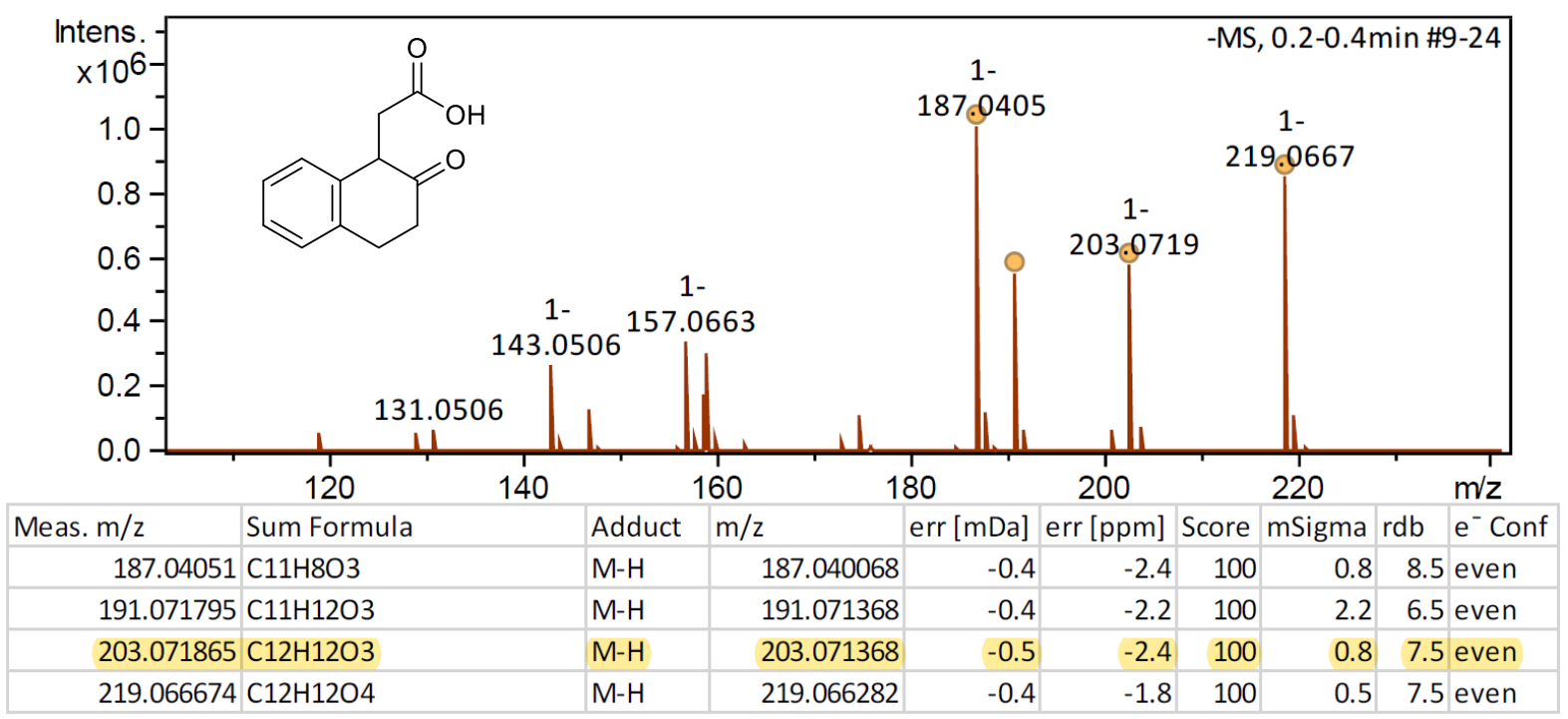

\section{2-(5-Methoxy-2-oxo-1,2,3,4-tetrahydronaphthalen-1-yl)acetic acid (11p)}

(ESI negative)

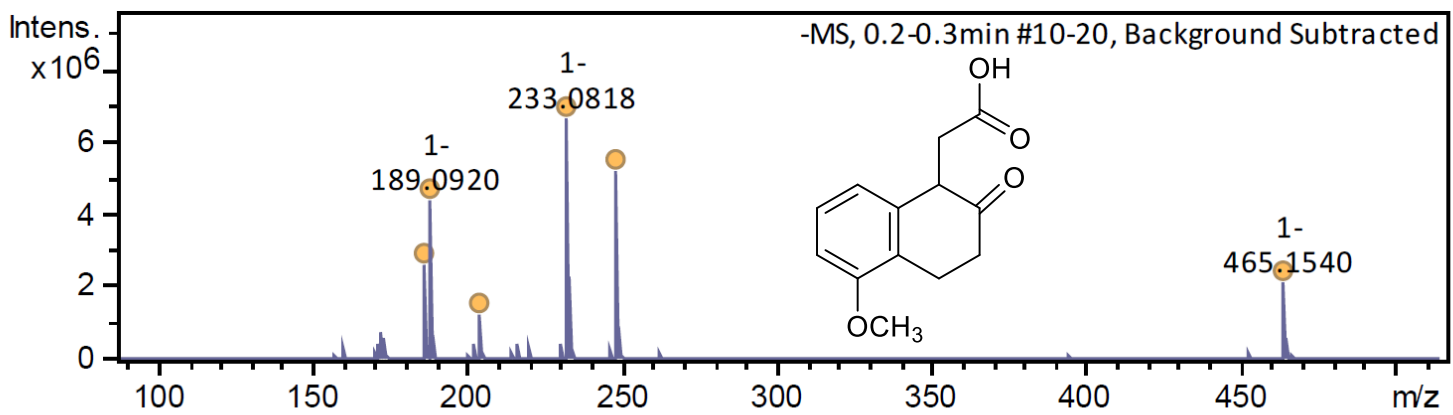

\begin{tabular}{|c|l|l|l|r|r|r|r|r|r|}
\hline Meas. $\mathrm{m} / \mathrm{z}$ & Sum Formula & Adduct & $\mathrm{m} / \mathrm{z}$ & err [mDa] & err [ppm] & Score & mSigma & rdb & $\mathrm{e}^{-}$Conf \\
\hline 187.076285 & $\mathrm{C} 12 \mathrm{H} 12 \mathrm{O} 2$ & $\mathrm{M}-\mathrm{H}$ & 187.076453 & 0.2 & 0.9 & 100 & 618.8 & 7.5 even \\
\hline 189.091986 & $\mathrm{C} 12 \mathrm{H} 14 \mathrm{2}$ & $\mathrm{M}-\mathrm{H}$ & 189.092103 & 0.1 & 0.6 & 100 & 10.8 & 6.5 even \\
\hline 205.086846 & $\mathrm{C} 12 \mathrm{H} 14 \mathrm{O} 3$ & $\mathrm{M}-\mathrm{H}$ & 205.087018 & 0.2 & 0.8 & 100 & 2.2 & 6.5 even \\
\hline 233.081849 & $\mathrm{C} 13 \mathrm{H} 14 \mathrm{H}$ & $\mathrm{M}-\mathrm{H}$ & 233.081932 & 0.1 & 0.4 & 100 & 117.5 & 7.5 even \\
\hline 249.076635 & $\mathrm{C} 13 \mathrm{H} 14 \mathrm{O} 5$ & $\mathrm{M}-\mathrm{H}$ & 249.076847 & 0.2 & 0.9 & 100 & 17.5 & 7.5 even \\
\hline 465.153958 & $\mathrm{C} 26 \mathrm{H} 26 \mathrm{O} 8$ & $\mathrm{M}-\mathrm{H}$ & 465.155491 & 1.5 & 3.3 & 100 & 0.4 & 14.5 even \\
\hline
\end{tabular}




\section{2-(6-Bromo-2-oxo-1,2,3,4-tetrahydronaphthalen-1-yl)acetic acid (11q)}

(APPI negative)

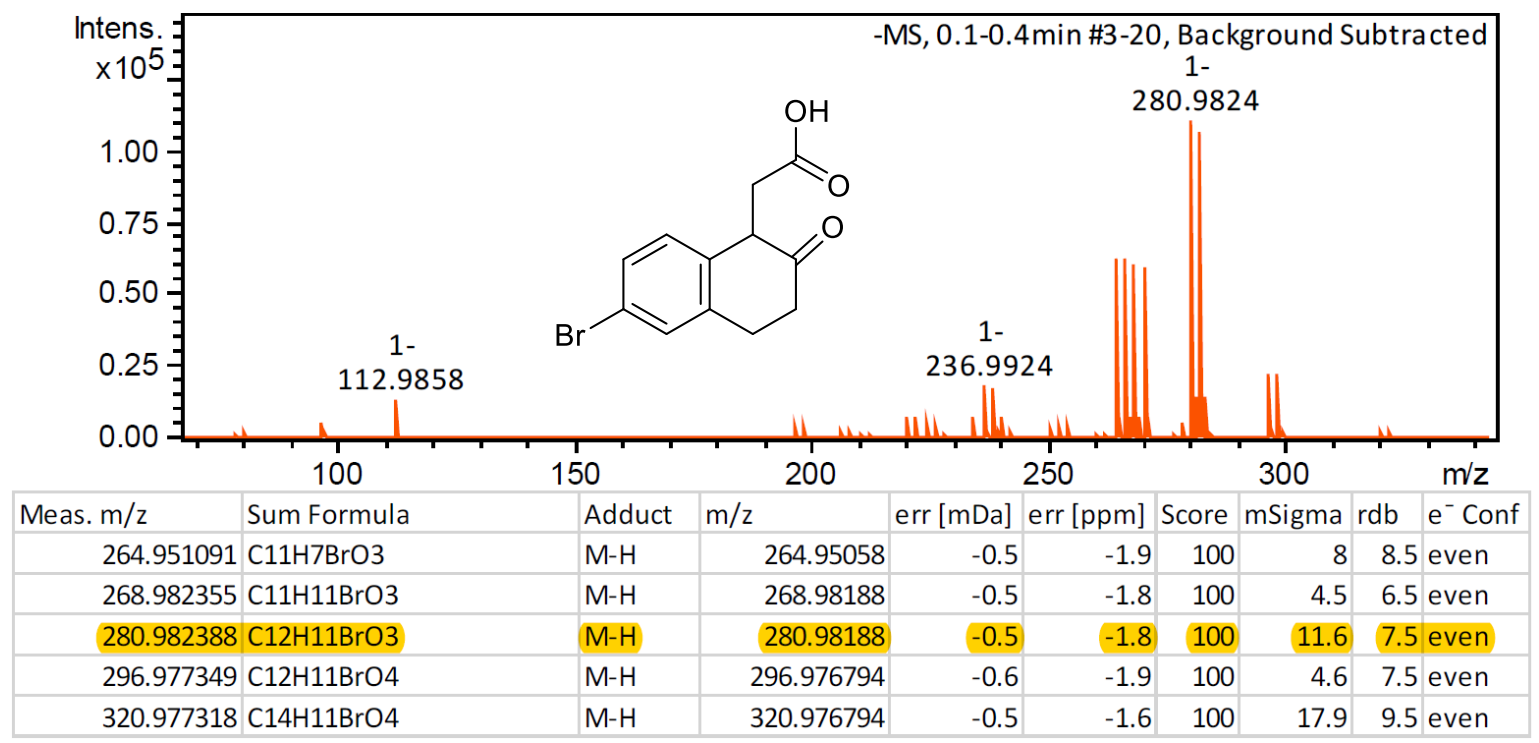

\section{2-(6-Chloro-2-oxo-1,2,3,4-tetrahydronaphthalen-1-yl)acetic acid (11r)}

(APPI negative)

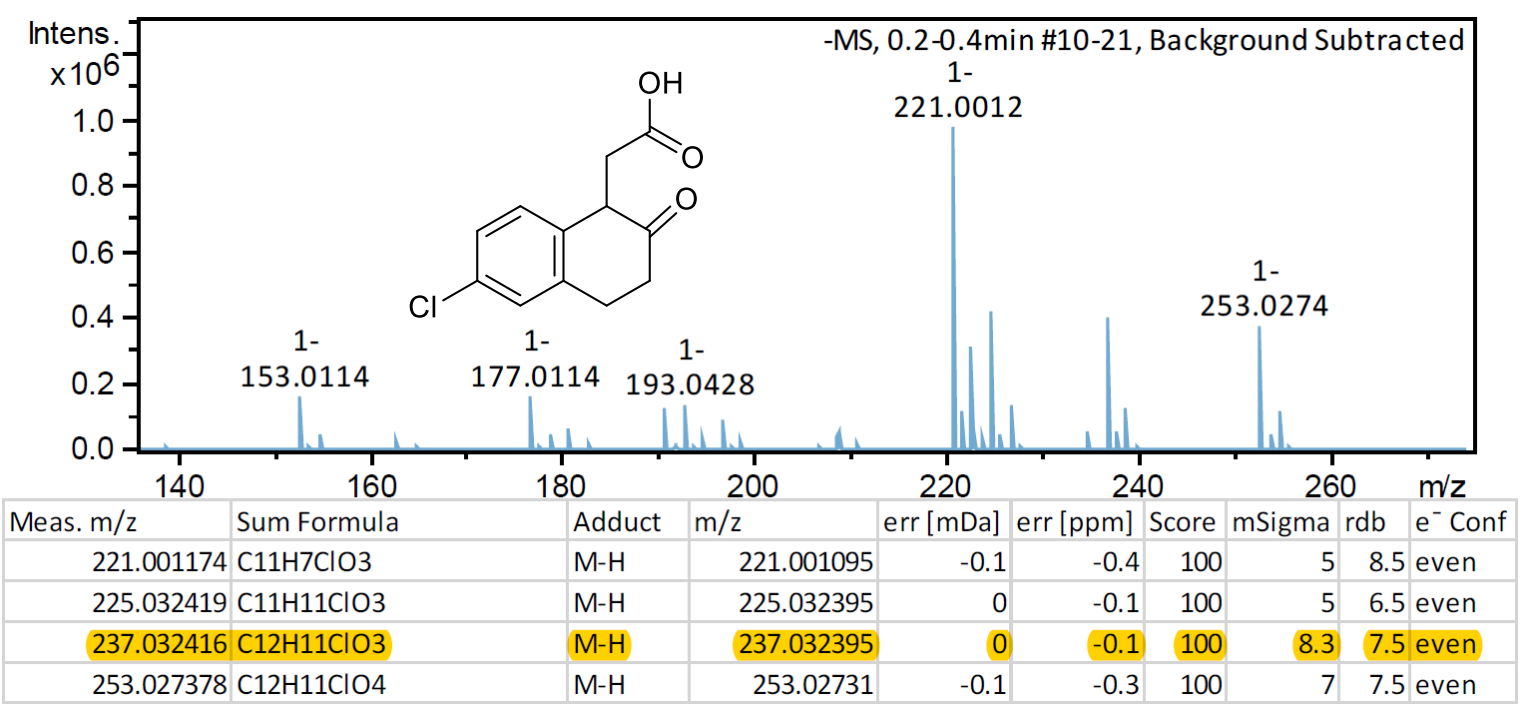




\section{2-(2-Oxo-1,2,3,4-tetrahydronaphthalen-1-yl)propanoic acid (14a)}

(ESI positive)

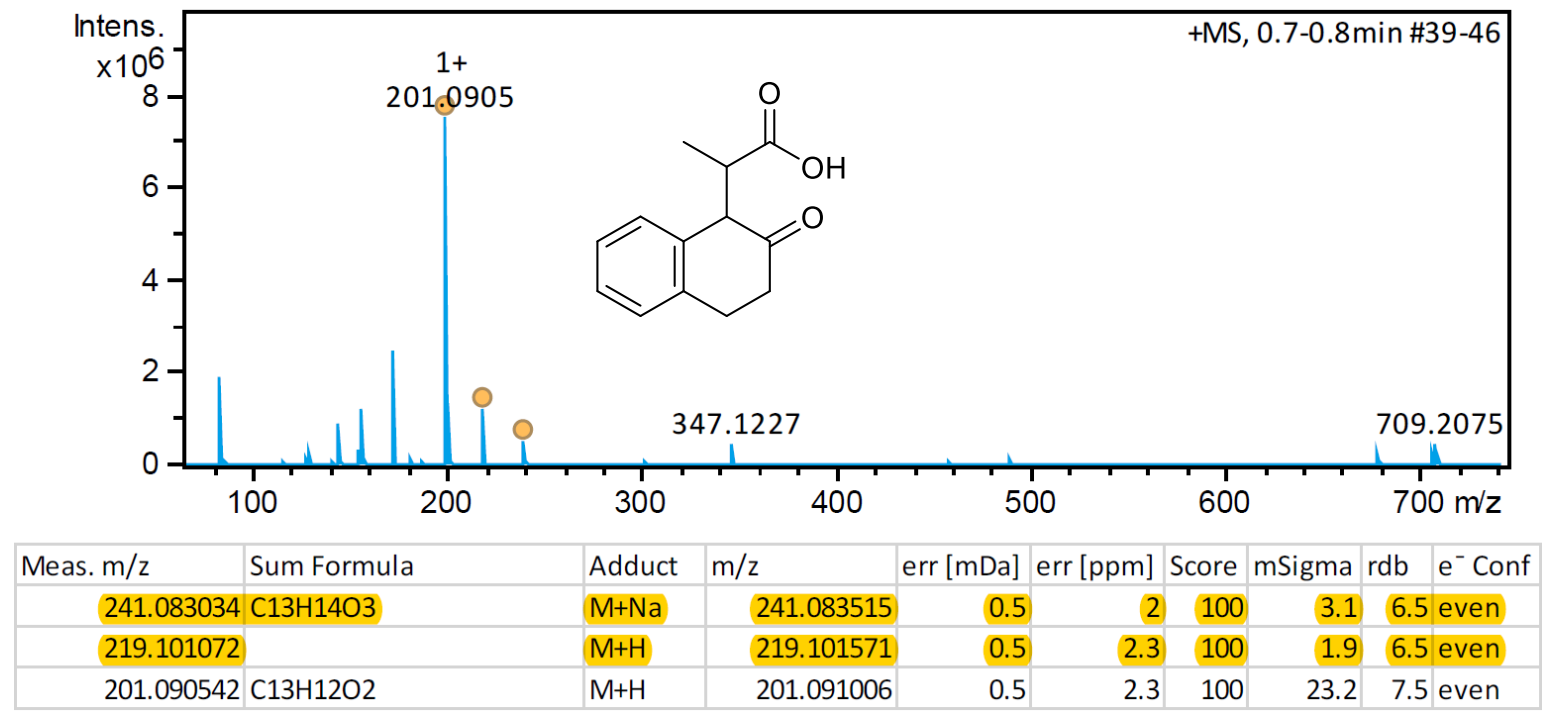

\section{2-(2-Oxo-1,2,3,4-tetrahydronaphthalen-1-yl)butanoic acid (14b)}

(ESI positive)

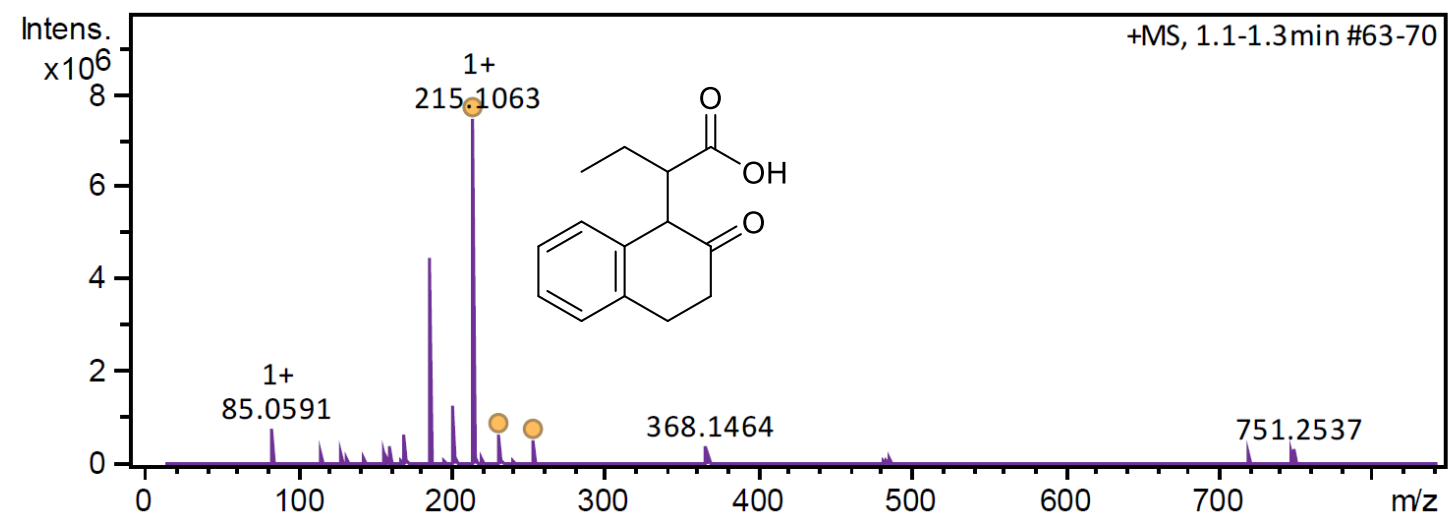

\begin{tabular}{|c|l|l|r|r|r|r|r|r|r|r|}
\hline Meas. $\mathrm{m} / \mathrm{z}$ & Sum Formula & Adduct & $\mathrm{m} / \mathrm{z}$ & err [mDa] & err [ppm] & Score & mSigma & rdb & $\mathrm{e}^{-}$Conf \\
\hline 255.098781 & $\mathrm{C} 14 \mathrm{H} 1603$ & $\mathrm{M}+\mathrm{Na}$ & 255.099165 & 0.4 & 1.5 & 100 & 0.1 & 6.5 even \\
\hline 233.116778 & $\mathrm{M}+\mathrm{H}$ & 233.117221 & 0.4 & 1.9 & 100 & 5.7 & 6.5 even \\
\hline 215.106299 & $\mathrm{C} 14 \mathrm{H} 1402$ & $\mathrm{M}+\mathrm{H}$ & 215.106656 & 0.4 & 1.7 & 100 & 33.6 & 7.5 even \\
\hline
\end{tabular}




\section{2-(2-Oxo-1,2,3,4-tetrahydronaphthalen-1-yl)-3-phenylpropanoic acid (14c)}

(APPI positive)

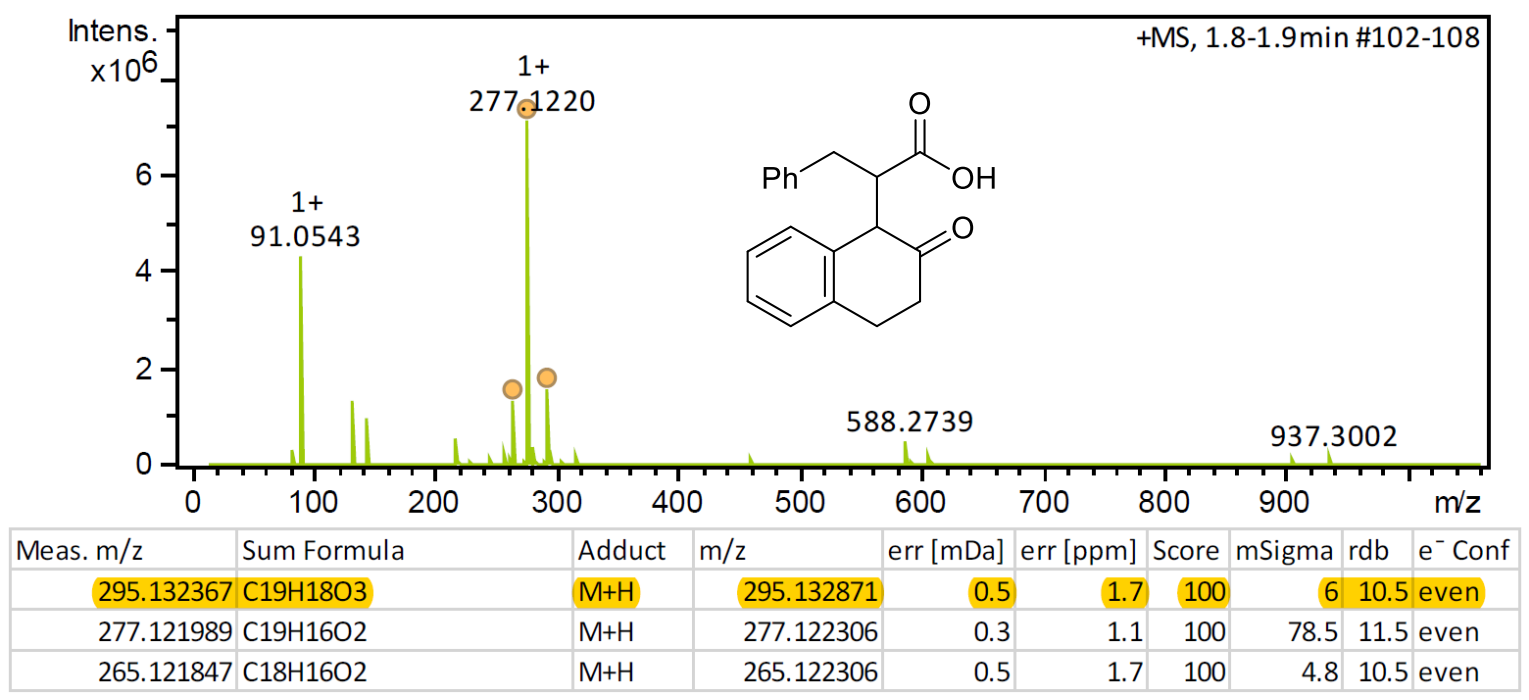

2-(2-Oxo-1,2,3,4-tetrahydronaphthalen-1-yl)-2-phenylacetic acid (14d)

(ESI positive)

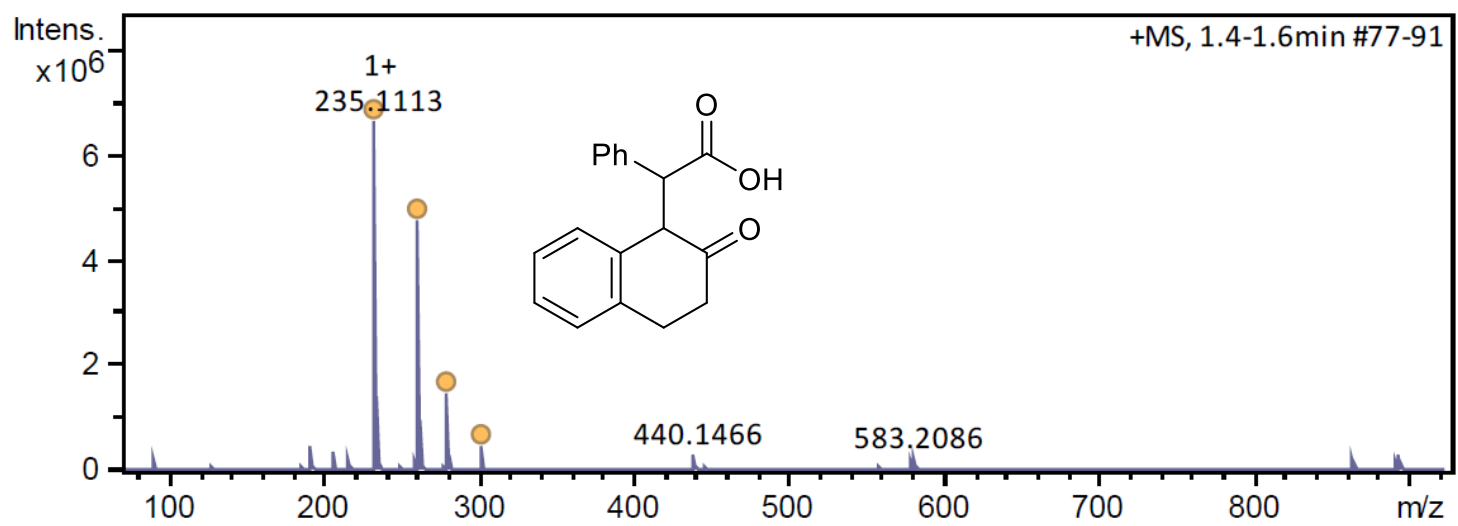

\begin{tabular}{|c|c|c|c|c|c|c|c|c|c|}
\hline Meas. $\mathrm{m} / \mathrm{z}$ & Sum Formula & Adduct & $\mathrm{m} / \mathrm{z}$ & err [mDa] & err [ppm] & Score & mSigma & $\mathrm{rdb}$ & $\mathrm{e}^{-}$Conf \\
\hline 303.098763 & $\mathrm{C} 18 \mathrm{H} 16 \mathrm{O} 3$ & $\mathrm{M}+\mathrm{Na}$ & 303.099165 & 0.4 & 1.3 & 100 & 2.4 & 10.5 & even \\
\hline 281.116807 & & $\mathrm{M}+\mathrm{H}$ & 281.117221 & 0.4 & 1.5 & 100 & 4.3 & 10.5 & even \\
\hline 263.106183 & $\mathrm{C} 18 \mathrm{H} 14 \mathrm{O} 2$ & $\mathrm{M}+\mathrm{H}$ & 263.106656 & 0.5 & 1.8 & 100 & 0.8 & 11.5 & even \\
\hline 235.111278 & $\mathrm{C} 17 \mathrm{H} 14 \mathrm{O}$ & $\mathrm{M}+\mathrm{H}$ & 235.111742 & 0.5 & 2 & 100 & 15.2 & 10.5 & even \\
\hline
\end{tabular}




\section{2-(6-Bromo-2-oxo-1,2,3,4-tetrahydronaphthalen-1-yl)-2-phenylacetic acid (14e)}

(ESI positive)

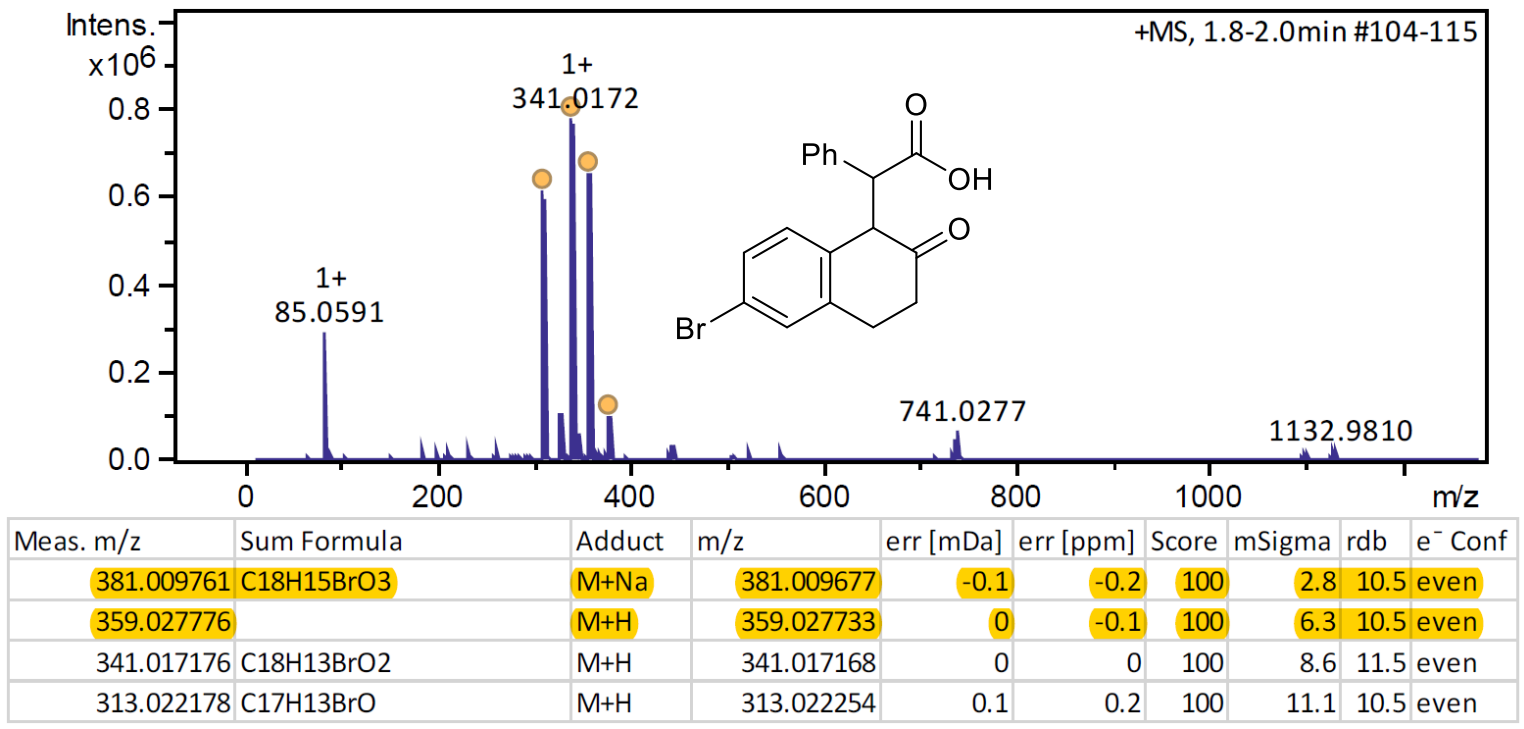

\section{2-(6-Bromo-2-oxo-1,2,3,4-tetrahydronaphthalen-1-yl)-2-phenylacetic acid (14f)}

(ESI positive)

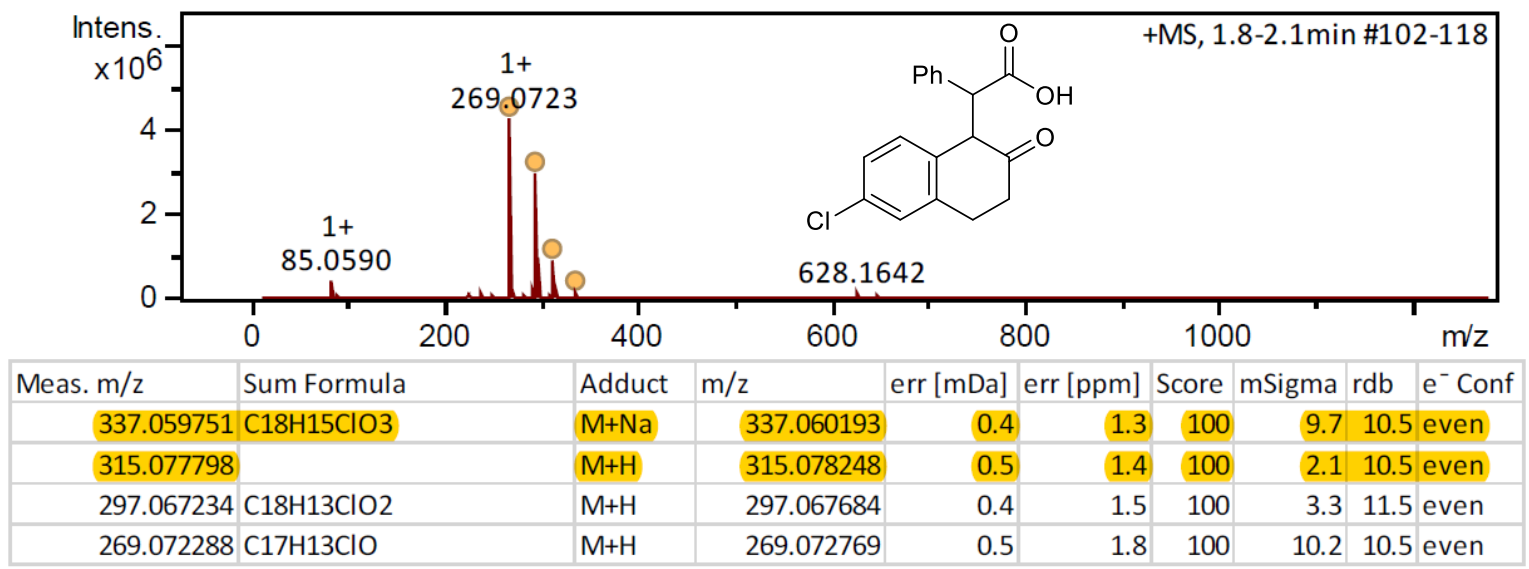


2-(8-Methoxy-2-oxo-1,2,3,4-tetrahydronaphthalen-1-yl)-2-phenylacetic acid (14g)

(ESI positive)

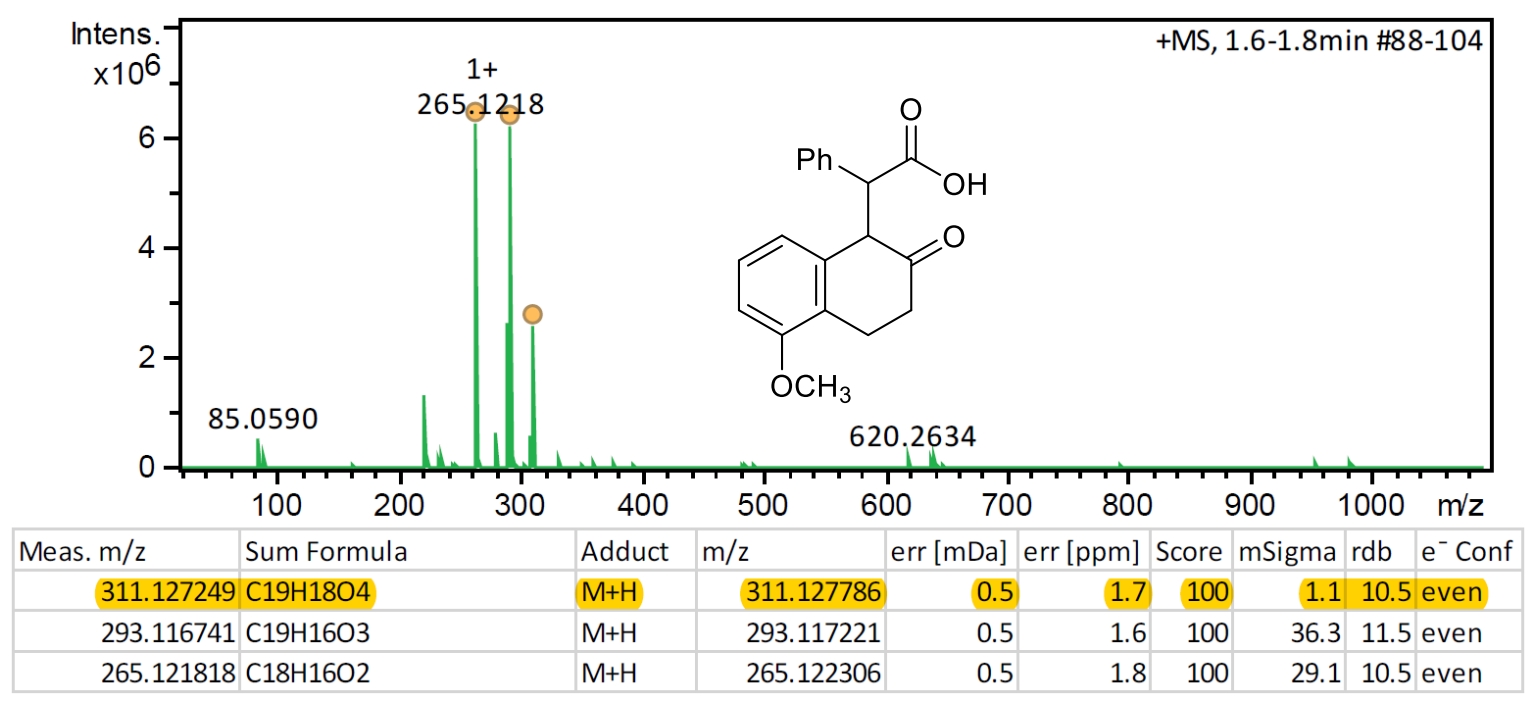

2-(2-Chlorophenyl)-2-(2-oxo-1,2,3,4-tetrahydronaphthalen-1-yl)acetic acid (14h)

(ESI positive)

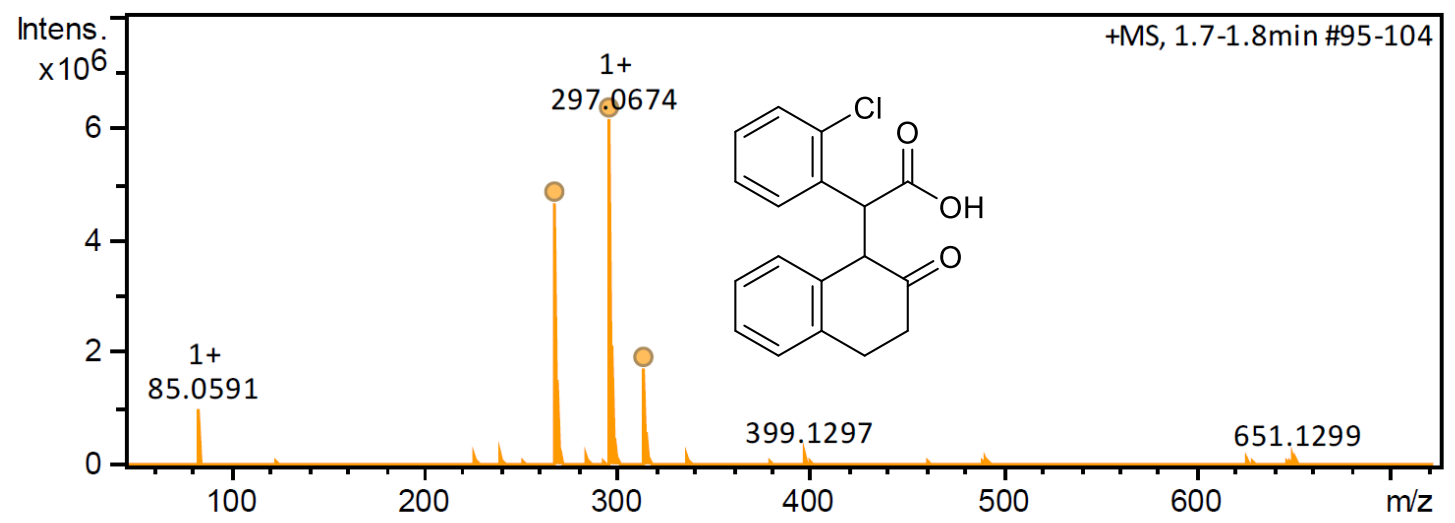

\begin{tabular}{|r|l|l|r|r|r|r|r|r|r|r|r|r|}
\hline Meas. $\mathrm{m} / \mathrm{z}$ & Sum Formula & Adduct & $\mathrm{m} / \mathrm{z}$ & err [mDa] & err [ppm] & Score & mSigma & rdb & $\mathrm{e}^{-}$Conf \\
\hline 315.078018 & $\mathrm{C} 18 \mathrm{H} 15 \mathrm{ClO} 3$ & $\mathrm{M}+\mathrm{H}$ & 315.078248 & 0.2 & 0.7 & 100 & 5.6 & 10.5 even \\
\hline 297.06742 & $\mathrm{C} 18 \mathrm{H} 13 \mathrm{ClO} 2$ & $\mathrm{M}+\mathrm{H}$ & 297.067684 & 0.3 & 0.9 & 100 & 5.4 & 11.5 even \\
\hline 269.072408 & $\mathrm{C} 17 \mathrm{H} 13 \mathrm{ClO}$ & $\mathrm{M}+\mathrm{H}$ & 269.072769 & 0.4 & 1.3 & 100 & 4.9 & 10.5 even \\
\hline
\end{tabular}


2-(7-Methoxy-2-oxo-1,2,3,4-tetrahydronaphthalen-1-yl)-2-phenylacetic acid (14i)

(FD)

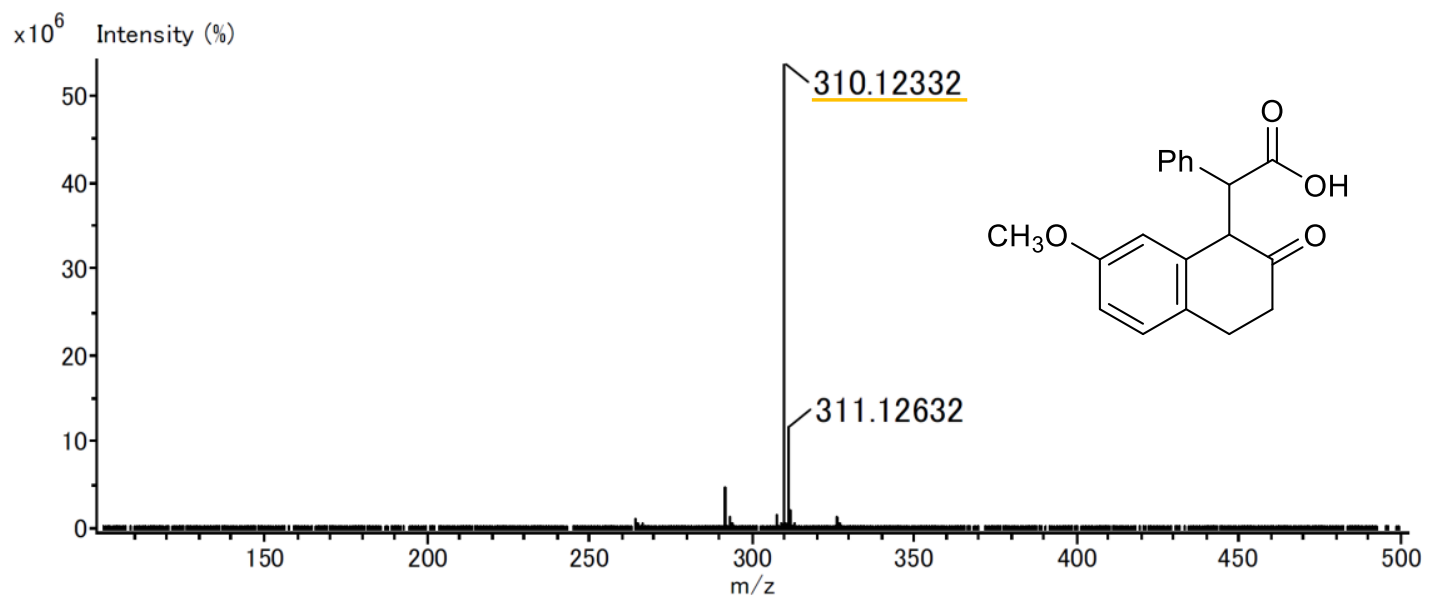

\section{2-(1-Oxo-1,2,3,4-tetrahydronaphthalen-2-yl)-2-phenylacetic acid (17a)}

(APCI positive)

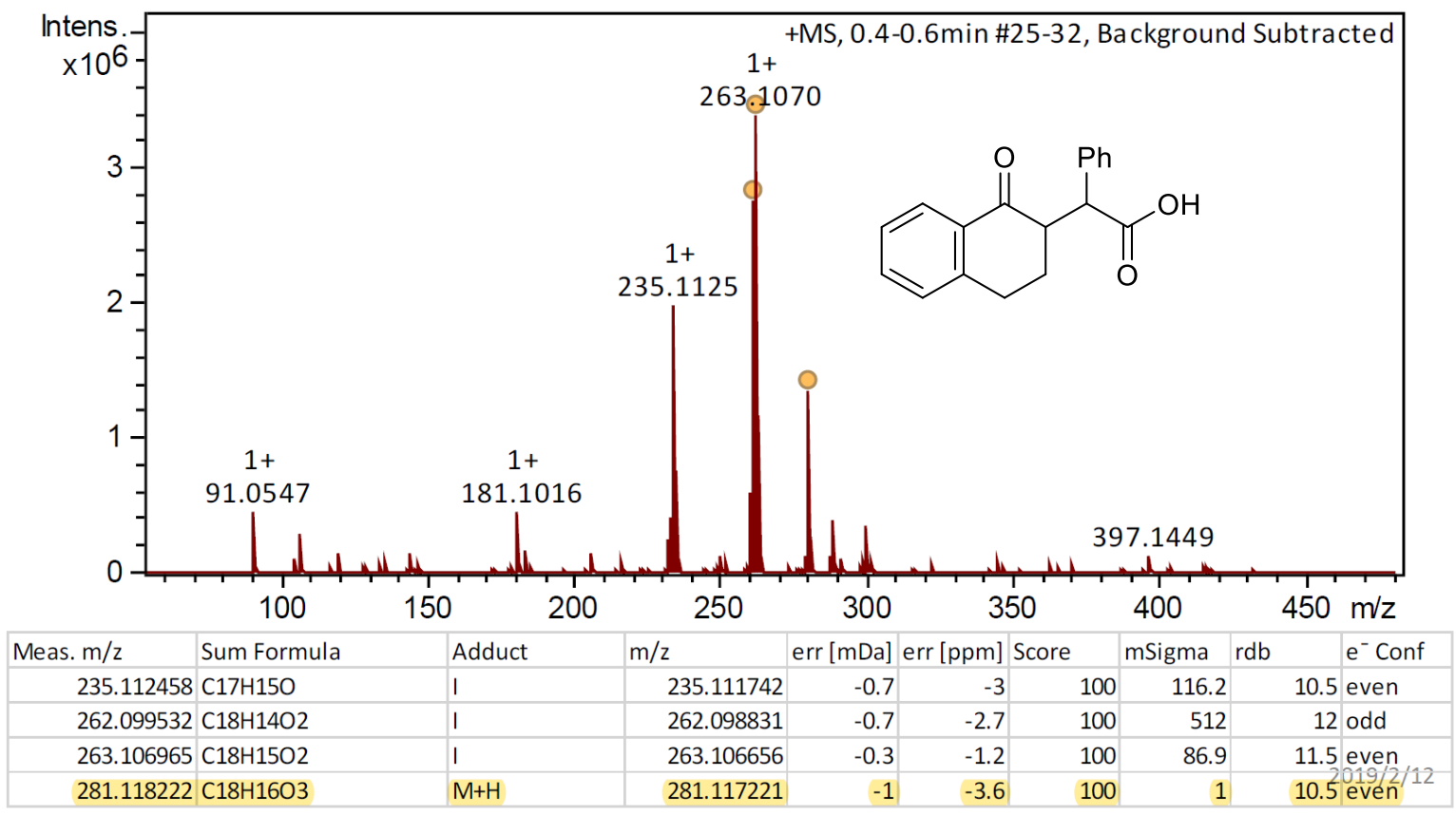


2-(7-Chloro-1-oxo-1,2,3,4-tetrahydronaphthalen-2-yl)-2-phenylacetic acid (17b)

(APCI positive)

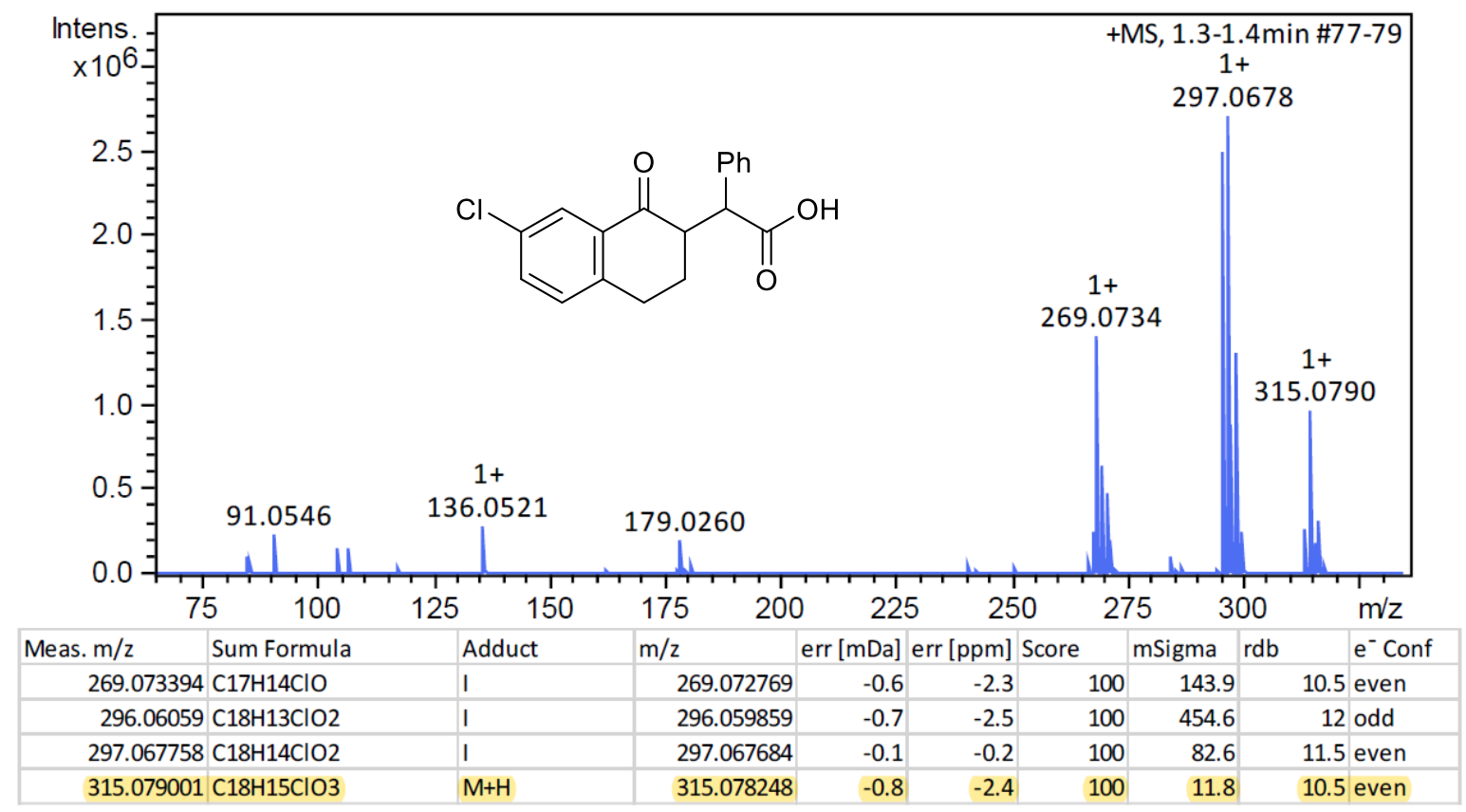

2-(1-Oxo-1,2,3,4-tetrahydronaphthalen-2-yl)propanoic acid (17c)

(FD)

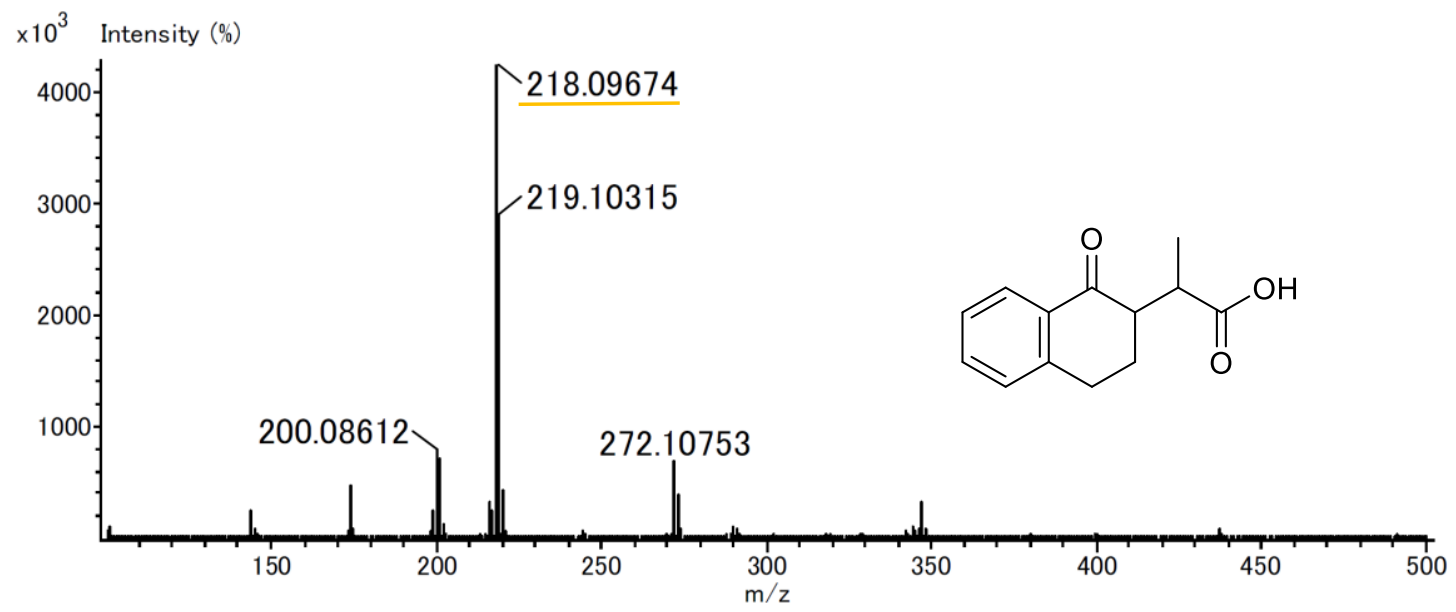


(1R,2R)-2-Methyl-1,2,3,4-tetrahydronaphthalen-1-ol (9a)

(FI)

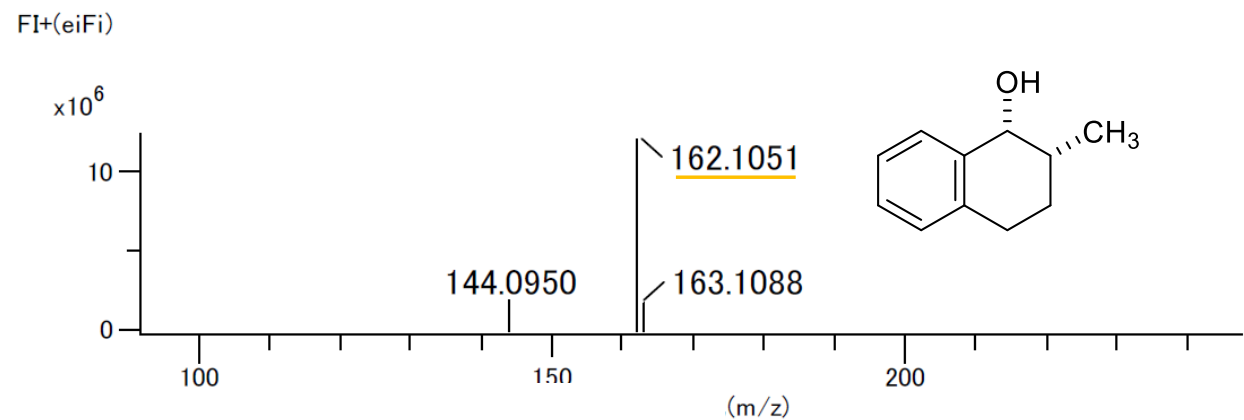

\section{$(1 R, 2 R)-2-M e t h y l-2,3-d i h y d r o-1 H-i n d e n-1-o l ~(9 b)$}

(FI)

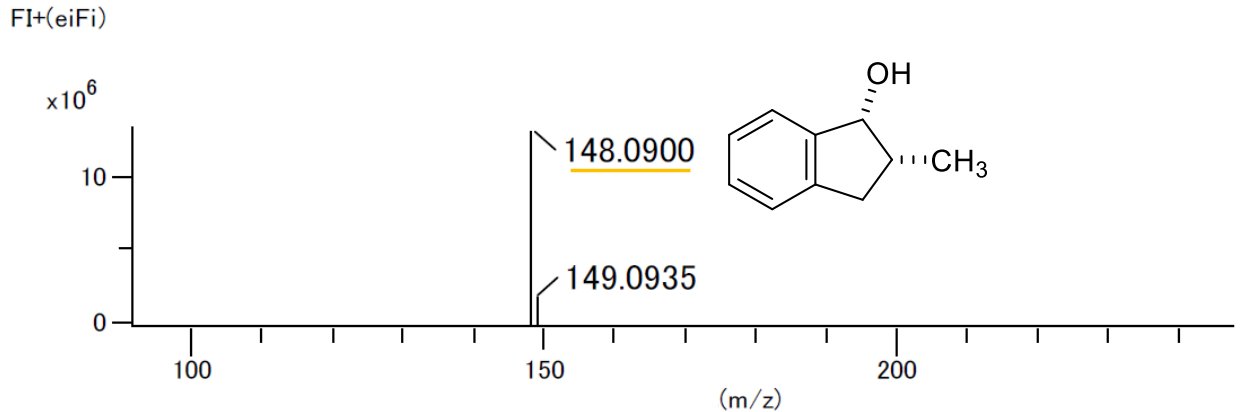


(5R,6R)-6-Methyl-6,7,8,9-tetrahydro-5H-benzo[7]annulen-5-ol (9c)

(FI)

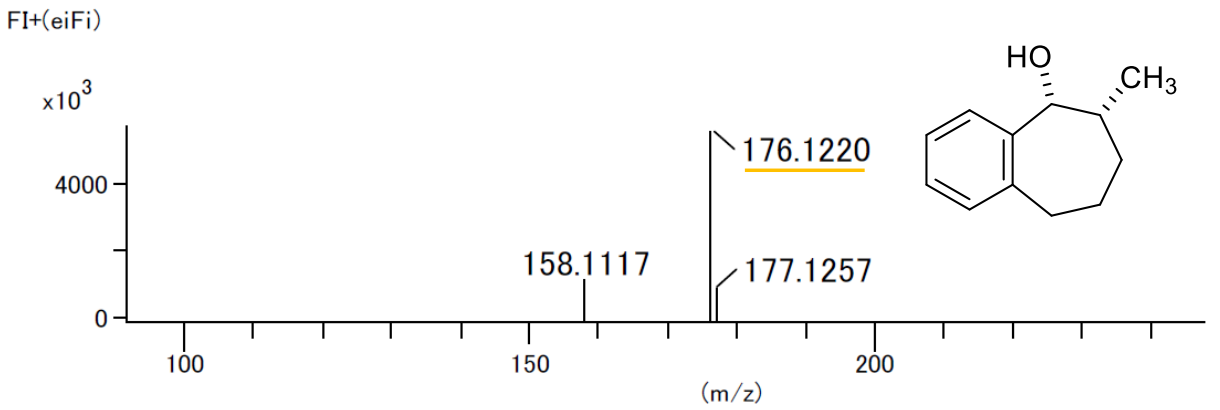

Methyl (1R,2R)-1-hydroxy-1,2,3,4-tetrahydronaphthalene-2-carboxylate (9d)

(FI)

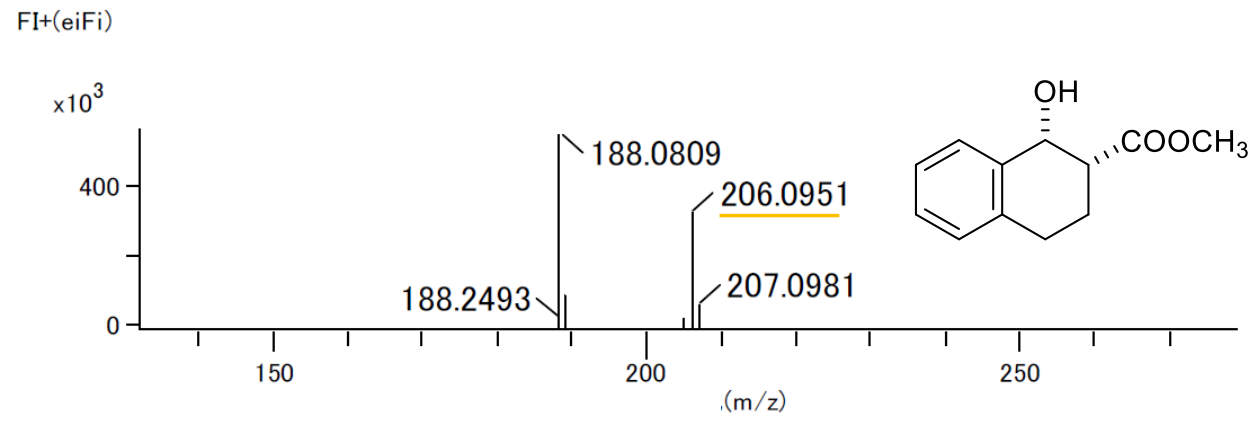




\section{(3aS,9bR)-3a,4,5,9b-Tetrahydronaphtho[1,2-b]furan-2(3H)-one (13a)}

(ESI positive)

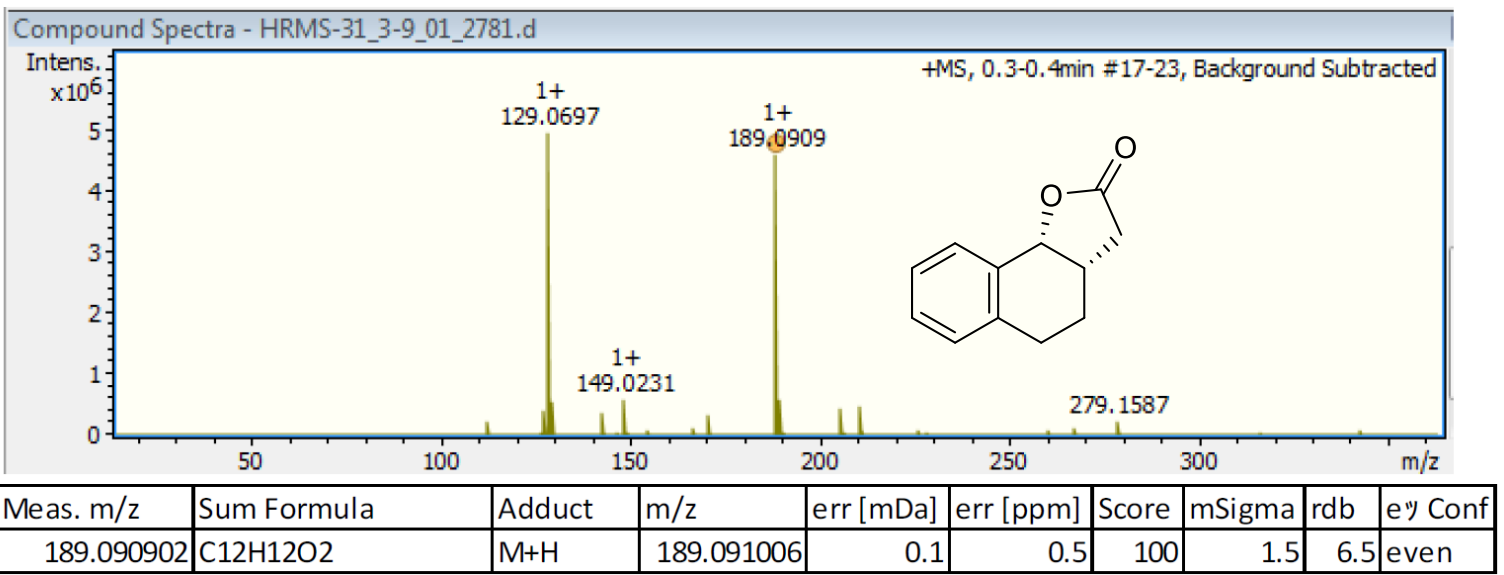

(3aS,9bR)-8-Methoxy-3a,4,5,9b-tetrahydronaphtho[1,2-b]furan-2(3H)-one (13b)

(APCI positive)

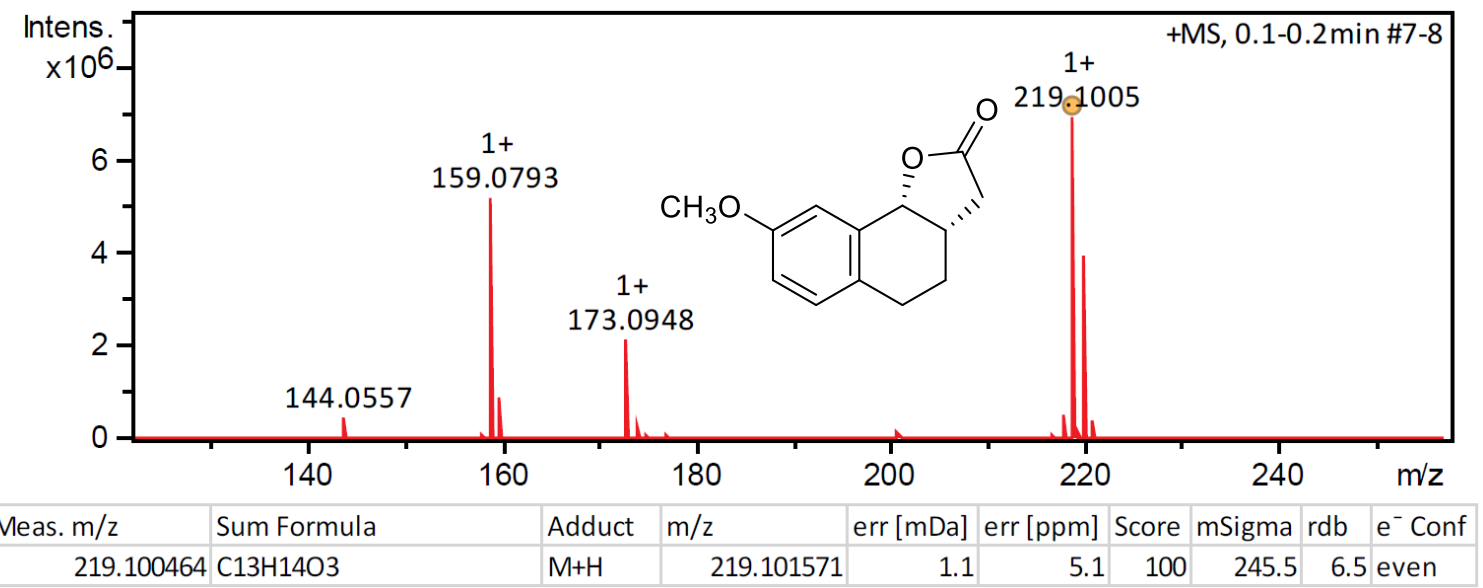




\section{(3aS,9bR)-8-Chloro-3a,4,5,9b-tetrahydronaphtho[1,2-b]furan-2(3H)-one (13c)}

(APCI positive)

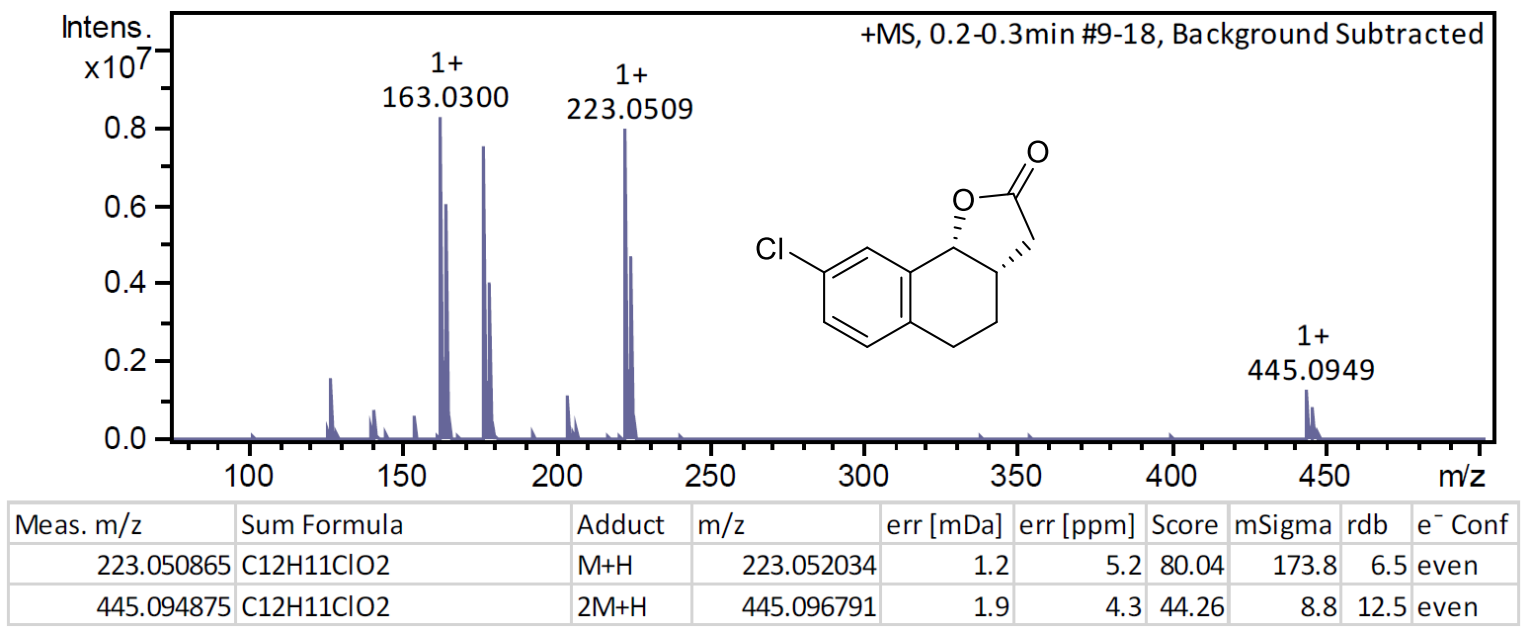

(3aS,9bR)-8-Bromo-3a,4,5,9b-tetrahydronaphtho[1,2-b]furan-2(3H)-one (13d)

(ESI positive)

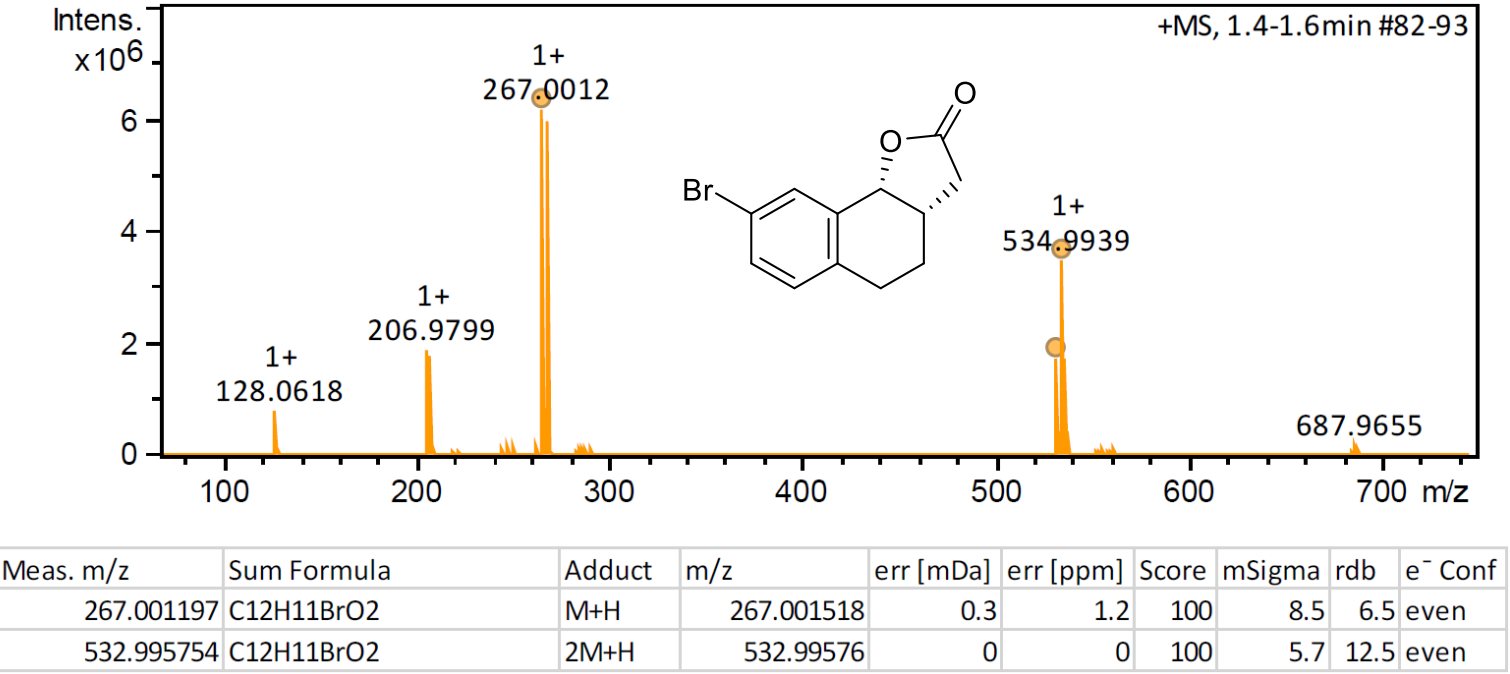


(3aS,9bR)-9-Bromo-8-methoxy-3a,4,5,9b-tetrahydronaphtho[1,2-b]furan-2(3H)-one (13e)

(ESI positive)

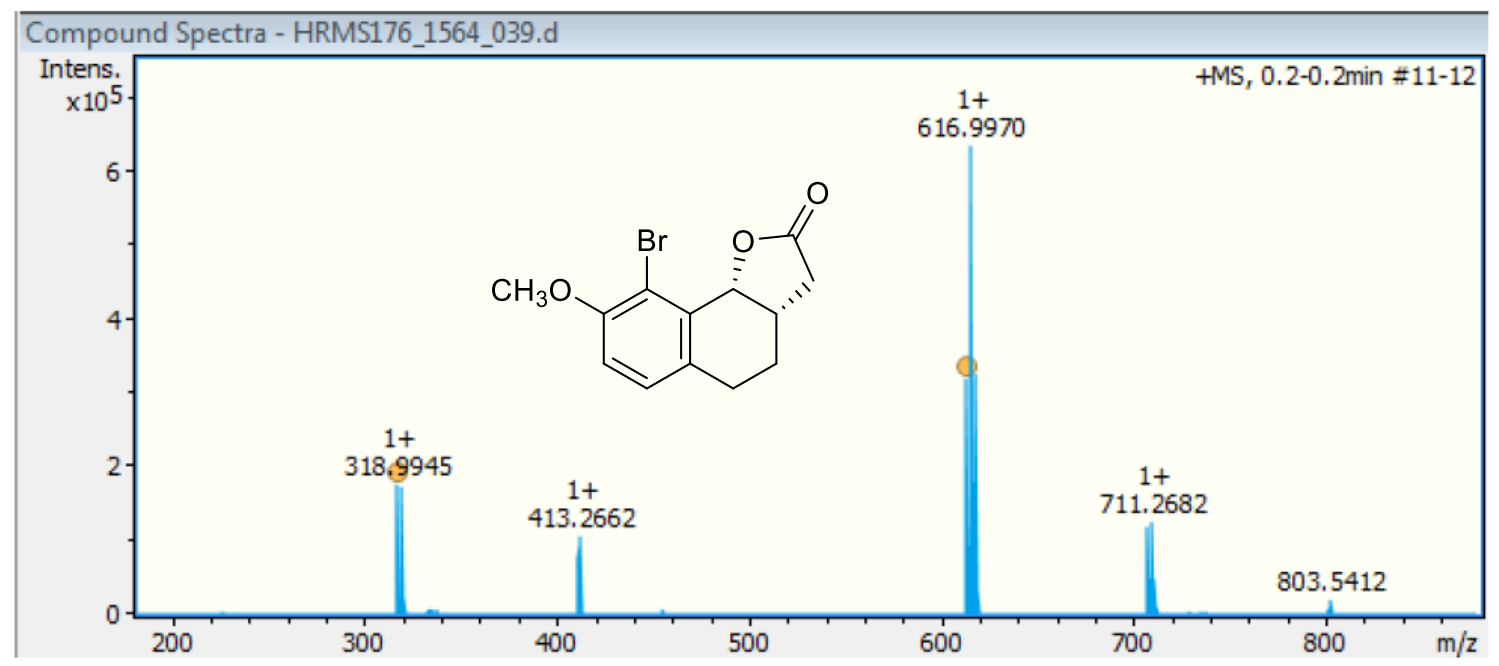

\begin{tabular}{|c|c|c|c|r|r|r|r|r|l|}
\hline Meas. $\mathrm{m} / \mathrm{z}$ & Sum Formula & Adduct & \multicolumn{1}{c|}{$\mathrm{m} / \mathrm{z}$} & $\begin{array}{c}\text { err } \\
{[\mathrm{mDa}]}\end{array}$ & \multicolumn{1}{c|}{$\begin{array}{c}\text { err } \\
{[\mathrm{ppm}]}\end{array}$} & Score & $\mathrm{mSigma}$ & $\mathrm{rdb}$ & $\mathrm{e}^{-}$Conf \\
\hline 318.9945 & $\mathrm{C} 13 \mathrm{H} 13 \mathrm{BrO3}$ & $\mathrm{M}+\mathrm{Na}$ & 318.994 & -0.5 & -1.6 & 100 & 4 & 6.5 even \\
\hline 614.9988 & $\mathrm{C} 13 \mathrm{H} 13 \mathrm{BrO3}$ & $2 \mathrm{M}+\mathrm{Na}$ & 614.9988 & 0 & 0.1 & 100 & 4.3 & 12.5 & even \\
\hline
\end{tabular}

\section{(3aS,9bR)-7-Hydroxy-3a,4,5,9b-tetrahydronaphtho[1,2-b]furan-2(3H)-one (13f)}

(APCI positive)

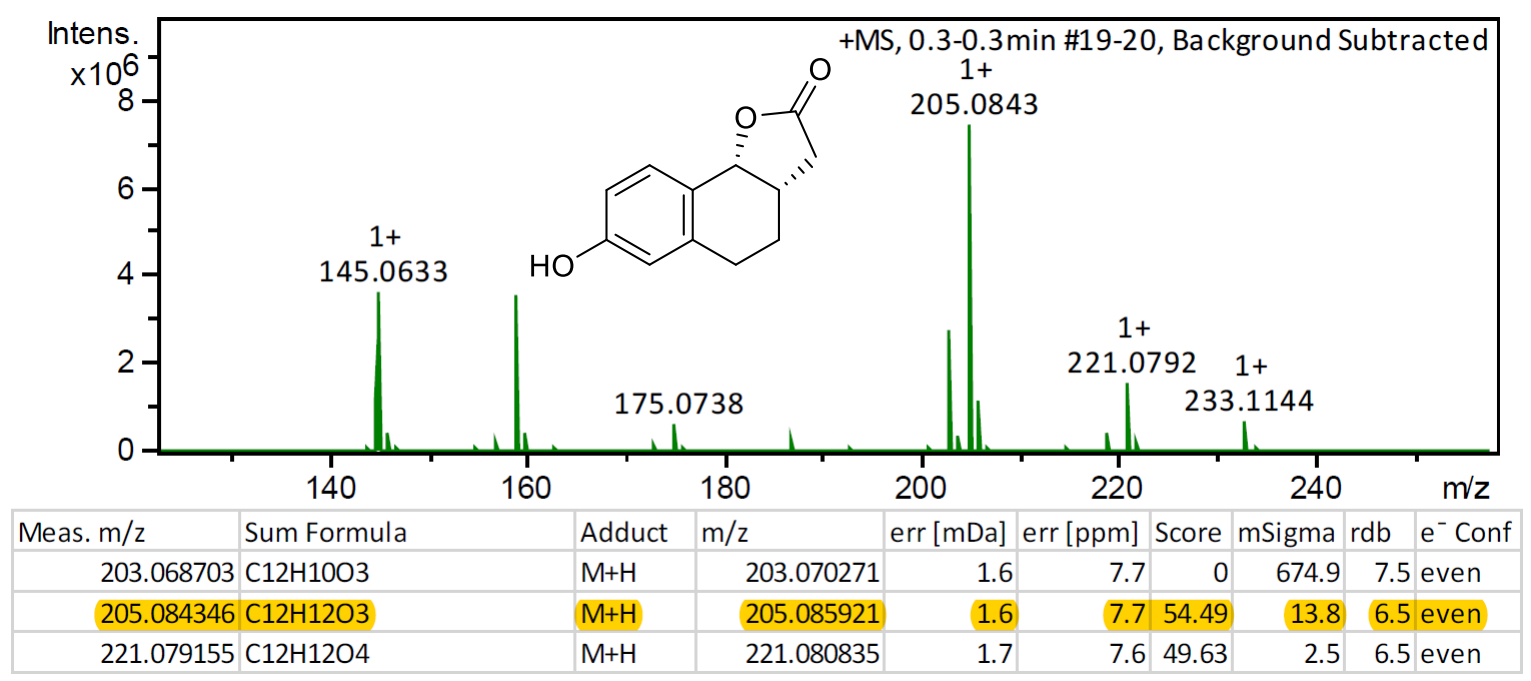




\section{(3aS,8bR)-3,3a,4,8b-Tetrahydro-2H-indeno[1,2-b]furan-2-one (13g)}

(ESI positive)

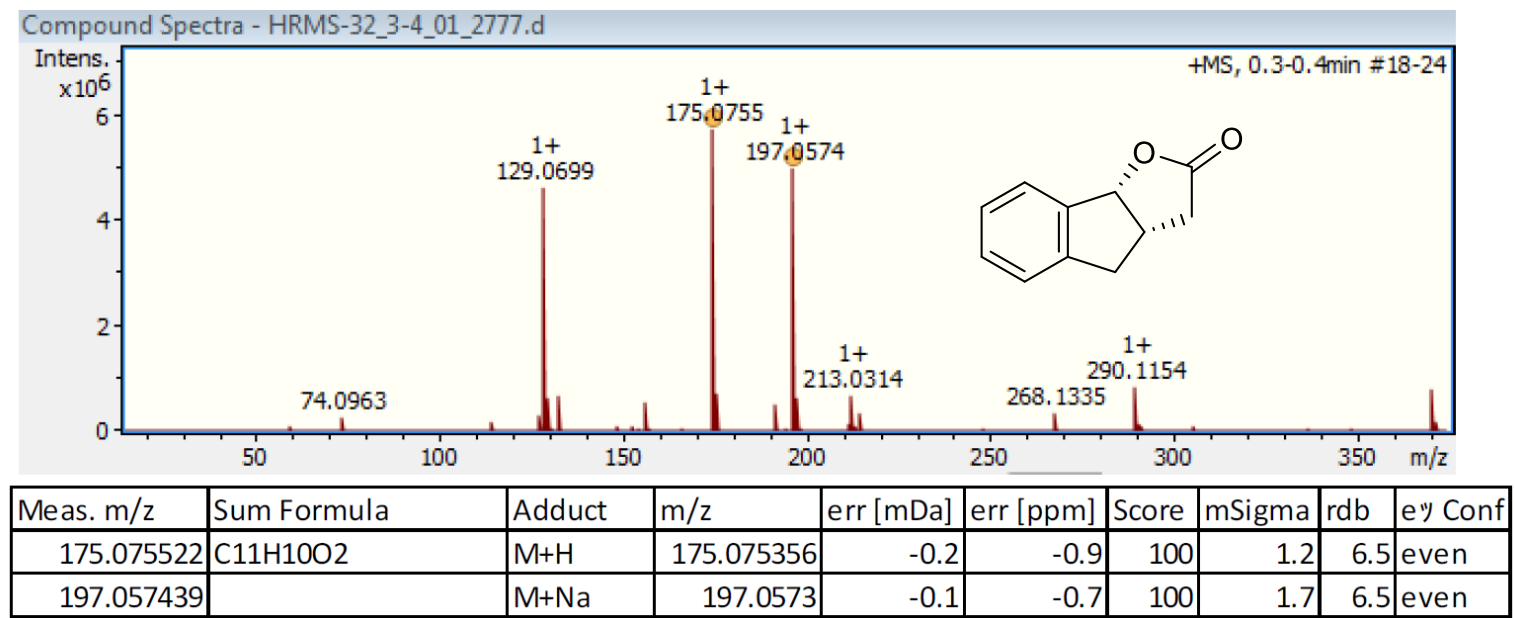

\section{(3aS,8bR)-6,7-Dimethoxy-3,3a,4,8b-tetrahydro- $2 H$-indeno[1,2-b]furan-2-one (13h)}

(APCI positive)

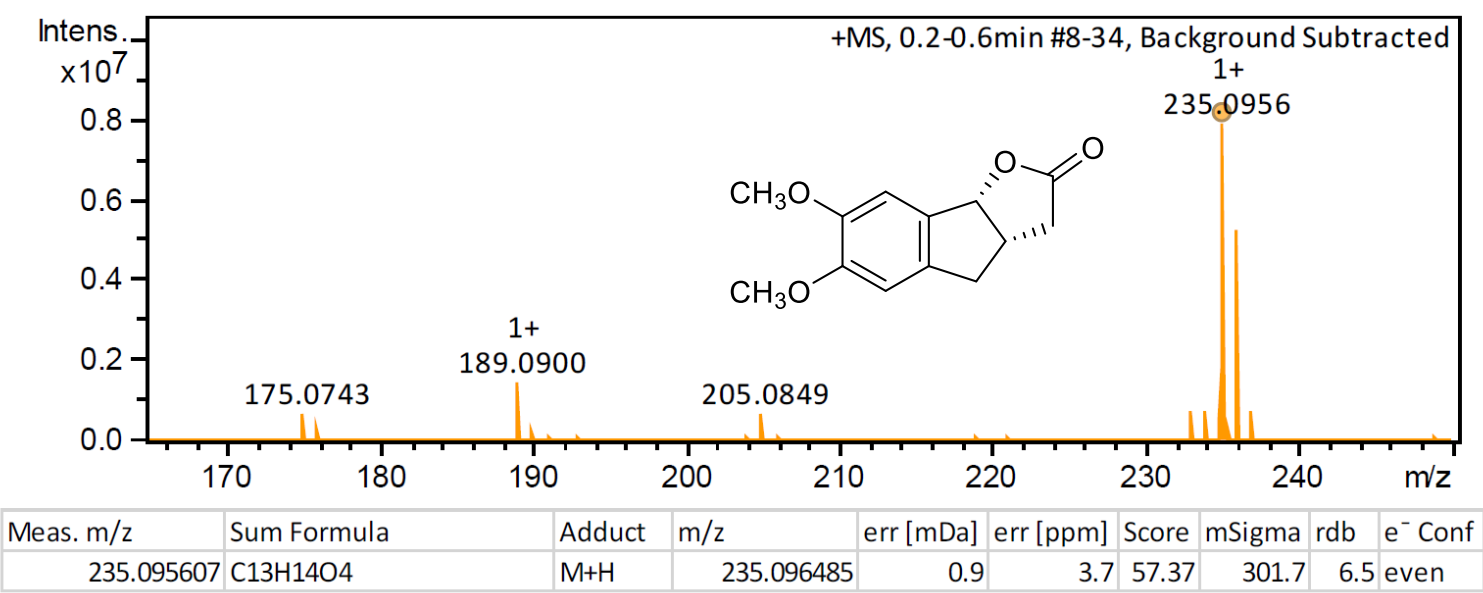


(3aS,8bR)-7-Fluoro-3,3a,4,8b-tetrahydro- $2 H$-indeno[1,2-b]furan-2-one (13i)

(APCI positive)

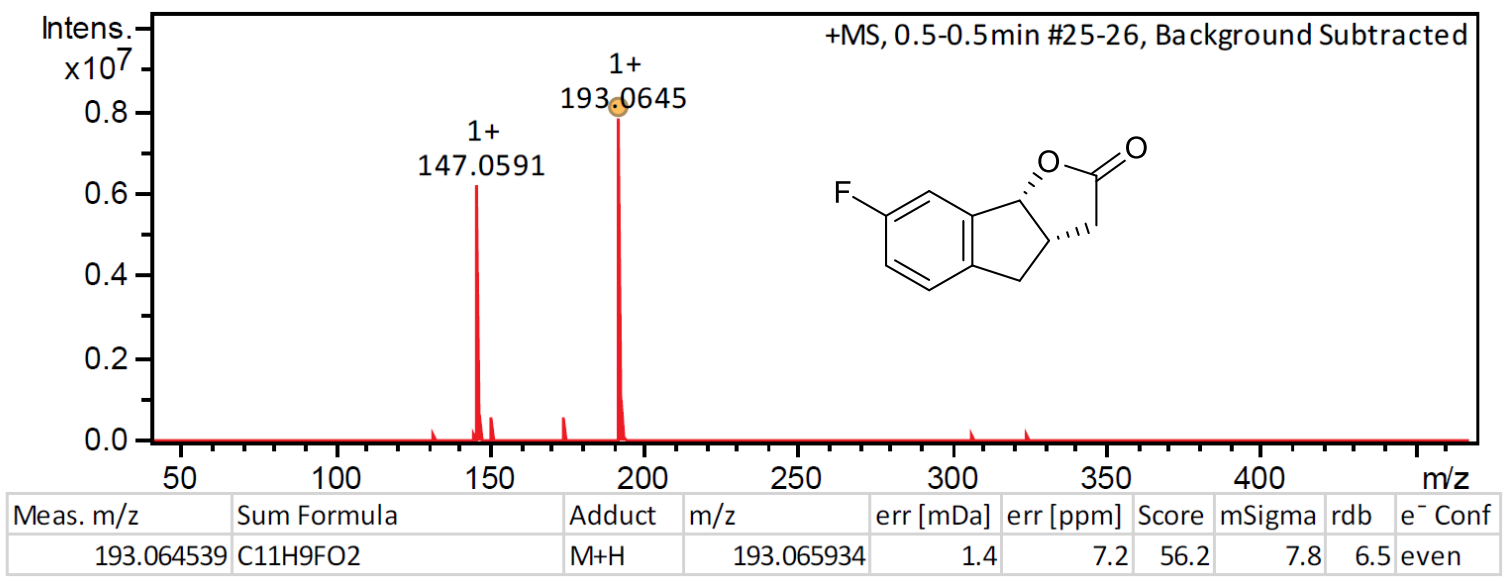

(3aS,8bR)-7-Chloro-3,3a,4,8b-tetrahydro-2H-indeno[1,2-b]furan-2-one (13j)

(ESI positive)

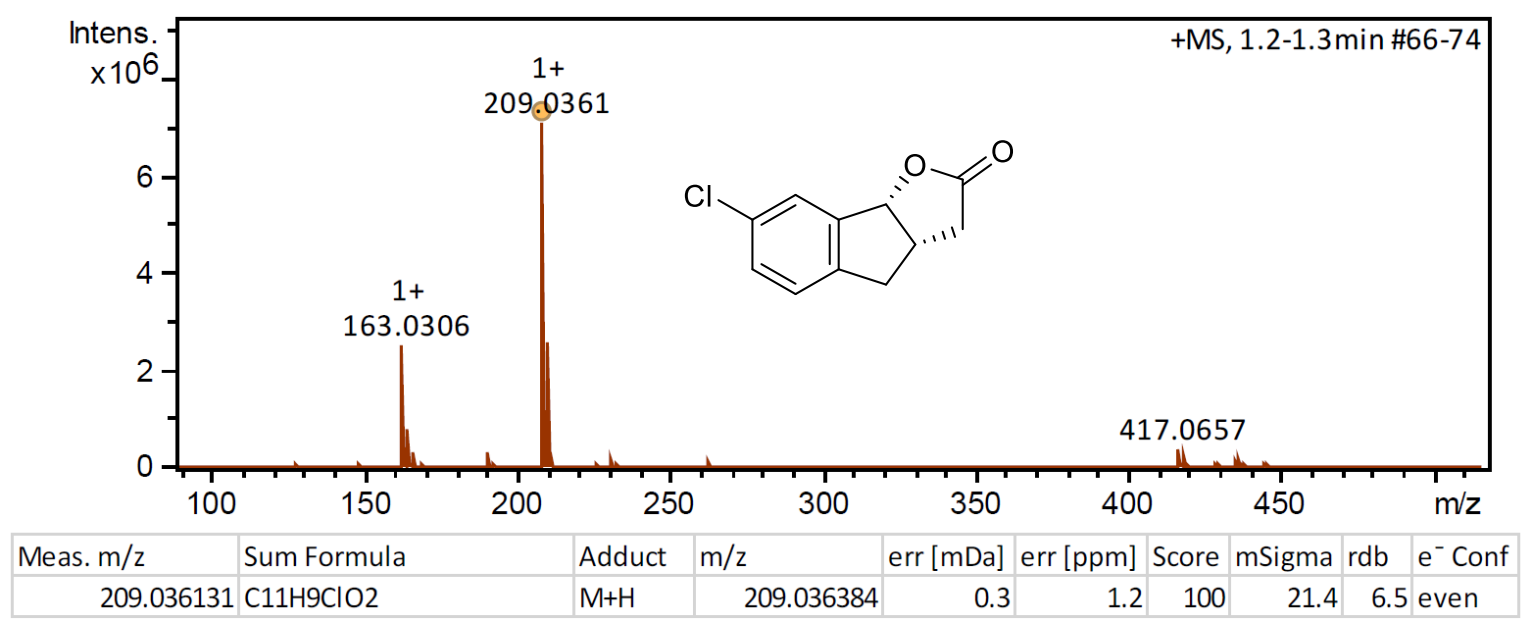


$(3 a S, 10 b R)-3,3 a, 4,5,6,10 b-H e x a h y d r o-2 H$-benzo[6,7]cyclohepta[1,2-b]furan-2-one (13k)

(FI)

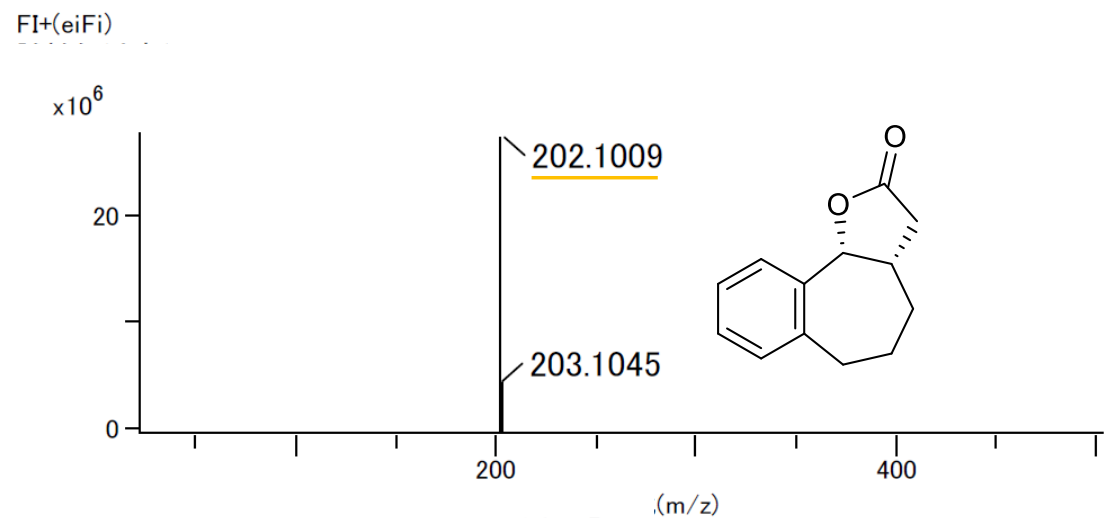

(4aS,10bR)-3,4,4a,5,6,10b-Hexahydro-2H-benzo[h]chromen-2-one (13I)

(ESI positive)

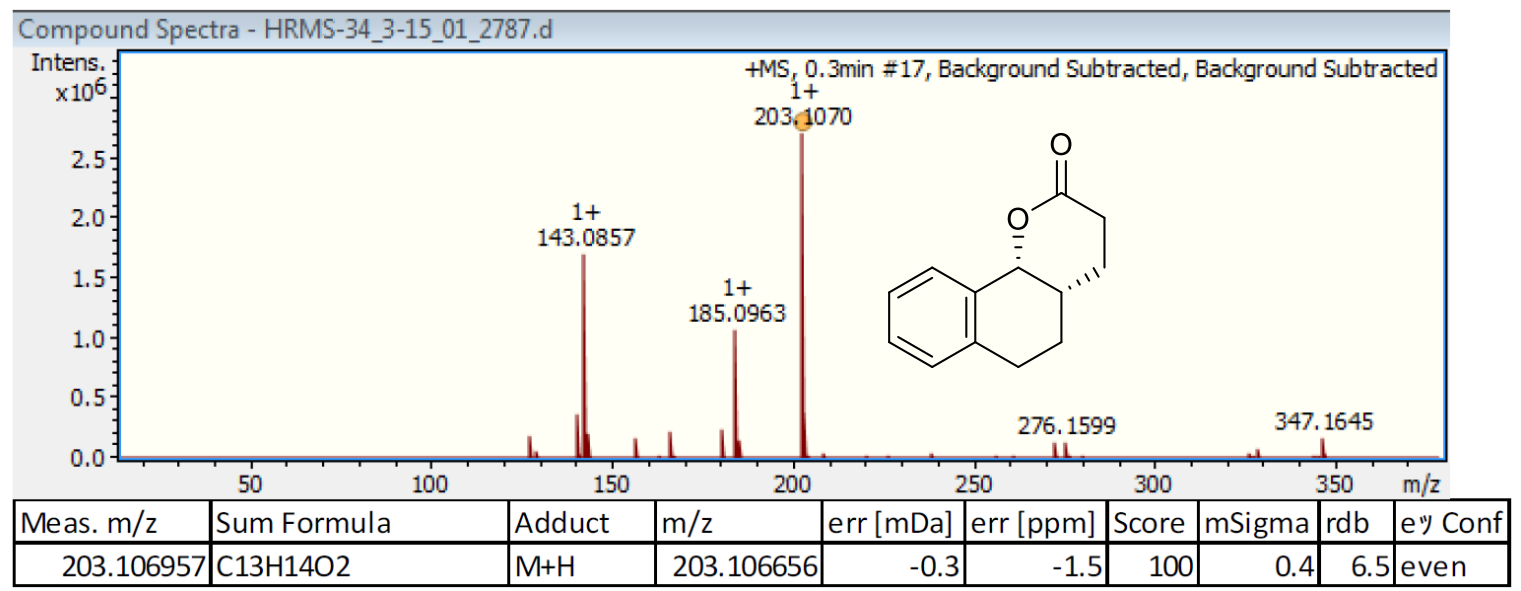


$(4 \mathrm{a} S, 10 \mathrm{~b} R)-9-M e t h o x y-3,4,4 a, 5,6,10 b-h e x a h y d r o-2 H$-benzo[ $h]$ chromen-2-one $(13 \mathrm{~m})$

(APCI positive)

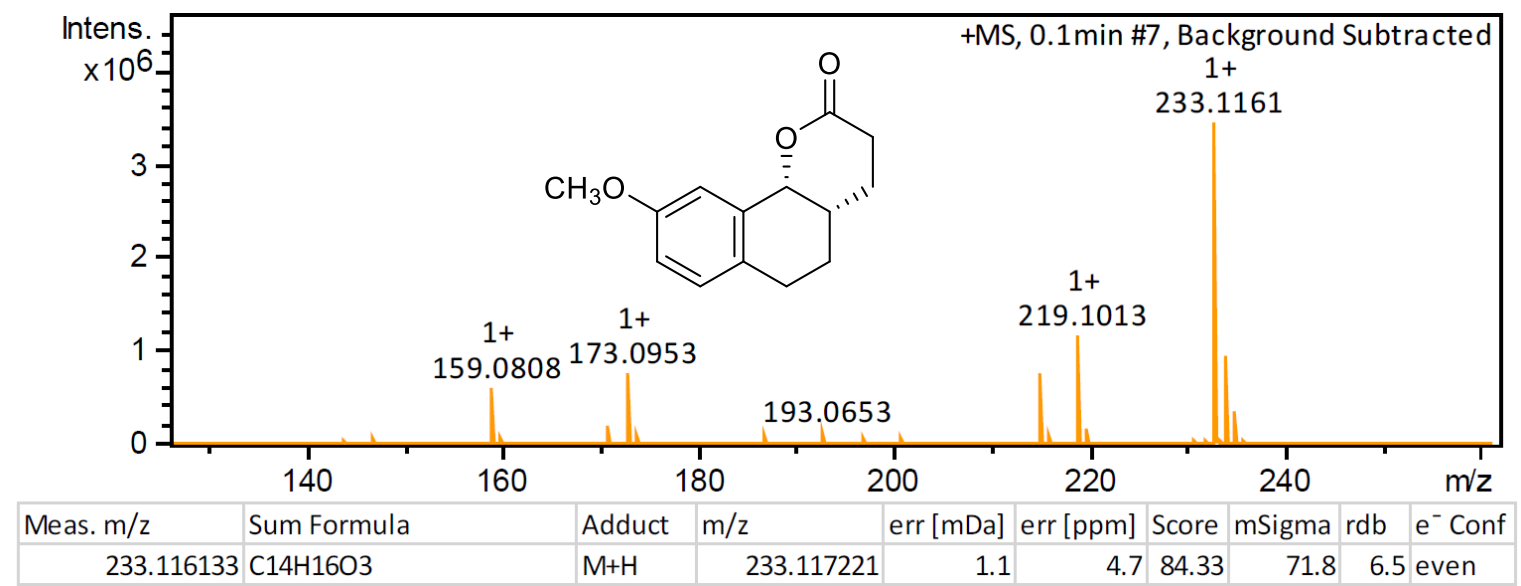

(4aS,10bR)-9-Chloro-3,4,4a,5,6,10b-hexahydro-2H-benzo[h]chromen-2-one (13n) (APCI positive)

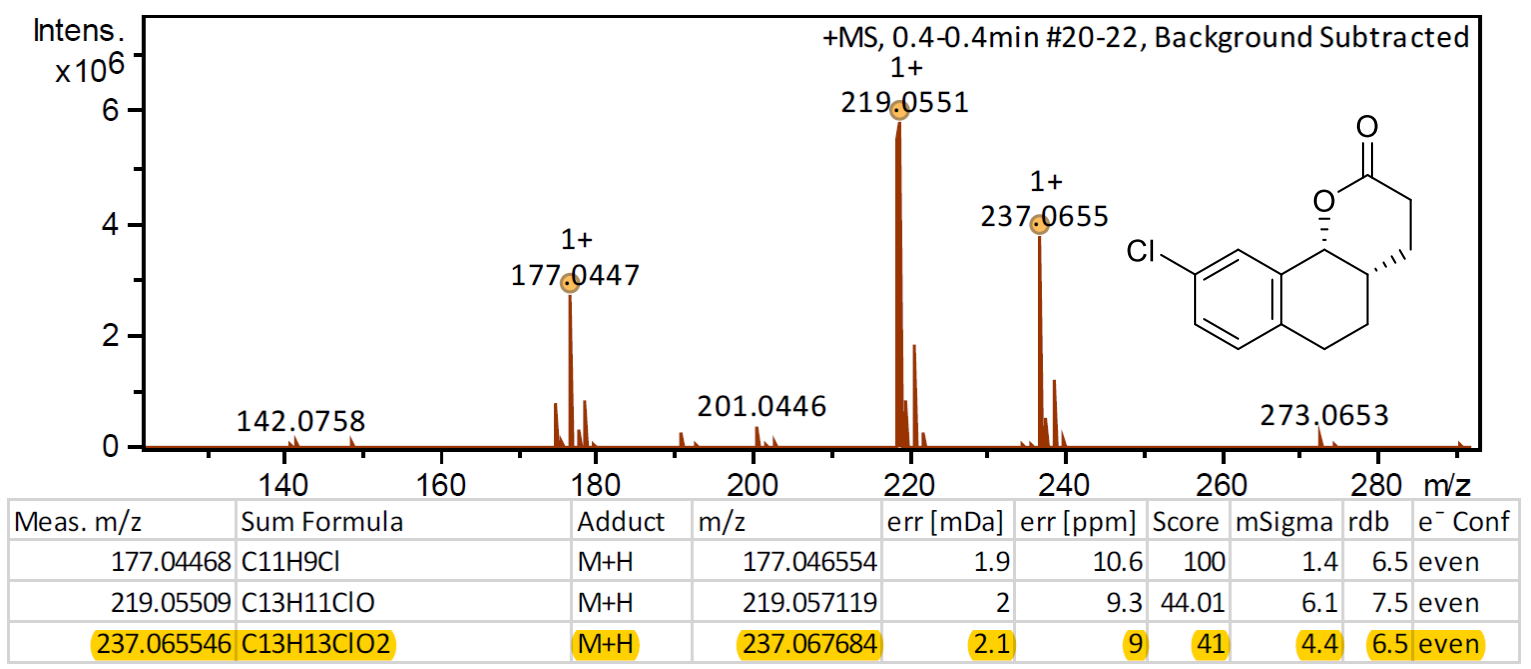




\section{$(3 \mathrm{a} R, 9 \mathrm{bS})-3 \mathrm{a}, 4,5,9 \mathrm{~b}-$ Tetrahydronaphtho[2,1-b]furan-2(1H)-one (13o)}

(ESI positive)

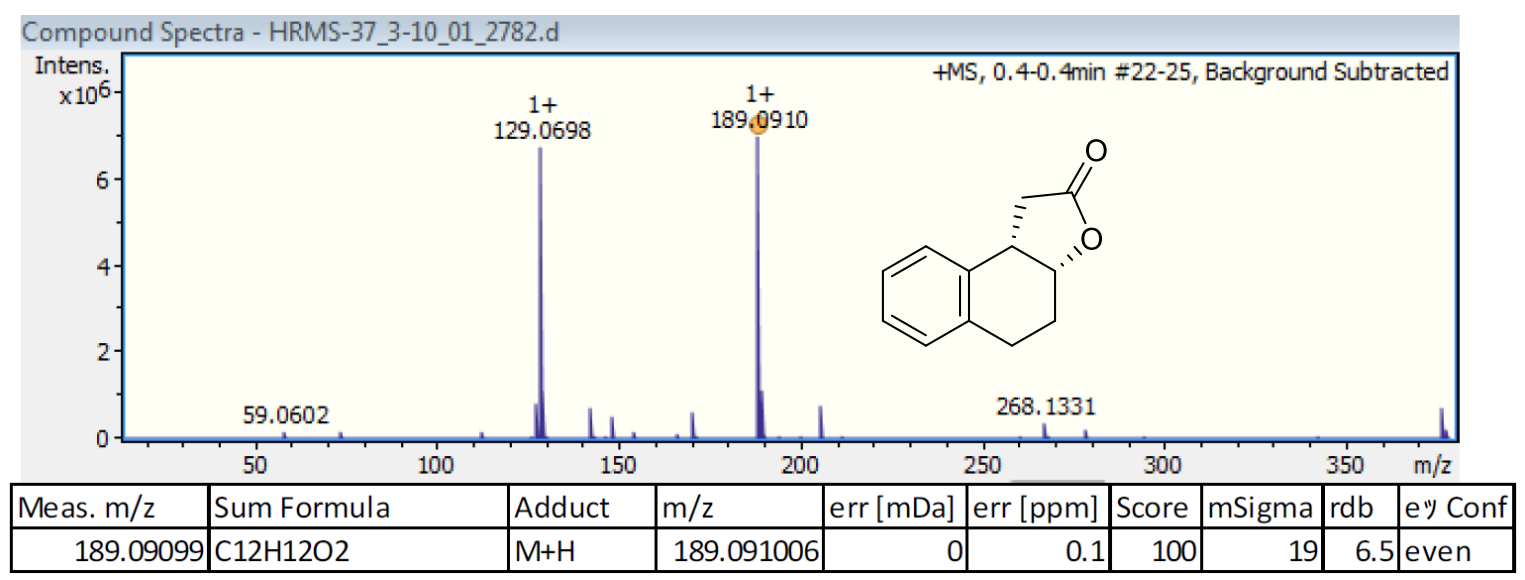

\section{$(3 \mathrm{a} R, 9 \mathrm{~b} S)-6-$ Methoxy-3a,4,5,9b-tetrahydronaphtho[2,1-b]furan-2(1H)-one (13p)}

(APCI positive)

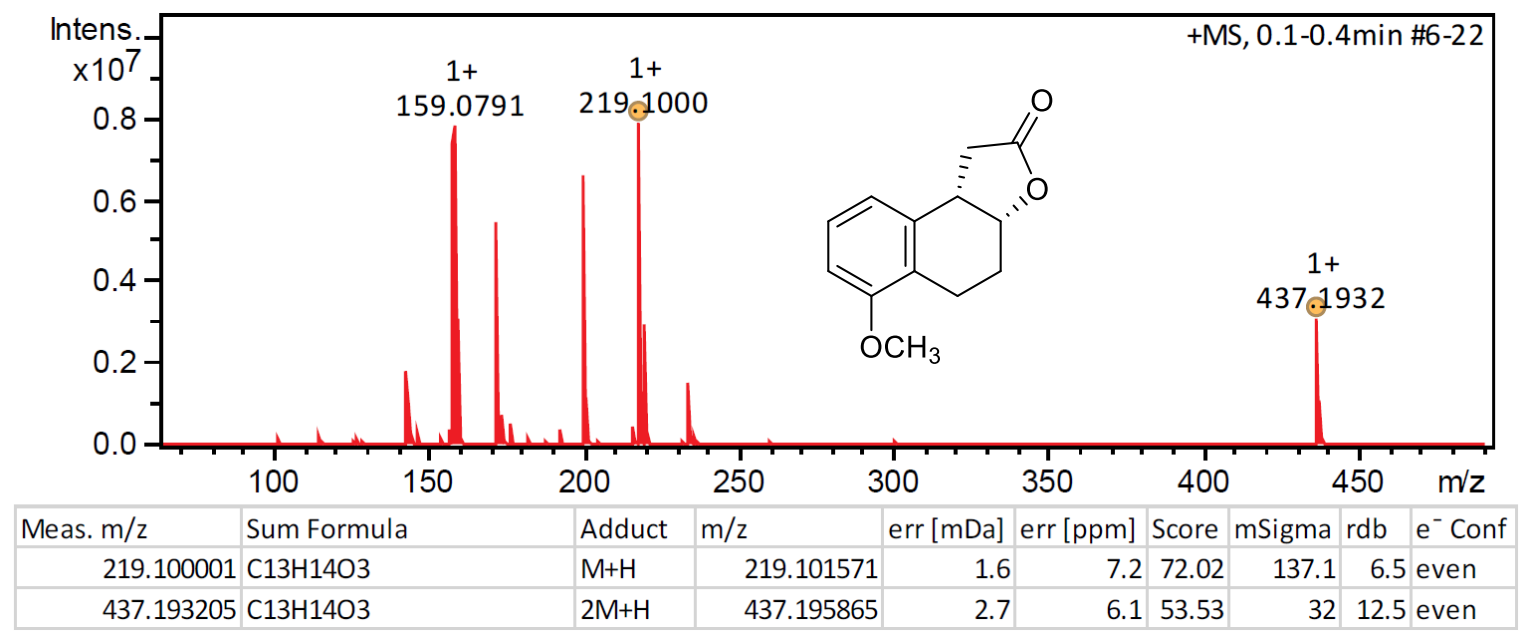


$(3 \mathrm{a} R, 9 \mathrm{bS})-7-B r o m o-3 a, 4,5,9 \mathrm{~b}-$ tetrahydronaphtho[2,1-b]furan-2(1H)-one (13q)

(ESI positive)

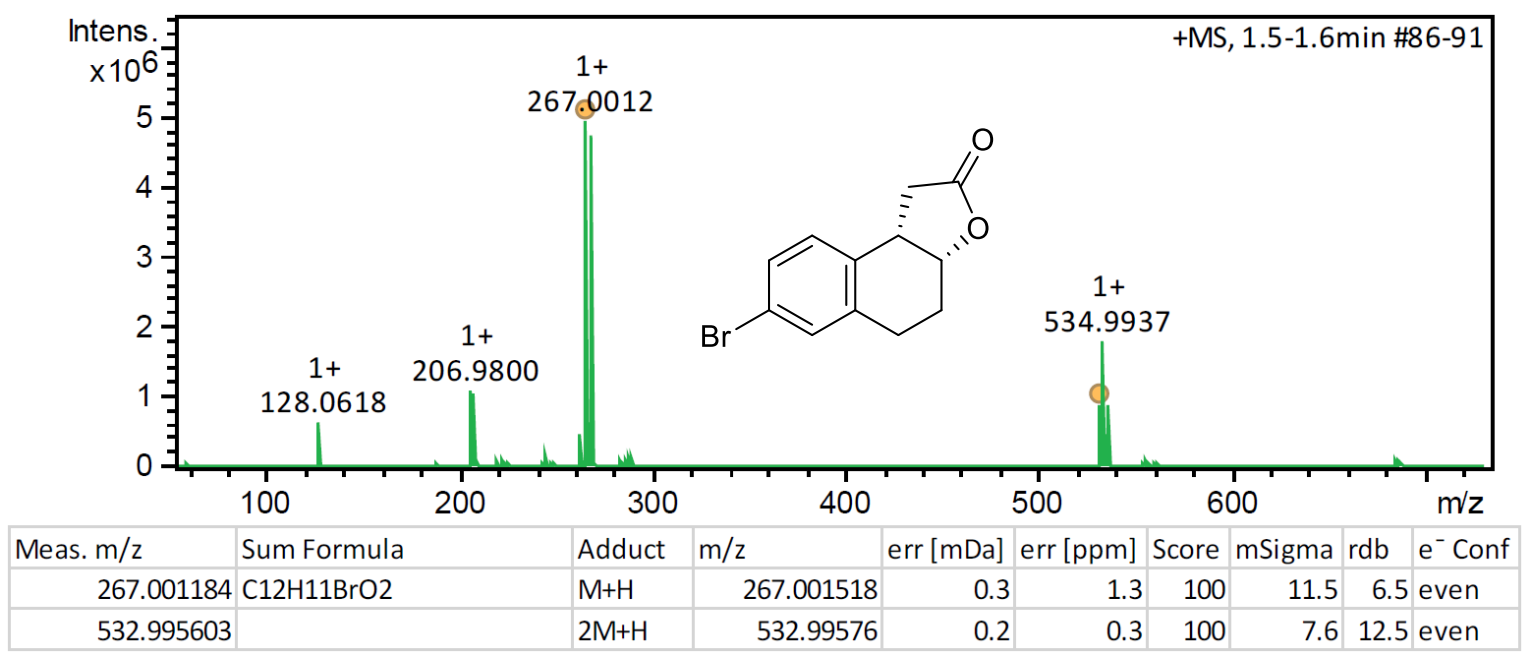

$(3 \mathrm{a} R, 9 \mathrm{bS})-7-C h l o r o-3 \mathrm{a}, 4,5,9 \mathrm{~b}-$ tetrahydronaphtho[2,1-b]furan-2(1H)-one $(13 \mathrm{r})$

(APCI positive)

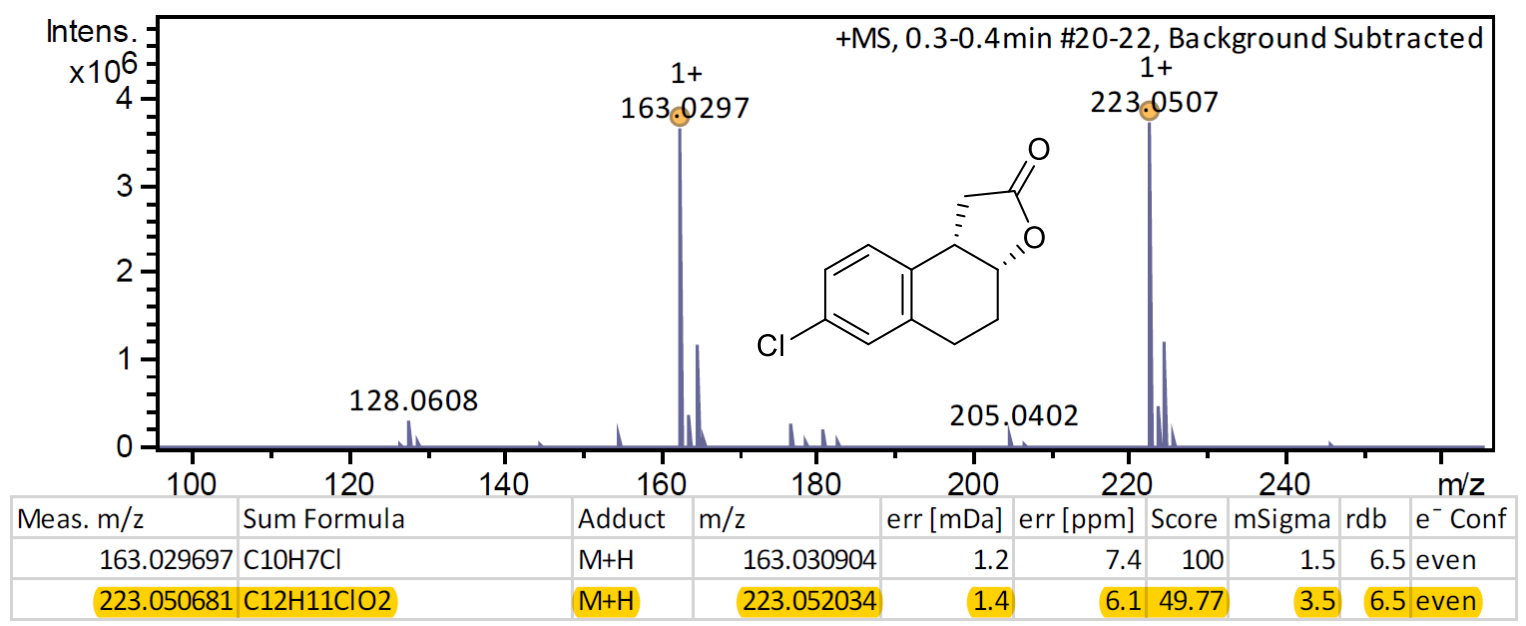


$(1 R, 3 a R, 9 b S)-1-M e t h y l-3 a, 4,5,9 b-t e t r a h y d r o n a p h t h o[2,1-b] f u r a n-2(1 H)$-one $(15 a)$

(ESI positive)

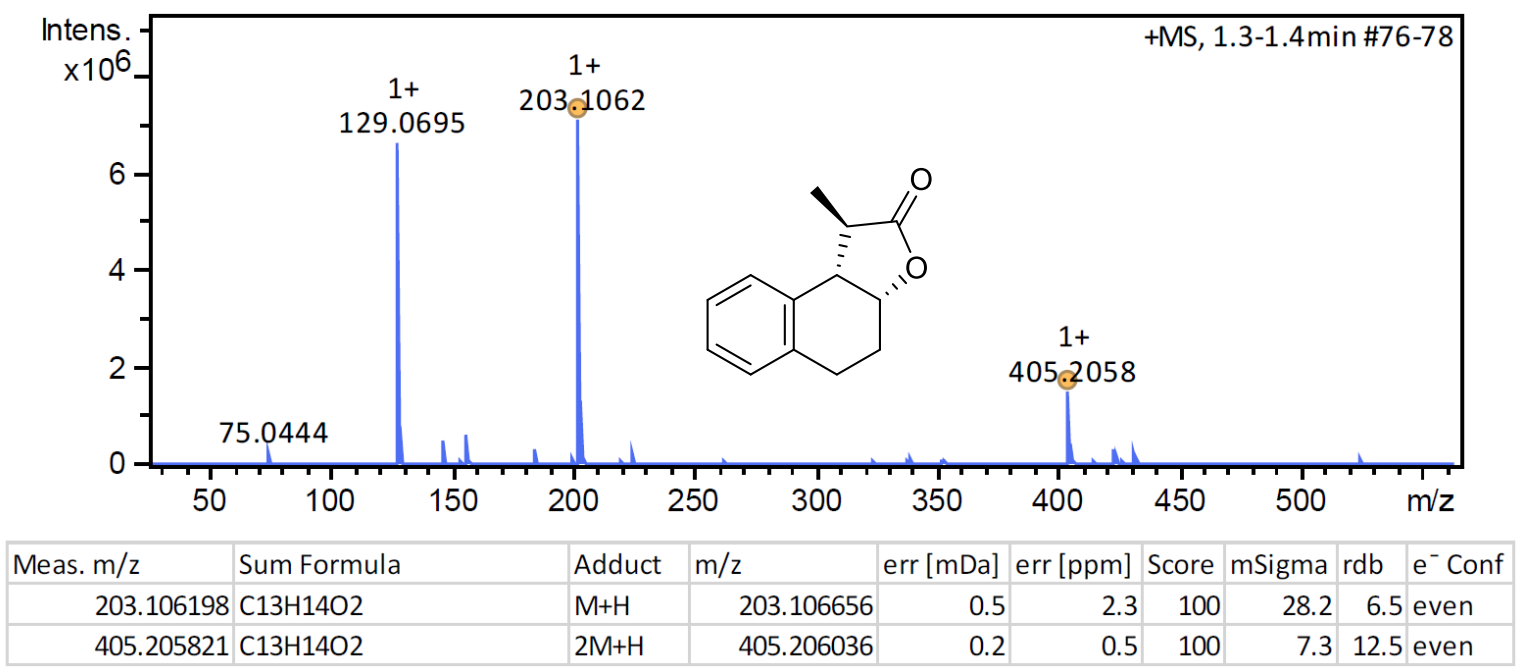

$(1 R, 3 \mathrm{a} R, 9 \mathrm{~b} S)-1-E t h y l-3 \mathrm{a}, 4,5,9 \mathrm{~b}-$ tetrahydronaphtho[2,1-b]furan-2(1H)-one (15b)

(ESI positive)

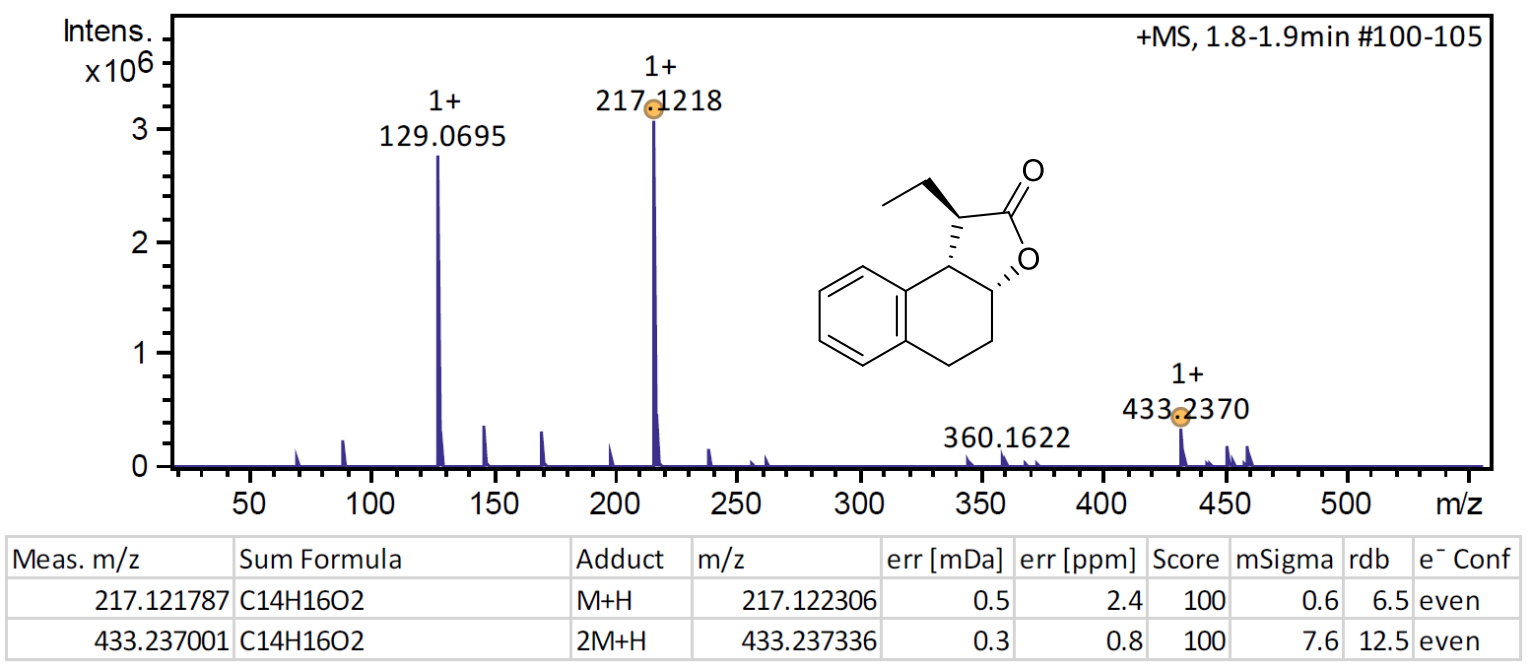


$(1 R, 3 \mathrm{a} R, 9 \mathrm{~b} S)-1-B e n z y l-3 \mathrm{a}, 4,5,9 \mathrm{~b}-$ tetrahydronaphtho[2,1-b]furan-2(1H)-one (15c)

(ESI positive)

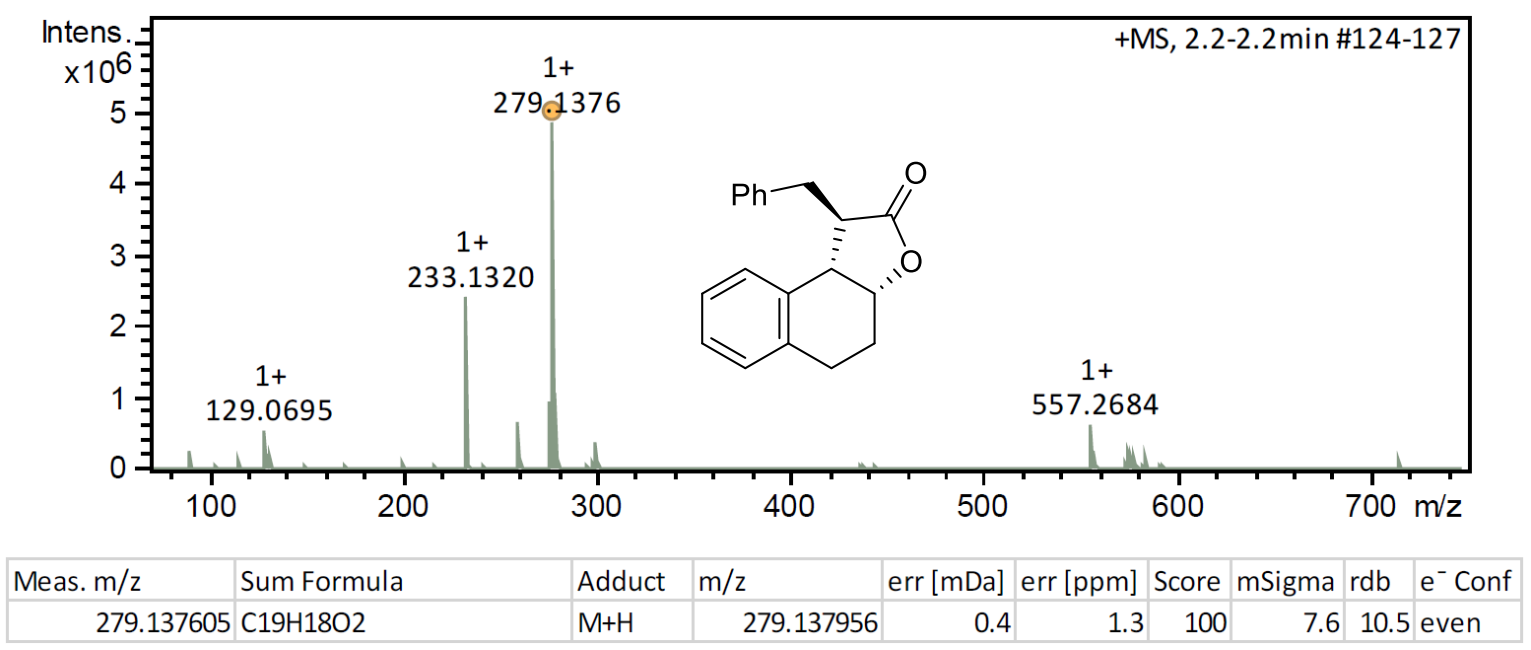

$(1 S, 3 a R, 9 b R)-1-P h e n y l-3 a, 4,5,9 b-t e t r a h y d r o n a p h t h o[2,1-b] f u r a n-2(1 H)-o n e ~(15 d)$

(ESI positive)

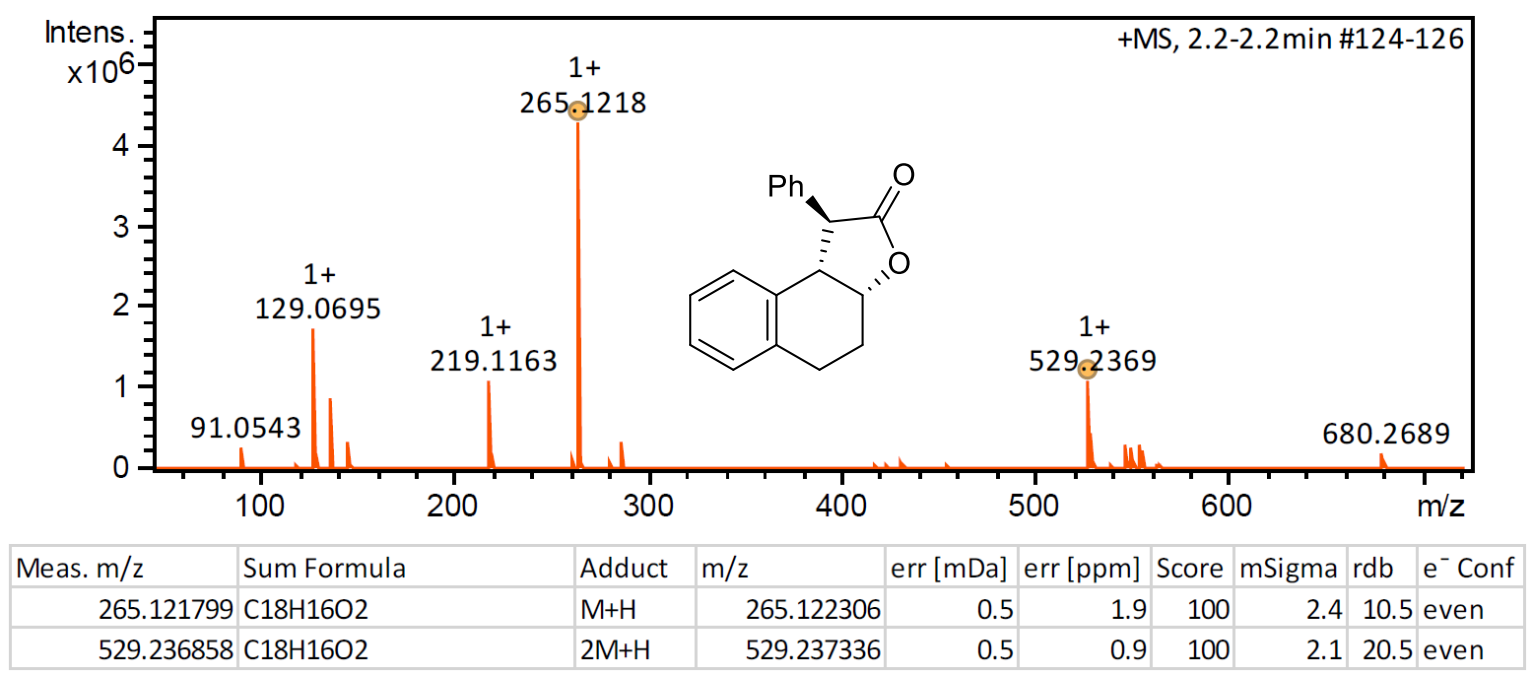


$(1 S, 3 a R, 9 b S)-7-B r o m o-1-p h e n y l-3 a, 4,5,9 b-t e t r a h y d r o n a p h t h o[2,1-b] f u r a n-2(1 H)-o n e$ (15e)

(ESI positive)

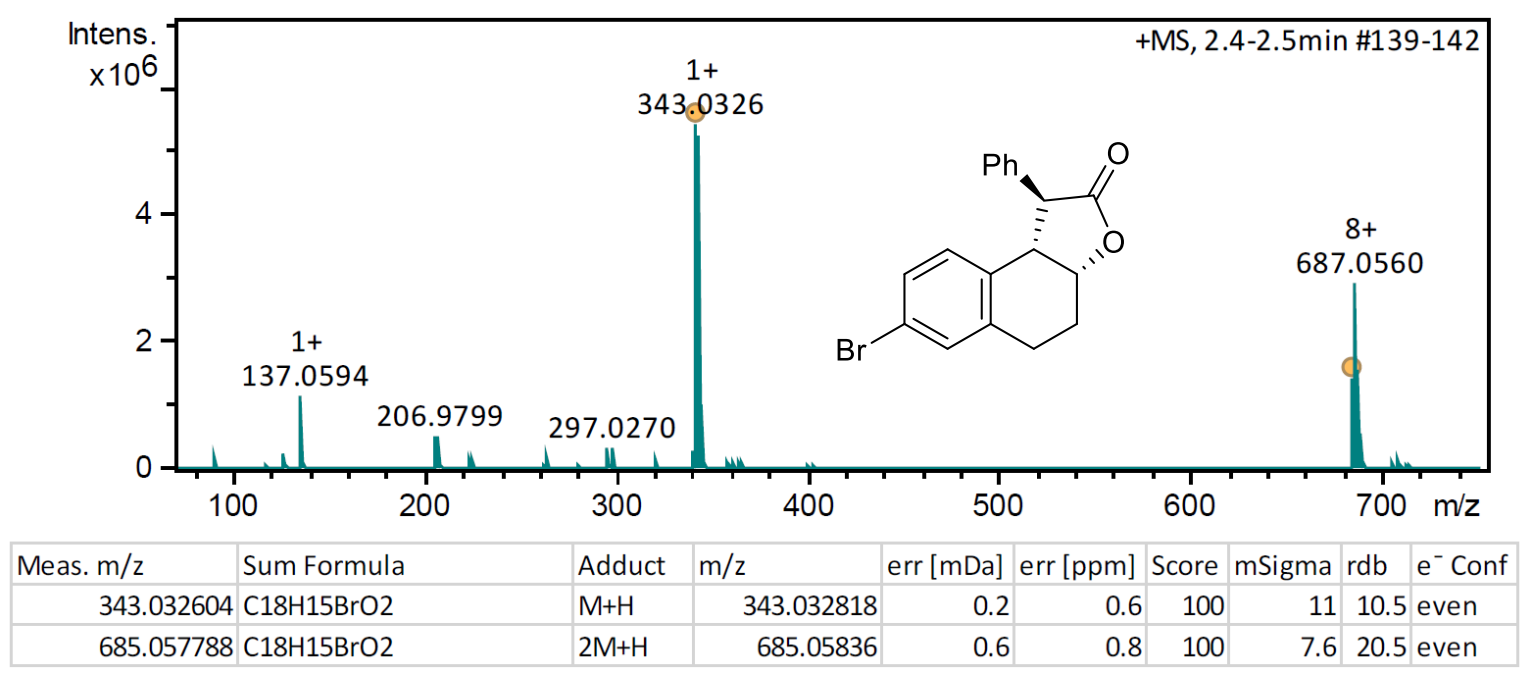

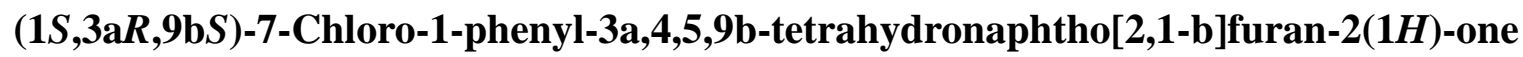
(15f)

(ESI positive)

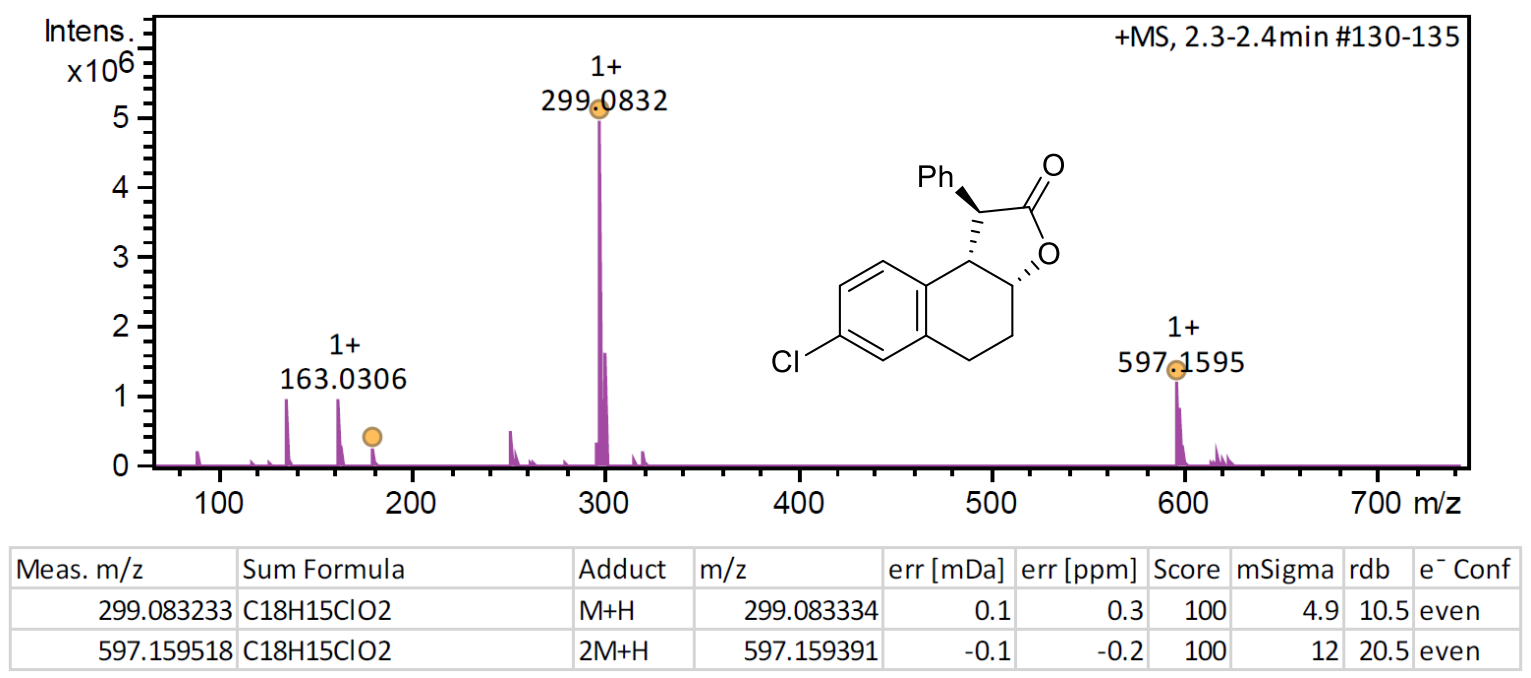


$(1 S, 3 a R, 9 b S)-9-M e t h o x y-1-p h e n y l-3 a, 4,5,9 b-t e t r a h y d r o n a p h t h o[2,1-b]$ furan-2(1H)-on e (15g)

(ESI positive)

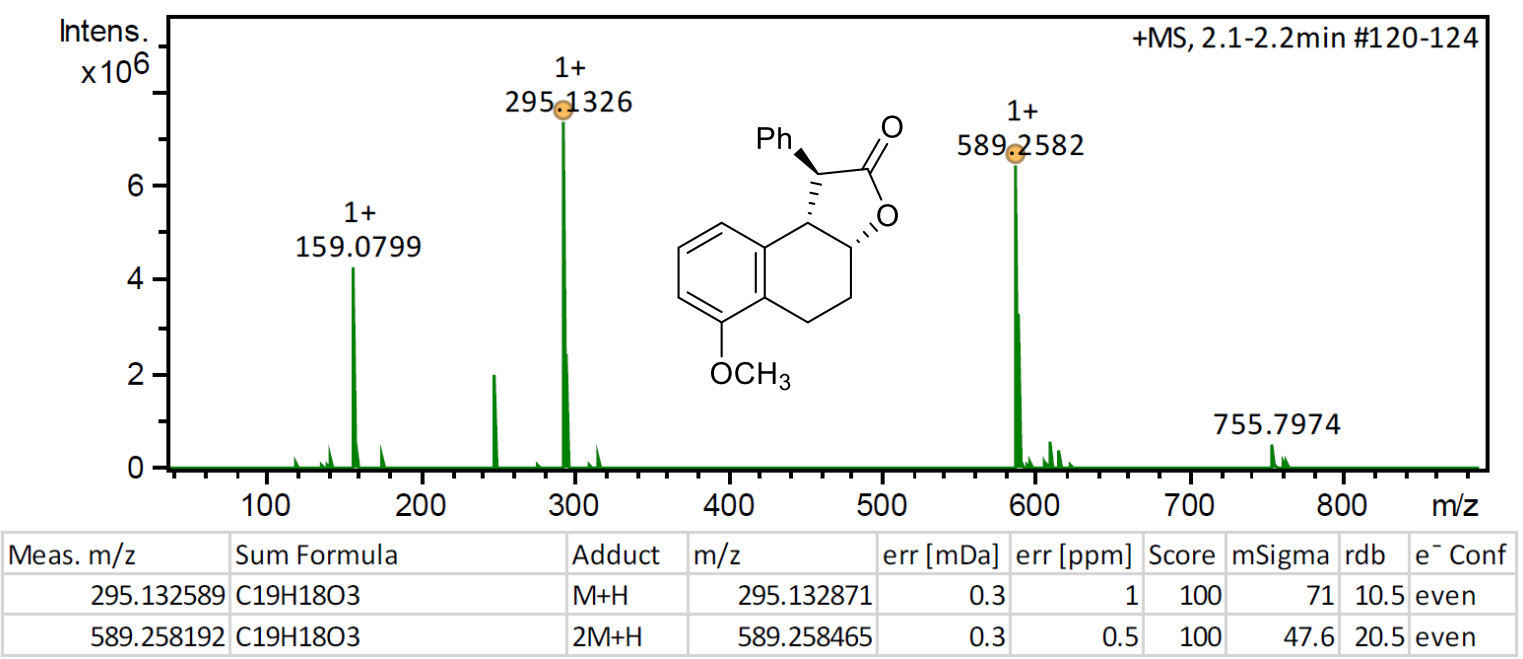

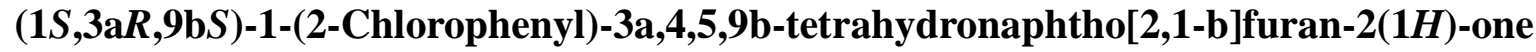
(15h)

(ESI positive)

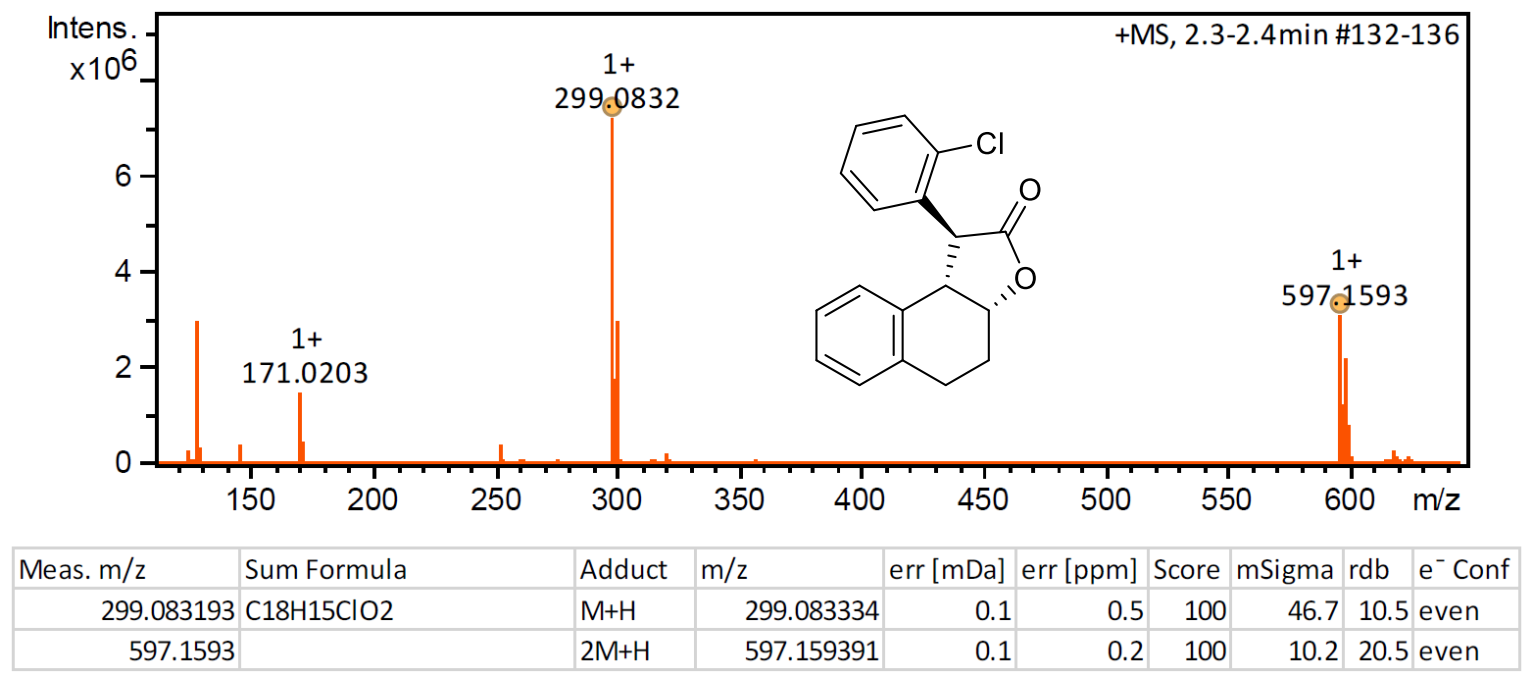


$(1 R, 3 a S, 9 b R)-8-M e t h o x y-1-p h e n y l-3 a, 4,5,9 b-t e t r a h y d r o n a p h t h o[2,1-b]$ furan-2(1H)-on e (7)

(APCI positive)

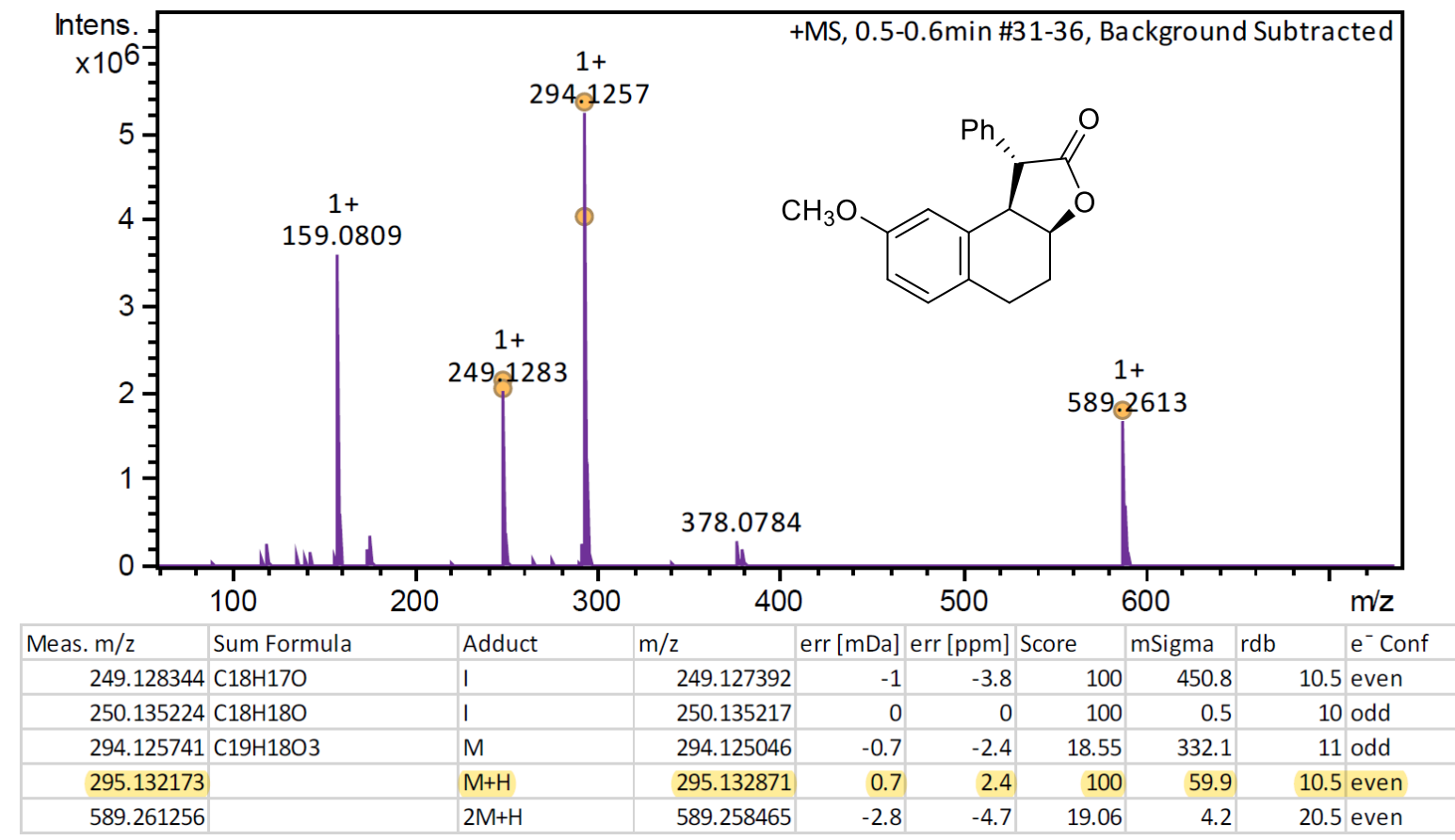

\section{(3R,3aS,9bR)-3-Phenyl-3a,4,5,9b-tetrahydronaphtho[1,2-b]furan-2(3H)-one (18a)}

(APCI positive)

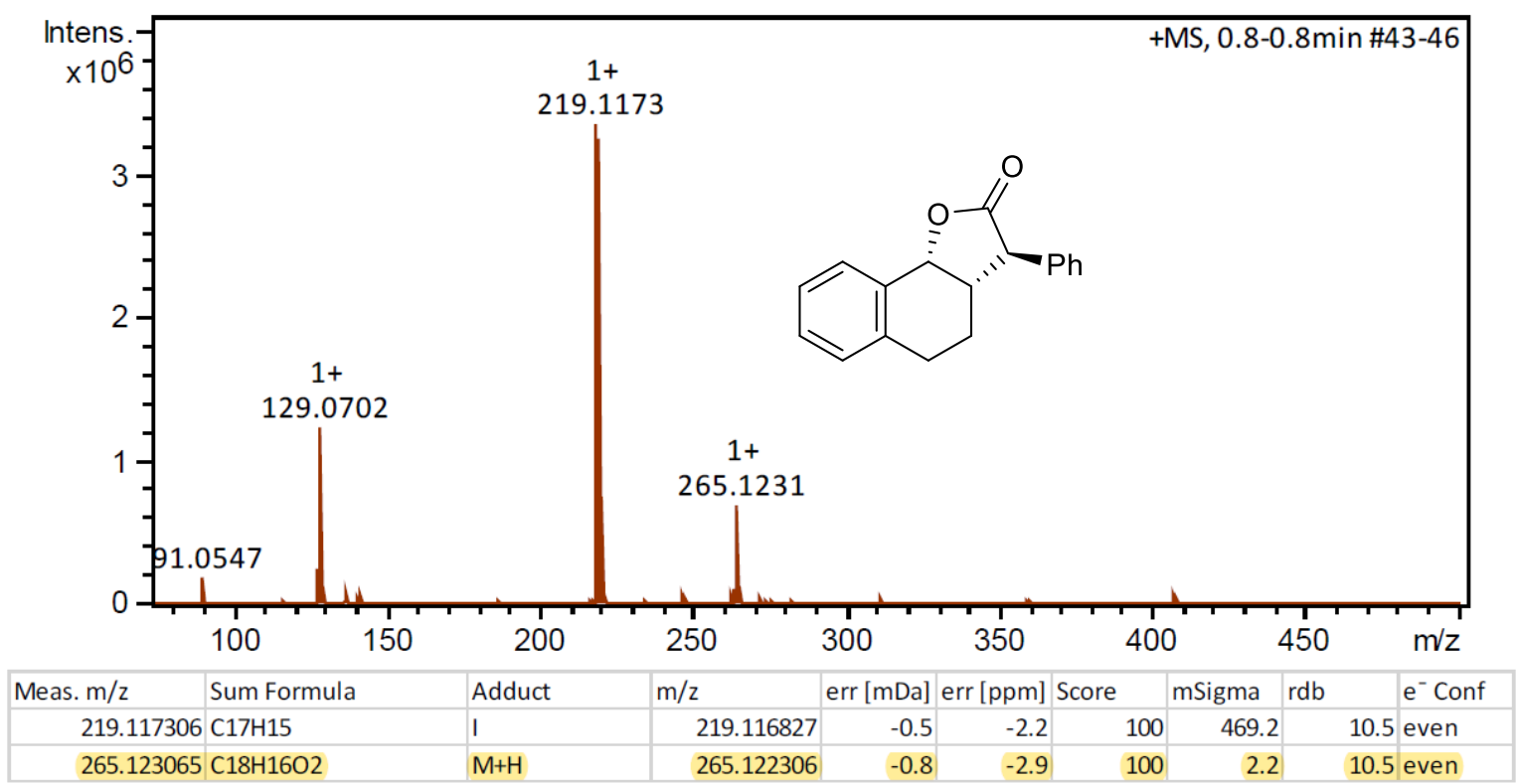


$(3 R, 3 \mathrm{a} S, 9 \mathrm{~b} R)-8$-Chloro-3-phenyl-3a,4,5,9b-tetrahydronaphtho[1,2-b]furan-2(3H)-one (18b)

(APCI positive)

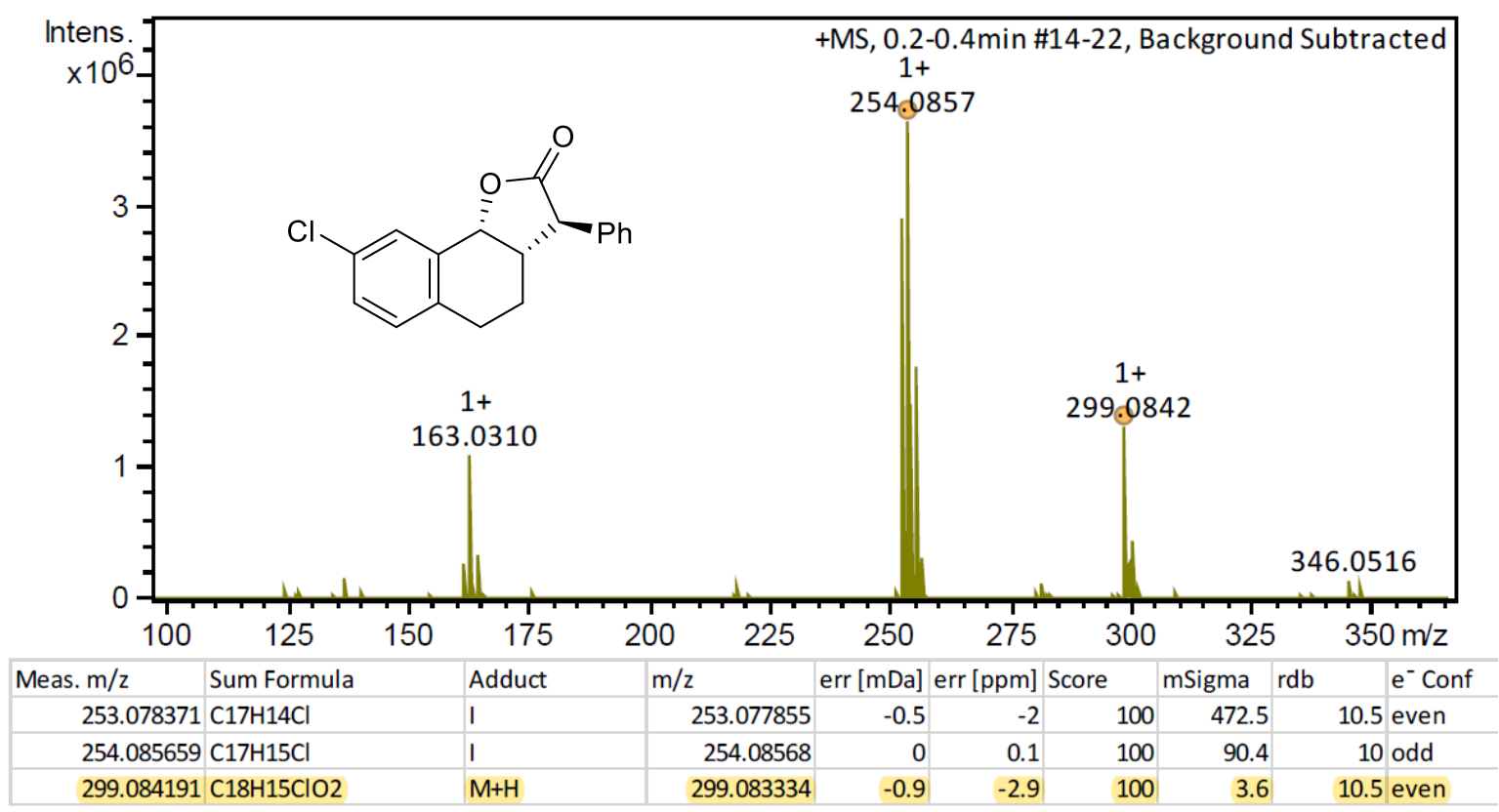

(3aS,9bR)-3-Methylene-3a,4,5,9b-tetrahydronaphtho[1,2-b]furan-2(3H)-one (20)

(APCI positive)

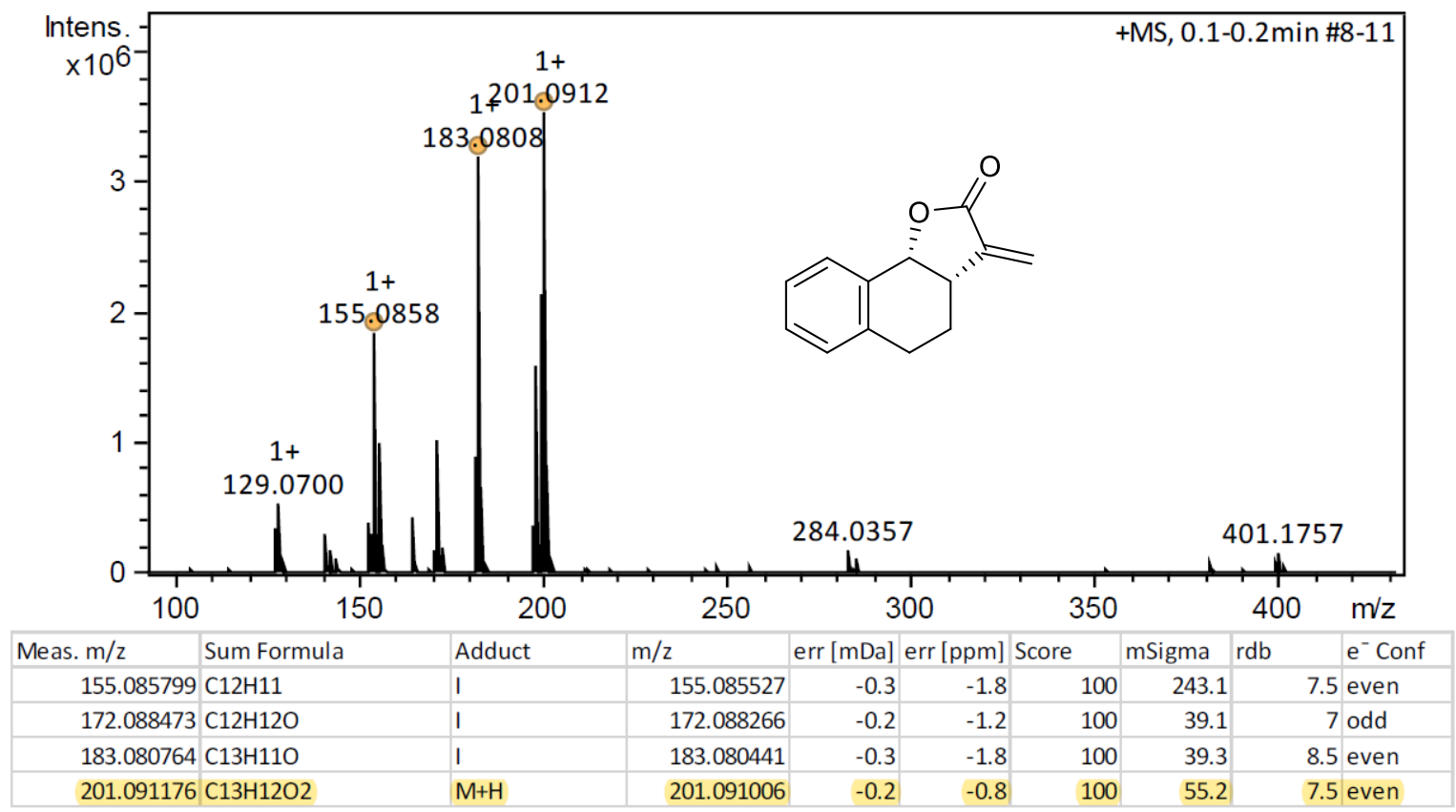


(3S,3aS,9bR)-3-Methyl-3a,4,5,9b-tetrahydronaphtho[1,2-b]furan-2(3H)-one (18c)

(APCI positive)

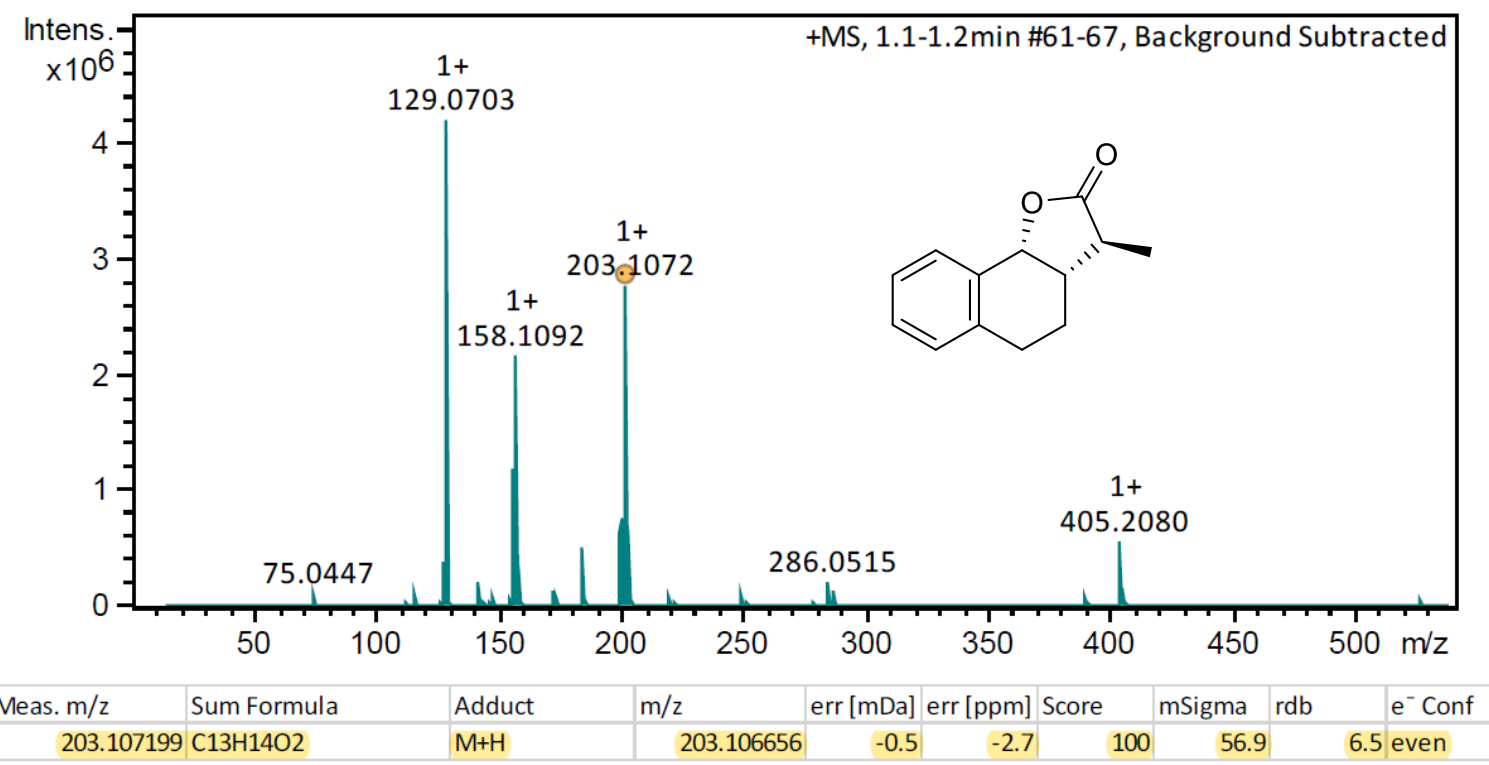

\section{$(3 R, 3 \mathrm{a} S, 9 \mathrm{~b} R)-3-M e t h y l-3 \mathrm{a}, 4,5,9 \mathrm{~b}-$ tetrahydronaphtho[1,2-b]furan-2(3H)-one (19c)}

(APCI positive)

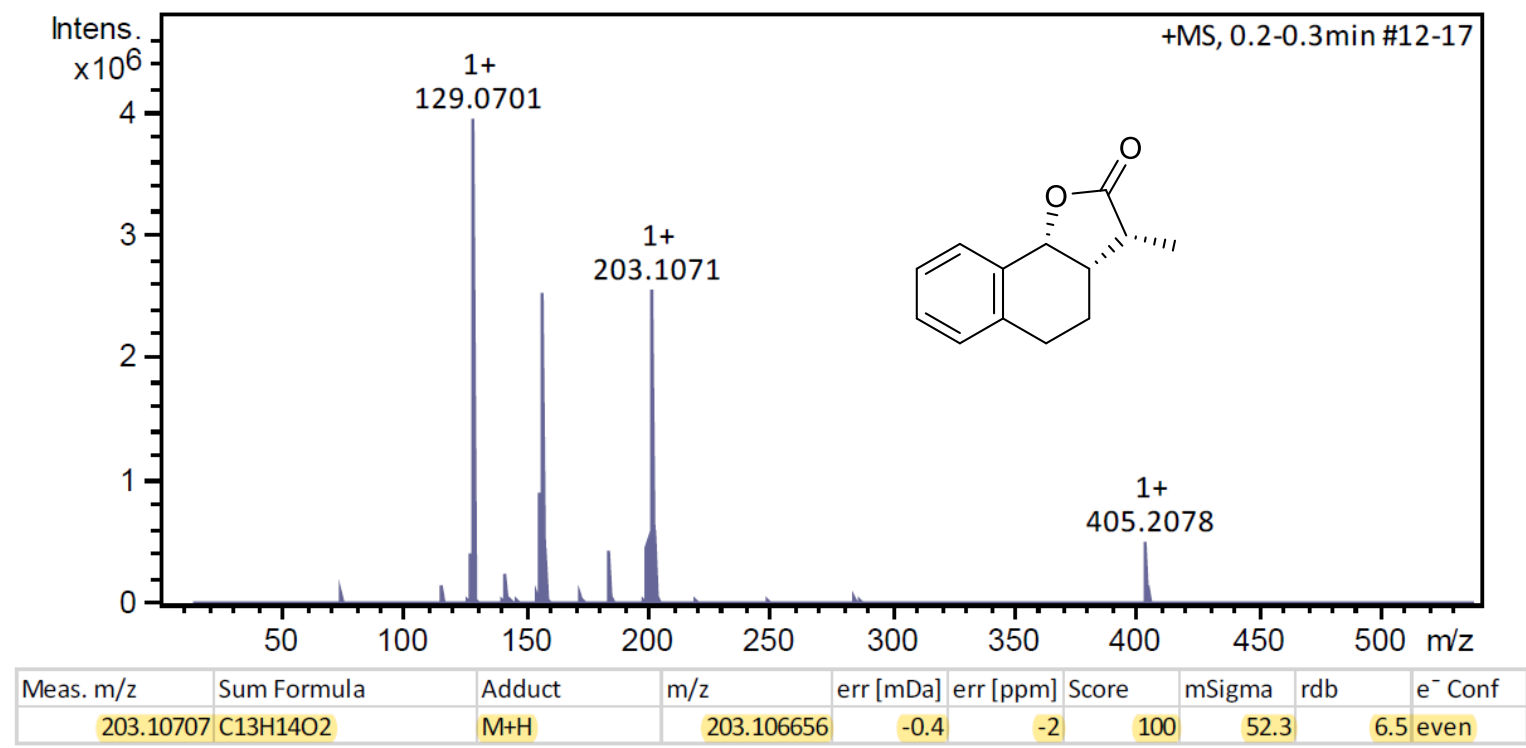




\section{2-(4-Methyl-2-oxocyclohex-3-en-1-yl)acetic acid (23)}

(APCI positive)

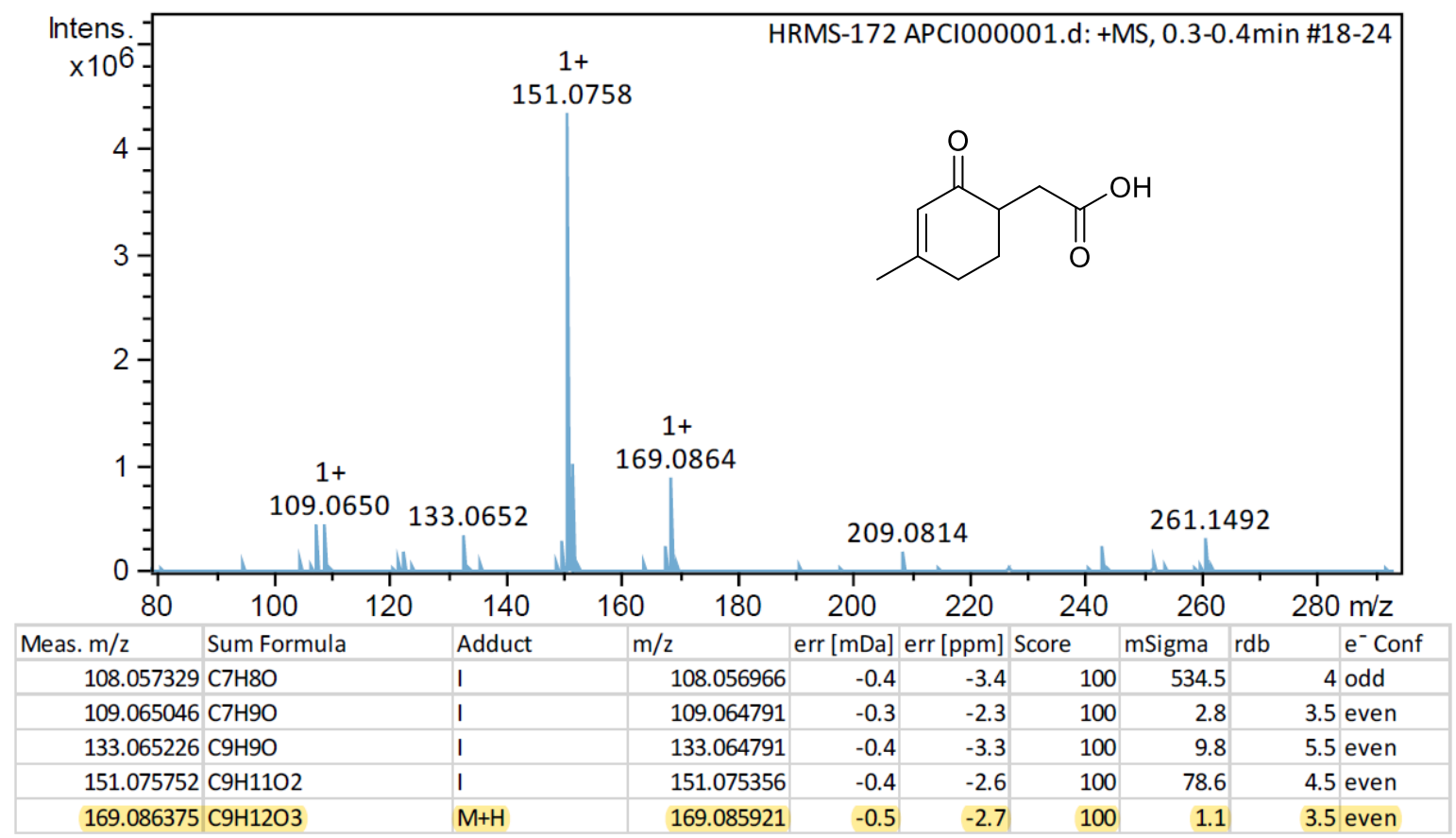

\section{(3aS,7aR)-6-Methyl-3a,4,5,7a-tetrahydrobenzofuran-2(3H)-one (24)}

(APCI positive)

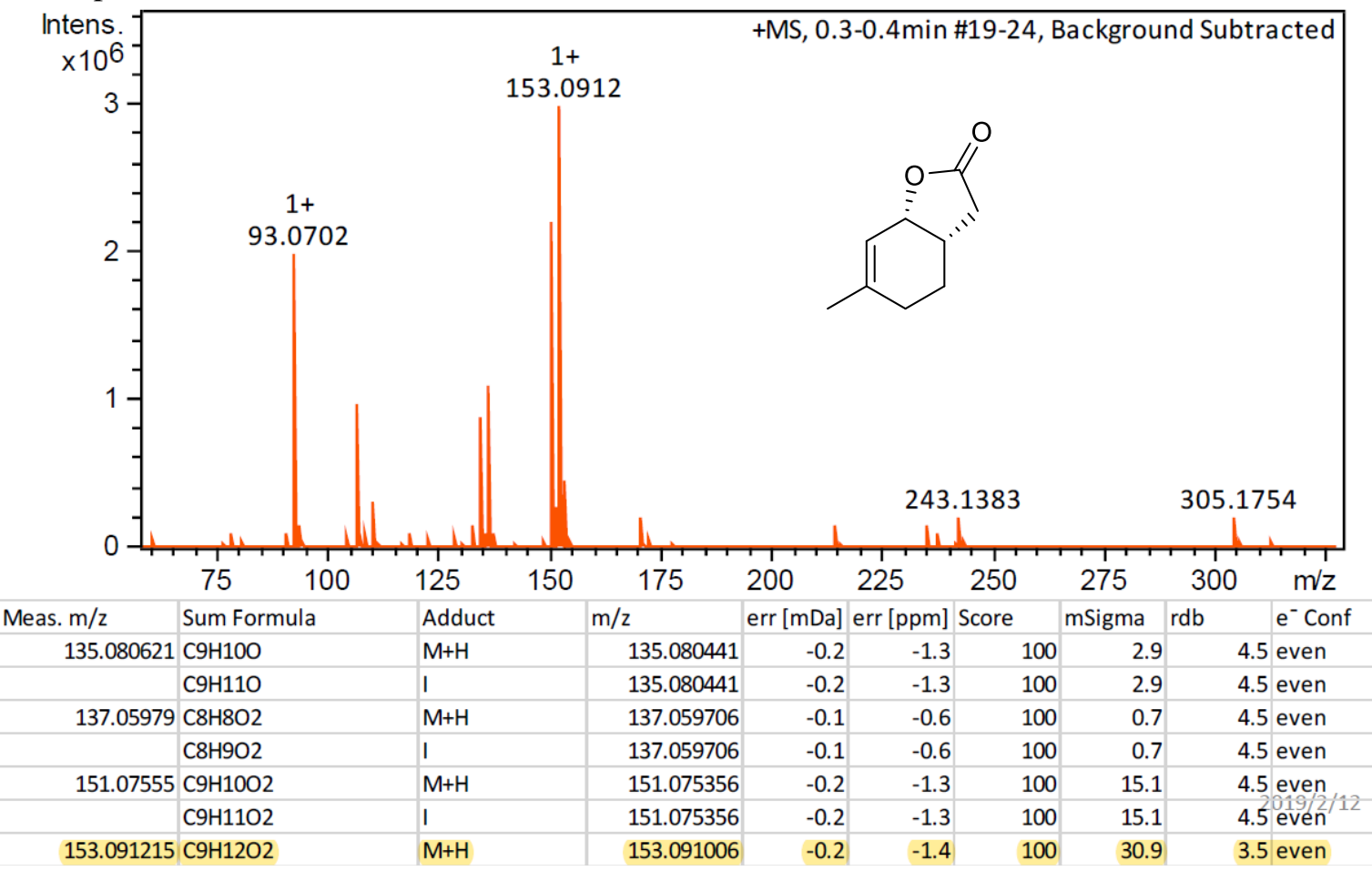


(3aS,7aS)-6-Methyl-3-methylene-3a,4,5,7a-tetrahydrobenzofuran-2(3H)-one (25)

(APCI positive)

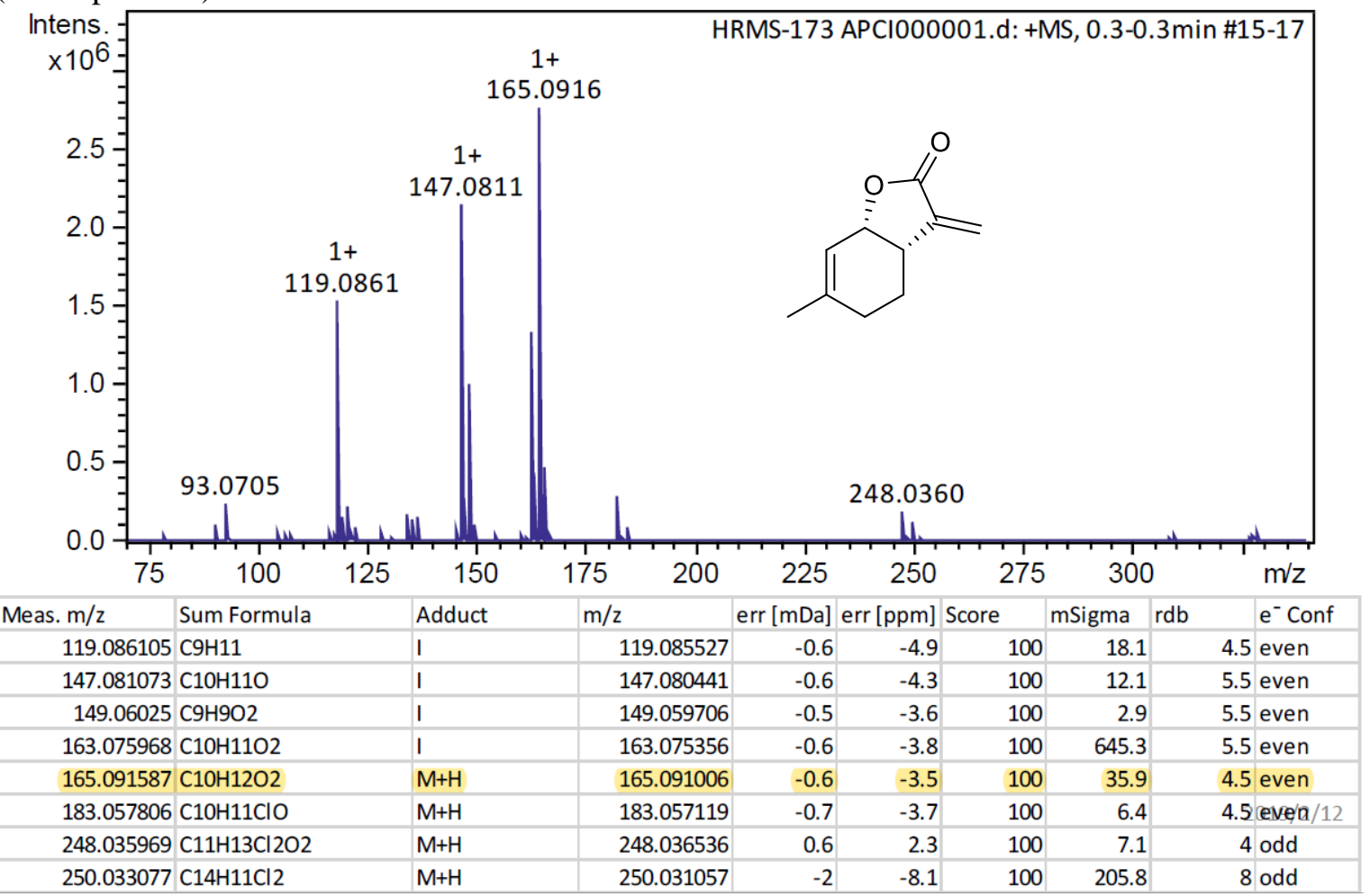

(3S,3aS,7aR)-3,6-Dimethyl-3a,4,5,7a-tetrahydrobenzofuran-2(3H)-one (Wine Lactone) $((3 S)-6)$

(APCI positive)

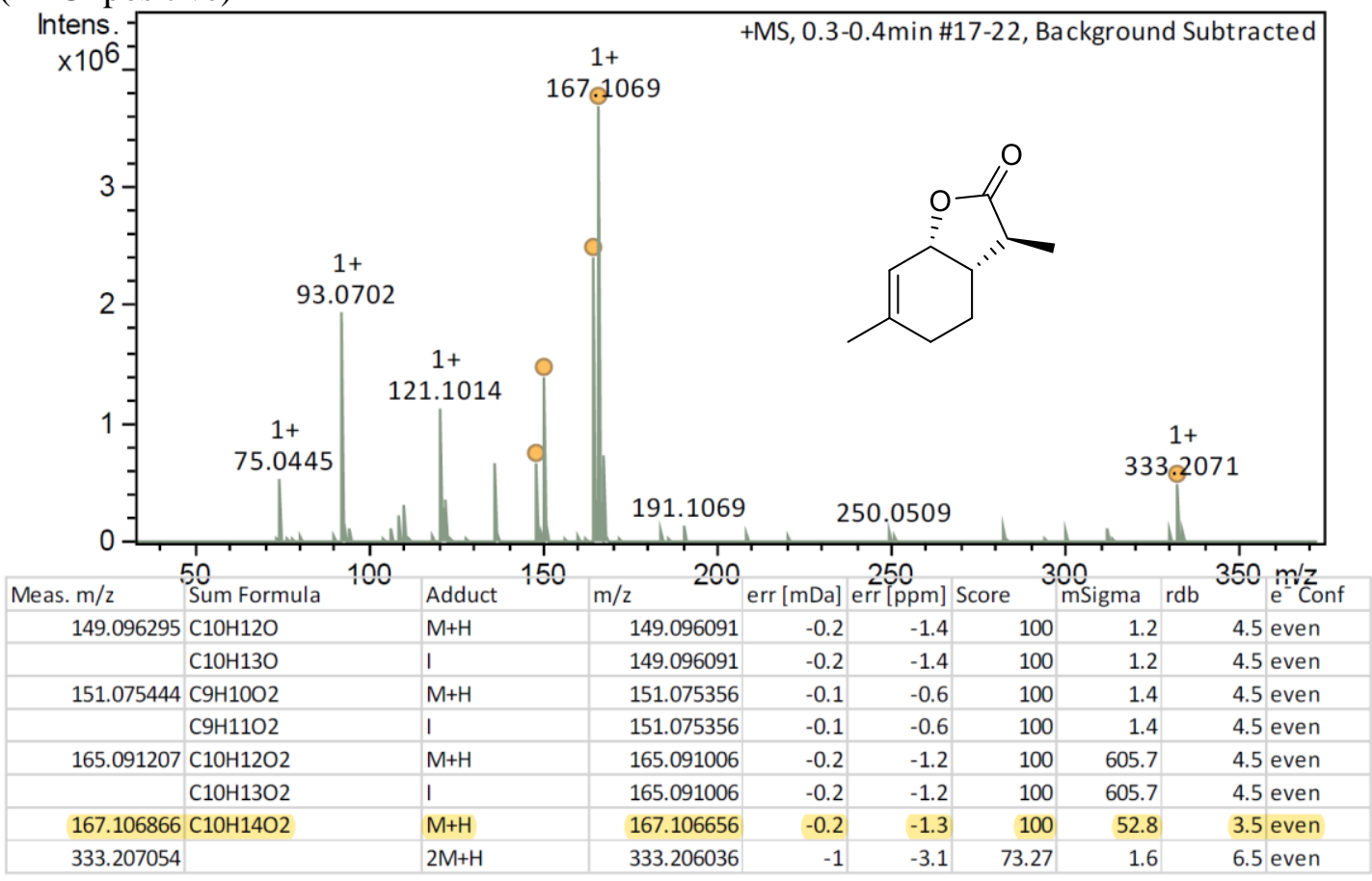


(3R,3aS,7aR)-3,6-Dimethyl-3a,4,5,7a-tetrahydrobenzofuran-2(3H)-one (Diastereomer of Wine Lactone) $((3 R)-6)$

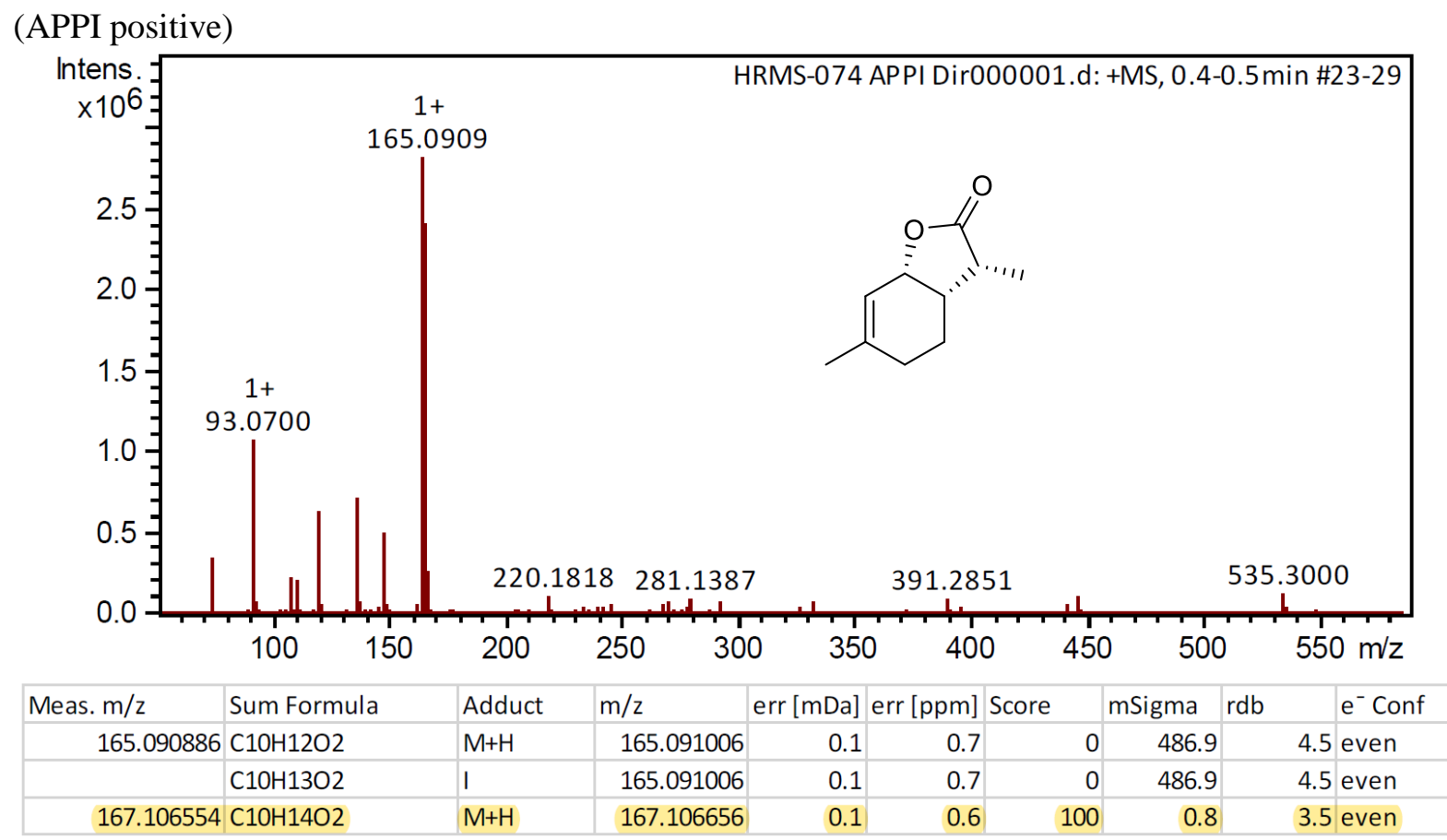

\section{$N$-((1R,2R)-2-Amino-1,2-diphenylethyl)-2,4,6-triisopropylbenzenesulfonamide $((R, R)$-TIPPs-DPEN)}

(APCI positive)

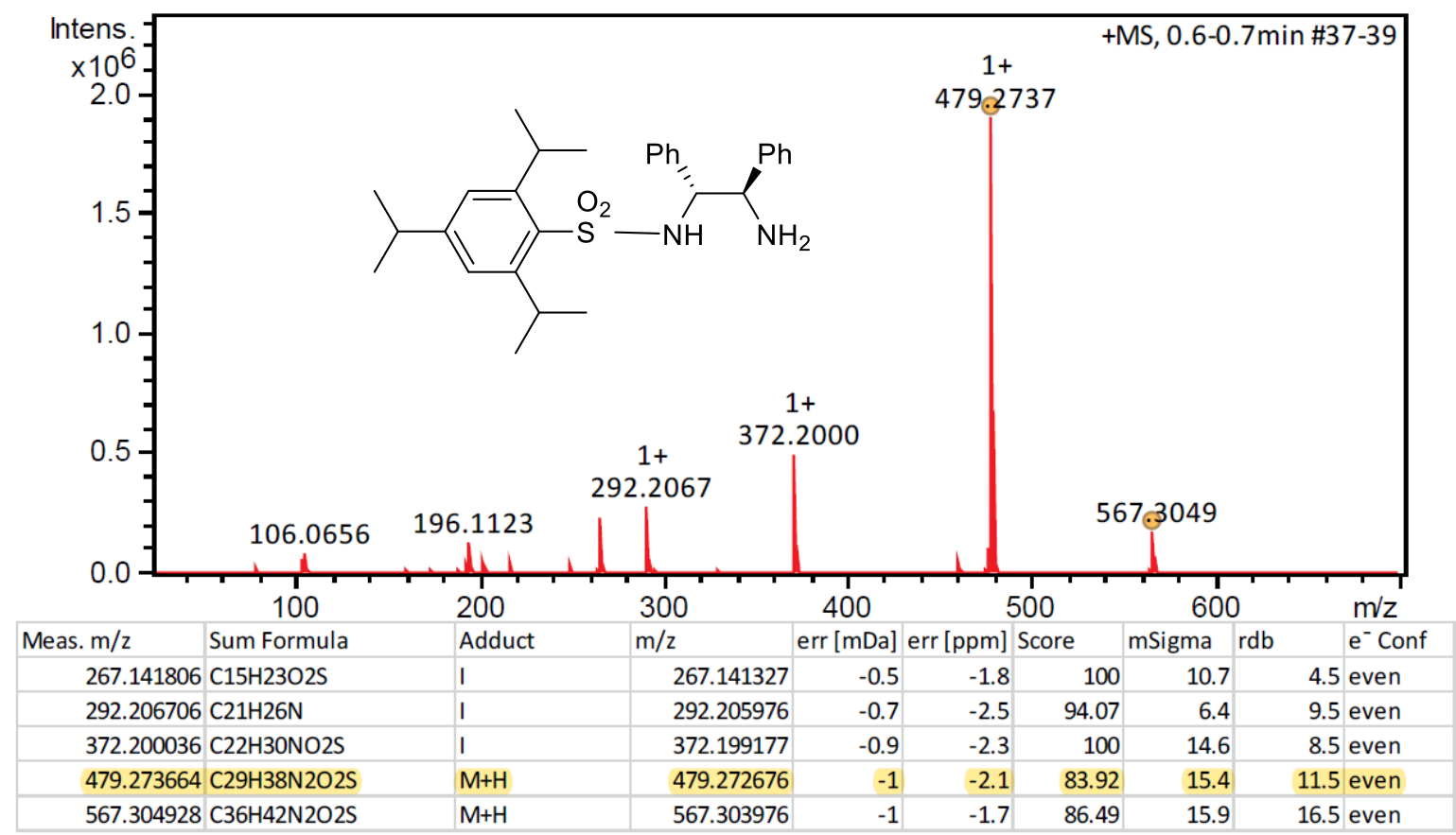


2,4,6-Triisopropyl- $N$-((1R,2R)-2-((2-((4-methylcyclohexa-1,4-dien-1-yl)methoxy)ethyl) amino)-1,2-diphenylethyl)benzenesulfonamide

(APCI positive)

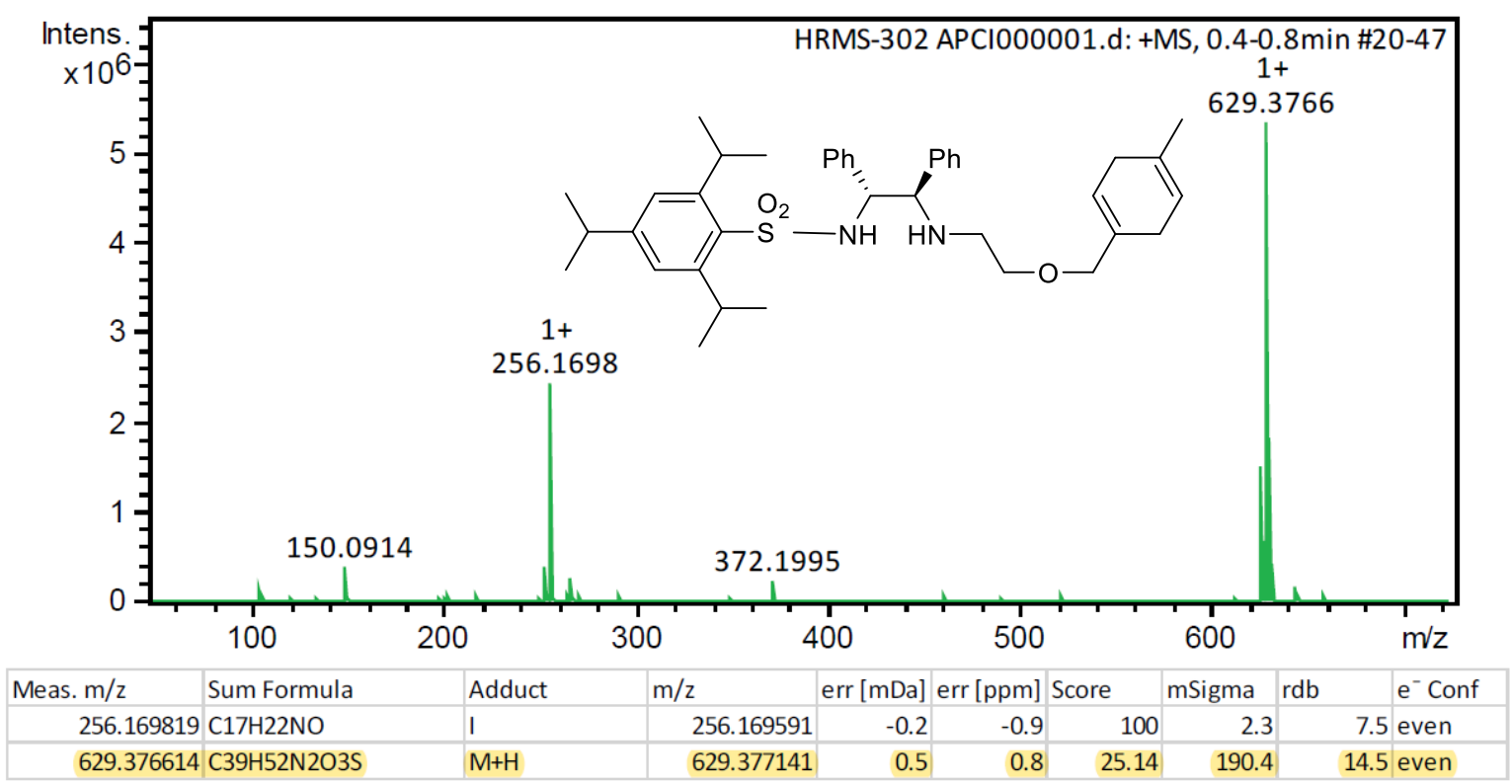

\section{$(R, R)$-TIPPs-DENEB $((R, R)-5)$}

(FD)

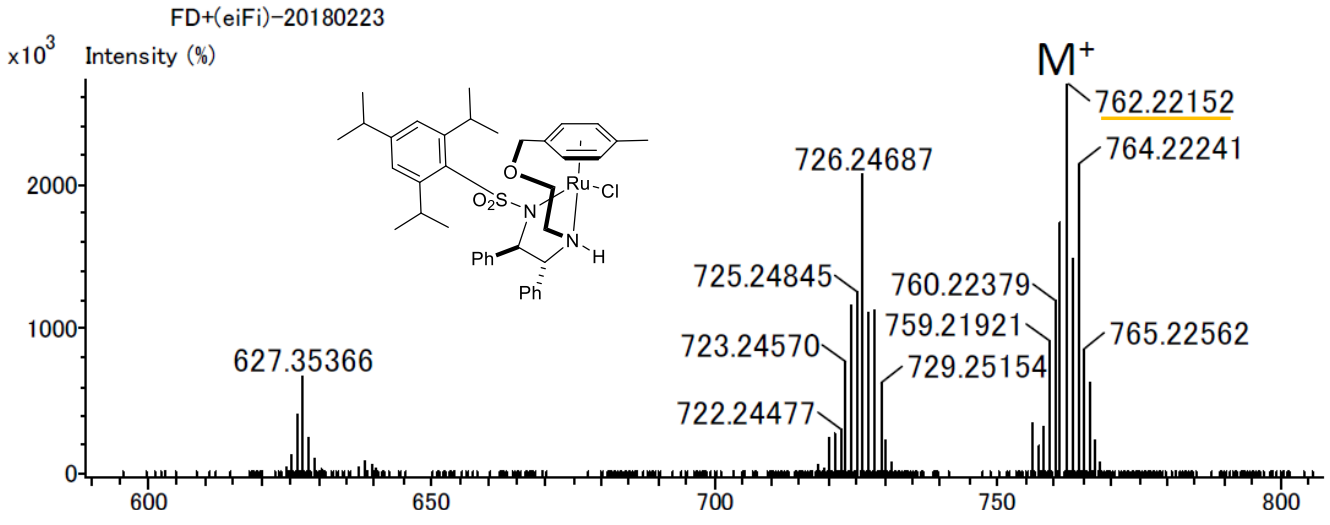

